\title{
US
}

University of Sussex

\section{Measurement of $\Delta m_{32}^{2}$ and $\sin ^{2} \theta_{23}$ using Muon Neutrino and Antineutrino Beams in the NOvA Experiment}

Tristan Blackburn

Submitted for the degree of Doctor of Philosophy

University of Sussex

21st February 2019 


\section{Declaration}

I hereby declare that this thesis has not been and will not be submitted in whole or in part to another University for the award of any other degree. 
UNIVERSITY OF SUSSEX

Tristan Blackburn, Doctor of Philosophy

$\underline{\text { MeAsurement of } \Delta m_{32}^{2} \text { And } \sin ^{2} \theta_{23} \text { In the NOvA Experiment Using }}$

Muon Neutrino And Antineutrino Beams

\begin{abstract}
$\underline{\text { ABSTRACT }}$
NOvA is a long-baseline neutrino oscillation experiment located in the mid-west United States. It consists of two functionally identical tracking calorimeters, known as the near and far detector, that measure neutrino interactions induced by the NuMI beam at baselines of $1 \mathrm{~km}$ and $810 \mathrm{~km}$. NuMI can be configured to provide a muon neutrino or antineutrino beam. Analysis of $\nu_{\mu}+\bar{\nu}_{\mu}$ disappearance allows constraint of the $\left|\Delta m_{32}^{2}\right|$ and $\sin ^{2} \theta_{23}$ oscillation parameters.

This thesis presents the first NOvA disappearance results using both neutrino and antineutrino data - previous NOvA analyses have only used neutrino beam data. Two analysis improvements are delineated in dedicated chapters - the design of selection criteria to identify events that are fully contained in the far detector and the optimization of particle identification selection criteria in a multi-dimensional parameter space.

The full-detector equivalent beam exposures used for this thesis are $8.85 \times 10^{20}$ and $6.91 \times 10^{20}$ protons on target for neutrino and antineutrino data respectively. Under the assumption of a normal (inverted) neutrino mass hierarchy, analysis of the data gives $\Delta m_{32}^{2}=+2.49_{-0.07}^{+0.09} \times 10^{-3} \mathrm{eV}^{2}\left(\Delta m_{32}^{2}=-2.54 \pm 0.08 \times 10^{-3} \mathrm{eV}^{2}\right)$ and $\sin ^{2} \theta_{23}=0.59 \pm 0.03$ $\left(\sin ^{2} \theta_{23}=0.44 \pm 0.03\right)$. Maximal mixing $\left(\sin ^{2} \theta_{23}=0.5\right)$ is disfavoured at the $1.7 \sigma$ level.
\end{abstract}




\section{Acknowledgements}

I am forever indebted to my supervisor Professor Jeff Hartnell who has given me the opportunity to work alongside the Sussex team as well as both the NOvA and DUNE collaborations. Throughout my years at Sussex his sagacious advice, attention to detail and immense patience have been invaluable and I am very thankful to have had such a supportive supervisor.

It has been a pleasure to work alongside the members of both the DUNE and NOvA collaborations. This thesis would not have been possible without the countless hours of work that they have contributed. In particular, I would like to thank fellow Sussex student Diana Méndez for the work she has done for NOvA - I am indebted to her many contributions and exemplary research.

I would also like to thank my examiners Dr Jaroslaw Nowak and Dr Clark Griffith. Their contributions in both time and expertise have been greatly appreciated.

The entire neutrino physics group at Sussex has been a pleasure to work with. Jonathan Davies, Matthew Tamsett, Michael Baird and Bruno Zamorano all offered hours upon hours of advice on top of being great company. I would have had a much harder time without them. My fellow students never failed to make my time at Sussex enjoyable. I would like to express my thanks to Luke Vinton, Diana Méndez, Tyler Alion, Alex Booth, Mark Stringer, Fabio Tresoldi, Fabrizio Miano, Nicola Abraham, Oliver Winston, Emma Zee, James Waterfield, Yusufu Shehu, Zara Grout and Ed Leming.

My time living in Brighton has been full of ups and downs and I am eternally grateful for the friendship of the wonderful people I have lived with - James Moody, Daniel Wigmore, Oliver Winston, Ant Hayes and Tyler Alion. Without each and every one of these people I would have been unable to complete this thesis.

During my time at Fermilab I met many people who have had significant positive impacts on my life. I would like to thank Tyler Alion, Andrew Gasbarro, Kevin Wood, Karl Warburton, Oksana Streltsova, Michael Wallbank, Rob Ainsworth, Diana Méndez, Luke Vinton, Jonathan Davies, Jenny Wallbank, David Pável, Rob Fine, Mônica Nunes, Stefano Tognini, Mateus Carneiro, Xuan Chen, Alex Booth and Nicola McConkey. I have 
spent hundreds of hours in the company of these people and they never cease to make me smile.

Whilst at Fermilab I met my girlfriend, Jessica Burlage, whose support and love has been immeasurable. The last four years of my life have been infinitely brighter with her besides me.

Finally, I would like to thank my family Phil Blackburn, Blandina Blackburn and Verity Blackburn. They have all provided constant support over the last four years for the small price of partial sanity. I love them dearly and am very appreciative of all they have done for me. 


\section{Preface}

The structure of this thesis, the general content of each chapter and the authors original contributions, are outlined here.

Chapter 1 serves as an introduction, outlining the thesis and its motive.

Chapter 2 contains a description of the underlying theory relevant to the content of this thesis and a review of current experimental results. This chapter has been written using many sources.

Chapter 3 describes the NOvA experiment. This chapter has been written using many sources and the author's personal knowledge.

Chapter 4 delineates the analysis methodology used to produce the results presented in this thesis. The detector hardware, computing infrastructure, reconstruction software and systematic uncertainty are all described. This chapter has been written using many sources and comprises work from many NOvA collaborators.

Chapter 5 describes a body of work performed by the author to redesign and optimize the selection criteria used to identify well contained events in the far detector. The new containment criteria were officially adopted by the NOvA collaboration and used for the results presented at the 'Neutrino 2018' conference (soon to be published) as well as in the published neutrino-only 2018 analyses.

Chapter 6 describes a body of work performed by the author to design and implement an optimization technique to maximize analysis sensitivity. The optimization was performed on three NOvA proprietary particle identification algorithms to identify selection boundaries. The determined selection boundaries were officially adopted by the NOvA collaboration and used in the results shown at the 'Neutrino 2018' conference.

Chapter 7 presents the NOvA $\nu_{\mu}+\bar{\nu}_{\mu}$ disappearance results - part of the official body of NOvA results produced from the joint $\nu_{e}\left(\bar{\nu}_{e}\right)$ appearance $+\nu_{\mu}\left(\bar{\nu}_{\mu}\right)$ disappearance analysis. The results are the product of many people's work, with the author's most significant personal contributions described in chapters 5 and 6 . The NOvA CAFAna fitting and analysis framework was used for all plots and results shown in this chapter and was first extended to divide the antineutrino data into quantiles by the author. The production of the intermediate systematics files was principally performed by Diana Mendez with input from the wider collaboration.

Chapter 8 summarises and concludes the thesis. 


\section{Contents}

1 Introduction $\quad 1$

2 Neutrino Physics 3

2.1 Discovery of the Neutrino . . . . . . . . . . . . . . 3

2.2 The Neutrino Model . . . . . . . . . . . . . . . . . . . . . . . . . . 4

2.2.1 The Solar Neutrino Problem, Super-Kamiokande and SNO . . . . . 6

2.2.2 Neutrino Interactions, Oscillations and the PMNS matrix . . . . . 9

2.2.3 Neutrino Oscillation Probability in Vacuum . . . . . . . . . . . . 10

2.3 Neutrino Masses and Hierarchies . . . . . . . . . . . . . . . . . . . . . 12

$2.4 \mathrm{CP}$ and T Violation in Neutrino Oscillations . . . . . . . . . . . . . . . 12

2.4 .1 The Matter Effect . . . . . . . . . . . . . . . . . 13

2.5 Neutrino Oscillation Experiments . . . . . . . . . . . . . . . . . . 14

2.6 Current Measurements of PMNS Matrix Parameters . . . . . . . . . . . . . 15

2.6.1 Measurement of $\theta_{12}$ and $\Delta m_{21}^{2} \ldots \ldots \ldots \ldots \ldots$

2.6.2 Measurement of $\theta_{13} \ldots \ldots \ldots \ldots \ldots$

2.6.3 Measurement of $\theta_{23}$ and $\left|\Delta m_{32}^{2}\right| \ldots \ldots \ldots \ldots$

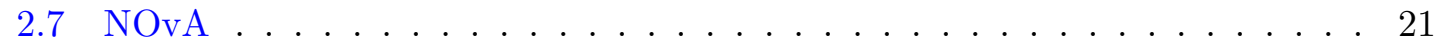

2.7 .1 Neutrino Interactions in NOvA . . . . . . . . . . . . . . 22

2.7.2 Background Sources . . . . . . . . . . . . . . . . 23

2.8 Beyond the Standard Model Majorana particles and The Seesaw Mechanism 29

2.9 Summary of Current Results . . . . . . . . . . . . . . . . . . 30

$3 \quad$ The NOvA Experiment 32

3.1 The NuMi Beam . . . . . . . . . . . . . . . . . . . . . . 33 
3.1 .1 The Magnetic Focussing Horns . . . . . . . . . . . . . . . . . 33

3.1 .2 Off-axis Design . . . . . . . . . . . . . . . 34

3.2 The NOvA Detectors . . . . . . . . . . . . . . . . . . 36

3.2 .1 Experimental Design . . . . . . . . . . . . . . 36

3.2 .2 The NOvA Near Detector . . . . . . . . . . . . . . . 43

3.2.3 The NOvA Far Detector . . . . . . . . . . . . . . . . . . . 44

4 NOvA Analysis Methodology and Systematic Uncertainties $\quad 47$

4.1 Monte Carlo Simulation . . . . . . . . . . . . . . . . . . . . 47

4.1 .1 Beam Flux Corrections . . . . . . . . . . . . . . . . . 49

4.1.2 Neutrino Interaction Modelling and Cross-Section Corrections . . . . 50

4.2 Event Reconstruction . . . . . . . . . . . . . . . . . . . 53

4.3 Selection and Background . . . . . . . . . . . . . 56

4.4 Analysis Software . . . . . . . . . . . . . . . . 63

4.5 Near to Far Detector Extrapolation . . . . . . . . . . . . . . . . . . . . 63

4.6 Constraints and Confidence Limit Contours . . . . . . . . . . . . . . . 64

4.7 Energy Resolution Binning . . . . . . . . . . . . . . . . 65

4.8 Systematic Uncertainties . . . . . . . . . . . . . . . . . . . . 69

4.8.1 Muonic and Hadronic Energy Estimation . . . . . . . . . . . . . 69

4.8 Cross sections and FSI . . . . . . . . . . . . 70

4.8 .3 Normalization. . . . . . . . . . . . . . . . 70

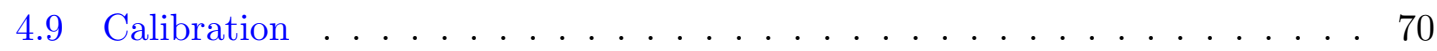

5 Containment Analysis Improvements $\quad 73$

5.1 Previous Analysis FD Containment Cuts . . . . . . . . . . . . . . . . . 74

5.2 Optimization Methodology . . . . . . . . . . . . . . 75

5.3 Containment Distributions and Selection Optimization . . . . . . . . . 76

5.3.1 Re-Optimization of Containment Variables Introduced in Previous Analyses . . . . . . . . . . . . . . . 76

5.3.2 Optimization of Containment Variables Introduced in 2018 . . . . . 81

5.4 Results of Optimization . . . . . . . . . . . . . . . 85

5.4 .1 Validation of FD Containment . . . . . . . . . . . 86

5.5 Cosmic Pile-Up . . . . . . . . . . . . . . . . . . . . . . 87

5.6 Conclusion . . . . . . . . . . . . . . . . . . . . . . . 93 
6 PID Selection Analysis Improvements

6.1 PID Selection Boundary Optimization Methodology . . . . . . . . . . . 95

6.1.1 Quantile Boundaries and Pre-Selection . . . . . . . . . . . . 95

6.1.2 Event Distributions and Oscillation Weights . . . . . . . . . 96

6.1.3 Assessed Selection Boundary Definitions . . . . . . . . . . . . . . 101

6.1.4 Tuning PID Cut Values . . . . . . . . . . . . . . . . . . . 102

6.2 Results of Optimization . . . . . . . . . . . . . . . . 106

6.2.1 Previous PID Selection Performance . . . . . . . . . . . . . 106

6.2 .2 Simple Optimization . . . . . . . . . . . . . . 107

6.2.3 Per Hadronic-Energy Quantile Optimization . . . . . . . . . . . . 110

6.3 Validation of Optimization . . . . . . . . . . . . . . . . 113

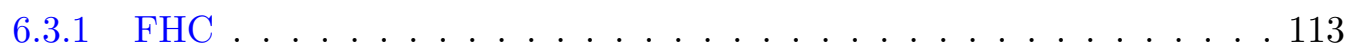

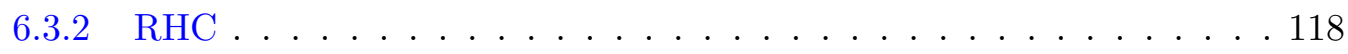

6.4 Conclusion . . . . . . . . . . . . . . . . . . . . 123

$\begin{array}{llr}7 & \text { Results } & \mathbf{1 2 4}\end{array}$

7.1 Near Detector Distributions . . . . . . . . . . . . . . . . . 125

7.2 Far Detector Data . . . . . . . . . . . . . . . . . . . 159

$7.2 .1 \quad$ Far Detector Distributions . . . . . . . . . . . . . . . 169

7.2.2 Fitting the Far Detector Neutrino Energy Spectrum . . . . . . . . . 201

7.2.3 The Matter Effect \& Combined Octant-Hierarchy Sensitivity . . . . 203

7.3 Systematic Uncertainty Analysis . . . . . . . . . . . . . . . . . 206

7.4 Future Prospects . . . . . . . . . . . . . . . . . . . . . . . . 220

8 Conclusion $\quad 222$

$\begin{array}{lr}\text { Appendices } & \mathbf{2 2 4}\end{array}$

A Containment and Pile-Up From Cosmics 225

A.0.1 2017 Analysis Datasets . . . . . . . . . . . . . 225

A.0.2 2018 Analysis Datasets . . . . . . . . . . . . . . . . 231

B List of Software Used for the PID Cut Optimization $\quad \mathbf{2 4 0}$

$\begin{array}{ll}\text { Bibliography } & 242\end{array}$ 


\section{List of Tables}

$2.11 \sigma$ confidence intervals for physics parameters in the normal mass hierarchy. 25

2.2 Three-flavour oscillation parameters from fit to global data. The results are presented where reactor fluxes have been left free in the fit and short baseline reactor data with $L \lesssim 100 \mathrm{~m}$ are included. The numbers in the 1 st (2nd) column are obtained assuming normal hierarchy (inverse hierarchy). The 3rd column is the best fit across both hierarchies. Table taken from [1],

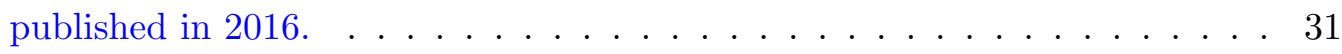

3.1 NOvA liquid scintillator chemical composition $[2] . \ldots \ldots \ldots$. . . . . . 41

4.1 Number of neutrino energy bins used for different energy regimes. The predicted number of ND neutrino events in each bin category for FHC and RHC are shown, as well as the associated estimated background, in the last four columns. FHC data is scaled to $8.03 \times 10^{20} \mathrm{POT}$ and RHC data is scaled to $3.10 \times 10^{20}$ POT. . . . . . . . . . . . . . . . 66

5.12017 analysis containment cuts, employed by previous analyses. . . . . . . . 74

5.2 Summary table of the 2018 analysis FD containment cuts. Both the reoptimized 2017 cuts and the new prong-based criteria are described. . . . . 85

5.3 RMS and means for 2017 and 2018 (reconstructed ${ }_{\nu_{\mu} E}-$ true $_{\nu_{\mu} E}$ )/true $\operatorname{tr}_{\nu_{\mu} E}$ $\nu_{\mu}$ energy distributions. . . . . . . . . . . . 8

5.4 Table showing the $\frac{\text { cosmic overlay MC }}{\text { single event MC }}$ ratio of signal event counts when using the 2017 and 2018 containment criteria. The use of the 2018 containment criteria universally reduces the effect of pile-up in all data periods. . . . . . 93 
6.1 Breakdown of the evaluated PID boundaries. The minimum value, maximum value and step size in each score is shown. All possible permutations of the boundaries are assessed in the optimization. The total number of steps for each score is also shown. . . . . . . . . . . . . . . . . 101

6.2 Summary of the 2018 per quantile PID selection boundaries. The FHC and RHC bounds are shown on the left and right respectively. . . . . . . . . . 103

6.3 Summary of the 2018 simple PID selection boundaries. Hand tuned to maximally reduce reliance on the BDT. . . . . . . . . . . . . . 106

6.4 Breakdown of signal and background events for the FHC analysis using the previous PID selection criteria and NOvA's $8.85 \times 10^{20}$ POT FHC-only best

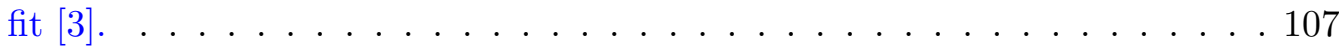

6.5 Breakdown of signal and background events for the RHC analysis using the previous PID selection criteria and NOvA's $8.85 \times 10^{20}$ POT FHC-only best fit $[3] \ldots \ldots \ldots \ldots \ldots \ldots$

6.6 FHC breakdown of signal and background events when applying the simple cut optimization and a comparison with the previous analysis results shown in Table 6.4. . . . . . . . . . . . . . . . . . . . . 108

6.7 RHC breakdown of signal and background events when applying the simple cut optimization and a comparison with the previous analysis results shown in Table 6.5. . . . . . . . . . . . . . . . . . . . . . 109

6.8 FHC breakdown of signal and background events when applying the per hadronic energy fraction cut optimization to FHC and a comparison with the previous analysis results as shown in Table 6.4 . . . . . . . . . . . . 111

6.9 RHC breakdown of signal and background events when applying the per hadronic energy fraction cut optimization and a comparison with the previous analysis results as shown in Table $6.5 \ldots$. . . . . . . . . . . . . 112

7.1 NOvA ND FHC simulated $\nu_{\mu}+\bar{\nu}_{\mu}$ CC candidate event interaction types. Numbers scaled to data POT. . . . . . . . . . . . . . . . . . . . . . 128

7.2 NOvA ND RHC simulated $\nu_{\mu}+\bar{\nu}_{\mu} \mathrm{CC}$ candidate event interaction types. Numbers scaled to data POT. . . . . . . . . . . . . . . . . . . . 129

7.3 ND $\nu_{\mu}+\bar{\nu}_{\mu} \mathrm{CC}$ candidate events and share of total events within each quantile in data and MC. FHC is shown on the top, and RHC on the bottom. . . . . . . . . . . . . . . . . . . . . 130 
7.4 MC oscillation channel breakdown for the total FHC dataset and for the total RHC dataset. The $20188.85 \times 10^{20}$ POT FHC-only analysis best fit is used. . . . . . . . . . . . . . . . . . . . . 160

7.5 FHC MC oscillation channel breakdown for each $E_{h a d} / E_{\nu}$ quantile under the assumption the $20188.85 \times 10^{20}$ POT FHC-only analysis best fit. . . . 161

7.6 RHC MC oscillation channel breakdown for each $E_{h a d} / E_{\nu}$ quantile under the assumption the $20188.85 \times 10^{20}$ POT FHC-only analysis best fit. . . . 162

$7.7 \nu_{\mu}+\bar{\nu}_{\mu}$-CC candidate events in each $E_{h a d} / E_{\nu}$ quantile in data and MC. FHC is shown in the top table, RHC in the bottom. The predicted events are calculated assuming neutrino oscillations at NOvA's $20188.85 \times 10^{20} \mathrm{POT}$ FHC-only [3] best fit point. The predicted events include both the beam and the cosmic ray background. . . . . . . . . . . . . . . 164

7.8 Breakdown of systematic and statistical uncertainty contributions on the values of $\sin ^{2} \theta_{23}$ and $\Delta m_{32}^{2}$ as measured by the $\nu_{\mu}+\bar{\nu}_{\mu}$ disappearrance analysis. . . . . . . . . . . . . . . . . . . . 206

7.9 Ten most significant systematic pulls on the FHC fit (left), and RHC fit (right), ordered by absolute size of the pull. . . . . . . . . . . . 209

7.10 Top ten systematic pulls in the combined FHC+RHC fit ordered by absolute size of the pull. The individual RHC and FHC pulls are restated for comparison. . . . . . . . . . . . . . . . . . . . . 210 


\section{List of Figures}

2.1 Theoretical muon to neutrino Feynman diagram assuming non-distinct neut-

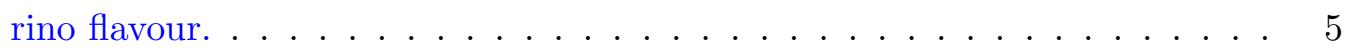

2.2 Feynman diagrams of weak force interactions with neutrinos. . . . . . . 8

2.3 Diagram of the two possible mass hierarchies. . . . . . . . . . . . . . . 12

2.4 Feynman diagrams showing the $\mathrm{CC}$ and $\mathrm{NC}$ weak interactions contributing towards the matter effect. . . . . . . . . . . . . . . . . 14

2.5 KamLAND results of measured survival probability of $\overline{\nu_{e}}$ against $L_{0} / E$. . 16

2.6 KamLAND contour plot showing the allowed regions in the $\Delta m_{21}^{2}$ against

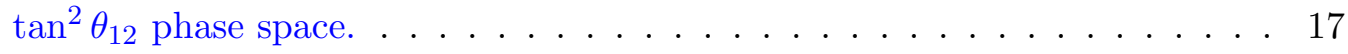

2.7 Daya Bay measurement of the electron antineutrino survival probability against. the ratio of the effective propagation distance, $\mathrm{L}_{e f f}$. to the average electron antineutrino energy, $<\mathrm{E}_{\nu}>\ldots \ldots \ldots \ldots$

2.8 Daya Bay's $\left|\Delta m_{e e}^{2}\right|$ vs. $\sin ^{2} \theta_{13}$ contour with $68.3 \%, 95.5 \%$ and $99.7 \%$ confidence levels. . . . . . . . . . . . . . . . . . . . . . . . 19

2.9 MINOS confidence limit contours in $\Delta m_{32}^{2}$ vs. $\sin ^{2} \theta_{23}$ for both mass hier-

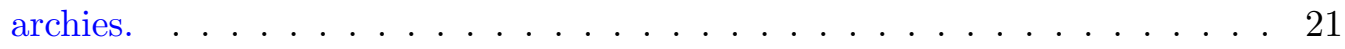

$2.10 \mathrm{~T} 2 \mathrm{~K}$ contour in $\Delta m_{32}^{2}$ against $\sin ^{2} \theta_{23}$ with snapshot of other experiments contours at the time of the $\mathrm{T} 2 \mathrm{~K}$ publication.

2.11 Simulated NOvA FD event distributions after selection, separated by inter-

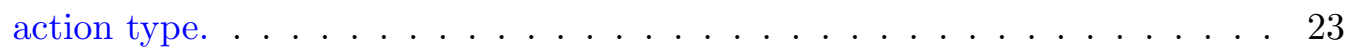

2.12 NOvA candidate $\nu_{\mu}$ events distribution. . . . . . . . . . 25

2.13 NOvA contour in $\Delta m_{32}^{2}$ against $\sin ^{2} \theta_{23}$ shown alongside the result from

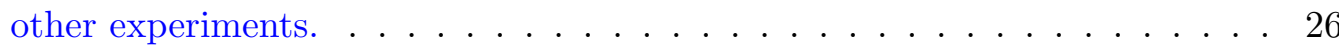

2.14 NOvA comparison of the candidate $\nu_{e}$ energy spectra of in the FD using the best fit prediction. . . . . . . . . . . . . . . . 26 
2.15 Significance at which each value of $\left|\Delta m_{32}^{2}\right|$ and $\sin ^{2} \theta_{23}$ is disfavoured in the normal or inverted mass hierarchy. . . . . . . . . . . . . . 27

2.16 NOvA $\nu_{e}$ candidate events compared to prediction as a function of $\delta_{\mathrm{CP}}$ with best fit values of both mass hierarchies . . . . . . . . . . . . . 27

2.17 NOvA significance at which each value of $\delta_{\mathrm{CP}}$ is disfavoured in each mass hierarchy scenario. . . . . . . . . . . . . . . . . 28

$2.18 \sin ^{2} \theta_{23}$ vs. $\delta_{\mathrm{CP}}$ parameter space consistent with the joint NOvA analysis. $\quad 28$

2.19 The feynman diagram for neutrinoless double-beta decay. . . . . . . . . . 29

3.1 Cartoon representation of the NuMI beam facility. [4] . . . . . . . . . 33

3.2 Diagram of the magnetic focusing horns operating in forward horn current, low energy configuration mode. . . . . . . . . . . . . . . . 34

3.3 Energy distributions using the medium energy NuMI beam configuration under assumption of differing off-axis angles $\theta$ at a $810 \mathrm{~km}$ baseline. . . . . 35

3.4 Rate of $\nu_{\mu}$-CC events vs neutrino energy in the absence of oscillations. . . . 37

3.5 Simulated energy distributions of $\nu_{\mu}$-CC events, $\nu_{e}$ events and NC events with and without oscillation modelling. . . . . . . . . . . . . 37

3.6 NOvA's near and far detectors shown to scale with respect to each other. . 38

3.7 Diagram of a NOvA cell comprised of an extruded PVC tube that is filled with liquid scintillator. . . . . . . . . . . . . . . . . . . . 39

3.8 An end on view of an extrusion consisting of 16 NOvA cells. Taken from [5]. 40

3.9 Diagram of a NOvA extrusion module. . . . . . . . . . . . . . . . . 40

3.10 Photo of a NOvA APD with a 32 pixel array. . . . . . . . . . . . . . 41

3.11 Diagram of the site on which the ND is located. . . . . . . . . . . . 45

3.12 Technical drawing of the NOvA near detector and cavern. . . . . . . . . . . 45

4.1 Default cross section in GENIE for $\nu_{\mu}$ charged current scattering with an isoscalar target. . . . . . . . . . . . . . . . . . . . . . 5 51

4.2 FHC reconstructed neutrino energy distributions for each interaction type before and after the application of the cross-section tune. . . . . . . . . 53

4.3 RHC reconstructed neutrino energy distributions for each interaction type before and after the application of the cross-section tune. . . . . . . . . . 54

4.4 Event display of reconstructed MC tracks identified by the NOvA Kalman tracker algorithm. . . . . . . . . . . . . . . . . . 55 
4.5 Reconstructed Muon Identification (ReMId) scores for muon neutrino charged current signal events and neutral current background events. . . . . . . . 60

4.6 Convolutional Visual Network (CVN) distributions for muon neutrino charged current event identification. . . . . . . . . . . . . . . . 61

4.7 Signal and cosmic background events passing each selection criteria. . . . . 63

$4.8 E_{h a d} / E_{\nu}$ against reconstructed neutrino energy, showing $E_{\text {had }} / E_{\nu}$ boundaries. 67

4.9 FHC significance of maximal mixing $\left(\sin ^{2} \theta_{23}=0.5\right)$ rejection against number of $E_{\text {had }} / E_{\nu}$ quantiles. . . . . . . . . . . . . . 68

4.10 RHC significance of maximal mixing $\left(\sin ^{2} \theta_{23}=0.5\right)$ rejection against number of $E_{\text {had }} / E_{\nu}$ quantiles. . . . . . . . . . . . . . 68

4.11 The near detector profiles of the reconstructed and true energy ratios as a function of $\mathrm{W}$ for $\mathrm{X}$-view hits before and after calibration. . . . . . . . . 71

4.12 The far detector profiles of the reconstructed and true energy ratios as a function of $\mathrm{W}$ for the $\mathrm{Y}$-view hits before and after calibration. . . . . . . . . 72

5.1 Distribution and $\frac{\text { Signal }}{\text { Cosmic Background }}$ ratio for the Kalman Forward Cell variable. 78

5.2 Distribution and $\frac{\text { Signal }}{\text { Cosmic Background }}$ ratio for the Kalman Backward Cell vari-

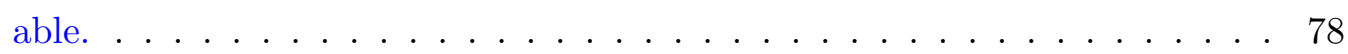

5.3 Distribution and $\frac{\text { Signal }}{\text { Cosmic Background }}$ ratio for the Cosmic Forward Cell variable. 79

5.4 Distribution and $\frac{\text { Signal }}{\text { Cosmic Background }}$ ratio for the Cosmic Back Cell variable. . 79

5.5 Distribution and $\frac{\text { Signal }}{\text { Cosmic Background }}$ ratio for the Planes to Front variable. . . 80

5.6 Distribution and $\frac{\text { Signal }}{\text { Cosmic Background }}$ ratio for the Planes to Back variable. . . . 80

5.7 Distribution and $\frac{\text { Signal }}{\text { Cosmic Background }}$ ratio for the Min Prong Distance to FD Top variable. . . . . . . . . . . . . . . . . . . . 82

5.8 Distribution and $\frac{\text { Signal }}{\text { Cosmic Background }}$ ratio for the Min Prong Distance to FD Bottom variable. . . . . . . . . . . . . . . . . . 82

5.9 Distribution and $\frac{\text { Signal }}{\text { Cosmic Background }}$ ratio for the Min Prong Distance to FD Front variable. . . . . . . . . . . . . . . . . . 83

5.10 Distribution and $\frac{\text { Signal }}{\text { Cosmic Background }}$ ratio for the Min Prong Distance to FD Back variable. . . . . . . . . . . . . . . . . 83

5.11 Distribution and $\frac{\text { Signal }}{\text { Cosmic Background }}$ ratio for the Min Prong Distance to FD East variable. . . . . . . . . . . . . . . . . . . . . 84

5.12 Distribution and $\frac{\text { Signal }}{\text { Cosmic Background }}$ ratio for the Min Prong Distance to FD West variable. . . . . . . . . . . . . . . . . . . . 84 
5.13 Energy distribution summed across periods 1,2, 3 and 5 of selected $\nu_{\mu}$ events using the 2017 and 2018 analysis containment criteria. . . . . . . . 87

$5.14 \nu_{\mu}$ energy distributions in periods $1(\mathrm{a})$ and $2(\mathrm{~b}) \ldots \ldots \ldots 8$

$5.15 \nu_{\mu}$ energy distributions In periods $3(\mathrm{a})$ and $5(\mathrm{~b}) \ldots \ldots \ldots 8$

5.16 Distribution of $\left(\right.$ reconstructed $_{\nu_{\mu} E}-$ true $\left._{\nu_{\mu} E}\right) /$ true $_{\nu_{\mu} E} \nu_{\mu}$ energy. Using the 2017 and 2018 analysis containment criteria. . . . . . . . . . . . 89

5.17 Cosmic energy spectra for 2017 and 2018 Containment criteria. . . . . . . . 89

5.18 Distribution of event interaction types using the 2017 analysis(a) and 2018 analysis (b) containment criteria. . . . . . . . . . . . . 90

5.19 Distribution of event types selected by the 2018 analysis containment but cut when using the 2017 analysis criteria. . . . . . . . . . . . . 91

$5.20 \nu_{\mu}$ reconstructed energy distributions produced using the 2017 and 2018 containment criteria on the period 1 dataset. . . . . . . . . . . . . 92

$5.21 \nu_{\mu}$ reconstructed energy distributions produced using the 2017 and 2018 containment criteria across the total dataset. . . . . . . . . . . . . . 92

6.1 Ratios of oscillated $\nu_{\mu}+\bar{\nu}_{\mu}$-CC over unoscillated $\nu_{\mu}+\bar{\nu}_{\mu}$-CC events for forward horn current $\mathrm{MC}$ files. . . . . . . . . . . . . . . . . . 97

6.2 The $\nu_{\mu}+\bar{\nu}_{\mu}$-CC and cosmic distributions in 3D-PID space of events passing the selection criteria for $\mathrm{FHC}$ and for RHC . . . . . . . . . . . . 98

6.3 The FHC $\nu_{\mu}+\bar{\nu}_{\mu}$-CC and cosmic distributions in 3D-PID space for each $E_{\text {had }} / E_{\nu}$ quantile. . . . . . . . . . . . . . . . . . 99

6.4 The RHC $\bar{\nu}_{\mu}$-CC and cosmic distributions in 3D-PID space for each $E_{\text {had }} / E_{\nu}$ quantile. . . . . . . . . . . . . . . . . . . 100

6.5 The FHC figure of merit $\left(\frac{s i g}{\sqrt{s i g+b k g}}\right)$ against total background for 6069 sets of cuts each representing one set of PID cuts analyzed. . . . . . . . . . . . 104

6.6 The RHC figure of merit $\left(\frac{s i g}{\sqrt{s i g+b k g}}\right)$ against total background for 6069 sets of cuts each representing one set of PID cuts analyzed. . . . . . . . . . 105

6.7 The FHC $\nu_{\mu}+\bar{\nu}_{\mu}$-CC distributions for each hadronic energy fraction quantile shown for each set of PID cuts analysed with the additional 2017 CVN selection added. . . . . . . . . . . . . . . . . . . . 115

6.8 The FHC $\nu_{\mu}+\bar{\nu}_{\mu}$-CC ratios, with respect to the 2017 cuts, for each hadronic energy fraction quantile shown for each set of PID cuts analysed with the additional 2017 CVN selection added. . . . . . . . . . . . . 115 
6.9 The FHC cosmic distributions for each hadronic energy fraction quantile shown for each set of PID cuts analysed with the additional 2017 CVN selection added. . . . . . . . . . . . . . . . . . 116

6.10 The FHC NC distributions for each hadronic energy fraction quantile shown for each set of PID cuts analysed with the additional 2017 CVN selection added. . . . . . . . . . . . . . . . . . . . 116

6.11 The FHC distributions of $\nu_{\mu}+\bar{\nu}_{\mu}$-CC, NC, other beam background and cosmic events summed across quantiles for each set of PID cuts analysed with the additional $2017 \mathrm{CVN}$ selection added. . . . . . . . . . . . 117

6.12 The total FHC $\nu_{\mu}+\bar{\nu}_{\mu}$-CC ratio, with respect to the 2017 cuts, shown for each set of PID cuts analysed with the additional 2017 CVN selection added.117

6.13 The RHC $\bar{\nu}_{\mu}$-CC distributions for each hadronic energy fraction quantile shown for each set of PID cuts analysed with the additional 2017 CVN selection added. . . . . . . . . . . . . . . . . . . . . . . . . 120

6.14 The $\mathrm{RHC} \bar{\nu}_{\mu}$-CC ratios, with respect to the 2017 cuts, for each hadronic energy fraction quantile shown for each set of PID cuts analysed with the additional 2017 CVN selection added. . . . . . . . . . . . . 120

6.15 The RHC cosmic distributions for each hadronic energy fraction quantile shown for each set of PID cuts analysed with the additional 2017 CVN selection added. . . . . . . . . . . . . . . . . . . . 121

6.16 The RHC NC distributions for each hadronic energy fraction quantile shown for each set of PID cuts analysed with the additional 2017 CVN selection

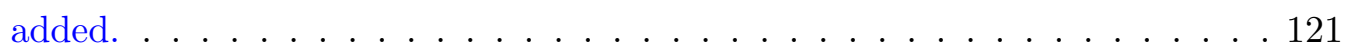

6.17 The RHC distributions of $\bar{\nu}_{\mu}$-CC, NC, other beam background and cosmic events summed across quantiles for each set of PID cuts analysed with the additional 2017 CVN selection added. . . . . . . . . . . . . . . 122

6.18 The total $\mathrm{RHC} \bar{\nu}_{\mu}$-CC ratio, with respect to the 2017 cuts, shown for each set of PID cuts analysed with the additional 2017 CVN selection added. . . 122

7.1 FHC and RHC interaction type distributions of $\nu_{\mu}\left(\bar{\nu}_{\mu}\right) \mathrm{CC}$ candidate events in the Near Detector. . . . . . . . . . . . . . . . . 126

7.2 FHC and RHC interaction type distributions of selected $\nu_{\mu} \mathrm{CC}$ events in the Near Detector separated by $E_{\text {had }} / E_{\nu}$ quantile. . . . . . . . . . . . 127

7.3 ND POT normalized FHC distributions of $\nu_{\mu}$ energy for each $E_{h a d} / E_{\nu}$ quantile. . . . . . . . . . . . . . . . . . . . . . . 138 
7.4 ND POT normalized RHC distributions of $\bar{\nu}_{\mu}$ energy for each $E_{h a d} / E_{\nu}$ quantile. . . . . . . . . . . . . . . . . . . . 138

7.5 ND area normalized FHC distributions of $\nu_{\mu}$ energy, muon energy, muon energy per hit and Kalman track length for the entire data set. . . . . . . . 139

7.6 ND area normalized RHC distributions of $\nu_{\mu}$ energy, muon energy, muon energy per hit and Kalman track length for the entire data set. . . . . . . . 139

7.7 ND area normalized FHC distributions of the $\nu_{\mu}$ energy for each $E_{h a d} / E_{\nu}$ quantile. . . . . . . . . . . . . . . . . . . . . . . . 140

7.8 ND area normalized RHC distributions of the $\nu_{\mu}$ energy for each $E_{h a d} / E_{\nu}$ quantile. . . . . . . . . . . . . . . . . . . . . . . . . 140

7.9 ND area normalized FHC distributions of the muon energy for each $E_{h a d} / E_{\nu}$ quantile. . . . . . . . . . . . . . . . . . . . . . . . 141

7.10 ND area normalized RHC distributions of the muon energy for each $E_{h a d} / E_{\nu}$ quantile. . . . . . . . . . . . . . . . . . . . . . . 141

7.11 ND area normalized FHC distributions of the muon energy per hit for each $E_{\text {had }} / E_{\nu}$ quantile.

7.12 ND area normalized RHC distributions of the muon energy per hit for each $E_{\text {had }} / E_{\nu}$ quantile. . . . . . . . . . . . . . . . 142

7.13 ND area normalized FHC distributions of the Kalman track length for each $E_{\text {had }} / E_{\nu}$ quantile. . . . . . . . . . . . . . . 143

7.14 ND area normalized RHC distributions of the Kalman track length for each $E_{\text {had }} / E_{\nu}$ quantile.

7.15 ND area normalized FHC distributions of hadronic energy, fraction of hadronic energy, hadronic energy per hit \& no of hits in slice for the entire data set. . . . . . . . . . . . . . . . . . . . . . 144

7.16 ND area normalized RHC distributions of hadronic energy, fraction of hadronic energy, hadronic energy per hit \& no of hits in slice for the entire data set. . . . . . . . . . . . . . . . . . . . . . . . . . . 144

7.17 ND area normalized FHC distributions of the hadronic energy for each

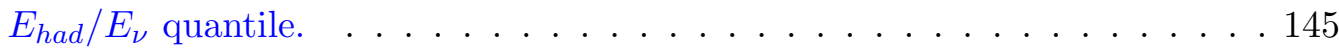

7.18 ND area normalized RHC distributions of the hadronic energy for each $E_{\text {had }} / E_{\nu}$ quantile. . . . . . . . . . . . . . . 145

7.19 ND area normalized FHC distributions of the fraction of hadronic energy for each $E_{\text {had }} / E_{\nu}$ quantile. . . . . . . . . . . . . . . . . 146 
7.20 ND area normalized RHC distributions of the fraction of hadronic energy for each $E_{h a d} / E_{\nu}$ quantile. . . . . . . . . . . . . . . . 146

7.21 ND area normalized FHC distributions of the hadronic energy per hit for each $E_{\text {had }} / E_{\nu}$ quantile. . . . . . . . . . . . . . . . 147

7.22 ND area normalized RHC distributions of the hadronic energy per hit for each $E_{\text {had }} / E_{\nu}$ quantile. . . . . . . . . . . . . . . . . 147

7.23 ND area normalized FHC distributions of the no of hits in slice for each $E_{\text {had }} / E_{\nu}$ quantile. . . . . . . . . . . . . . . . 148

7.24 ND area normalized RHC distributions of the no of hits in slice for each $E_{\text {had }} / E_{\nu}$ quantile. . . . . . . . . . . . . . 148

7.25 ND area normalized FHC distributions of no of hits in Kalman track, cosmic track, CVN 2017 \& CVN 2018 for the entire data set. . . . . . . . . . . 149

7.26 ND area normalized RHC distributions of no of hits in Kalman track, cosmic track, CVN 2017 \& CVN 2018 for the entire data set. . . . . . . . . . . . 149

7.27 ND area normalized FHC distributions of the no of hits in Kalman track for each $E_{\text {had }} / E_{\nu}$ quantile. . . . . . . . . . . . . . 150

7.28 ND area normalized RHC distributions of the no of hits in Kalman track for each $E_{h a d} / E_{\nu}$ quantile. . . . . . . . . . . . . . 150

7.29 ND area normalized FHC distributions of the cosmic track for each $E_{h a d} / E_{\nu}$ quantile. . . . . . . . . . . . . . . . . . . . . . 151

7.30 ND area normalized RHC distributions of the cosmic track for each $E_{h a d} / E_{\nu}$ quantile. . . . . . . . . . . . . . . . . . . 151

7.31 ND area normalized FHC distributions of the CVN 2017 for each $E_{h a d} / E_{\nu}$ quantile. . . . . . . . . . . . . . . . . . . . . 152

7.32 ND area normalized RHC distributions of the CVN 2017 for each $E_{\text {had }} / E_{\nu}$

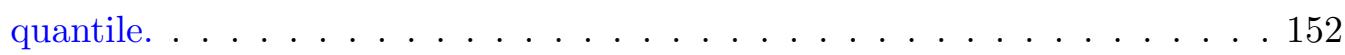

7.33 ND area normalized FHC distributions of the CVN 2018 for each $E_{\text {had }} / E_{\nu}$ quantile. . . . . . . . . . . . . . . . . . . . . . . 153

7.34 ND area normalized RHC distributions of the CVN 2018 for each $E_{h a d} / E_{\nu}$ quantile. . . . . . . . . . . . . . . . . . . . 153

7.35 ND area normalized FHC distributions of ReMId, ReMId fractional plane likelihood, ReMId scattering angle likelihood \& ReMId $\frac{d E}{d x}$ likelihood for the entire data set. . . . . . . . . . . . . . . . . . . . . . . 154 
7.36 ND area normalized RHC distributions of ReMId, ReMId fractional plane likelihood, ReMId scattering angle likelihood \& ReMId $\frac{d E}{d x}$ likelihood for the entire data set. . . . . . . . . . . . . . . . . . . 154

7.37 ND area normalized FHC distributions of the ReMId for each $E_{h a d} / E_{\nu}$ quantile. . . . . . . . . . . . . . . . . . . . . 155

7.38 ND area normalized RHC distributions of the ReMId for each $E_{h a d} / E_{\nu}$

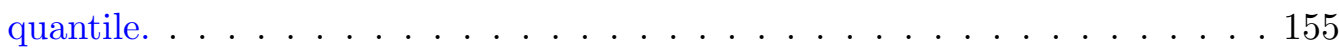

7.39 ND area normalized FHC distributions of the ReMId fraction of planes along the track consistent with having minimum ionizinglike $\frac{d E}{d x}$ for each

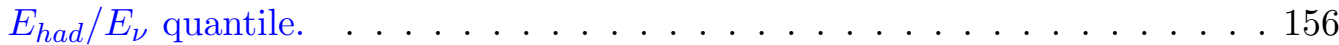

7.40 ND area normalized RHC distributions of the ReMId fraction of planes along the track consistent with having minimum ionizinglike $\frac{d E}{d x}$ for each $E_{h a d} / E_{\nu}$ quantile.

7.41 ND area normalized FHC distributions of the ReMId scattering angle likelihood for each $E_{h a d} / E_{\nu}$ quantile. . . . . . . . . . . . . . 157

7.42 ND area normalized RHC distributions of the ReMId scattering angle likelihood for each $E_{h a d} / E_{\nu}$ quantile. . . . . . . . . . . . . . 157

7.43 ND area normalized FHC distributions of the ReMId $\frac{d E}{d x}$ likelihood for each

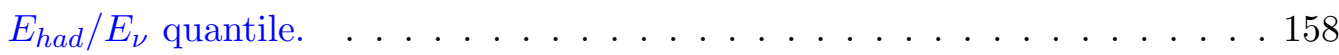

7.44 ND area normalized RHC distributions of the ReMId $\frac{d E}{d x}$ likelihood for each

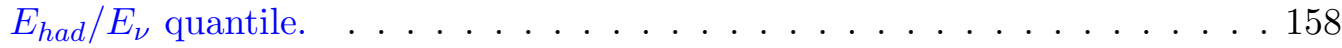

7.45 FHC FD $\nu_{\mu}$-energy distributions for the total dataset, unoscillated prediction and oscillated prediction. . . . . . . . . . . . . . . . 165

7.46 RHC FD $\nu_{\mu}$-energy distributions for the total dataset, unoscillated prediction and oscillated prediction. . . . . . . . . . . . . . 166

7.47 FHC FD $\nu_{\mu}$-energy distributions in each $E_{h a d} / E_{\nu}$ quantile for the unoscillated prediction, oscillated prediction and as observed in data. . . . . . . 167

7.48 RHC FD $\nu_{\mu}$-energy distributions in each $E_{h a d} / E_{\nu}$ quantile for the unoscillated prediction, oscillated prediction and as observed in data. . . . . . . . 167

7.49 FHC FD ratios of the $\frac{\text { oscillated }_{M C}}{\text { unoscillated }} \nu_{\mu}+\nu_{\mu}$-CC distributions and of the oscillated $_{\text {Data }} \nu_{\mu}+\nu_{\mu}$-CC distributed $\nu$ dibutions. . . . . . . . . . . . . 168

7.50 RHC FD ratios of the $\frac{\text { sscillated }_{M C}}{\text { unoscillated }} \nu_{\mu}+\nu_{\mu}$-CC distributions and of the $\frac{\text { oscillated }_{\text {Data }}}{\text { unoscillated }} \nu_{\mu}+\nu_{\mu}$-CC distributions. . . . . . . . . . . . 168

7.51 FHC FD reconstructed $\nu_{\mu}$-energy distributions for the total dataset. . . . . 173 
7.52 RHC FD reconstructed $\bar{\nu}_{\mu}$-energy distributions for the total dataset. $\ldots 173$

7.53 FHC FD reconstructed $\nu_{\mu}$-energy distributions for each $E_{h a d} / E_{\nu}$ quantile. . 174

7.54 RHC FD reconstructed $\bar{\nu}_{\mu}$-energy distributions for each $E_{h a d} / E_{\nu}$ quantile. . 174

7.55 FHC FD reconstructed $\mu^{-}$-energy distributions for the total dataset. . . . 175

7.56 RHC FD reconstructed $\mu^{+}$-energy distributions for the total dataset. . . . 175

7.57 FHC FD reconstructed $\mu^{-}$-energy distributions for each $E_{\text {had }} / E_{\nu}$ quantile. . 176

7.58 RHC FD reconstructed $\mu^{+}$-energy distributions for each $E_{\text {had }} / E_{\nu}$ quantile. 176

7.59 FHC FD reconstructed $\mu^{-}$-energy per hit distributions for the total dataset. 177

7.60 RHC FD reconstructed $\mu^{+}$-energy per hit distributions for the total dataset. 177

7.61 FHC FD reconstructed $\mu^{-}$-energy per hit distributions for each $E_{\text {had }} / E_{\nu}$

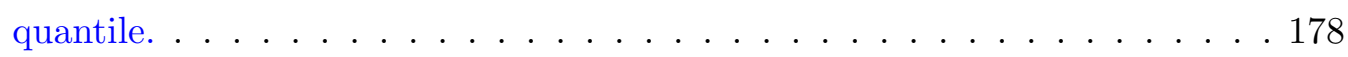

7.62 RHC FD reconstructed $\mu^{+}$-energy per hit distributions for each $E_{\text {had }} / E_{\nu}$ quantile. . . . . . . . . . . . . . . . . . . . . . 178

7.63 FHC FD reconstructed hadronic-energy distributions for the total dataset. 179

7.64 RHC FD reconstructed hadronic-energy distributions for the total dataset. 179

7.65 FHC FD reconstructed hadronic-energy distributions for each $E_{h a d} / E_{\nu}$ quantile.180

7.66 RHC FD reconstructed hadronic-energy distributions for each $E_{\text {had }} / E_{\nu}$ quantile. . . . . . . . . . . . . . . . . . . 180

7.67 FHC FD reconstructed hadronic-energy per hit distributions for the total dataset. . . . . . . . . . . . . . . . . . . . . . 181

7.68 RHC FD reconstructed hadronic-energy per hit distributions for the total dataset. . . . . . . . . . . . . . . . . . . . . . 181

7.69 FHC FD reconstructed hadronic-energy per hit distributions for each $E_{\text {had }} / E_{\nu}$ quantile. . . . . . . . . . . . . . . . . . . . 182

7.70 RHC FD reconstructed hadronic-energy per hit distributions for each $E_{h a d} / E_{\nu}$ quantile. . . . . . . . . . . . . . . . . . . . 182

7.71 FHC FD hadronic fraction of total energy distributions for the total dataset.183

7.72 RHC FD hadronic fraction of total energy distributions for the total dataset.183

7.73 FHC FD hadronic fraction of total energy distributions for each $E_{\text {had }} / E_{\nu}$ quantile. . . . . . . . . . . . . . . . . . . . . . 184

7.74 RHC FD hadronic fraction of total energy distributions for each $E_{h a d} / E_{\nu}$ quantile. . . . . . . . . . . . . . . . . . . . 184

7.75 FHC FD distributions of the number of Kalman tracks for the total dataset. 185

7.76 RHC FD distributions of the number of Kalman tracks for the total dataset.185 
7.77 FHC FD distributions of the number of Kalman tracks for each $E_{\text {had }} / E_{\nu}$ quantile. . . . . . . . . . . . . . . . 186

7.78 RHC FD distributions of the number of Kalman tracks for each $E_{\text {had }} / E_{\nu}$ quantile. . . . . . . . . . . . . . . . . 186

7.79 FHC FD distributions of the Kalman track angle with respect to the beam for the entire data set. . . . . . . . . . . . . . . . . . . 187

7.80 RHC FD distributions of the Kalman track angle with respect to the beam for the entire data set. . . . . . . . . . . . . . . . . . . . 187

7.81 FHC FD distributions of the Kalman track angle with respect to the beam for each $E_{\text {had }} / E_{\nu}$ quantile. . . . . . . . . . . . . . . . 188

7.82 RHC FD distributions of the Kalman track angle with respect to the beam for each $E_{\text {had }} / E_{\nu}$ quantile. . . . . . . . . . . . . . . . 188

7.83 FHC FD distributions of the CVN PID score for the total dataset. $E_{\text {had }} / E_{\nu}$ quantiles. . . . . . . . . . . . . . . . . . . . . . . 189

7.84 RHC FD distributions of the CVN PID score for the total dataset. $E_{\text {had }} / E_{\nu}$ quantiles. . . . . . . . . . . . . . . . . . . . 189

7.85 FHC FD distributions of the CVN PID score for each $E_{\text {had }} / E_{\nu}$ quantile. . . 190

7.86 RHC FD distributions of the CVN PID score for each $E_{\text {had }} / E_{\nu}$ quantile. . . 190

7.87 FHC FD distributions of the 2017 (older) CVN PID score for the total dataset. . . . . . . . . . . . . . . . . . . . . . . 191

7.88 RHC FD distributions of the 2017 (older) CVN PID score for the total dataset. . . . . . . . . . . . . . . . . . . . . . . . . . 191

7.89 FHC FD distributions of the 2017 (older) CVN PID score for each $E_{\text {had }} / E_{\nu}$ quantile. . . . . . . . . . . . . . . . . . . . 192

7.90 RHC FD distributions of the 2017 (older) CVN PID score for each $E_{\text {had }} / E_{\nu}$ quantile. . . . . . . . . . . . . . . . . . . . . . . 192

7.91 FHC FD distributions of the ReMId PID score for the total dataset. $E_{\text {had }} / E_{\nu}$ quantiles. . . . . . . . . . . . . . . . . . . . . . 193

7.92 RHC FD distributions of the ReMId PID score for the total dataset. $E_{\text {had }} / E_{\nu}$ quantiles. . . . . . . . . . . . . . . . . . . 193

7.93 FHC FD distributions of the ReMId PID score for each $E_{h a d} / E_{\nu}$ quantile. . 194

7.94 RHC FD distributions of the ReMId PID score for each $E_{h a d} / E_{\nu}$ quantile. . 194

7.95 FHC FD distributions of the ReMId scattering angle likelihood for the entire data set. . . . . . . . . . . . . . . . . . . . . . 195 
7.96 RHC FD distributions of the ReMId scattering angle likelihood for the entire data set. . . . . . . . . . . . . . . . . . . 195

7.97 FHC FD distributions of the ReMId scattering angle likelihood score for each $E_{h a d} / E_{\nu}$ quantile. . . . . . . . . . . . . . 196

7.98 RHC FD distributions of the ReMId scattering angle likelihood score for

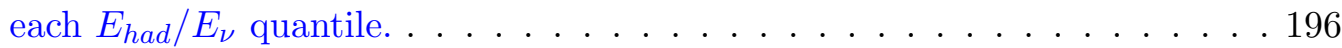

7.99 FHC FD distributions of the ReMId $\frac{d E}{d x}$ likelihood for the total dataset. . . 197

7.100RHC FD distributions of the ReMId $\frac{d E}{d x}$ likelihood for the total dataset. . . 197

7.101FHC FD distributions of the ReMId $\frac{d E}{d x}$ likelihood for each $E_{h a d} / E_{\nu}$ quantile.198 7.102RHC FD distributions of the ReMId $\frac{d E}{d x}$ likelihood for each $E_{h a d} / E_{\nu}$ quantile.198

7.103FHC FD distributions of the ReMId fraction of planes along the track consistent with having minimum ionizinglike $\frac{d E}{d x}$ likelihood for the total dataset. $E_{\text {had }} / E_{\nu}$ quantiles. . . . . . . . . . . . . . . . 199

7.104RHC FD distributions of the ReMId fraction of planes along the track consistent with having minimum ionizinglike $\frac{d E}{d x}$ likelihood for the total dataset. $E_{h a d} / E_{\nu}$ quantiles. . . . . . . . . . . . . . . . 199

7.105FHC FD distributions of the ReMId fraction of planes along the track consistent with having minimum ionizinglike $\frac{d E}{d x}$ likelihood for each $E_{h a d} / E_{\nu}$ quantile. . . . . . . . . . . . . . . . . . . . 200

7.106RHC FD distributions of the ReMId fraction of planes along the track consistent with having minimum ionizinglike $\frac{d E}{d x}$ likelihood for each $E_{h a d} / E_{\nu}$ quantile. . . . . . . . . . . . . . . . . . . . 200

7.107The statistics only and full systematics $90 \%$ confidence limit contours in $\Delta m_{32}^{2}$ vs. $\sin ^{2} \theta_{23}$ produced by the $\nu_{\mu}+\bar{\nu}_{\mu}$ disappearance analysis. . . . . 202

7.108The $90 \%$ full systematic confidence limit contours in $\Delta m_{32}^{2}$ vs. $\sin ^{2} \theta_{23}$ produced for $\mathrm{FHC}, \mathrm{RHC}$ and $\mathrm{FHC}+\mathrm{RHC}$ data. . . . . . . . . . . . . 202

7.109Significance vs. $\Delta m_{32}^{2}$ for the FHC data, for the RHC data and for the combined $\mathrm{FHC}+\mathrm{RHC}$ fits, assuming normal hierarchy. . . . . . . . . . . . . 203

7.110Significance vs. $\sin ^{2} \theta_{23}$ for the FHC data, for the RHC data and for the combined $\mathrm{FHC}+\mathrm{RHC}$ fits, assuming normal hierarchy. . . . . . . . . . . . . 204

7.111The $90 \%$ confidence limit contour in $\Delta m_{32}^{2}$ vs. $\sin ^{2} \theta_{23}$ produced by various experiments. 
7.112Uncertainty bar chart for $\Delta m_{32}^{2}$ showing each systematics positive and negative uncertainty on the measurement. Fake data was used and oscillated assuming the $8.85 \times 10^{20}$ POT FHC-only best fit [3] and normal hierarchy. 207

7.113Uncertainty bar chart for $\sin ^{2} \theta_{23}$ showing each systematics positive and negative uncertainty on the measurement. Fake data was used and oscillated assuming the $8.85 \times 10^{20}$ POT FHC-only best fit [3] and normal hierarchy. 207

7.114Bar chart comparison of systematic pulls in units of $\sigma \ldots \ldots \ldots$. . . 208

7.115Plot of $\Delta m_{32}^{2}$ vs. $\sin ^{2} \theta_{23}$ with the best fit shift caused by each calibration systematic shown. . . . . . . . . . . . . . . . . . 213

7.116Plot of $\Delta m_{32}^{2}$ vs. $\sin ^{2} \theta_{23}$ with the best fit shift caused by each PPFX flux systematic shown. . . . . . . . . . . . . . . . . . 214

7.117Plot of $\Delta m_{32}^{2}$ vs. $\sin ^{2} \theta_{23}$ with the best fit shift caused by each GENIE principal component analysis (PCA) systematic shown. . . . . . . . . . 215

7.118Plot of $\Delta m_{32}^{2}$ vs. $\sin ^{2} \theta_{23}$ with the best fit shift caused by each GENIE reweighting systematic shown. . . . . . . . . . . . . . . 216

7.119Plot of $\Delta m_{32}^{2}$ vs. $\sin ^{2} \theta_{23}$ with the best fit shift caused by each MEC systematic shown. . . . . . . . . . . . . . . . . . . . . 217

7.120Plot of $\Delta m_{32}^{2}$ vs. $\sin ^{2} \theta_{23}$ with the best fit shift caused by each normalization systematic shown. . . . . . . . . . . . . . . . . 218

7.121Plot of $\Delta m_{32}^{2}$ vs. $\sin ^{2} \theta_{23}$ with the best fit shift caused by each cross-section systematic shown. . . . . . . . . . . . . . . . . . . . 219

7.122Significance vs. time for the rejection of maximal mixing under different oscillation assumptions.

A.1 $\nu_{\mu}$ energy distributions in Period 1 using normal MC and cosmic overlay MC. kNumuQuality, kNumuNCRej and kNumuCosmicRej cuts applied. . 226

A.2 $\nu_{\mu}$ energy distributions in Period 1 using normal MC and cosmic overlay MC. kNumuQuality and kKirkSATuneFD (2016 BDT) cuts applied. . . . . 226

A.3 $\nu_{\mu}$ energy distributions in Period 2 using normal MC and cosmic overlay MC. kNumuQuality, kNumuNCRej and kNumuCosmicRej cuts applied. . . 227

A.4 $\nu_{\mu}$ energy distributions in Period 2 using normal MC and cosmic overlay MC. kNumuQuality and kKirkSATuneFD (2016 BDT) cuts applied. . . . . 227

A.5 $\nu_{\mu}$ energy distributions in Epoch $3 \mathrm{~b}$ using normal MC and cosmic overlay MC. kNumuQuality, kNumuNCRej and kNumuCosmicRej cuts applied. . . 228 
A.6 $\nu_{\mu}$ energy distributions in Epoch 3b using normal MC and cosmic overlay MC. kNumuQuality and kKirkSATuneFD (2016 BDT) cuts applied. . . . . 228

A.7 $\nu_{\mu}$ energy distributions in Epoch 3c using normal MC and cosmic overlay MC. kNumuQuality, kNumuNCRej and kNumuCosmicRej cuts applied. . . 229

A.8 $\nu_{\mu}$ energy distributions in Epoch 3c using normal MC and cosmic overlay MC. kNumuQuality and kKirkSATuneFD (2016 BDT) cuts applied. . . . . 229

A.9 $\nu_{\mu}$ energy distribution for all data periods using normal MC and cosmic overlay MC. kNumuQuality, kNumuNCRej and kNumuCosmicRej cuts applied. . . . . . . . . . . . . . . . . . . . . 2230

A.10 $\nu_{\mu}$ energy distribution for all data periods using normal $\mathrm{MC}$ and cosmic overlay MC. kNumuQuality and kKirkSATuneFD (2016 BDT) cuts applied. 230 A.11 $\nu_{\mu}$ energy distributions in Period 1 using normal MC and cosmic overlay MC. kNumuQuality, kNumuNCRej and kNumuCosmicRej cuts applied. . . 232 A.12 $\nu_{\mu}$ energy distributions in Period 1 using normal MC and cosmic overlay MC. kNumuQuality and kKirkTATuneFD (2016 BDT) cuts applied. . . . . 232 A.13 $\nu_{\mu}$ energy distributions in Period 1 using normal MC and cosmic overlay MC. kNumuQuality and kKirkTATuneFD2017 (2017 BDT) cuts applied. . . 233 A.14 $\nu_{\mu}$ energy distributions in Period 2 using normal MC and cosmic overlay MC. kNumuQuality, kNumuNCRej and kNumuCosmicRej cuts applied. . . 233 A.15 $\nu_{\mu}$ energy distributions in Period 2 using normal MC and cosmic overlay MC. kNumuQuality and kKirkTATuneFD (2016 BDT) cuts applied. . . . . 234 A.16 $\nu_{\mu}$ energy distributions in Period 2 using normal MC and cosmic overlay MC. kNumuQuality and kKirkTATuneFD2017 (2017 BDT) cuts applied. . . 234 A.17 $\nu_{\mu}$ energy distributions in Period 3 using normal MC and cosmic overlay MC. kNumuQuality, kNumuNCRej and kNumuCosmicRej cuts applied. . . 235 A.18 $\nu_{\mu}$ energy distributions in Period 3 using normal MC and cosmic overlay MC. kNumuQuality and kKirkTATuneFD (2016 BDT) cuts applied. . . . . 235 A.19 $\nu_{\mu}$ energy distributions in Period 3 using normal MC and cosmic overlay MC. kNumuQuality and kKirkTATuneFD2017 (2017 BDT) cuts applied. . . 236 A.20 $\nu_{\mu}$ energy distributions in Period 5 using normal MC and cosmic overlay MC. kNumuQuality, kNumuNCRej and kNumuCosmicRej cuts applied. . . 236 A.21 $\nu_{\mu}$ energy distributions in Period 5 using normal MC and cosmic overlay MC. kNumuQuality and kKirkTATuneFD (2016 BDT) cuts applied. . . . . 237 
A.22 $\nu_{\mu}$ energy distributions in Period 5 using normal MC and cosmic overlay MC. kNumuQuality and kKirkTATuneFD2017 (2017 BDT) cuts applied. . . 237 A.23 $\nu_{\mu}$ energy distributions for data summed across all data periods using normal MC and cosmic overlay MC. kNumuQuality, kNumuNCRej and kNumuCosmicRej cuts applied. . . . . . . . . . . . . . . . . . 238

A.24 $\nu_{\mu}$ energy distributions for data summed across all data periods using normal MC and cosmic overlay MC. kNumuQuality and kKirkTATuneFD (2016 BDT) cuts applied. . . . . . . . . . . . . . . . . 238

A.25 $\nu_{\mu}$ energy distributions for data summed across all data periods using normal MC and cosmic overlay MC. kNumuQuality and kKirkTATuneFD2017 (2017 BDT) cuts applied. . . . . . . . . . . . . . . . . 239 


\section{Chapter 1}

\section{Introduction}

Neutrinos are unusual particles. Discovered more than sixty years ago to remedy the violation of energy conservation in beta-decay, these elusive particles have turned up many fascinating new questions. Neutrino oscillation was discovered at the turn of the century a phenomenon by which neutrinos can change flavour in flight. This landmark result lead to a rich field of experimental physics, pioneered by several large scale collaborations.

The oscillation effect violates one of the tenets of the particle physics standard model. Namely that neutrinos are massless. The oscillation effect cannot manifest unless the neutrino masses differ. We know from experiment that at least two of the three leptonic neutrino flavours must have non-zero mass. Consequently, the standard model must be incomplete. Empirical evidence of beyond the standard model physics is only present in the neutrino sector.

The combined efforts of nuclear reactor, solar, atmospheric and long-baseline oscillation experiments have yielded constraints on the neutrino properties and the PMNS matrix parameters that govern oscillation. Nonetheless, there are many unanswered questions. Examples include the octant that the $\theta_{23}$ mixing angle occupies and the ordering of mass states (also known as the mass hierarchy). The charge-parity violating phase, necessitated by the oscillation matrix, is also unknown.

NOvA is a long-baseline neutrino oscillation experiment situated in the United States. Comprising two detectors and an accelerator muon (anti)neutrino beam facility, NOvA measures the rate of $\nu_{e}\left(\bar{\nu}_{e}\right)$ appearance and $\nu_{\mu}\left(\bar{\nu}_{\mu}\right)$ disappearance at baselines of $1 \mathrm{~km}$ and $810 \mathrm{~km}$. In doing so NOvA is able to probe the mass hierarchy, CP-violating phase, neutrino mixing angle $\theta_{23}$ and mass squared difference $\Delta m_{32}^{2}$. The $\nu_{e}$ appearance analysis is predominantly sensitive to the former two parameters. This thesis is focused on the combined $\nu_{\mu}+\bar{\nu}_{\mu}$ disappearance analysis, which is predominantly sensitive to $\theta_{23}$ and 


\section{$\Delta m_{32}^{2}$.}

NOvA's first $\bar{\nu}_{\mu}$ disappearance results are presented in this thesis. The antineutrino data is combined with existing neutrino data to produce NOvA's most sensitive disappearance measurement to date. The systematic and methodological improvements that have increased NOvA's measurement sensitivity and reduced background selection are detailed. The agreement between simulation and data is presented for both NOvA detectors. Two significant analysis improvements are detailed in dedicated chapters. The first body of work comprises a redesign of the far detector selection used to identify events wholly inside in the active detector volume. This was performed by migrating the selection to act on track objects, making the analysis more robust, and also optimizing cut values. The result was a significant decrease in the cosmic background with little to no compromise in signal selection. The second body of work comprises a multi-dimensional analysis of the machine learning particle identification algorithms NOvA utilises. This was done to determine the best combination of cuts for maximizing NOvA's sensitivity. A significant reduction in all background sources and a small increase in signal selection was achieved.

The resulting constraints on $\Delta m_{32}^{2}$ and $\sin ^{2} \theta_{23}$ are presented, competitive with the best in the world.

- Chapter 1 introduces the thesis and its contents.

- Chapter 2 contains an overview of the theoretical framework describing neutrino interactions and their oscillations.

- Chapter 3 describes the experimental apparatus of NOvA.

- Chapter 4 describes the methodology of the analysis.

- Chapter 5 \& Chapter 6 delineates analysis improvements performed by the author.

- Chapter 7 presents the data for both NOvA detectors and the analysis results.

- Chapter 8 concludes. 


\section{Chapter 2}

\section{Neutrino Physics}

The field of neutrino research is one the pillars of modern particle physics and has proved of great theoretical and experimental interest. Significant breakthroughs in the latter half of the 20th century culminated in the discovery of oscillation - courtesy of the SuperK and SNO experiments. This result could not be explained via the standard model and was rewarded with the 2015 Nobel Prize for physics. This chapter briefly covers the history, underlying theory and experimental results that have paved the way for the NOvA experiment.

\subsection{Discovery of the Neutrino}

The field of neutrino physics was born in the early 20th century. In 1914 James Chadwick observed the continuous nature of the $\beta$ decay energy distribution [6]. This result was problematic - it suggested the violation of energy and angular momentum conservation or some other new physics. It was not until 1930 that the correct theoretical solution was found. The energy deficit was caused by the presence of a particle that would later become known as the neutrino (Italian for "the little neutral one"). Postulated by Wolfgang Pauli, this particle would have to be small in mass and neutrally charged to fit observation. These properties explained the invisible loss of energy in $\beta$ decay but made the neutrino very difficult to detect. In 1934 Fermi [7] devised a theory of $\beta$ decay that involved this extra particle and the neutrino was given its name. Early estimates by Bethe and Peierls suggested that many light years of solid matter would be required to ensure a neutrino would interact [8].

The first direct evidence for the neutrino was provided by Cowan and Reines in 1956 [9]. A nuclear reactor was used as a neutrino source to observe inverse $\beta$ decay $\left(p+\bar{\nu} \rightarrow n+e^{+}\right)$. 
The produced in such an event first annihilates with an electron giving a prompt signal. This is then followed by a secondary signal which occurs after neutron capture. The delayed coincidence between these two signals was key to the discovery.

The $\nu_{e}$ was the only known neutrino until 1962 [10] when Brookhaven laboratory designed an experiment to look at muon production in neutrino interactions. The experiment used a spark chamber to detect neutrinos originating from a pion decay source. 34 muons were identified with energy greater than $300 \mathrm{MeV} / \mathrm{c}$. The $\nu_{\mu}$ was postulated as the analogous particle to the $\nu_{e}$ to explain this. The $\nu_{e}$ and $\nu_{\mu}$ particles were considered flavour distinct at this time. The discovery of the $\nu_{\tau}$ particle did not happen until 2000 courtesy of the Fermilab-based experiment DONUT [11]. This discovery was made by analysis of 203 neutrino interactions in nuclear emulsion targets. $4 \pm 0.34 \nu_{\tau}$ candidates were identified from $\tau$ lepton signatures. With the discovery of the $\nu_{\tau}$, a neutrino corresponding to each standard model lepton had been observed.

The total number of active light neutrinos was first measured by ALEPH in 1989 . ALEPH constrained there to be three such particles at the $98 \%$ confidence level through measurements of the mass and width of the Z-boson [12]. In 2005, ALEPH conclusively proved this result, showing the number of light neutrino species to be $2.9840 \pm 0.0082$ [13]. To do this ALEPH analyzed 18 million $\mathrm{Z}$ decays. The partial $\mathrm{Z}$ decay width attributable to neutrino pair production is proportional to the number of neutrino flavours.

\subsection{The Neutrino Model}

Neutrinos are neutrally charged particles with tiny mass that only interact weakly. At an experimentally observable scale this means that neutrinos cannot be tracked through their energy loss in matter. Consequently, the neutrino is indirectly detected via the charged particles produced or affected in weak interactions. The neutrino flavour is defined by the leptonic charged particle associated with a given charged-current weak interaction. For example the $\nu_{e}$ neutrino is defined as the neutrino state produced alongside a positron. The $\bar{\nu}_{e}$ interaction-flavour dependence was first observed in nuclear beta decay. When a positron was produced in combination with a neutrino, the final state lepton after the neutrino interacted would be an electron. It appeared that neutrinos produced in association with matter or antimatter were distinct. Beam neutrino experiments such as the Brookhaven experiment supported this theory as $\pi^{+} \rightarrow \mu^{+}+\nu_{\mu}$ decays always produced a muon in charged-current interactions. Additionally, the nonobservance of the process $\mu^{-} \rightarrow e^{-}+\gamma$ (the Feynman diagram for which can be admitted as shown 


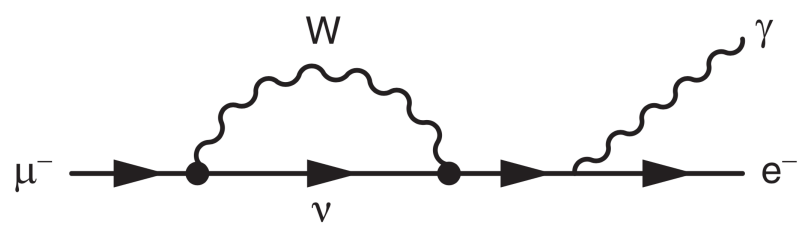

Figure 2.1: A possible path for a muon to produce an electron if the neutrino is not flavour distinct. [4]

in 2.1) suggested that the neutrino associated with $\mathrm{W} \mu^{-} \nu$ vertex was distinct from the neutrino associated with the $\mathrm{W} e^{-} \nu$ vertex. Additionally, chiral suppression of the neutrino interactions meant that the right handed matter and left handed anti-matter chiral states were never observed [14] - suggesting a massless particle. This body of evidence led physicists to conclude that the neutrino states $\left(\nu_{e}, \nu_{\mu}\right.$ and $\left.\nu_{\tau}\right)$ were massless and that the neutrino states were distinct.

Atmospheric Neutrinos The Earth's atmosphere is continuously bombarded with cosmic rays. The vast majority (95\%) of these are protons. Alpha particles make up approximately $5 \%$ and heavier nuclei and electrons make up less than 1\%. Hadronic showers are produced when a cosmic ray interacts with a nuclei in the atmosphere. Atmospheric neutrinos are produced from the decay of these hadrons. The dominant part of the decay chain is:

$$
\pi^{+} \rightarrow \mu^{+} \nu_{\mu}, \mu^{+} \rightarrow \mathrm{e}^{+} \nu_{e} \bar{\nu}_{\mu} \text { and } \pi^{-} \rightarrow \mu^{-} \bar{\nu}_{\mu}, \mu^{-} \rightarrow \mathrm{e}^{-} \bar{\nu}_{e} \nu_{\mu}
$$

At higher energies Kaon decay also becomes relevant. Detectors that target atmospheric neutrinos experience a neutrino flux with flight paths ranging in length from $15 \mathrm{~km}$ to $13,000 \mathrm{~km}$. The $15 \mathrm{~km}$ flight path interactions correspond to events entering the detector top down through the atmosphere. The 13,000 km flight path interactions enter the detector through the bottom face, having passed through the atmosphere and then through the earth. From the dominant decay processes shown in Equation 2.1, one would expect the ratio $\mathrm{R}=\frac{\nu_{\mu}+\bar{\nu}_{\mu}}{\nu_{e}+\bar{\nu}_{e}}$ to be equal to two. Super-Kamiokande measured this ratio at $0.61 \pm 0.03$ (stat.) \pm 0.05 (sys.), approximately a third of expectation.

Solar Neutrinos Nuclear fusion in the Sun produces a large flux of electron neutrinos. This rate is estimated from the standard solar model as $2 \times 10^{38} \nu_{e} s^{-1}$.

The standard solar model suggests three major neutrino sources:

1: $p$ p neutrinos $\left(p+p \rightarrow d+e^{+}+\nu_{e}, E_{\max .}=420 \mathrm{keV}\right)$. 
2: ${ }^{7} \mathrm{Be}$ neutrinos $\left({ }^{7} \mathrm{Be}+e^{-} \rightarrow{ }^{7} \mathrm{Li}+\nu_{e}, E_{\max .}=860 \mathrm{keV}\right)$.

3: ${ }^{8} \mathrm{~B}$ neutrinos $\left({ }^{8} \mathrm{~B}+e^{-} \rightarrow{ }^{8} \mathrm{Be}+e^{+}+\nu_{e}, E_{\max .}=14 \mathrm{MeV}\right)[15]$.

The main hydrogen burning process, known as the pp cycle, proceeds in three steps:

$$
\begin{gathered}
p+p \rightarrow \mathrm{D}+e^{+}+\nu_{e}, \\
\mathrm{D}+p \rightarrow{ }_{2}^{3} \mathrm{He}+\gamma, \\
{ }_{2}^{3} \mathrm{He}+{ }_{2}^{3} \mathrm{He} \rightarrow{ }_{2}^{4} \mathrm{He}+\mathrm{p}+\mathrm{p} .
\end{gathered}
$$

Neutrinos produced in the first process have low energies (less than $0.5 \mathrm{MeV}$ ) making them difficult to detect. The majority of solar neutrino experiments have focused on the detection of the higher-energy solar neutrinos from rarer fusion processes. The second highest energy solar neutrinos originate from the $\beta$-decay of boron- $8\left({ }^{8} \mathrm{~B}\right)$. Neutrinos are produced as a result of beta decay following the fusion of two helium nuclei. This process proceeds as follows:

$$
\begin{gathered}
{ }_{2}^{4} \mathrm{He}+{ }_{2}^{3} \mathrm{He} \rightarrow{ }_{4}^{7} \mathrm{Be}+\gamma, \\
{ }_{4}^{7} \mathrm{Be}+\mathrm{p} \rightarrow{ }_{5}^{8} \mathrm{~B}+\gamma,
\end{gathered}
$$

with the subsequent $\beta$-decay:

$$
{ }_{5}^{8} \mathrm{~B} \rightarrow{ }_{4}^{8} \mathrm{Be}^{*}+e^{+}+\nu_{e},
$$

with neutrino energies up to $15 \mathrm{MeV}$.

\subsubsection{The Solar Neutrino Problem, Super-Kamiokande and SNO}

Experimental data collected at Homestake in the 1960s suggested further depth to the neutrino model. The results would prove incompatible with the massless, flavour distinct neutrino model.

In 1968 Ray Davis et al. published a paper entitled "Search For Neutrinos From the Sun" [16]. Davis' experiment, located in a mine in South Dakota, employed a radiochemical technique to measure the flux of ${ }^{8} \mathrm{~B}$ and ${ }^{7} \mathrm{Be}$ solar electron neutrinos. It consisted of a tank of 615 tons of dry-cleaning fluid, $\mathrm{C}_{2} \mathrm{Cl}_{4}$. The solar neutrino flux was measured by counting the number of ${ }^{37} \mathrm{Ar}$ atoms produced in inverse $\beta$-decay resulting from ${ }^{37} \mathrm{Cl}+\nu \rightarrow e^{-}+{ }^{37} \mathrm{Ar}$ interactions. The ${ }^{37} \mathrm{Ar}$ atoms were extracted from the tank and counted through their subsequent radioactive decays.

Ray Davis expected the solar neutrino flux to yield $1.7 \frac{\text { interactions }}{\text { day }}$. This prediction came from a derivation by Bahcall et al. who pioneered the standard solar model [17]. In actuality, $0.48 \pm 0.04$ events were observed. This neutrino flux was approximately a 
third of the expectation, a deficit that became known as the 'Solar Neutrino Problem. The Homestake experiment was only sensitive to high energy ${ }^{8} \mathrm{~B}$ neutrinos. The deficit was later observed and confirmed by the radiochemical experiments SAGE [18] and GALLEX [15] - both of which were sensitive to a broader neutrino energy range. Ray Davis' Homestake result won the Nobel Prize in 2002.

It appeared that the standard solar model was either incorrect or that some fraction of the electron neutrinos produced in solar fusion did not survive propagation to Earth. Many solutions were posited but the solar neutrino deficit went unresolved for many years. Building on a previous theory that Pontecorvo authored, Maki, Nakagawa, and Sakata formulated a theory to explain the neutrino deficit and published their solution in 1968. Their answer was a 3 -flavour $\left(\nu_{e}, \nu_{\mu}\right.$ and $\left.\nu_{\tau}\right)$ oscillation model that described how neutrinos transform from one flavour eigenstate to another [19, 20, 21]. They postulated that oscillation manifests due to a varying superposition of a neutrino's quantum mass eigenstates during flight. Due to the neutrino's small interaction cross-section physicists had limited means of probing this theory for many years. In the late 1990's and early 2000's Super-Kamiokande and SNO finally provided the solution. Both experiments utilised massive detectors but while SNO focused almost entirely on solar neutrinos, Super-K targeted measurements of both solar and atmospheric neutrino sources. The combined effort from both experiments provided the first conclusive evidence of neutrino oscillation. The 2015 Nobel Prize in Physics was awarded to leading members of both collaborations for their discovery.

Super-Kamiokande is a 50,000-ton water Cherenkov detector. Surrounded by photomultiplier tubes (PMTs) capable of detecting single photons, Super-K detected solar neutrinos in the elastic scattering process shown in Figure 2.2. This process has the same initial and final state (an electron neutrino and electron) albeit with differing kinematics. Super$\mathrm{K}$ was sensitive down to electron neutrino energies of $5 \mathrm{MeV}$, below which the $\beta$-decay background of radioisotopes dominated the signal. Like Homestake this limited the study to, primarily, neutrinos produced in ${ }^{8} \mathrm{~B}$ decay. But unlike Homestake, Super-K was able to retain directional information from the incoming neutrino. The cross section for $\nu_{e^{-}}$ electron elastic scattering has a $\cos \theta$ dependence, where $\theta$ is the polar angle of the electron with respect to the direction of origin. The scattering angle of a neutrino is isotropic in the centre-of-mass frame, and thus because the COM frame is boosted in the direction of the incoming neutrino, the directional dependence of the interaction is maintained. In this way Super-K could determine the flux of electron neutrinos originating from the sun. The 

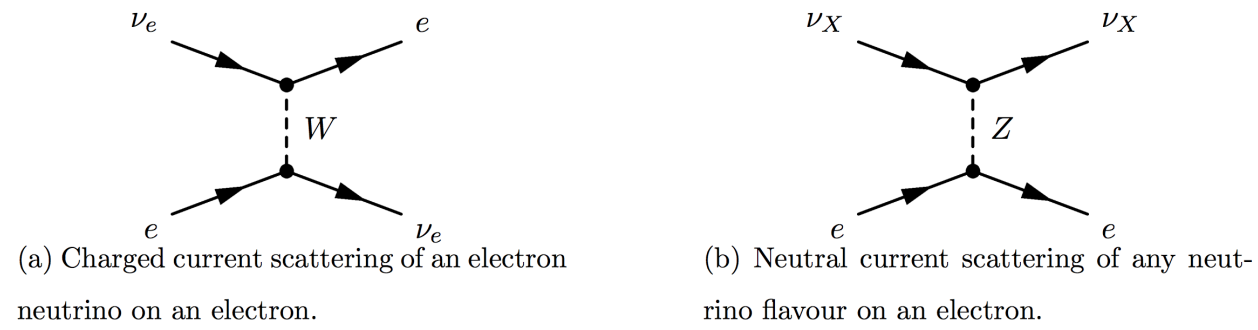

Figure 2.2: Feynman diagrams of the weak force neutrino interactions through the charged current and neutral current mediated by the $\mathrm{W}$ and $\mathrm{Z}$ bosons respectively - the two processes contributing to elastic scattering that Super-K observed. The lepton flavour, $l$, is conserved for both charged and neutral current interactions.

flux from the Sun was less than half of the expectation, confirming the result at Homestake and providing strong evidence for neutrino flavour oscillation [22].

The SNO experiment was built to measure both the electron neutrino and total neutrino flux from the sun [23]. It was similar in design to Super-K, consisting of 1000 tonnes of heavy water $\left(\mathrm{D}_{2} \mathrm{O}\right)$ inside a $12 \mathrm{~m}$ acrylic vessel, surrounded by 9600 PMTs. The use of heavy water, which has a low $(2.2 \mathrm{MeV})$ nuclear binding energy, allowed SNO to detect solar neutrinos through multiple processes, including the charged-current electron neutrino interaction, which was kinematically forbidden in Super-K. Each of these processes had a different dependency on the flux of electron, muon and tau neutrinos, allowing greater insight into the solar neutrino deficit. The experimental data from SNO revealed that the total flux of neutrinos from the sun is consistent with the standard solar model. However, the observed flux comprised a large $\nu_{e}$ deficit. The flux composition of this non- $\nu_{e}$ component was not measured but the results were compatible with the PMNS model. The SNO and Super-K experiments had made the discovery of neutrino oscillations.

The results published by SNO and Super-K lead to a wealth of experiments focused on characterising oscillation and determining neutrino properties. The oscillation parameters, matter-antimatter neutrino nature, the strength of the matter-neutrino interaction couplings, the neutrino mass, the mass origin and the absolute number of neutrino flavours have all been constrained [24]. The confirmation of the oscillation effect has opened new questions in the standard model regarding the origin of fermion masses and the relationship between quarks and leptons [25]. 


\subsubsection{Neutrino Interactions, Oscillations and the PMNS matrix}

When a neutrino interacts with matter it does so as one of the the three weak interaction eigenstates $\left(\nu_{e}, \nu_{\mu}\right.$ and $\left.\nu_{\tau}\right)$. The mediating particle may be the electrically charged $\mathrm{W}^{ \pm}$ gauge boson or the electrically neutral $\mathrm{Z}$ gauge boson. Figure 2.2 shows the lowest order Feynman diagrams for neutrino interactions mediated by each gauge boson, $\mathrm{W}$ and $\mathrm{Z}$. The electrically charged $\mathrm{W}$ boson interactions are known as charged current interactions. The neutral $\mathrm{Z}$ boson interactions are known as neutral current interactions. In the case of charged current interactions, the lepton flavour produced corresponds to the weak eigenstate flavour. Similarly a charged lepton producing a neutrino will yield a weak eigenstate neutrino of the same flavour.

Neutrino oscillation is a quantum phenomenon. The effect is best described by the relationship between the eigenstates of the weak interaction $\nu_{e}, \nu_{\mu}$ and $\nu_{\tau}$ and the eigenstates of the free particle Hamiltonian, known as the mass eigenstates, $\nu_{1}, \nu_{2}$ and $\nu_{3}$.

The basis of weak eigenstates can be related to the basis of mass eigenstates by the unitary matrix shown in Equation 2.4. This matrix is known as the Pontecorvo-MakiNakagawa-Sakata (PMNS) matrix.

$$
\begin{aligned}
U & =\left(\begin{array}{lll}
U_{e 1} & U_{e 2} & U_{e 3} \\
U_{\mu 1} & U_{\mu 2} & U_{\mu 3} \\
U_{\tau 1} & U_{\tau 2} & U_{\tau 3}
\end{array}\right) \\
& =\left(\begin{array}{ccc}
c_{13} c_{12} \\
-c_{23} s_{12}-s_{13} s_{23} c_{12} e^{i \delta} & c_{23} c_{12}-s_{13} s_{23} s_{12} e^{i \delta} & c_{13} s_{23} \\
s_{23} s_{12}-s_{13} c_{23} c_{12} e^{i \delta} & -s_{23} c_{12}-s_{13} c_{23} s_{12} e^{i \delta} & c_{13} c_{23}
\end{array}\right) \\
& =\left(\begin{array}{ccc}
1 & 0 & 0 \\
0 & c_{23} & s_{23} \\
0 & -s_{23} & c_{23}
\end{array}\right)\left(\begin{array}{ccc}
c_{13} & 0 & s_{13} e^{-i \delta} \\
0 & 1 & 0 \\
-s_{13} e^{i \delta} & 0 & c_{13}
\end{array}\right)\left(\begin{array}{ccc}
c_{12} & s_{12} & 0 \\
-s_{12} & c_{12} & 0 \\
0 & 0 & 1
\end{array}\right)
\end{aligned}
$$

where $s_{i j} \equiv \sin \theta_{i j}, c_{i j} \equiv \cos \theta_{i j}$ and $\delta$ is the $\mathrm{CP}$ violating phase. A non-zero and non- $\pi$ value of $\delta$ would give rise to charge-parity violation in the lepton sector [26].

From Equation 2.4 it can be seen that each weak eigenstate is a linear combination of the mass eigenstates defined by the relative charged-current weak interaction couplings of the mass eigenstates, and vice versa. As such a neutrino of definite weak flavour, $\alpha$, can be written as a linear combination of the mass states; 


$$
\left|\nu_{\alpha}>=\sum_{i=1}^{3} U_{\alpha i}^{*}\right| \nu_{i}>,
$$

where $U_{\alpha i}^{*}$ is the PMNS matrix element (Pontecorvo, Maki, Nakagawa, and Sakata) that encapsulates the coupling strength between the mass state $i$ and the flavour state $\alpha$.

The neutrino state propagates as a coherent linear superposition of the three mass eigenstates until the point of interaction, wherein it collapses to a weak eigenstate, producing a lepton of corresponding flavour. If the mass eigenstates are not of a common mass, a phase difference can exist between the different components of the wave-function and neutrino oscillation can occur. A neutrino created with a given flavour may interact at a later time to produce a lepton of different flavour.

The PMNS matrix can be described by three real parameters and a single phase. The unitarity of the PMNS matrix introduces six additional degrees of freedom in the form $\exp (\mathrm{i} \delta)$. However not all of these have physical relevance and thus five of them can be absorbed into the definition of the particles without consequence. The PMNS can therefore be expressed using three mixing angles $\theta_{12}, \theta_{23}$ and $\theta_{13}$ and a single complex phase $\delta$. It is commonly decomposed into the three separate 3 -by-3 matrices, expressed using the four aforementioned parameters, as shown in Equation 2.4.

\subsubsection{Neutrino Oscillation Probability in Vacuum}

The following derivation of the neutrino oscillation probability follows [24] and [27]. A neutrino is produced via a weak interaction as a flavour eigenstate. At time $t=0$ the flavour state, $\alpha$, can be written as the sum of the mass eigenstates, as in Equation 2.6

$$
\left|\nu_{\alpha}(t=0)>=\sum_{i=1}^{3} U_{\alpha i}^{*}\right| \nu_{i}>.
$$

As the neutrino propagates the mass states evolve. At a later time, $t$, we have,

$$
\left|\nu_{\alpha}(t)>=\sum_{i=1}^{3} U_{\alpha i}^{*} e^{-i p_{i} \cdot x}\right| \nu_{i}>,
$$

where $p_{i}$ is the four-momentum and $x$ the four-position of mass state $\nu_{i}$. At time the neutrino weakly interacts with matter in flavour state $\beta$,

$$
\begin{aligned}
<\nu_{\beta} \mid \nu_{\alpha}> & =\sum_{j=1}^{3} \sum_{i=1}^{3} U_{\beta j} U_{\alpha i}^{*} e^{-i p_{i} \cdot x}<\nu_{j} \mid \nu_{i}> \\
& =\sum_{j=1}^{3} U_{\beta j} U_{\alpha j}^{*} e^{-i p_{j} \cdot x} .
\end{aligned}
$$


Assuming all mass states have the same three-momentum $\mathbf{p}^{1}$,

$$
\begin{aligned}
p_{j} \cdot x & =E_{j} t-\mathbf{p} \cdot \mathbf{x} \\
& =t \sqrt{|\mathbf{p}|^{2}+m_{j}^{2}}-\mathbf{p} \cdot \mathbf{x}
\end{aligned}
$$

Since neutrinos are extremely light $\left(m_{\nu}<2 \mathrm{eV}[24]\right)$ we can make the approximations, $m_{j}<<E_{j}, t=L$ and $\mathbf{p} \cdot \mathbf{x}=|\mathbf{p}| L$. Using a binomial expansion we find,

$$
p_{j} \cdot x=|\mathbf{p}| L\left(1+\frac{m_{j}^{2}}{2|\mathbf{p}|^{2}}\right)-|\mathbf{p}| L=\frac{m_{j} L}{2 E}
$$

Combining Equations 2.8 and 2.10 we get $\left\langle\nu_{\beta} \mid \nu_{\alpha}\right\rangle=\sum_{j=1}^{3} U_{\beta j} U_{\alpha j}^{*} e^{-i \frac{m_{j} L}{2 E}}$.

The probability of observing the neutrino in flavour state $\beta$ after travelling distance $L$ given initial flavour state $\alpha$ is given in Equation 2.11 .

$$
\begin{aligned}
P_{\alpha \rightarrow \beta} & =\mid\left\langle\nu_{\beta}(t)\left|\nu_{\alpha}(t)>\right|^{2}\right. \\
& =\left(\sum_{j=1}^{3} U_{\beta j} U_{\alpha j}^{*} e^{-i \frac{m_{j} L}{2 E}}\right)\left(\sum_{i=1}^{3} U_{\beta i}^{*} U_{\alpha i} e^{i \frac{m_{i} L}{2 E}}\right) .
\end{aligned}
$$

Equation 2.12 then follows from Equation 2.11.

$$
\begin{aligned}
P_{\alpha \rightarrow \beta}=\delta_{\alpha \beta} & -4 \sum_{i>j} \mathfrak{R}\left[U_{\alpha i}^{*} U_{\alpha j} U_{\beta i} U_{\beta j}^{*}\right] \sin ^{2}\left(\frac{\Delta m_{i j}^{2}}{4 E} L\right) \\
& +2 \sum_{i>j} \mathfrak{I}\left[U_{\alpha i}^{*} U_{\alpha j} U_{\beta i} U_{\beta j}^{*}\right] \sin ^{2}\left(\frac{\Delta m_{i j}^{2}}{2 E} L\right),
\end{aligned}
$$

where $\Delta m_{i j}^{2} \equiv m_{i}^{2}-m_{j}^{2}$ and $\delta_{\alpha \beta}$ is the Kronecker delta. The equation shows that the neutrino oscillation probability depends on the parameters of the PMNS matrix and the value of the two sinusoidal arguments. The probability depends on the mass splittings $\Delta m_{21}^{2}, \Delta m_{31}^{2}, \Delta m_{32}^{2}$, and varies with the length of the baseline, $L$, and the energy of the neutrino beam, $E$. Only two of the mass splittings are independent and (with knowledge of the mass ordering) the third can be described in terms of the other two. The smaller mass splitting, $\Delta m_{21}^{2}$, is often referred to as the "solar mass splitting" due to its role in oscillation regarding neutrinos from the sun. While the larger weighted average of the other two, $\Delta m_{32 / 31}^{2}$, is often named the "atmospheric mass splitting", $\Delta m_{\text {atm }}^{2}$ due to its role in atmospheric neutrino oscillations.

\footnotetext{
${ }^{1} \mathrm{~A}$ more exact method is to use the wave packet treatment but this has been shown to produce the same result for our needs [28].
} 


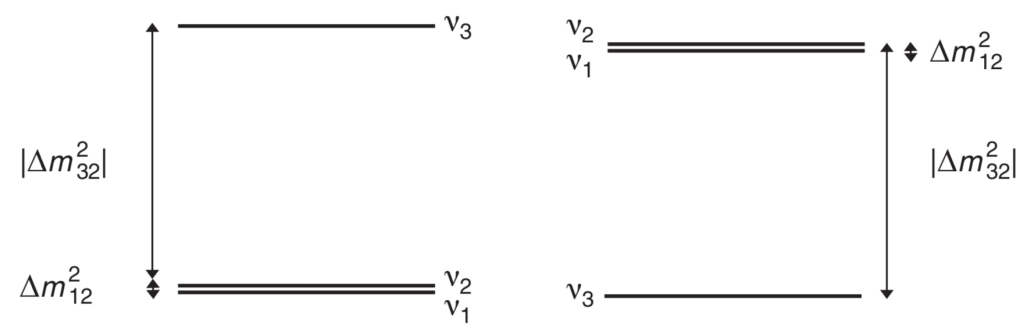

Figure 2.3: The two possible mass hierarchies. The normal is shown on the left, the inverted on the right. [4]

\subsection{Neutrino Masses and Hierarchies}

Experimental measurements of neutrino oscillations are not dependent on the absolute neutrino mass scale. At the time of writing, there are no direct measurements of the neutrino masses, just limits imposed by the currently available data. The bounds for neutrino mass are set by measurements of $\Delta m_{\text {atm }}^{2}=\Delta m_{32}^{2}$ and $\Delta m_{\text {sun }}^{2}=\Delta m_{21}^{2}$ from atmospheric and solar experiments respectively. As such, neutrino masses are known to be smaller than the electron mass by about six orders of magnitude. This has led to Higgs-alternative theories for the method of neutrino mass generation. The best known such theory is the Seesaw mechanism, which will be addressed later.

There are two remaining scenarios for the neutrino mass orderings shown in Figure 2.3. These have been labeled the normal hierarchy and the inverted hierarchy with $\mathrm{m}_{3}>\mathrm{m}_{2}$ and $\mathrm{m}_{3}<\mathrm{m}_{2}$ respectively. Previous experiments were not capable of distinguishing between these two possibilities. NOvA has been designed, in part, to answer this question.

Recent measurements of the neutrino mass squared differences have yielded;

$$
\begin{gathered}
\Delta m_{21}^{2}=\mathrm{m}_{2}^{2}-\mathrm{m}_{1}^{2} \approx 8 \times 10^{-5} \mathrm{eV}^{2} \\
\left|\Delta m_{32}^{2}\right|=\left|\mathrm{m}_{3}^{2}-\mathrm{m}_{2}^{2}\right| \approx 2.5 \times 10^{-3} \mathrm{eV}^{2}
\end{gathered}
$$

\subsection{CP and T Violation in Neutrino Oscillations}

$\mathrm{CP}$ violation is a known requirement for the observed baryonic-asymmetry of the universe [29]. QED and QCD interactions are both invariant under $\mathrm{C}$ and $\mathrm{P}$ transformations (though CP violation is possible in the QCD sector but has never been experimentally observed) and thus the weak interaction is the only $\mathrm{CP}$ violation admissible sector of the standard model. Furthermore, it is clear that the vector nature of both QCD and QED 
makes the respective matrix elements invariant under $\mathrm{T}$ transformations. CPT conservation is currently considered an exact symmetry of the universe. Any CP violation thus implies that $\mathrm{T}$ reversal symmetry is also violated.

The oscillation probability of a $\nu_{e}$ neutrino to a $\nu_{\mu}$ neutrino can be expressed using the elements of the 3x3 PMNS matrix, as well as the squared mass differences. Both the normal elements and complex conjugate elements are required, as necessary for the associated probability integrals. This probability is dependent on both the energy and propagation length of an initial neutrino. To obtain the probability of the reverse oscillation, i.e. that of a $\nu_{\mu}$ neutrino to a $\nu_{e}$ neutrino, one need simply exchange the labelling of the PMNS matrix elements such that $\mu \rightarrow e$ and vice versa. The matrix elements that appear in the expression for the first probability are the complex conjugates of the matrix elements that occur in the second probability. Hence, unless all elements of the PMNS matrix are real, time reversal symmetry may not be true for neutrino oscillations. The full mathematical treatment of this discussion is not shown for the sake of brevity.

One can use the same argument invoked above for CP symmetry. The CP operation transforms particles to their anti-particle states (or vice versa) and flips their handedness. Analogous to $\mathrm{T}$ symmetry, the probability of a $\nu_{e}$ oscillating to a $\nu_{\mu}$ can be shown as different to the probability of a $\bar{\nu}_{e}$ going to a $\bar{\nu}_{\mu}$ unless all matrix elements of the PMNS are real. Hence CP violation can be accommodated by neutrino oscillations. This result is somewhat expected as CPT is thought to be an exact symmetry of the universe. Neutrino oscillations violating $\mathrm{T}$ symmetry implies that they must also violate $\mathrm{CP}$ symmetry since neutrino oscillations are invariant under the combined action of CPT.

A measurement of the relative magnitude of the $\mathrm{CP}$ violation effect in neutrino oscillations can be provided by measuring the probability difference $\mathrm{P}\left(\nu_{\mu} \rightarrow \nu_{e}\right) \quad \mathrm{P}\left(\bar{\nu}_{\mu} \rightarrow \bar{\nu}_{e}\right)$.

\subsubsection{The Matter Effect}

When propagating through matter neutrinos experience what's known as the MikheyevSmirnov-Wolfenstein effect, often dubbed the matter effect. It describes weak force interactions neutrinos experience in the form of coherent forward scattering when propagating through matter. These interactions are non-negligible, despite the diminutive neutrino interaction cross sections, and has a measured effect on propagation at the energy scale relevant to NOvA and other long-baseline experiments.

Coherent forward scattering can occur through both charged current and neutral current weak interactions. Only the charged current interactions affect oscillation as the 
neutral current interactions do not affect flavour. The lowest-order Feynman diagrams which admit the scattering of neutrinos on electrons are shown in Figure 2.4. Figure 2.4c shows neutral current scattering. Figure 2.4a and Figure 2.4b show the charged current scattering diagrams. The $\nu_{e}$ is the only neutrino flavour in the $\mathrm{CC}$ diagrams because electrons are the only lepton found in matter. This charged current coherent scattering is different for $\nu_{e}$ and $\bar{\nu}_{e}$ and gives rise to a false CP-violation effect such that $\nu_{e}$ appearance is boosted (suppressed) for normal (inverted) hierarchy and vice versa for $\bar{\nu}_{e}$. For example, the additional normal hierarchy $\nu_{e}$ appearance increases $\nu_{\mu}$ disappearance and/or decreases $\nu_{\tau}$ appearance. This occurs because of the effect on the scattering amplitude of $\nu_{e}\left(\bar{\nu}_{e}\right)$ which results in an oscillation probability that differs from vacuum expectation. [30, 31].

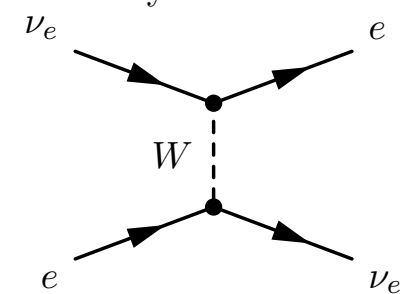

(a) Charge current scattering of a $\nu_{e}$ and electron.

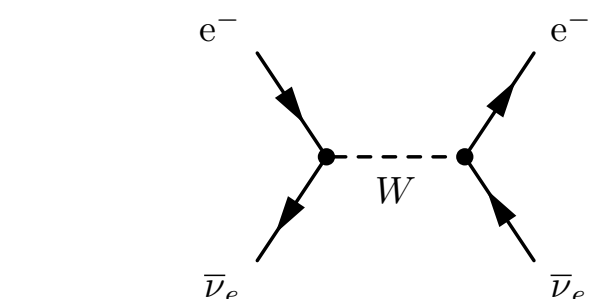

(b) Charge current scattering of a $\frac{\bar{\nu}_{e}}{\bar{\nu}_{e}}$ and electron.

(c) Neutral current scattering of a neutrino on an electron.

Figure 2.4: Feynman diagrams showing the $\mathrm{CC}$ and $\mathrm{NC}$ weak interactions contributing towards the matter effect.

\subsection{Neutrino Oscillation Experiments}

So far, the discussion of experimental oscillation results has been focused on solar neutrinos, atmospheric neutrinos and their respective historical significance. In recent years it has been long-baseline, accelerator and reactor experiments that have been responsible for much progress. Long-baseline neutrino oscillation experiments make two sets of meas- 
urements:

1. The appearance of a different charged lepton flavour from a beam of a given neutrino flavour.

2. The disappearance of a charged lepton flavour from a beam of the same neutrino flavour.

The features of a long-baseline neutrino experiment that make it suitable for physics discovery are discussed in the NOvA detector chapter (Chapter 3).

\subsection{Current Measurements of PMNS Matrix Parameters}

In the last twenty years significant contributions to the field have been made by myriad experiments. Reactor based oscillation experiments such as Daya Bay, KamLAND, RENO and Double Chooz alongside beam neutrino oscillation experiments such as MINOS, K2K, Super-K, T2K and NOvA have all examined the PMNS matrix and the oscillation effect. Advancements in hardware, simulation and analysis methodology have helped achieve some precise measurements. The latest results and experimental research are presented in this section.

\subsubsection{Measurement of $\theta_{12}$ and $\Delta m_{21}^{2}$}

A combination of reactor and solar neutrino experiments are used to constrain $\theta_{12}$ and $\Delta \mathrm{m}_{21}^{2} . \theta_{12}$ is most sensitively measured by solar experiments, chiefly SNO. $\Delta \mathrm{m}_{21}^{2}$ is most sensitively measured by reactor experiments, chiefly KamLAND.

Kamioka Liquid Scintillator Antineutrino Detector (KamLAND) is an underground experiment situated in the Japanese Alps, utilising 53 commercial nuclear reactors. The flux averaged baseline between the detector and reactors is $180 \mathrm{~km}$ [32]. The reactors produce $\bar{\nu}_{e}$ from the decay of radioactive fission products in the nuclear fuel. KamLAND measures the $\bar{\nu}_{e}$ flux from the reactors. The detector comprises a $13 \mathrm{~m}$ diameter transparent balloon filled with 1 kton of ultra-pure liquid scintillator. It is surrounded by non-scintillating oil [33].

Figure 2.5 shows the measured survival probability of electron antineutrinos against $\mathrm{L} / \mathrm{E}$ (the flux-averaged baseline divided by the neutrino energy). KamLAND's L/E range is sufficient to observe the oscillatory shape of the neutrino survival probability that arises due to oscillation. Figure 2.6 shows the confidence limit contours resulting from the fit of three-flavour oscillations to the KamLAND data. KamLAND measured $\Delta m_{21}^{2}=$ 


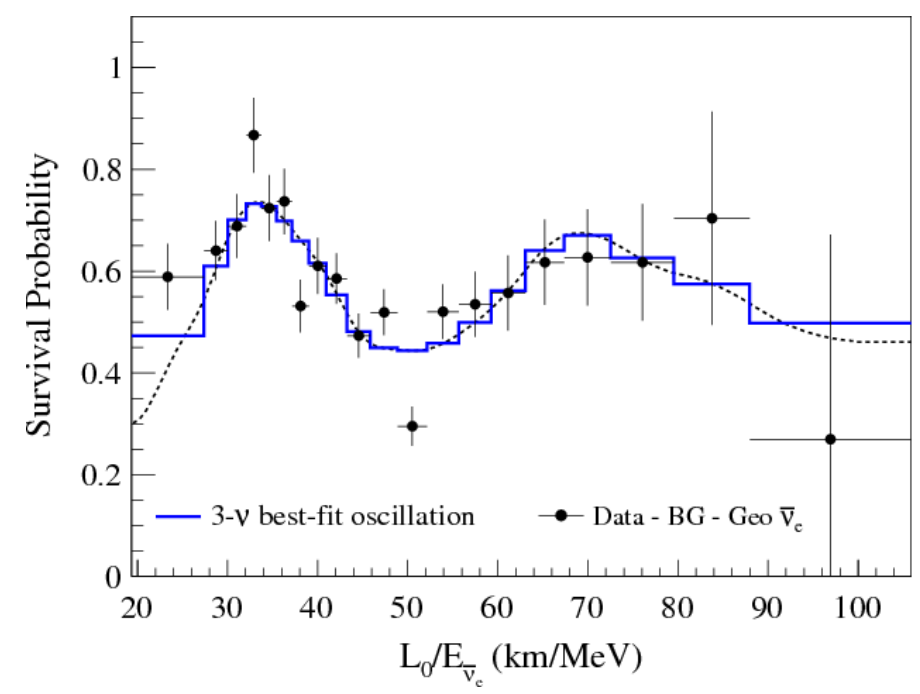

Figure 2.5: Measured survival probability of $\overline{\nu_{e}}$ against $L_{0} / E$ (effective baseline divided by the neutrino energy). KamLAND data is represented by the black data points. The three flavour oscillation fit to data is represented by the blue histogram. $\theta_{13}$ has been constrained in the fit by accelerator and short baseline reactor experiments. Figure from [34].

$7.59 \pm 0.21 \times 10^{-5} \mathrm{eV}^{2}$ and $\tan ^{2} \theta_{12}=0.47_{-0.05}^{+0.06}$ [34]. Regions allowed by KamLAND, the solar experiments and a combined analysis are shown by the black, blue and colour shaded regions respectively. It can be immediately seen that the KamLAND reactor result best constrains $\Delta m_{21}^{2}$ and that the solar result, primarily contributed to by SNO, best constrains $\theta_{12}$.

\subsubsection{Measurement of $\theta_{13}$}

The measurement of a non-zero $\theta_{13}$ occurred recently, in 2012. The Daya Bay experiment was the first to measure a non-zero value to greater than $5 \sigma$ confidence level [35]. This result was markedly significant for the field of neutrino research - a value of zero would have prohibited the possibility of $\mathrm{CP}$ violation through the $\delta_{C P}$ phase. This result also opened a door allowing the neutrino mass hierarchy and $\theta_{23}$ octant to be probed.

The Daya Baya neutrino experiment is a reactor based nuclear power complex located in China. Six reactor cores with a total thermal energy of 17.4 GWh provide a strong flux of electron antineutrinos. There are three underground experimental halls (EHs) - two near halls and one far hall. The near-hall detectors measure the neutrino flux from the reactor cores with almost no effect from $\theta_{13}$ whereas the far-hall detectors measure the oscillation effect caused by the $\theta_{13}$ mixing angle. Each near hall contains two antineutrino detectors and the far hall contains four detectors. 


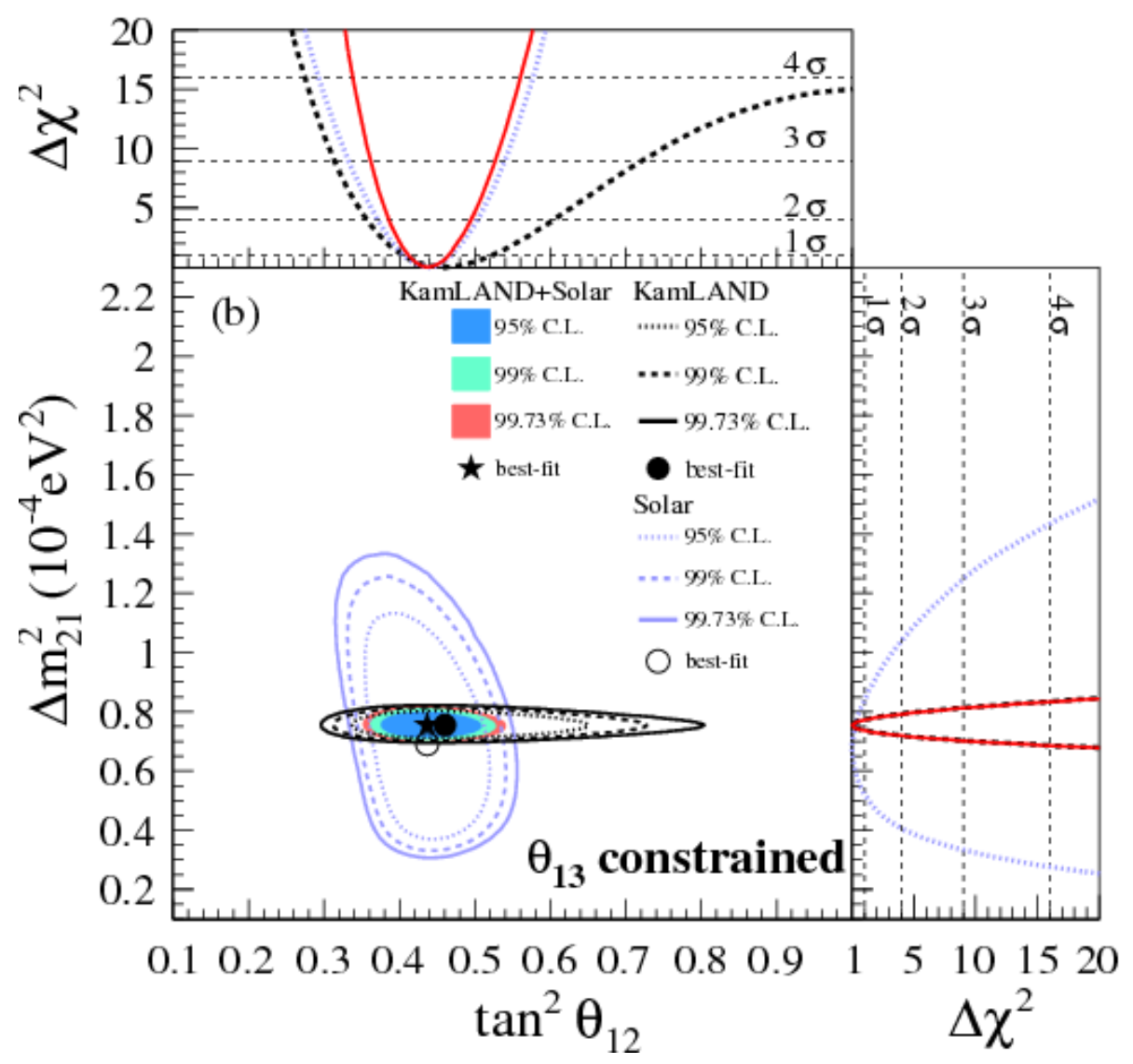

Figure 2.6: Contour plot showing the allowed regions in the $\Delta m_{21}^{2}$ against $\tan ^{2} \theta_{12}$ phase space. The blue and black contours show the regions allowed by the KamLAND and Solar experiments respectively. A combined analysis of the solar and KamLAND data is shown by the colour shaded regions. The value of $\theta_{13}$ is constrained by the accelerator and the short baseline reactor experiments. Figure taken from [34]. 
Daya Bay detectors comprise a large vessel holding 20 tons of liquid scintillator loaded with gadolinium. The vessels are outfitted with photo-multiplier tube arrays. Electron antineutrinos are detected by the inverse $\beta$-decay reaction $\nu_{e}+\mathrm{p} \rightarrow e^{+}+n$. The positron ionizes the scintillator and the subsequent annihilation of the positron with an electron yields two prompt gammas. The low-energy neutron scatters in the liquid scintillator until it is captured by a gadolinium nucleus. The neutron capture, which occurs on a timescale of $30 \mu \mathrm{s}$, produces gammas from $\mathrm{n}+\mathrm{Gd} \rightarrow \mathrm{Gd} * \rightarrow \mathrm{Gd}+\gamma$ processes. The gammas from both the annihilation process and neutron capture produce Compton scattered electrons. These electrons ionise the liquid scintillator producing scintillation light. The coincidence of a prompt pulse of scintillation light from the annihilation and a delayed pulse from the neutron capture $10100 \mu \mathrm{s}$ later is the experimental signature of a $\bar{\nu}_{e}$ interaction. Neutrino energy is estimated from the light yield.

Figure 2.7 shows the electron antineutrino survival probability against the effective propagation distance divided by the average antineutrino energy. The effective propagation distance is calculated by equating the actual flux to an effective flux using a single baseline [36]. The measured survival probabilities at experiment halls 1, 2 and 3 are shown by the blue, green and black data-points respectively. A best fit to the data measured in the experiment halls is shown by the solid red line. The regions allowed (in the $\left|\Delta m_{e e}^{2}\right|$ vs. $\sin ^{2} \theta_{13}$ plane) by the fit to the data is shown in the contour plot in Figure 2.8 where $\left|\Delta m_{e e}^{2}\right|$ is the effective mass-squared difference defined as

$$
\left|\Delta \mathrm{m}_{e e}^{2}\right|=\left|\Delta \mathrm{m}_{31}^{2}\right| \pm\left(\left|\Delta \mathrm{m}_{21}^{2}\right|-\Delta \mathrm{m}_{\phi}^{2}\right)
$$

where

$$
\phi=\tan ^{-1}\left(\frac{\sin 2 \Delta_{21}}{\cos 2 \Delta_{21}+\tan ^{2} \theta_{12}}\right)
$$

and

$$
\Delta \mathrm{m}_{\phi}^{2}=\phi \times \frac{4 E}{L}
$$

The Daya Bay analysis results gave $\sin ^{2} 2 \theta_{13}=0.084 \pm 0.005$ and $\left|\Delta m_{e e}^{2}\right|=(2.42 \pm$ $0.11) \times 10^{-3} \mathrm{eV}^{2}[36]$.

\subsubsection{Measurement of $\theta_{23}$ and $\left|\Delta m_{32}^{2}\right|$}

Long-baseline neutrino oscillation experiments have the best measurement sensitivity to $\theta_{23}$ and $\left|\Delta m_{32}^{2}\right|$. The experiments MINOS, Super-K, T2K and NOvA have all made measurements of these parameters. These experiments are characterised by beam energies of between 0.5 and $10 \mathrm{GeV}$ and baselines of order hundreds of kilometres. 


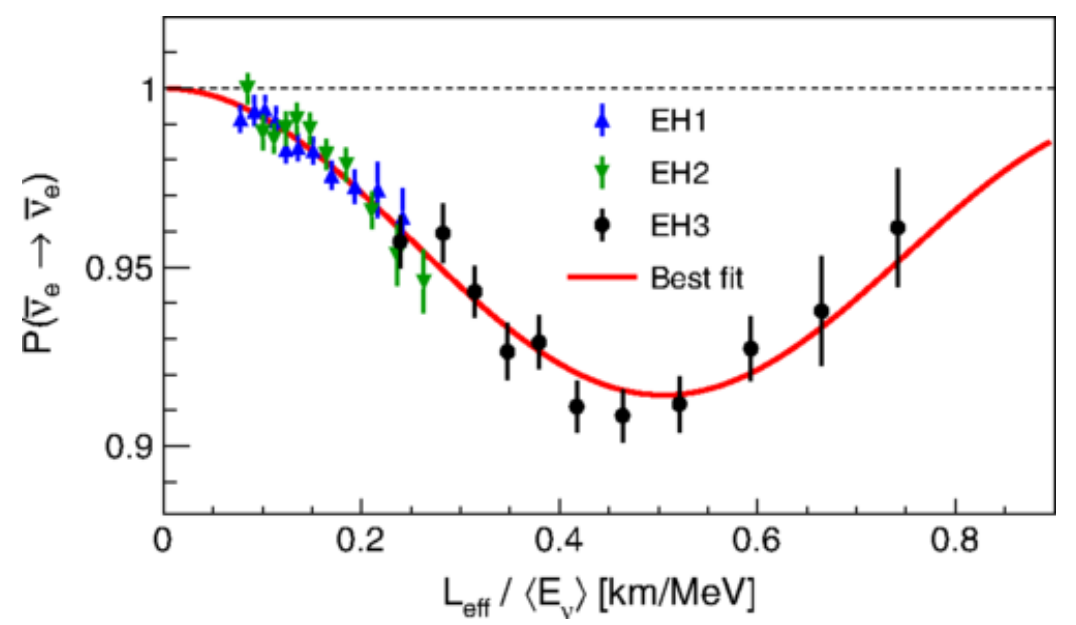

Figure 2.7: Measurement by Daya Bay of the electron antineutrino survival probability vs. the ratio of the effective propagation distance, $\mathrm{L}_{\text {eff }}$. to the average electron antineutrino energy, $\left\langle\mathrm{E}_{\nu}\right\rangle$. The measurements taken with the detectors situated in experiment halls (EH) 1, 2 and 3 are shown by the blue, green and black data points respectively. The best fit to the data is shown by the solid red line. Figure taken from [36].

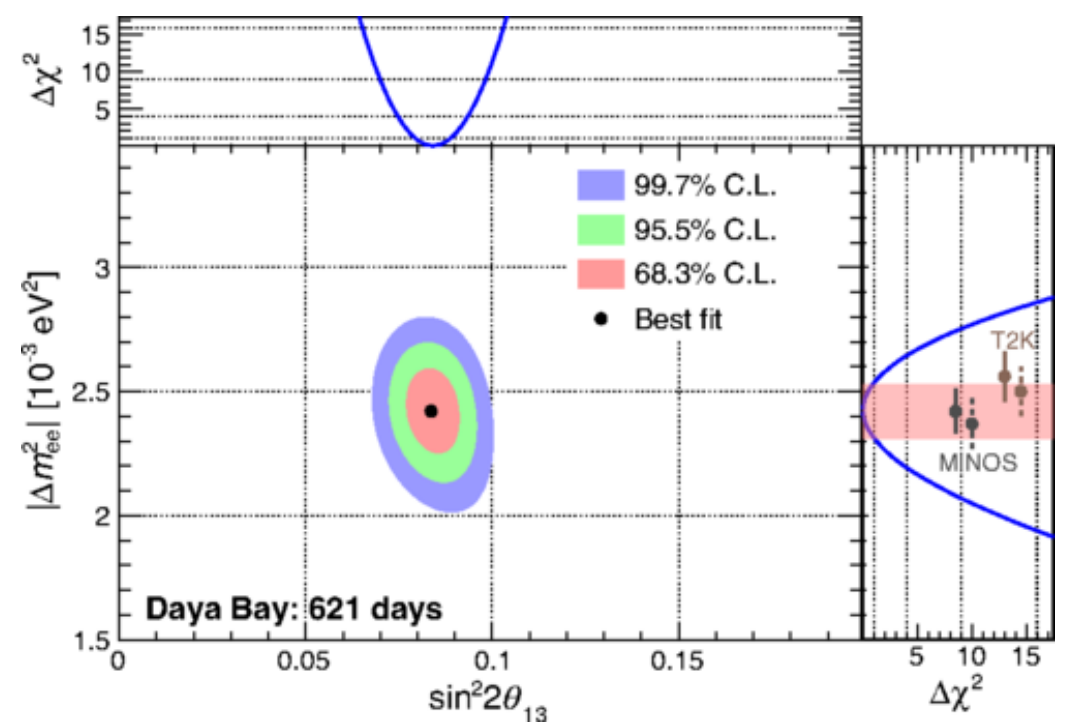

Figure 2.8: The Daya Bay experiments measured confidence limit conour. The contour plot shows the allowed regions in the $\left|\Delta m_{e e}^{2}\right|$ vs. $\sin ^{2} \theta_{13}$ plane. Regions allowed with $68.3 \%, 95.5 \%$ and $99.7 \%$ confidence levels are shown by the red, green and blue regions. Figure taken from [36]. 
MINOS was a long-baseline neutrino oscillation experiment that preceded NOvA at Fermilab. It comprised a near detector $1 \mathrm{~km}$ downstream from the accelerator neutrino source and a far detector $735 \mathrm{~km}$ downstream. The priority measurements made by MINOS were $\sin ^{2} \theta_{23}$ and $\Delta m_{32}^{2}$.

The neutrino source used by MINOS was produced by the Neutrinos at the Main Injector (NuMI) beam facility that is currently used by NOvA. For MINOS, NuMI produced a $0.3 \mathrm{MW}$ beam of muon (anti)neutrinos. The neutrino energy regime was $15 \mathrm{GeV}$ and peaked at $3 \mathrm{GeV}$. The 1000 ton MINOS near detector is located $1 \mathrm{~km}$ from the beam source and the 5400 ton MINOS far detector is located in a mine in Northern Minnesota, $735 \mathrm{~km}$ from the beam source. The detectors are relatively simple, consisting of planes of iron, which provide the bulk of the mass, interleaved with planes of $4 \mathrm{~cm}$ wide strips of plastic scintillator. When a charged particle traverses the scintillator, light is produced. This scintillation light is transmitted to small PMTs using optical fibres that are embedded in the scintillator. The MINOS detector was magnetised, allowing measurement of particle charge-sign and muon momenta (from the curvature of muon tracks originating from $\nu_{\mu}$ $N \rightarrow \mu^{-} X$ interactions). The amount of scintillation light produced gives a measure of the energy originating from the hadronic recoil system. Hence, on an event-by-event basis, the neutrino energy is reconstructed by summing the muonic and hadronic energy.

Figure 2.9 shows contours constraining the allowed regions in measured parameter space given a fit of theory to the appearance and disappearance probability distributions. The results from MINOS yielded values of $\left|\Delta m_{32}^{2}\right|=[2.28-2.46] \times 10^{-3} \mathrm{eV}^{2}$ (68\% C.L.) and $\sin ^{2} \theta_{23}=0.35-0.65$ (90\% C.L.) [37]. The bottom right plot in Figure 2.9 shows the $\log$ likelihood of a fit to the data for each value of $\sin ^{2} \theta_{23}$. The data from MINOS indicates a preference for the lower octant value of $\sin ^{2} \theta_{23}=0.41$ with maximal mixing disfavoured by $-2 \Delta \log (\mathcal{L})=1.54$ (or $\sigma=1.24$ ) [37]. MINOS had low sensitivity to the mass hierarchy but preferred the inverted mass hierarchy - the normal hierarchy disfavoured by 0.23 units of $-2 \Delta \log (\mathcal{L})$.

The Tokai to Kamioka (T2K) experiment is a long-baseline neutrino experiment located in Japan, that was preceded by K2K. T2K comprises three detectors that measure the flux of accelerator neutrinos produced by the J-PARC facility. This beam is predominately composed of muon neutrinos created using a high-intensity proton accelerator. Two of the three detectors are situated $2.5^{\circ}$ off-axis from the beamline with the third detector situated on-axis. Of the two off-axis detectors a near detector sits $280 \mathrm{~m}$ downstream from the neutrino beam source and the far detector sits $295 \mathrm{~km}$ downstream from the 

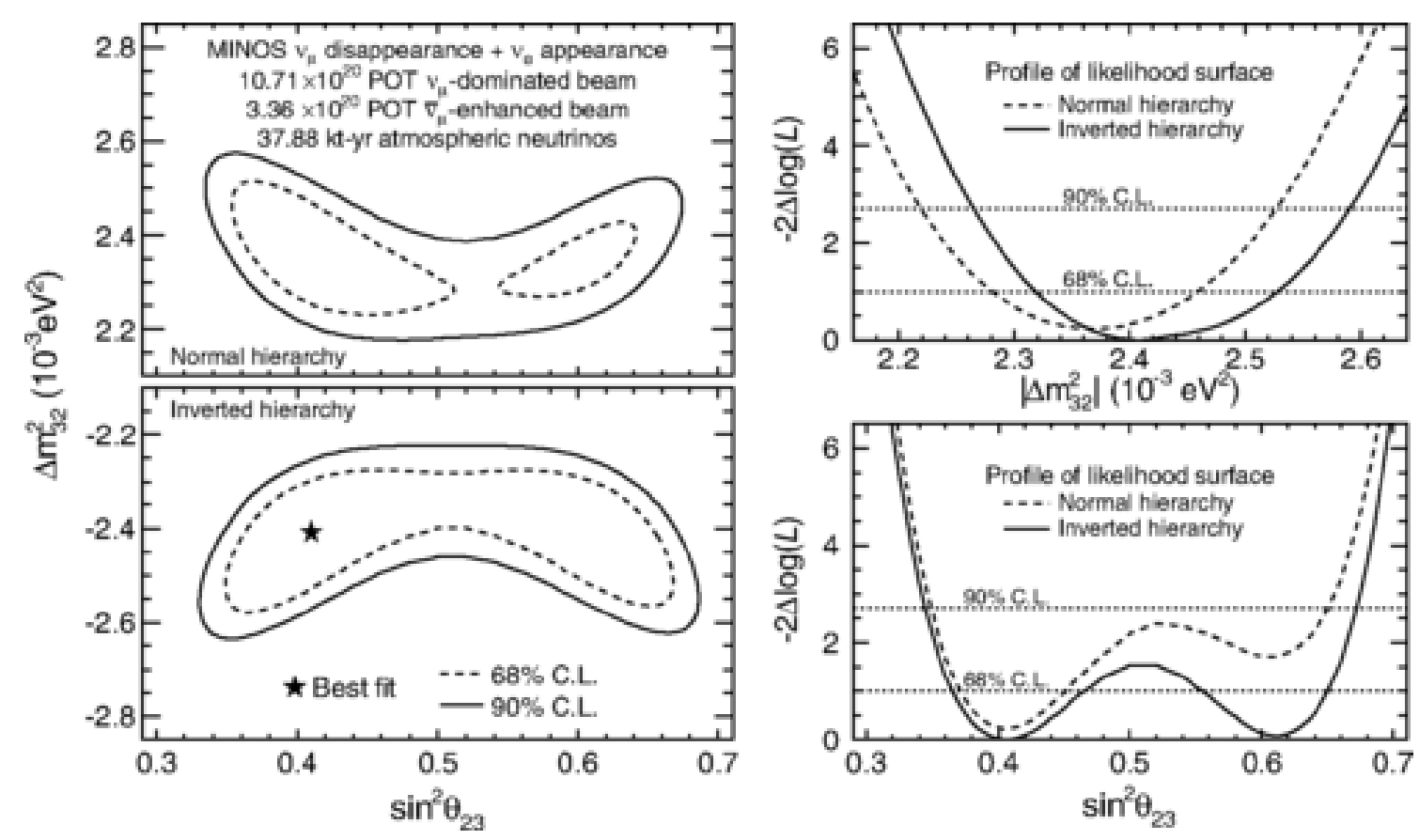

Figure 2.9: MINOS confidence limits. The left-hand plots show the confidence contours for $\Delta m_{32}^{2}$ vs. $\sin ^{2} \theta_{23}$ for the normal mass hierarchy (top) and the inverted mass hierarchy (bottom). The best fit point is indicated by the star. The right-hand plots show the one dimensional likelihood profiles as functions of $\Delta m_{32}^{2}$ (top) and $\sin ^{2} \theta_{23}$ (bottom), where the normal and inverted ordering are shown by the dotted and solid lines respectively. Figure taken from [37].

beam source. The third detector (on-axis) acts as a second near detector and is located $280 \mathrm{~m}$ downstream from the beam source. The two near detectors are used to measure the flux and composition of the neutrino beam. The utilization of separate on and off axis near detectors facilitates the parameterization of beam properties. T2K measures both the muon neutrino disappearance and electron neutrino appearance probabilities. T2K data disfavours the inverted hierarchy at 0.86 units of $\Delta \chi^{2}$. T2K favours a point close to maximal mixing $\left(\sin ^{2} \theta_{23}=0.5\right)$ at $\sin ^{2} \theta_{23}=0.527$ [38]. Figure 2.10 shows the confidence limit contour resulting from the joint disappearance and appearance analysis.

\section{$2.7 \quad$ NOvA}

This thesis focusses on NOvA's first $\nu_{\mu}+\bar{\nu}_{\mu}$ disappearance analysis. The previous analysis comprised $8.85 \times 10^{20} \mathrm{POT}$ worth of $\nu_{\mu}$ disappearance data and no $\bar{\nu}_{\mu}$ data. The results of the previous analysis were published in early 2018 and are presented in this section, 


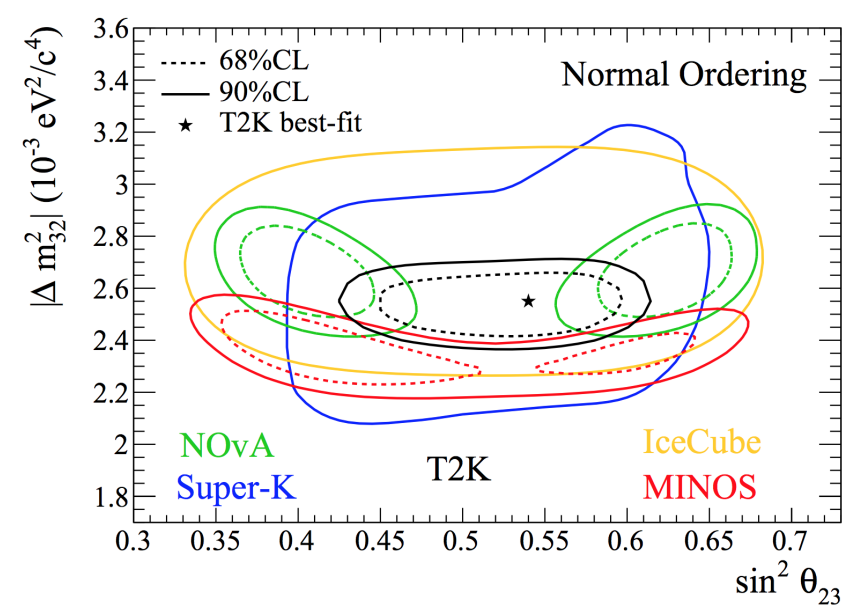

Figure 2.10: $\Delta m_{32}^{2}$ against $\sin ^{2} \theta_{23}$ confidence contours for T2K (black lines), MINOS (red lines), Super Kamiokande (blue line) and NOvA's 2016 (second) analysis (green). The confidence limits in the normal and inverted mass hierarchies are shown in the top and bottom plots respectively. Confidence limits of $68 \%$ and $90 \%$ are shown by the dashed and solid lines respectively. Figure taken from [38].

preceded by a short discussion of the neutrino interactions and background sources that NOvA experiences.

\subsubsection{Neutrino Interactions in NOvA}

Interactions in NOvA are dominated by resonant scattering (RES), quasi-elastic scattering (QE), meson exchange current (MEC) and deep inelastic scattering (DIS) events. NOvA also experiences coherent pion production events, but at a much reduced rate. The distribution of event types in NOvA is governed by the neutrino energy. The RES, QE and DIS interactions occur via the exchange of weak force mediating gauge bosons.

Neutrino interactions occur more frequently with nucleons than with atomic electrons. This is due to an effective increase in the interaction cross section due to its proportional relationship with centre-of-mass energy. For fixed target experiments this energy is approximately $2 \mathrm{~m} E_{\nu}$, where $\mathrm{m}$ is the mass of the target particle and hence interactions with the larger nucleon are more frequent than with atomics electrons.

Figure 2.11 shows the charged current FD event distribution against reconstructed energy. The figure shows the total MC sample distribution and the individual distributions from QE, DIS and RES interactions.

Quasi-elastic scattering events are characterised by a neutrino scattering off a nucleon. The nucleon is usually ejected from the nucleus. Charged current QE neutrino interactions 


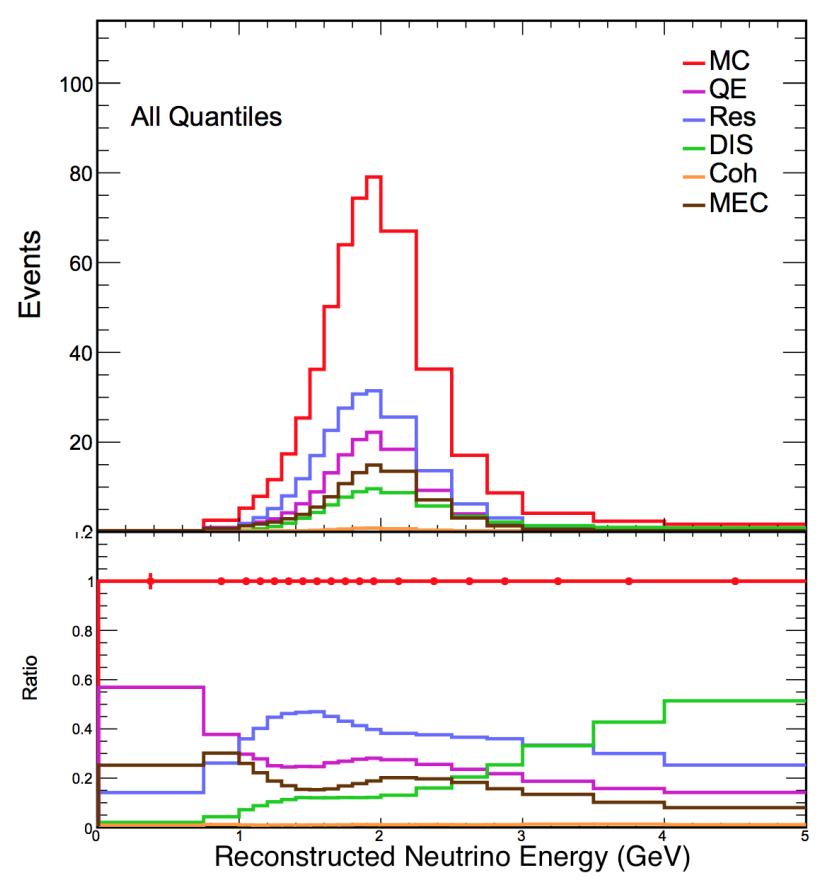

Figure 2.11: Simulated NOvA FD event distributions after selection, separated by interaction type. Total MC is shown in red, quasi-elastic events in purple, resonance events in blue, deep inelastic scattering in green, coherent pion production events in orange and MEC events in dark brown

take the general form: $\nu_{l}+n \rightarrow l^{-}+p$, where $l$ is the lepton flavour, $n$ is a neutron and $p$ is a proton.

Resonance interactions are characterised by a neutrino causing excitation of the nucleon. This excitation puts the nucleon in a resonance state which takes the general form: $\nu_{l}+N \rightarrow l+R$, where $\mathrm{N}$ is the target nucleon and $\mathrm{R}$ is the resonance state. This resonant state then decays creating a hadronic shower. Deep inelastic scattering events are characterised by a sufficiently high-energy neutrino that can resolve the quarks within the nucleon. These events take the general form: $\nu_{l}+N \rightarrow l+X$, where $\mathrm{X}$ is the hadronic recoil system. Hadronization always occur in DIS events as the nucleus is subject to QCD when a constituent quark is acted upon.

\subsubsection{Background Sources}

The NOvA disappearance analysis experiences three major beam backgrounds. These originate from $\nu_{e}, \nu_{\tau}$ and neutral current $(\mathrm{NC})$ events. $\mathrm{NC}$ events leave the lepton flavour unchanged and are thus treated as a background source in oscillation analyses - they carry no information on the neutrino flavour. 
Neutral current events are identified by the absence of an outgoing muon from the interaction vertex. Signal selection efficiencies constrain the rejection of NC events. Outgoing mesons can be mistaken for muons.

The cosmogenic background dominates at the far detector. While most cosmic events are well characterised and easily rejected, the sheer volume of cosmic activity introduces impurity in selection. The methods used to remove the background events from the analysis are discussed in Section 4.3.

$\nu_{\mu}$ disappearance Post selection, $126 \nu_{\mu}$-CC candidates were observed in the FD. The prediction in the absence of oscillations was $720.3_{-47.0}^{+67.4}$ inclusive of an expected background of 5.8 cosmic ray background events and 3.4 beam background neutrino events.

Figure 2.12 shows the observed energy spectrum of observed $\nu_{\mu}$ events. The spectrum has a dip at approximately $1.6 \mathrm{GeV}$; the dip's depth gives information about $\sin ^{2} \theta_{23}$ and $\Delta m_{32}^{2}$ corresponds to the dips position. The systematic error is smaller in the high energy regime due to a reduced beam \& cosmic background. Additionally, in the high energy regime long track length events, which are are easier to estimate energy for, are more common (provided the fraction of hadronic energy in the event is low).

Figure 2.13 shows the $90 \%$ confidence level contours for $\Delta m_{32}^{2}$ and $\sin ^{2} \theta_{23}$ for NOvA, T2K, MINOS, IceCube, and Super-Kamiokande. No experiments are inconsistent with maximal mixing. Maximal mixing of $\theta_{23}$ is disfavoured to $0.8 \sigma$.

$\nu_{e}$ appearance Post selection, $66 \nu_{e} \mathrm{CC}$ candidate events were observed in the far detector inclusive of an expected background of $20.3 \pm 2.0$ events. The estimated background composition was 7.3 beam $\nu_{e}$ CC events, $6.4 \mathrm{NC}$ events, $1.3 \nu_{\mu} \mathrm{CC}$ events, $0.4 \nu_{\tau} \mathrm{CC}$ events, and 4.9 cosmic events.

The $\nu_{e}$ analysis is binned according to the Convolutional Visual Network (CVN) score a neural network trained to identify neutrino candidates ${ }^{2}$. Figure 2.14 shows the identified $\nu_{e}$ energy spectrum for the three CVN classifier bins and for the peripheral sample. The expected background contributions and the best fit predictions are also shown. ${ }^{3}$

Figure 2.15 shows the significance at which each value of $\left|\Delta m_{32}^{2}\right|$ and $\sin ^{2} \theta_{23}$ is disfavoured in the normal and inverted mass hierarchy scenarios. Figure 2.16 shows the number of identified $\nu_{e}$ candidate events and the expectation for the best fit values of $\Delta m_{32}^{2}$ and $\sin ^{2} \theta_{23}$ as a function of $\delta_{\mathrm{CP}}$, for the two possible mass hierarchies. Figure 2.17 shows the significance at which each value of $\delta_{\mathrm{CP}}$ is disfavoured in each mass hierarchy scenario. In

\footnotetext{
${ }^{3}$ The CVN score and other selection metrics are detailed fully in Chapter 4.
} 


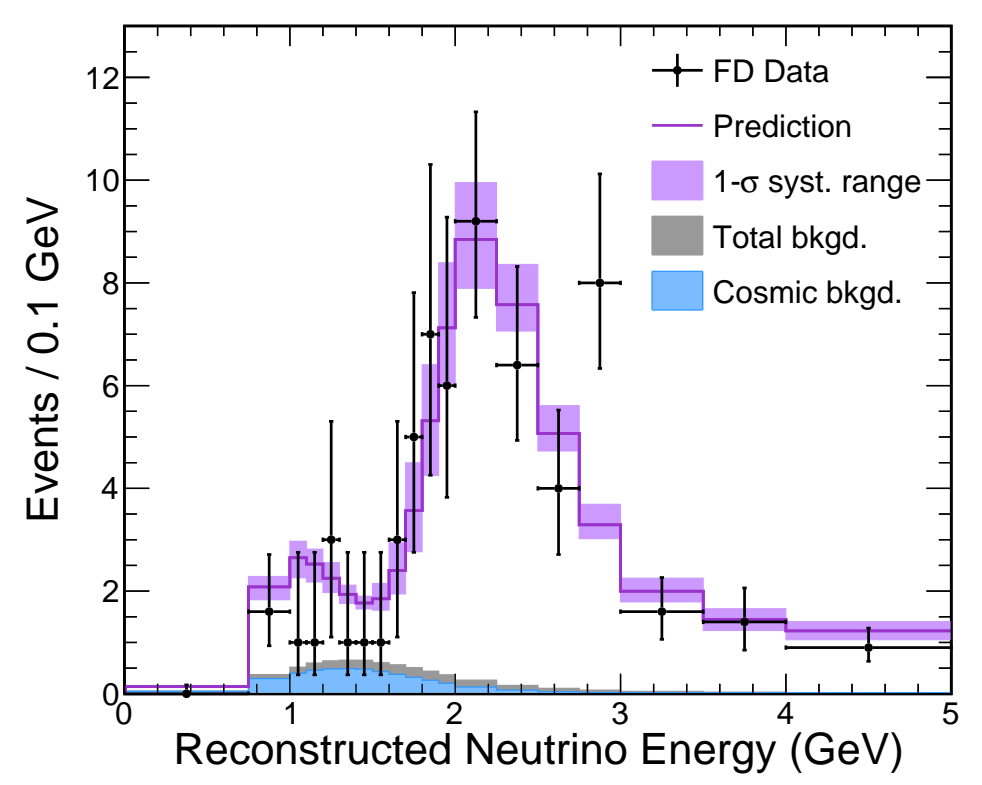

Figure 2.12: The energy spectra of candidate $\nu_{\mu}$ events in data and simulation. The $1 \sigma$ systematic range is shown in purple. The spectra of background sources is also shown, the cosmogenic background is shown in blue and the total background in gray. Figure from $[3]$.

Figure 2.16 and Figure 2.17 two curves are shown in the normal mass hierarchy, one for each of the $\theta_{23}$ octants, corresponding to the near degeneracy shown in the right plot of Figure 2.15. Only one curve is shown for the inverted mass hierarchy since there is only one minimum, which occurs in the upper octant. Figure 2.18 shows the 1,2 and $3 \sigma$ confidence level contours in $\sin ^{2} \theta_{23}$ and $\delta_{\mathrm{CP}}$ produced from the joint $\nu_{\mu}$ disappearance $+\nu_{e}$ appearance fit. Table 2.1 shows the $1 \sigma \Delta m_{32}^{2}, \sin ^{2} \theta_{23}$, and $\delta_{\mathrm{CP}}$ confidence intervals.

Table 2.1: $1 \sigma$ confidence intervals for physics parameters in the normal mass hierarchy.

\begin{tabular}{lc}
\hline \hline Parameter (units) & $1 \sigma$ interval $(\mathrm{s})$ \\
\hline$\Delta m_{32}^{2}\left(10^{-3} \mathrm{eV}^{2} / c^{4}\right)$ & {$[2.37,2.52]$} \\
$\sin ^{2} \theta_{23}$ & {$[0.43,0.51]$ and $[0.52,0.60]$} \\
$\delta_{\mathrm{CP}}(\pi)$ & {$[0,0.12]$ and $[0.91,2]$} \\
\hline \hline
\end{tabular}




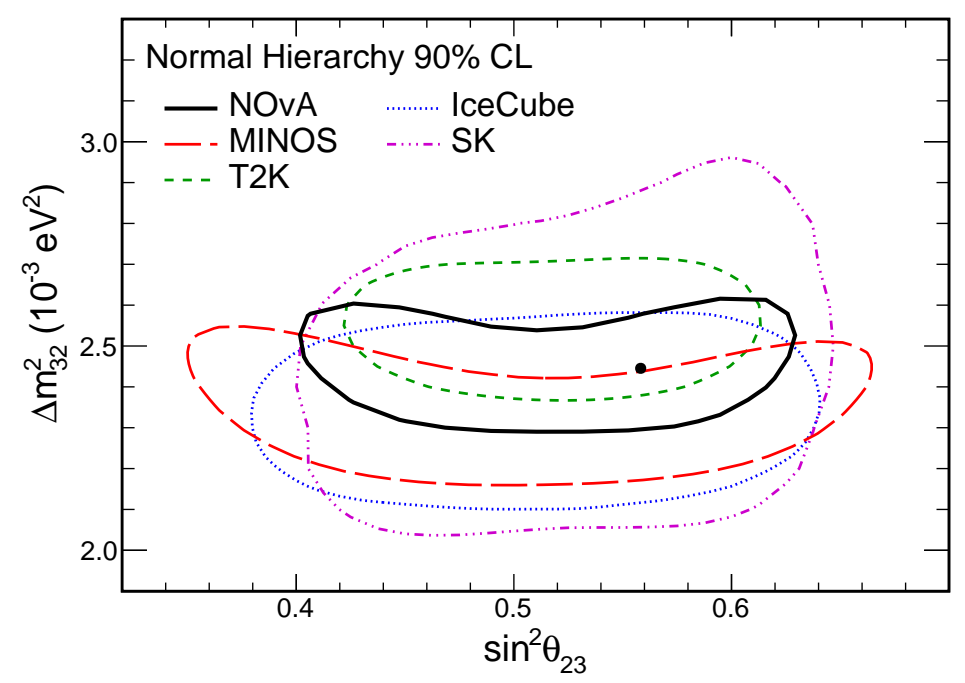

Figure 2.13: Comparison of various experiments $90 \%$ confidence level limits in $\Delta m_{32}^{2}$ vs. $\sin ^{2} \theta_{23}$ for this result (black line; best-fit value, black point), T2K (green dashed), MINOS (red dashed), IceCube (blue dotted) and Super-Kamiokande (purple dash-dotted).

Figure from [3].

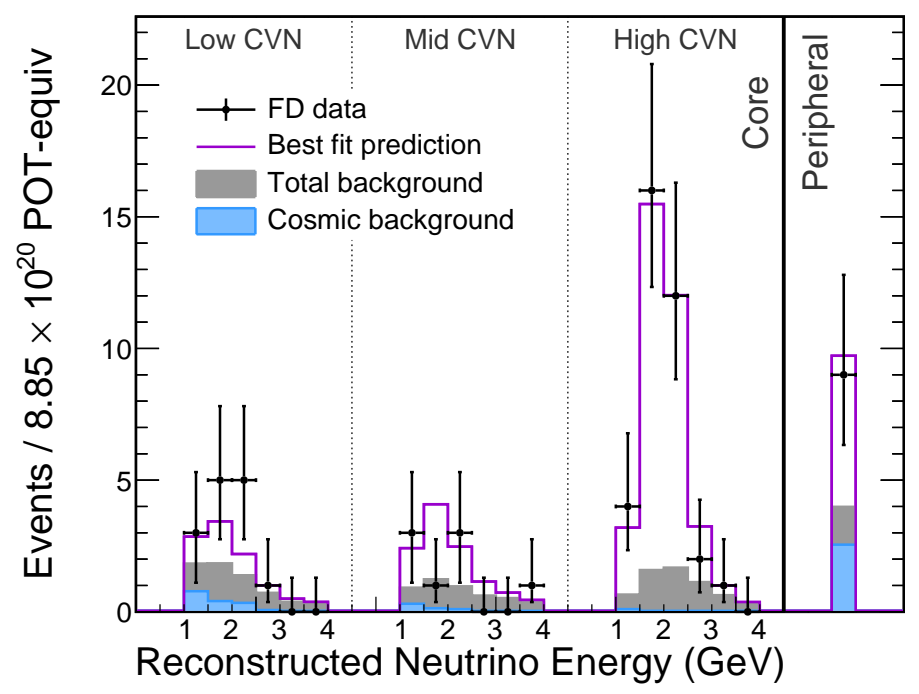

Figure 2.14: Comparison of the neutrino energy spectra of selected $\nu_{e}$ candidates in the FD data (black dots) with the best fit prediction (purple lines) in the three CVN classifier bins and the peripheral sample. The total expected background (gray, upper) and the cosmic component of it (blue, lower) are shown as shaded areas. The events in the peripheral bin have energy between 1 and $4.5 \mathrm{GeV}$.

Results NOvA published the measurements described in this section in 2018. The analysis gave $\Delta m_{32}^{2}=2.44_{-0.07}^{+0.08} \times 10^{-3} \mathrm{eV}^{2}$ and $\sin ^{2} \theta_{23}=0.56_{-0.04}^{+0.04}$. The data disfavoured maximal mixing at $0.8 \sigma$ significance. The inverted mass hierarchy was disfavoured at the 

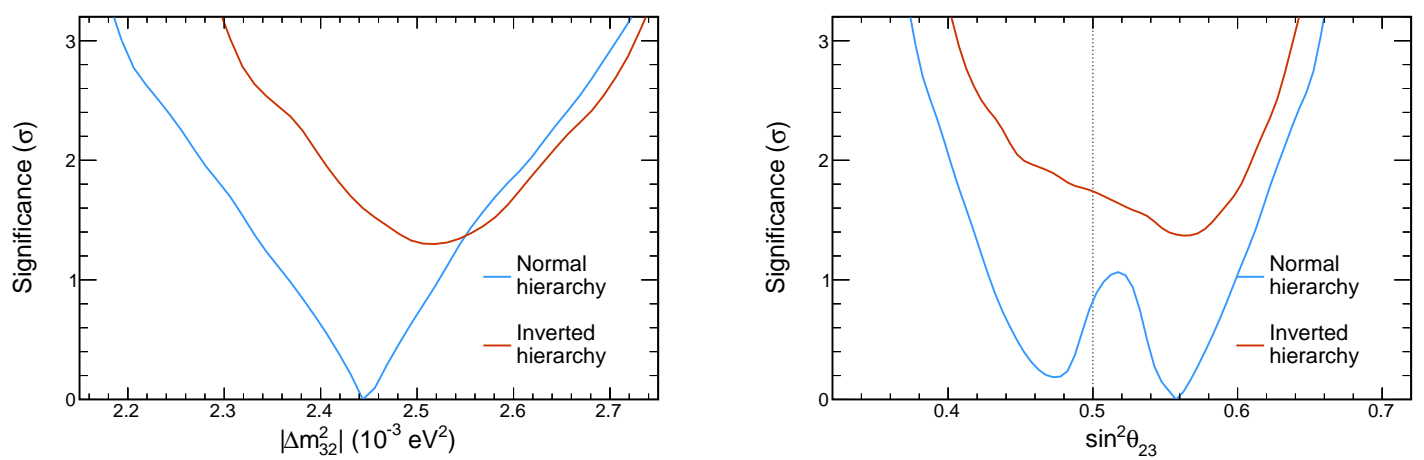

Figure 2.15: Significance at which each value of $\left|\Delta m_{32}^{2}\right|$ (left) and $\sin ^{2} \theta_{23}$ (right) is disfavoured in the normal (blue, lower) or inverted (red, upper) mass hierarchy.

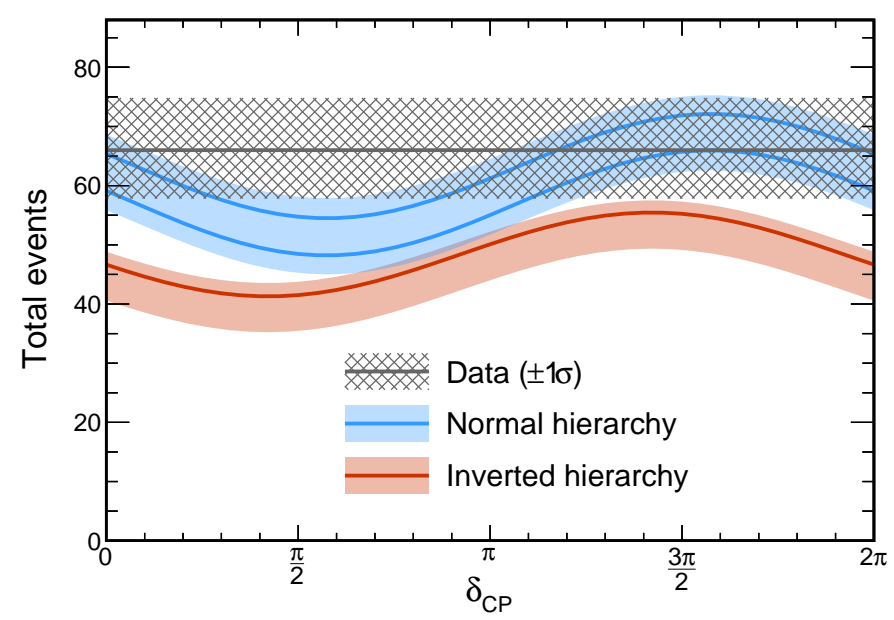

Figure 2.16: Total number of $\nu_{e}$ candidate events observed in the FD (gray) compared to the prediction as a function of $\delta_{\mathrm{CP}}$. The coloured lines correspond to the best fit values of $\sin ^{2} \theta_{23}$ and $\Delta m_{32}^{2}$ with the upper two curves (blue) representing two octants in the normal mass hierarchy $\left(\Delta m_{32}^{2}>0\right)$ and the lower curve (red) the inverted hierarchy $\left(\Delta m_{32}^{2}<0\right)$. The colour bands correspond to $0.43 \leq \sin ^{2} \theta_{23} \leq 0.60$. All other parameters are held fixed at the best-fit values.

95\% confidence level for all choices of the other oscillation parameters. 


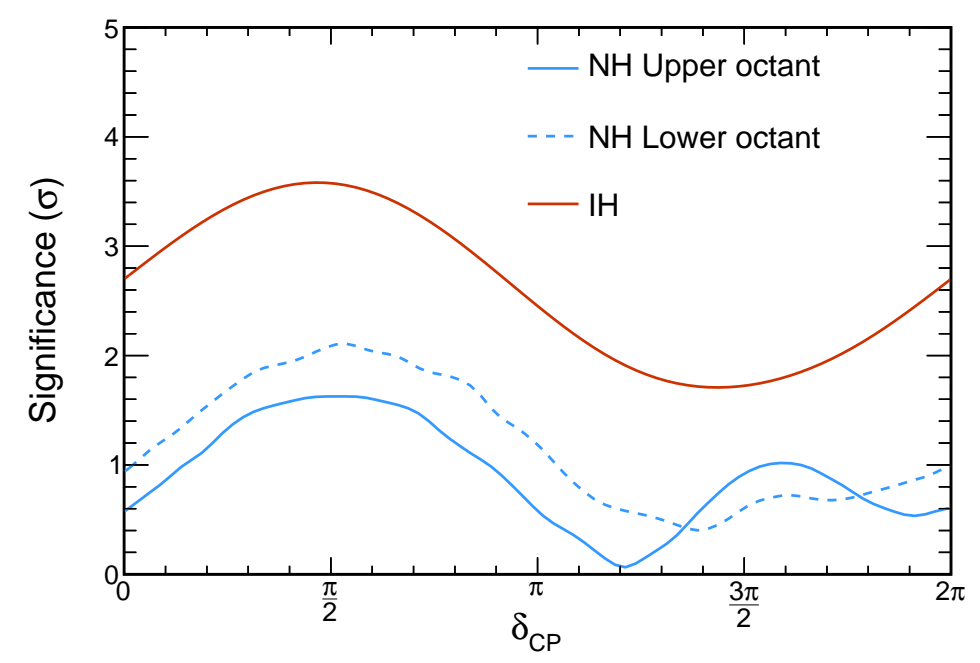

Figure 2.17: Significance at which each value of $\delta_{\mathrm{CP}}$ is disfavoured in the normal (blue, lower) or inverted (red, upper) mass hierarchy. The normal mass hierarchy is divided into upper (solid) and lower (dashed) $\theta_{23}$ octants corresponding to the near degeneracy in $\sin ^{2} \theta_{23}$.

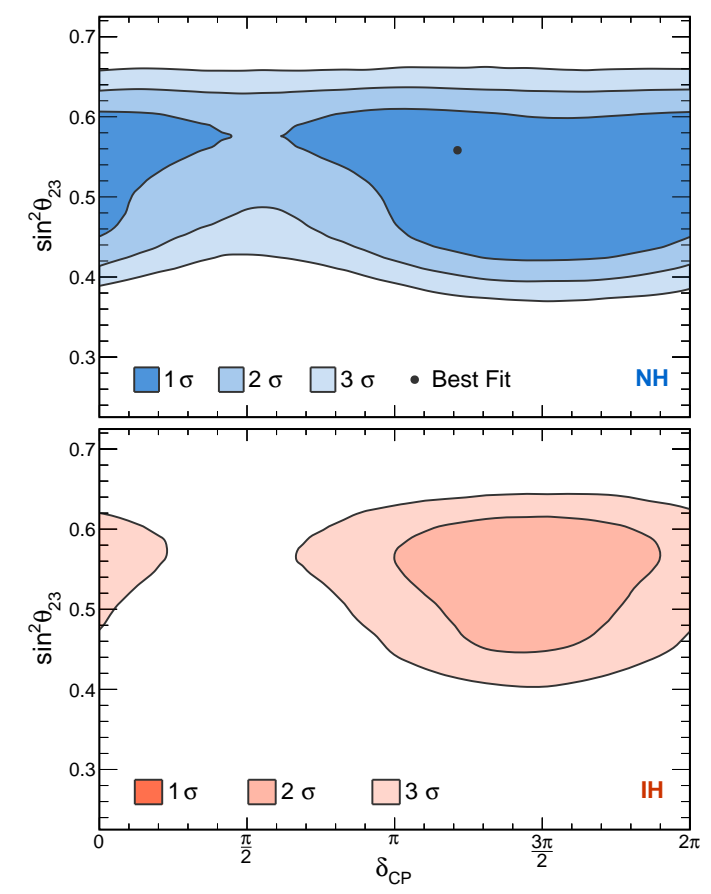

Figure 2.18: $\sin ^{2} \theta_{23}$ vs. $\delta_{\mathrm{CP}}$ parameter space consistent with the joint $\nu_{\mu}$ disappearance $+\nu_{e}$ appearance NOvA analysis. The top panel corresponds to normal mass hierarchy $\left(\Delta m_{32}^{2}>0\right)$ and the bottom panel to inverted hierarchy $\left(\Delta m_{32}^{2}<0\right)$. 


\subsection{Beyond the Standard Model Majorana particles and The Seesaw Mechanism}

The right-handed chiral neutrino states do not couple to the electroweak gauge bosons or the gluons. In other words, they do not participate in any standard model interactions. There is no experimental evidence to suggest they exist. Neutrino oscillations, as discussed previously, have shown that the neutrinos are not massless and, therefore, there must be some mass term to accommodate them in the standard model Lagrangian. As is the case for the quarks, the neutrino masses may be introduced to the standard model using the conjugate Higgs doublet. However this is somewhat unsatisfactory; the neutrino masses are over six orders of magnitude smaller than the other fermions. Theorists have answered this with alternative mechanisms for mass generation in the neutrino sector.

A comprehensive overview of Lagrangian field theory is not provided in this review. Suffice it to say that because the right-handed neutrinos and left-handed antineutrinos transform as singlets under SM gauge transformations, only terms containing these can be added to the Lagrangian without breaking gauge invariance. One can construct a mass term from the right-handed neutrino fields and the left-handed antineutrino fields that maintains the SM local gauge invariance. This term is referred to as the Majorana mass term. It implies the existence of direct couplings between matter and antimatter and so a similar Majorana mass term would not be accommodated by the leptons - charge conservation would be violated. This problem is not present for neutrinos. In fact, neutrinos are neutral and the possibility that they are their own anti-particle is a consequence of introducing such a term. Particles of this nature are referred to as Majorana particles, as opposed to Dirac particles. SNO+, the upgrade to SNO in Sudbury, is one experiment looking to answer the question of Majorana neutrino existence by attempting to observe neutrinoless double-beta decay. The Feynman diagram is shown in Figure 2.19.

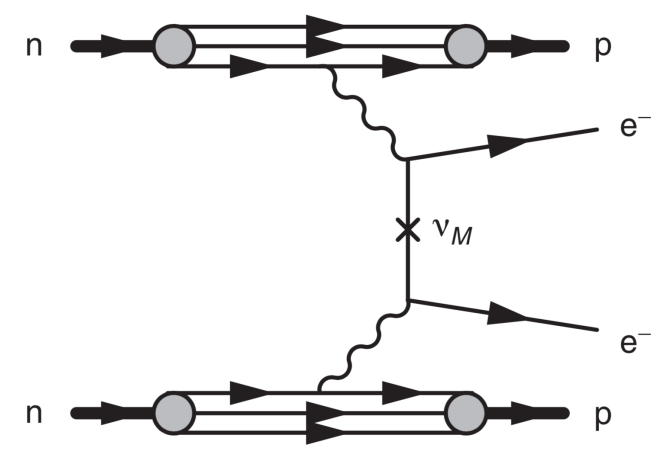

Figure 2.19: The feynman diagram for neutrinoless double-beta decay. 
If $\mathrm{SNO}+$, or another experiment, confirms the Majorana nature of neutrinos, this opens the door for the seesaw mechanism of neutrino mass generation. By creating a term with both a Dirac and Majorana component one can construct the most general renormalizable Lagrangian for neutrino masses. The physical states of such a system can be obtained from the Lie Algebra of the $\mathrm{U}(1) \times \mathrm{SU}(2)$ gauge symmetry, in an analogous fashion to that done to obtain the physical states of the neutral gauge bosons. The masses of the physical neutrino states are simply the eigenvalues of the mass matrix M,

$$
\mathcal{L}_{D M}=-\frac{1}{2}\left[\begin{array}{ll}
\bar{\nu}_{L} & \bar{\nu}_{R}^{c}
\end{array}\right]\left[\begin{array}{cc}
0 & m_{D} \\
m_{D} & M
\end{array}\right]\left[\begin{array}{c}
\nu_{L}^{c} \\
\nu_{R}
\end{array}\right]+\text { h.c. }
$$

where $m_{D}$ is the Dirac mass and $\mathrm{M}$ is the Majorana mass. In the seesaw mechanism the Dirac mass terms for the neutrino are set to be of similar size as for the other fermions. Then, one need only solve the characteristic determinant equation $(\operatorname{det}(\mathrm{M}-\lambda \mathrm{I}))$ to find the neutrino masses. In doing so one recovers two neutrino masses the first giving a light state neutrino and the other giving a heavy state neutrino. If the Majorana mass is made sufficiently large then the lighter of two neutrino states becomes of the correct order, in accordance with current neutrino observations. This relationship between the light and heavy neutrino states is where the seesaw mechanism gets its name. As you increase the Majorana mass term, the low mass neutrino state becomes ever lighter. The seesaw mechanism predicts that for each of the neutrino generations there is one such light neutrino state, corresponding to the currently observed neutrinos, and an associated massive neutrino state.

\subsection{Summary of Current Results}

Table 2.2 contains a summary of current constraints on each of the oscillation parameters. 


\begin{tabular}{l|cc|cc|c}
\hline \hline & \multicolumn{2}{|c|}{ Normal Ordering $\left(\Delta \chi^{2}=0.97\right)$} & \multicolumn{2}{|c|}{ Inverted Ordering (best fit) } & Any Ordering \\
\hline & bfp $\pm 1 \sigma$ & $3 \sigma$ range & bfp $\pm 1 \sigma$ & $3 \sigma$ range & $3 \sigma$ range \\
\hline $\sin ^{2} \theta_{12}$ & $0.304_{-0.012}^{+0.013}$ & $0.270 \rightarrow 0.344$ & $0.304_{-0.012}^{+0.013}$ & $0.270 \rightarrow 0.344$ & $0.270 \rightarrow 0.344$ \\
$\theta_{12} /^{\circ}$ & $33.48_{-0.75}^{+0.78}$ & $31.29 \rightarrow 35.91$ & $33.48_{-0.75}^{+0.78}$ & $31.29 \rightarrow 35.91$ & $31.29 \rightarrow 35.91$ \\
$\sin ^{2} \theta_{23}$ & $0.452_{-0.028}^{+0.052}$ & $0.382 \rightarrow 0.643$ & $0.579_{-0.037}^{+0.025}$ & $0.389 \rightarrow 0.644$ & $0.385 \rightarrow 0.644$ \\
$\theta_{23} /{ }^{\circ}$ & $42.3_{-1.6}^{+3.0}$ & $38.2 \rightarrow 53.3$ & $49.5_{-2.2}^{+1.5}$ & $38.6 \rightarrow 53.3$ & $38.3 \rightarrow 53.3$ \\
$\sin ^{2} \theta_{13}$ & $0.0218_{-0.0010}^{+0.0010}$ & $0.0186 \rightarrow 0.0250$ & $0.0219_{-0.0010}^{+0.0011}$ & $0.0188 \rightarrow 0.0251$ & $0.0188 \rightarrow 0.0251$ \\
$\theta_{13} /{ }^{\circ}$ & $8.50_{-0.21}^{+0.20}$ & $7.85 \rightarrow 9.10$ & $8.51_{-0.21}^{+0.20}$ & $7.87 \rightarrow 9.11$ & $7.87 \rightarrow 9.11$ \\
$\delta_{\mathrm{CP}} /{ }^{\circ}$ & $306_{-70}^{+39}$ & $0 \rightarrow 360$ & $254_{-62}^{+63}$ & $0 \rightarrow 360$ & $0 \rightarrow 360$ \\
\hline \hline
\end{tabular}

Table 2.2: Three-flavour oscillation parameters from fit to global data. The results are presented where reactor fluxes have been left free in the fit and short baseline reactor data with $L \lesssim 100 \mathrm{~m}$ are included. The numbers in the 1st (2nd) column are obtained assuming normal hierarchy (inverse hierarchy). The 3rd column is the best fit across both hierarchies. Table taken from [1], published in 2016. 


\section{Chapter 3}

\section{The NOvA Experiment}

The NOvA (NuMI off-axis $\nu_{e}$ appearance) experiment was designed to make precise measurements of electron neutrino appearance and muon neutrino disappearance. The NuMI (Neutrinos at the Main Injector) facility at Fermilab provides the neutrino source.

NOvA uses two detectors to measure neutrino energy and flavour composition at fixed distances from the neutrino beam source. The near detector sits $1.015 \mathrm{~km}$ from the NuMI target. The far detector sits $810 \mathrm{~km}$ from the NuMI target. Both detectors are functionally identical, comprising highly-granular PVC tubing filled with liquid scintillator, but differ in a few ways, such as total mass. The NOvA detectors are situated 14.6 mrad away from the central axis of the NuMI beam. At this location, the neutrino flux has a narrow energy distribution with a central peak at approximately $1.9 \mathrm{GeV}$. This energy peak is close to the first oscillation maximum at NOvA's far detector and the tightened distribution helps remove high energy background events. Because both detectors sit level with local gravity, they are angled with respect to the beam. The beam is angled down by $3^{\circ}$ at the near detector and up by $3^{\circ}$ at the far detector. Analysis of both the observed ND (anti)neutrino energy spectrum and simulated near detector data is used to perform systematic extrapolation to the far detector as well as measure the beam composition.

The implemented design of NOvA closely resembles that of the one described in the technical design report (TDR) [5]. This chapter discusses the features of the experiment most pertinent to the analysis that follows in later chapters. Chiefly addressed is the design of the two detectors (near and far) and the neutrino beam. 


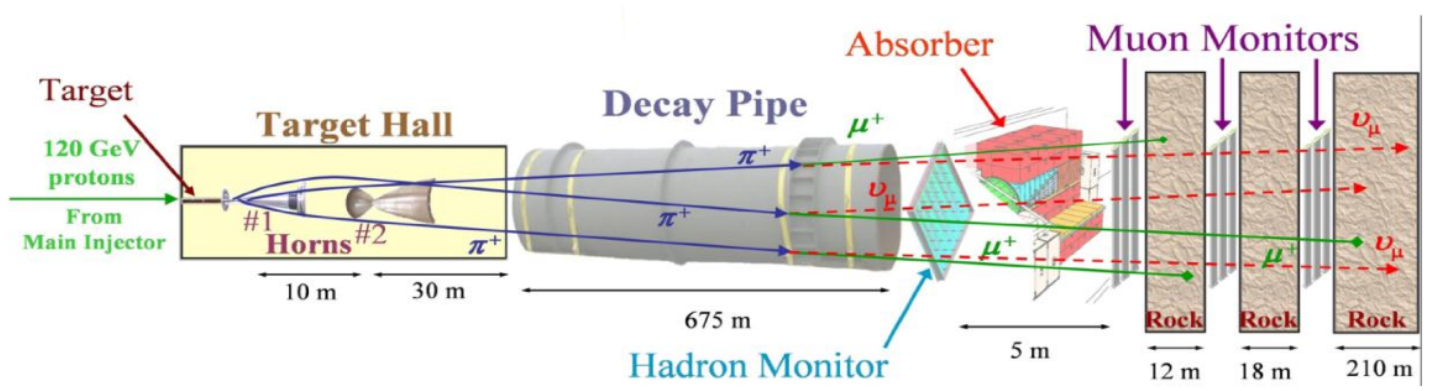

Figure 3.1: Cartoon representation of the NuMI beam facility. [4]

\subsection{The NuMI Beam}

The source of neutrino flux NOvA utilizes is provided by the Fermilab NuMI beam [4]. The main injector accelerates protons up to $120 \mathrm{GeV}$, grouped into six batches, and then emits them in a $10 \mu \mathrm{s}$ long window. The number of protons per spill is approximately $4.8 \times 10^{13}$ and the spills occur at approximately $1.33 \mathrm{~s}$ intervals. The protons are fired at a $0.95 \mathrm{~m}$ long graphite target, creating a cascade of mesons that produce neutrinos as a decay product.

A cartoon representation of the NuMI beam facility is shown in Figure 3.1. It shows the main components - the target hall, decay pipe, hadron monitor, absorber and muon monitors. The target hall is where the protons collide with the graphite target yielding secondary particles. These secondary particles are primarily comprised of pions but a small kaon component is present as well. Two magnetic horns then focus the charged mesons (the charge sign can be chosen) into a $675 \mathrm{~m}$ long helium filled decay pipe. This affords the mesons enough time to decay. Following this, a hadron absorber and over $200 \mathrm{~m}$ of rock stop any remaining hadrons and charged leptons producing a neutrino-only flux. The NOvA near detector sits just after the rock in an alcove $100 \mathrm{~m}$ underground. The beam then continues through the earth's crust for $810 \mathrm{~km}$ before arriving at the far detector, the farthest downstream site of measurement for the beam.

\subsubsection{The Magnetic Focussing Horns}

The mesons that emerge from the target hall are shaped into a beam using two parabolic magnetic horns. The horns act as a lens to focus the mesons. The focal length of this lens is governed by the meson momenta and strength of the magnetic field. These horns have two general operational modes forward horn current (FHC) and reverse horn current (RHC) - which select positively or negatively charged mesons respectively. This mechanism works due to the horn's ability to have current passed through them in either 


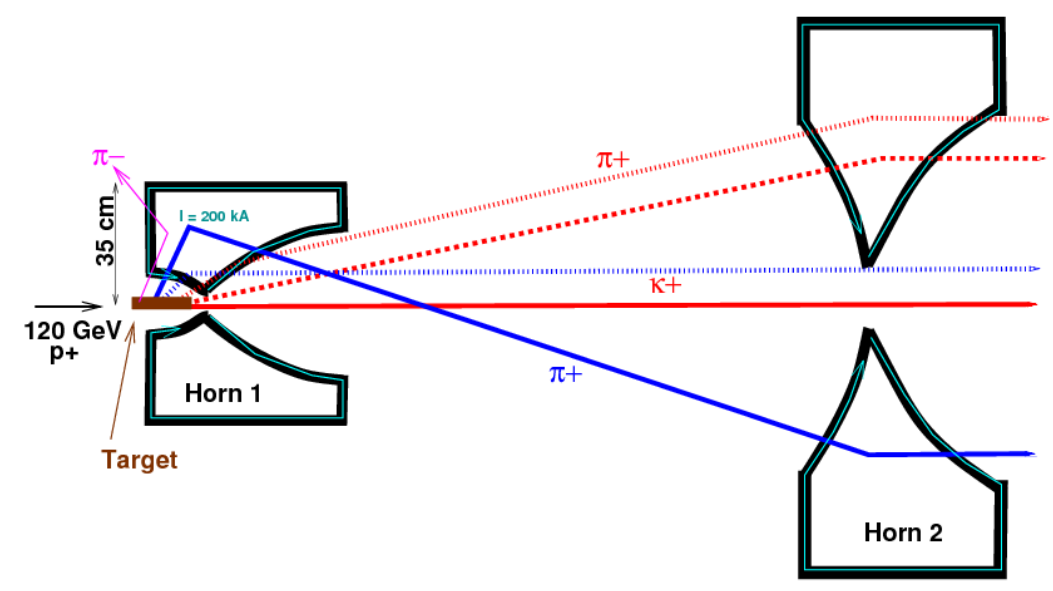

10 meters

Figure 3.2: Diagram of the magnetic focusing horns operating in forward horn current, low energy configuration mode. The two parabolic magnetic horns focus incoming positively charged mesons. The mesons may be over or under focused by horn 1. The trajectories are adjusted by horn 2 to correct for focussing errors in horn 1 . NOvA is run in the medium energy configuration where horn separation is increased to $13 \mathrm{~m}$ and the target moved out. Diagram taken from [4].

direction, changing the magnetic field direction and, subsequently, the sign of particles focused. FHC produces a flux of primarily neutrinos (94.1\%), RHC a flux of primarily antineutrinos (92.9\%). Figure 3.2 shows a simple schematic of the horns (labeled 1 and 2) and beam target. The energy of the beam is tuneable by varying the distance between the target, horn 1 and horn 2. During design, low, medium and high energy configurations were developed each affecting total beam flux and the width and mean of the energy distribution. NOvA operates using the medium-energy configuration due to this producing the highest neutrino flux at the approximate oscillation maximum of $2 \mathrm{GeV}$. In the medium energy configuration horn 1 is positioned $1.3 \mathrm{~m}$ downstream of the target and horn 2 is situated $13 \mathrm{~m}$ downstream with respect to the front face of horn 1.

\subsubsection{Off-axis Design}

Both NOvA detectors are positioned at 14 mrad off-axis with respect to the beam. This has the effect of reducing the overall event rate at both near and far detector but causes the neutrino flux at the oscillation maximum to increase whilst also reducing the background. A discussion of the underlying physics follows.

The decay of pions, such as those produced by the NuMI beamline, can be described by two-body decay kinematics, producing a neutrino and muon. In the rest frame of any 


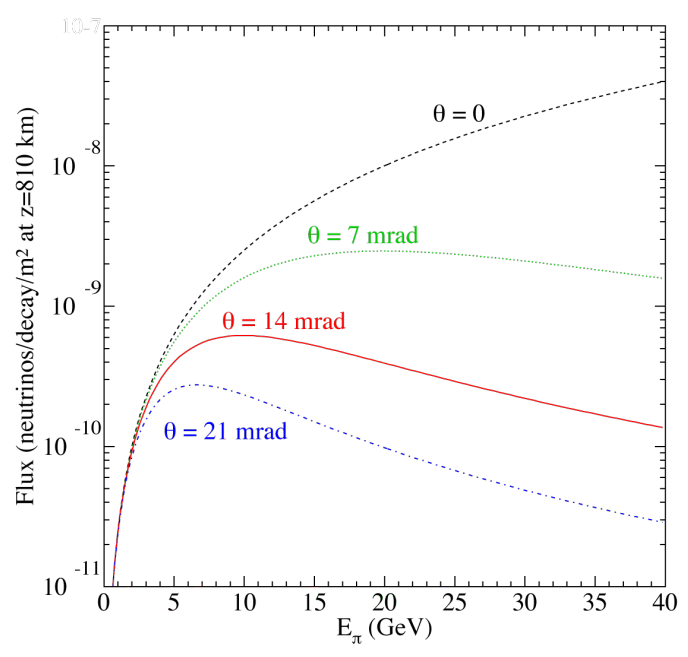

(a) Neutrino flux vs. pion energy.

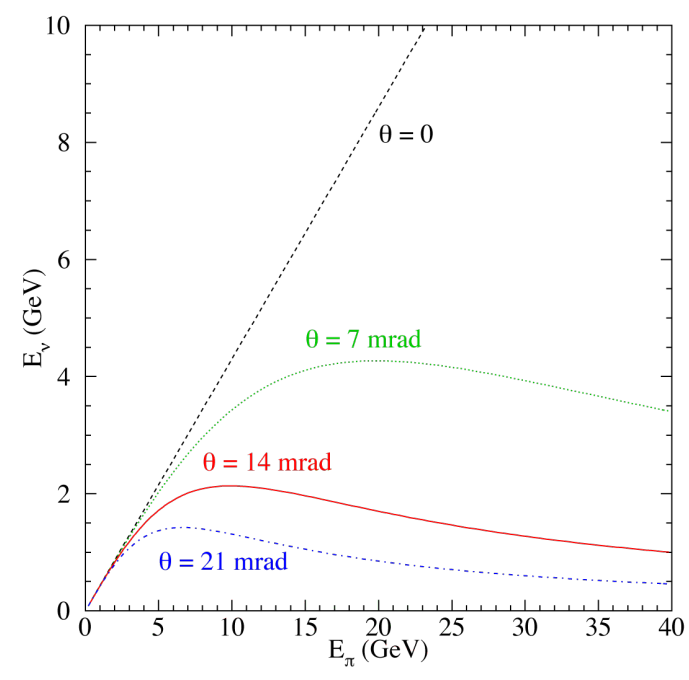

(b) Neutrino energy vs. pion energy.

Figure 3.3: Distributions for the medium energy NuMI beam configuration as viewed from a location off-axis by an angle $\theta, 810 \mathrm{~km}$ from the NuMI target. Plot 3.3a shows the neutrino flux as a function of the energy of the parent pion for different off-axis angles. Plot 3.3b shows the neutrino energy as a function of the parent pion energy for different off-axis angles. This diagram was taken from [5].

given particle its decay is isotropic, whereas in the laboratory frame the daughter particles are boosted in the direction of the parent. For small angles, the flux $(\Phi)$ per pion decay $\left(\pi \rightarrow \nu_{\mu}+\mu\right)$ is given by

$$
\Phi=\left(\frac{2 \gamma}{1+\gamma^{2} \theta^{2}}\right)^{2} \frac{A}{4 \pi z^{2}}
$$

and the energy $\left(E_{\nu}\right)$ of neutrinos produced is given by

$$
E_{\nu}=\frac{0.43 E_{\pi}}{1+\gamma^{2} \theta^{2}}
$$

where $E_{\pi}$ is the energy of the parent pion, $m_{\pi}$ the mass of the parent pion, $\gamma=E_{\pi} / m_{\pi}, \theta$ the angle between the parent pion and outgoing neutrino direction, $A$ is the cross-sectional area and $z$ is the distance from the pion decay vertex.

For the medium energy NuMI beam configuration Figure 3.3 shows $\Phi$ (Equation 3.1) and $E_{\nu}$ (Equation 3.2) plotted as functions of pion energy. Four off-axis angles are shown in this figure $(\theta=21$ mrads, $\theta=14$ mrads, $\theta=7$ mrads and $\theta=0$ mrads $)$ with the NOvA off-axis angle $\theta=14$ mrads shown in red. Figure $3.3 \mathrm{~b}$ shows that when using the medium energy NuMI beam configuration and a 14 mrad off-axis detector the neutrino energy does not have a strong dependence on the parent pion energy. 
Figure 3.4 shows the neutrino event rate as a function of the $\nu_{\mu}$ energy when utilising the low-energy (Figure 3.4a) and medium-energy (Figure 3.4b) NuMI beam configurations. The distributions are shown for various off-axis angles (the angles correspond to the ones shown in Figure 3.3). The plots show a decrease in the energy spectrum mean and a narrowing of the energy spectrum width as the off-axis angle is increased. Additionally, Figure $3.4 \mathrm{~b}$ shows the peak at $1.9 \mathrm{GeV}$ with a neutrino rate approximately four times greater than the on-axis equivalent. The first oscillation maximum occurs at about 1.6 GeV given $\Delta \mathrm{m}_{32}^{2}=2.45 \times 10^{-3} \mathrm{eV}^{2}$ and NOvA's baseline. In addition to the benefit of increasing the expected neutrino flux at the oscillation maximum, placing the detector off-axis helps reduce the neutral-current background. Neutral current interactions are a prevalent background source whose event topologies can be hard to distinguish from electron showers produced by $\nu_{e}$ charged current events. In such neutral current events, the neutrino carries a significant amount of the energy away. The remaining energy tends to "feed down" to lower energies. An off-axis narrow band beam helps circumvent this problem by significantly reducing the flux of higher energy neutrinos. The majority of the remaining neutral current energy spectrum is pushed below the signal region of the $\nu_{e}$ appearance analysis. Figure 3.5 shows the number of $\nu_{\mu}$ CC, $\nu_{e} \mathrm{CC}$ and NC events as a function of energy. It is apparent that the majority of neutral current events (black) are shifted below the signal region (red-hatched).

\subsection{The NOvA Detectors}

This section delineates the design of the NOvA detectors. First, the general detector architecture is described followed by a detailed description of the near and far detectors.

\subsubsection{Experimental Design}

As with other long-baseline neutrino oscillation experiments, NOvA employs two functionally identical, but volumetrically differing, detectors - a near and far. The detector similarity, in physical structure and instrumentation, helps minimize the impact of systematic uncertainties in the analysis.

NOvA takes measurements of $\nu_{e}$ appearance and of $\nu_{\mu}$ disappearance. A key experimental requirement is separation of $\nu_{e} \mathrm{CC}$ interactions from neutral-current (NC) events or other CC events that contain a $\pi^{0}$ and thus, the detectors are optimized for electron- $\gamma$ separation. They are constructed from carbon and other low Z materials which facilitates particle identification in scenarios where $\pi^{0}$ 's mimic electron showers. The detectors have 


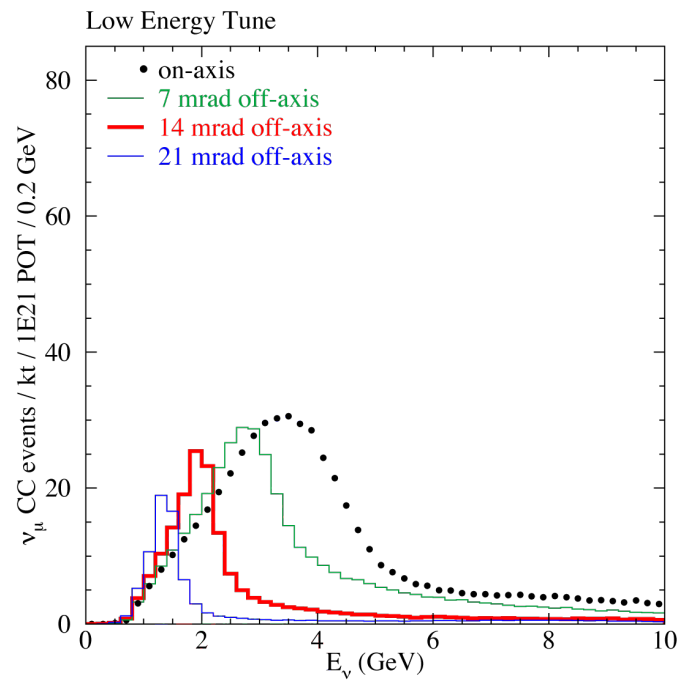

(a) Neutrino energy distribution for the low energy NuMI beam configuration.

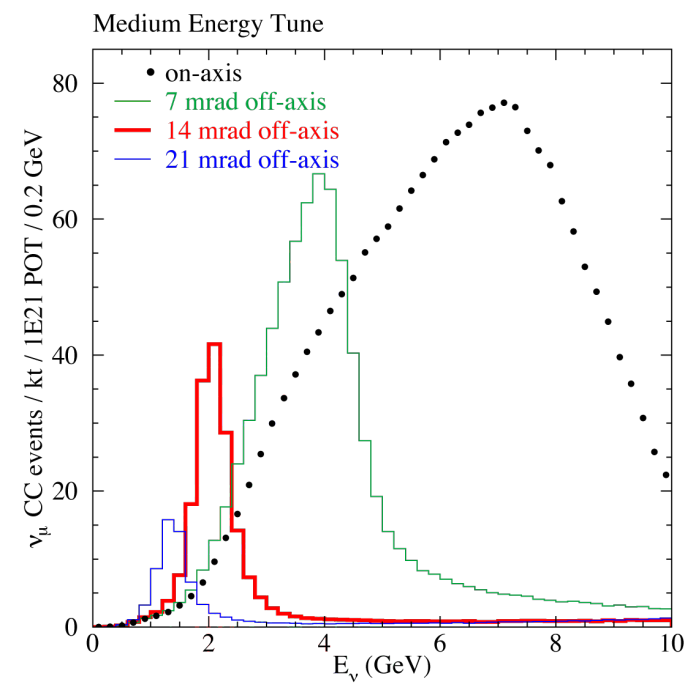

(b) Neutrino energy distribution for the medium energy NuMI beam configuration.

Figure 3.4: Rate of charged current $\nu_{\mu}$ events vs neutrino energy in the absence of oscillations. These distributions correspond to a detector at various off-axis angles that is $800 \mathrm{~km}$ from the NuMI target. This diagram is taken from [5].

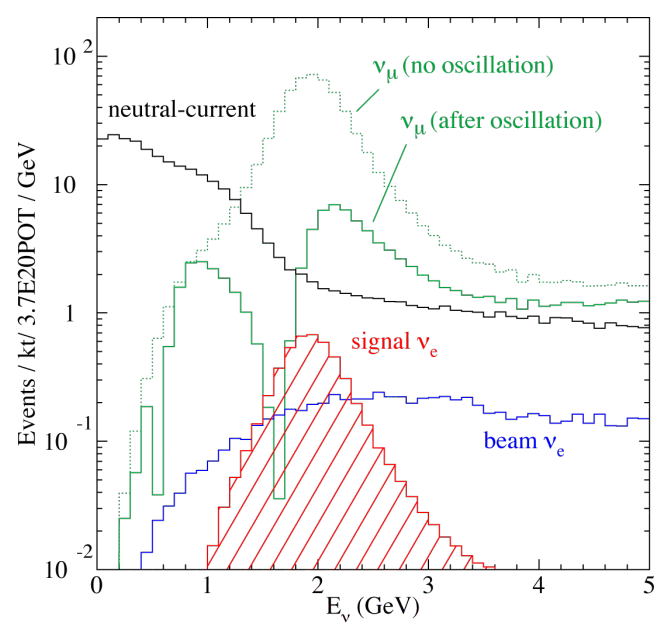

Figure 3.5: Neutrino energy distributions of $\nu_{\mu}$ charged current events (with and without oscillations), signal events from $\nu_{e}$ oscillation, intrinsic beam $\nu_{e}$ events and neutral current events. These plots were produced with simulated Monte-Carlo data. The simulation assumes an off-axis position of $12 \mathrm{~km}$ at $810 \mathrm{~km}, \Delta m_{32}^{2}=2.5 \times 10^{-3} \mathrm{eV}^{2}, \sin ^{2}\left(2 \theta_{23}\right)=1.0$ and $\sin ^{2}\left(2 \theta_{13}\right)=0.1$. Diagram taken from [5]. 


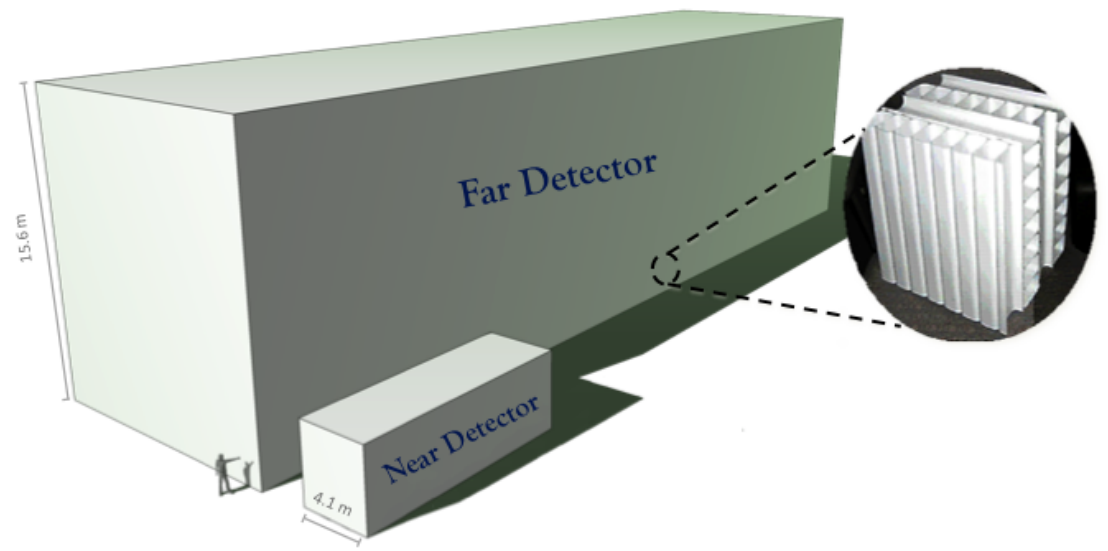

Figure 3.6: NOvA's near and far detectors shown to scale with respect to each other. A cartoon person is included as a reference point. The near detector is approximately $16 \mathrm{~m}$ long, the far detector $60 \mathrm{~m}$. The inset depicts the orthogonal, alternating plane structure.

a radiation length [24] of approximately $40 \mathrm{~cm}$, equivalent to the depth (width) of 7 (10) units of NOvA granularity. Event identification and separation is accomplished through analysis of the event topologies and charge depositions in the detector.

Figure 3.6 shows a scale diagram of the two NOvA detectors. The few differences between near and far detector exist to account for proximity to the NuMI beam and detector depth relative to ground level. The far detector sits on the surface, with a small rock overburden, whereas the near detector is situated $100 \mathrm{~m}$ underground. A significant design difference is the presence of a "muon catcher" in the near detector, which is responsible for extending the energy range of events that can be captured by the near detector. Additionally, The near detector uses a faster electronic sampling rate to reduce the effect of pile-up.

Both NOvA detectors comprise extruded PVC "cells" that act as the basic units of geometric, and hence readout, division. These cells span either the full width or the full height of the relevant detector and are glued together side-by-side to comprise a detector plane. The planes are arranged in alternating, orthogonal layers that are oriented perpendicular to the neutrino beam. A schematic of this structure is shown by the inset in Figure 3.6. This architecture allows for three-dimensional reconstruction of any particle that traverses multiple planes. The NOvA detectors are comprised of blocks made from planes. Each far detector block is made from 32 planes of cells. Each near detector block is made from 24 planes of cells. These blocks are put together to form the complete physical structure of the detectors. 


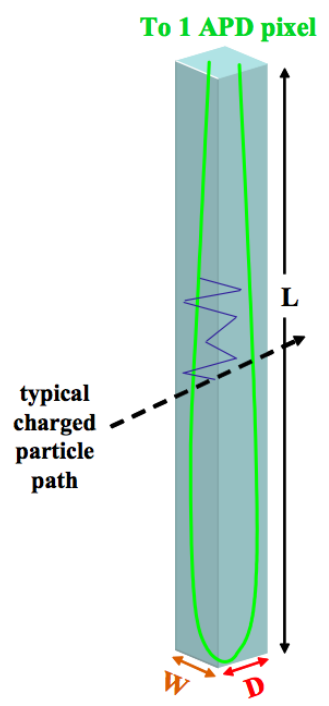

Figure 3.7: Diagram of a NOvA cell comprised of an extruded PVC tube that is filled with liquid scintillator. A looped wavelength-shifting fibre sits inside. A charged particle is shown traversing the cell. Taken from [5].

Figure 3.7 shows a NOvA cell and describes its components. Each cell is filled with liquid scintillator and fitted with a looped wavelength-shifting fibre. This requires that the length of the fibre is at least twice that of the PVC cell itself. This looping allows captured light to travel bi-directionally along the fibre where it is read out at the instrumented side of the cell by a single pixel of an Avalanche Photo Diode (APD) array. Each pixel is thus attached to both ends of the fibre.

The thickness of the cell walls varies between $2 \mathrm{~mm}$ and $4.5 \mathrm{~mm}$. The thicker walls help ensure the detectors' structural integrity. The smaller two dimensions of each cell measure $5.9 \mathrm{~cm}$ along the beam direction and $3.8 \mathrm{~cm}$ perpendicular to the beam direction. The longest dimension corresponds to the full width of the relevant detector. Far detector cells measure $15.5 \mathrm{~m}$ in length, near detector cells measure $3.6 \mathrm{~m}$.

A NOvA extrusion, comprising 16 cells, is shown from an end-on view in Figure 3.8. Each extrusion has cross-section dimensions of $63.5 \mathrm{~cm}$ by $6.6 \mathrm{~cm}$. Figure 3.9 shows an extrusion module made from two extrusions. Each module has a total of 32 cells, an end plate, a side seal, a manifold cover, a snout and an electronics box. The liquid scintillator is contained in the cell by sealing the module at both ends. At one end a manifold cover is employed which also guides the 32 wavelength-shifting fibres onto the corresponding APD pixels. At the other end an end plate and side seal are used. A plane is comprised from multiple extrusion modules glued together.

By mass, liquid scintillator comprises $65 \%$ of the NOvA detectors. Table 3.1 details 


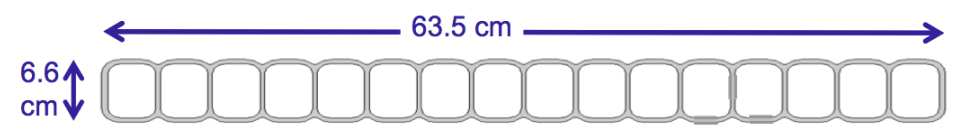

Figure 3.8: An end on view of an extrusion consisting of 16 NOvA cells. Taken from [5].

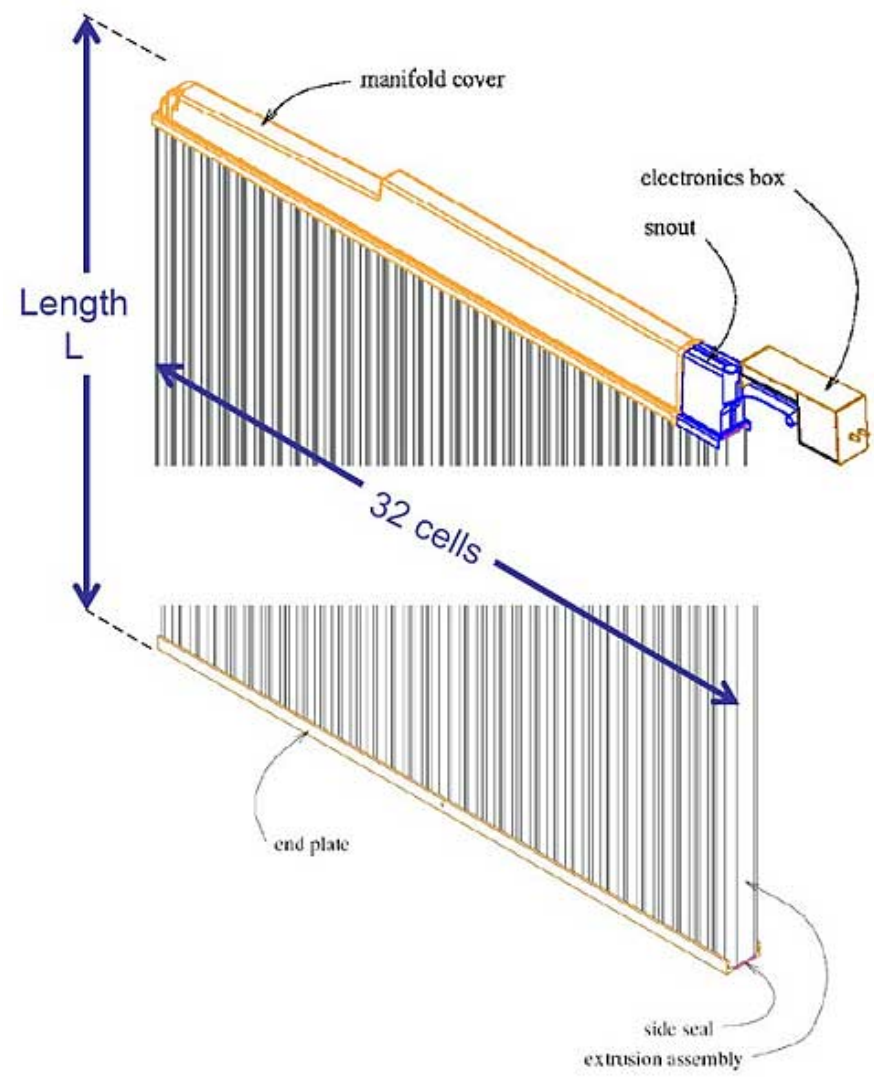

Figure 3.9: Diagram of a NOvA extrusion module. It is constructed from two extrusions of 16 cells, an end plate, a side seal, a manifold cover, a snout and an electronics box. Diagram from [5]. 


\begin{tabular}{ccc}
\hline \hline Component & Purpose & Mass fraction \% \\
\hline mineral oil & solvent & 94.63 \\
pseudocumene & scintillant & 5.23 \\
PPO & waveshifter & 0.14 \\
bis-MSB & waveshifter & 0.0016 \\
stadis-425 & anti-static agent & 0.0010 \\
tocopherol & antioxidant & 0.0010 \\
\hline \hline
\end{tabular}

Table 3.1: NOvA liquid scintillator chemical composition [2].

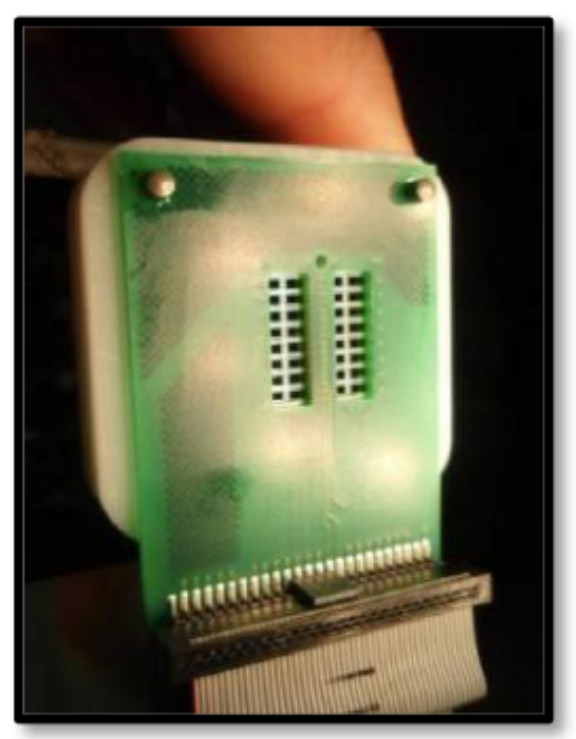

Figure 3.10: Photo of a NOvA APD with a 32 pixel array.

the composition of the liquid scintillator utilised. Mineral oil is the principal component at $94.63 \%$ followed by the scintillant pseudocumene at $5.23 \%$. Pseudocumene emits light with a wavelength spectrum peaked at approximately $375 \mathrm{~nm}$. PPO and bis-MSB act as wavelength shifting chemical additives to shift the spectrum such that it is optimized for absorption by the wavelength-shifting fibres (400-450 nm).

Scintillation light produced by charged particle excitation is absorbed and re-emitted along the wavelength shifting fibre until it reaches the APD readout. The APD converts the light yield into an electronic signal. Each of the 32 APD pixels is connected to both ends of a single wavelength-shifting fibre in a single cell. Each APD reads out to a front end board that digitizes the electronic signals for the data acquisition system to handle. Figure 3.10 shows a photograph of a NOvA APD. The glossy white lining of the PVC cells 
helps maximise the light that is collected in the fibre by means of being highly reflective.

The cores of each fibre are made from a mixture of polystyrene and wavelength-shifting R27 dye at a concentration of $300 \mathrm{ppm}$. Each wavelength-shifting fibre has a diameter of $0.7 \mathrm{~mm}$. Two outer coatings contribute $3 \%(0.021 \mathrm{~mm})$ of the diameter. The inner part of the fibre is an acrylic layer of PMMA and the outer coating is fluor-acrylic ${ }^{1}$. The liquid scintillator emits light in the 400 - $450 \mathrm{~nm}$ wavelength range. The fibre absorbs this light and the R27 dye shifts the wavelength range to 490 - $550 \mathrm{~nm}$. Light attenuation, at a factor of about 10, occurs along the fibre resulting in preferential survival of light in the 520 - $550 \mathrm{~nm}$ wavelength range.

APD's are used due to their high $(85 \%)$ quantum efficiency at the light wavelength range $520-550 \mathrm{~nm}$, which corresponds to the light that exits the fibre. The APDs have a thermal noise background that is reduced by thermo-electric coolers. These coolers keep each APD at $-15^{\circ} \mathrm{C}$.

The NOvA Data Acquisition System NOvA's electronics operate as a continuous readout system. The entirety of the detector APD information is coordinated onto a single data stream. This data stream is then transmitted onto the memory bank of buffer farm computers for temporary storage. The DAQ's primary purpose is to manage this process. This temporary data is sorted by software which decides on whether to permanently store or delete it. External triggers are also used to decide on the acceptance/rejection of data. The accelerator signal from Fermilab, which indicates a NuMI beam spill, is an example of one such trigger.

The most upstream components of the DAQ are the front end boards (FEBs). The FEBs have a one to one mapping with each APD. The FEB handles the shaping, timestamping and pedestal-subtraction for each signal. This digitized signal is passed to a data concentrator module (DCM) interfaced with up to 64 FEBs. A DCM collates all information it receives in a $50 \mu s$ window called a "microslice". The data from each microslice is then passed to the buffer farm.

The NuMI Spill The NOvA far detector is situated on the surface. This greatly increases the detector activity rate and reduces the signal to cosmic ray background ratio. A data-driven trigger capable of capturing all neutrino events would be costly to implement

\footnotetext{
${ }^{1}$ The fibre have a lower refractive index than the core. This disparity facilitates the total internal reflection of light within the fibre and so helps maximize the $\gamma$ yield by capturing the photons before they undergo further interaction.
} 
- the disk space requirement to record all data would be untenable. An external trigger incident with the NuMI beam spill is used instead. For each NuMI beam spill a trigger signal is time stamped and a network packet is delivered to both NOvA detectors. The $2.8 \mathrm{~ms}$ neutrino time of flight to the far detector is corrected for. This trigger indicates when to record data to permanent storage. A NuMI spill trigger has two parts - a $T_{0}$ time stamp and a $\Delta T$ duration. When the DAQ sees a trigger, the buffer nodes are scanned for all microslices from all DCMs that are incident with the trigger window. This incidence is defined as the time between $T_{0}$ and $T_{0}+\Delta T$. This data is then written to disk for long term storage. For NuMI beam spills $T_{0}$ occurs approximately $218 \mu s$ before the $10 \mu s$ beam spill and $\Delta T$ is set to $500 \mu s$. These values of $T_{0}$ and $\Delta T$ help ensure that the beam data is roughly centred in the much larger readout window. Event windows called sidebands look at detector activity outside of the beam spill. These sidebands allow analysis of background events under identical detector conditions. They also provide insurance against the NOvA timing system drifting out of synchronisation.

Timing System It is essential to have synchronised and accurate hardware to record data. NOvA uses a timing system to ensure that the internal clocks of DAQ hardware components remain coordinated. The near and far detector (separated by $810 \mathrm{~km}$ ) must be precisely time aligned. This is achieved through an absolute wall clock derived from a GPS signal. This clock was verified with an atomic clock that was synced to the MINOS detector (which was known to be synchronised with the NuMI beam) [39]. The atomic clock was moved from the Soudan mine to the NOvA far detector and back again [40].

Two identical timing systems are employed by the NOvA detectors. A primary system is utilised by both, with the other system acting as a backup. Both systems use a master timing unit directly connected to the GPS antenna. This master unit provides the clock ticks for the entire timing system. It also has reset and re-synchronization options which can be used when needed. Timing stamps are delivered from the most upstream to the most downstream DAQ component - starting at the DCMs before being distributed to the FEBs. Timing offsets between DCMs, due to different cable lengths, can be calibrated offline by utilisation of this master signal. These offsets have been studied and validated by comparing hit times along tracks that cross DCM boundaries [41].

\subsubsection{The NOvA Near Detector}

The NOvA near detector is positioned $1.015 \mathrm{~km}$ downstream from the NuMI target, onsite at Fermilab. Figure 3.11 shows the detector's position in relation to the MINOS 
hall alongside the MINOS shaft and NuMI beamline axis. The near detector is $105 \mathrm{~m}$ underground and therefore experiences a reduced cosmic ray background relative to the far detector. The neutrino beam travels downward at an angle of $3^{\circ}$.

The near detector's proximity to the beam means it experiences a far higher neutrino interaction rate than the far detector. The near detector sees approximately 5-10 neutrino interactions per $10 \mu s$ beam spill window. To address this, the near detector electronics sample each APD pixel every $125 \mathrm{~ns}$ compared to the far detectors sampling frequency of $500 \mathrm{~ns}$. This improves the detector's time resolution and thus helps with separating events that overlap due to data pile-up.

The near detector is functionally equivalent to its far detector counterpart but with two differences in infrastructure to accommodate its proximity to the NuMI target and smaller size:

- An increased electronic sampling rate relative to the far detector.

- The presence of a muon catcher to assist in muon containment.

In the near detector 20,192 cells are arranged in 214 cell planes. The fully active part of the detector has dimensions of $4.2 \mathrm{~m} \mathrm{x} 4.2 \mathrm{~m} \times 12 \mathrm{~m}$. The muon catcher extends the longest dimension to $15.8 \mathrm{~m}$. Figure 3.12 shows a schematic of the NOvA near detector, including the Near Detector cavern, access ways, the fully active detector blocks and the muon catcher.

For the sake of muon containment, the ND has a muon catcher at its most downstream end. The muon catcher has a unique construction compared with other elements of the NOvA detectors, made from a series of stacked cell planes and steel. A pair of one vertically-oriented and one horizontally-oriented scintillator plane is interleaved with one $10 \mathrm{~cm}$-thick plane of steel. In total, there are 11 pairs of scintillator planes separated by 10 steel planes in the muon catcher. The vertical planes are constructed from 3 extrusion modules. The horizontal planes are constructed from 2 extrusion modules. This disparity means that the muon catcher's steel and scintillator planes are shorter in height, but equally wide, to the rest of the detector.

\subsubsection{The NOvA Far Detector}

The NOvA far detector is positioned $810 \mathrm{~km}$ downstream from the NuMI target at Ash River, Minnesota. The detector bottom sits approximately $10 \mathrm{~m}$ below the surface, $372 \mathrm{~m}$ above sea level. The far detector is level with local gravity but off-axis from the neutrino 


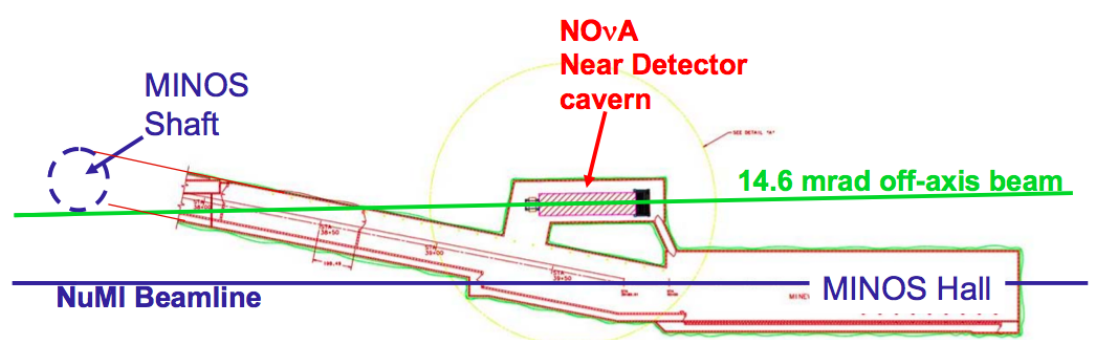

Figure 3.11: Diagram of the site on which the ND is located. The off-axis position is visible in green with respect to the beamline shown in blue. [4].

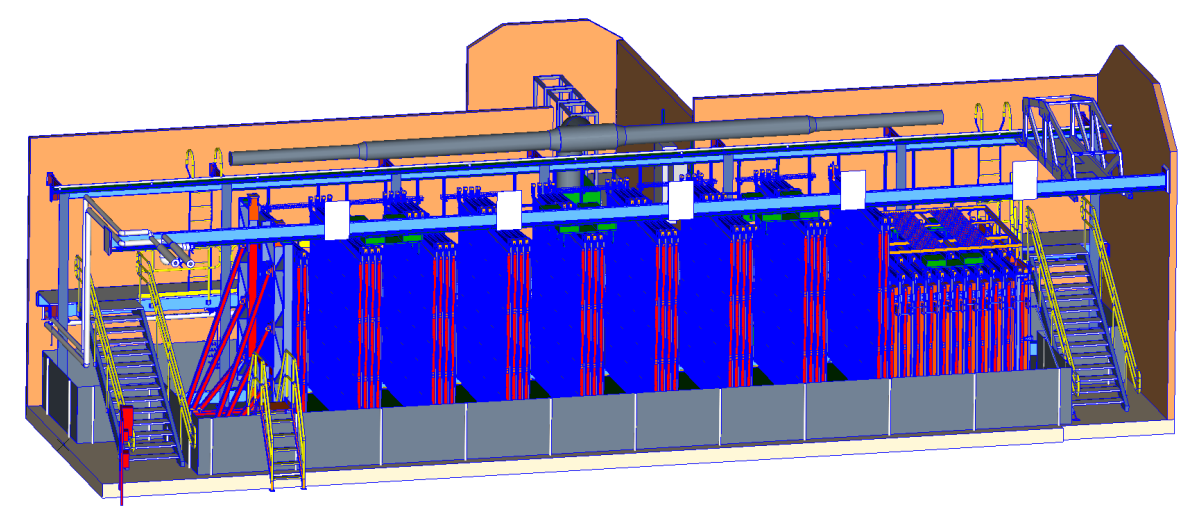

Figure 3.12: Technical drawing of the NOvA near detector and cavern. The NuMI beam enters from the left. The muon catcher planes are located on the downstream side of the detector. Note that only some of the planes have been drawn to aid visualisation of the detector layout. 
beam which points up at an angle of $3^{\circ}$. The total mass of the far detector is 14,000 tonnes comprising 896 planes constructed from a total of 344,064 $15.5 \mathrm{~m}$ long cells.

The surface position of the far detector results in a significant cosmic ray background flux entering the detector. The far detector sees approximately $60-70$ cosmic rays in a 550 $\mu s$ window - spread over approximately 17 times more channels than the near detector. This rate would be higher were it not for presence of a rock shielding overburden that sits on a building that houses the detector. The overburden has the effect of reducing the cosmic ray background by a factor 1600. To this end, approximately 9 radiation lengths $(15 \mathrm{~cm})$ of barite were placed above the detector. Additionally, the NOvA detector building comprises a $122 \mathrm{~cm}$ thick concrete enclosure that forms the roof and walls above ground. The combined effect of the barite and concrete provides 12 radiation lengths worth of shielding.

This thesis uses NOvA data taken from seven separate running periods, labelled period 1 through period 7. The far detector architecture is alike in all of these running periods save for period 1 and period 2 which recorded data from a partially instrumented detector. This was due to the modular construction of the far detector in which sections of the detector, called diblocks, were installed and instrumented sequentially during data collection. Period 1 also utilised 'coarse timing' with lower resolution to digitise the data read out from the far detector APDs. All other periods, and all near detector data, utilised 'fine timing' which provides better resolution of the hit time and consequently reduces the contribution of noise hits to the calorimetric energy. From period 3 onwards the far detector was operated in high gain mode, where the APD gain was increased by $50 \%$. These changes in the running conditions of the detectors are modelled in the simulation.

Periods 1, 2, 3 \& 5 comprise forward horn current data in which a neutrino beam was produced by NuMI. Periods $4,6 \& 7$ comprise reverse horn current data in which an antineutrino beam was produced by NuMI. 


\section{Chapter 4}

\section{NOvA Analysis Methodology and Systematic Uncertainties}

This chapter details the $\nu_{\mu}+\bar{\nu}_{\mu}$ disappearance analysis methodology. The process by which interaction information is extracted from raw data is called reconstruction. It associates hits on an APD to specific particle interactions within the detector based on spatial proximity and time incidence, allowing segmentation of the data into events. The groups of hits in these events can be used to extract event topology, identify specific interaction channels and estimate particle energy.

A detailing of the NOvA Monte-Carlo simulation is presented first, followed by an overview of the reconstruction process. The method by which the systematic uncertainties are estimated and addressed is described last.

\subsection{Monte Carlo Simulation}

NOvA utilises several software packages to simulate the experiment. The $\nu_{\mu}+\bar{\nu}_{\mu}$ disappearance analysis uses simulation to predict event distributions in the NOvA detectors, develop analysis methods, analyse performance of existing analysis methods and study systematic uncertainties. The NuMI beam, detectors, particle interactions, particle propagation and detector response are all simulated.

The simulation chain starts with the production and dispersion of the NuMI beam. The beam simulation models the production of hadrons from proton collisions on a graphite target. It then simulates the resulting decays in the target hall and decay volume that produce a neutrino beam. A detailed model of the beamline is used to this end. GEANT4 is responsible for geometry modelling of the target, horns, target hall, and decay volume. 
The PPFX package, developed by the MINERvA collaboration for the NuMI beam, is used to correct the neutrino flux according to constraints on the hadron spectrum obtained in thin-target hadroproduction data [42] - see Section 4.1.1 for a complete detailing of the PPFX flux weighting process. Secondary particles produced from the initial proton-target interaction are propagated through the geometry where they either continue to interact or decay. Neutrinos produced from decay products of particles are all saved for downstream simulation.

The simulated beam flux files are used as inputs to GENIE [43] to simulate neutrino interactions within the detector and surrounding volumes. The neutrino interaction rate, type of interaction, interaction kinematics and the interaction topology are all modelled. GENIE simulates these interactions using neutrino interaction cross sections combined with probabilistic sampling of the simulated beam neutrino rate. GENIE also determines the final particles emerging from the nucleus following an interaction. Delineation of the cross-section weighting process is given in Section 4.1.2. After GENIE has determined the final state particles of a given interaction, propagation and interaction of those particles through the detector geometry is simulated by GEANT4. The energy depositions in each cell are then passed to NOvA's proprietary readout simulation software.

The readout response of NOvA to energy depositions is modelled using internally developed software. Two such pieces of software are used $[44,45]$. The first of these modules starts its simulation from energy deposition in the NOvA cell and finishes its simulation as photons are collected at the APD. The second module simulates the FEB response to the signals provided by the APDs. The resultant APD signal is a combination of the photons arriving at the APD and the noise modelling of the APD itself.

The first step in the simulation of detector response is modelling the propagation of photons in the scintillator. Following this, photon capture on the fiber, transport along the fiber and electronic response are simulated.

The cells are assumed to be identical to allow the use of general templates for modelling energy deposition. A per-cell simulation would be too computationally intensive. The template function determines the rate of photon capture on the fiber as a function of the position at which the fiber captures it. The number of photons incident on the APD as a function of time is determined by fiber transportation. Half of the photons captured propagate in each direction along the fiber. The average number of photons arriving at the APD is determined from an attenuation curve that was measured in bench tests. The number of photoelectrons produced by the APD are calculated utilising Poisson sampling 
with the quantum efficiency accounted for. APD noise is modelled as a log-normal distribution because it extends beyond Poisson statistics. The time of photoelectron liberation is determined by the energy deposition time and the photon propagation time through the fiber. Photon transport time in the fiber is calculated from a ray tracing simulation.

The front end electronics are also simulated. Analogue signals are generated for all cells with photoelectron signals at the point of photoelectron creation. The shape of this signal can be used to determine the total number of photoelectrons. Pile-up in cells is solved by modelling a signal for each energy deposition, and the summed result across all signals. The signals are then digitized at a sampling rate of $125 \mathrm{~ns}$ and $500 \mathrm{~ns}$ for the near and far detector respectively. Noise is added randomly from a distribution of noise hits as measured from real data cosmic events. Hits are created from simulated electronic responses to model those used in the data acquisition system. Once hits have been simulated, the simulation is complete and data can be treated as equivalent to real data, albeit with associated simulation information.

Cosmic rays are simulated to aid calibration and provide information for accurate reconstruction of muon events. The CRY package is used to model cosmic ray interactions in the detector. CRY simulates cosmic ray particle shower distributions from tables derived from full simulations of primary cosmic rays in the atmosphere. From this simulation CRY determines a list of particles that cross the simulated detector volume within the trigger window [46].

The modelled interaction volumes, at both the near and far sites, includes the material surrounding the detector, as well as the detector itself. The near detector is exposed to a high rate of particles induced by neutrino interactions in the rock upstream of the detector, outside the detector hall. This results in event pile-up wherein particle interactions that occur inside the detector are incident in time with detector activity caused by external neutrino induced activity. Rock interactions are simulated independently, due to computational limitations, and the resulting data is then randomly overlaid with detector events to match total detector activity to expectation. Due to statistical limitation the rock interactions are reused many times in the ND. Far detector data is simulated such that every trigger window has a neutrino or rock activity.

\subsubsection{Beam Flux Corrections}

The NuMI beamline produces neutrinos through proton-graphite hadroproduction and subsequent decay. Neutrino predictions are subject to significant uncertainty due to the 
complexity of QCD-affected hadroproduction and hadron attenuation in beamline materials and optics. This section describes the Package to Predict the Flux (PPFX) beam corrections used by NOvA to reduce this uncertainty.

PPFX is NuMI experiment agnostic software package that applies two types of correction weight to the flux. The first corrects for mis-modelling of interaction probability (the attenuation correction) and the second corrects for mis-modelling of post-interaction daughter particle production (the interaction correction) [42].

PPFX weights are given by

$$
\text { weight }_{i}=\frac{N_{i}^{\text {data }}}{N_{i}^{M C}}
$$

where $\mathrm{N}$ is taken from the data or MC cross-section given the projectile, target, produced hadron and event kinematics. The label $i$ refers to the initial and final state system and each $N_{i}$ term holds information on the hadron production dataset bin, the uncertainty $\sigma_{i}$ and the covariance with respect to other bins in the dataset.

Particle Attenuation Correction Interaction rate is dependent on cross-section and the extent of material through which the particle propagates. If an interaction does not occur, PPFX applies a correction to the particle survival probability. If an interaction does occur then the correction is applied to the survival probability of the particle up until the interaction point.

Interaction Correction PPFX uses FLUKA to remove residual energy dependence at the NuMI-appropriate energy range of 12-120 GeV. Thanks to the joint effort of thin target experiments invariant double differential cross sections are available as functions of Feynman-x $\left(x_{F}\right)$ and transverse momentum $\left(p_{T}\right)$. PPFX interaction corrections are invoked directly from these measurements. Where measurement data is limited or not available, pure theoretical models are used to adjust or estimate the corrections. The associated uncertainty is estimated by comparison with other datasets.

\subsubsection{Neutrino Interaction Modelling and Cross-Section Corrections}

This section starts with a brief overview of the cross-section dependent GENIE and GEANT4 simulations that model interactions and particle propagation in the detector. This is followed by a description of the cross-section tune used for the $2018 \nu_{\mu}+\bar{\nu}_{\mu}$ disappearance analysis.

Figure 4.1 shows the default $\nu_{\mu}$ charged current cross section as a function of neutrino energy as used in GENIE. The black data points are experimental measurements, the 


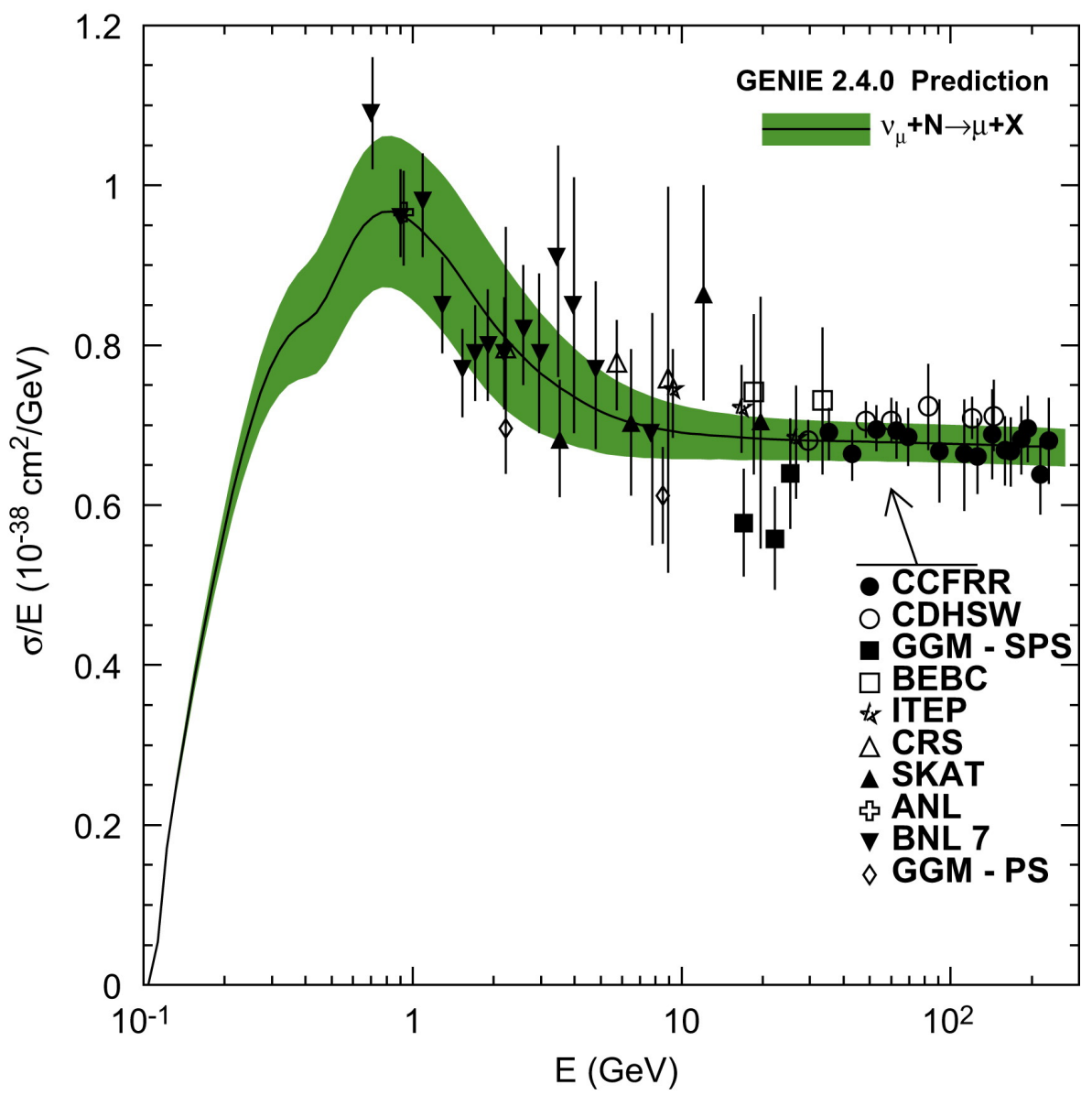

Figure 4.1: Default cross section in GENIE for $\nu_{\mu}$ charged current scattering with an isoscalar target. The shaded green band shows the estimated uncertainty on the free nucleon cross section and the various black points correspond to data from various experiments, as described by the legend. Figure taken from [43].

black curve is the fit of standard model theory to the observed data and the green shaded band is the associated estimated uncertainty. Following a simulated neutrino interaction, GENIE simulates the propagation of the resulting primary particles through the nucleus (where appropriate), including inter-nuclear scattering and absorption. After GENIE or CRY has determined the final state particles of a given interaction, propagation and interaction within the detector are simulated by GEANT4. This simulation models further interactions that occur within the detector and the decay of final state particles using GEANT4's particle cross sections.

As of 2016 Meson Exchange Current (MEC) processes have been included in the NOvA cross-section model. Meson exchange current (MEC) events involve the emission of two nucleons following the interaction of a weak boson from the leptonic current. Other experiments have provided evidence that MEC interactions contribute to the neutrino 
interaction event rate and thus need to be accommodated [47]. NOvA's near detector data supports this hypothesis [48]. The inclusion of these corrections commenced from the 2016 analysis [49]. This was done at the GENIE level where a model of MEC processes was used to simulate how neutrinos scatter from nucleon pairs within the nucleus via a meson exchange current between nucleons. The semi-empirical model used was initially motivated by observations in electron on nucleus scattering [50].

The $2018 \nu_{\mu}+\bar{\nu}_{\mu}$ disappearance analysis incorporates many updates to the crosssection modelling used by previous analyses. The changes to the cross-section tune were motivated by a general drive to lessen the impact of cross-section systematics but also to incorporate antineutrino data and better address MEC events. The cross section tune is performed first by analysis of theory and data (internal and external) followed by adjustment for non-MEC activity. MEC activity is incorporated into the model by a final set of tweaks.

$M_{A}$ CC QE GENIE is used to calculate the quasi-elastic cross-section. It uses the single axial nucleon dipole form factor - constrained by deuterium data. GENIE then corrects for the Pauli blocking effect and a random phase approximation (RPA) is applied.

QE \& RES RPA The need for a quasi-elastic random phase approximation has been well established by the MINERvA collaboration's measurements of NuMI interactions [42]. Current calculations, taken from Rik Gran [51], only exist for the QE RPA, though the effect is thought to act in low momentum transfer events such as pion production through resonance. An RPA correction to resonance events is thus applied by using a crude generalization of the QE RPA as a function of $Q^{2}$.

DIS Deep inelastic scattering corrections are simulated by two independent GENIE models that operate at different regimes of total invariant mass $(\mathrm{W})$ - either side of $\mathrm{W}=1.7 \mathrm{GeV}$. A normalization, based on the data, is applied to correct for the uncertainty introduced by this model mixing which scales up all DIS events across the energy regime, with $\mathrm{W}>1.7 \mathrm{GeV}$, by $10 \%$.

MEC MEC activity is difficult to accurately predict, with disagreement between the best theoretical models and experimental data. In the intermediate hadronic energy regime, between QE and RES, MEC dominates the total cross-section uncertainty and NOvA uses ND data to estimate its impact. After all non-MEC cross-section corrections are applied MC is tuned to data and the MEC contribution is estimated by a simultaneous fit to 
q0 and q3 under the assumption that the majority of the remaining data-MC disparity is attributable to MEC.

Antineutrinos The QE RPA correction is applied universally for neutrino and antineutrino data. MEC is tuned separately for neutrinos and for antineutrinos. The wrong-sign $\nu_{\mu}$ component of the RHC data uses the MEC tuning of the FHC $\nu_{\mu}$ signal. The DIS tune is only applied to neutrinos as few antineutrino events occupy the $\mathrm{W}>1.7 \mathrm{GeV}$ region and the observed discrepancy was only visible in FHC. The RES RPA correction is applied to both neutrino and antineutrino events but estimated from different calculations.

Figure 4.2 and Figure 4.3 show the reconstructed neutrino energy distributions for each interaction type before and after the application of the cross-section tune for FHC and RHC respectively. There is an appreciable improvement in data-MC agreement after application of the cross-section corrections.
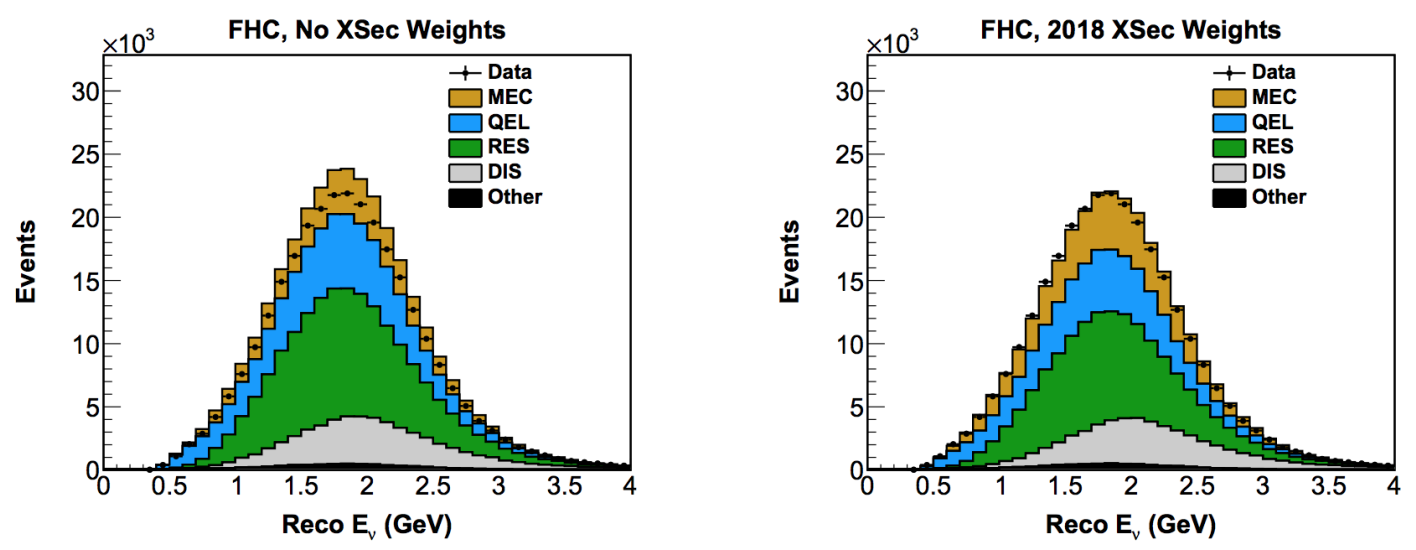

Figure 4.2: FHC reconstructed neutrino energy distributions for each interaction type before (left) and after (right) the application of the cross-section tune. Data is shown by black circles, MEC in brown, QE in blue, RES in green, DIS in gray and other interaction types in shaded black. Figure taken from [52].

\subsection{Event Reconstruction}

Reconstruction involves the production of many event sub-structures that categorize hits into objects such as tracks, showers and events. The process starts with the identification of APD signals above a noise-filtering threshold. These hits are then grouped into collections incident in space and time - a process called slicing [53, 54]. This helps separate unrelated interactions and aids noise discrimination. Each slice is then processed through track 

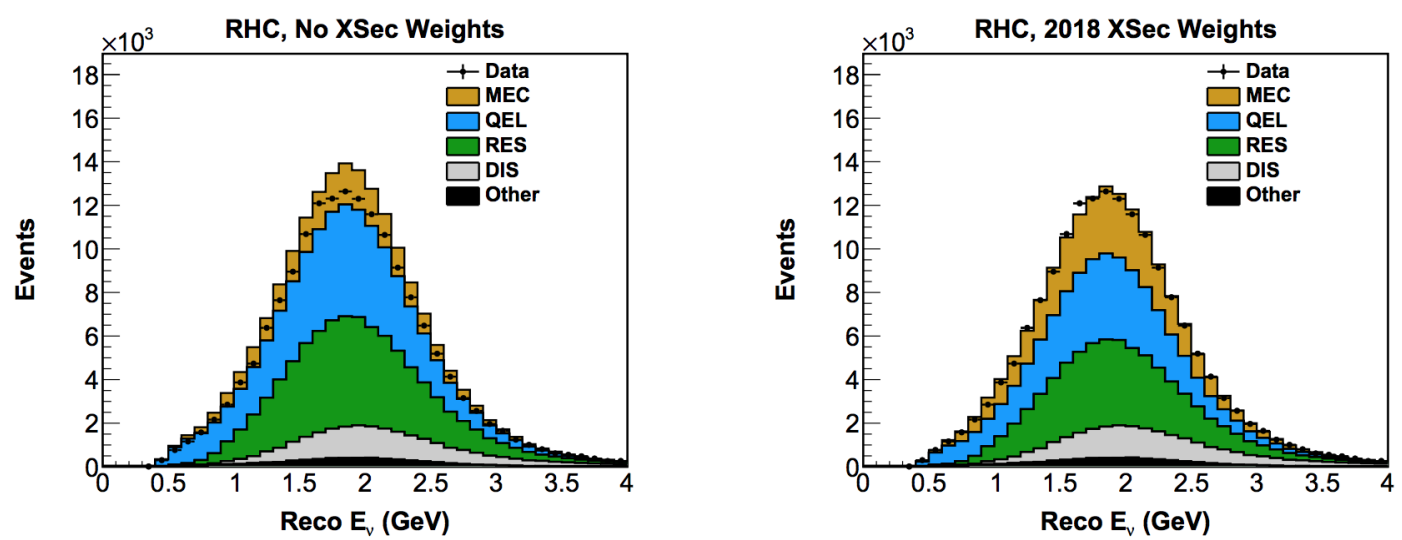

Figure 4.3: RHC reconstructed neutrino energy distributions for each interaction type before (left) and after (right) the application of the cross-section tune. Data is shown by black circles, MEC in brown, QE in blue, RES in green, DIS in gray and other interaction types in shaded black. Figure taken from [52].

identification to map the flight path of particles that deposited energy in the detector. Tracks are then used to reconstruct events and identify analysis candidates [53].

The process by which hits are associated to a given track is known as track finding. First, small segments of track called seeds are formed by identifying detector activity with two hits less than 4 cells apart. These seeds are used to estimate the position and gradient of a track and a Kalman filter is used to elongate the track by adding hits that fit its current topology. A hit is selected for addition to a track if it is less than $8 \chi^{2}$ units away from the track prediction based on a linear fit where the $\chi^{2}$ is calculated per degree of freedom. Upon addition of new hits to a track, the position and direction of the track are recalculated. This process is repeated until no new hits can be added.

Track identification proceeds from the farthest downstream end of the detector. Statistically, this is where a given NuMI beam neutrino interaction is most likely to be maximally disparate, and so most separable. The reconstruction then proceeds upstream towards the point of least separation, i.e. the interaction vertex or vertices. Track propagation concludes when no further hits are consistent with the track projections or if the probability of a gap existing in a track from one hit to the next is below $\frac{1}{1 \times 10^{5}}$. After the tracks have been maximally propagated upstream, the propagation is run in the downstream direction to collect additional hits that may have been missed. A third and final propagation is then run, proceeding upstream, to separate tracks near the interaction vertex. Track finding continues this process of hit assignment and propagation until no new tracks can be formed in a slice. A NOvA specific implementation of the Kalman filter algorithm is 
used to identify tracks in this manner $[55,56]$.

Figure 4.4 shows a reconstructed event in the NOvA far detector in which the tracks have been identified using the Kalman track algorithm. The top and bottom halves of the figure show the XZ and YZ detector planes respectively. Each of these has a twodimensional trajectory that can be aligned to identify a three-dimensional track [56]. The hit time and associated hit charge are shown as histograms in the bottom left and right respectively. Different coloured lines represent uniquely identified tracks. Well reconstructed tracks are characterised by particles with long and straight trajectories. Such tracks are typically dominated by small angle multiple scattering with some large scattering angles resulting from strong or electromagnetic interactions [56].

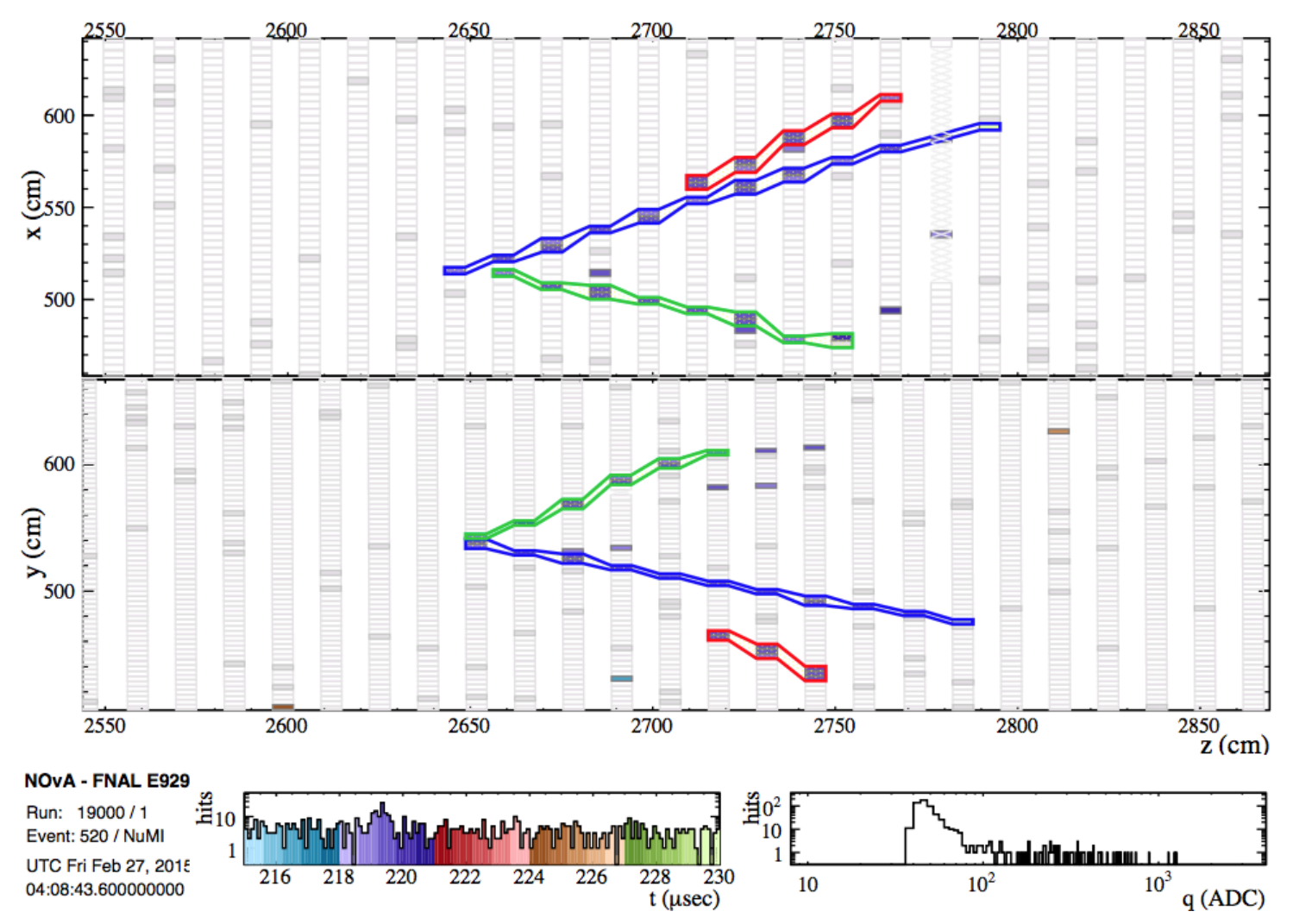

Figure 4.4: Reconstructed MC tracks identified by the NOvA Kalman tracker algorithm. Uniquely identified tracks are shown by the different coloured (red, blue and green) lines. The XZ and YZ detector planes are shown in the top and bottom half of the figure respectively. Figure taken from NOvA's Kalman track technical note [56].

Accurate topological information is required to characterise the energy, momenta and trajectory of particles identified in the detector. Total neutrino energy $\left(\mathrm{E}_{\nu_{\mu}\left(\bar{\nu}_{\mu}\right)}\right)$ is calculated as the sum of hadronic $\left(\mathrm{E}_{\text {had. }}\right)$ and muonic $\left(\mathrm{E}_{\mu}^{-(+)}\right)$energy,

$$
E_{\nu_{\mu}\left(\bar{\nu}_{\mu}\right)}=E_{\mu^{-(+)}}+E_{h a d}
$$


where $\mathrm{E}_{\mu}^{-(+)}$is calculated from the length of the muonic track, and $\mathrm{E}_{\text {had }}$ is calculated from the sum of the remaining energy deposited in the detector.

\subsection{Selection and Background}

NOvA uses an on-the-surface far detector and an underground near detector. The dominant far detector background source is from cosmic ray events. The most significant beam background source is from $\mathrm{NC}$ events with smaller background contributions from $\nu_{e} \mathrm{CC}$ and $\nu_{\tau}$ CC events.

Data Quality Selection The data quality cuts are the first selection criteria applied. They act at the run, subrun, and spill levels. These cuts streamline the volume of data processed downstream by removing events with difficult or impossible to analyse characteristics. These cuts are determined by the Data Quality Working Group [57].

Using general nomenclature (CAF nomenclature ${ }^{1}$ ), the data quality cuts are:

- Number of hits in slice (slc.nhit) $>20$

- Number of cosmic tracks identified (trk.ncosmic) $>0$

- Muon likelihood score (sel.remid.pid) >0

- Number of continous planes hit (slc.ncontplanes) $>4$

The first of these cuts removes events with fewer than 20 reconstructed hits as they lack the spatial information for accurate reconstruction. Events with few hits are characteristic of low energy events and are therefore unlikely to have originated from a beam neutrino. The second cut ensures that events have a minimum of one 'cosmic-tracker' identified track. The cosmic tracker specifically identifies highly linear tracks (not just cosmic origin tracks) - events for which no such tracks can be identified are likely to be low energy or swamped by noise.

The third cut ensures a ReMId PID (a metric for neutral current rejection) exists for at least one 3D Kalman track in a slice. The ReMId score can be interpreted as a measure of a track's muon-likelihood. By requiring a ReMId score of $>0$, events with poorly reconstructed tracks are removed.

The fourth cut requires a slice to contain hits in four consecutive planes. This cut ensures data with geometric gaps, and therefore inconsistencies for analysis, is removed. Addi-

\footnotetext{
${ }^{1}$ See Chapter 4, Section 4.4 for an overview of CAF.
} 
tionally, given the orientation of the incoming beam neutrinos, it removes vertical muons that are highly unlikely to come from the NuMI source.

Containment Selection The primary function of the containment cuts is to select events that are fully inside the active volumes of the near and far detector. Ideally, selected events do not exit the detector and leave an energy signature that corresponds to true energy, making them simpler to accurately reconstruct. Detector-exiting events likely deposit energy near the detector edges, a feature shared by cosmic background events, and so the containment cuts have a second function - to reject cosmic and rock events. Rock events occur when the $\nu$ interaction vertex is external to the detector but energy depositions occur within the detector due to the propagation of a daughter particle(s). Rock events predominantly originate from muon propagation and are often referred to as rock muons. The containment cuts do not prioritise cosmic rejection. Instead, a separate family of cosmic rejection cuts are used to this end. Nonetheless, the function of the containment and cosmic rejection selections are entangled.

Far detector containment operates at the hit, prong (track-like object) and Kalman track level. The containment criteria employed for NOvA's 2018 analysis were re-optimized and updated by the author - the process for which is detailed in Chapter 5. Cosmic and Kalman tracks are required to cross a certain number of detector cells when extrapolated from their end points to the detector edges. The exact number of cells varies depending on the direction of extrapolation and the track finding algorithm, Cosmic or Kalman, that identified the track. Hits are required to be further than 1 plane from the front or back of the detector. Prongs (which can be thought of as a mid-reconstruction track objects) are also required to be a sufficient distance from each of the detector edges. This is performed on a per edge basis to ensure that the functionality of any given cut is minimally biased by the cosmic activity experienced at a different edge.

Near detector containment is performed using an independent set of cuts to the far detector to accommodate differences in detector design, size and surface depth. The only functional difference between detectors is the presence of the muon catcher in the near detector. The near detector is also $100 \mathrm{~m}$ underground and so experiences a significantly reduced cosmic background with respect to the far detector. Near detector containment operates on reconstructed showers, Kalman tracks and muon catcher activity. Showers are required to be sufficiently far from each detector edge. This is calibrated for each edge of the detector independently to avoid bias due to local detector activity over tightening cuts. The muon catcher is required to solely contain activity from the primary muon 
track to ensure that energy depositions within the detector are reconstructable. For the same reason, the primary muon track has to exit into the muon catcher at a height the muon catcher can see. Analogous Kalman cuts to the FD are also used to ensure track projections are sufficiently far from the detector edges. The reconstructed location of the interaction vertex is required to be in the main part of the detector, not the muon catcher. The first and last detector planes are also cut on - removing all events with hits in the outermost two planes [58].

Particle Identification Selectors Three machine-learning algorithms are used for particle identification in NOvA analyses. Reconstructed Muon Identification (ReMId) scores a track's muon-likelihood, Convolutional Visual Network (CVN) uses event topology to score neutrino-event likelihood and the cosmic Boosted Decision Tree (BDT) scores the likelihood an event is not of cosmic origin. The BDT is a tree-based implementation of gradient boosting, ReMId is an implementation of a k-nearest-neighbour 'lazy learning' algorithm and CVN, the most algorithmically complicated, is an implementation of a GPU-trained convolutional neural network. Expansive overviews of the underlying algorithmic methodologies used by each score are not within the scope of this thesis. For thorough descriptions of the BDT, ReMId and CVN scores refer to the internal NOvA documents [59], [60] and [61] respectively. All three algorithms are used to facilitate signal-background discrimination but they operate on different event characteristics. The selection boundaries of these algorithms were optimised by the author, the process for which is described in Chapter 6.

ReMId ReMId is a k-nearest neighbour classifier [62] that is used to compute a track's muon likelihood [63]. The four input features to the algorithm are track length, $\frac{d E}{d x}$ likelihood, scattering angle likelihood and fraction of planes along the track consistent with having minimum ionizinglike $\frac{d E}{d x}$.

Muons have characteristic $\frac{d E}{d x}$ loss as a function of energy prescribed by the Bethe-Bloch equation. Charged pions lose energy through hadronic scattering as well as Bethe-Bloch processes. The log-likelihood (LL) that a particle of type i created the energy deposition profile of a given track is defined as

$$
L L_{i}^{E}=\frac{1}{N_{\text {Plane }}} \sum j P_{j}^{i}
$$

where $\frac{d E}{d x}$ is measured at plane $j$ and the probability of a particle of type $i$ to have the measured $\frac{d E}{d x}, P_{j}^{i}$ is calculated as a function of the distance from the track end from a 
sample histogram. The number of planes in which the $\frac{d E}{d x}$ is measured $\left(N_{\text {Plane }}\right)$ normalizes the LL among tracks of different lengths.

The scattering LL variable is analogous to the $\frac{d E}{d x}$ LL but characterises tracks by their scattering instead of $\frac{d E}{d x}$. Any curvature in reconstructed tracks is due to scattering because the NOvA detectors are not magnetized. Curvature in muon tracks is attributed to small angle multiple scattering with infrequent Coulomb scattering leading to hard scatters. Charged pions undergo the same scattering processes but have additional scattering from hadronic interactions. Looking at the scattering of the reconstructed track as a function of the distance from the end of the track therefore gives a handle on the muon-likeness of a given track. The scattering LL is defined as

$$
L L_{i}^{\text {Scatt }}=\frac{1}{N_{\text {Scatt }}} \sum j P_{j}^{i}
$$

where $P_{j}^{i}$ is the probability, as a function of the distance from the end of the track, of the particle of type i to have a measured scatter at position j. The $N_{S c a t t}$ factor is applied to normalize the LL among tracks of different lengths, defined by the number of identified scattering processes along the track.

ReMId is scored for each event - the PID distribution is shown in Figure 4.5 for the highest PID valued track for each slice in the FD. The data in the plot is normalized to $18 \times 10^{20}$ protons on target and comes from an independent sample from the training data. A containment cut ensures the start and stop points of the track are $50 \mathrm{~cm}$ from the detector edge. Muon neutrino charged current events are shown in black, and neutral current background events are shown in red. The selected $\nu_{\mu}+\bar{\nu}_{\mu}$-CC events exhibit a distinct peak in ReMId at a score of $\approx 1$. In any given event the highest ReMId scoring Kalman track is selected as the primary muon track. To qualify as a $\nu_{\mu}\left(\bar{\nu}_{\mu}\right)$ charged current candidate event the ReMId score of the primary track must exceed 0.70. This selection gives a far detector signal efficiency and purity of $81 \%$ and $95 \%$ respectively for well contained events under the assumption of oscillation parameters $\Delta m_{32}^{2}=2.5 \times$ $10^{-3} \mathrm{eV}^{2}$ and $\sin ^{2} \theta_{23}=0.5[63,60]$.

CVN Convolutional Visual Network [64] (CVN) is a neural network image classifier that is used for selecting muon neutrino charged current events. As inputs CVN takes two 2D event displays, with a charge dimension, and identifies muon neutrino charged current events from topological characteristics alone - mapped to signal likelihood by the neural networks internal weightings. CVN's score distribution is shown in Figure 4.6. Muon neutrino charged current events are shown in green, and neutral current background 


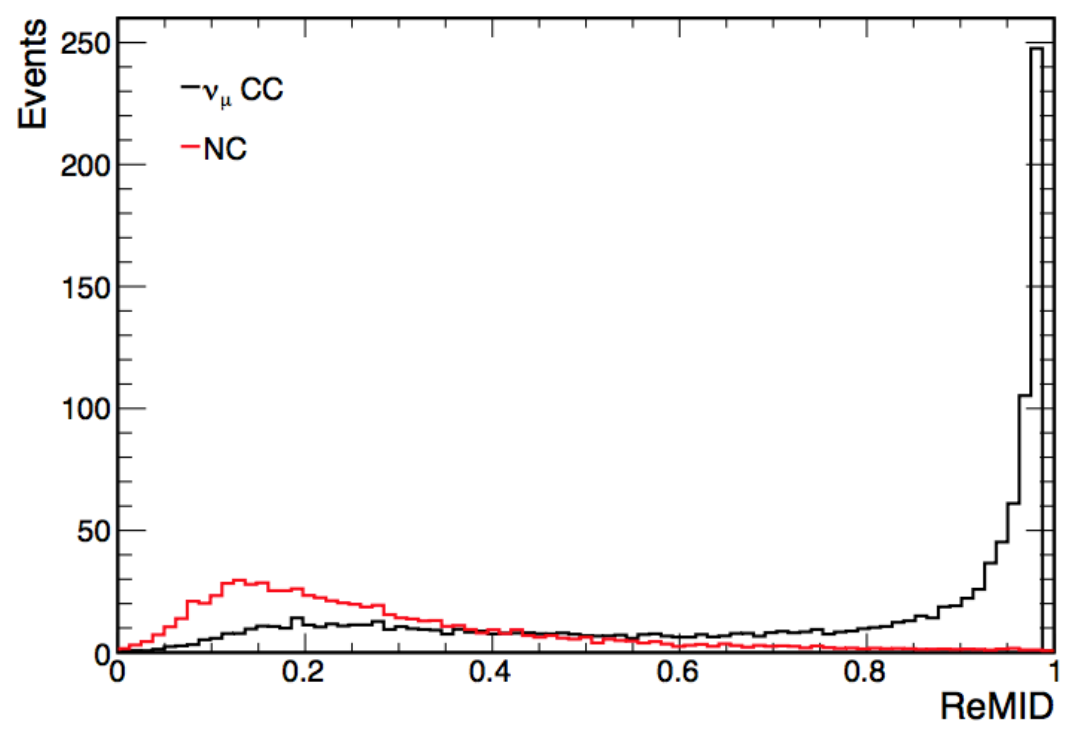

Figure 4.5: Reconstructed Muon Identification (ReMId) scores for muon neutrino charged current signal events (black) and neutral current background events (red). For the standard analysis events are required to have a ReMId score of 0.70 or greater. Figure taken from [60].

events in blue. The appearance $\nu_{e}$ and inherent beam $\nu_{e}$ events are shown by in purple and pink respectively [64]. Two CVN scores are used by NOvA in the latest analysis. The primary analysis selection acts through the CVN algorithm that was trained in 2018 but, due to developmental differences, a very loose CVN score from the 2017 training is also applied for the purpose of background rejection.

Cosmic BDT Additional cosmic rejection is done using a cut on the beam spill window and a boosted decision tree (BDT) that operates on the Kalman track with the highest ReMId score. To understand the BDT, an overview of the co-ordinate system is required. The Z-axis runs parallel to the beam, through the longest detector dimension. The lowest value represents the closest detector edge downstream from the beamline and the highest value in $\mathrm{Z}$ is the furthest. The $\mathrm{X}$-axis runs between the upright walls of the detector housing facility that are orthogonal to the Z-axis. The Y-axis runs, lowest to highest value, from the detector edge sitting on the floor to the detector edge closest to the ceiling. The BDT estimates the likelihood a particle is not of cosmic origin [65] using eleven variables:

1. The angle of the track relative to the NuMI beam direction - larger angles are more 


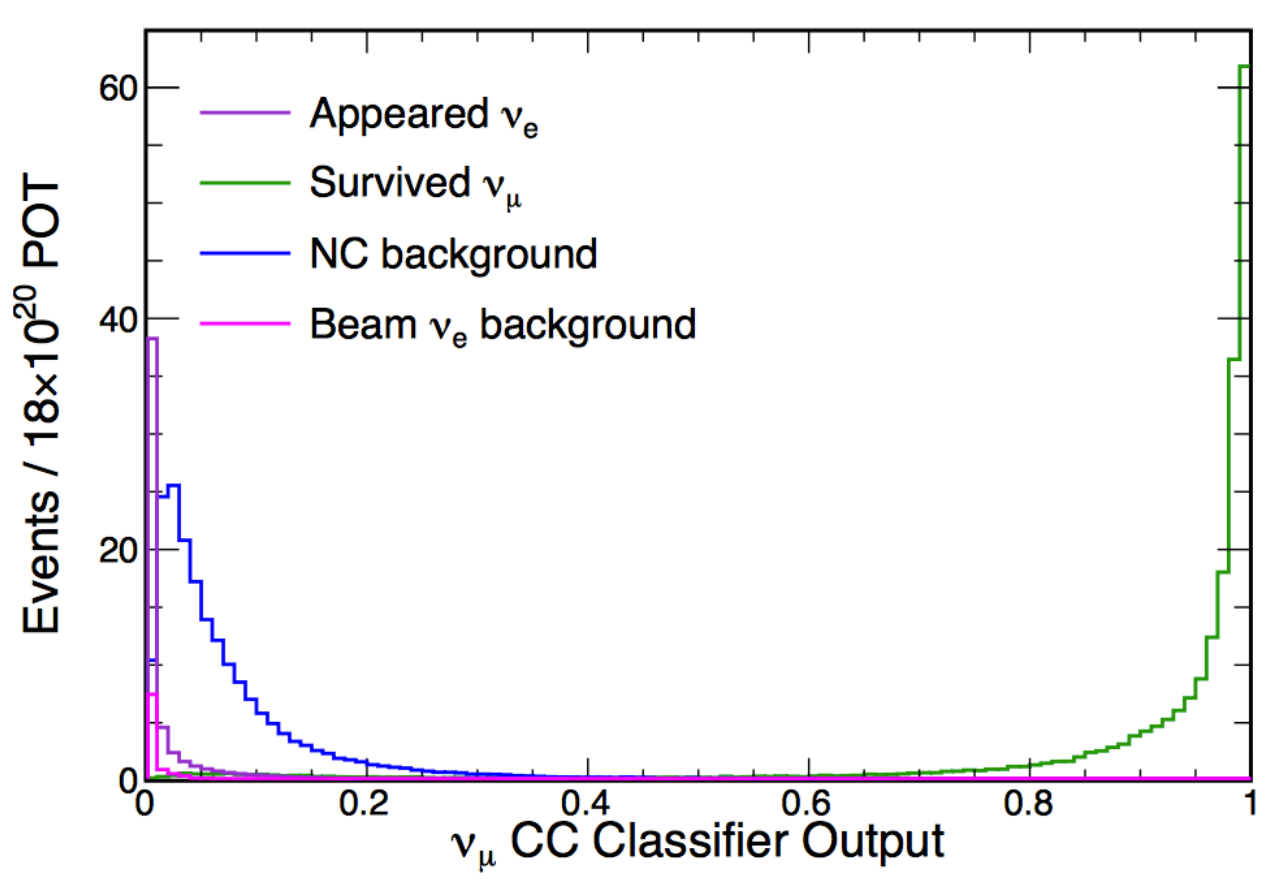

Figure 4.6: Convolutional Visual Network (CVN) distributions for muon neutrino charged current event identification. Muon neutrino charged current events are shown in green. The backgrounds of neutral current events are shown in blue, appearance $\nu_{e}$ events in purple histogram and inherent beam $\nu_{e}$ events are shown in pink. Figure taken from [64].

likely to be of cosmogenic origin.

2. The y-direction of the track - events running parallel to the y-axis are more likely to be of cosmogenic origin.

3. Maximum height of activity within the detector - cosmic origin events are more likely to deposit energy at the top of the detector.

4. The number of hits on the track - events with more than 400 hits are likely cosmogenic.

5. Length of the track - the track length of beam originating events is not likely to exceed a threshold.

6. The number of cells crossed by projections from the end (start) of the track to the furthest (nearest) edge of the detector - cosmogenic events are more likely to be close to the detector edges.

7. The estimated scattering divided by the track length - cosmogenic events exhibit characteristic scattering. 
8. Number of hits in slice - events with more than 400 hits are likely cosmogenic.

9. Visible calorimetric energy per hit in the slice - beam events are energy constrained.

10. Minimum y-position of activity in the slice - the top down nature of most cosmic events means they are more likely to deposit energy at the top of the detector.

11. The number of 3D Kalman tracks in the slice - cosmogenic events sometimes contain many more tracks than events of beam origin.

Spill Selection A 'good spill' selection is used to ensure that the NuMI beam was operating in a state that provides analysable data. The selection requires: spill time ( $<0.5$ seconds), spill POT $\left(>2 \times 10^{12}\right)$, horn current $(-202 \mathrm{kA}<I<-198 \mathrm{kA})$, proton beam position on NuMI target $(-2 \mathrm{~mm}<\operatorname{pos}(x, y)<2 \mathrm{~mm})$ and beam width $(0.57 \mathrm{~mm}<\operatorname{width}(x, y)<1.58 \mathrm{~mm})[66,67]$.

Data Concentrator Module Selection The data quality selection removes events with problems in one or more data concentrator modules. The cuts require that: no data concentrator modules completely drop out during the spill, that the detector is not out of sync and that the fraction of hits outside the NuMI beam spill window (in cells affected by light leaking) is less than $45 \%$ [68]. The DCM edge metric is used to decide whether the detector is out of sync - indicated by an excess number of tracks stopping at DCM edge boundaries [69].

Beam and Cosmic Timing Selection The beam backgrounds are estimated using detector specific simulation. Events that pass selection without containing a true muon or anti-muon neutrino are determined as background. The cosmic background is estimated using far detector data taken outside of the beam spill window. Two such cosmic window samples are used. The first sample is taken using the timing sidebands of the data collected with the NuMI spill trigger. This sample matches the exposure of the detector to the NuMI beam but is statistically limited. The second sample is taken using a pulser trigger where data is collected during a $550 \mu$ s window outside of the beam-spill window. This second sample contains more events and provides better statistics but does not so closely match the far detector running conditions. Figure 4.7 shows the estimated number of cosmic background and signal events after each successive selection is applied to the sample. 


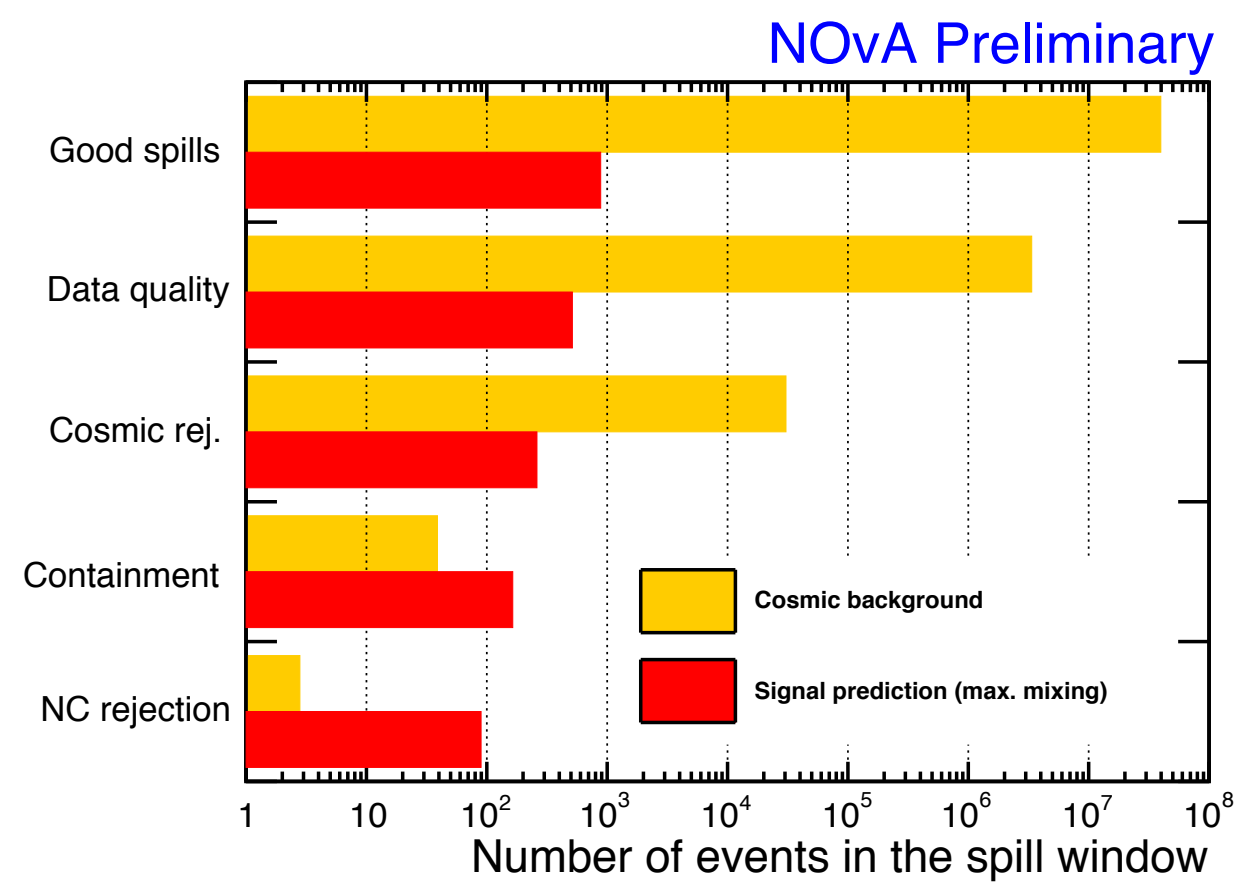

Figure 4.7: The number of signal events (red) and cosmic background events (yellow) surviving each successive analysis selection. The signal is estimated from the simulation and the cosmic background is estimated from the timing sidebands of the NuMI trigger. Figure taken from [70].

\subsection{Analysis Software}

NOvA's Common Analysis Format [71] (CAFAna) software framework is used to produce the results detailed in this thesis. CAFAna builds on top of the data analysis framework ROOT, containing experiment specific programming objects in addition to the standard ROOT library. CAFAna streamlines data handling in NOvA by having common algorithms for producing or manipulating data objects in the reconstruction chain. CAFAna takes individual reconstructed events as an input but fitting uses histogram objects to lessen the required computing time. This high computing efficiency allows for easy comparison of results to new or updated analyses and fast reproduction of old results.

\subsection{Near to Far Detector Extrapolation}

CAFAna includes functionality to extrapolate near detector to far detector - a means of systematic error reduction that allows estimation of FD event rates. In the extrapolation process near detector data is compared with MC and disparities are identified. These differences are then translated to the FD using a combination of far detector MC and 
conversion matrices. CAFAna allows the application, adjustment and extrapolation of systematic uncertainties using systematically shifted template distributions. Minuit [72] is used to fit to the data and accounts for the systematic uncertainties using an oscillation adjusted systematic template spectra.

The extrapolation process begins with the subtraction of expected background from the near detector data distribution. The background expectation is estimated from simulation. A matrix which converts reconstructed energy to true energy is then utilised to produce a true energy spectrum. The true spectrum is then scaled to the far detector using the simulated $\frac{\text { unoscillated }}{\text { oscillated }}$ distribution ratio. This scaling also models differing acceptances between detectors and differing experienced flux at each detector. This scaled spectrum is then re-converted into a reconstructed energy spectrum using a far detector conversion matrix. As with the near detector's reconstructed-to-true matrix, the far detector matrix is calculated from simulation. The reconstructed far detector spectrum is then summed with the far detector beam and cosmic background expectations. The beam and cosmic backgrounds are calculated from simulation and data respectively. The background added far detector spectrum acts as the baseline comparison for the neutrino energy distribution observed in data [49].

\subsection{Constraints and Confidence Limit Contours}

Constraints on oscillation parameters are measured by comparing far detector data with prediction. For each combination of oscillation parameters considered the Poisson loglikelihood is calculated by comparison of data and prediction using Equation 4.5. The first sum runs over all bins analysed. The number of predicted events is given by $\nu_{i}$, the number of observed events by $n_{i}$, the vector of oscillation parameters by $\vec{\theta}$ and the vector of systematic nuisance parameters by $\vec{\delta}$. The last sum gives the log-likelihood due to systematics where $\delta_{i}$ is the value of the systematic and $\sigma_{i}$ is the error. The best fit is found by minimization of the log-likelihood. A $\Delta L L$ score, which represents the difference in likelihood of any given point with respect to the best fit, is used to set confidence limits. The confidence limit in $\Delta m_{32}^{2}-\sin ^{2} \theta_{23}$ phase space is calculated as a two parameter fit. The other PMNS parameters are treated as penalty terms using the constraints set by external experiments. The extent to which a parameter set may be rejected is calculated by $\sqrt{\Delta L L}$. 


$$
\ln \lambda(\vec{\theta}, \vec{\delta})=-2 \sum_{i=1}^{N}\left[\nu_{i}(\vec{\theta}, \vec{\delta})-n_{i}+n_{i} \ln \frac{n_{i}}{\nu_{i}(\vec{\theta}, \vec{\delta})}\right]+\sum_{i=1}^{M} \frac{\delta_{i}^{2}}{\sigma_{i}^{2}}
$$

The analysis sensitivity can be probed before data is observed through use of highstatistics simulated neutrino data using the Asimov approach [73]. The simulated data can be mock-oscillated with any chosen set of parameters. A contour can be constructed from such fake-data and so the analysis sensitivity for a given choice of oscillation parameters can be studied a priori.

\subsection{Energy Resolution Binning}

NOvA utilises energy resolution binning - events are split into four quantiles according to the fraction of hadronic energy comprising the total energy in a given event and into 19 bins according to reconstructed $\nu_{\mu}\left(\bar{\nu}_{\mu}\right)$ energy.

Table 4.1 shows the $\nu_{\mu}+\bar{\nu}_{\mu}$-CC energy binning breakdown for the 2018 joint analysis. The binning is optimized to produce the highest sensitivity without introducing untenable computational overhead - granularity increases around the analysis-paramount oscillation maximum. 


\begin{tabular}{|c|c|c|c|c|c|}
\hline $\begin{array}{c}\text { Neutrino Energy } \\
\text { Range }(\mathrm{GeV})\end{array}$ & Number of Bins & $\begin{array}{l}\text { Neutrino ND Simulated } \\
\text { Neutrino Event Count }\end{array}$ & $\begin{array}{c}\text { Antineutrino ND Simulated } \\
\text { Neutrino Event Count }\end{array}$ & $\begin{array}{c}\text { FHC ND Simulated } \\
\text { Background Event Count }\end{array}$ & $\begin{array}{c}\text { RHC ND Simulated } \\
\text { Background Event Count }\end{array}$ \\
\hline $0-0.75$ & 1 & $1.80 \times 10^{3}$ & $4.02 \times 10^{2}$ & $3.51 \times 10^{1}$ & $7.49 \times 10^{0}$ \\
\hline $0.75-1$ & 1 & $2.65 \times 10^{4}$ & $4.86 \times 10^{3}$ & $2.93 \times 10^{2}$ & $5.07 \times 10^{1}$ \\
\hline $1-2$ & 10 & $1.06 \times 10^{6}$ & $2.10 \times 10^{5}$ & $3.74 \times 10^{3}$ & $6.25 \times 10^{2}$ \\
\hline $2-3$ & 4 & $2.73 \times 10^{5}$ & $5.82 \times 10^{4}$ & $5.18 \times 10^{2}$ & $6.50 \times 10^{1}$ \\
\hline $3-4$ & 2 & $1.34 \times 10^{4}$ & $3.15 \times 10^{3}$ & $4.91 \times 10^{1}$ & $8.27 \times 10^{0}$ \\
\hline $4-5$ & 1 & $9.10 \times 10^{2}$ & $2.34 \times 10^{2}$ & $9.69 \times 10^{0}$ & $3.09 \times 10^{0}$ \\
\hline Total $0-5$ & 19 & $1.38 \times 10^{6}$ & $2.77 \times 10^{5}$ & $4.64 \times 10^{3}$ & $7.60 \times 10^{2}$ \\
\hline
\end{tabular}

Table 4.1: Number of neutrino energy bins used for different energy regimes. The predicted number of ND neutrino events in each bin category for FHC and RHC are shown, as well as the associated estimated background, in the last four columns. FHC data is scaled to $8.03 \times 10^{20} \mathrm{POT}$ and RHC data is scaled to $3.10 \times 10^{20}$ POT. 
Resolution worsens as the percentage of hadronic energy present in an event increases due to the more spatially dispersive, shorter track length interactions that hadronic systems tend to undergo. Binning separates high resolution events from lower resolution events. This in turn reduces the energy measurement uncertainty for events with a lower fractional percentage of hadronic energy.

Figure 4.8 shows the $2 \mathrm{D}$ distribution of $E_{h a d} / E_{\nu}$ against reconstructed neutrino energy, with total events on the Z-axis. The neutrino energy boundaries are visible from the colour separation in adjacent bins and the $E_{h a d} / E_{\nu}$ boundaries, as used in the present analysis, are shown explicitly for FHC (blue solid line) and RHC (dashed pink line).

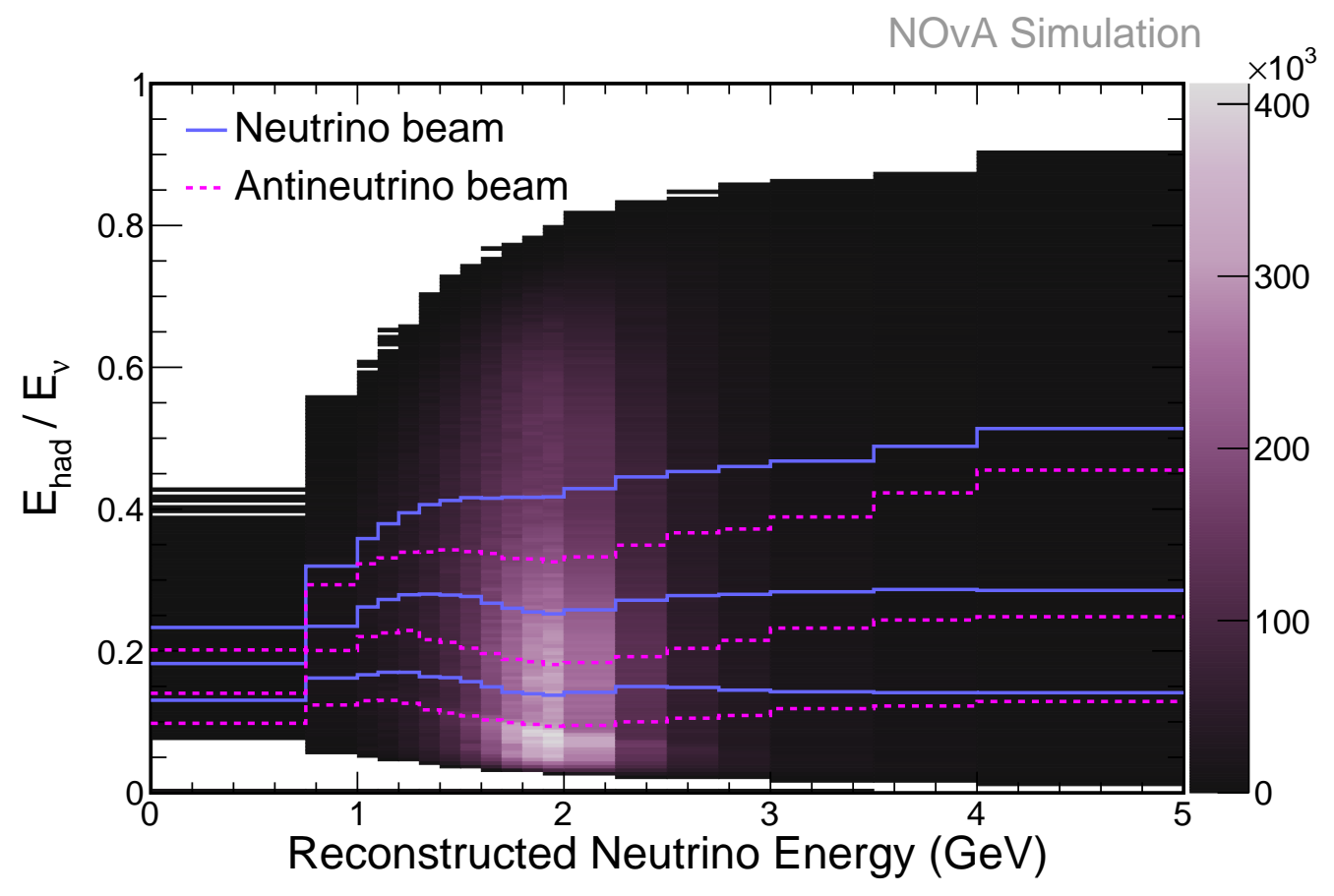

Figure 4.8: $E_{\text {had }} / E_{\nu}$ against reconstructed neutrino energy, with total events on the Zaxis. The variable bin width in neutrino energy can be seen. The bin boundaries for the quantiles are shown for FHC and RHC by the blue solid and pink dashed lines respectively.

Figure 4.9 and Figure 4.10 show the FHC and RHC plots of significance of maximal mixing $\left(\sin ^{2} \theta_{23}=0.5\right)$ rejection against number of $E_{\text {had }} / E_{\nu}$ quantiles respectively. Figure 4.9 was taken from [74] and shows the FHC result. Figure 4.10 was produced by the author to determine whether RHC would require different binning to FHC. It is clear from the two plots that analysis sensitivity plateaus past four bins and so four quantiles are used for both horn currents. 


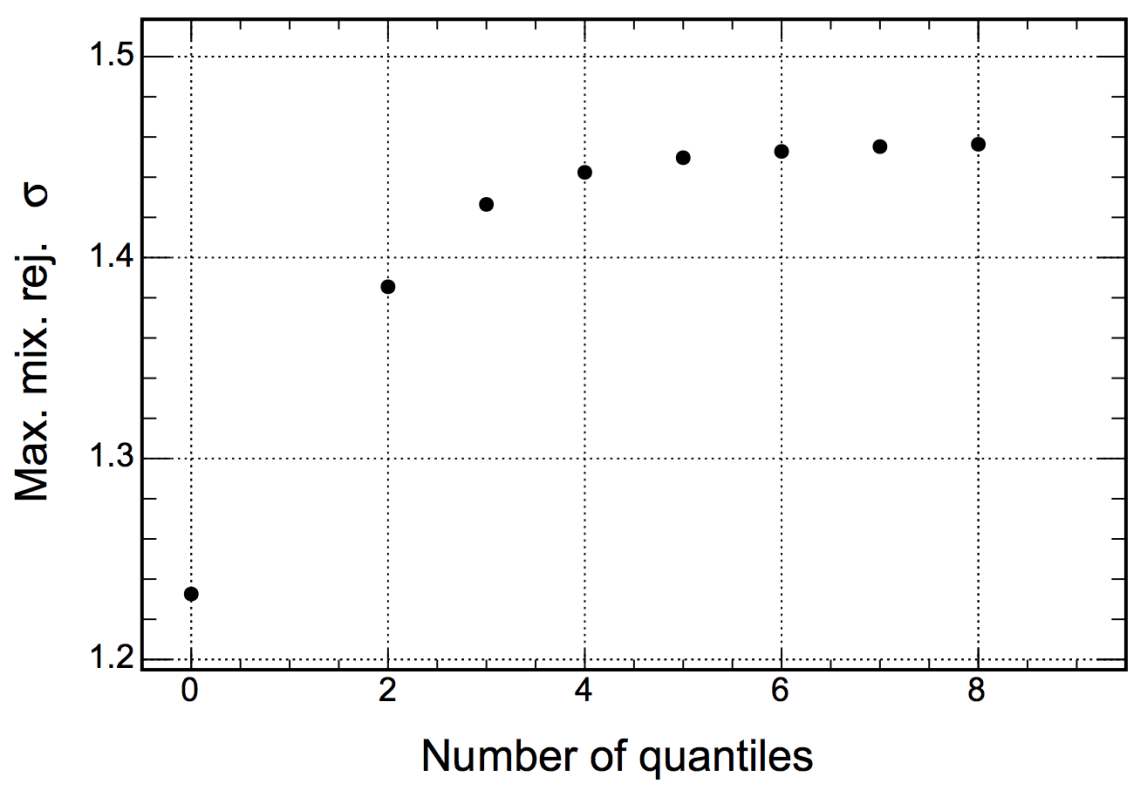

Figure 4.9: FHC significance of maximal mixing $\left(\sin ^{2} \theta_{23}=0.5\right)$ rejection against number of $E_{h a d} / E_{\nu}$ quantiles. NOvAs 2017 analysis sensitivity which uses only a single bin is shown by the point where quantiles $=0$.

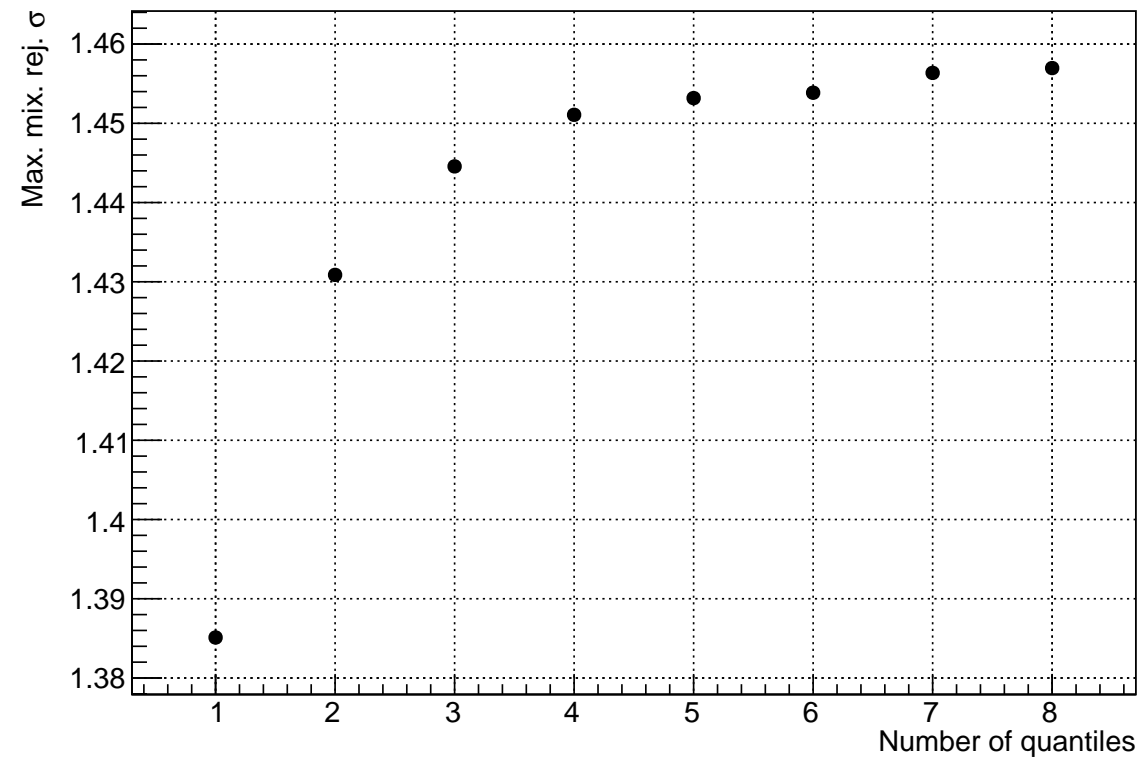

Figure 4.10: RHC significance of maximal mixing $\left(\sin ^{2} \theta_{23}=0.5\right)$ rejection against number of $E_{\text {had }} / E_{\nu}$ quantiles. 


\subsection{Systematic Uncertainties}

NOvA is capable of precision measurement, greatly aided by the use of two detectors to reduce systematic uncertainty. Any mis-modelling of the beam flux, cross-section or detector response can introduce systematic error. This section outlines the systematic uncertainties, describing the source and treatment of each one.

Potential systematic uncertainties are evaluated by re-weighting events, or by generating new event samples. These systematically shifted samples are then processed through the full reconstruction chain and the impact on measurement with respect to the nominal sample can be used to estimate the significance of the systematic.

\subsubsection{Muonic and Hadronic Energy Estimation}

The relationship between muon range and muon kinetic energy gives a handle on an events muonic energy. Accordingly, NOvA estimates muon energy using reconstructed track length. Errors in the estimation of muonic energy are evaluated separately for each detector as well as for the ND muon catcher. All correlated and uncorrelated errors between the detector volumes are also assessed. The ND error, the FD error and the fully correlated error between detectors are all estimated at less than $1 \%$. The dominant contributor to these errors comes from GEANT4's treatment of the Fermi density effect. Other contributors include the externally measured mean excitation energies of elements, the detector material composition and chemical binding effects - though these contributions are significantly less impactful than the error driven by GEANT4. The uncorrelated errors between detectors are contributed to by both scaling effects (which arise due to detector mass accounting) and neutron pile-up in the ND. The absolute error in the muon catcher is dominated by the density effect and the effect of neutron capture pile-up [75].

The uncertainty associated with calorimetric energy calibration is the primary contributor to the overall uncertainty in energy response. Visible hadronic energy is defined as the sum of energy depositions arising from all visible cell hits that are not associated with the muon track - $E_{v i s}^{\text {had }}$. Hadronic energy reconstruction is difficult as neutral particles do not form visible tracks. Additionally, not all of the kinetic energy carried by a charged particle causes energy depositions. Neutrons are particularly problematic as they leave no visible energy signature and are more common in antineutrino interactions. Consequently, $E_{v i s}^{h a d}$ provides an incomplete picture of hadronic kinematics. See Section 4.1.2 for an overview of the models NOvA uses to treat interaction and cross-sections uncertainties. A data-MC comparison of proton energy is used to define an uncertainty of $\pm 5 \%$ on the 
energy scale of hadronic showers. The profiles of $\frac{d E}{d x}$ against the distance to the track end were compared in data and MC for both protons and muons. The best agreement with data was achieved by scaling the MC by $95 \%$ for protons [76, 77].

\subsubsection{Cross sections and FSI}

Neutrino oscillation measurement is reliant on well characterised interaction cross-sections. Final State Interactions (FSI) which affect the hadronic system after the initial primary scattering of a neutrino on a nucleus also contribute significantly to the uncertainty.

Recent measurements of neutrino cross sections have necessitated modifications to the treatment of NOvA's cross-section uncertainties. GENIE is still used to estimate most cross-section and FSI systematics [78] but additional uncertainties are introduced to the model. One of these is applied to the energy and momentum-transfer dependence of quasi-elastic CC scattering and MEC events. These modifications account for longrange nuclear correlations [79], neutrino energy dependence, energy-transfer dependence, final-state nucleon-nucleon pair composition [51, 80], the observed quasi-elastic axial mass excess, non-resonant pion production involving three or more pions, radiative corrections and second-class currents in quasi-elastic-CC events.

\subsubsection{Normalization}

There is an uncertainty associated with imperfections in the simulated model of near detector pile-up. The uncertainty is addressed by comparing signal selection efficiencies in simulation and data, with an additional simulated event overlaid onto each event in the sample. The total uncertainty arising from pile-up is estimated at the $1.3 \%$ level. The overall normalization systematic is produced by adding the individual uncertainties in quadrature.

\subsection{Calibration}

Calibration is important for all NOvA analyses. The goal is to accurately determine the detector's response to a given deposit of energy. The amount of light captured by a given fiber in a cell is calibrated such that it corresponds to the energy deposition within that cell. Calibration is divided into two branches - relative and absolute. Relative calibration is performed first, wherein variations in cell response and variations along fiber length are accounted for. After relative calibration, all hits can be analysed as uniform structures, 
with no positional variance. Absolute calibration then computes a scale factor, from MC and real data, to map detector response to an energy deposition recording.

The general calibration methodology is to use cosmic ray muons to perform a cellby-cell calibration which can probe the effect of light attenuation in the fibers. Biases introduced by hits that fall below the readout threshold or energy depositions originating from self-shielding effects are accounted for using simulation. This is done on a perdetector, per-cell basis for each view. Muons that stop in the detector, well characterized by energy loss, are then used as standard candles for absolute calibration.

Figure 4.11 and Figure 4.12 show the y view detector response in terms of reconstructed over true energy before and after absolute calibration for the near and far detector respectively. $\mathrm{W}$ is the distance from the cell central point along the fiber. Slope and offset disparities between unity and $\frac{E_{\text {reconstructed }}}{E_{\text {true }}}$ are used to create datasets to evaluate the associated systematic uncertainty caused by calibration [81].

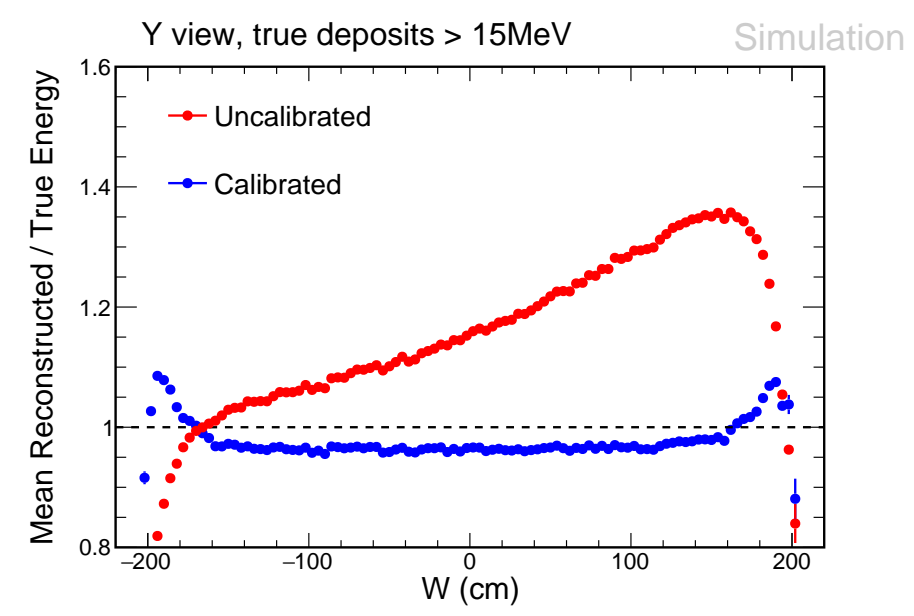

Figure 4.11: The near detector profiles of the reconstructed and true energy ratios as a function of $\mathrm{W}$ for the $\mathrm{x}$ view hits before and after calibration. The red curve in these plots is the ratio of the mean of reconstructed to the true energies before calibration and blue curve is after the calibration. Each point shows the mean of the reconstructed to the true energy ratio distribution at that $\mathrm{W}$ value and the error bar is a measure of the width of that distribution $\left(\frac{R M S}{\sqrt{N}}\right)$. 


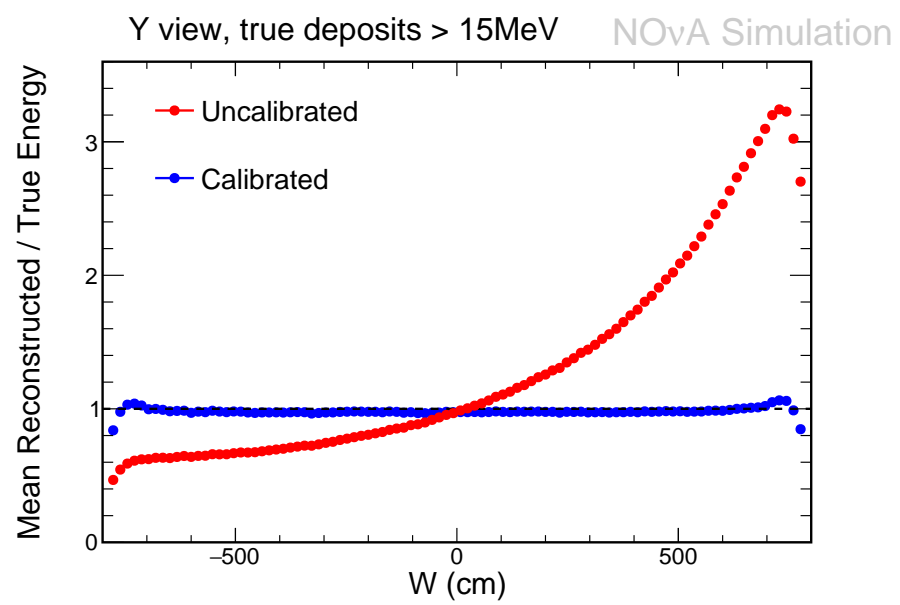

Figure 4.12: The far detector profiles of the reconstructed and true energy ratios as a function of $\mathrm{W}$ for the $\mathrm{y}$ view hits before and after calibration. The red curve in these plots is the ratio of the mean of reconstructed to the $\mathrm{t}$ rue energies before calibration and blue curve is after the calibration. Each point shows the mean of the reconstructed to the true energy ratio distribution at that $\mathrm{W}$ value and the error bar is a measure of the width of that distribution $\frac{R M S}{\sqrt{N}}$. 


\section{Chapter 5}

\section{Containment Analysis}

\section{Improvements}

In the $\nu_{\mu}+\bar{\nu}_{\mu}$ disappearance analysis a set of FD containment cuts are used to select candidate events completely inside the active fiducial volume of the detector. These containment cuts operate on the distance between event features and the detector edges - if an event has activity close to the detector edges it is rejected on the basis that one or more final state particles may have exited the detector. Activity close to the detector edges is a common feature of both detector-exiting and cosmic background events, and so the containment cuts also serve as a cosmic veto. Issues that arise from uncertainties in reconstruction, APD noise and event pile-up cause inefficiencies to be introduced into the analysis, making robust containment optimization challenging.

This chapter describes the previously used FD containment cuts, the methodology used to optimize the current containment criteria and the results of this optimization. The optimization was done following the $6.05 \times 10^{20}$ POT 2017 analysis [49] using neutrino (FHC) simulation only - antineutrino data had not yet been introduced to the analysis. However, due to the topological nature of the variables containment operates on, the selection is agnostic to neutrino or antineutrino interactions. The optimization process allowed changes to the experimental running conditions and analysis methodology to be reflected in the containment decision boundaries. A new cut acting on the six faces of the far detector was also introduced to replace a cut with a noise susceptibility and thus improve the selection robustness.

The selection criteria developed through the work described in this chapter were adopted and used in the $2018 \nu_{\mu}+\bar{\nu}_{\mu}$ disappearance analysis - the results of which are presented in Chapter 7. The selection was also used in both of the published 2018 NOvA 
analyses.

\subsection{Previous Analysis FD Containment Cuts}

The containment cuts used for the $6.05 \times 10^{20}$ POT 2017 [49] analysis are shown in Table 5.1. The cells from edge cut helps ensure that selected events are sufficiently far from the FD borders and therefore less susceptible to energy loss due to particles exiting the detector. This cut operates on cells at the hit level, such that an event is rejected if it contains any hits less than 2 cells away from a detector edge.

The planes to detector front (back) cut also acts on hits ensuring that events are sufficiently far, in units of planes, from the most upstream (downstream) end of the FD. Events are required to be more than one plane from the detector front and more than one plane from the detector back to pass selection.

The Kalman forward and backward cell projection cuts ensure the reconstructed 3D Kalman tracks in a given event are sufficiently far from projecting out of the detector. The Kalman tracks are extrapolated, at both ends, to the FD edges - ten or more cells must be crossed by each extrapolation path for an event to pass selection.

The cosmic forward and backward cell projection cuts ensure the reconstructed cosmictracker tracks in a given event are sufficiently far from projecting out of the detector. As with the Kalman projection cuts, identified cosmic tracks are extrapolated to the FD edges. The number of cells crossed by each extrapolation before the FD edge is hit is required to be greater than zero.

\begin{tabular}{|cc|}
\hline \hline $\begin{array}{c}\text { Name of Cut } \\
(C A F)\end{array}$ & $\begin{array}{c}2017 \text { Value } \\
(\text { planes } / \text { cells })\end{array}$ \\
\hline Number of cells from edge (slc.ncellsfromedge) & $>1$ \\
Planes to Detector Front (planestofront) & $>1$ \\
Planes to Detector Back (planestoback) & $>1$ \\
Kalman Forward Cell Projection (sel.contain.kalfwdcell) & $>10$ \\
Kalman Backward Cell Projection (sel.contain.kalbakcell) & $>10$ \\
Cosmic Forward Cell Projection (sel.contain.cosfwdcell) & $>0$ \\
Cosmic Backward Cell Projection (sel.contain.cosbakcell) & $>0$ \\
\hline \hline
\end{tabular}

Table 5.1: 2017 analysis containment cuts, employed by previous analyses. 


\subsection{Optimization Methodology}

The FD containment cuts have been optimized using a threefold approach. First, the $\nu_{\mu}$-CC signal and cosmic background distributions for each containment variable were evaluated and the minimum cut threshold identified as the point at which signal exceeds background. For variables whose $\frac{\text { Signal }}{\text { Cosmic Background }}$ ratio plateaued, the containment cut was tuned to select all events occurring in a regime equal to or above the plateau point. Finally, the overall effect on the $\nu_{\mu}$-CC signal and cosmic background spectra was assessed by comparing the signal and background event counts when running the analysis with each containment criteria. Further checks were performed by assessing the interaction type distributions and the $\nu_{\mu}$ energy resolution, defined as $\left(\right.$ reconstructed $\left._{\nu_{\mu} E}-\operatorname{true}_{\nu_{\mu} E}\right) / \operatorname{true}_{\nu_{\mu} E}$.

The containment cuts operate on distances measured in various units - centimetres, detector cells and detector planes. Events with activity too close to the detector edges are rejected. Due to this, containment criteria act in a way that reduces the effective detector volume. In NOvA this effective reduction is small, with only the outermost regions of the detector cut on. However, cuts on variables strongly correlated with an event's distance from the upper detector face are more strictly placed - removing a greater fraction of effective detector volume. This is due to the high cosmic flux that the detector top experiences, which causes the $\frac{\text { Signal }}{\text { Cosmic Background }}$ denominator to increase, decreasing the figure of merit.

Pre-Selection Certain preselection criteria are applied throughout the optimization. The data quality cuts were applied as described in Section 4.3. Additionally, the following criteria were applied:

- The ReMId score (sel.remid.pid) $>0.70$

- Cosine of primary track angle with respect to the beam (sel.cosrej.anglekal) $>0.5$.

These preselection cuts remove large numbers of easily rejected cosmics, ensuring that only the more significant cosmic events inform the optimization. They also mirror the preselection used for training the 2018 analysis BDT [82]. The cosmic rejection BDT cuts themselves were not applied. This choice was statistically motivated to allow enough of the cosmic spectra to be present in the distribution for better assessment of cut placement. The full BDT selection would leave too few cosmic events to inform the optimization. 


\subsection{Containment Distributions and Selection Optimization}

This section presents the containment variable distributions and $\frac{\text { Signal }}{\text { Cosmic Background }}$ ratios that drive the cut placements. The selection boundaries are shown superimposed onto each distribution and ratio plot. The variables that have been recycled from the previous criteria and re-optimized are presented first.

The variables introduced to the 2018 containment to replace the 'cells from edge' cut are then shown. The 'cells from edge' cut was used to reject events based on the distance (in cells) of the outermost hit from the nearest detector edge. This cut was replaced by a more robust set of cuts that are better at handling noise. These cuts act on the minimum distance of each far detector edge to a track-like reconstruction object called a prong. Prongs are constructed from hits that are local in time and space, making the new cuts less susceptible to noise. Six new cuts were introduced - one for each FD edge. These cuts use 'LiveGeometry' which models the realtime detector variance caused by hardware maintenance, installation and adjustment. This allows for uniform functionality across all detector configurations and across all data taking periods.

\subsubsection{Re-Optimization of Containment Variables Introduced in Previous Analyses}

The distributions and $\frac{\text { Signal }}{\text { Cosmic Background }}$ ratios for variables that were used in both the previous analysis containment criteria and the updated containment criteria are shown in Figure 5.1 through to Figure 5.6. The orange dashed line shows the 2017 analysis cut, the green dotted line shows the optimized cut for the 2018 analysis. The cut placements reflect the general methodology outlined in the previous section - a combination of hand tuning and quantitative analysis, depending on the distribution. All events to the left of these lines are cut and all events to the right are selected ${ }^{1}$.

Figure 5.1 shows the distribution and $\frac{\text { Signal }}{\text { Cosmic Background }}$ ratio of the 'Kalman Forward Cell' variable. Figure 5.2 shows the distribution and $\frac{\text { Signal }}{\text { Cosmic Background }}$ ratio of the 'Kalman Backward Cell' variable. Both variables exhibit similar distributions with signal-cosmic separation best realized from projections of approximately 6 cells or greater for each variable. These cuts were consequently changed from $>10$ cells to $>6$ cells.

\footnotetext{
${ }^{1}$ Figure 5.1 through to Figure 5.6 were made using NOvASoft version S1705-31. The dataset employed was the 2018 analysis cosmic overlay nonswap FD MC: prod_caf_R17-03-01-prod3recog_fd_genie_nonswap_fhc_nova_v08_Period_v1 where 'Period' is the relevant data running period, as described on a per plot basis.
} 
Figure 5.3 shows the distribution and $\frac{\text { Signal }}{\text { Cosmic Background }}$ ratio of the 'Cosmic Forward Cell' variable. Figure 5.4 shows the distribution and $\frac{\text { Signal }}{\text { Cosmic Background }}$ ratio of the 'Cosmic Backward Cell' variable. The forward cell projection distribution shows no room for improvement, plateaued at $>0$ cells and was kept unchanged with respect to previous analyses. The backward cell projection distribution indicates a loose cut, letting through a non-negligible background, with a low signal to cosmic ratio in the 1 to 6 cell region. The 'Cosmic Backward Cell' projection was resultantly tightened to $>7$ cells from a previous value of $>0$ cells. The backward cell projection has a far greater population of cosmogenic events than the forward cell projection. This is due to the highly down-going nature of cosmic events. The backward projection extrapolation acts towards the detector top where the cosmic population is highest and thus this distribution exhibits a far greater cosmic ratio than the cosmic forward projection.

Figure 5.5 shows the distribution and $\frac{\text { Signal }}{\text { Cosmic Background }}$ ratio of the 'Planes to Front'

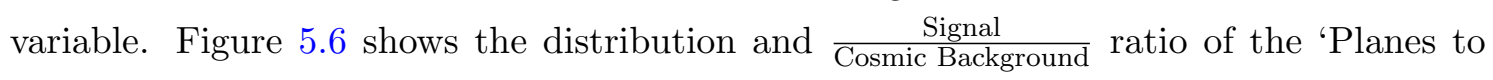
Back' variable. Both variables exhibit similar distributions with signal-cosmic separation already optimized at the cut value used in previous analyses ( $>1$ plane). No changes were made to the cuts on these variables.

Figure 5.1, Figure 5.2, Figure 5.3 and Figure 5.4 exhibit peaks at approximately 3 cells/planes in both the cosmic and $\nu_{\mu}$-CC distributions. Naively, one may expect these peaks to occur at 0 but there are several factors contributing to the offset. The preselection removes many events that occur in the low value regions of these distributions. The track finding algorithms also rely on a likelihood method that favours track identification further from the edges, where hits are generally more populous. Finally, track extrapolations are agnostic to which detector edge is reached resulting in a flattened 1D distribution, constructed from 3D topology that favours minimum extrapolations just above zero.

The cosmic distributions shown in Figure 5.5 and Figure 5.6 exhibit a step like behaviour in which successive planes are alternately populated by a constant magnitude after the $\frac{\text { signal }}{\text { background }}$ plateau. This is likely due to the orthogonal plane arrangement of detector cells. Cosmogenic events are often highly down-going and detector planes that are parallel to the incoming particle are therefore likely to contain a slightly greater number of cosmic events. This theory has not been explicitly studied. 


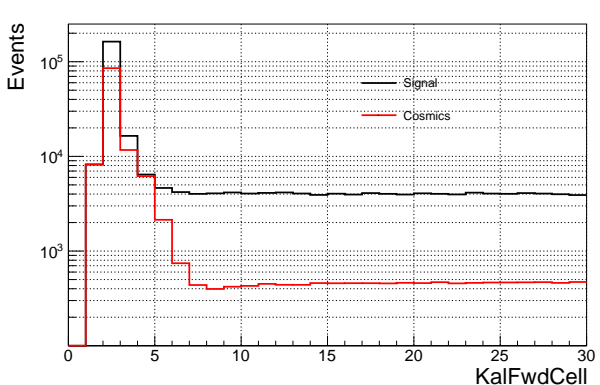

(a)

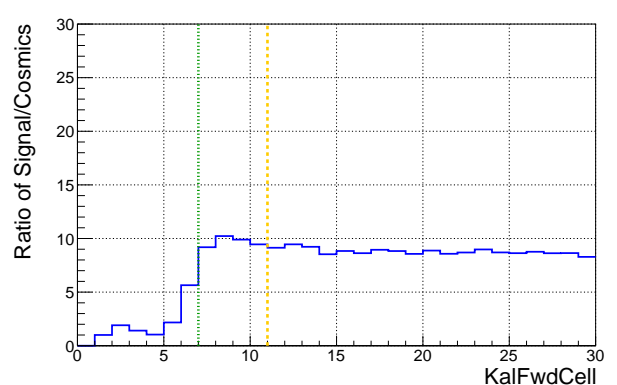

(b)

Figure 5.1: Distribution(a) and $\frac{\text { Signal }}{\text { Cosmic Background }}$ ratio(b) for the Kalman Forward Cell variable. The orange dashed line shows the 2017 analysis cut, the green dotted line shows the optimized cut for the 2018 analysis. All events to the left of these lines are cut and all events to the right are selected.

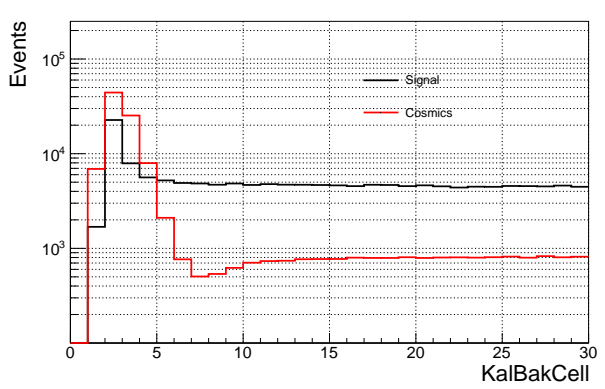

(a)

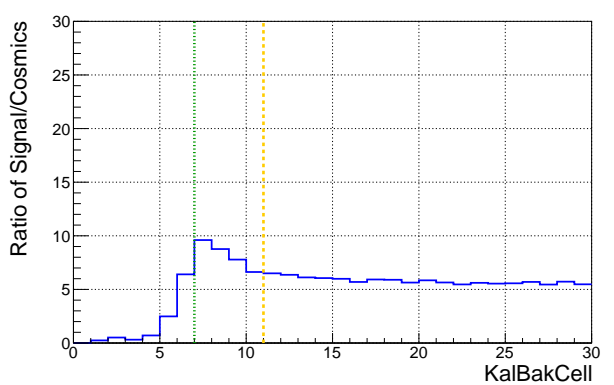

(b)

Figure 5.2: Distribution(a) and $\frac{\text { Signal }}{\text { Cosmic Background }}$ ratio(b) for the Kalman Backward Cell variable. See Figure 5.1 for a detailed description of the legend. 


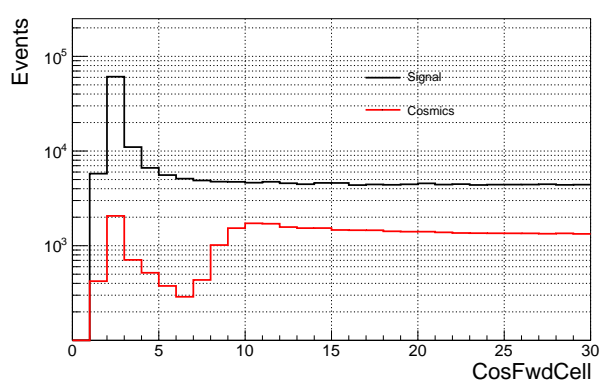

(a)

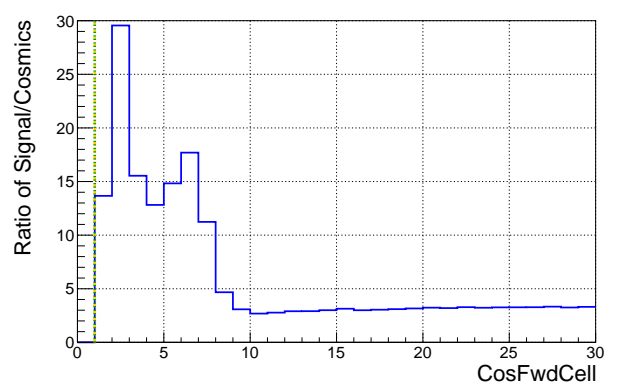

(b)

Figure 5.3: Distribution(a) and $\frac{\text { Signal }}{\text { Cosmic Background }}$ ratio(b) for the Cosmic Forward Cell variable. See Figure 5.1 for a detailed description of the legend. Here the previous and updated selection are at the same value and so the orange and green lines overlap.

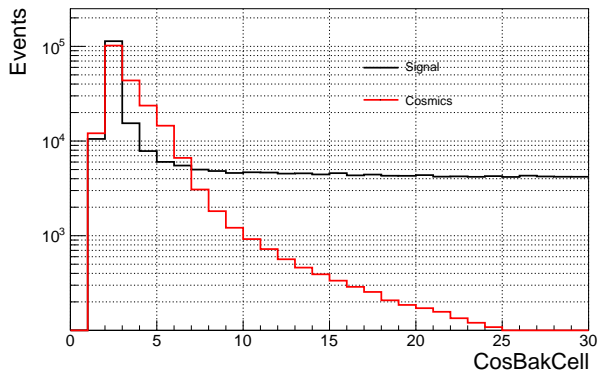

(a)

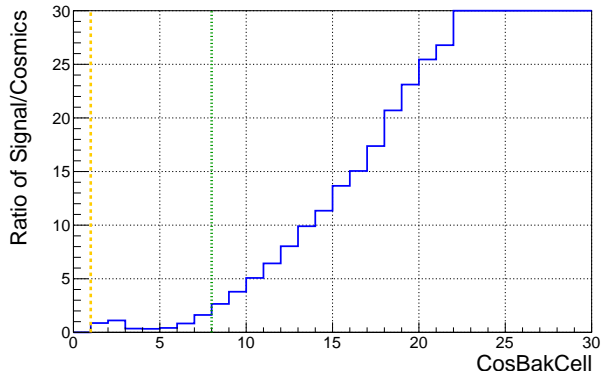

(b)

Figure 5.4: Distribution(a) and $\frac{\text { Signal }}{\text { Cosmic Background }}$ ratio(b) for the Cosmic Back Cell variable. See Figure 5.1 for a detailed description of the legend. 


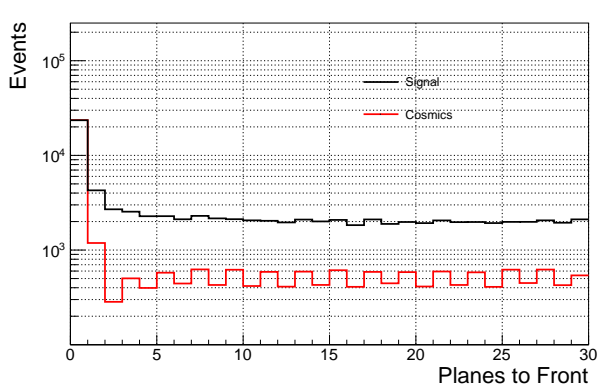

(a)

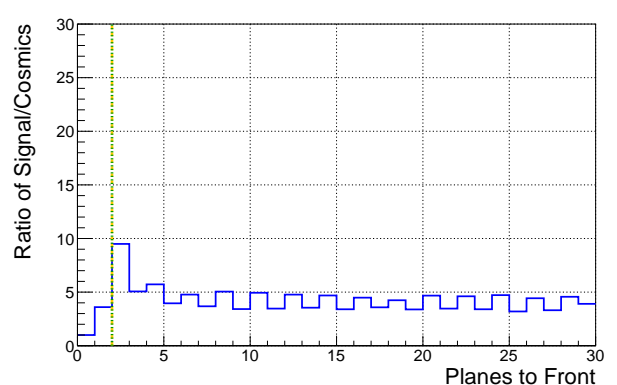

(b)

Figure 5.5: Distribution(a) and $\frac{\text { Signal }}{\text { Cosmic Background }}$ ratio(b) for the Planes to Front variable. See Figure 5.1 for a detailed description of the legend. Here the previous and updated selection are at the same value and so the orange and green lines overlap.

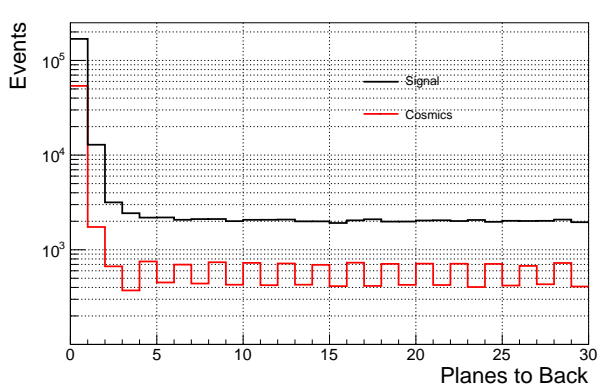

(a)

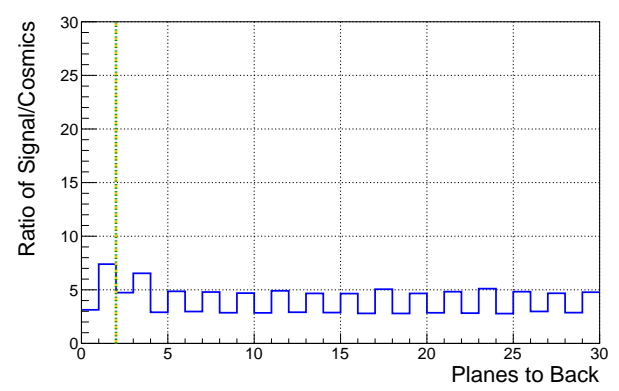

(b)

Figure 5.6: Distribution(a) and $\frac{\text { Signal }}{\text { Cosmic Background }}$ ratio(b) for the Planes to Back variable. See Figure 5.1 for a detailed description of the legend. Here the previous and updated selection are at the same value and so the orange and green lines overlap. 


\subsubsection{Optimization of Containment Variables Introduced in 2018}

Figure 5.7 through to Figure 5.12 show the distribution and $\frac{\text { Signal }}{\text { Cosmic Background }}$ ratio plots for the new variables introduced in the 2018 containment selection. As before, the green dotted line shows the optimized cut for the 2018 analysis. All events to the left of this line are cut and all events to the right are selected.

Figure 5.7 shows the distribution and $\frac{\text { Signal }}{\text { Cosmic Background }}$ ratio of the prong distance to the detector top edge. The top of the detector is subject to a lot more cosmic activity than the other detector edges due to the downward bias of cosmogenic events. As such, the distribution does not exhibit a distinct plateau and the selection has been chosen to aggressively cut deep into the detector at the $60 \mathrm{~cm}$ mark to ensure a 20:1 signal to background ratio.

Figure 5.8 shows the distribution and $\frac{\text { Signal }}{\text { Cosmic Background }}$ ratio of the prong distance to the detector bottom edge. The bottom face of the detector experiences the smallest cosmogenic background flux with the maximum point in the ratio occuring at $12 \mathrm{~cm}$ - this point was chosen for the cut.

Figure 5.9 shows the distribution and $\frac{\text { Signal }}{\text { Cosmic Background }}$ ratio of the prong distance to the detector front edge. The front face of the detector experiences a significant cosmogenic background flux with the plateau point in the sensitivity ratio occuring at $12 \mathrm{~cm}$, a conservative cut selection of $18 \mathrm{~cm}$ was chosen due to the significant background component at this edge.

Figure 5.10 shows the distribution and $\frac{\text { Signal }}{\text { Cosmic Background }}$ ratio of the prong distance to detector back edge. The back face of the detector also experiences a significant cosmogenic background flux with the plateau point in the sensitivity ratio occuring at $12 \mathrm{~cm}$, a conservative cut selection of $18 \mathrm{~cm}$ was chosen due to the significant background component at this edge.

Figure 5.11 and Figure 5.12 show the distribution and $\frac{\text { Signal }}{\text { Cosmic Background }}$ ratio ratios of the prong distance to detector east and west edge respectively. These cuts were found best optimized at $16 \mathrm{~cm}$ and $12 \mathrm{~cm}$ for the east and west edge cuts respectively ${ }^{2}$.

\footnotetext{
${ }^{2}$ Figure 5.7 through to Figure 5.12 were made using NOvASoft version S1705-31. The dataset employed was the 2018 analysis cosmic overlay nonswap FD MC: prod_caf_R17-03-01-prod3recog_fd_genie_nonswap_fhc_nova_v08_Period_v1 where 'Period' is the relevant data running period, as described on a per plot basis.
} 


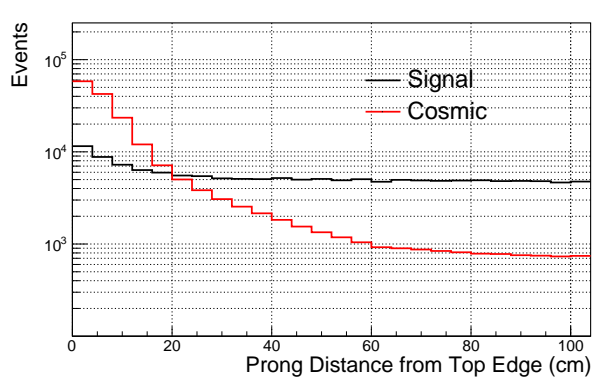

(a)

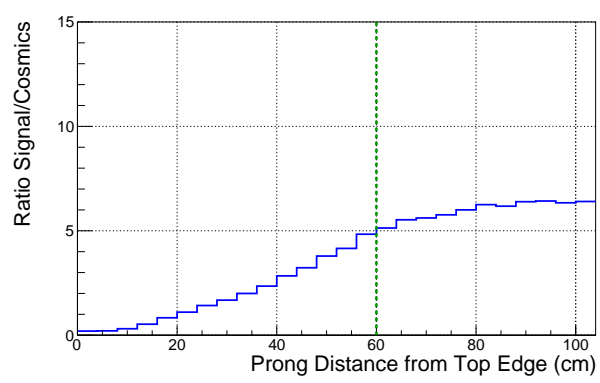

(b)

Figure 5.7: Distribution(a) and $\frac{\text { Signal }}{\text { Cosmic Background }}$ ratio(b) for the Min Prong Distance to FD Top variable. The green dashed line shows the optimized cut for the 2018 analysis. All events to the left of this line are cut and all events to the right are selected.

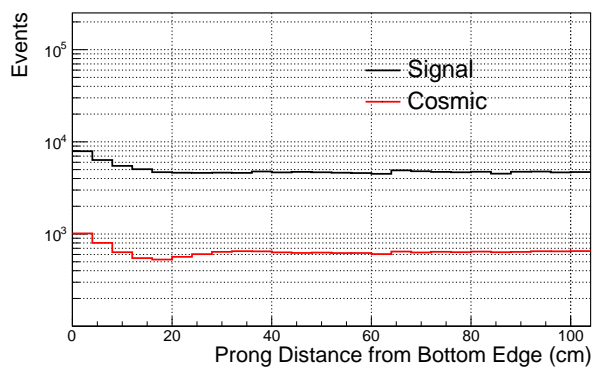

(a)

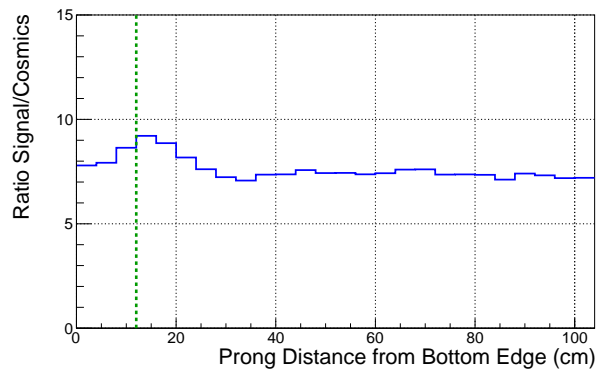

(b)

Figure 5.8: Distribution(a) and $\frac{\text { Signal }}{\text { Cosmic Background }}$ ratio(b) for the Min Prong Distance to FD Bottom variable. See Figure 5.7 for a detailed description of the legend. 


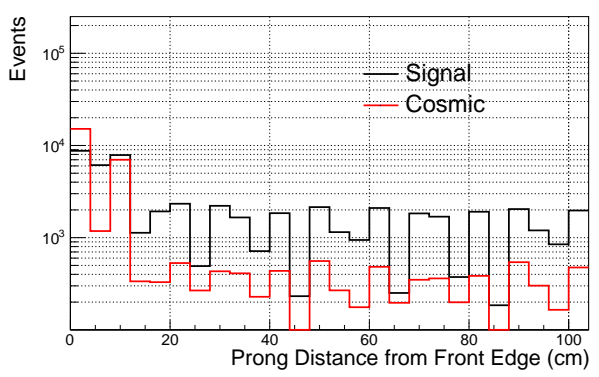

(a)

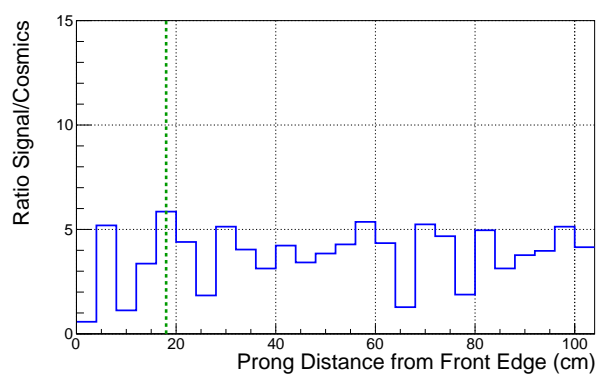

(b)

Figure 5.9: Distribution(a) and $\frac{\text { Signal }}{\text { Cosmic Background }}$ ratio(b) for the Min Prong Distance to FD Front variable. See Figure 5.7 for a detailed description of the legend.

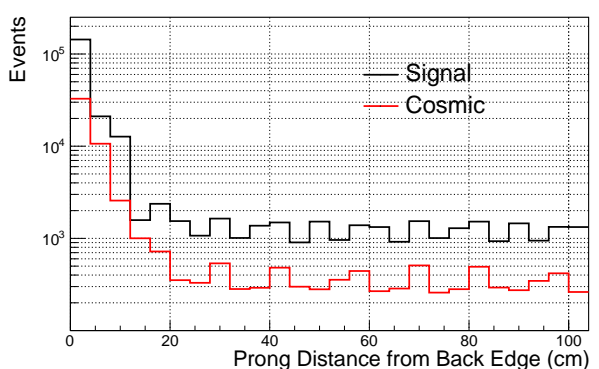

(a)

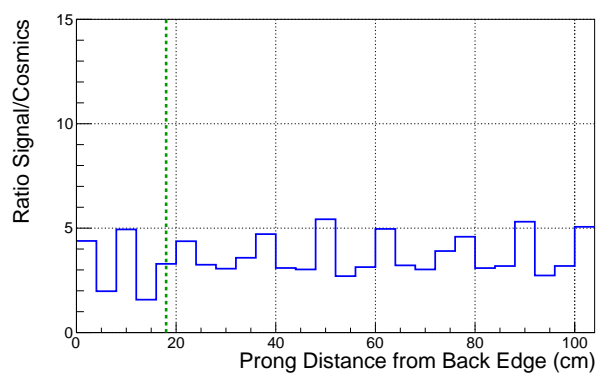

(b)

Figure 5.10: Distribution(a) and $\frac{\text { Signal }}{\text { Cosmic Background }}$ ratio(b) for the Min Prong Distance to FD Back variable. See Figure 5.7 for a detailed description of the legend. 


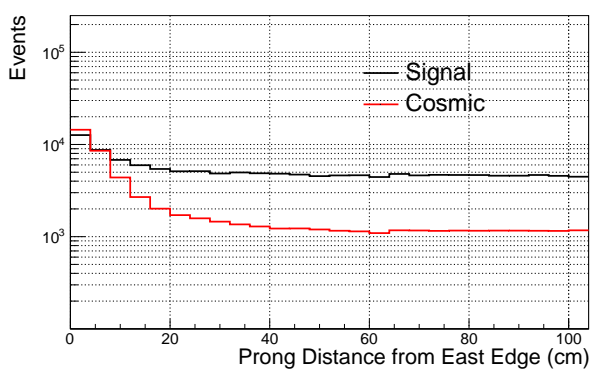

(a)

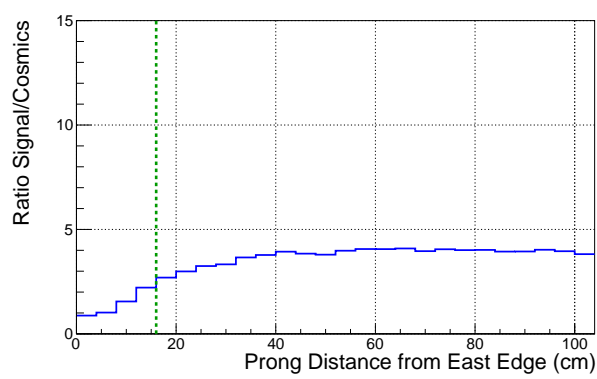

(b)

Figure 5.11: Distribution(a) and $\frac{\text { Signal }}{\text { Cosmic Background }}$ ratio(b) for the Min Prong Distance to FD East variable. See Figure 5.7 for a detailed description of the legend.

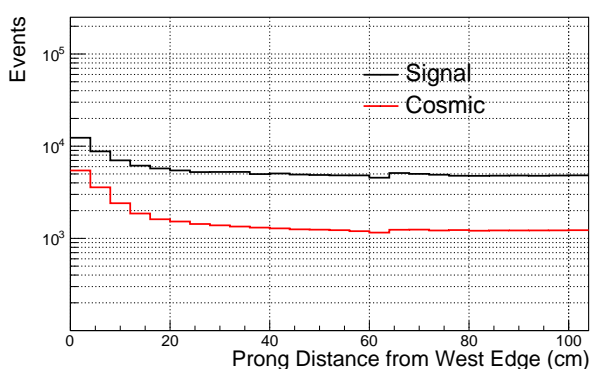

(a)

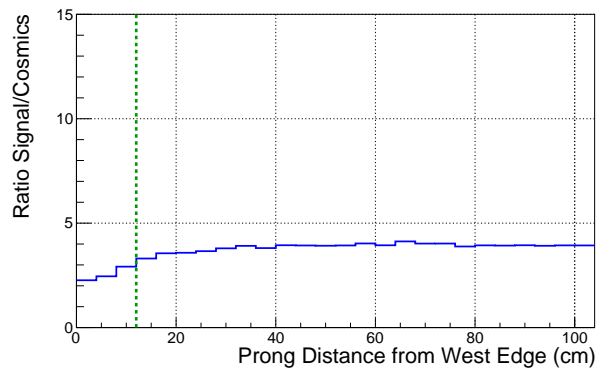

(b)

Figure 5.12: Distribution(a) and $\frac{\text { Signal }}{\text { Cosmic Background }}$ ratio(b) for the Min Prong Distance to FD West variable. See Figure 5.7 for a detailed description of the legend. 


\subsection{Results of Optimization}

A summary of the re-optimized containment criteria and of the optimized FD prong containment criteria, as employed by the 2018 analysis, is given in Table 5.2.

\begin{tabular}{|ccc|}
\hline \hline Name of Cut & $\begin{array}{c}2017 \text { Value } \\
\text { (Units) }\end{array}$ & $\begin{array}{c}2018 \text { Value } \\
\text { (Units) }\end{array}$ \\
\hline Number of cells from detector edge & $>1$ (Cells) & Deleted \\
Forward projection of Kalman track to detector edge & $>10$ (Cells) & $>6$ (Cells) \\
Backward projection of Kalman track to detector edge & $>10$ (Cells) & $>6$ (Cells) \\
Forward projection of Cosmic track to detector edge & $>0$ (Cells) & $>0($ Cells) \\
Backward projection of Cosmic track to detector edge & $>0$ (Cells) & $>7($ Cells $)$ \\
Number of planes to detector front & $>1($ Planes $)$ & $>1($ Planes) \\
Number of planes to detector back & $>1($ Planes $)$ & $>1($ Planes) \\
Track (prong) distance to FD top edge & N/A & $>60(\mathrm{~cm})$ \\
Track (prong) distance to FD bottom edge & N/A & $>12(\mathrm{~cm})$ \\
Track (prong) distance to FD east edge & N/A & $>16(\mathrm{~cm})$ \\
Track (prong) distance to FD west edge & N/A & $>12(\mathrm{~cm})$ \\
Track (prong) distance to FD front edge & N/A & $>18(\mathrm{~cm})$ \\
Track (prong) distance to FD back edge & N/A & $>18(\mathrm{~cm})$ \\
\hline \hline
\end{tabular}

Table 5.2: Summary table of the 2018 analysis FD containment cuts. Both the reoptimized 2017 cuts and the new prong-based criteria are described. 


\subsubsection{Validation of FD Containment}

To assess the effectiveness of the twelve 2018 analysis cuts listed in Table 5.2 the event energy distributions, event interaction type distributions and $\nu_{\mu}$ energy resolutions distribution were evaluated and compared to the previous FD containment criteria ${ }^{3}$.

Figure 5.13 shows the $\nu_{\mu}$-CC MC signal energy spectra summed across data periods 1, 2, 3 and 5 for the previous analysis containment and for the 2018 analysis containment. Whilst the RMS and mean of these distributions exhibit negligibly small differences, the overall number of neutrino events selected increases by $0.1 \%\left(9.12 \times 10^{5} \mathrm{MC}\right.$ events with 2018 analysis containment, as opposed to $9.11 \times 10^{5} \mathrm{MC}$ events using previous containment criteria).

Figure 5.14 shows the $\nu_{\mu}$-CC MC signal energy spectra using the previous analysis containment and the 2018 analysis containment. The left and right plot correspond to data recorded during period 1 and period 2 respectively. Figure 5.15 shows the analogous spectra with the left and right plot corresponding to data recorded during period 3 and period 5 respectively. All periods exhibit a small gain in $\nu_{\mu}$-CC signal when using the 2018 containment criteria, summing to a $0.1 \%$ gain in overall signal. This gain is largely contributed to by period 1 which sees a $5.6 \% \nu_{\mu}$-CC signal increase with the newer containment criteria. This is due to the lower gain and coarser sampling rate used in this period which made the previous containment criteria vulnerable to noise. This vulnerability was reduced in the 2018 selection.

Figure 5.16 shows the $\nu_{\mu}$-CC MC signal (reconstructed ${ }_{\nu_{\mu} E}-$ true $_{\nu_{\mu} E}$ )/true $\nu_{\mu} E$ energy distribution. There is negligible difference between the 2017 and 2018 mean and RMS see Table 5.3. This indicates that our ability to reconstruct energy has not been adversely affected by the introduction of the new containment criteria.

Figure 5.17 shows the cosmic energy distribution. The 2017 distribution is shown in black, 2018 in red. The previous analysis identified 3021 cosmic events as $\nu_{\mu}$ candidates as opposed to 1366 cosmics identified as $\nu_{\mu}$ candidates when using the 2018 containment criteria. This is equivalent to a $55 \%$ reduction in the cosmic background ${ }^{4}$.

Figure 5.18 show the $\nu_{\mu}$-CC MC signal event type distributions for the 2017 and 2018

\footnotetext{
${ }^{3}$ Figure 5.18 through to Figure 5.16 were made using NOvASoft version S1705-31. The dataset employed was the 2018 analysis cosmic overlay nonswap FD MC: prod_caf_R17-03-01-prod3recog_fd_genie_nonswap_fhc_nova_v08_Period_v1 where 'Period' is the relevant data running period, as described on a per plot basis.

${ }^{4}$ The cosmic dataset used to produce Figure 5.17 is: prod_caf_R17-03-01-prod3recoh_fd_cosmic_full_v1_goodruns where "full" refers to the complete dataset across all periods.
} 


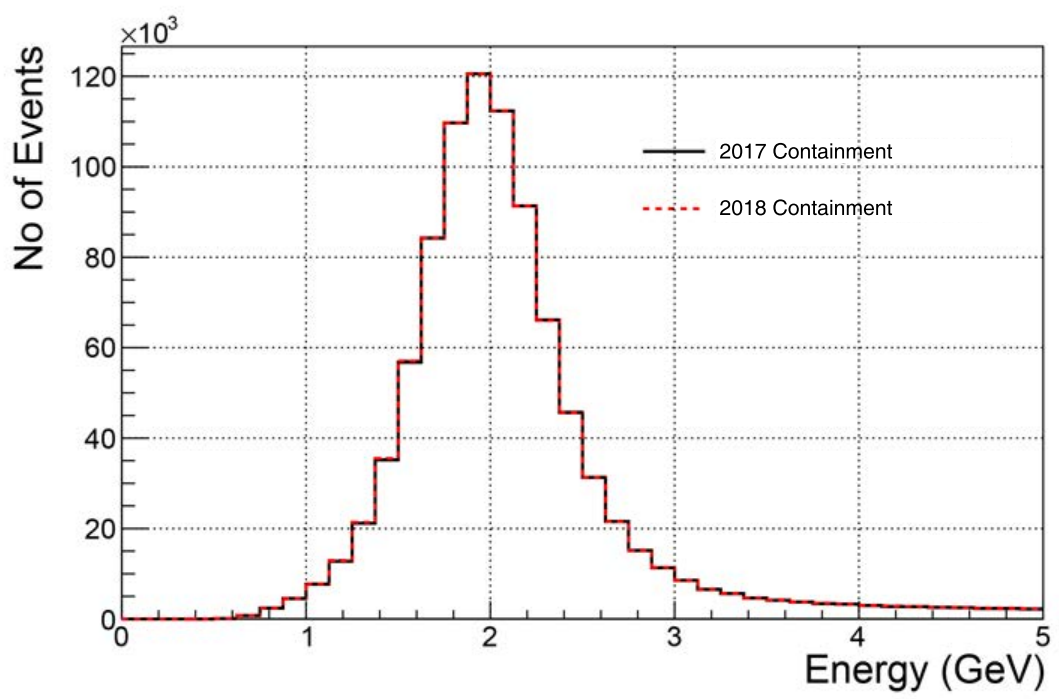

Figure 5.13: Energy distribution of selected $\nu_{\mu}$ events using the 2017(black) and 2018(red) analysis containment criteria across all periods. An increase of $0.1 \%$ in total neutrino event count was achieved.

analysis after application of the respective containment. The interaction type distribution of $\nu_{\mu}$-CC MC signal events that are selected by the 2018 containment but not selected by the 2017 containment is shown in Figure 5.19. There is no appreciable change to the percentage contribution of event types between analyses. This indicates that there is minimal bias with regard to event selection between the containment methodologies.

\begin{tabular}{|lll|}
\hline \hline & Mean & RMS \\
\hline 2017 & -0.049 & 0.092 \\
2018 & -0.049 & 0.093 \\
\hline
\end{tabular}

Table 5.3: RMS and means for 2017 and 2018 (reconstructed $\nu_{\nu_{\mu} E}-$ true $_{\nu_{\mu} E}$ )/true $\operatorname{tr}_{\nu_{\mu}} \nu_{\mu}$ energy distributions.

\subsection{Cosmic Pile-Up}

Two formats of simulated data were in use by the NOvA experiment at the time the work detailed in this chapter was conducted. The first was a MC in which each event contained a single neutrino. The second was a cosmic overlay MC in which a minimum bias trigger of the same time duration was superimposed onto the simulated event to better replicate reality. The cosmic overlay simulation is the standardized MC format presently used in 


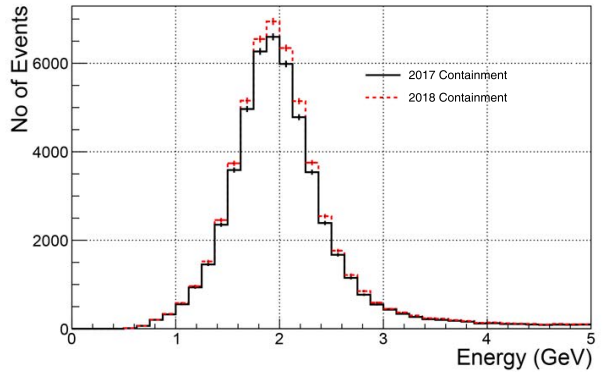

(a) Energy distribution of selected $\nu_{\mu}$ events using the 2017(black) and 2018(red) analysis containment criteria in period 1. 50859 neutrino events were selected using the 2017 analysis containment. 53724 events were selected using the 2018 analysis containment, corresponding to a $5.6 \%$ increase.

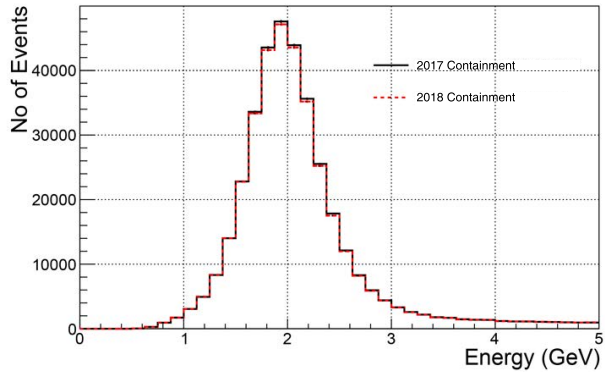

(b) Energy distribution of selected $\nu_{\mu}$ events using the 2017(black) and 2018(red) analysis containment criteria in period 2. 358869 neutrino events were selected using the 2017 analysis containment. 355903 events were selected using the 2018 analysis containment, corresponding to a $0.8 \%$ decrease.

Figure 5.14: $\nu_{\mu}$ energy distributions in periods $1(\mathrm{a})$ and $2(\mathrm{~b})$.

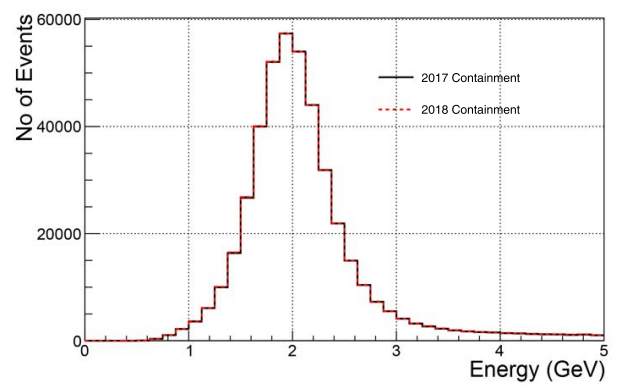

(a) Energy distribution of selected $\nu_{\mu}$ events using the 2017(black) and 2018(red) analysis containment criteria in period 3. 434515 neutrino events were selected using the 2017 analysis containment. 435602 events were selected using the 2018 analysis containment, corresponding to a $0.3 \%$ increase.

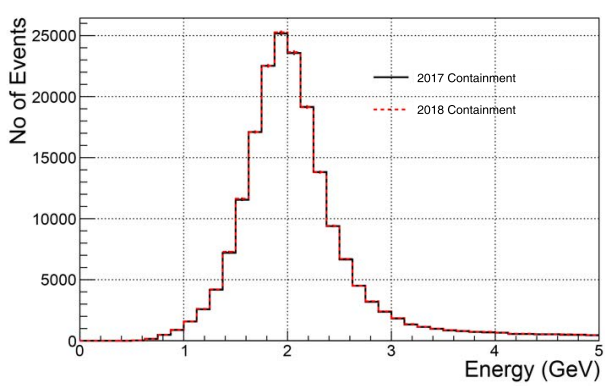

(b) Energy distribution of selected $\nu_{\mu}$ events using the 2017(black) and 2018(red) analysis containment criteria in period 5. 188642 neutrino events were selected using the 2017 analysis containment. 189479 events were selected using the 2018 analysis containment, corresponding to a $0.4 \%$ increase.

Figure 5.15: $\nu_{\mu}$ energy distributions In periods 3(a) and 5(b). 


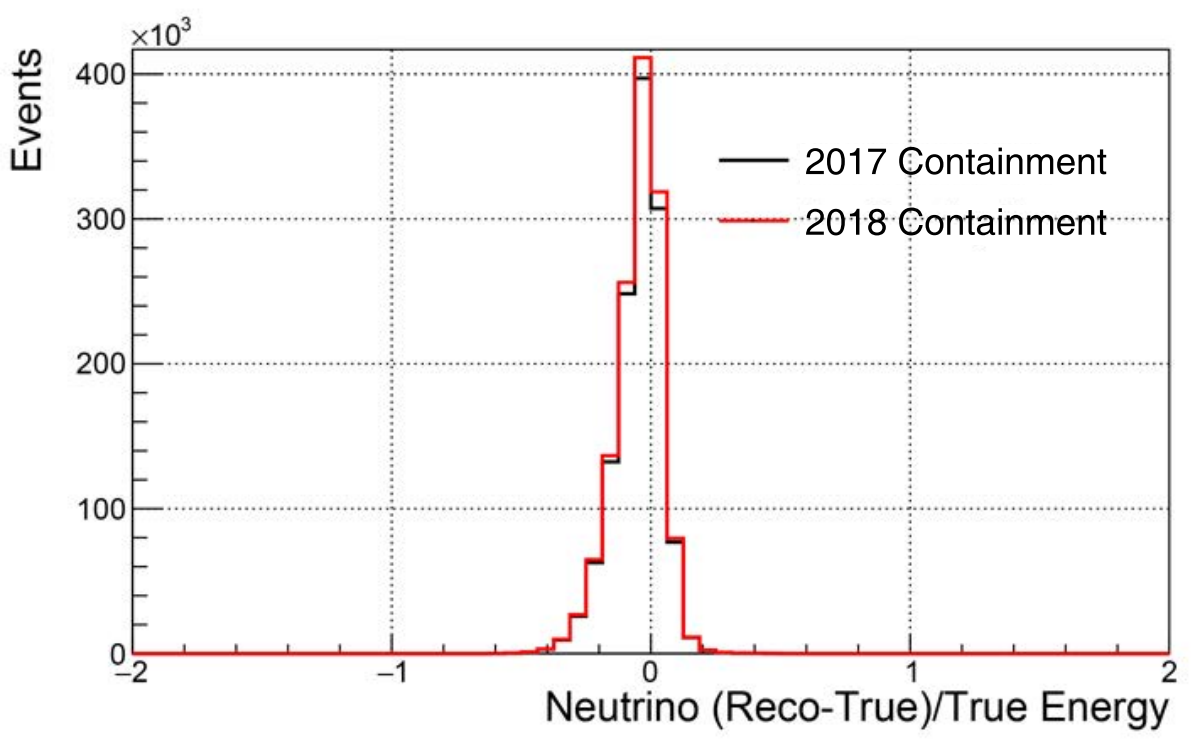

Figure 5.16: Distribution of (reconstructed $\left.{ }_{\nu_{\mu} E}-\operatorname{true}_{\nu_{\mu} E}\right) / \operatorname{true}_{\nu_{\mu} E} \nu_{\mu}$ energy. Using the 2017(black) and 2018(red) analysis containment criteria. There is negligible difference in the RMS and mean between distributions. The increase in events seen when using the 2018 containment is generally centred about zero, indicating increased selection efficiency.

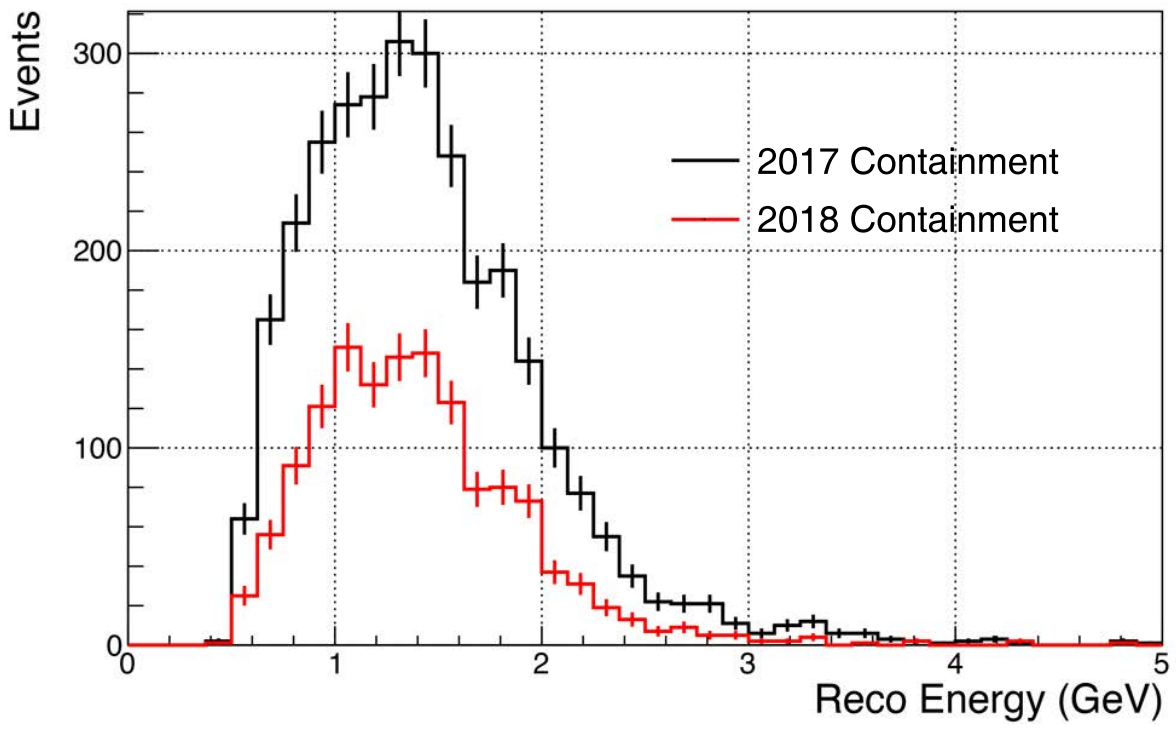

Figure 5.17: Cosmic energy spectra for 2017(black) and 2018(red) Containment criteria. The previous analysis identifies 3021 cosmic events as $\nu_{\mu}$ events. Changing to the 2018 containment, and holding all other cuts the same, 1366 cosmics are identified as $\nu_{\mu}$ events. This is equivalent to a $55 \%$ reduction in the cosmic background. 
Event Type Distribution using 2017 Containment

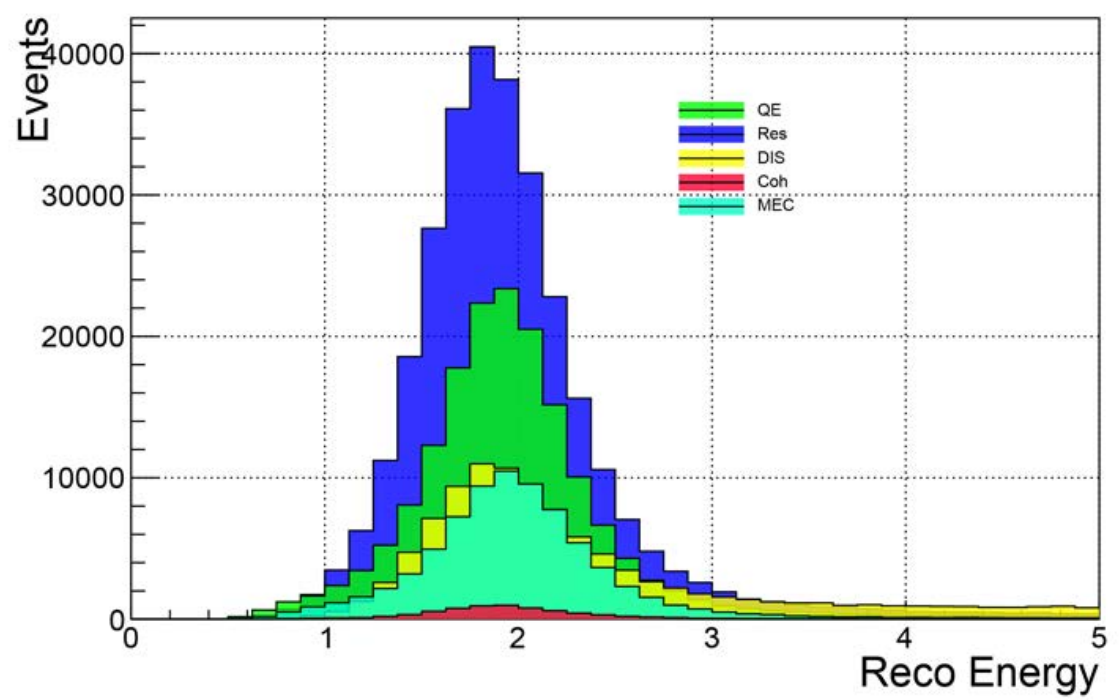

(a)

Event Type Distribution using 2018 Containment

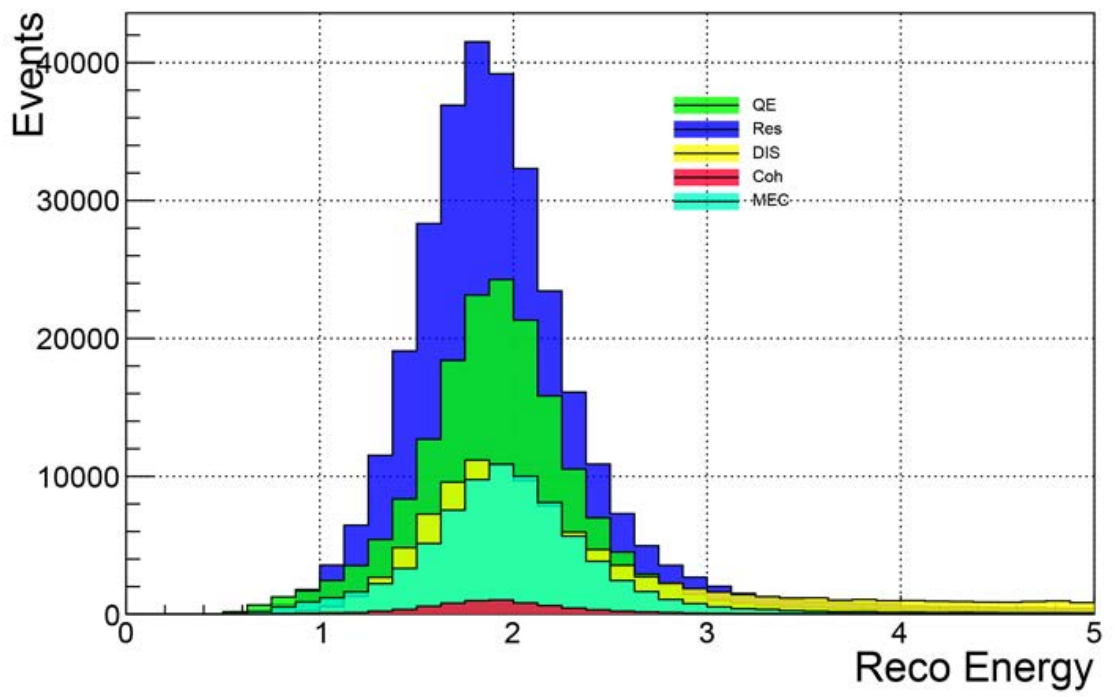

(b)

Figure 5.18: Distribution of event interaction types using the 2017 analysis(a) and 2018 analysis(b) containment criteria. 


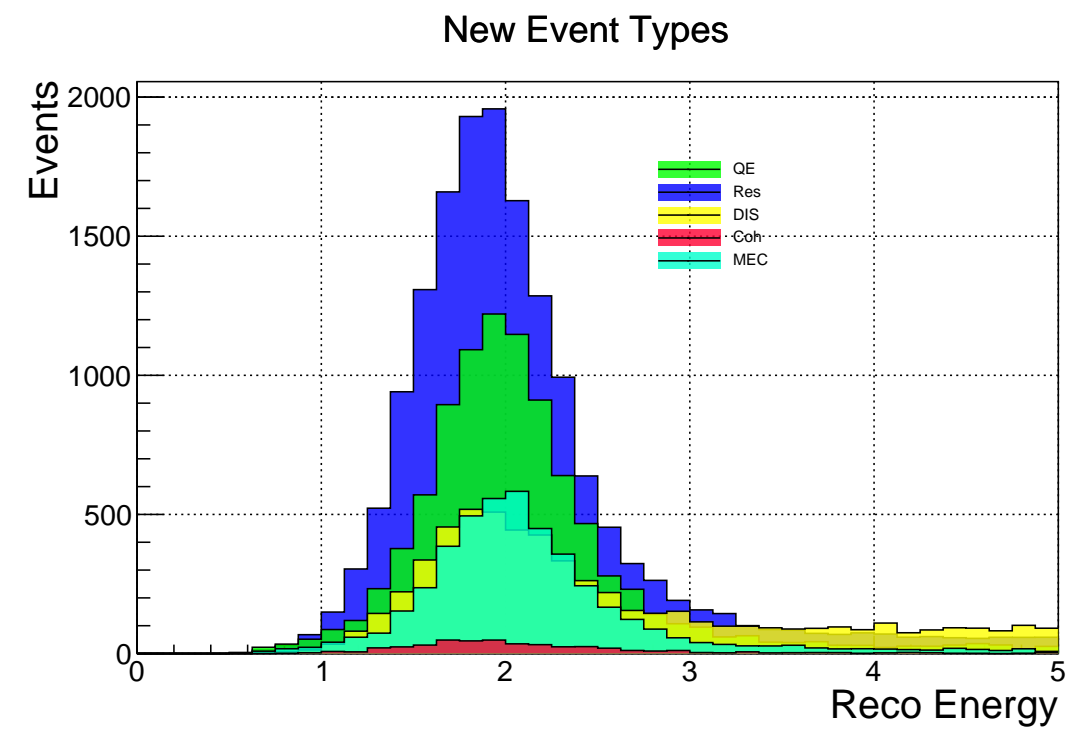

Figure 5.19: Distribution of event types selected by the 2018 analysis containment but cut when using the 2017 analysis criteria.

NOvA. When cosmic overlay MC was first introduced to the analysis about $3.1 \%$ of signal events were lost because of pile-up with cosmic activity. The containment criteria were identified as a potential contributor to this signal loss. This section details a series of work by the author to test this hypothesis by comparing the signal event count obtained after application of the 2017 and 2018 containment criteria.

In each figure the left and right plot show two $\nu_{\mu}$ energy distributions - one produced by running the analysis over the single-event $\mathrm{MC}$ and the second by running over the cosmic overlay MC. The left plot is produced using the 2017 containment criteria and the right plot by using the 2018 criteria. The distributions are shown for two data taking periods the full dataset and the period 1 distributions. Period 1 is highlighted as it exhibits the greatest improvement. The cosmic and neutral current rejection criteria applied in these plots were not used in the 2018 analysis and represented an intermediate selection based on a combination of particle-identification score cuts. The currently implemented criteria were designed by the author and are detailed thoroughly in Chapter 6. Appendix A contains a full breakdown of the plots for all data taking periods.

Figure 5.20 shows the period $1 \nu_{\mu}$-CC energy distribution when using the 2017 (left) and 2018 (right) containment criteria. Introduction of the 2018 containment reduces the effect of signal loss due to pile-up from $9.5 \%$ to $5.1 \%$. Figure 5.21 shows the full dataset $\nu_{\mu}$-CC energy distribution using the 2017 (left) and 2018 (right) containment criteria. The 2018 containment reduces the total effect of signal loss due to pile-up from $3.1 \%$ to $1.4 \%$. 


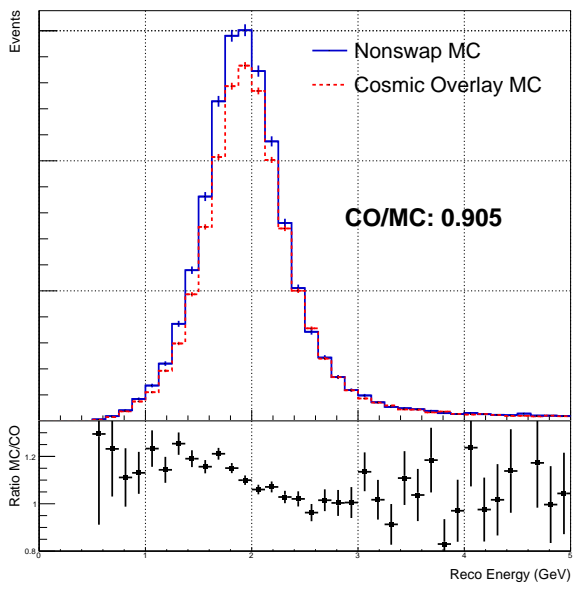

(a) $\nu_{\mu}$ energy distributions using 2017 analysis containment

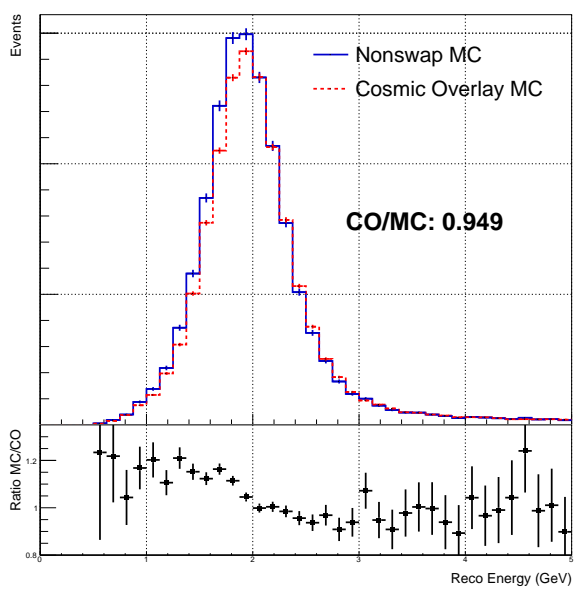

(b) $\nu_{\mu}$ energy distributions using 2018 analysis containment

Figure 5.20: $\nu_{\mu}$ reconstructed energy distributions produced using the 2017 and 2018 containment criteria on the period 1 dataset. The blue line shows the distribution when run over the single event MC dataset. The red dashed line shows the distribution when run over the cosmic overlay MC. The $\frac{\operatorname{cosmic} \text { overlay MC }}{\text { single event MC }}$ event count ratio is given on each plot, below the legend.

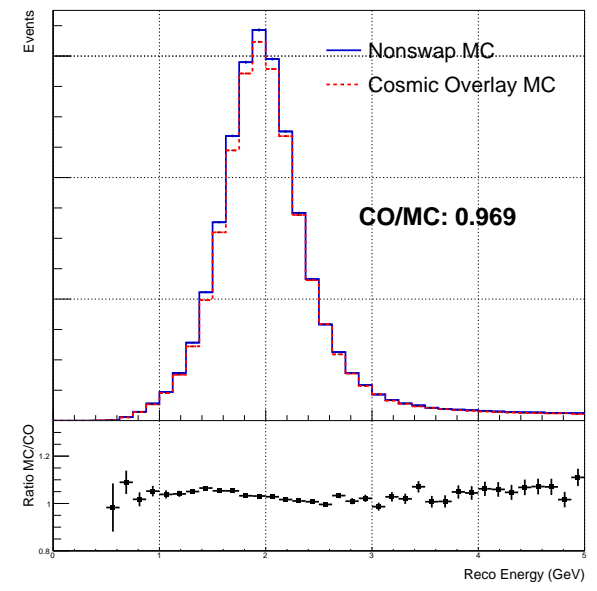

(a) $\nu_{\mu}$ energy distributions using 2017 analysis containment

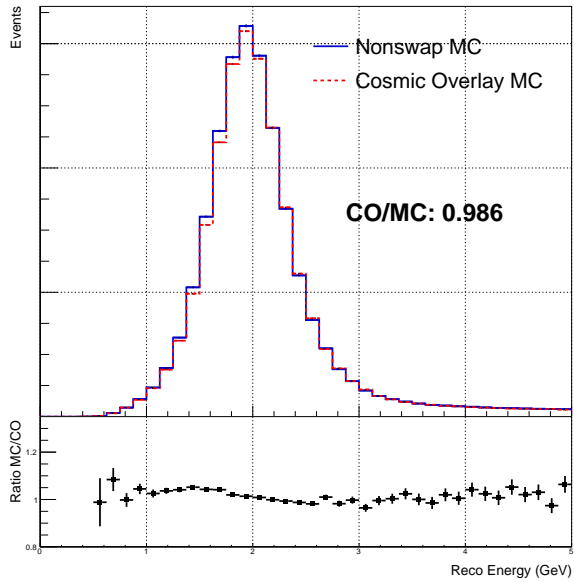

(b) $\nu_{\mu}$ energy distributions using 2018 analysis containment

Figure 5.21: $\nu_{\mu}$ reconstructed energy distributions produced using the 2017 and 2018 containment criteria across the total dataset. The blue line shows the distribution when run over the single event MC dataset. The red dashed line shows the distribution when run over the cosmic overlay MC. The $\frac{\text { cosmic overlay MC }}{\text { single event MC }}$ event count ratio is given on each plot, below the legend. 
Table 5.4 shows the $\frac{\text { cosmic overlay MC }}{\text { single event MC }}$ signal event count ratios for each data period. The ratios obtained using both the 2017 and 2018 containment criteria are shown. The use of the 2018 containment criteria reduces the effect of cosmic pile-up in every data period.

\begin{tabular}{|c|c|c|c|c|}
\hline Period & $\begin{array}{l}2017 \text { Containment } \\
\frac{\text { cosmic overlay MC }}{\text { single event MC }}\end{array}$ & $\begin{array}{l}2018 \text { Containment } \\
\frac{\text { cosmic overlay MC }}{\text { single event MC }}\end{array}$ & $\begin{array}{c}2017 \text { Signal } \\
\text { Pile-Up Loss (\%) }\end{array}$ & $\begin{array}{c}2018 \text { Signal } \\
\text { Pile-Up Loss (\%) }\end{array}$ \\
\hline 1 & 0.905 & 0.949 & 9.5 & 5.1 \\
\hline 2 & 0.983 & 0.991 & 1.7 & 0.9 \\
\hline 3 & 0.966 & 0.983 & 3.4 & 1.7 \\
\hline 5 & 0.960 & 0.980 & 4.0 & 2.0 \\
\hline full & 0.969 & 0.986 & 3.1 & 1.4 \\
\hline
\end{tabular}

Table 5.4: Table showing the $\frac{\text { cosmic overlay MC }}{\text { single event MC }}$ ratio of signal event counts when using the 2017 and 2018 containment criteria. The use of the 2018 containment criteria universally reduces the effect of pile-up in all data periods.

\subsection{Conclusion}

The FD selection criteria used by the $\nu_{\mu}+\bar{\nu}_{\mu}$ disappearance analysis to select well contained events has been re-optimized by the author. This updated containment was adopted by the experiment and used in the $2018 \nu_{\mu}+\bar{\nu}_{\mu}$ disappearance analysis as well as in the $8.85 \times 10^{20}$ POT 2018 analysis [3].

A single cut from the previous containment criteria was removed and replaced by six prong based cuts - one for each detector edge. All variables were optimized by evaluating the respective distributions and corresponding $\frac{\text { Signal }}{\text { Cosmic Background }}$ ratio.

The 2018 containment increases the number of selected signal events across all periods by approximately $0.1 \%$ and significantly decreases the selected cosmic background by $55 \%$. When using the more realistic cosmic overlay sample, the 2018 containment increases the total number of selected signal events by approximately $1.9 \%$. The cosmic background reduction when using the 2018 containment for the cosmic overlay sample is the same as for the old MC (55\%). The average energy resolution has remained essentially unchanged. When the 2017 containment criteria is used $3.1 \%$ of signal events are lost due to pile-up with cosmics. Introduction of the 2018 containment reduces the cosmic pile-up signal loss to $1.4 \%$. 


\section{Chapter 6}

\section{PID Selection Analysis Improvements}

The $\nu_{\mu}+\bar{\nu}_{\mu}$ disappearance analysis applies selection boundaries in three particle identification (PID) scores to help identify $\nu_{\mu}+\bar{\nu}_{\mu}$-CC signal and discriminate background events. ReMId scores a tracks muon-likelihood, the cosmic BDT scores the likelihood an event is of cosmic origin and CVN scores $\nu_{\mu}+\bar{\nu}_{\mu}$-CC event likelihood from interaction topology. For a more detailed description of each algorithm see Section 4.3.

The PID algorithms have been updated and re-optimized since the $6.05 \times 10^{20} \mathrm{POT}$ 2017 analysis [49] to accommodate $\bar{\nu}_{\mu}$ data and improve general performance. Consequently, the PID selection cuts required tuning. This chapter describes work performed by the author to optimize the PID selection criteria. The selection boundaries produced from this work have been adopted by the official NOvA analysis and the NOvA $\nu_{\mu}+\bar{\nu}_{\mu}$ disappearance results that incorporate them are presented in Chapter 7. The research presented here supports the latest internal analysis summary document [83]. The 1st, 2nd and 3rd analysis documents contain a comprehensive detailing of the previous PID cut implementations and are found in [84], [85] and [59] respectively.

The optimization was performed simultaneously in ReMId vs. CVN vs. BDT PIDspace using a new methodology that was developed by the author. Analysis sensitivity was quantified using a figure of merit (FOM) based on signal and background counts. Three targets were outlined - increasing the number of well reconstructed $\nu_{\mu}+\bar{\nu}_{\mu}$-CC events selected, decreasing the number of background events and minimizing the analysis dependence on the cosmic BDT that is only used in the FD. 


\subsection{PID Selection Boundary Optimization Methodology}

The CVN, BDT and ReMId scores are designed to perform different functions and may uniquely reject or accept any given event. However, the algorithms do have partially overlapping functionality due to correlations in the input parameters. The univariate approach to optimization would therefore fail to capture the optimum selection boundaries. To address this, the PID cuts were optimized using a multidimensional approach which only considered the combined effect of a given set of cuts. The $\nu_{\mu}+\bar{\nu}_{\mu}$-CC signal, neutral current background, other beam background and cosmic background spectra were assessed at more than 6000 cut value combinations. The total MC event counts in each $E_{\text {had }} / E_{\nu}$ quantile were calculated and saved to a file. These trees were used as the input to the optimization with the $\frac{\text { signal }}{\text { background }}$ and $\frac{\text { signal }}{\sqrt{\text { signal+background }}}$ FOM's acting as metrics for assessing performance.

\subsubsection{Quantile Boundaries and Pre-Selection}

The PID optimization utilizes identical pre-selection to the main disappearance analysis, varying only the PID selection boundaries. An overview of the complete selection criteria used is given in this section.

Candidate $\nu_{\mu}+\bar{\nu}_{\mu}$-CC events are split into four approximately equi-statistical quantiles according to hadronic energy fraction. Instead of calculating unique quantile boundaries for each set of analyzed PID cuts, a standard set of $E_{\text {had }} / E_{\nu}$ quantile boundaries were universally used. The chosen boundaries were calculated using the previous PID selection criteria, wherein each PID score must exceed 0.5. This method was favoured over using boundaries generated at each cut combination because of the huge computational demands in doing so. For the validation process $E_{h a d} / E_{\nu}$ boundaries were generated for each set of cuts that were implemented into the analysis software framework ${ }^{1}$.

The data quality cuts, determined by the Data Quality Working Group, are applied at the run, subrun, and spill levels [57]. The full quality cut selection is applied as described in Section 4.3.

Loose PID and cosmic rejection cuts were also applied to remove easily-rejected cosmogenic events, events with a significant transverse momentum fraction and events with energy exceeding the $\nu_{\mu}+\bar{\nu}_{\mu}$-CC signal regime. These cuts were universally applied throughout the PID analysis. In general (CAF) nomenclature they read as follows:

\footnotetext{
${ }^{1}$ For a comprehensive overview of the datasets and software used, see Appendix B.
} 
- Cosine of Kalman track angle with respect to the beam (sel.cosrej.anglekal) $>0.5$.

- Number of hits contained in the slice (slc.nhit) $<400$.

- Fractional transverse momentum with respect to beam direction (sel.nuecosrej.pngptp) $<0.9$.

The first cut ensures that Kalman tracks highly orthogonal to the beam direction are discarded due to the high likelihood that such events are cosmogenic. The second cut ensures that the event slice contains less than 400 hundred hits. Events with this many hits would be highly unlikely candidate events given the $\nu_{\mu}+\bar{\nu}_{\mu}$-CC signal energy regime. The final cut acts on transverse momentum and has been reintroduced to the current analysis having been last used in the 2017 analysis. This cut ensures the transverse momentum of prongs with respect to the beam is not a huge fraction of the total - which would generally indicate a background-like event. At least $10 \%$ of the total momentum must be in the direction of the beam.

In addition to the cuts outlined above, minimum thresholds for the PID cuts were assessed to clean the data, producing a smaller analysis sample with a faster computational turnover. These cuts were conservatively implemented and only reject events in a highly background dominated regime. In general (CAF) nomenclature they read as follows:

- Cosmic BDT score (sel.cosrej.numucontpid) > 0.40

- ReMId score of primary track (sel.remid.pid) $>0.10$

- CVN score (sel.cvnProd3Train.numuid) > 0.10

\subsubsection{Event Distributions and Oscillation Weights}

The MC outlined in Section 6.1.1 comprises unoscillated events in the NOvA far detector. CAFAna has the capability to calculate neutrino oscillation weights for each event but for technical reasons this option was not implementable. However, the effect of oscillation can not be negated as it changes the number of signal events without affecting the background. To remedy this, oscillation weights were generated for each $E_{h a d} / E_{\nu}$ quantile using the previous analysis PID selection criteria to create ratio plots of the oscillated $\nu_{\mu}+\bar{\nu}_{\mu}$-CC energy over the equivalent unoscillated spectrum. These ratio histograms were then provided as weights to the PID histograms, allowing event populations to be scaled to a good oscillation approximation at the NOvA best fit point. The FHC oscil- 
lation plots are shown in Figure 6.1, the RHC equivalents are very similar and are not shown for succintness.
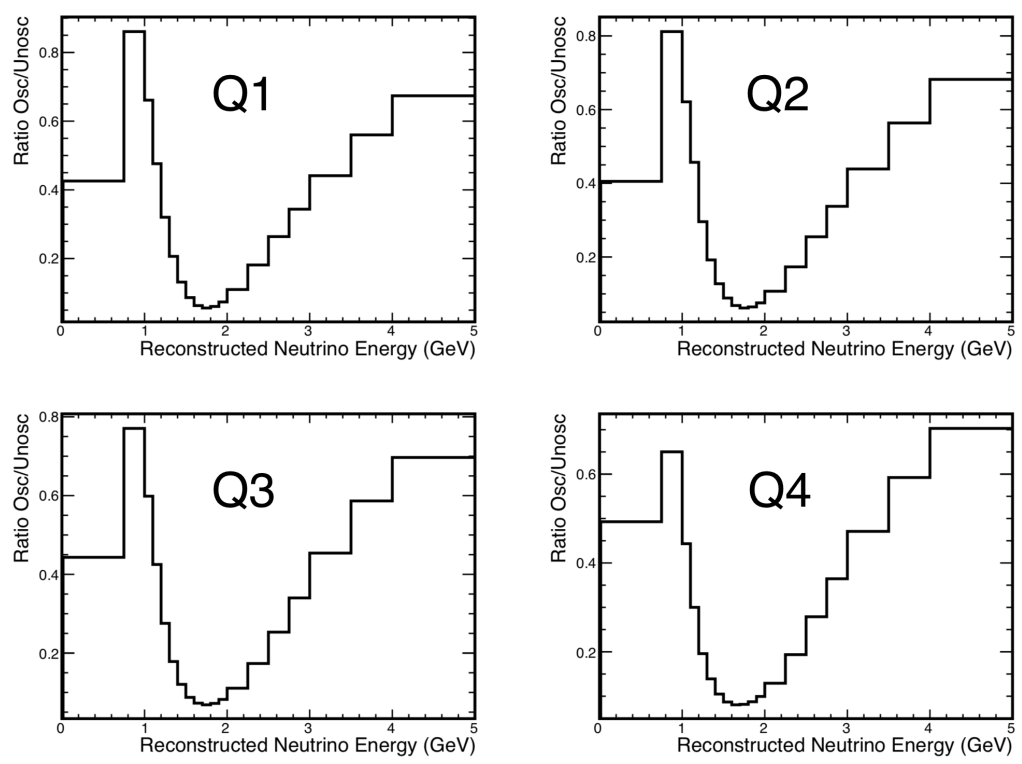

Figure 6.1: Ratios of oscillated $\nu_{\mu}+\bar{\nu}_{\mu}$-CC over unoscillated $\nu_{\mu}+\bar{\nu}_{\mu}$-CC events for forward horn current MC files. Top left is the lowest $E_{\text {had }} / E_{\nu}$ quantile. Top-right is the $2^{\text {nd }}$ lowest $E_{\text {had }} / E_{\nu}$ quantile. Bottom-left is the $2^{n d}$ highest $E_{h a d} / E_{\nu}$ quantile. Bottomright is the highest $E_{h a d} / E_{\nu}$ quantile.

Using the oscillation weights from Figure 6.1, histograms were produced for the different signal and background components present in the $\nu_{\mu}+\bar{\nu}_{\mu}$-CC analysis. The FHC and RHC $\nu_{\mu}+\bar{\nu}_{\mu}$-CC and cosmic distributions in 3D-PID space are shown summed across all $E_{\text {had }} / E_{\nu}$ quantiles in Figure 6.2. The $E_{\text {had }} / E_{\nu}$ quantile breakdowns are given for FHC and RHC in Figure 6.3 and Figure 6.4 respectively. 

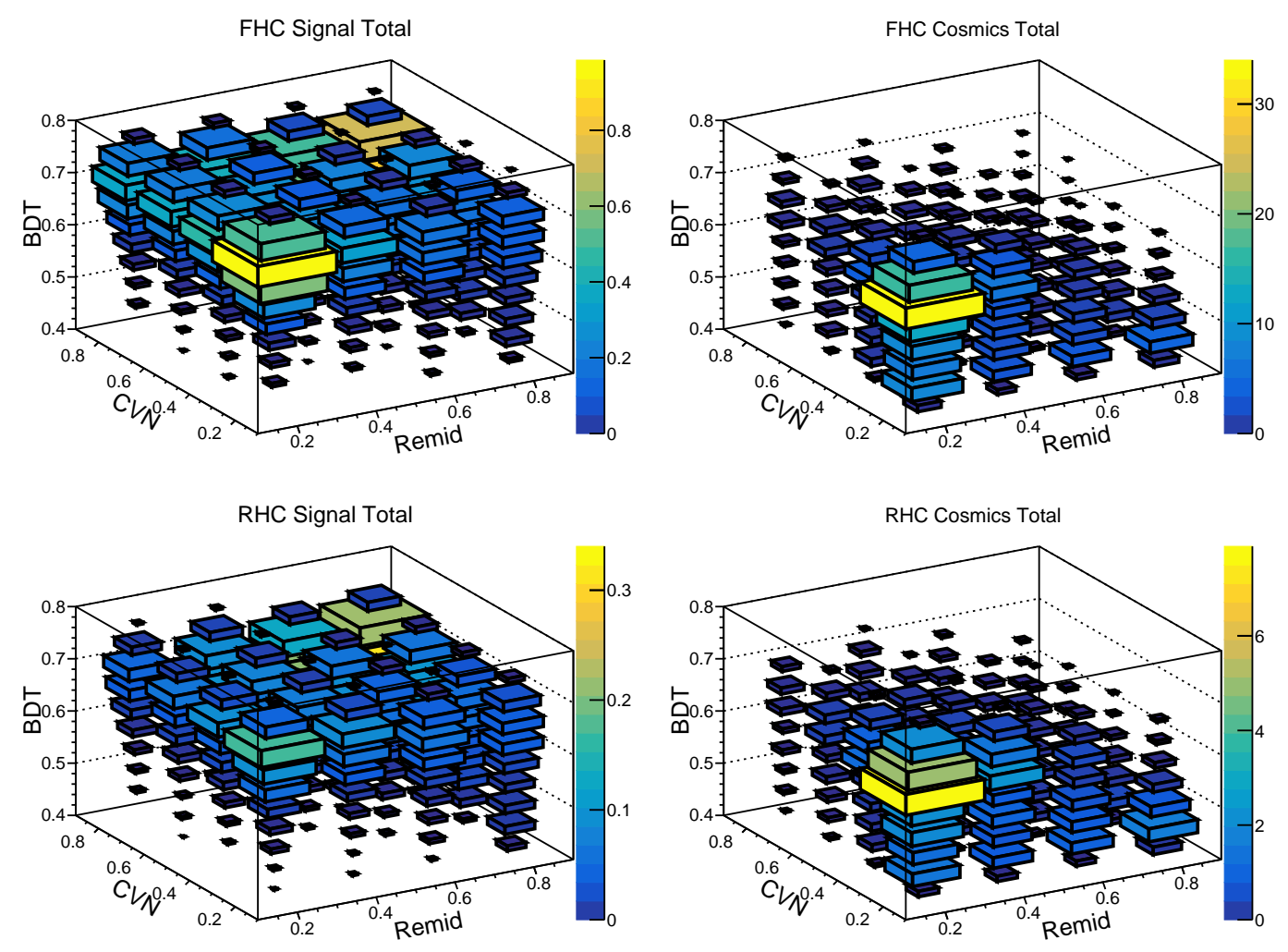

Figure 6.2: The $\nu_{\mu}+\bar{\nu}_{\mu}$-CC (left) and cosmic (right) total distributions in 3D-PID space of events passing selection for FHC and for RHC. The fourth axis, represented by colour, shows the population in each bin. The volume of each bin is also proportional to its population. The top left plot shows the FHC $\nu_{\mu}+\bar{\nu}_{\mu}$-CC signal total, the bottom left plot shows the RHC $\bar{\nu}_{\mu}$-CC signal total, the top right plot shows the FHC cosmic background total and the bottom right plot shows the RHC cosmic background total. 

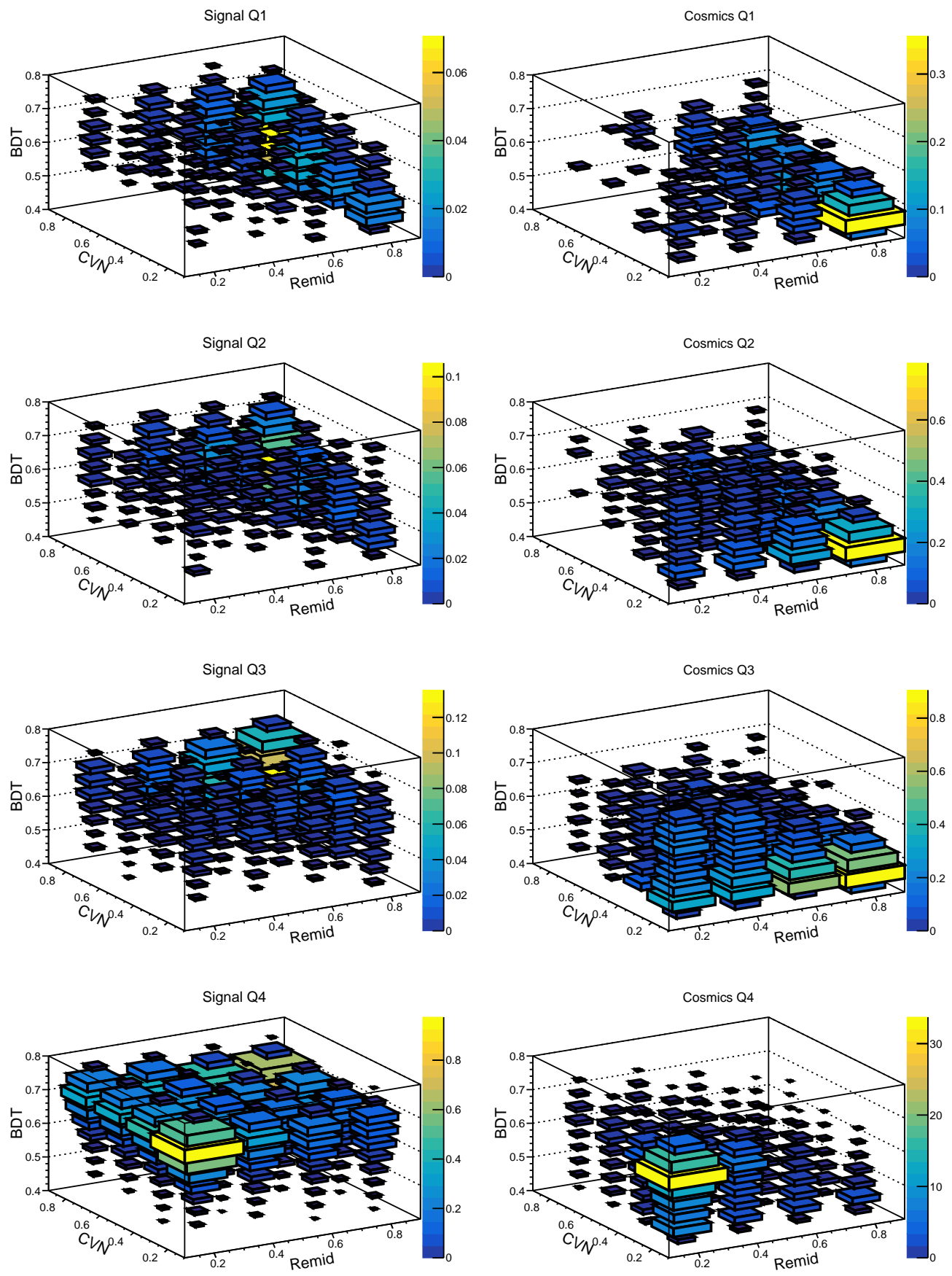

Figure 6.3: The FHC $\nu_{\mu}+\bar{\nu}_{\mu}$-CC (left) and cosmic background (right) distributions in 3D-PID space, shown for each $E_{\text {had }} / E_{\nu}$ quantile. The four plots on each side run top to bottom from lowest to highest $E_{h a d} / E_{\nu}$ quantile. The fourth axis, represented by colour, shows the population in each bin. The volume of each bin is also proportional to its population. 

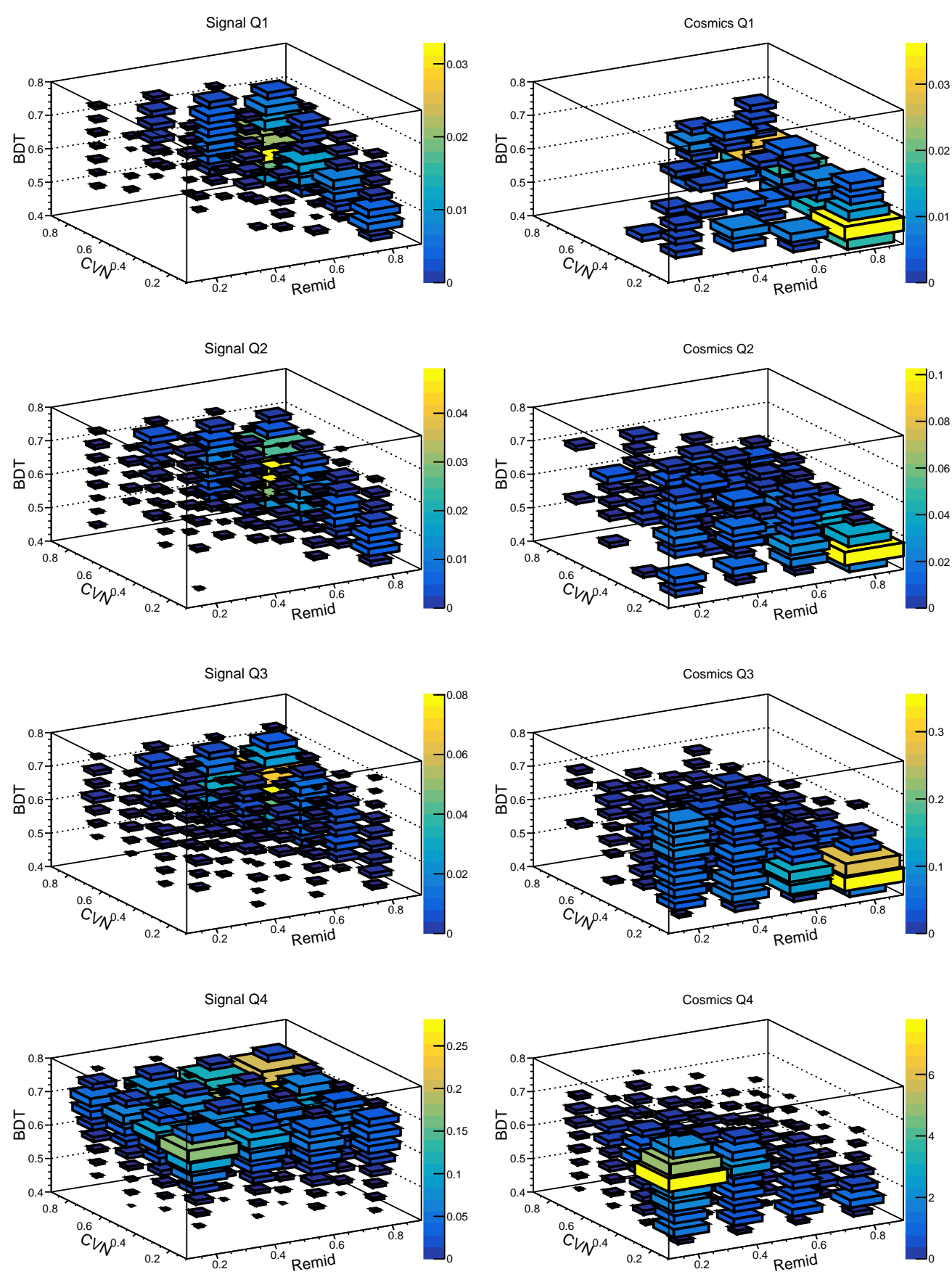

Figure 6.4: The RHC $\bar{\nu}_{\mu}$-CC (left plots) and cosmic background (right plots) distributions in 3D-PID space, shown for each $E_{\text {had }} / E_{\nu}$ quantile. The four plots on each side run top to bottom from lowest to highest $E_{h a d} / E_{\nu}$ quantile. The fourth axis, represented by colour, shows the population in each bin. The volume of each bin is also proportional to its population. 


\subsubsection{Assessed Selection Boundary Definitions}

The PID optimization process was carried out for all boundary permutations described in Table 6.1.

\begin{tabular}{|cccc|}
\hline \multicolumn{4}{|c|}{ ReMID } \\
\hline Min & Max & Step Size & Total Steps \\
\hline 0.10 & 0.90 & 0.05 & 17 \\
\hline
\end{tabular}

\begin{tabular}{|cccc|}
\hline \multicolumn{4}{|c|}{ CVN } \\
\hline Min & Max & Step Size & Total Steps \\
\hline 0.10 & 0.90 & 0.05 & 17 \\
\hline
\end{tabular}

\begin{tabular}{|cccc|}
\hline \multicolumn{4}{|c|}{ Cosmic BDT } \\
\hline Min & Max & Step Size & Total Steps \\
\hline 0.40 & 0.60 & 0.01 & 21 \\
\hline
\end{tabular}

\section{Total Permutations: 6069}

Table 6.1: Breakdown of the evaluated PID boundaries. The minimum value, maximum value and step size in each score is shown. All possible permutations of the boundaries are assessed in the optimization. The total number of steps for each score is also shown.

The total number of cut permutations assessed by the optimization is $17 \times 17 \times 21=$ $6069^{2}$. For each hadronic energy fraction quantile all 6069 event counts were calculated and saved. The calculation integrated the sum total of weighted events in each PID histogram for the range specified by a given permutation of cuts. These integrals were produced separately for each major analysis interaction $-\nu_{\mu}+\bar{\nu}_{\mu}$-CC, NC, other beam background and cosmic background. In addition, two figures of merit were also saved to the binary tree - $\frac{\text { signal }}{\text { background }}$ and $\frac{\text { signal }}{\sqrt{\text { signal+background }}}$ where signal is defined as the $\nu_{\mu}+\bar{\nu}_{\mu}$-CC event count integral and background is defined as the event count integral of the summed cosmic, NC and other beam background distributions.

\footnotetext{
${ }^{2}$ Each set of integrals was also calculated in $1 \mathrm{GeV}$ wide energy bins, ranging from 0 to $5 \mathrm{GeV}$. However, optimization at this level was later concluded too susceptible to bias due to a lack of cosmics passing the event selection.
} 


\subsubsection{Tuning PID Cut Values}

Tuning produced two sets of PID cut boundaries. The first set is optimized quantitatively with no hand tuning and produces a unique boundary for each horn current and for $E_{\text {had }} / E_{\nu}$ quantile. The second is a simple case in which identical PID cuts are used for FHC, RHC and across all $E_{\text {had }} / E_{\nu}$ quantiles. This second criteria was motivated to remove reliance on the cosmic BDT - a cut only used in the FD.

The optimization was performed by mass comparison of the FOM's produced by application of each cut selection. The $\frac{\text { signal }}{\sqrt{\text { signal+background }}}$ FOM, signal count and total background were compared to the equivalent values produced when using the 2017 PID selection. Fig-

ure 6.5 and Figure 6.6 show the $\frac{\text { signal }}{\sqrt{\text { signal+background }}}$ against total background plots for FHC and RHC respectively. The flatness visible in these plots is indicative of the potential for reducing background without reducing analysis measurement sensitivity. Each plot is marked with three points, representing relevant cut permutations - the circle represents the 2017 PID selection, the cross represents the 2018 simple selection and the star represents the 2018 per quantile selection.

Optimization was performed for each horn current and each $E_{h a d} / E_{\nu}$ quantile separately. Of the set of cuts that had greater FOM's than those achieved by the previous analysis, the single highest scoring $\frac{\text { signal }}{\sqrt{\text { signal+background }}}$ combination was first identified. Optimization then proceeded by summing the total background for all other cut sets and accepting all permutations with a lower total background than the best scoring selection. To avoid bias towards a statistically limited cosmic sample any selected cut set had to correspond to a populated region - defined as there being at least two cut permutations in every adjacent bin in both the $\frac{\text { signal }}{\sqrt{\text { signal+background }}}$ and total background domains. Of these selections, the boundary choice that produced the minimum total background whilst maintaining a figure of merit within $0.5 \%$ of the best scoring case was identified as the optimized PID boundary. An optimum selection boundary was produced for each $E_{\text {had }} / E_{\nu}$ quantile following this method. The cut boundaries for each PID algorithm in each $E_{h a d} / E_{\nu}$ quantile is given in Table 6.2. 


\begin{tabular}{|c|ccc|ccc|}
\hline & \multicolumn{3}{|c|}{ FHC } & \multicolumn{3}{c|}{ RHC } \\
\hline$E_{\text {had }} / E_{\nu}$ Quantile & BDT & RID & CVN & BDT & RID & CVN \\
\hline Lowest & 0.56 & 0.60 & 0.55 & 0.47 & 0.40 & 0.80 \\
Second-Lowest & 0.56 & 0.60 & 0.55 & 0.48 & 0.40 & 0.65 \\
Second-Highest & 0.58 & 0.60 & 0.55 & 0.56 & 0.40 & 0.60 \\
Highest & 0.59 & 0.60 & 0.55 & 0.59 & 0.65 & 0.50 \\
\hline
\end{tabular}

Table 6.2: Summary of the 2018 per quantile PID selection boundaries. The FHC and RHC bounds are shown on the left and right respectively. 
Q1

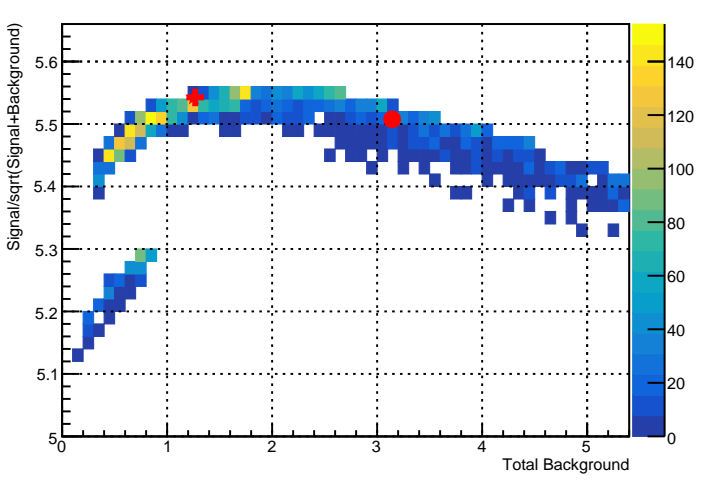

Q3

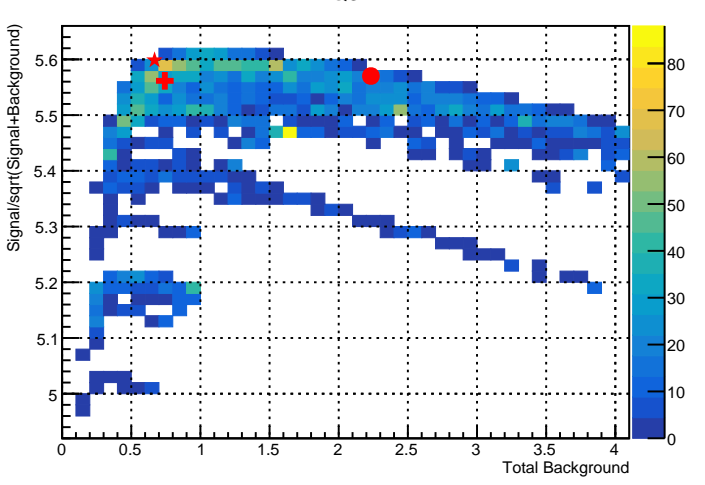

Q2

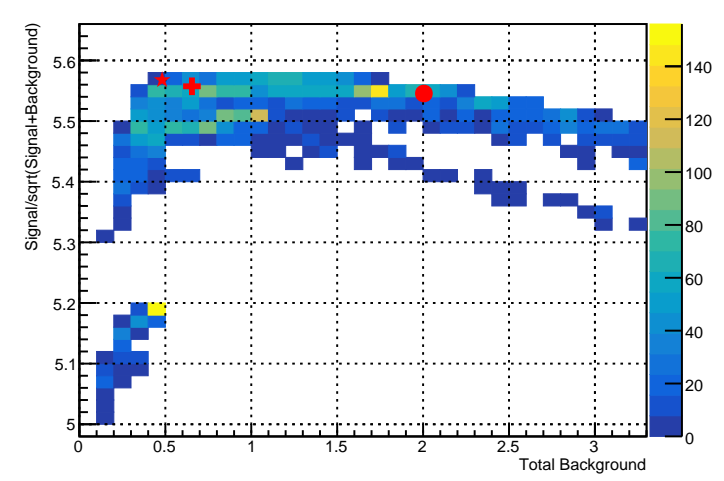

Q4

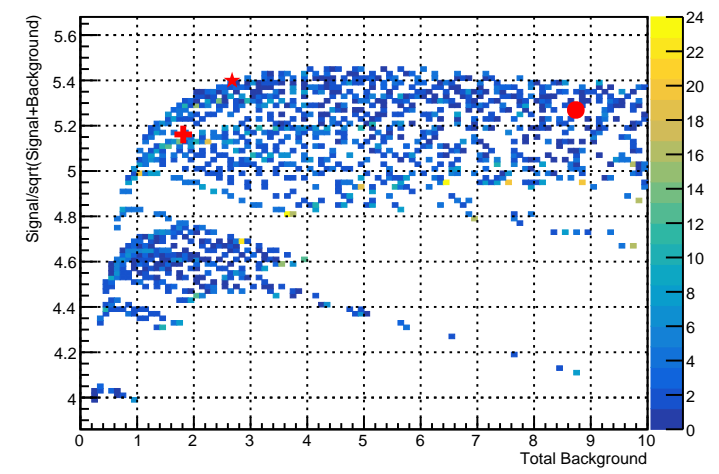

Figure 6.5: The FHC figure of merit $\left(\frac{s i g}{\sqrt{s i g+b k g}}\right)$ against total background for 6069 sets of cuts each representing one set of PID cuts analyzed. The lowest (top-left), second-lowest (top-right), second-highest (bottom-left) and highest (bottom-right) hadronic energy fraction quantiles are shown. The circle represents the 2017 point, the cross the universal cut case and the star the per hadronic energy fraction quantile cuts. 
Q1

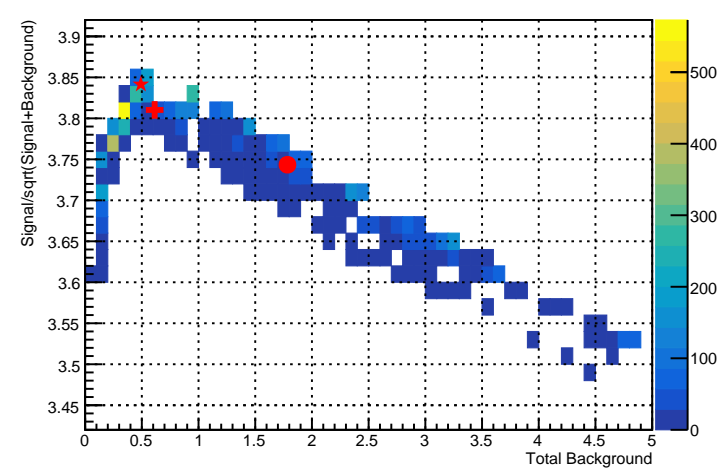

Q3

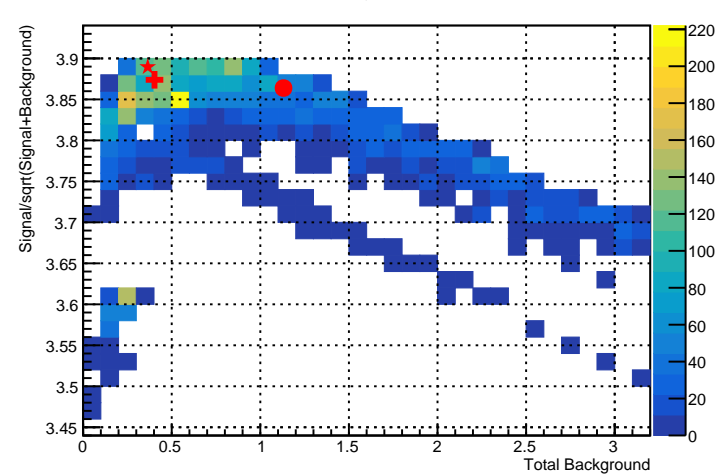

Q2

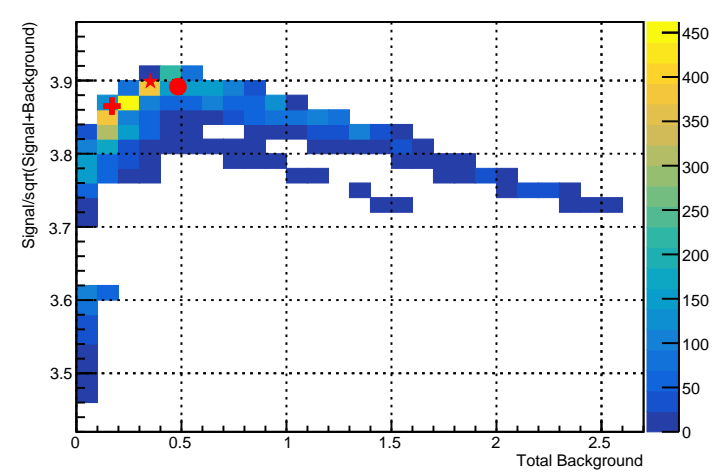

Q4

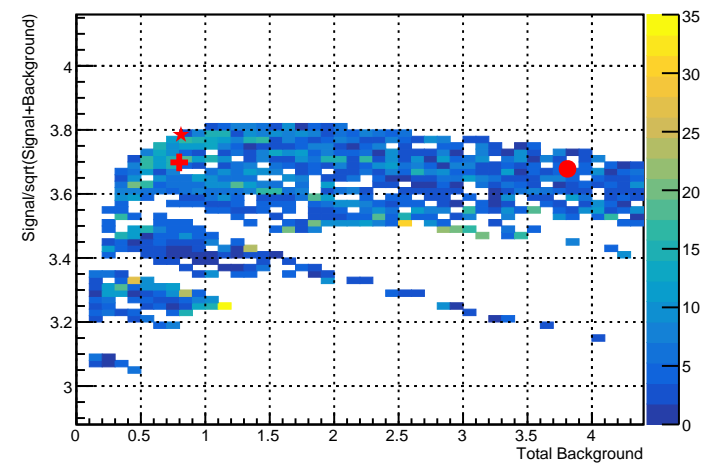

Figure 6.6: The RHC figure of merit $\left(\frac{s i g}{\sqrt{s i g+b k g}}\right)$ against total background for 6069 sets of cuts each representing one set of PID cuts analyzed. The lowest (top-left), second-lowest (top-right), second-highest (bottom-left) and highest (bottom-right) hadronic energy fraction quantiles are shown. The circle represents the 2017 point, the cross the universal cut case and the star the per hadronic energy fraction quantile cuts. 
The cosmic BDT selection is only applied at the FD - the ND does not experience a significant cosmic flux. For this reason a simple cut selection was produced to reduce reliance on the BDT, favouring harsher cuts in CVN and ReMId. This selection could be universally applied to all quantiles and was agnostic to neutrino or antineutrino data. A binary tree and additional macro allowed almost instantaneous production of FOM metrics for any cut combination, facilitating hand scanning for optimum boundaries. The per quantile optimization provided an optimum FOM for the hand tune to target. The simple cuts produced from this tune are given in Table 6.3.

\begin{tabular}{|ccc|}
\hline \multicolumn{3}{|c|}{ FHC and } \\
\hline BDT & RID & CVN \\
\hline 0.53 & 0.70 & 0.70 \\
\hline
\end{tabular}

Table 6.3: Summary of the 2018 simple PID selection boundaries. Hand tuned to maximally reduce reliance on the BDT.

\subsection{Results of Optimization}

This section details the optimization metrics as produced at each optimized set of selection boundaries. The $\nu_{\mu}+\bar{\nu}_{\mu}$-CC signal, other beam background, cosmic background, neutral current, total background, $\frac{s i g}{b k g}$ and $\frac{s i g}{\sqrt{s i g+b k g}}$ are given for each optimized cut set. The equivalent figures for the previous analysis are introduced first for the sake of comparison.

Alongside the 2018 CVN selector, the 2017 CVN selector is still used by the analysis because of its enhanced cosmic rejection in the lowest $E_{\text {had }} / E_{\nu}$ quantile [82]. This cut is very loose $(\mathrm{CVN} 2017>0.1)$ and only serves to remove certain background events. The added background rejection gained from using the 2017 CVN selector was found after the work performed in this chapter was completed. The optimization results shown in this section have been updated to include this additional cut.

\subsubsection{Previous PID Selection Performance}

Table 6.4 and Table 6.5 show a breakdown of signal and background events selected by the $8.85 \times 10^{20}$ POT FHC-only analysis Methodology for FHC and RHC respectively. The analysis methodology that produced the figures in these tables was optimized for FHC only as RHC data had not yet been introduced. A full detailing of the cuts used is not merited in this document but is contained in [83] which gives a comprehensive overview 
of the analysis.

\begin{tabular}{|cccccc|cc|}
\hline \multicolumn{7}{|c|}{ FHC Event Breakdown - 2017 PID Selection } \\
\hline$E_{\text {had }} / E_{\nu}$ Quantile & $\nu_{\mu}+\bar{\nu}_{\mu}$-CC & Other Beam Bkg & Cosmics & NC & Total Bkg & $\frac{s i g}{b k g}$ & $\frac{s i g}{\sqrt{s i g+b k g}}$ \\
\hline Lowest & 29.82 & 0.01 & 0.13 & 0.06 & 0.20 & 152.38 & 5.44 \\
Second-Lowest & 30.20 & 0.01 & 0.11 & 0.14 & 0.26 & 117.17 & 5.47 \\
Second-Highest & 30.63 & 0.02 & 0.47 & 0.33 & 0.83 & 36.92 & 5.46 \\
Highest & 32.49 & 0.10 & 4.92 & 1.94 & 6.95 & 4.67 & 5.17 \\
\hline Total & 123.21 & 0.14 & 5.60 & 2.46 & 8.20 & 15.02 & 10.75 \\
\hline
\end{tabular}

Table 6.4: Breakdown of signal and background events for the FHC analysis using the previous PID selection criteria and NOvA's $8.85 \times 10^{20}$ POT FHC-only best fit [3].

\begin{tabular}{|cccccc|cc|}
\hline \multicolumn{7}{|c|}{ RHC Event Breakdown - 2017 PID Selection } \\
\hline$E_{\text {had }} / E_{\nu}$ Quantile & $\nu_{\mu}+\bar{\nu}_{\mu}$-CC & Other Beam Bkg & Cosmics & NC & Total Bkg & $\frac{s i g}{b k g}$ & $\frac{s i g}{\sqrt{s i g+b k g}}$ \\
\hline Lowest & 13.53 & 0.00 & 0.02 & 0.01 & 0.03 & 393.06 & 3.67 \\
Second-Lowest & 14.17 & 0.00 & 0.03 & 0.03 & 0.06 & 240.15 & 3.76 \\
Second-Highest & 14.68 & 0.00 & 0.16 & 0.10 & 0.26 & 56.28 & 3.80 \\
Highest & 15.54 & 0.04 & 2.84 & 0.81 & 3.69 & 4.21 & 3.54 \\
\hline Total & 57.96 & 0.05 & 3.06 & 0.95 & 4.05 & 14.30 & 7.36 \\
\hline
\end{tabular}

Table 6.5: Breakdown of signal and background events for the RHC analysis using the previous PID selection criteria and NOvA's $8.85 \times 10^{20}$ POT FHC-only best fit [3].

\subsubsection{Simple Optimization}

Table 6.6 and Table 6.7 show the overall impact on the $\nu_{\mu}+\bar{\nu}_{\mu}$-CC signal, other beam background, cosmic background and neutral current when using the previous PID and new simple PID selection criteria. The FHC result gave a significant decrease in total background $(68 \%)$ at the cost of a small loss $(5 \%)$ in $\nu_{\mu}+\bar{\nu}_{\mu}$-CC signal. The RHC result also gave a significant decrease in total background (75\%) with almost no cost to $\bar{\nu}_{\mu}$-CC signal. The $\frac{\text { signal }}{\sqrt{\text { signal+background }}}$ FOM remained essentially unchanged for FHC and increased by $2.0 \%$ for RHC. 


\begin{tabular}{|cccccc|cc|}
\hline \multicolumn{7}{|c|}{ FHC Simple Optimization } \\
\hline$E_{\text {had }} / E_{\nu}$ Quantile & $\nu_{\mu}+\bar{\nu}_{\mu}$-CC & Other Beam Bkg & Cosmics & NC & Total Bkg & $\frac{s i g}{b k g}$ & $\frac{s i g}{\sqrt{s i g}+b k g}$ \\
\hline Lowest & 32.23 & 0.00 & 0.31 & 0.07 & 0.38 & 84.33 & 5.64 \\
Second-Lowest & 29.10 & 0.01 & 0.16 & 0.10 & 0.28 & 105.01 & 5.37 \\
Second-Highest & 29.34 & 0.02 & 0.21 & 0.24 & 0.47 & 62.98 & 5.37 \\
Highest & 26.40 & 0.03 & 0.75 & 0.71 & 1.49 & 17.74 & 5.00 \\
\hline & 123.21 & 0.14 & 5.60 & 2.46 & 8.20 & 15.02 & 10.75 \\
\hline 2017 & 117.07 & 0.07 & 1.42 & 1.12 & 2.61 & 44.80 & 10.70 \\
$\frac{2018}{2017}$ Ratio & 0.95 & 0.46 & 0.25 & 0.46 & 0.32 & 2.98 & 1.00 \\
\hline
\end{tabular}

Table 6.6: FHC breakdown of signal and background events when applying the simple cut optimization and a comparison with the previous analysis results - shown in Table 6.4 . 


\begin{tabular}{|c|c|c|c|c|c|c|c|c|}
\hline \multicolumn{9}{|c|}{ RHC Simple Optimization } \\
\hline$E_{\text {had }} / E_{\nu}$ Quantile & $\bar{\nu}_{\mu}+\nu_{\mu}-\mathbf{C C}$ & Other & Beam Bkg & Cosmics & $\mathrm{NC}$ & Total Bkg & $\frac{s i g}{b k g}$ & $\frac{s i g}{\sqrt{s i g+b k g}}$ \\
\hline Lowest & 15.27 & & 0.00 & 0.07 & 0.02 & 0.08 & 187.86 & 3.90 \\
\hline Second-Lowest & 14.18 & & 0.00 & 0.04 & 0.04 & 0.08 & 186.84 & 3.76 \\
\hline Second-Highest & 14.24 & & 0.00 & 0.14 & 0.08 & 0.22 & 65.85 & 3.75 \\
\hline Highest & 13.68 & & 0.02 & 0.36 & 0.30 & 0.68 & 20.24 & 3.61 \\
\hline \multicolumn{9}{|c|}{ Totals } \\
\hline 2017 & 57.96 & & 0.05 & 3.06 & 0.95 & 4.05 & 14.30 & 7.36 \\
\hline 2018 & 57.38 & & 0.02 & 0.60 & 0.43 & 1.05 & 54.68 & 7.51 \\
\hline$\frac{2018}{2017}$ Ratio & 0.99 & & 0.41 & 0.20 & 0.46 & 0.26 & 3.82 & 1.02 \\
\hline
\end{tabular}

Table 6.7: RHC breakdown of signal and background events when applying the simple cut optimization and a comparison with the previous analysis results - shown in Table 6.5. 


\subsubsection{Per Hadronic-Energy Quantile Optimization}

Table 6.8 and Table 6.9 show the overall impact on the $\nu_{\mu}+\bar{\nu}_{\mu}$-CC signal, other beam background, cosmic background and neutral current when using the previous PID selection and when using the per horn-current, per $E_{h a d} / E_{\nu}$ quantile, PID selection. The FHC result gave a significant decrease in total background (65\%) at the cost of a small loss $(2.5 \%)$ in $\nu_{\mu}+\bar{\nu}_{\mu}$-CC signal. The RHC result also gave a significant decrease in total background $(76 \%)$ and a marginal increase $(1 \%)$ in $\bar{\nu}_{\mu}$-CC signal. The $\frac{\text { signal }}{\sqrt{\text { signal }+ \text { background }}}$ FOM increased by $0.7 \%$ and by $2.9 \%$ for $\mathrm{FHC}$ and $\mathrm{RHC}$ respectively. 


\begin{tabular}{|ccccccc|cc|}
\hline \multicolumn{7}{|c|}{ FHC Per Quantile Optimization } \\
\hline$E_{\text {had }} / E_{\nu}$ Quantile & $\nu_{\mu}+\bar{\nu}_{\mu}$-CC & Other Beam Bkg & Cosmics & NC & Total Bkg & $\frac{s i g}{b k g}$ & $\frac{s i g}{\sqrt{s i g+b k g}}$ \\
\hline Lowest & 32.31 & 0.00 & 0.31 & 0.07 & 0.39 & 83.22 & 5.65 \\
Second-Lowest & 29.10 & 0.01 & 0.11 & 0.11 & 0.23 & 126.22 & 5.37 \\
Second-Highest & 29.77 & 0.01 & 0.12 & 0.29 & 0.42 & 70.21 & 5.42 \\
Highest & 29.00 & 0.05 & 0.68 & 1.17 & 1.90 & 15.25 & 5.22 \\
\hline & 123.21 & 0.14 & 5.60 & 2.46 & 8.20 & 15.02 & 10.75 \\
2017 & 120.17 & 0.08 & 1.23 & 1.64 & 2.94 & 40.81 & 10.83 \\
2018 & 0.98 & 0.54 & 0.22 & 0.67 & 0.36 & 2.72 & 1.01 \\
\hline
\end{tabular}

Table 6.8: FHC breakdown of signal and background events when applying the per hadronic energy fraction cut optimization to FHC and a comparison with the previous analysis results as shown in Table 6.4. 


\begin{tabular}{|c|c|c|c|c|c|c|c|c|}
\hline \multicolumn{9}{|c|}{ RHC Per Quantile Optimization } \\
\hline$E_{\text {had }} / E_{\nu}$ Quantile & $\bar{\nu}_{\mu}+\nu_{\mu}-\mathbf{C C}$ & Other & Beam Bkg & Cosmics & $\mathrm{NC}$ & Total Bkg & $\frac{s i g}{b k g}$ & $\frac{s i g}{\sqrt{s i g+b k g}}$ \\
\hline Lowest & 15.36 & & 0.00 & 0.06 & 0.02 & 0.08 & 198.54 & 3.91 \\
\hline Second-Lowest & 14.49 & & 0.00 & 0.05 & 0.05 & 0.10 & 150.80 & 3.79 \\
\hline Second-Highest & 14.38 & & 0.00 & 0.14 & 0.09 & 0.23 & 63.59 & 3.76 \\
\hline Highest & 14.11 & & 0.02 & 0.13 & 0.43 & 0.58 & 24.14 & 3.68 \\
\hline \multicolumn{9}{|c|}{ Totals } \\
\hline 2017 & 57.96 & & 0.05 & 3.06 & 0.95 & 4.05 & 14.30 & 7.36 \\
\hline 2018 & 58.33 & & 0.02 & 0.38 & 0.58 & 0.98 & 59.29 & 7.57 \\
\hline$\frac{2018}{2017}$ Ratio & 1.01 & & 0.44 & 0.12 & 0.61 & 0.24 & 4.15 & 1.03 \\
\hline
\end{tabular}

Table 6.9: RHC breakdown of signal and background events when applying the per hadronic energy fraction cut optimization and a comparison with the previous analysis results as shown in Table 6.5. 


\subsection{Validation of Optimization}

\subsubsection{FHC}

Figure 6.7 shows the $\nu_{\mu}+\bar{\nu}_{\mu}$-CC energy distributions for each $E_{h a d} / E_{\nu}$ quantile using the old PID selection, the simple PID selection and the per quantile PID selection. A small increase in selected signal is evident across the energy regime for both of the updated PID selections in the lowest two $E_{h a d} / E_{\nu}$ quantiles. The second-highest $E_{\text {had }} / E_{\nu}$ quantile exhibits almost no difference in signal selection when using any selection criteria. The highest $E_{\text {had }} / E_{\nu}$ quantile exhibits a $\nu_{\mu}+\bar{\nu}_{\mu}$-CC signal decrease across the energy regime. This is due to the larger fraction of hadronic energy contained in these events causing event features indicative of background. The corresponding ratio plots for each spectra with the 2017 spectra acting as the denominator is given in Figure 6.8. The signal increase in the lower two quantiles and decrease in the highest quantile are all evident.

Figure 6.9 shows the cosmic energy distributions for each $E_{h a d} / E_{\nu}$ quantile using the old PID selection, the simple PID selection and the per quantile PID selection. The lower two $E_{h a d} / E_{\nu}$ quantiles have a minute cosmic background and so the spectra are statistically limited - the background integrals nonetheless decrease for both quantiles. The upper two $E_{h a d} / E_{\nu}$ quantiles exhibit a clear decrease in cosmic background selection, with the highest quantile showing a significant decrease.

Figure 6.10 shows the NC energy distributions for each $E_{\text {had }} / E_{\nu}$ quantile using the old PID selection, the simple PID selection and the per quantile PID selection. As the $E_{\text {had }} / E_{\nu}$ quantile increases, the updated selections both show a decreasing NC selection with respect to the 2017 PID selection. The lowest $E_{h a d} / E_{\nu}$ quantile exhibits little difference between the 2017 and the simple selection, with the per quantile selection marginally outperforming both (selecting marginally fewer NC). The second lowest and second highest quantiles show appreciable decreases in $\mathrm{NC}$ rejection when using either of the updated selections. The highest quantile exhibits the largest gain in NC rejection. The per quantile selection outperforms the others in every quantile with a particularly significant NC reduction in the highest $E_{h a d} / E_{\nu}$ quantile.

Figure 6.11 shows the $\nu_{\mu}+\bar{\nu}_{\mu}$-CC signal (top-left), NC background (top-right), other beam background (bottom-left) and cosmic energy spectra summed across all $4 E_{\text {had }} / E_{\nu}$ quantiles using the old PID selection, the simple PID selection and the per quantile PID selection. The marginal $5 \%$ decrease in $\nu_{\mu}+\bar{\nu}_{\mu}$-CC signal is visible. All background sources are reduced with both new selections. The cosmic and other beam background spectra show minimal difference between the per quantile and simple selection. The NC 
background is appreciably lowered using the per quantile selection compared to either of the other criteria.

Figure 6.12 shows the total ratio plot for the $\nu_{\mu}+\bar{\nu}_{\mu}$-CC spectra shown in the top-left of Figure 6.11 with the 2017 spectra acting as the denominator. 

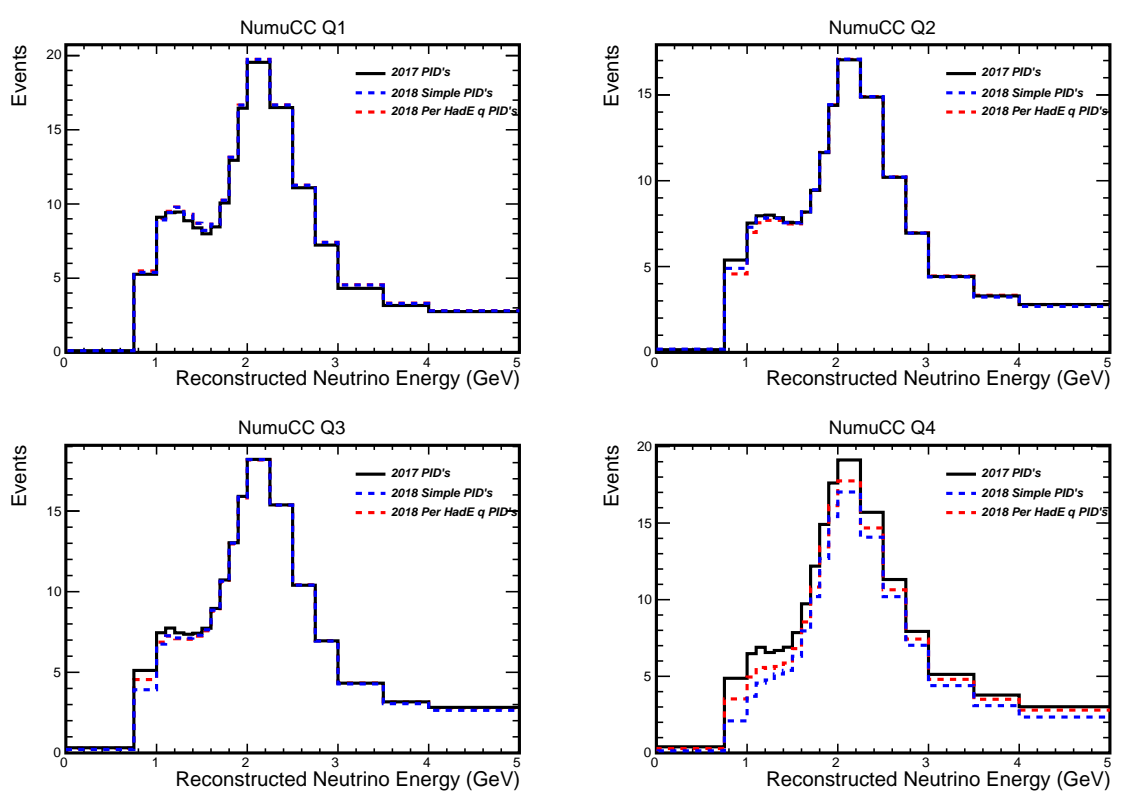

Figure 6.7: The FHC $\nu_{\mu}+\bar{\nu}_{\mu}$-CC distributions for each hadronic energy fraction quantile when using the 2017 PID cuts (black), the 2018 simple cuts (blue) and the 2018 per $E_{\text {had }} / E_{\nu}$ quantile cuts (red).
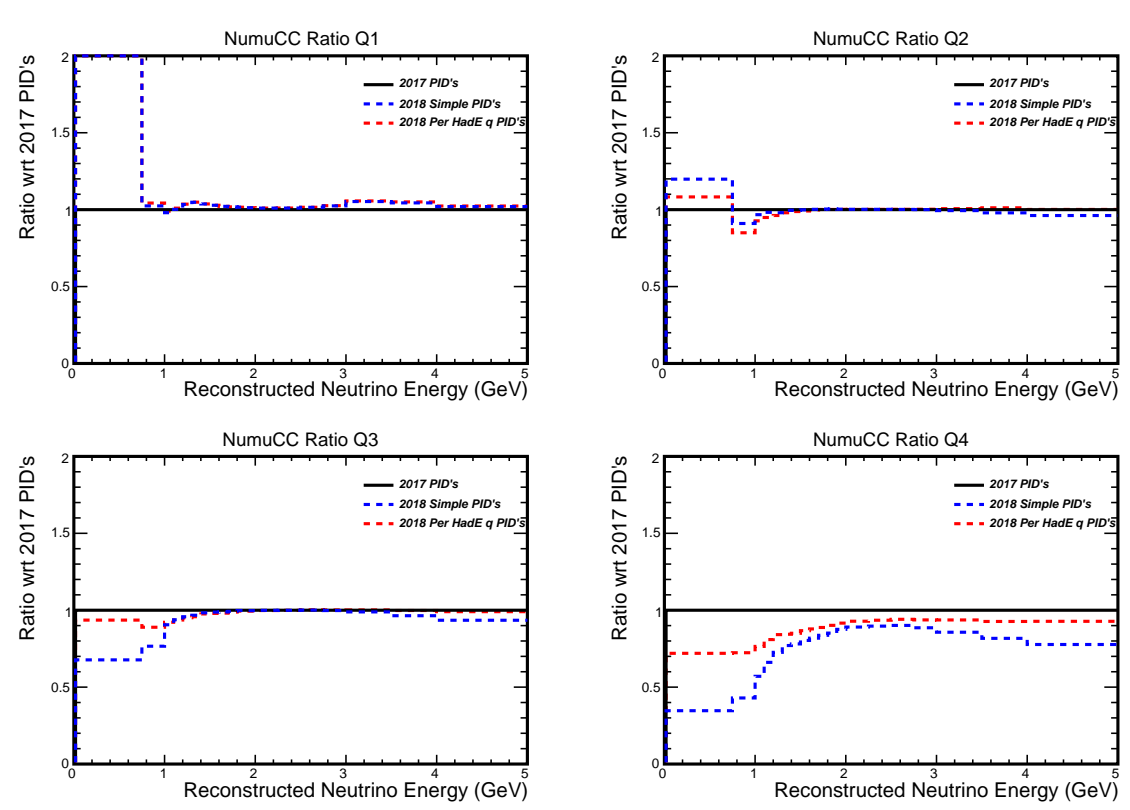

Figure 6.8: The FHC $\nu_{\mu}+\bar{\nu}_{\mu}$-CC ratios, with respect to the 2017 cuts, for each hadronic energy fraction quantile when using the 2017 PID cuts (black), the 2018 simple cuts (blue) and the 2018 per $E_{h a d} / E_{\nu}$ quantile cuts (red). 

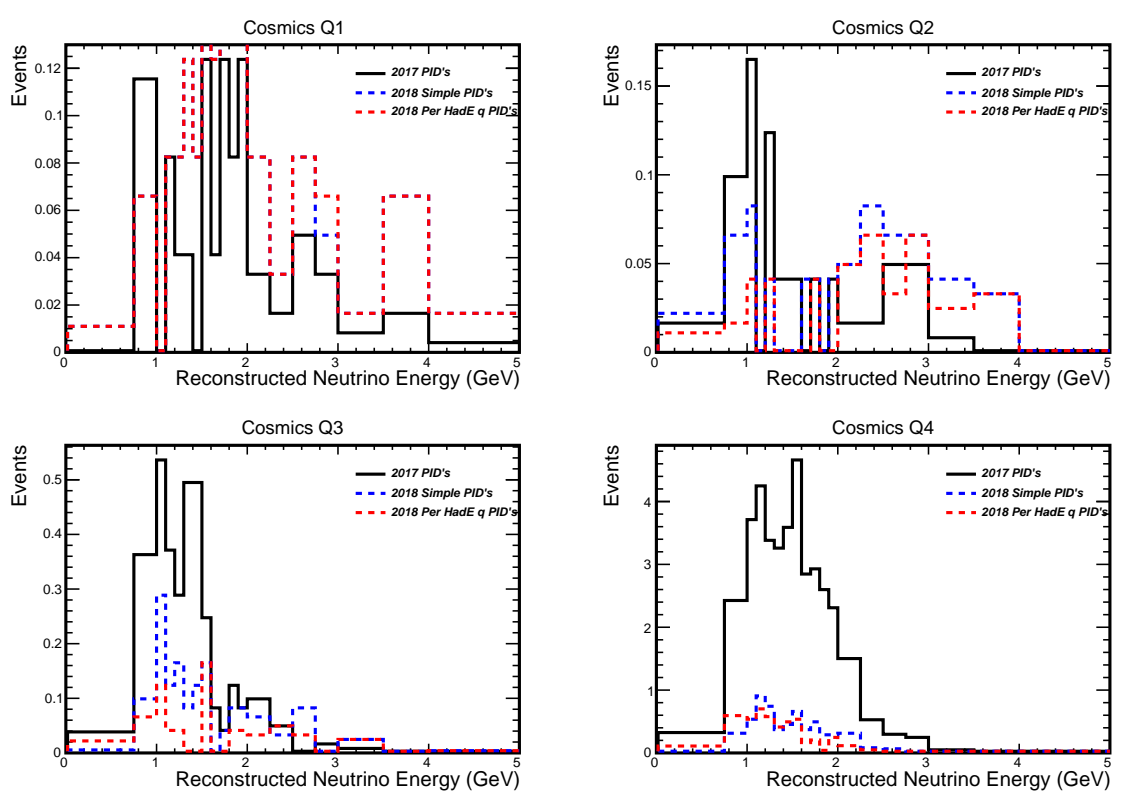

Figure 6.9: The FHC cosmic distributions for each hadronic energy fraction quantile when using the 2017 PID cuts (black), the 2018 simple cuts (blue) and the 2018 per $E_{h a d} / E_{\nu}$ quantile cuts (red).
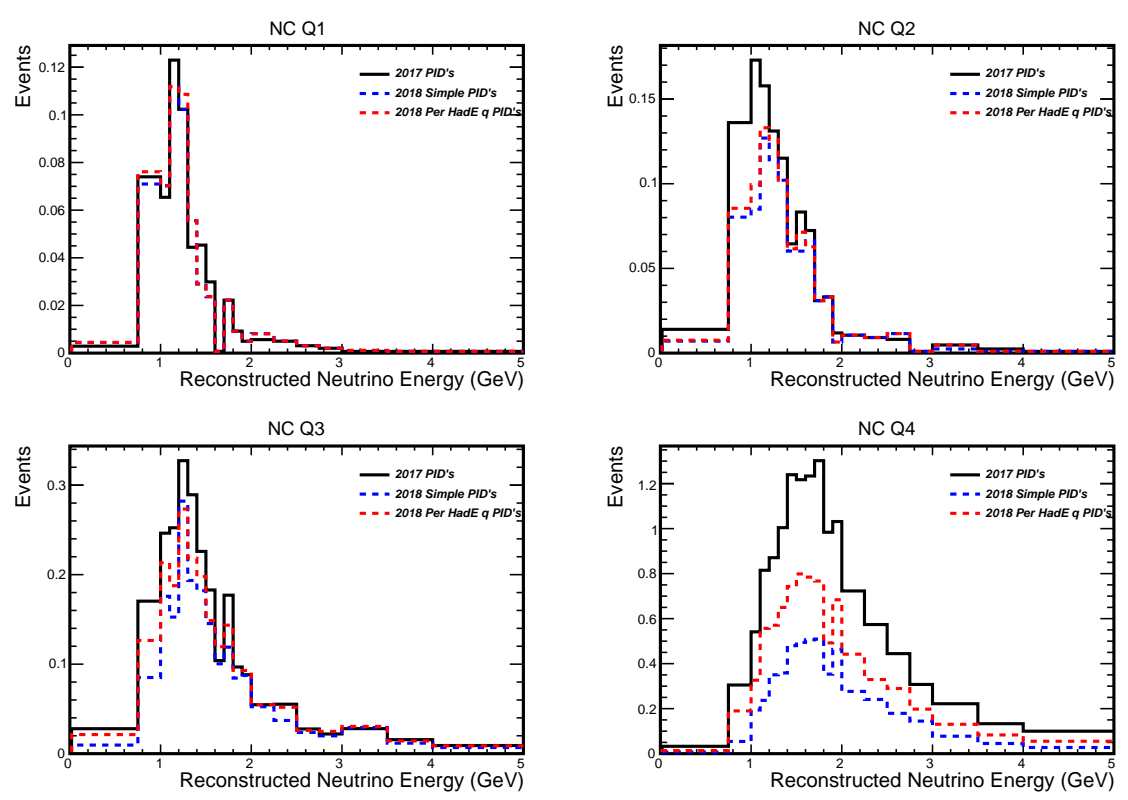

Figure 6.10: The FHC NC distributions for each hadronic energy fraction quantile when using the 2017 PID cuts (black), the 2018 simple cuts (blue) and the 2018 per $E_{h a d} / E_{\nu}$ quantile cuts (red). 

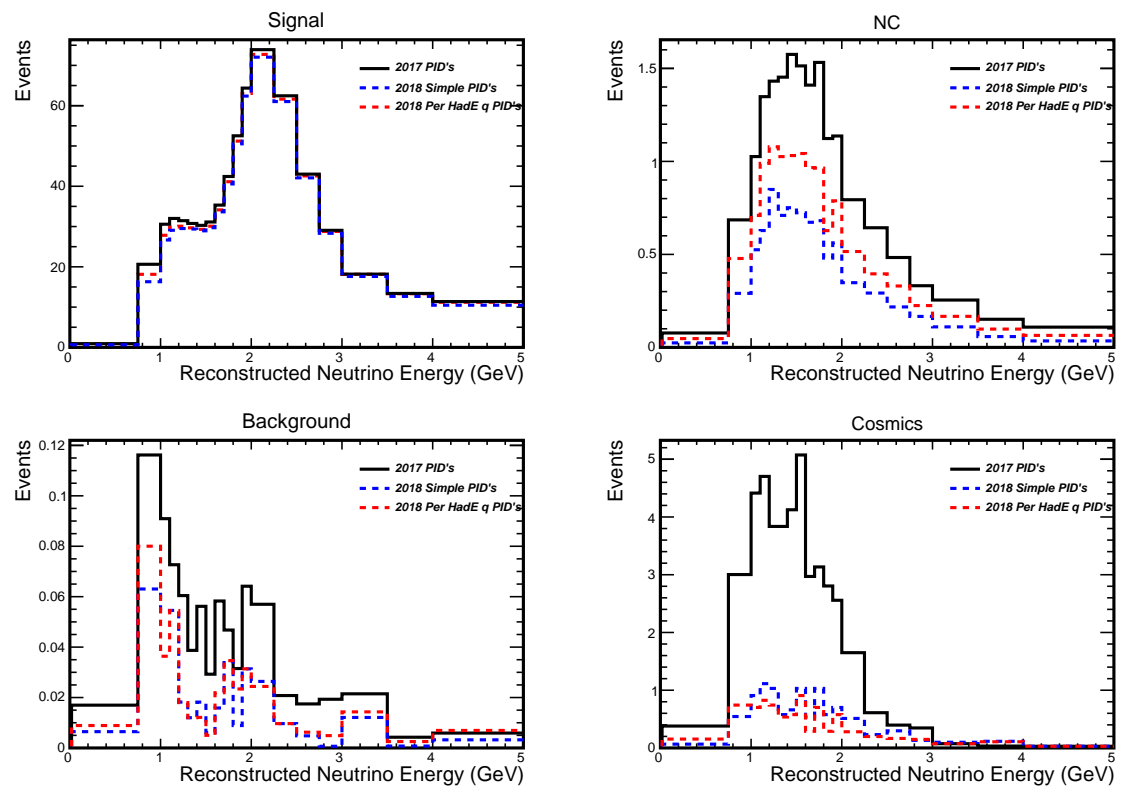

Figure 6.11: The FHC total $\nu_{\mu}+\bar{\nu}_{\mu}$-CC (top-left), NC (top-right), Other Beam Background (bottom-left) and Cosmic (bottom-right) when using the 2017 PID cuts (black), the 2018 simple cuts (blue) and the 2018 per $E_{\text {had }} / E_{\nu}$ quantile cuts (red).

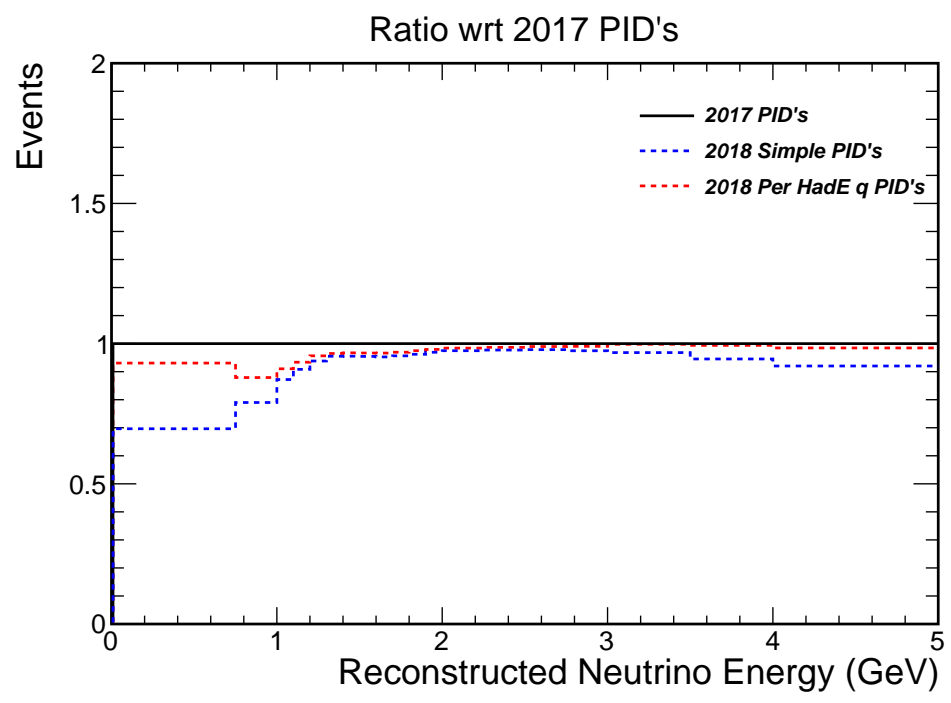

Figure 6.12: The total FHC $\nu_{\mu}+\bar{\nu}_{\mu}$-CC ratio, with respect to the 2017 cuts, when using the 2017 PID cuts (black), the 2018 simple cuts (blue) and the 2018 per $E_{h a d} / E_{\nu}$ quantile cuts (red). 


\subsubsection{RHC}

Figure 6.13 shows the $\bar{\nu}_{\mu}$-CC energy distributions for each $E_{h a d} / E_{\nu}$ quantile using the old PID selection, the simple PID selection and the per quantile PID selection. A universal increase in signal is visible across the energy regime for both of the updated PID selections for the lowest two $E_{h a d} / E_{\nu}$ quantiles - significantly the gain is largely in the 1-2 GeV energy regime, where the first oscillation maximum occurs. The second-highest $E_{h a d} / E_{\nu}$ quantile exhibits little signal selection difference when using different selection criteria. The highest $E_{h a d} / E_{\nu}$ quantile exhibits a $\bar{\nu}_{\mu}$-CC signal decrease across the energy regime. The corresponding ratio plots for each spectra with the 2017 spectra acting as the denominator are given in Figure 6.14. The signal increase in the lower two quantiles and decrease in the highest quantile are all clearly visible.

Figure 6.15 shows the cosmic energy distributions for each $E_{h a d} / E_{\nu}$ quantile using the old PID selection, the simple PID selection and the per quantile PID selection. As with the FHC data, the lower two $E_{\text {had }} / E_{\nu}$ quantiles have a small cosmic background and so the spectra are statistically limited - the background integrals decrease for both quantiles. The upper two $E_{h a d} / E_{\nu}$ quantiles exhibit a clear decrease in the cosmic background selection, with the highest quantile showing a significant decrease. The simple selection outperforms the per quantile selection in the highest $E_{h a d} / E_{\nu}$ quantile - performance disparities are indistinguishable in the other quantiles.

Figure 6.16 shows the NC energy distributions for each $E_{h a d} / E_{\nu}$ quantile using the old PID selection, the simple PID selection and the per quantile PID selection. As the $E_{\text {had }} / E_{\nu}$ quantile increases, the updated selections both show a decreasing NC selection with respect to the 2017 PID selection. The lowest $E_{\text {had }} / E_{\nu}$ quantile exhibits a small decrease in NC rejection when using either updated selection. The second-lowest $E_{\text {had }} / E_{\nu}$ quantile exhibits a small decrease in NC rejection when using the simple selection and a small increase in NC rejection for the per quantile selection. The upper two quantiles, which experience the largest NC background, both show significantly less NC events being selected with the updated criteria. The per quantile cuts outperform the simple and 2017 cuts - akin to the FHC trend.

Figure 6.17 shows the $\bar{\nu}_{\mu}$-CC signal (top-left), NC background (top-right), other beam background (bottom-left) and cosmic energy spectra summed across all $4 E_{h a d} / E_{\nu}$ quantiles using the old PID selection, the simple PID selection and the per quantile PID selection. The small $1 \%$ increase in $\bar{\nu}_{\mu}$-CC signal is visible in the $0.8-1.5 \mathrm{GeV}$ region. All background sources are reduced when using either family of updated PID selections. 
Figure 6.18 shows the total ratio plot for the $\bar{\nu}_{\mu}$-CC spectra shown in the top-left of Figure 6.17 with the 2017 spectra acting as the denominator. 

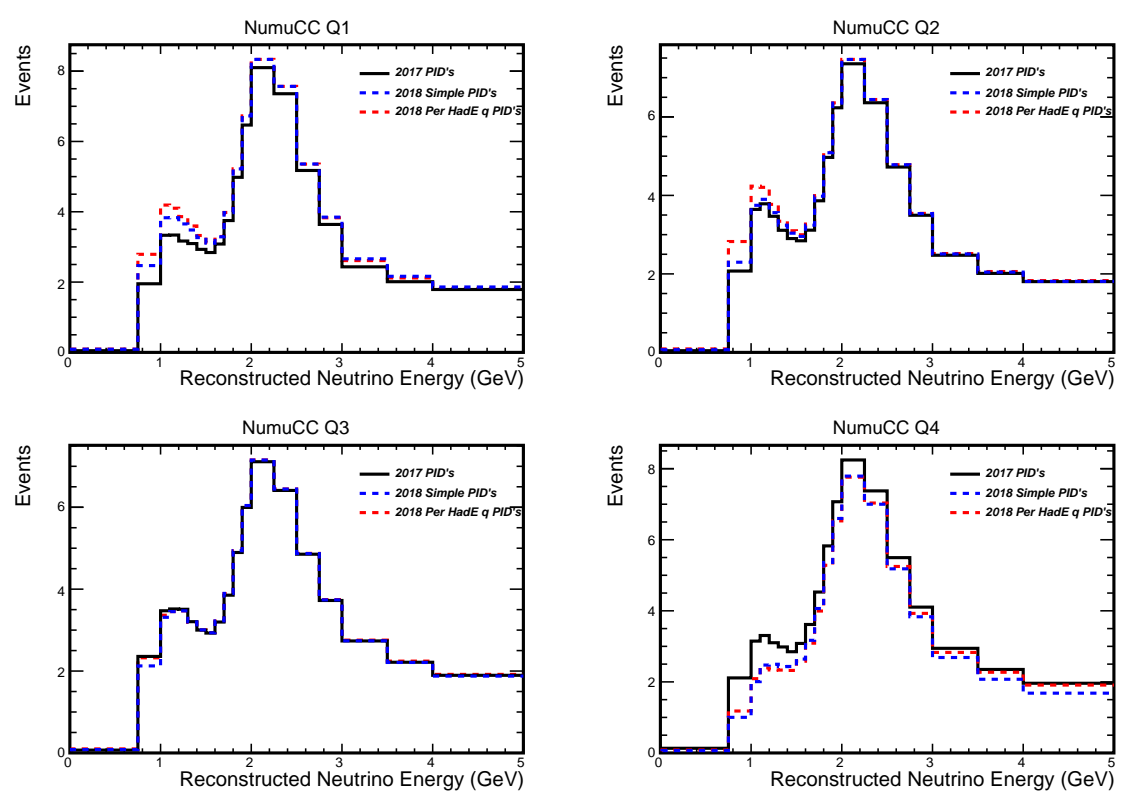

Figure 6.13: The RHC $\bar{\nu}_{\mu}$-CC distributions for each hadronic energy fraction quantile when using the 2017 PID cuts (black), the simple 2018 cuts (blue) and the 2018 per quantile cuts (red).
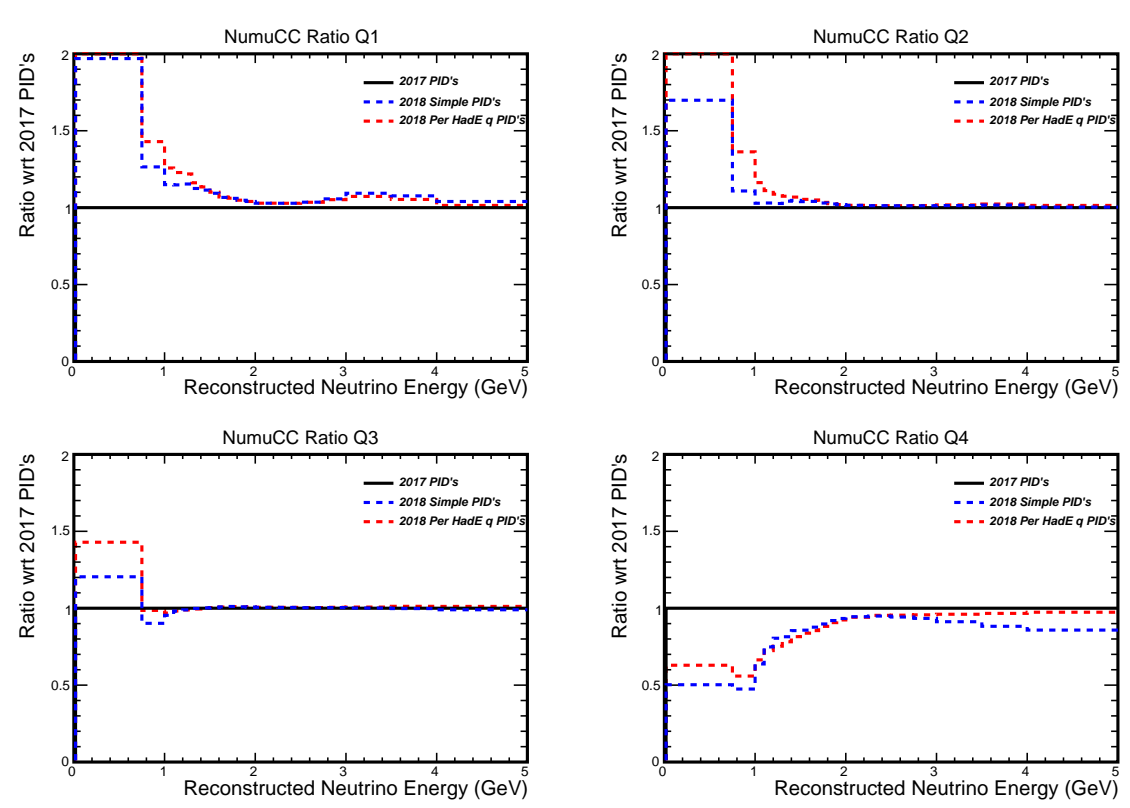

Figure 6.14: The RHC $\bar{\nu}_{\mu}$-CC ratios, with respect to the 2017 cuts, for each hadronic energy fraction quantile when using the 2017 PID cuts (black), the simple 2018 cuts (blue) and the 2018 per quantile cuts (red). 

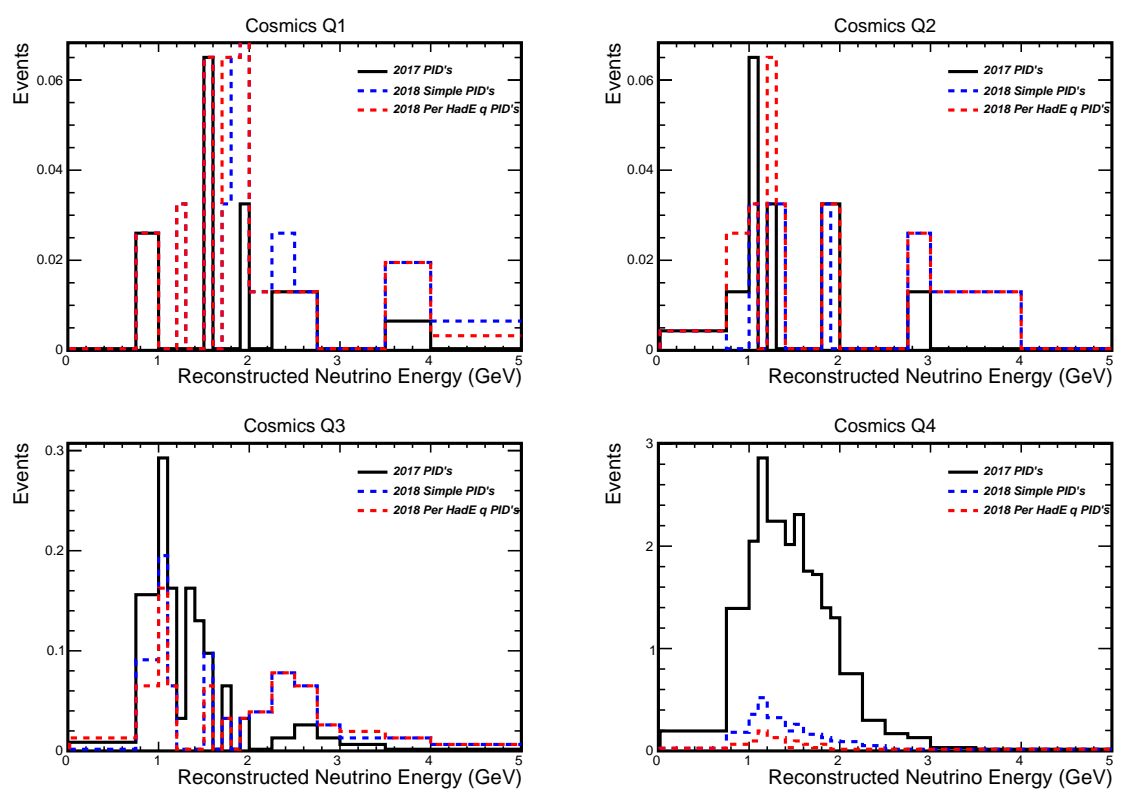

Figure 6.15: The RHC cosmic distributions for each hadronic energy fraction quantile when using the 2017 PID cuts (black), the simple 2018 cuts (blue) and the 2018 per quantile cuts (red).
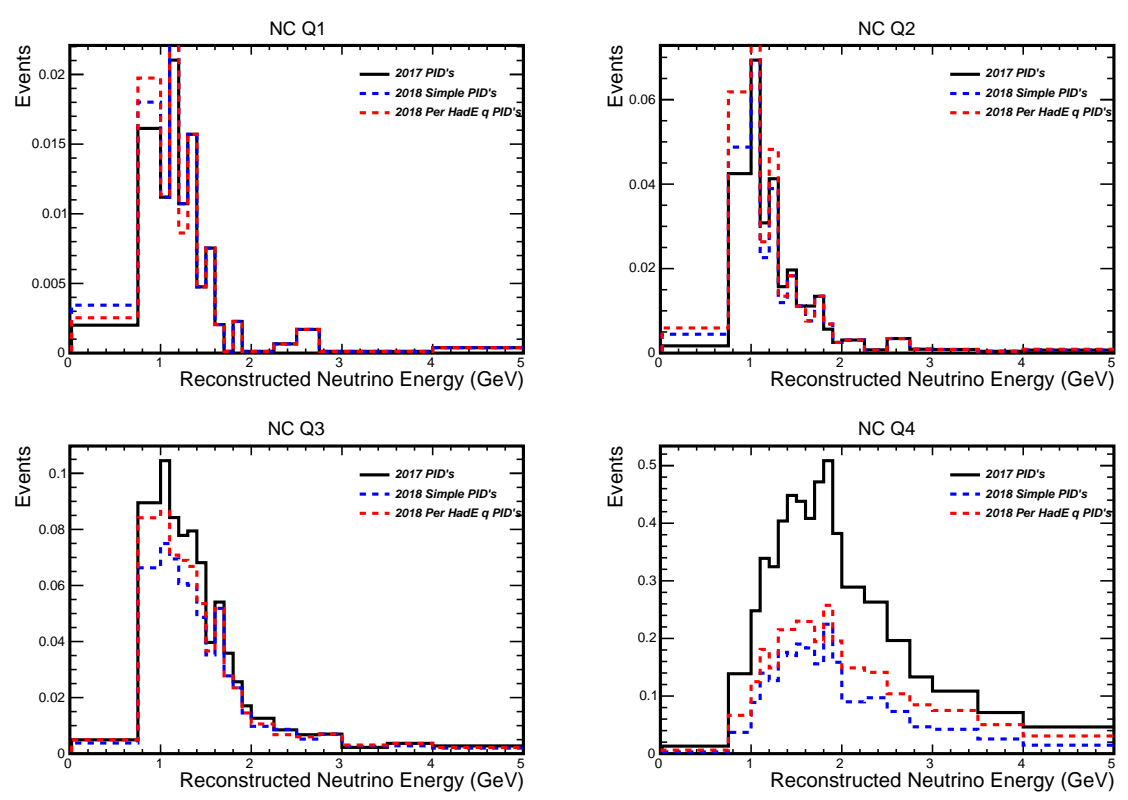

Figure 6.16: The RHC NC distributions for each hadronic energy fraction quantile when using the 2017 PID cuts (black), the simple 2018 cuts (blue) and the 2018 per quantile cuts (red). 

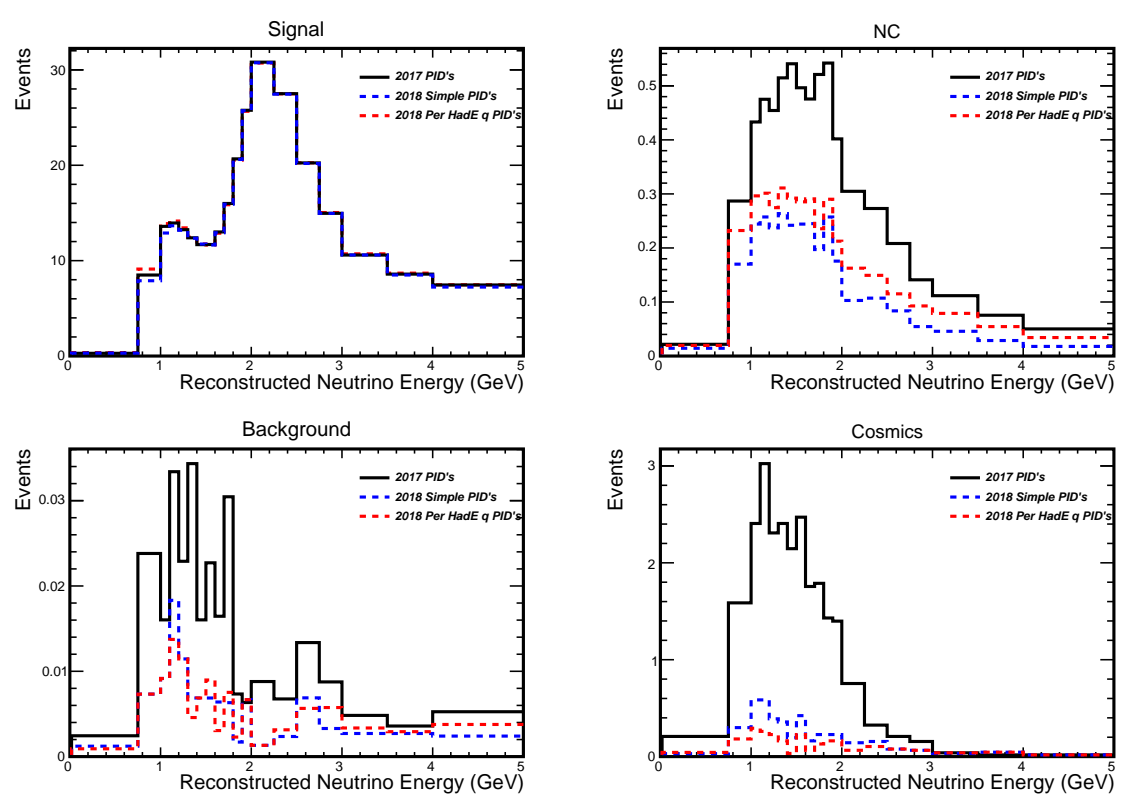

Figure 6.17: The total RHC $\bar{\nu}_{\mu}$-CC (top-left), NC (top-right), Other Beam Background (bottom-left) and Cosmic (bottom-right) when using the 2017 PID cuts (black), the simple 2018 cuts (blue) and the 2018 per quantile cuts (red).

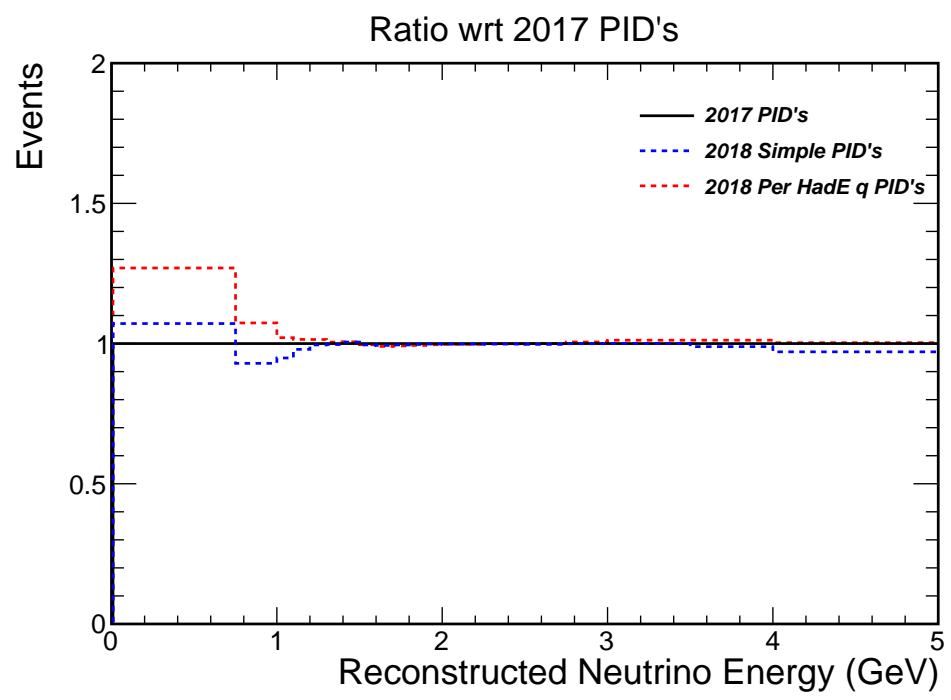

Figure 6.18: The total $\mathrm{RHC} \bar{\nu}_{\mu}$-CC ratio, with respect to the 2017 cuts, when using the 2017 PID cuts (black), the simple 2018 cuts (blue) and the 2018 per quantile cuts (red). 


\subsection{Conclusion}

The PID selection boundaries used by the $\nu_{\mu}+\bar{\nu}_{\mu}$ disappearance analysis have been reoptimized by the author using a new methodology. The aim was to maintain or improve analysis sensitivity while reducing all backgrounds. By producing extremely condensed datasets in low memory computing objects a large number of cut permutations could be quickly evaluated using a simple $\frac{\text { signal }}{\sqrt{\text { signal+background }}}$ FOM. This allowed discovery of a cut combination that substantially reduced the background in both FHC and RHC.

All three PID acceptance boundaries were changed and the updated values were adopted by the official NOvA analysis. The BDT was shifted from an acceptance of $>0.50$ to an acceptance of $>0.53$. The new CVN selector has been fixed at an acceptance of $>0.70$ as opposed to $>0.50$ in the previous analysis. Similarly, ReMId has been fixed at an acceptance of $>0.70$ as opposed to $>0.50$ in the previous analysis. In addition to the implementation of single valued cuts which can be uniformly used across FHC, RHC and all $E_{h a d} / E_{\nu}$ quantiles, separate optimizations were found with separate BDT, CVN and ReMId cuts for each hadronic energy fraction quantile and for each horn current. This per quantile optimization may be used in future analyses.

The optimized PID selection boundaries decrease the number of selected $\nu_{\mu}+\bar{\nu}_{\mu}$-CC signal events in both FHC and RHC - by $5 \%$ by $1 \%$ respectively. The total selected background has been significantly reduced by approximately $65 \%$ in $\mathrm{FHC}$ and $75 \%$ in $\mathrm{RHC}$ respectively and overall analysis sensitivity has gone up. 


\section{Chapter 7}

\section{Results}

NOvA has now collected data from both NuMI horn current configurations. The Forward Horn Current (FHC) configuration produces a beam of primarily neutrinos, whereas the Reverse Horn Current (RHC) configuration produces a beam of primarily antineutrinos. The first $\nu_{\mu}+\bar{\nu}_{\mu}$ disappearance results using data collected from both configurations are presented in this chapter.

Chapter 5 and Chapter 6 detail select analysis improvements produced by the author. These improvements happened over the course of two analyses. Chapter 5 details updates first incorporated into the 2018 [3] analysis and Chapter 6 details improvements that were implemented for the results given in this chapter that were first released at the 2018 'NEUTRINO' conference [86].

The data used for the present analysis was recorded over the course of five years, spanning February 2013 through April 2018. The far detector data corresponds to a full 14 ktonne FHC exposure of $8.85 \times 10^{20}$ protons on target (POT) [3] and an RHC exposure of $6.91 \times 10^{20}$ POT.

Data from each detector is presented separately ${ }^{1}$. Near detector data-MC comparisons, inclusive of systematic uncertainty bands, are shown first. Far detector distributions, inclusive of statistics-dominated errors are shown next. Finally, the fit to the far detector muon (anti)neutrino energy spectrum is performed using data recorded from the neutrino and antineutrino beams. Constraints on the oscillation parameters $\left|\Delta m_{32}^{2}\right|$ and $\sin ^{2} \theta_{23}$ are produced.

The plots shown in this chapter are made using data that, at the time of writing, has not been published in any major NOvA collaboration paper. As such the header has been

\footnotetext{
${ }^{1}$ All plots in this section are made using the latest cross section and beam flux weights as described in Chapter 4 by Section 4.1.1 and Section 4.1.2 respectively.
} 
modified, in this chapter, to include the 'NOvA Preliminary' tag. This choice has been made over inclusion on a plot-by-plot basis to facilitate readability and layout.

\subsection{Near Detector Distributions}

The agreement between MC and data for the most pertinent analysis variables in the near detector is described herein. Distributions are presented for the entire RHC or FHC dataset followed by the respective breakdown for the total dataset. Plots are arranged such that forward and reverse horn current are side-by-side for direct comparison.

Assessment of the level of agreement in these variables is performed before far detector data results are observed. This helps mitigate any significant inconsistencies that may be present in the near detector, informing the analysis of far detector data. After the full analysis selection criteria and tuned cross-section weights are applied the observed disparity between MC and data normalizations is approximately $1 \%$.

Interaction type breakdowns for FHC and RHC are shown in Figure 7.1. Per-quantile breakdowns follow in Figure 7.2. These plots are produced using simulated data. The overall breakdown of events is similar between horn currents. FHC contains a higher relative proportion of resonance events and a lower relative proportion of quasi-elastic events. The per quantile breakdowns show similar distributions but RHC and FHC have notable differences in the lower two $E_{h a d} / E_{\nu}$ quantiles. The lowest quantile contains more quasi-elastic and less resonance events in RHC than in FHC. The second lowest quantile exhibits the largest FHC-RHC difference. In RHC the first, second and third most dominant type of interaction come from MEC, quasi-elastic and resonance events respectively. In FHC the first, second and third most dominant type of interaction come from resonance, MEC and quasi-elastics respectively.

A comprehensive quantitative breakdown of the ND interaction types is given for FHC in Table 7.1 and for RHC in Table 7.2. The tables show the absolute number of simulated events selected by the analysis, the percentage of each interaction type relative to the total number of events in that quantile, the percentage of events of each type relative to the overall total and the percentage of each type of event relative to the overall total of that interaction type. 

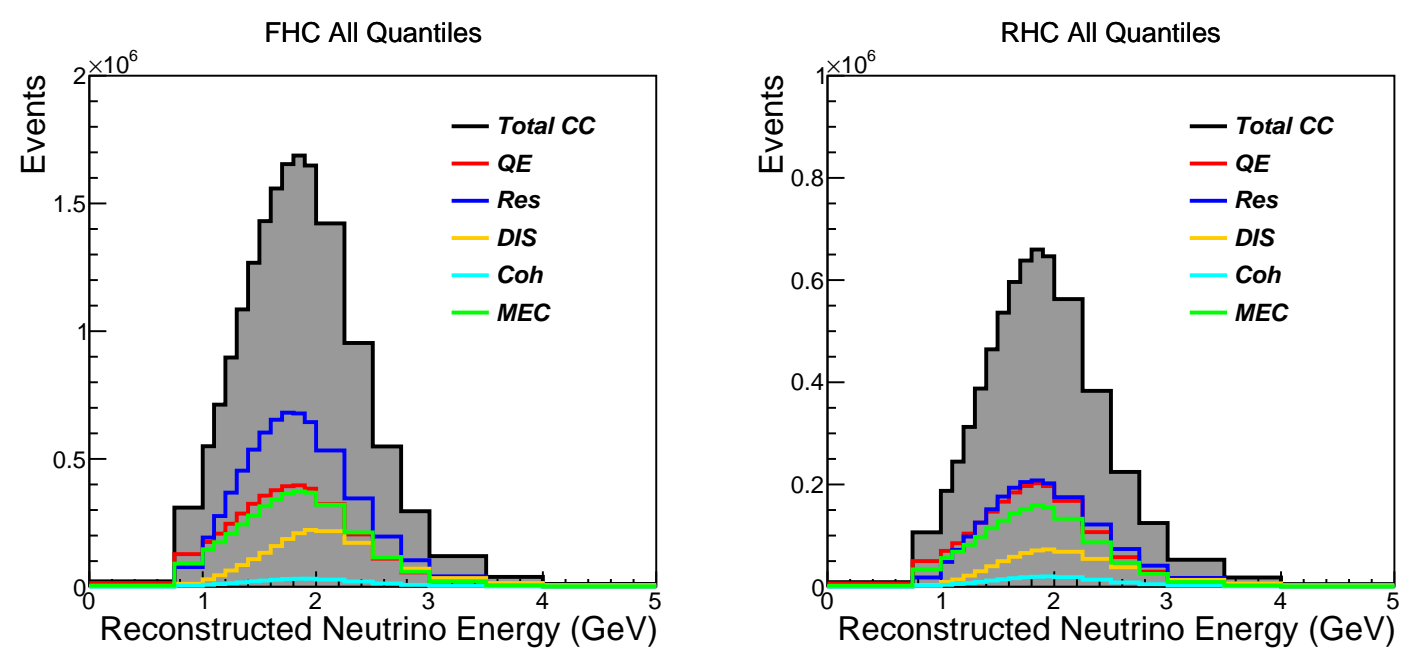

Figure 7.1: FHC (left) and RHC (right) interaction type distributions of selected $\nu_{\mu}+\bar{\nu}_{\mu}$ CC candidate events in the Near Detector. Plots are shown for the total respective dataset. 

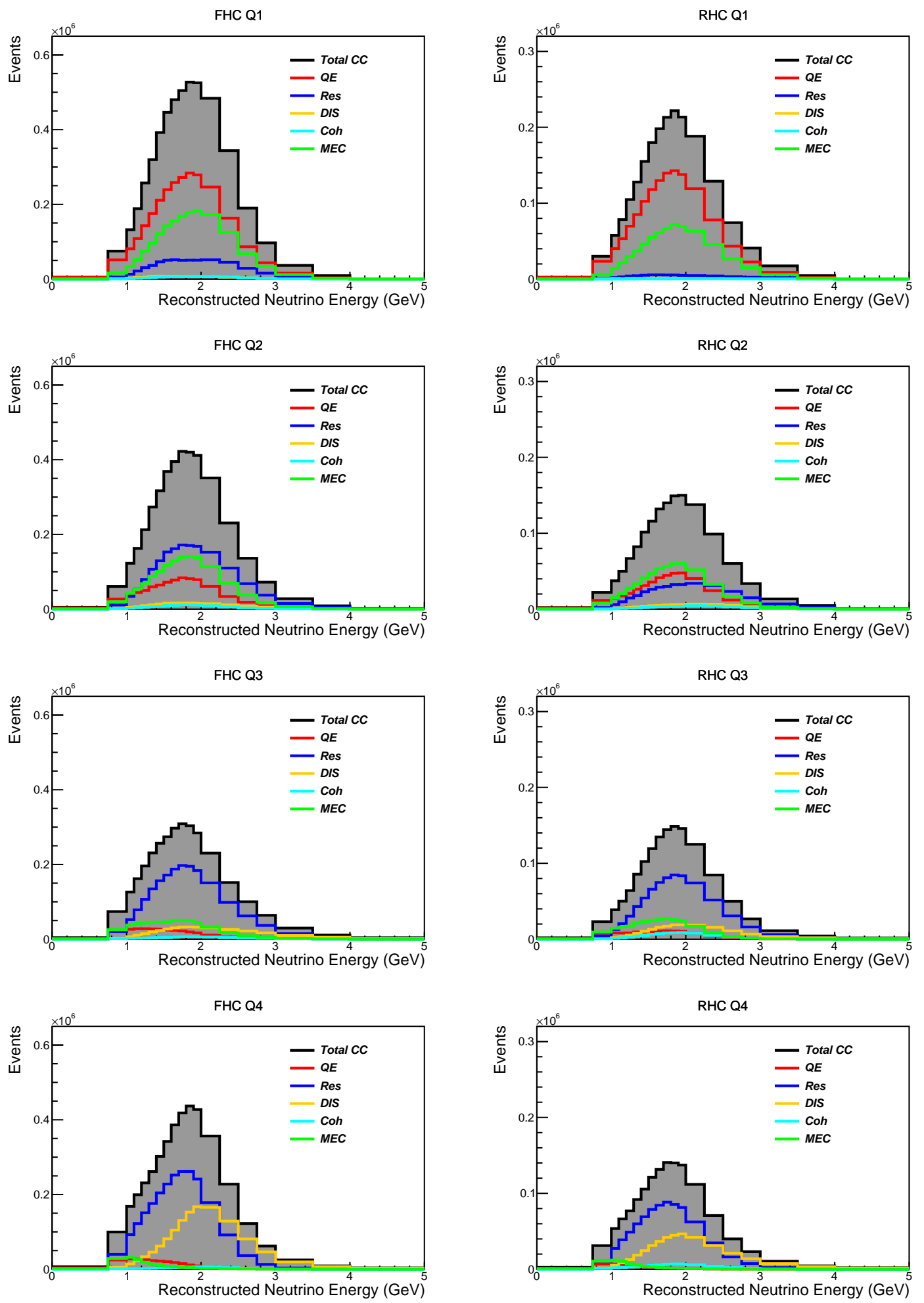

Figure 7.2: FHC (left) and RHC (right) interaction type distributions of selected $\nu_{\mu}+\bar{\nu}_{\mu}$ $\mathrm{CC}$ candidate events in the Near Detector. Plots are shown for each $E_{\text {had }} / E_{\nu}$ quantile, running from top to bottom as lowest to highest fractional hadronic energy, labelled Q1 through Q4. 


\begin{tabular}{|ccccccc|}
\hline \hline Quantile & $\nu_{\mu}$-CC & QE & Res & DIS & Coh & MEC \\
\hline Q1 & $7.02 \times 10^{05}$ & $3.66 \times 10^{05}$ & $8.21 \times 10^{04}$ & $1.18 \times 10^{04}$ & $9.95 \times 10^{03}$ & $2.32 \times 10^{05}$ \\
Q2 & $5.48 \times 10^{05}$ & $1.07 \times 10^{05}$ & $2.29 \times 10^{05}$ & $2.6 \times 10^{04}$ & $1.39 \times 10^{04}$ & $1.72 \times 10^{05}$ \\
Q3 & $4.27 \times 10^{05}$ & $3.94 \times 10^{04}$ & $2.49 \times 10^{05}$ & $5.77 \times 10^{04}$ & $9.9 \times 10^{03}$ & $7.05 \times 10^{04}$ \\
Q4 & $5.6 \times 10^{05}$ & $3.16 \times 10^{04}$ & $2.92 \times 10^{05}$ & $2.05 \times 10^{05}$ & $7.89 \times 10^{03}$ & $2.32 \times 10^{04}$ \\
Total & $2.24 \times 10^{06}$ & $5.44 \times 10^{05}$ & $8.52 \times 10^{05}$ & $3.01 \times 10^{05}$ & $4.17 \times 10^{04}$ & $4.97 \times 10^{05}$ \\
\hline \hline & Percentages relative to each quantiles $\nu_{\mu}$ CC candidate events & \\
Quantile & $\nu_{\mu}$-CC & QE & Res & DIS & Coh & MEC \\
\hline Q1 & 100 & 52.1 & 11.7 & 1.68 & 1.42 & 33.1 \\
Q2 & 100 & 19.6 & 41.8 & 4.75 & 2.54 & 31.3 \\
Q3 & 100 & 9.24 & 58.4 & 13.5 & 2.32 & 16.5 \\
Q4 & 100 & 5.64 & 52.2 & 36.6 & 1.41 & 4.14 \\
Total & 100 & 24.3 & 38.1 & 13.4 & 1.86 & 22.2 \\
\hline \hline Q4 & 25 & 5.81 & 34.3 & 68.2 & 18.9 & 4.66 \\
\hline Quantile & $\nu_{\mu}$-CC & QE & Res & DIS & Coh & MEC \\
\hline Q1 & 31.4 & 16.4 & 3.67 & 0.528 & 0.445 & 10.4 \\
Q2 & 24.5 & 4.79 & 10.2 & 1.16 & 0.623 & 7.67 \\
Q3 & 19.1 & 1.76 & 11.1 & 2.58 & 0.443 & 3.15 \\
Q1 & 25 & 1.41 & 13.1 & 9.18 & 0.353 & 1.04 \\
\hline \hline
\end{tabular}

Table 7.1: NOvA ND FHC simulated $\nu_{\mu}+\bar{\nu}_{\mu}$ CC candidate event interaction types. Numbers scaled to data POT. 


\begin{tabular}{|c|c|c|c|c|c|c|}
\hline \multicolumn{7}{|c|}{ Absolute numbers } \\
\hline Quantile & $\bar{\nu}_{\mu}-\mathrm{CC}$ & $\mathrm{QE}$ & Res & DIS & Coh & $\mathrm{MEC}$ \\
\hline Q1 & $2.84 \times 10^{05}$ & $1.81 \times 10^{05}$ & $1 \times 10^{04}$ & $2.28 \times 10^{03}$ & 786 & $9.01 \times 10^{04}$ \\
\hline Q2 & $2.02 \times 10^{05}$ & $5.84 \times 10^{04}$ & $5.38 \times 10^{04}$ & $1.09 \times 10^{04}$ & $6.24 \times 10^{03}$ & $7.28 \times 10^{04}$ \\
\hline Q3 & $1.92 \times 10^{05}$ & $1.67 \times 10^{04}$ & $1.04 \times 10^{05}$ & $2.82 \times 10^{04}$ & $1.04 \times 10^{04}$ & $3.26 \times 10^{04}$ \\
\hline Q4 & $1.87 \times 10^{05}$ & $7.04 \times 10^{03}$ & $1.01 \times 10^{05}$ & $6.19 \times 10^{04}$ & $8.61 \times 10^{03}$ & $8.89 \times 10^{03}$ \\
\hline Total & $8.65 \times 10^{05}$ & $2.63 \times 10^{05}$ & $2.68 \times 10^{05}$ & $1.03 \times 10^{05}$ & $2.6 \times 10^{04}$ & $2.04 \times 10^{05}$ \\
\hline \multicolumn{7}{|c|}{ Percentages relative to each quantiles $\bar{\nu}_{\mu} \mathrm{CC}$ candidate events } \\
\hline Quantile & $\bar{\nu}_{\mu}-\mathrm{CC}$ & $\mathrm{QE}$ & Res & DIS & Coh & MEC \\
\hline Q1 & 100 & 63.6 & 3.52 & 0.802 & 0.277 & 31.8 \\
\hline Q2 & 100 & 28.9 & 26.6 & 5.41 & 3.09 & 36 \\
\hline Q3 & 100 & 8.7 & 54.2 & 14.7 & 5.4 & 17 \\
\hline Q4 & 100 & 3.76 & 53.8 & 33.1 & 4.6 & 4.75 \\
\hline Total & 100 & 30.4 & 31 & 11.9 & 3.01 & 23.6 \\
\hline \multicolumn{7}{|c|}{ Percentages relative to total $\bar{\nu}_{\mu} \mathrm{CC}$ candidate events } \\
\hline Quantile & $\bar{\nu}_{\mu}-\mathrm{CC}$ & $\mathrm{QE}$ & Res & DIS & Coh & MEC \\
\hline Q1 & 32.8 & 20.9 & 1.16 & 0.263 & 0.0909 & 10.4 \\
\hline Q2 & 23.4 & 6.75 & 6.22 & 1.26 & 0.722 & 8.42 \\
\hline Q3 & 22.2 & 1.93 & 12 & 3.26 & 1.2 & 3.76 \\
\hline Q4 & 21.6 & 0.814 & 11.6 & 7.15 & 0.996 & 1.03 \\
\hline \multicolumn{7}{|c|}{ Relative to interaction type total } \\
\hline Quantile & $\bar{\nu}_{\mu}-\mathrm{CC}$ & $\mathrm{QE}$ & Res & DIS & Coh & MEC \\
\hline Q1 & 32.8 & 68.8 & 3.73 & 2.2 & 3.02 & 44.1 \\
\hline Q2 & 23.4 & 22.2 & 20.1 & 10.6 & 24 & 35.6 \\
\hline Q3 & 22.2 & 6.34 & 38.7 & 27.3 & 39.8 & 15.9 \\
\hline Q4 & 21.6 & 2.68 & 37.5 & 59.9 & 33.1 & 4.35 \\
\hline
\end{tabular}

Table 7.2: NOvA ND RHC simulated $\nu_{\mu}+\bar{\nu}_{\mu}$ CC candidate event interaction types. Numbers scaled to data POT. 
The number of $\nu_{\mu}+\bar{\nu}_{\mu} \mathrm{CC}$ candidate events in both data and MC is shown for FHC and RHC in Table 7.3. Naively, each quantile is expected to contain approximately $25 \%$ of the total events in both data and MC. This assumption proves false as the $E_{h a d} / E_{\nu}$ quantile boundaries are determined from FD, not ND, simulation. The overall MC-data agreement is good and any remaining data-MC disparity is largely addressed by the extrapolation procedure. In FHC the lowest and second highest $E_{h a d} / E_{\nu}$ quantiles contain $31.9 \%$ and $18.6 \%$ of the events respectively. In RHC the lowest and highest $E_{h a d} / E_{\nu}$ quantiles contain $35.6 \%$ and $19.3 \%$ respectively. FHC consists of approximately $1.9 \times 10^{6}$ events in data and MC whereas RHC consists of approximately $0.4 \times 10^{6}$ events. The FHC and RHC event rates correspond to approximately $0.25 \times 10^{6}$ and $0.13 \times 10^{6}$ events for every $1 \times 10^{20}$ POT exposure from the NuMI beam respectively.

\begin{tabular}{|c|cc|cc|}
\hline FHC & \multicolumn{2}{|c|}{$\nu_{\mu}+\bar{\nu}_{\mu}$ CC events } & \multicolumn{2}{c|}{$\frac{\nu_{\mu}+\bar{\nu}_{\mu} \text { CC events }}{\text { total events }}$} \\
\hline$E_{\text {had }} / E_{\nu}$ quantile & Data & $\mathrm{MC}$ & Data & $\mathrm{MC}$ \\
\hline Lowest & $6.10 \times 10^{05}$ & $5.95 \times 10^{05}$ & 0.319 & 0.314 \\
Second lowest & $4.59 \times 10^{05}$ & $4.64 \times 10^{05}$ & 0.240 & 0.245 \\
Second highest & $3.56 \times 10^{05}$ & $3.62 \times 10^{05}$ & 0.186 & 0.191 \\
Highest & $4.85 \times 10^{05}$ & $4.76 \times 10^{05}$ & 0.254 & 0.251 \\
\hline Total & $1.911 \times 10^{06}$ & $1.896 \times 10^{06}$ & 1 & 1 \\
\hline
\end{tabular}

\begin{tabular}{|c|cc|cc|}
\hline $\mathbf{R H C}$ & \multicolumn{2}{|c|}{$\nu_{\mu}+\bar{\nu}_{\mu}$ CC events } & \multicolumn{2}{|c|}{$\frac{\nu_{\mu}+\bar{\nu}_{\mu} \mathrm{CC} \text { events }}{\text { total events }}$} \\
\hline$E_{\text {had }} / E_{\nu}$ quantile & Data & $\mathrm{MC}$ & Data & $\mathrm{MC}$ \\
\hline Lowest & $1.37 \times 10^{05}$ & $1.27 \times 10^{05}$ & 0.356 & 0.328 \\
Second lowest & $9.31 \times 10^{04}$ & $9.07 \times 10^{04}$ & 0.241 & 0.233 \\
Second highest & $8.10 \times 10^{04}$ & $8.56 \times 10^{04}$ & 0.210 & 0.220 \\
Highest & $7.46 \times 10^{04}$ & $8.46 \times 10^{04}$ & 0.193 & 0.218 \\
\hline Total & $3.86 \times 10^{05}$ & $3.88 \times 10^{05}$ & 1 & 1 \\
\hline
\end{tabular}

Table 7.3: ND $\nu_{\mu}+\bar{\nu}_{\mu}$ CC candidate events and share of total events within each quantile in data and MC. FHC is shown on the top, and RHC on the bottom.

The ND data-MC comparison plots shown in this section have a standardized format and legend. Data is shown in black, with associated statistical errors. Simulation is shown in red and the associated systematic uncertainty is represented by the red shaded region. The simulated background that passes selection is represented by the blue histogram. The per quantile breakdown plots also share a common quantile arrangement. The top- 
left plot is always the lowest $E_{h a d} / E_{\nu}$ quantile, the top-right plot is the second lowest $E_{h a d} / E_{\nu}$ quantile, the bottom-left plot is the second highest $E_{h a d} / E_{\nu}$ quantile and the bottom-right the highest $E_{h a d} / E_{\nu}$ quantile.

Uncertainty is estimated by assessing the combination of $1 \sigma$ systematic shifts that results in the largest uncertainty band. The full systematic uncertainty is estimated by combination with the statistical uncertainty - achieved by addition in quadrature. High statistics MC means that the effect of the statistical uncertainty is relatively small compared to the systematic uncertainty in the ND.

Figures 7.3 and 7.4 show the POT-normalized $\nu_{\mu}$ energy distributions, split by $E_{h a d} / E_{\nu}$ quantile, for FHC and RHC respectively. The POT-normalized plots demonstrate the overall normalization difference alongside the full uncertainty band for each $E_{h a d} / E_{\nu}$ quantile. The plots that follow the POT normalized plots are all area normalized - showing the shape-only uncertainties on each distribution.

Area normalized ND distributions, using the entire dataset, are presented in figures that contain four different variables. These are given for FHC data first, with the equivalent RHC figure immediately after. On the following pages the individual quantile plots for each variable and for each horn current are then presented. In total 160 ND distribution plots are shown - 80 per horn current (16 total distributions and 64 quantile distributions). The plots shown are grouped into broad categories of energy, track and particle identification variables and are presented in this order.

Figure 7.5 and Figure 7.6 show the distributions of $\nu_{\mu}$-energy, muonic-energy, muon energy per hit and Kalman track length for FHC and RHC respectively. A discussion of each variable follows.

Reconstructed Neutrino Energy The FHC reconstructed $\nu_{\mu}$-energy distribution (top left of Figure 7.5) exhibits good data-MC agreement. The means of the distributions are $1.906 \mathrm{GeV}$ in data and $1.904 \mathrm{GeV}$ in simulation, less than $0.2 \%$ apart. All data points sit inside the $1 \sigma$ tolerance of their respective bin. The RHC reconstructed $\nu_{\mu}$-energy distribution (top left of Figure 7.6) also shows good data-MC agreement with all data points inside the $1 \sigma$ tolerance of their respective bin. The RHC distribution means are $1.942 \mathrm{GeV}$ in data and $1.938 \mathrm{GeV}$ in simulation - sitting slightly higher than observed in FHC.

The per quantile FHC reconstructed $\nu_{\mu}$-energy distributions are shown in Figure 7.7. In all four quantiles data-MC agreement is good - all data points sit inside the simulated $1 \sigma$ systematic error. Again, the strength of the agreement visibly declines in the two highest 
$E_{h a d} / E_{\nu}$ quantiles, where simulation under-predicts below $2 \mathrm{GeV}$ and over-predicts above. This discrepancy stays inside the uncertainty bound. The lower three quantiles exhibit less than $0.2 \%$ discrepancy between data and MC means, and the highest less than $0.5 \%$. The per RHC quantile reconstructed $\nu_{\mu}$-energy distributions are shown in Figure 7.8. Like FHC, all four quantiles show good data-MC agreement - all data points, bar one, sit inside the simulated $1 \sigma$ systematic error and all means agree within $1 \%$. The strength of the agreement visibly declines as the $E_{\text {had }} / E_{\nu}$ quantile increases. As with FHC, simulation under-predicts energy for events below $2 \mathrm{GeV}$ and over-predicts above. This offset is visible from the second lowest $E_{\text {had }} / E_{\nu}$ quantile. This discrepancy stays inside the uncertainty bound across all quantiles, except for an isolated bin at approximately $2 \mathrm{GeV}$ in the highest $E_{\text {had }} / E_{\nu}$ quantile.

Overall NOvA's ability to reconstruct neutrino energy has improved with respect to the previous analysis [3]. The agreement in both RHC and FHC suggests that our reconstruction methodology, as well as improvements made to the modelling of hadronic and muonic energy deposits, has produced a more accurate determination of energy. Improvements made to selection criteria have also substantially reduced the background and the impact of misidentified neutrino events has been lessened.

Reconstructed Muon Energy The FHC and RHC reconstructed $\mu$-energy distributions also show good data-MC agreement. The FHC and RHC distributions are shown summed across all $E_{\text {had }} / E_{\nu}$ quantiles in the top right of Figure 7.5 and Figure 7.6 respectively. The FHC agreement is noticeably better in the sub $1.5 \mathrm{GeV}$ region but almost all RHC data points fall within the $1 \sigma$ systematic uncertainty. The means agree to about $0.3 \%$ and $1.9 \%$ for the FHC and RHC beam configurations respectively.

Figure 7.9 and Figure 7.10 show the respective $E_{\text {had }} / E_{\nu}$ breakdowns of the muon energy distributions for FHC and RHC. Across all four FHC quantiles the means agree to less than $0.7 \%$ with the equivalent RHC quantiles agreeing within $1.2 \%$. The vast majority of data is within the $1 \sigma$ systematic uncertainty across the energy regime.

Overall NOvA's ability to reconstruct muonic energy has improved with respect to the previous analysis [3]. Whereas the previous analysis exhibited some disagreement in the highest $E_{h a d} / E_{\nu}$ quantiles, this disagreement is no longer present in the current analysis. This is likely due to improvements in event characterisation and muonic energy reconstruction. The simulated detector geometry has also been updated and is more accurate with respect to the analysis published in 2016 [49]. PDG values used to estimate associated uncertainties have also been carefully reviewed and updated where appropriate. This has 
aided in reducing uncertainties arising from mass accounting, errors arising from elemental composition $\frac{d E}{d x}$ modelling, errors caused by density effects and errors in application of the Bethe equation. The reconstruction of muonic energy has also been aided by a software patch that NOvA collaborator Matthew Strait submitted to GEANT4. This update improved the accuracy of GEANT4's simulation of energy loss for all particles [75]. All of the above factors are likely contributors to NOvA's improved muon energy reconstruction.

Reconstructed Hadronic Energy Similar to the muonic and neutrino energy spectra, the hadronic energy distributions exhibit good data-MC agreement. The FHC and RHC distributions are shown summed across all $E_{h a d} / E_{\nu}$ quantiles in the top-left of Figure 7.15 and Figure 7.16 respectively. All FHC data points fall within the $1 \sigma$ systematic uncertainty. All RHC data points, bar one in the low energy regime (sub $0.1 \mathrm{GeV}$ ), also fall within the $1 \sigma$ systematic uncertainty. The means agree to about $0.4 \%$ and $5.8 \%$ for the FHC and RHC beam configurations respectively.

Figure 7.17 and Figure 7.18 show the respective $E_{\text {had }} / E_{\nu}$ breakdowns of the hadronic energy distributions for FHC and RHC. Across all four FHC quantiles the means agree to less than $0.4 \%$ with the equivalent RHC quantiles agreeing within $3.6 \%$ meaning that the difference in the overall distribution arises due to the $10 \%$ under and over estimation of $\mathrm{MC}$ events in the lowest and highest $E_{h a d} / E_{\nu}$ quantile respectively. The vast majority of data is within the $1 \sigma$ systematic uncertainty across the energy regime. FHC exhibits slightly tighter agreement.

NOvA's ability to reconstruct hadronic energy has improved with respect to the previous analysis [3]. Figure 7.17 and Figure 7.18 both exhibit great agreement. This improvement likely happened as a result of improvements to hadronic shower modelling. These improvements have principally affected estimations of the energy carried by neutral particles, invisible to the NOvA detectors, such as neutrons. Hadronic energy estimation is still one of the largest sources of error in total energy estimation in NOvA and updates to modelling complicated hadronic showers are continuously in progress.

Energy Per Hit and Hadronic Energy Fraction As well as the muonic, hadronic and neutrino energies, the muonic energy per hit, hadronic energy per hit and hadronic energy fraction (used to determine $E_{h a d} / E_{\nu}$ quantile boundaries) are assessed.

The overall muonic energy per hit is shown in Figure 7.5 and Figure 7.6, with the per $E_{\text {had }} / E_{\nu}$ breakdowns shown in Figure 7.11 and Figure 7.12 for FHC and RHC respectively. The overall hadronic energy per hit is shown in Figure 7.15 and Figure 7.16, with the per 
$E_{h a d} / E_{\nu}$ breakdowns shown in Figure 7.21 and Figure 7.22 for FHC and RHC respectively. The overall hadronic energy fraction is shown in Figure 7.15 and Figure 7.16, with the per $E_{h a d} / E_{\nu}$ breakdowns shown in Figure 7.19 and Figure 7.20 for FHC and RHC respectively.

The total and per-quantile muonic and hadronic energy per hit distributions generally show some level of MC-data agreement but there are inconsistencies. Most data falls within the systematic uncertainty bound. The data generally seems to have a lower reconstructed muonic energy per hit than expectation and higher reconstructed hadronic energy per hit. For both the muonic and hadronic energy per hit, the second lowest hadronic energy quantile exhibits the worst agreement. Agreement in the the hadronic energy per hit distributions (shown for FHC and RHC in Figure 7.11 and Figure 7.12) is bad but agreement in the hadronic energy distributions (shown for FHC and RHC in Figure 7.17 and Figure 7.18) is good. The mis-modelling of neutrons in simulation may cause this discrepancy and would not show up in the uncertainty band. It may also be due to the differing interaction types that occupy each quantile for FHC and RHC, as shown in Figure 7.2. The muonic energy per hit shows worse agreement than the hadronic, with few data points sitting inside the $1 \sigma$ systematic range. The shape of the muonic energy per hit distribution closely matches that of simulation but it is centred slightly lower in energy per hit. The muonic energy scale systematic is pinned using track length alone and does not incorporate the $5 \%$ calibration uncertainty used for the hadronic component, hence the error bands shown in the muonic energy per hit distributions are not inclusive of the calibration uncertainty. The hadronic energy per hit shows good data-MC agreement with most data points (outside of the second lowest $E_{\text {had }} / E_{\nu}$ ) sitting inside the $1 \sigma$ uncertainty range. The FHC data generally shows better agreement than the RHC.

NOvA's ability to reconstruct hadronic and muonic energy per hit has improved with respect to the previous analysis [3]. However, there is still some disagreement. The overall energy estimations for neutrino, hadronic \& muonic components are good. However, the per hit disagreement suggests that some hits may be mis-associated as muonic or hadronic, likely near the interaction vertex. This is supported by the fact that prediction tends to overestimate the muonic energy per hit and to underestimate the hadronic energy per slice. The disagreement in muonic energy per hit is consistently caused by prediction overestimation across all quantiles. However, the hadronic energy per slice only exhibits noteworthy disagreement in the second-lowest $E_{\text {had }} / E_{\nu}$ quantile, particularly for RHC. This suggests that the dominance of MEC events in this quantile, and the associated uncertainty in their reconstruction, may be causing the discrepancy. The disagreements 
shown in Figure 7.11, Figure 7.12, Figure 7.21 and Figure 7.22, whilst not covered by the shape-only uncertainty, are covered by the total uncertainty when POT-normalized ${ }^{2}$.

The hadronic energy fraction plots show very tight data-MC agreement for both FHC and RHC. The summed and per quantile distributions show universal agreement, with all data falling inside the $1 \sigma$ range for both beam types. The notable exception is in the lowest RHC $E_{h a d} / E_{\nu}$ quantile, which shows good agreement but with some data outside the respective uncertainty bound. This result follows from the tight agreement exhibited by the neutrino and hadronic energy distributions.

Track Variables Hits incident in time and space are organized into structures such as tracks and slices - the process for which is described in Chapter 4. Four such distributions are presented in this section - The number of hits in the slice, the number of hits on the primary track, the length of the primary track and the angle of the primary track with respect to the beam.

The primary track length distributions are shown in Figure 7.5, and Figure 7.6 with the per $E_{\text {had }} / E_{\nu}$ breakdowns shown in Figure 7.13 and Figure 7.14 for FHC and RHC respectively.

The distribution of the number of hits contained in a slice is shown in Figure 7.15 and Figure 7.16 with the per $E_{h a d} / E_{\nu}$ breakdowns shown in Figure 7.23 and Figure 7.24 for FHC and RHC respectively.

The distribution of the number of hits contained in a track is shown in Figure 7.25 and Figure 7.26 with the per $E_{\text {had }} / E_{\nu}$ breakdowns shown in Figure 7.27 and Figure 7.28 for FHC and RHC respectively.

The distribution of the primary track angle with respect to the beam is shown in Figure 7.25 and Figure 7.26 with the per $E_{h a d} / E_{\nu}$ breakdowns shown in Figure 7.29 and Figure 7.30 for FHC and RHC respectively.

The primary track length, hits per slice, hits per track and track angle distributions exhibit good MC-data agreement for both horn currents, across all quantiles. There are very minor disagreements in certain bins of the hits per slice plots which may cause the discrepancy in the muonic/hadronic energy per hit distributions, discussed above. The coordinates of the start and end points of tracks were shown to be well reconstructed in the previous analysis [87]. It follows that the track length, hits per slice, hits per track and track angle plots exhibit strong agreement in this analysis as hit thresholds and the method by which hits are associated to tracks have both remained unchanged.

\footnotetext{
${ }^{2}$ See Figures 7.3 and 7.4 to see the total uncertainty on the neutrino energy distribution.
} 
ReMId and CVN The particle identification variables CVN and ReMId are assessed. Both the 2017 and 2018 CVN variables are used in the analysis and so both distributions are shown. Also shown is the ReMId score and three of the variables that it is trained on $\frac{d E}{d x}$ likelihood, scattering angle likelihood and fraction of planes along the track consistent with having minimum ionizinglike $\frac{d E}{d x}$.

The total 2017 and 2018 CVN score distributions are shown in Figure 7.25 and Figure 7.26 for FHC and RHC respectively. The 2017 CVN score and 2018 CVN score are shown as a per $E_{h a d} / E_{\nu}$ quantile breakdown in Figure 7.31(Figure 7.32) and Figure 7.33(Figure 7.34), for $\mathrm{FHC}(\mathrm{RHC})$ respectively.

The simulation-data agreement is good for both CVN scores in RHC, FHC, across all quantiles and on a per quantile basis. All data falls inside the $1 \sigma$ range of the respective bin. Notably, the 2018 CVN score shows better agreement than the 2017 CVN score did when used in the previous analysis [87]. This suggests that the re-training of CVN for the present analysis produced a more reliable PID score. Note that the 2017 score shown in this analysis was trained on different data than the simulated data that was used in this analysis and the most fair comparison is of the score shown in Figure 7.33 to the one shown in the previous analysis $[3,87]$.

The total ReMId score and associated training variables are shown in Figure 7.35 and Figure 7.36 for FHC and RHC respectively. The ReMId score, ReMId fraction of planes along the track consistent with having minimum ionizinglike $\frac{d E}{d x}$, ReMId scattering angle likelihood and ReMId $\frac{d E}{d x}$ likelihood are shown per $E_{h a d} / E_{\nu}$ quantile in Figure 7.37(Figure 7.38), Figure 7.39(Figure 7.40), Figure 7.41(Figure 7.42), Figure 7.43(Figure 7.44), for $\mathrm{FHC}(\mathrm{RHC})$ respectively.

The agreement between simulation and data is good for the ReMId score. The overall score distributions show tight agreement in both horn current configurations and across all quantiles. The ReMId fraction of planes along the track consistent with having minimum ionizinglike $\frac{d E}{d x}$ shows similar agreement across all quantiles for both horn currents.

There is some disagreement between simulation and data for the ReMId $\frac{d E}{d x}$ likelihood and ReMId scattering likelihood. This follows from the observed disagreement in the muon energy per hit which shows a lower expectation than observed in data. A lower muonic energy per hit translates to lower track $\frac{d E}{d x}$ and a therefore more muonic like particle will be identified by ReMId. Both variables agree well when summed across all quantiles but the lower two $E_{\text {had }} / E_{\nu}$ quantiles exhibit slight disagreement. The disagreement is strongest for the $\frac{d E}{d x}$ variable and is consistent across horn currents - simulation under-estimates 
the number of events left of the peak, and over-estimates right of the peak. The overall disparity is nonetheless small. The scattering likelihood shows tight agreement in the upper two $E_{h a d} / E_{\nu}$ quantiles. Overall, the shapes of all ReMId distributions closely agree between simulation and data.

Summary In general the ND distributions show good data-MC agreement when summed across the $E_{h a d} / E_{\nu}$ quantiles. On a per quantile basis the vast majority of distributions exhibit good agreement but there are some disparities - notably for the muonic energy per hit, the hadronic energy per hit and the ReMId training variables. These inconsistencies are generally local to individual quantiles, with the total distributions showing better agreement. However, the muonic and hadronic energy per hit spectra are incorrectly centred, suggestive of a small modelling error that is covered by uncertainties. The overall similarity between data and simulation represents an improvement with respect to previous NOvA analyses [3, 49, 88, 89]. 


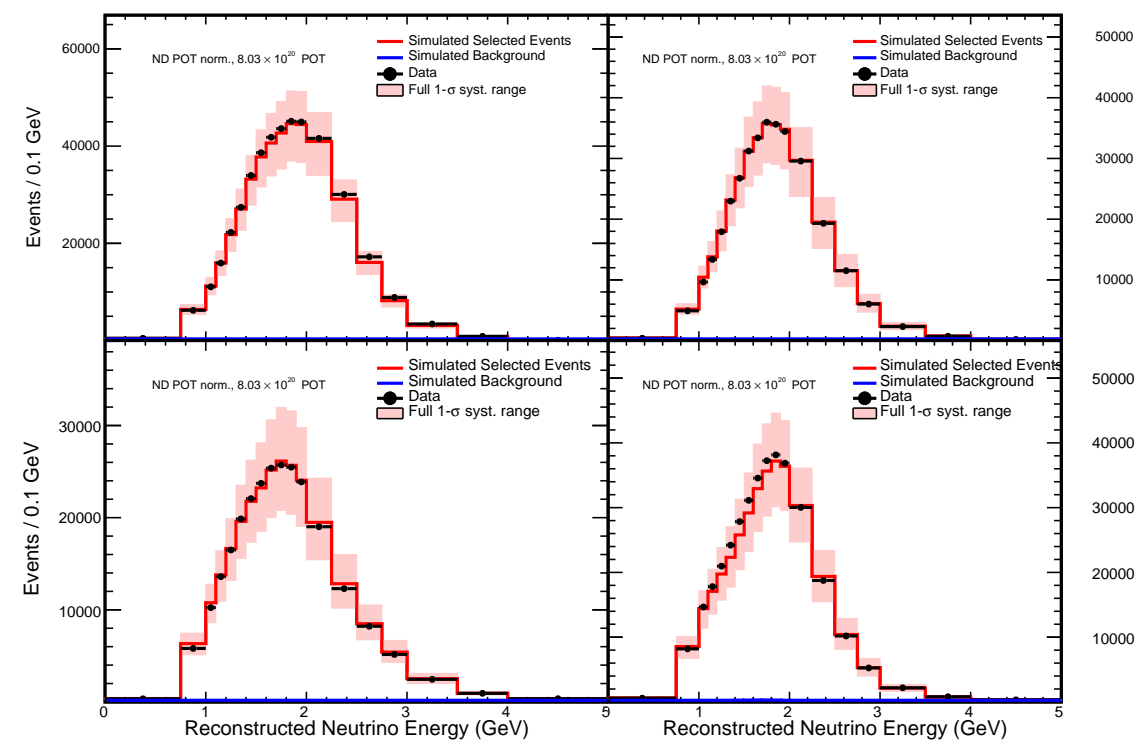

Figure 7.3: POT normalized FHC distributions of $\nu_{\mu}$ energy for each $E_{h a d} / E_{\nu}$ quantile. $\mathrm{MC}$ and data events passing selection are shown by the red histogram and black points respectively. The MC systematic uncertainty is shown by the shaded red region. The MC background after selection is shown by the blue histogram.

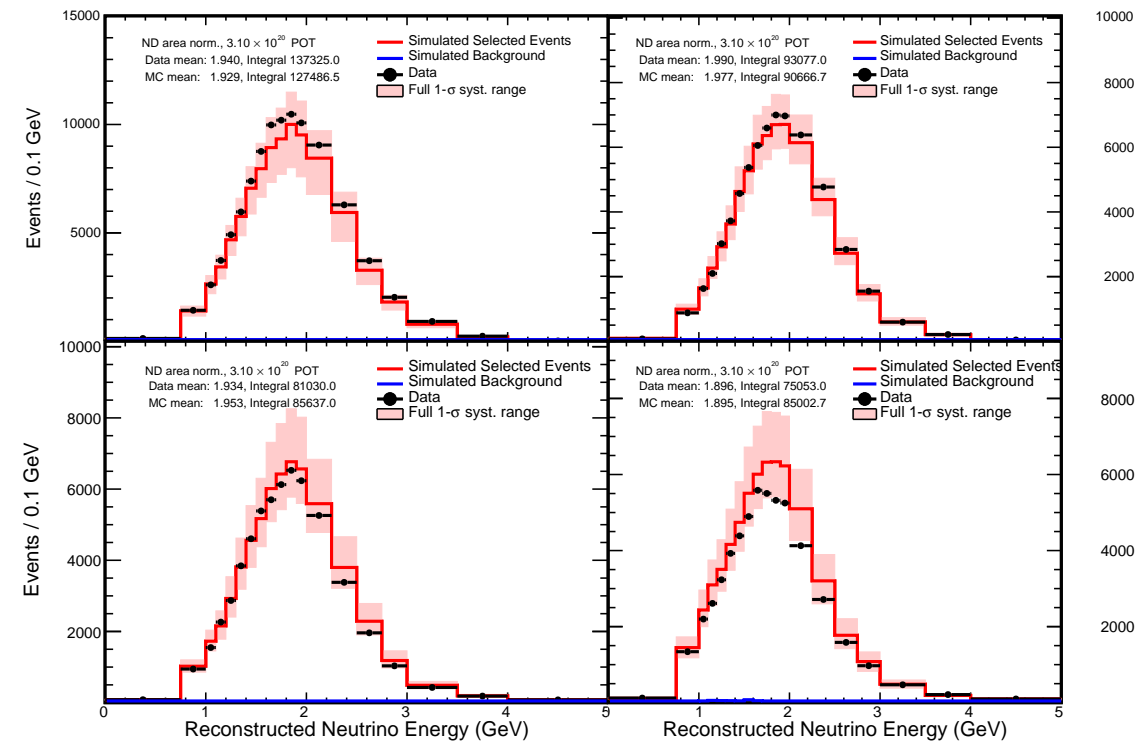

Figure 7.4: POT normalized RHC distributions of $\nu_{\mu}$ energy for each $E_{h a d} / E_{\nu}$ quantile. MC and data events passing selection are shown by the red histogram and black points respectively. The MC systematic uncertainty is shown by the shaded red region. The MC background after selection is shown by the blue histogram. 

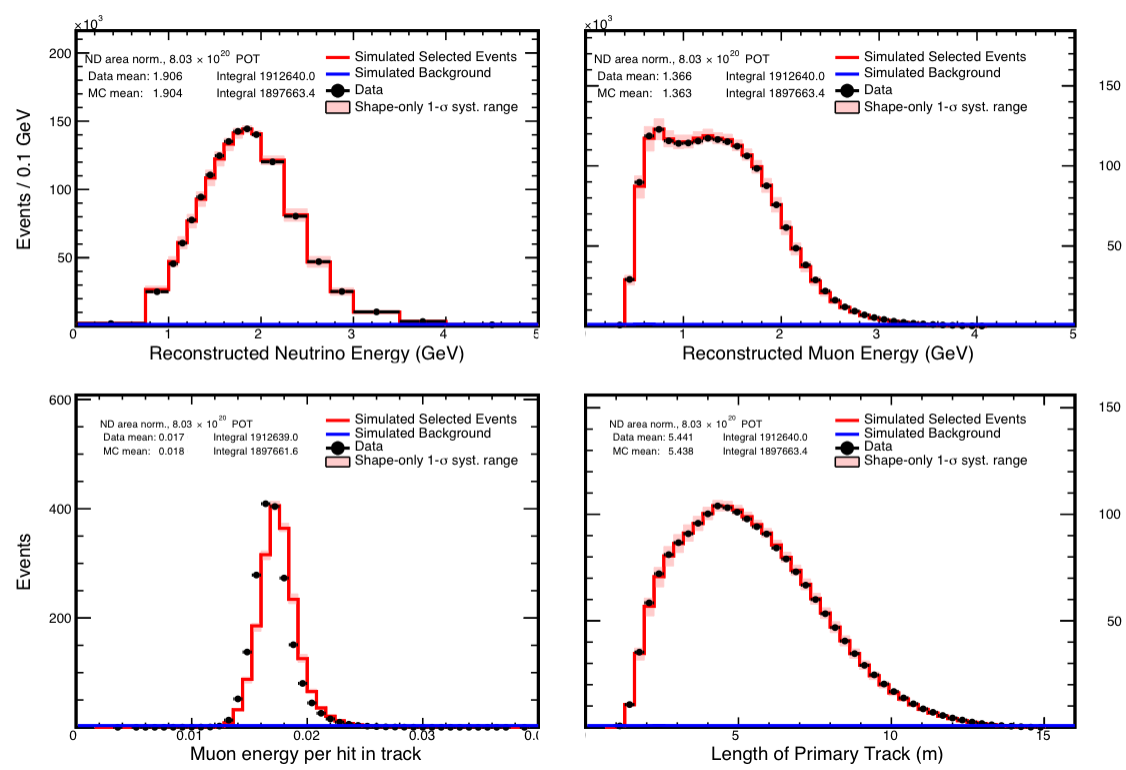

Figure 7.5: Area normalized FHC distributions of $\nu_{\mu}$ energy, muon energy, muon energy per hit and Kalman track length for the entire data set. MC and data events passing selection are shown by the red histogram and black points respectively. The systematic uncertainty in the distribution of the simulated events is shown by the shaded red region enclosing the red histogram. The simulated background events passing the selection are shown by the blue histogram.
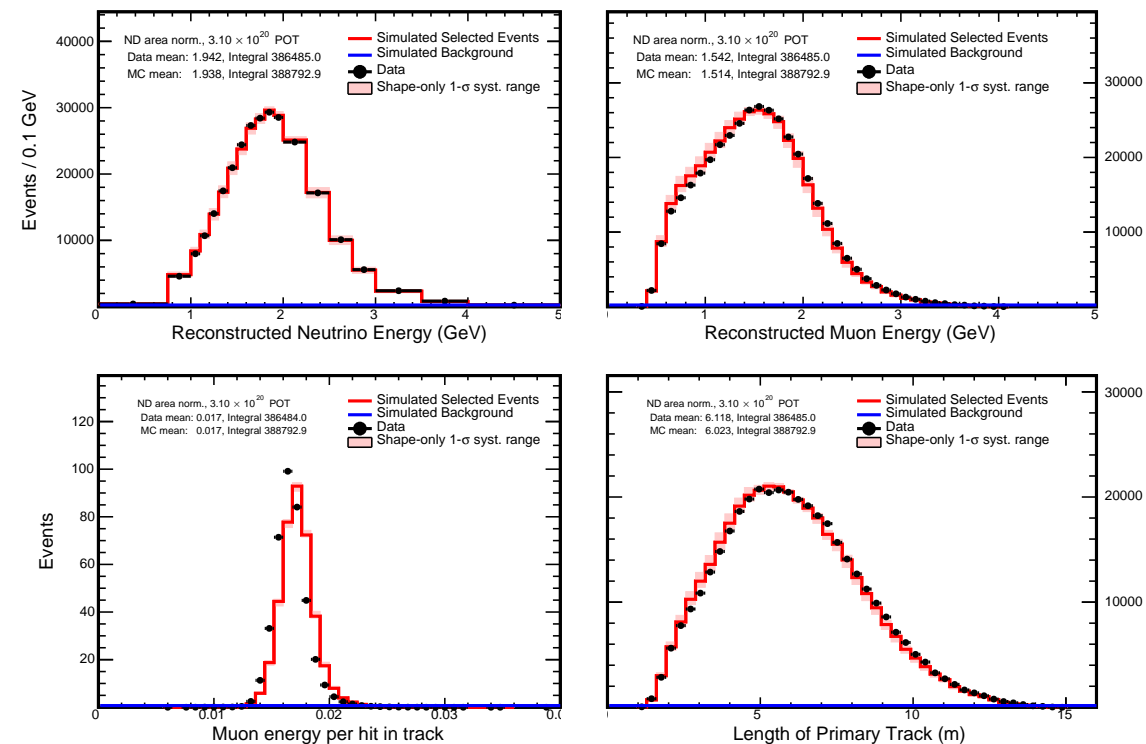

Figure 7.6: Area normalized RHC distributions of $\nu_{\mu}$ energy, muon energy, muon energy per hit and Kalman track length for the entire data set. MC and data events passing selection are shown by the red histogram and black points respectively. The systematic uncertainty in the distribution of the simulated events is shown by the shaded red region enclosing the red histogram. The simulated background events passing the selection are shown by the blue histogram. 


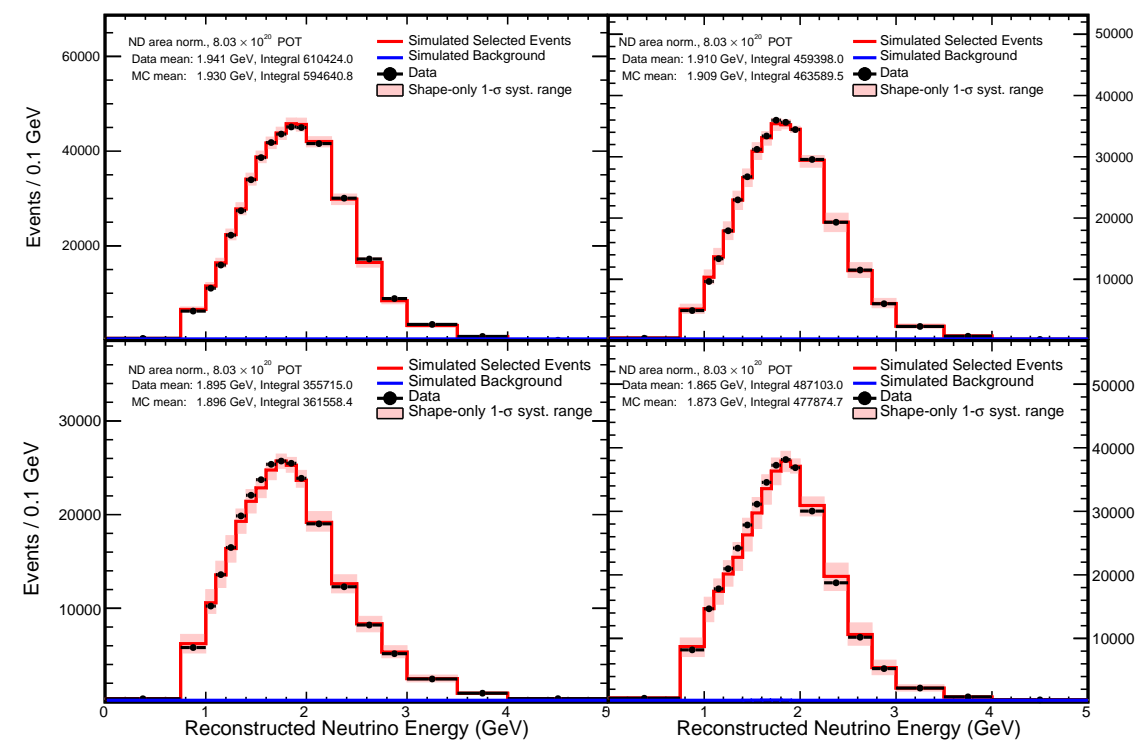

Figure 7.7: Area normalized FHC distributions of the $\nu_{\mu}$ energy for each $E_{h a d} / E_{\nu}$ quantile. $\mathrm{MC}$ and data events passing selection are shown by the red histogram and black points respectively. The MC systematic uncertainty is shown by the shaded red region. The MC background after selection is shown by the blue histogram.

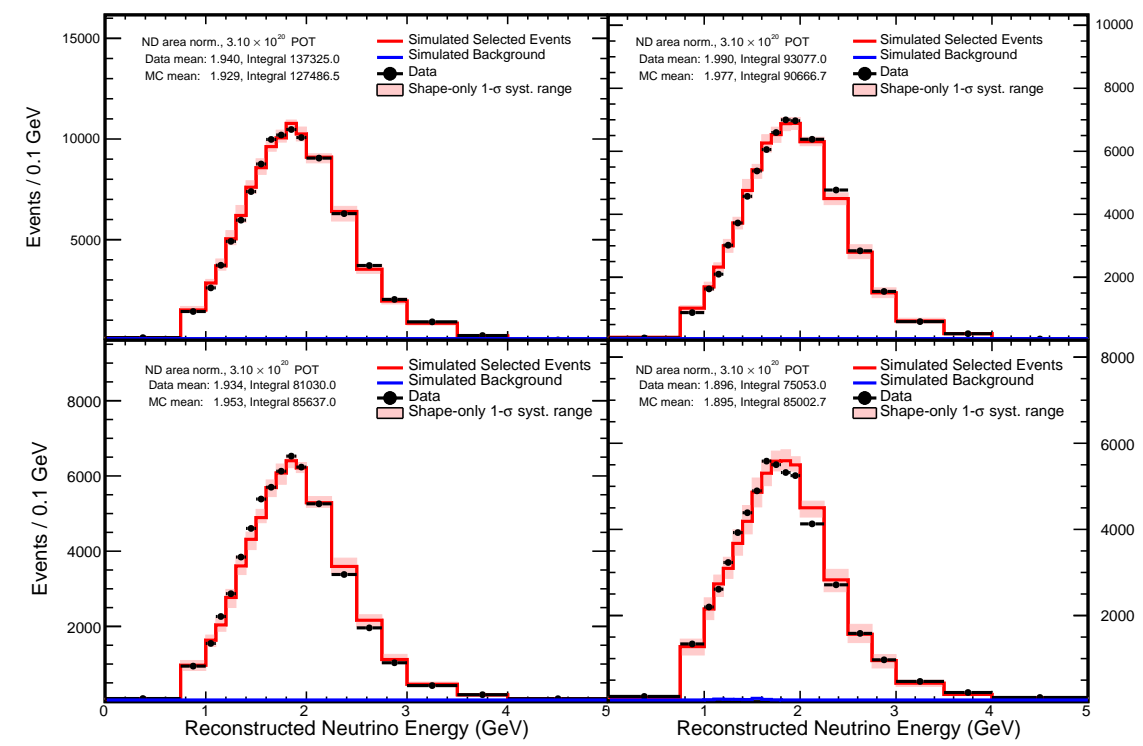

Figure 7.8: Area normalized RHC distributions of the $\nu_{\mu}$ energy for each $E_{h a d} / E_{\nu}$ quantile. $\mathrm{MC}$ and data events passing selection are shown by the red histogram and black points respectively. The MC systematic uncertainty is shown by the shaded red region. The MC background after selection is shown by the blue histogram. 


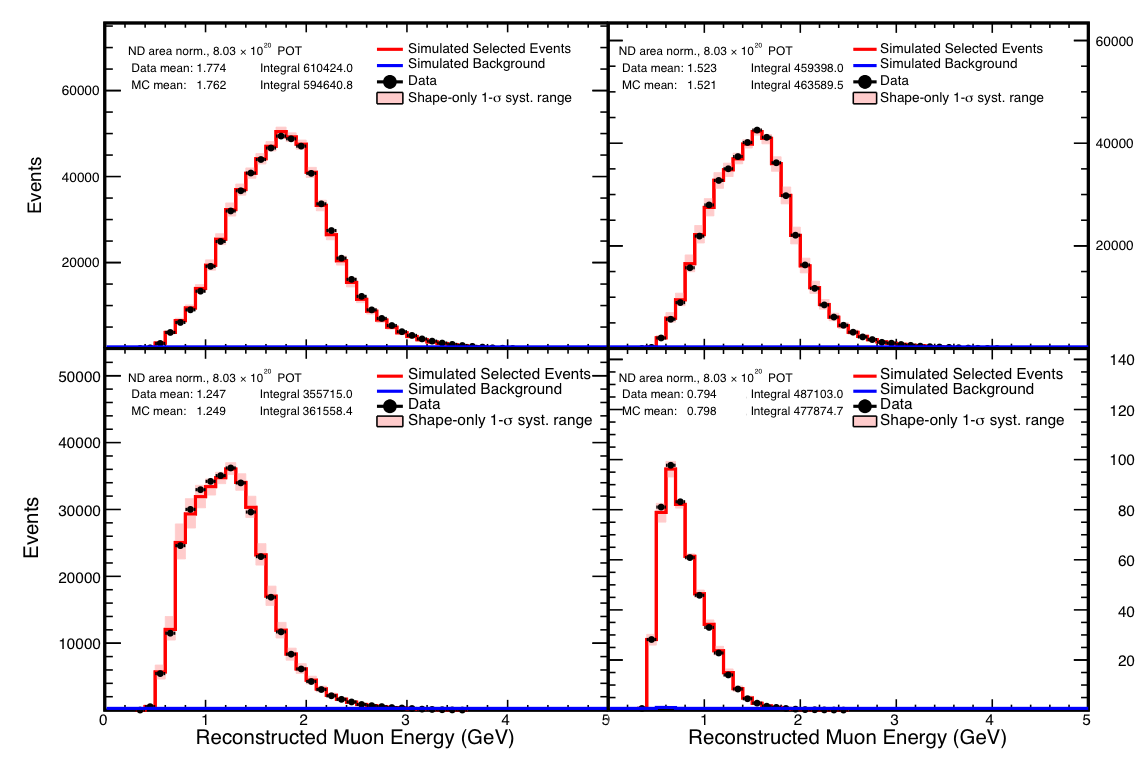

Figure 7.9: Area normalized FHC distributions of the muon energy for each $E_{h a d} / E_{\nu}$ quantile. $\mathrm{MC}$ and data events passing selection are shown by the red histogram and black points respectively. The MC systematic uncertainty is shown by the shaded red region. The MC background after selection is shown by the blue histogram.

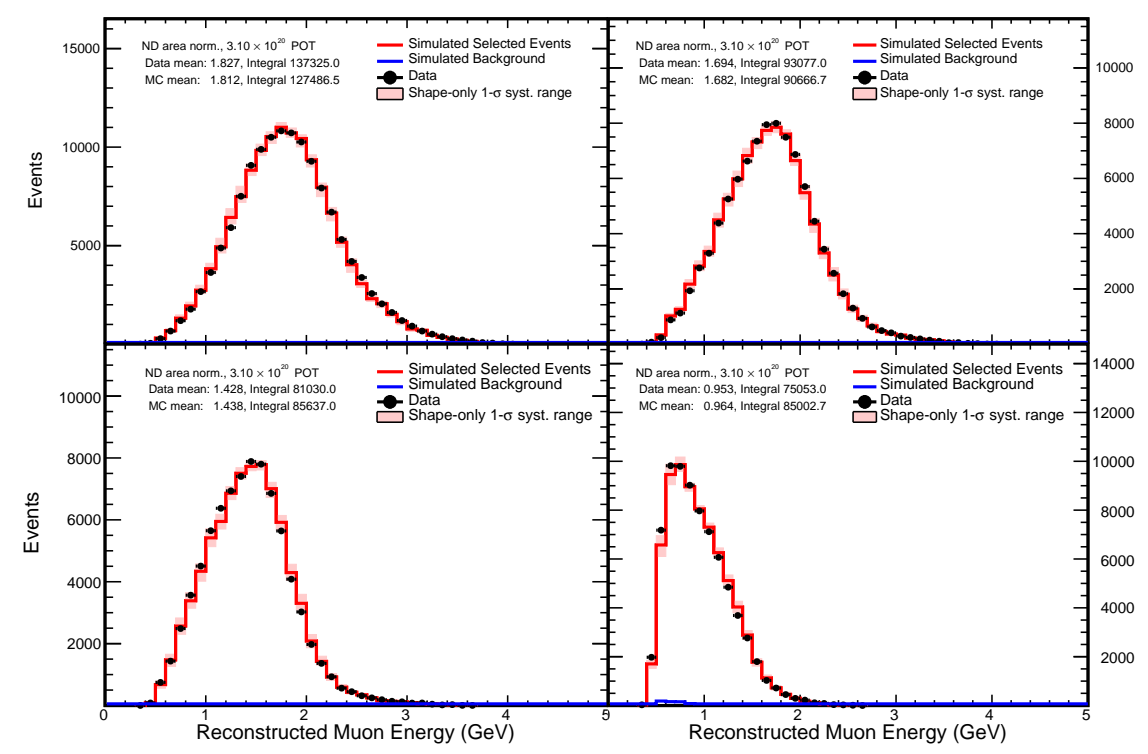

Figure 7.10: Area normalized RHC distributions of the muon energy for each $E_{h a d} / E_{\nu}$ quantile. MC and data events passing selection are shown by the red histogram and black points respectively. The MC systematic uncertainty is shown by the shaded red region. The MC background after selection is shown by the blue histogram. 


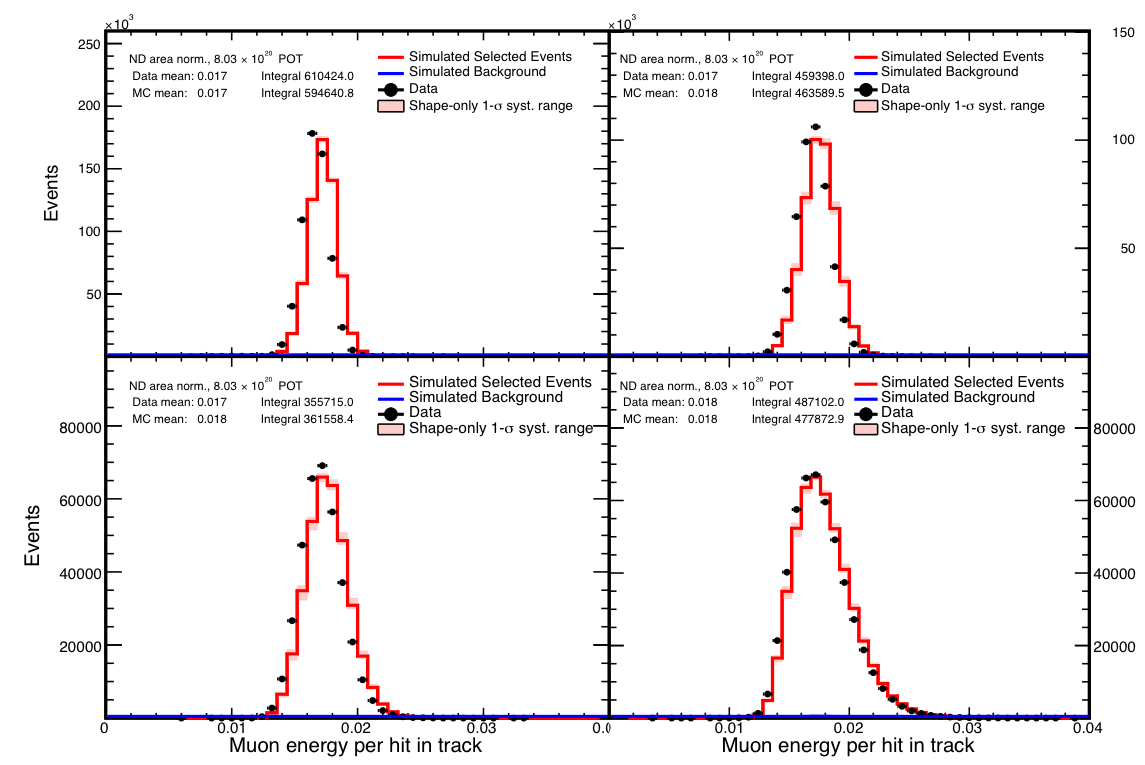

Figure 7.11: Area normalized FHC distributions of the muon energy per hit for each $E_{\text {had }} / E_{\nu}$ quantile. MC and data events passing selection are shown by the red histogram and black points respectively. The MC systematic uncertainty is shown by the shaded red region. The MC background after selection is shown by the blue histogram.

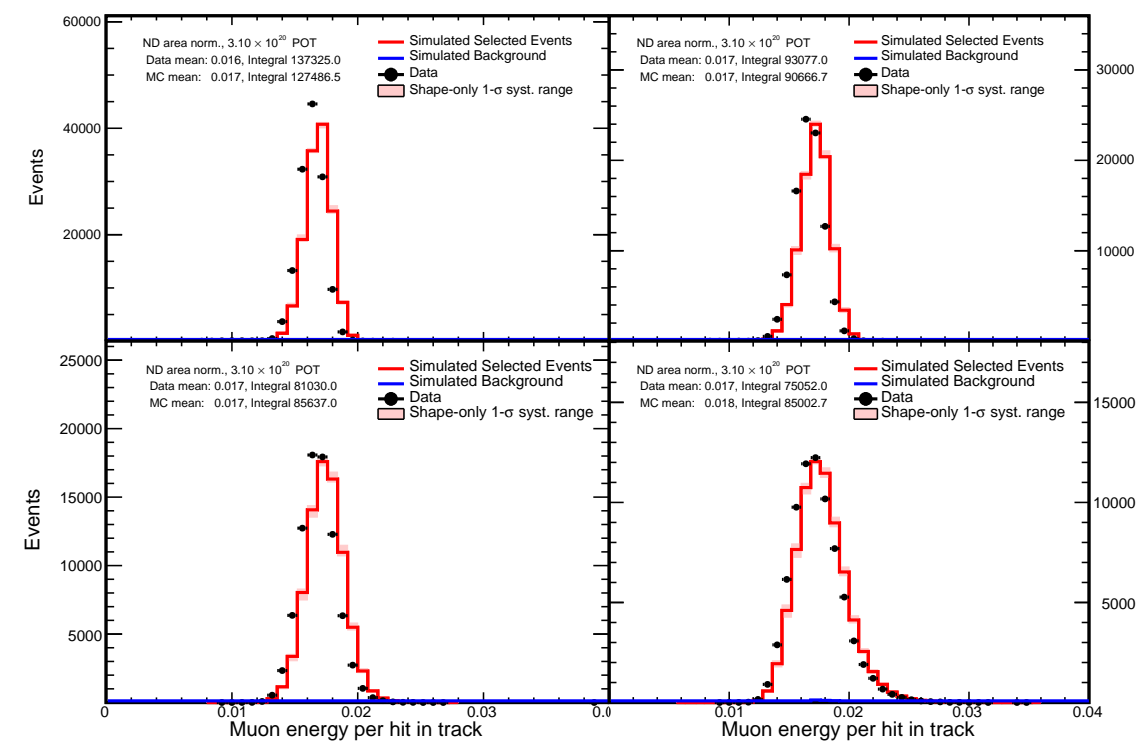

Figure 7.12: Area normalized RHC distributions of the muon energy per hit for each $E_{\text {had }} / E_{\nu}$ quantile. MC and data events passing selection are shown by the red histogram and black points respectively. The MC systematic uncertainty is shown by the shaded red region. The MC background after selection is shown by the blue histogram. 


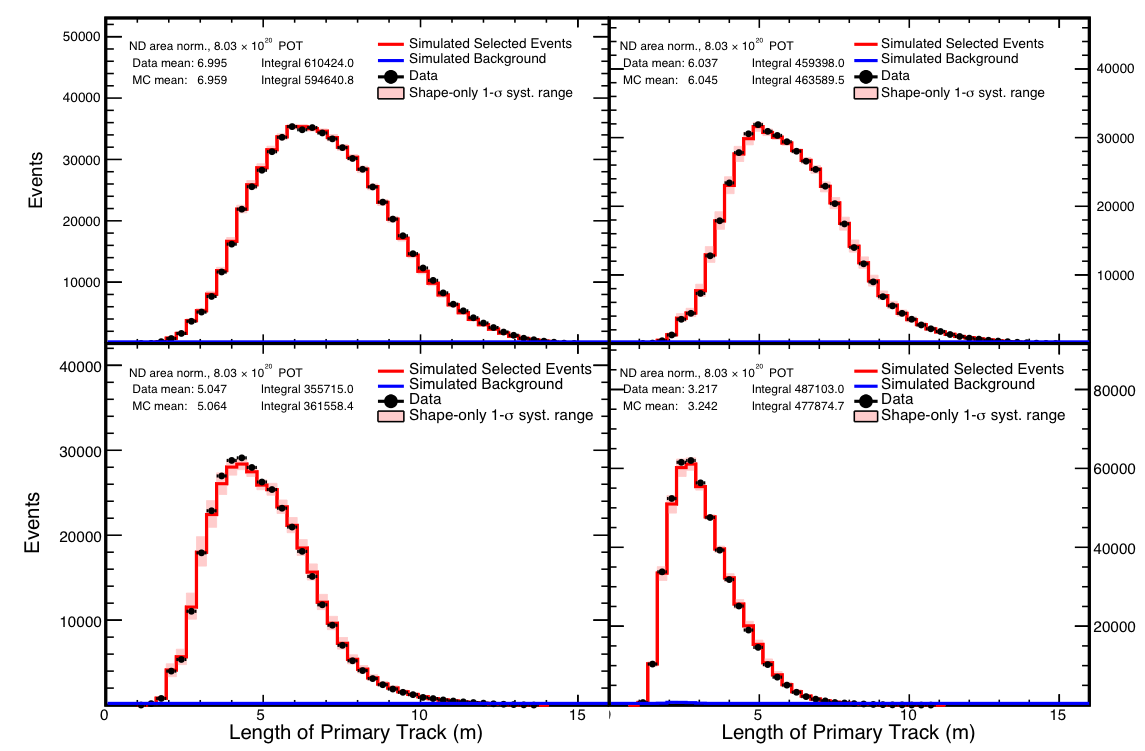

Figure 7.13: Area normalized FHC distributions of the Kalman track length for each $E_{\text {had }} / E_{\nu}$ quantile. MC and data events passing selection are shown by the red histogram and black points respectively. The MC systematic uncertainty is shown by the shaded red region. The MC background after selection is shown by the blue histogram.

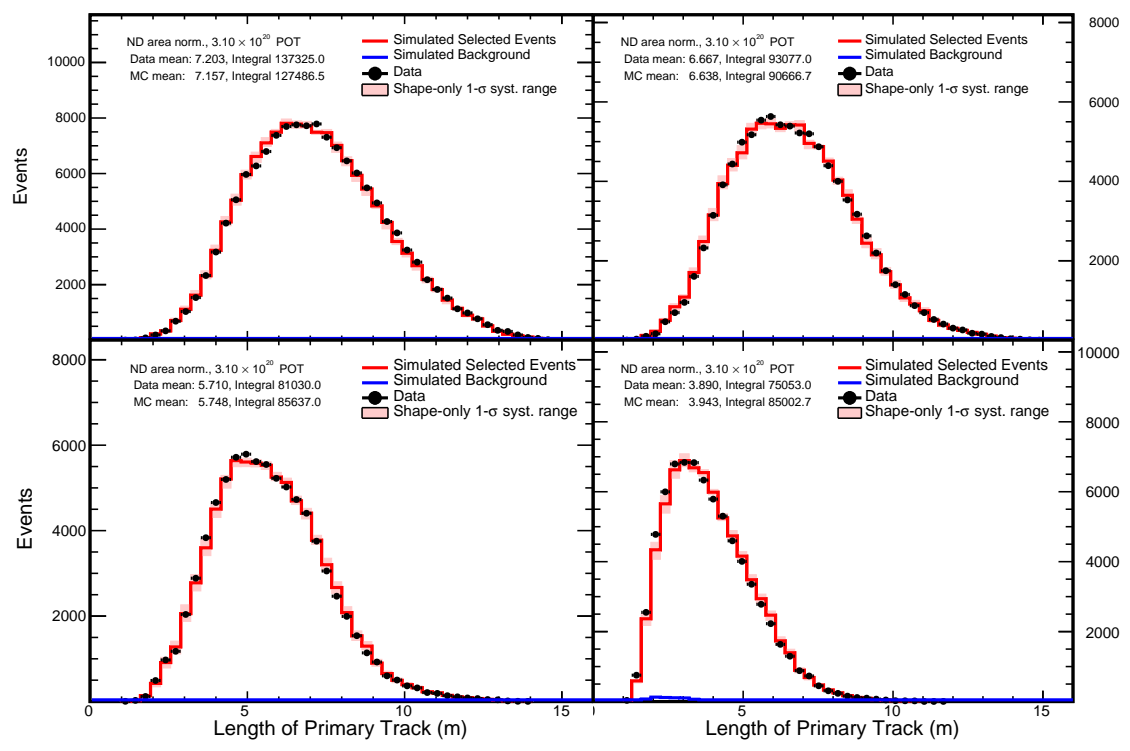

Figure 7.14: Area normalized RHC distributions of the Kalman track length for each $E_{\text {had }} / E_{\nu}$ quantile. MC and data events passing selection are shown by the red histogram and black points respectively. The MC systematic uncertainty is shown by the shaded red region. The MC background after selection is shown by the blue histogram. 

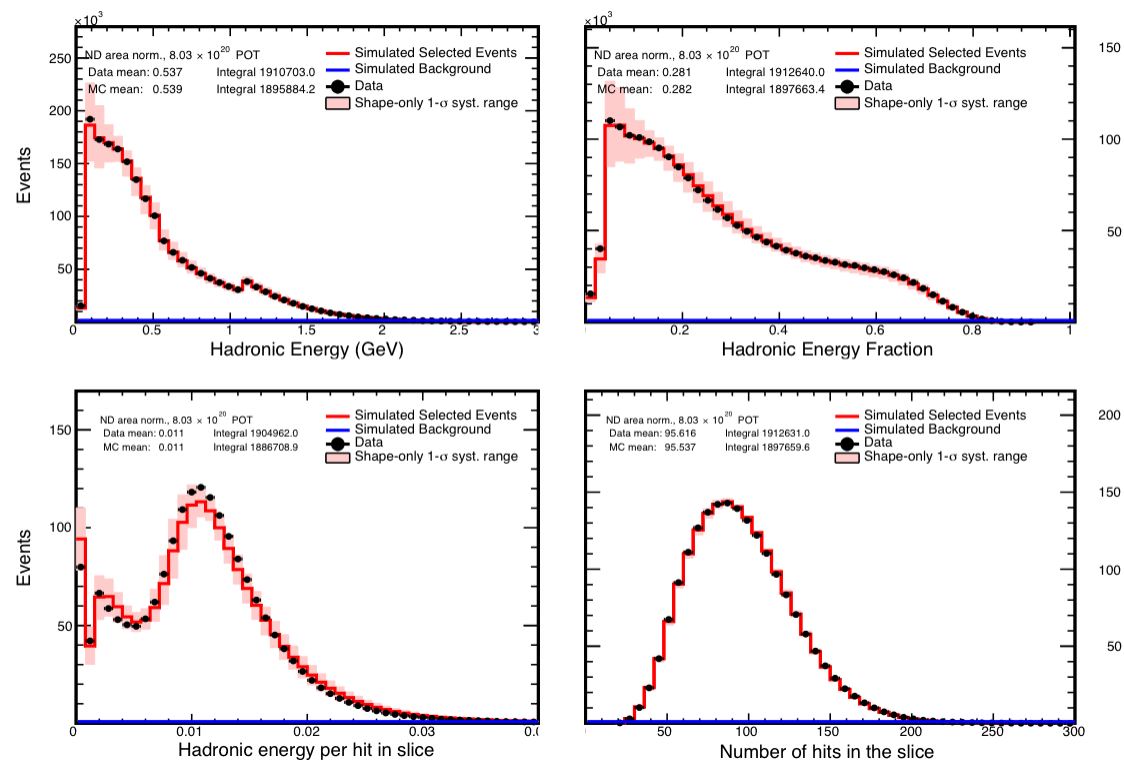

Figure 7.15: Area normalized FHC distributions of hadronic energy, fraction of hadronic energy, hadronic energy per hit \& no of hits in slice for the entire data set. MC and data events passing selection are shown by the red histogram and black points respectively. The systematic uncertainty in the distribution of the simulated events is shown by the shaded red region enclosing the red histogram. The simulated background events passing the selection are shown by the blue histogram.
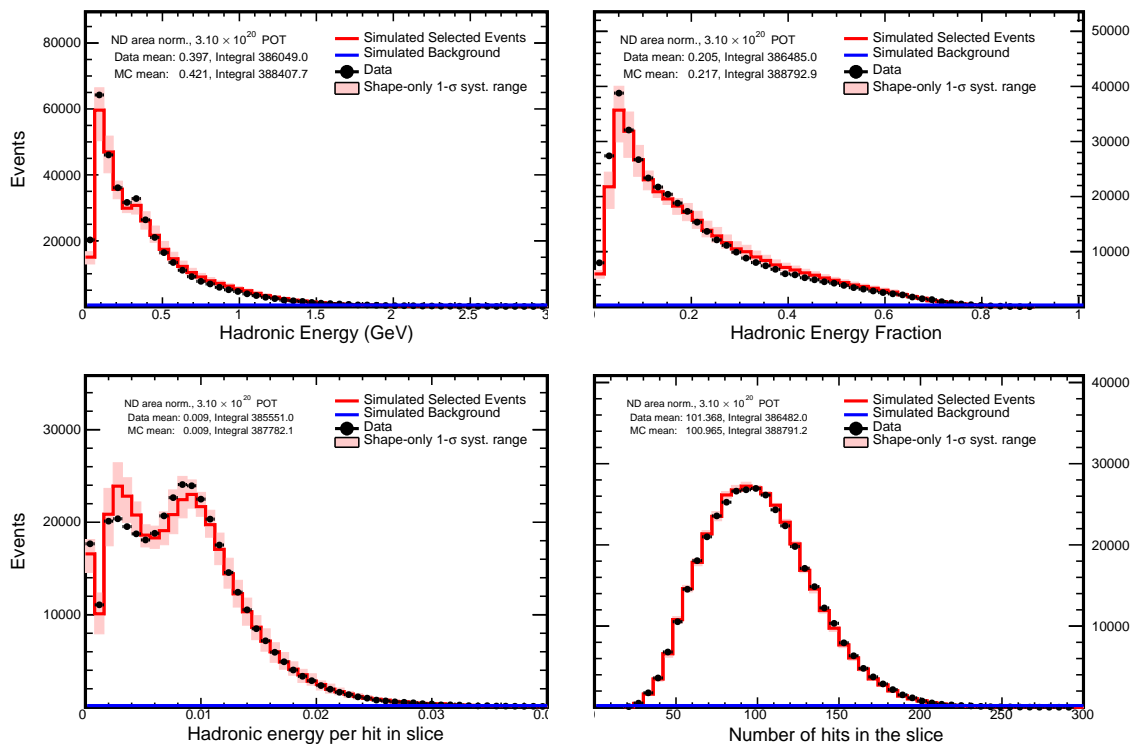

Figure 7.16: Area normalized RHC distributions of hadronic energy, fraction of hadronic energy, hadronic energy per hit \& no of hits in slice for the entire data set. MC and data events passing selection are shown by the red histogram and black points respectively. The systematic uncertainty in the distribution of the simulated events is shown by the shaded red region enclosing the red histogram. The simulated background events passing the selection are shown by the blue histogram. 


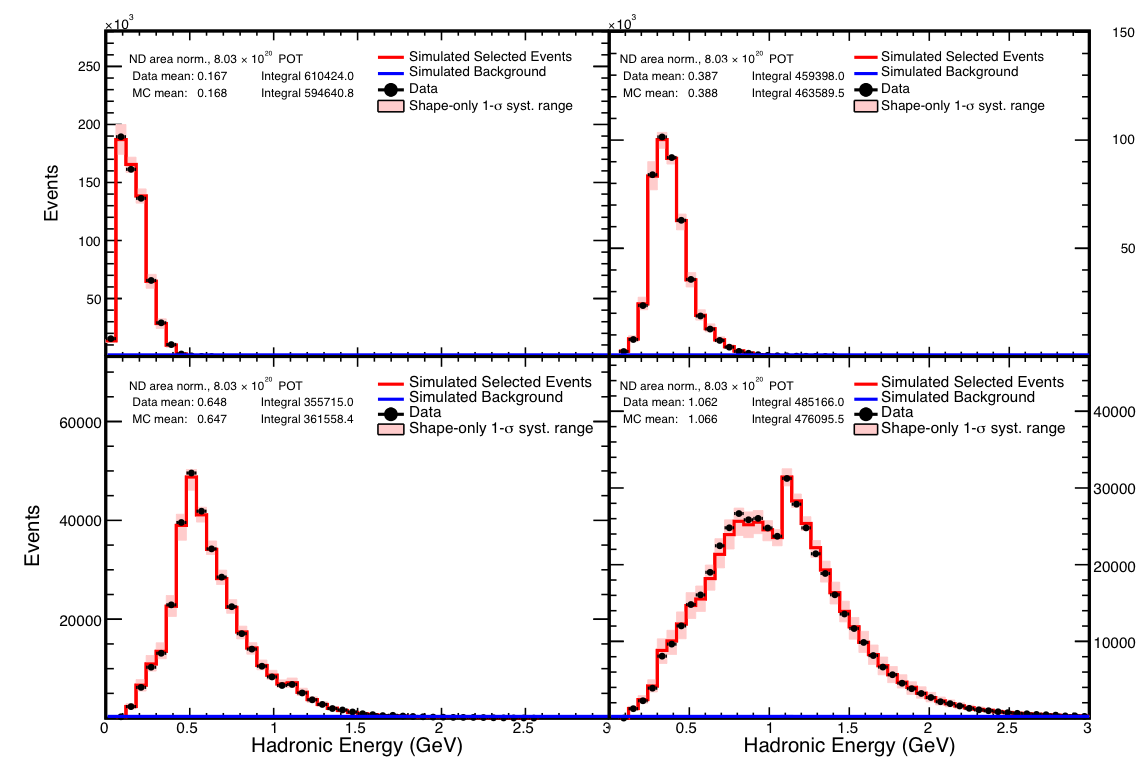

Figure 7.17: Area normalized FHC distributions of the hadronic energy for each $E_{h a d} / E_{\nu}$ quantile. MC and data events passing selection are shown by the red histogram and black points respectively. The MC systematic uncertainty is shown by the shaded red region. The MC background after selection is shown by the blue histogram.

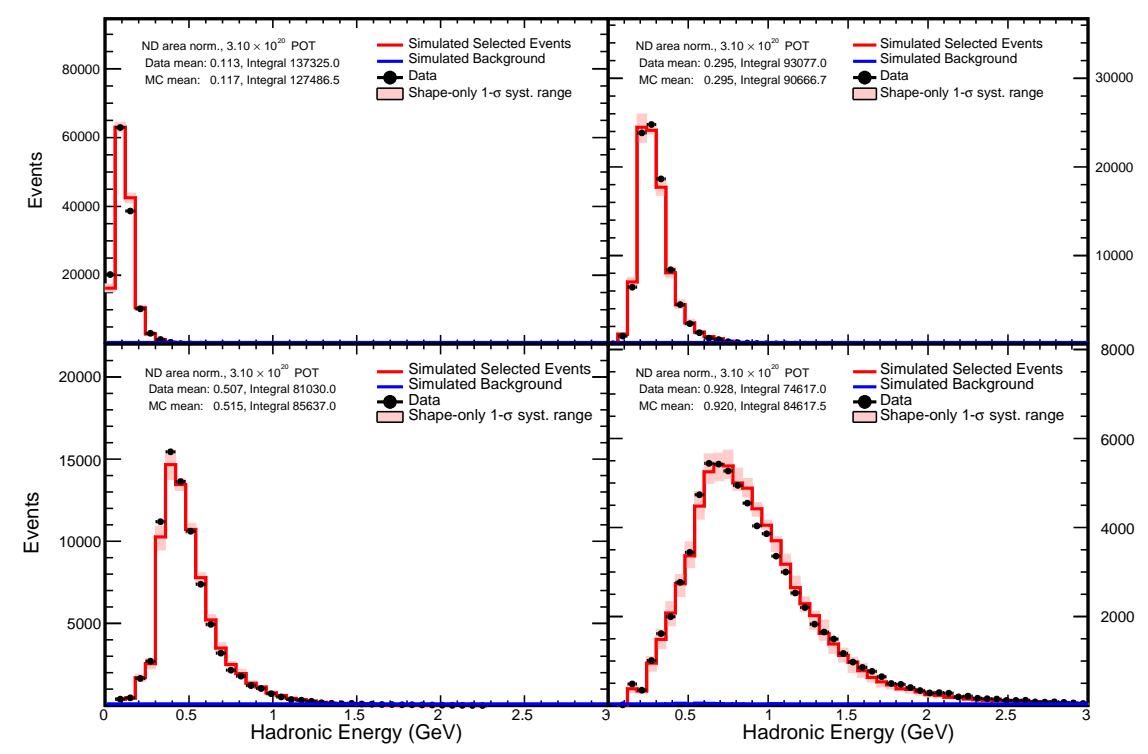

Figure 7.18: Area normalized RHC distributions of the hadronic energy for each $E_{h a d} / E_{\nu}$ quantile. MC and data events passing selection are shown by the red histogram and black points respectively. The MC systematic uncertainty is shown by the shaded red region. The MC background after selection is shown by the blue histogram. 


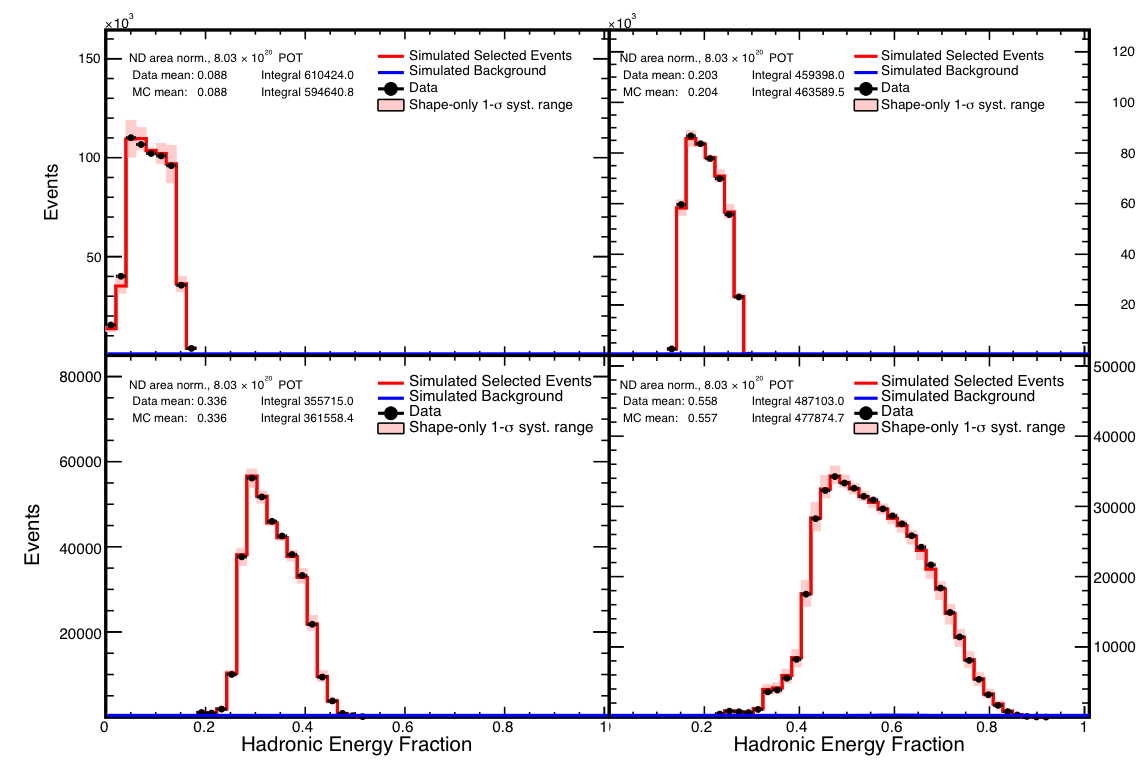

Figure 7.19: Area normalized FHC distributions of the fraction of hadronic energy for each $E_{\text {had }} / E_{\nu}$ quantile. MC and data events passing selection are shown by the red histogram and black points respectively. The MC systematic uncertainty is shown by the shaded red region. The MC background after selection is shown by the blue histogram.

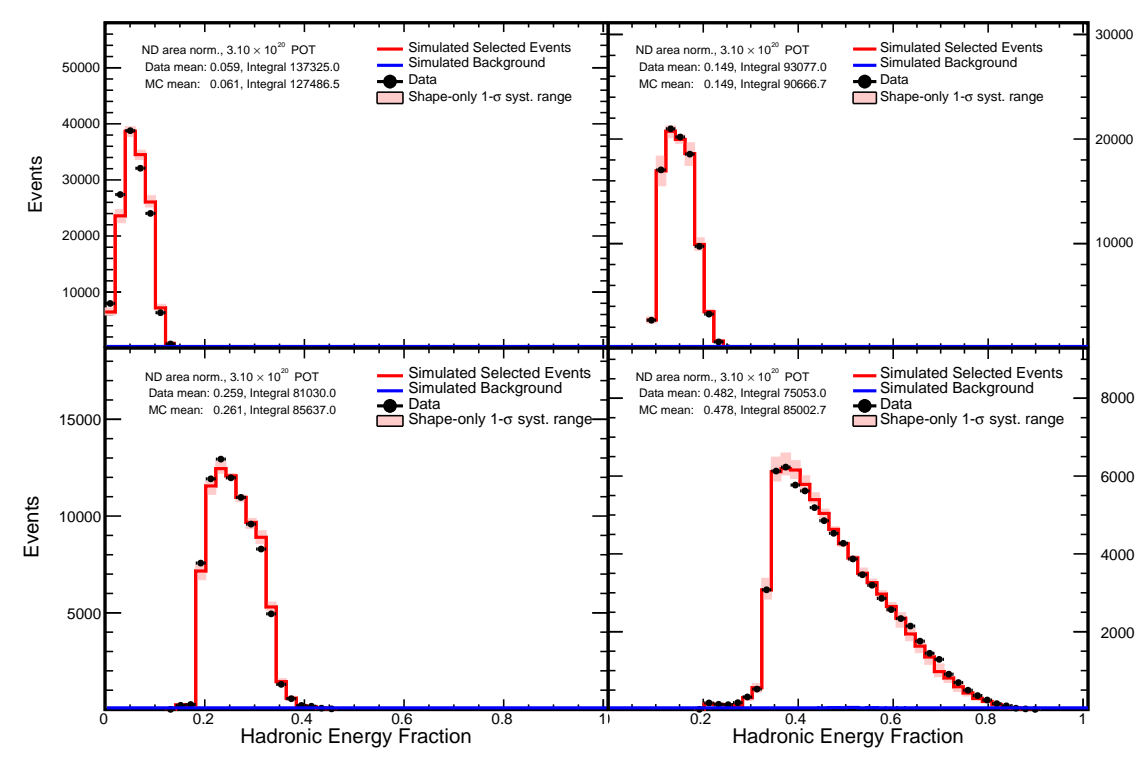

Figure 7.20: Area normalized RHC distributions of the fraction of hadronic energy for each $E_{\text {had }} / E_{\nu}$ quantile. MC and data events passing selection are shown by the red histogram and black points respectively. The MC systematic uncertainty is shown by the shaded red region. The MC background after selection is shown by the blue histogram. 


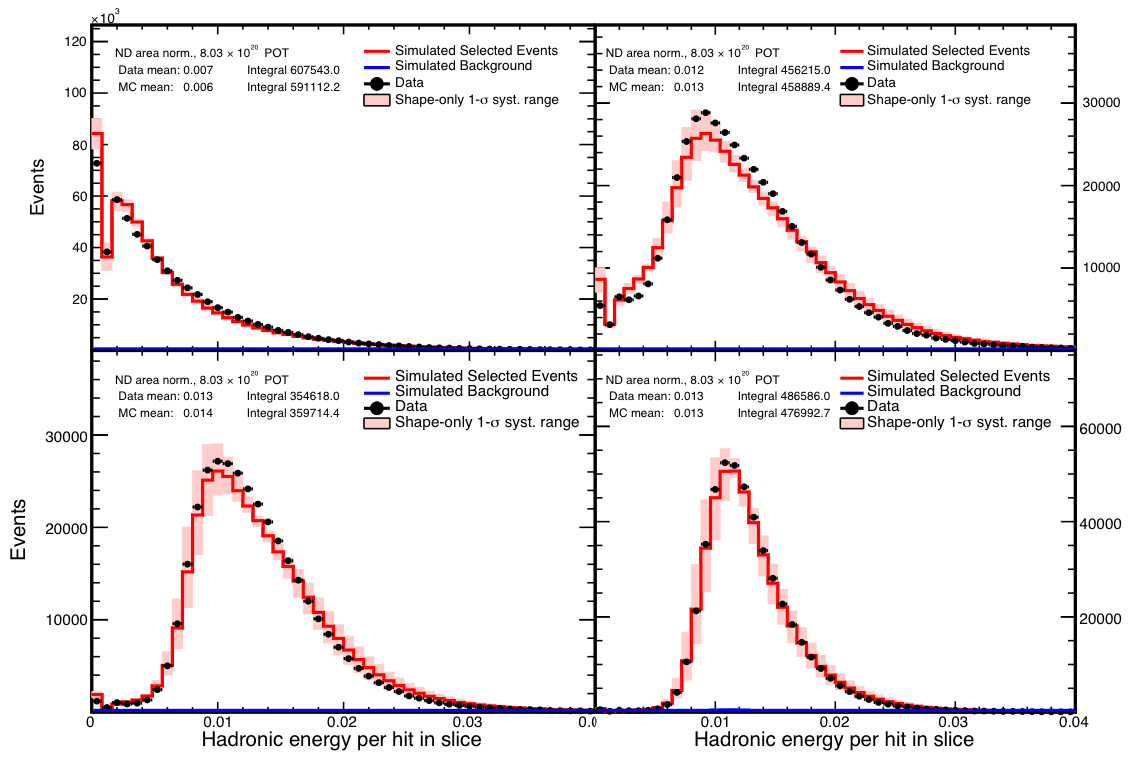

Figure 7.21: Area normalized FHC distributions of the hadronic energy per hit for each $E_{\text {had }} / E_{\nu}$ quantile. $\mathrm{MC}$ and data events passing selection are shown by the red histogram and black points respectively. The MC systematic uncertainty is shown by the shaded red region. The MC background after selection is shown by the blue histogram.

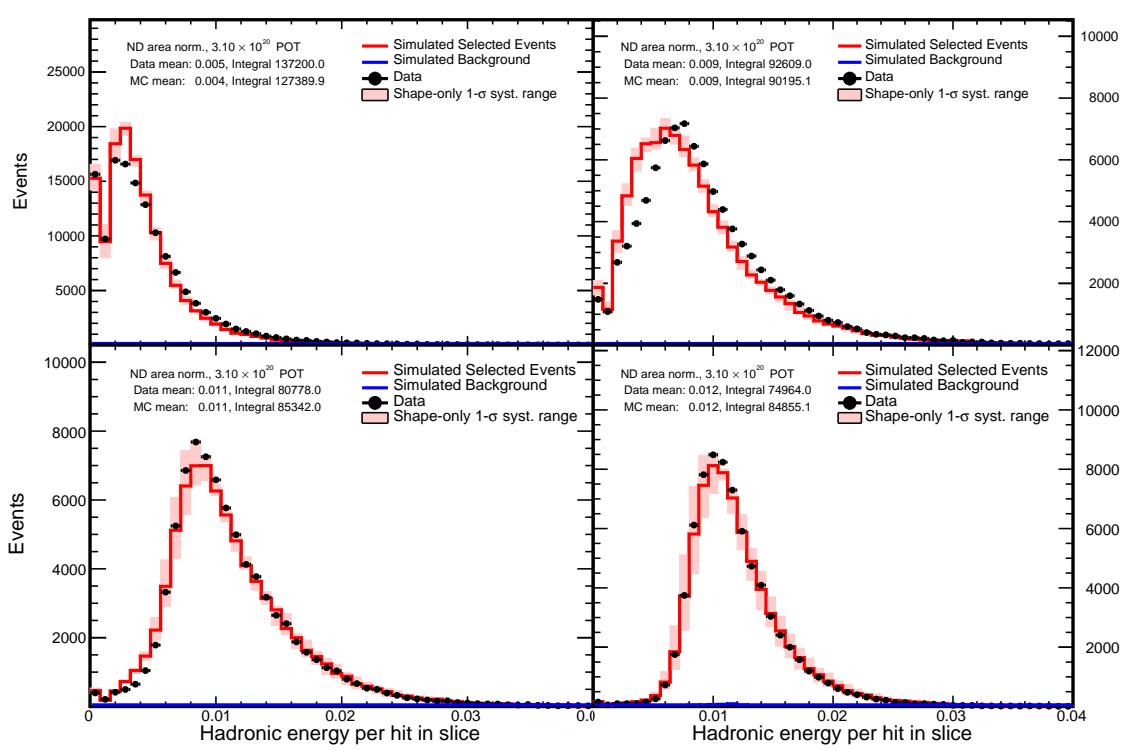

Figure 7.22: Area normalized RHC distributions of the hadronic energy per hit for each $E_{\text {had }} / E_{\nu}$ quantile. MC and data events passing selection are shown by the red histogram and black points respectively. The MC systematic uncertainty is shown by the shaded red region. The MC background after selection is shown by the blue histogram. 


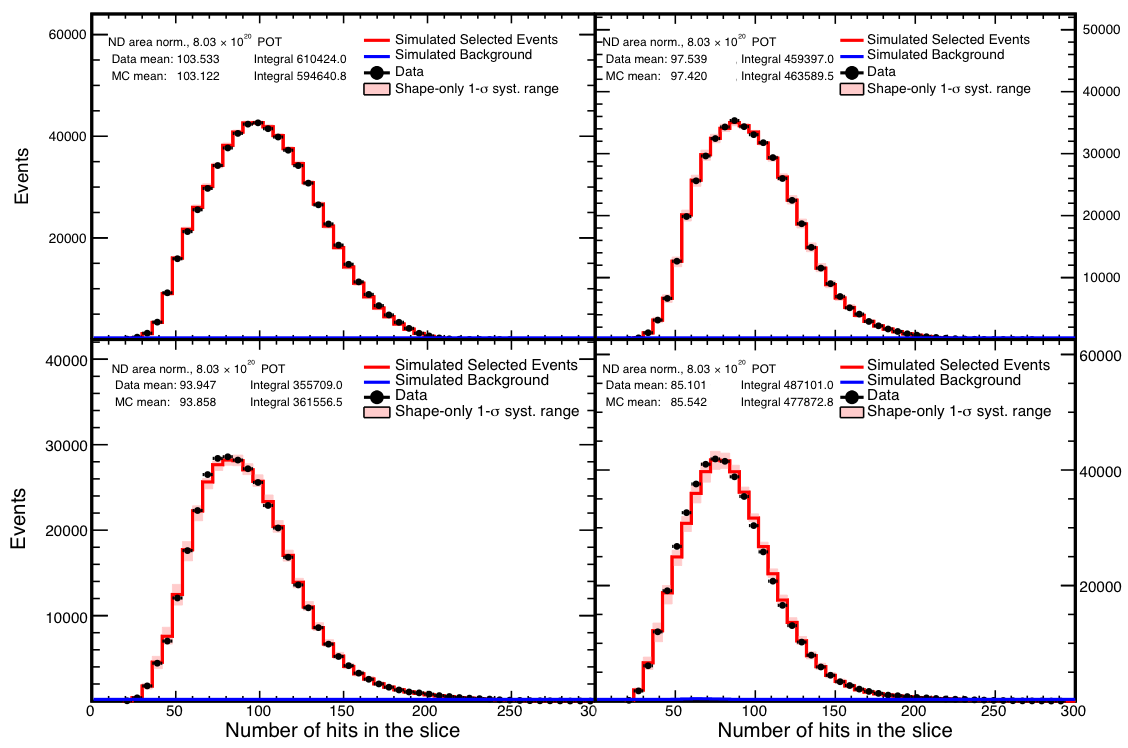

Figure 7.23: Area normalized FHC distributions of the no of hits in slice for each $E_{h a d} / E_{\nu}$ quantile. MC and data events passing selection are shown by the red histogram and black points respectively. The MC systematic uncertainty is shown by the shaded red region. The MC background after selection is shown by the blue histogram.

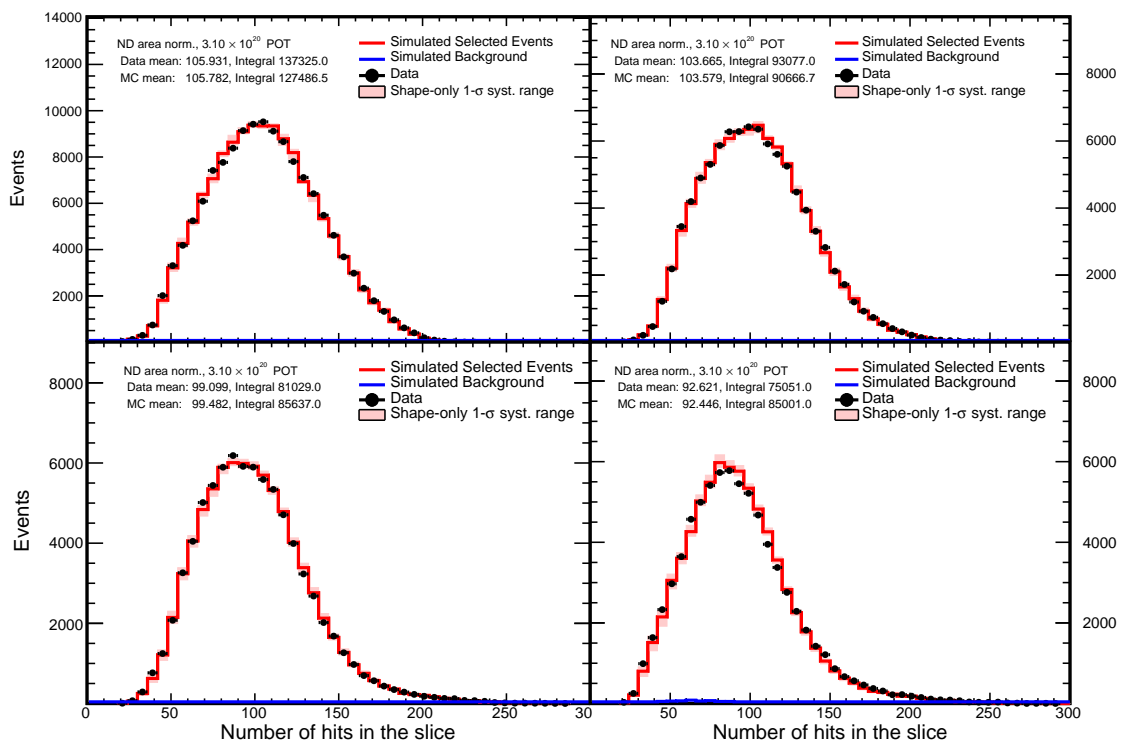

Figure 7.24: Area normalized RHC distributions of the no of hits in slice for each $E_{\text {had }} / E_{\nu}$ quantile. MC and data events passing selection are shown by the red histogram and black points respectively. The MC systematic uncertainty is shown by the shaded red region. The MC background after selection is shown by the blue histogram. 

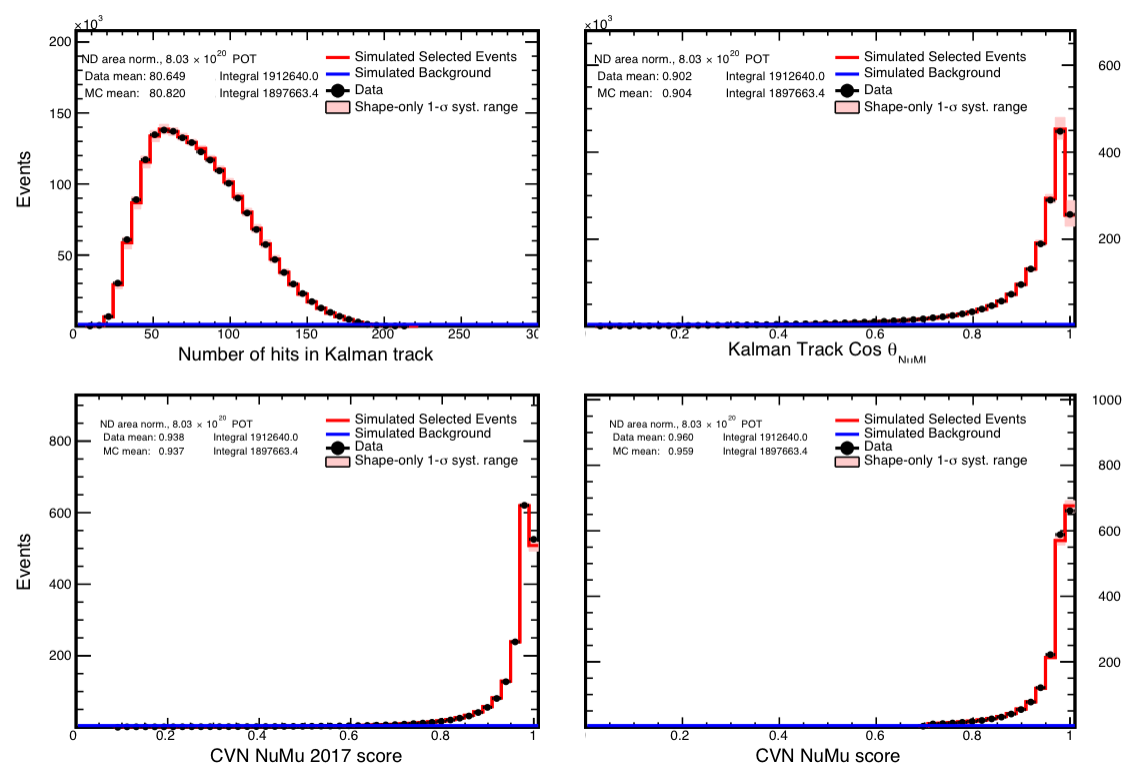

Figure 7.25: Area normalized FHC distributions of no of hits in Kalman track, cosmic track, CVN 2017 \& CVN 2018 for the entire data set. MC and data events passing selection are shown by the red histogram and black points respectively. The systematic uncertainty in the distribution of the simulated events is shown by the shaded red region enclosing the red histogram. The simulated background events passing the selection are shown by the blue histogram.
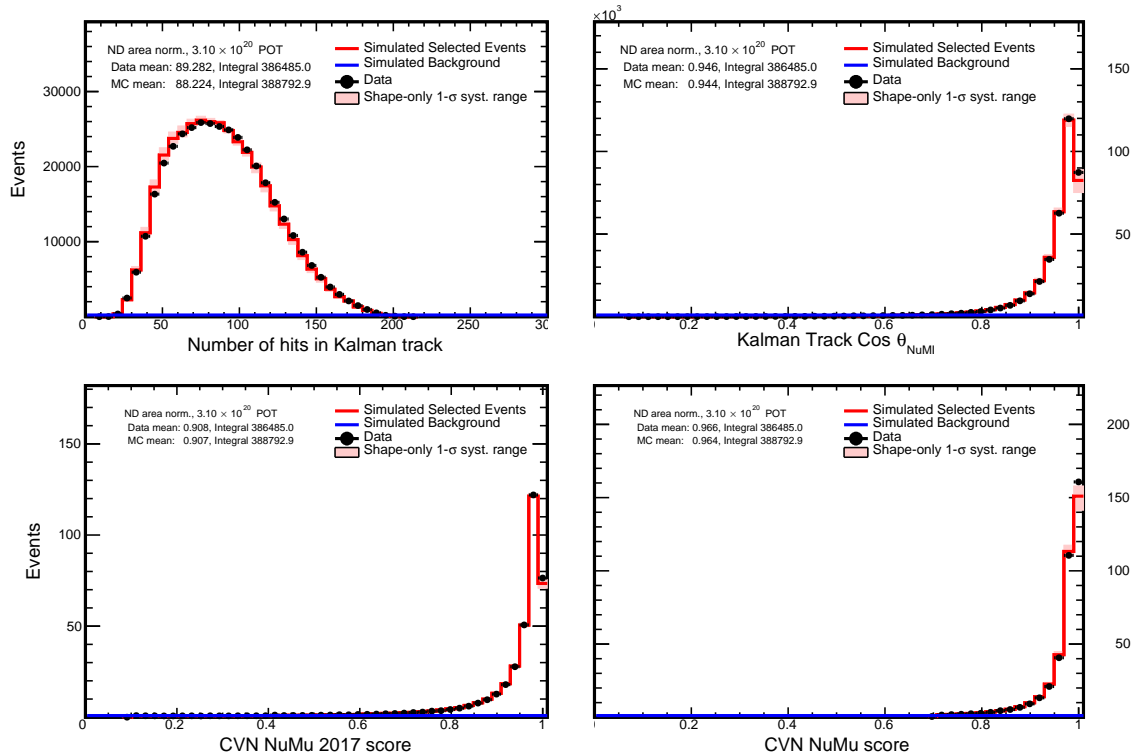

Figure 7.26: Area normalized RHC distributions of no of hits in Kalman track, cosmic track, CVN 2017 \& CVN 2018 for the entire data set. MC and data events passing selection are shown by the red histogram and black points respectively. The systematic uncertainty in the distribution of the simulated events is shown by the shaded red region enclosing the red histogram. The simulated background events passing the selection are shown by the blue histogram. 


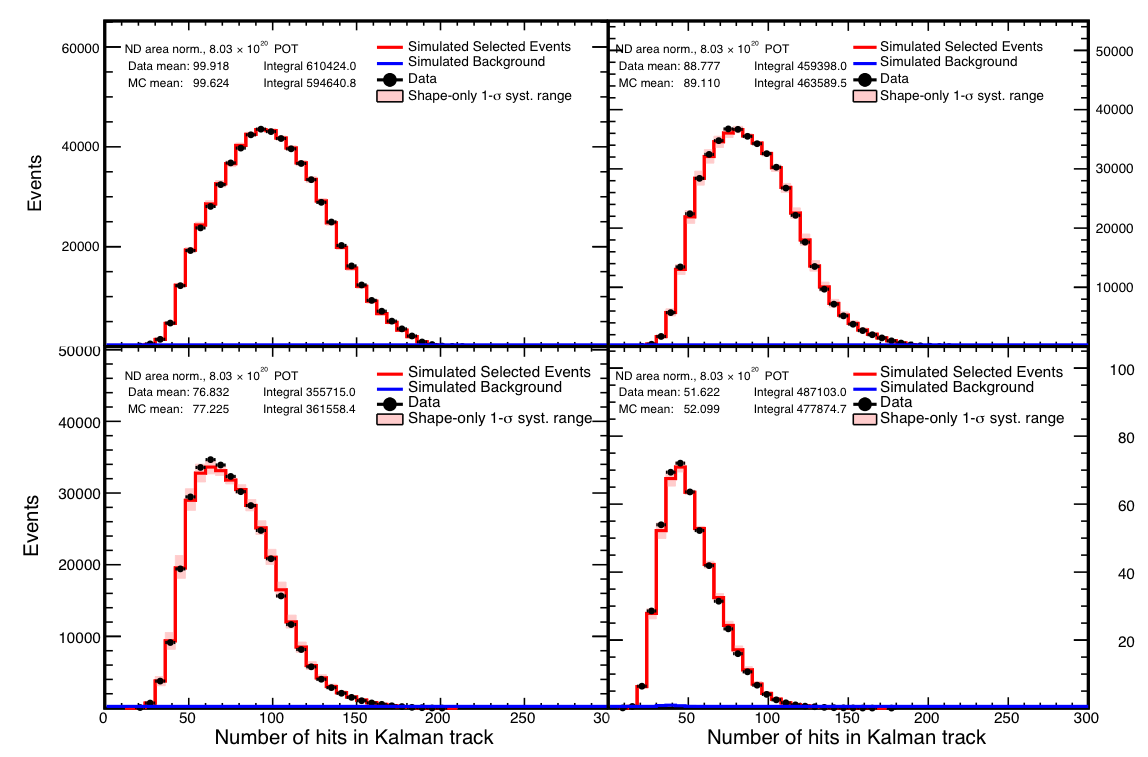

Figure 7.27: Area normalized FHC distributions of the no of hits in Kalman track for each $E_{\text {had }} / E_{\nu}$ quantile. MC and data events passing selection are shown by the red histogram and black points respectively. The MC systematic uncertainty is shown by the shaded red region. The MC background after selection is shown by the blue histogram.

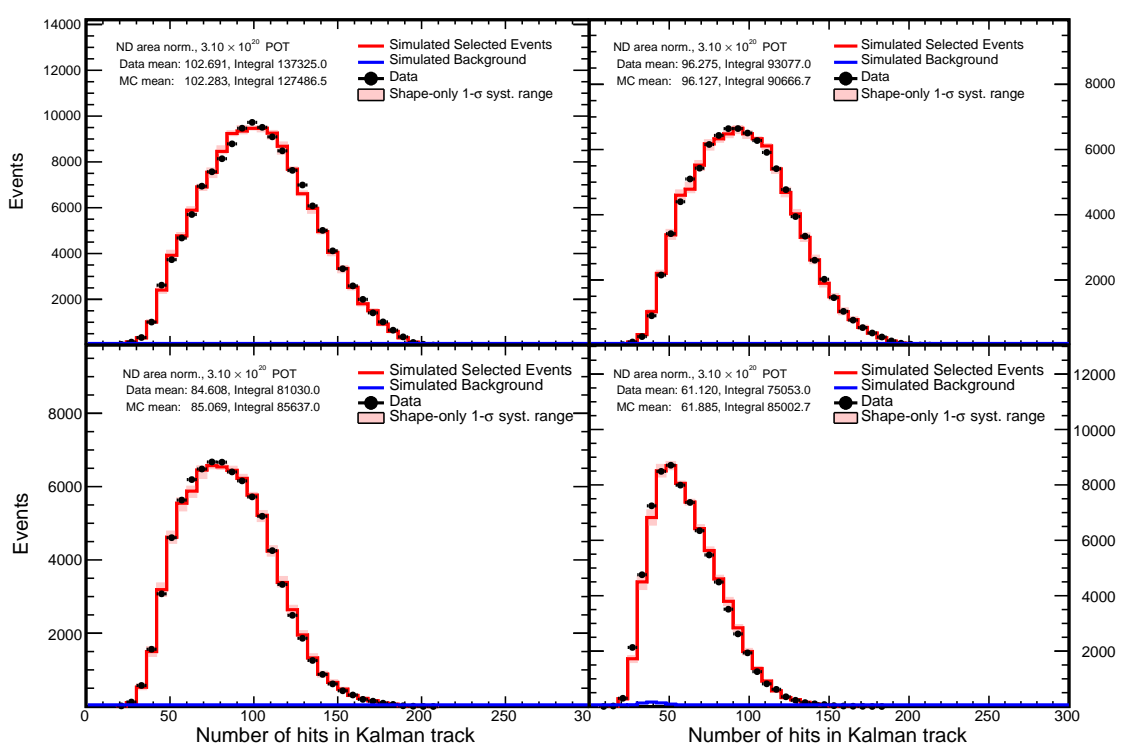

Figure 7.28: Area normalized RHC distributions of the no of hits in Kalman track for each $E_{\text {had }} / E_{\nu}$ quantile. MC and data events passing selection are shown by the red histogram and black points respectively. The MC systematic uncertainty is shown by the shaded red region. The MC background after selection is shown by the blue histogram. 


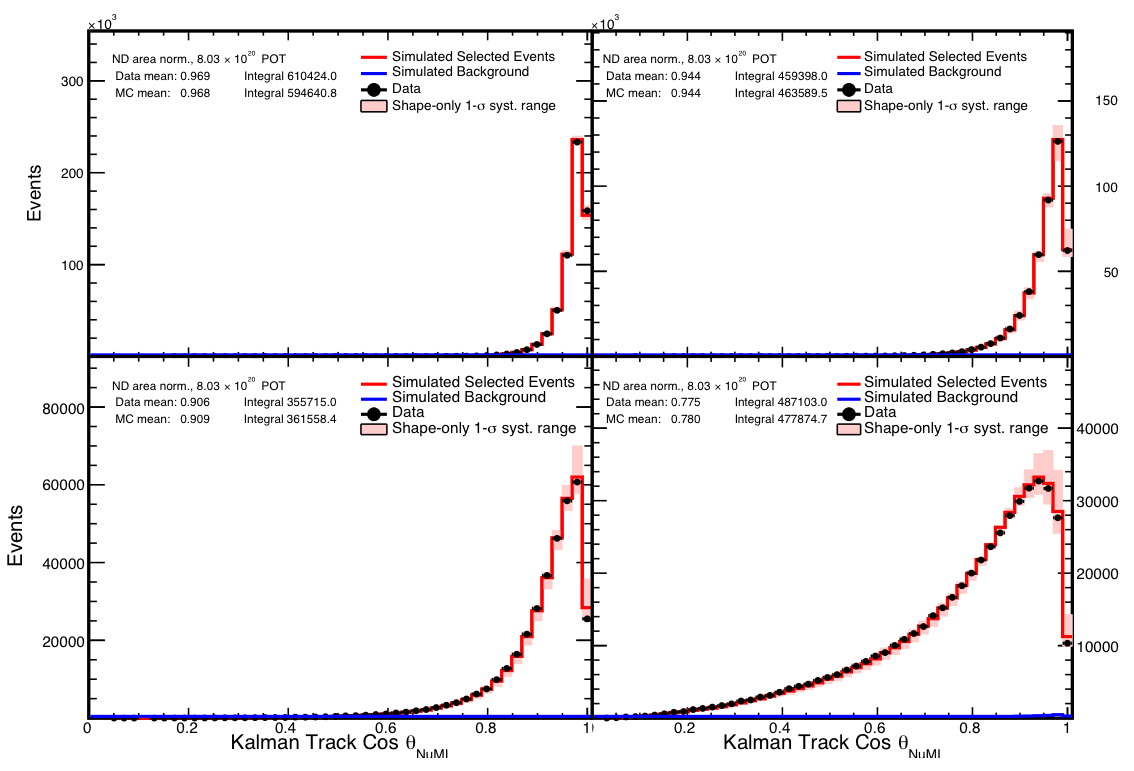

Figure 7.29: Area normalized FHC distributions of the cosmic track for each $E_{h a d} / E_{\nu}$ quantile. MC and data events passing selection are shown by the red histogram and black points respectively. The MC systematic uncertainty is shown by the shaded red region. The MC background after selection is shown by the blue histogram.

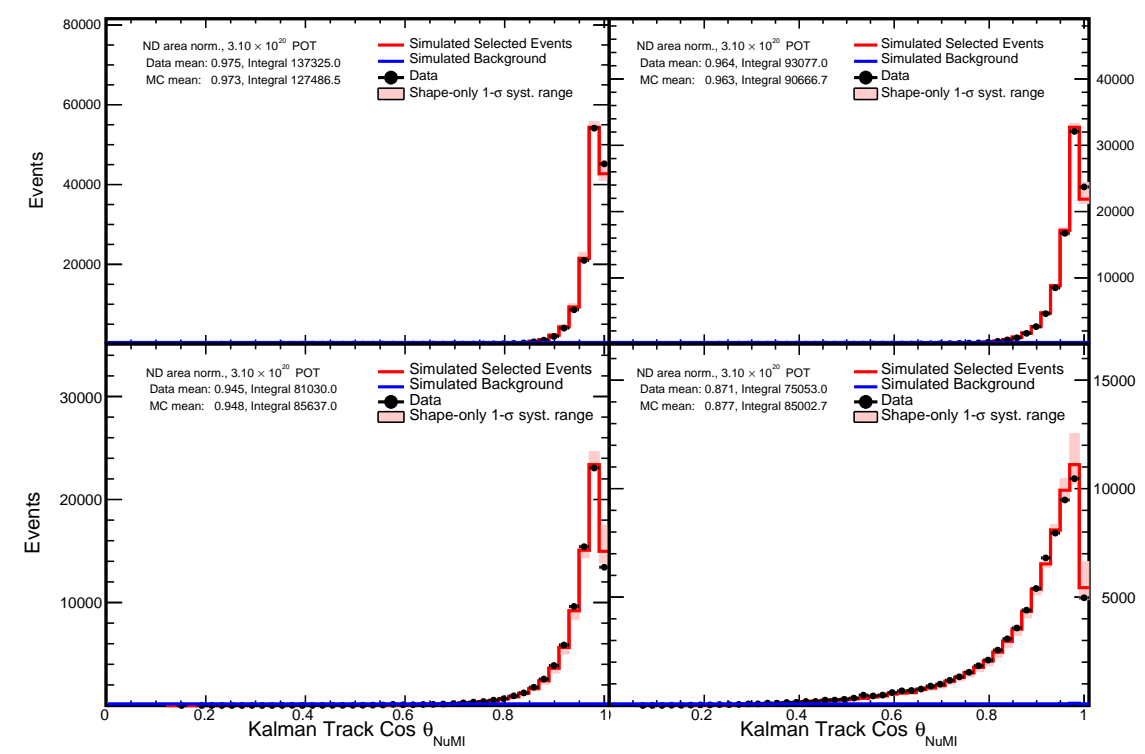

Figure 7.30: Area normalized RHC distributions of the cosmic track for each $E_{h a d} / E_{\nu}$ quantile. MC and data events passing selection are shown by the red histogram and black points respectively. The MC systematic uncertainty is shown by the shaded red region. The MC background after selection is shown by the blue histogram. 


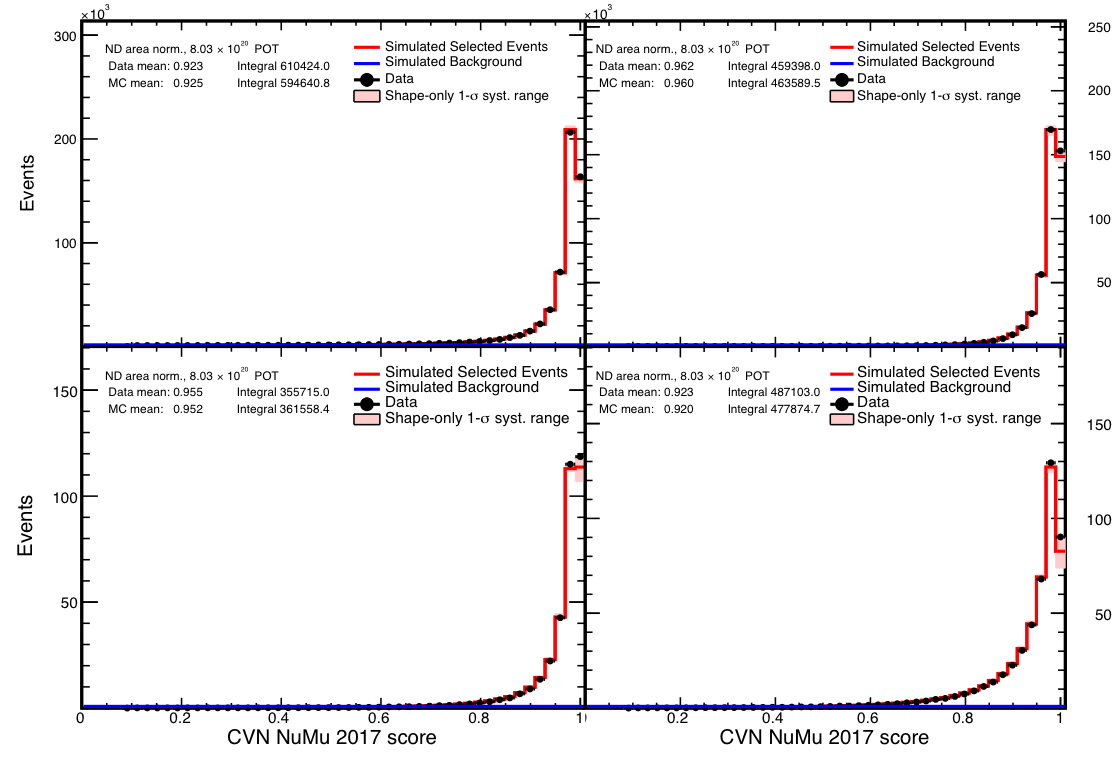

Figure 7.31: Area normalized FHC distributions of the CVN 2017 for each $E_{\text {had }} / E_{\nu}$ quantile. $\mathrm{MC}$ and data events passing selection are shown by the red histogram and black points respectively. The MC systematic uncertainty is shown by the shaded red region. The MC background after selection is shown by the blue histogram.

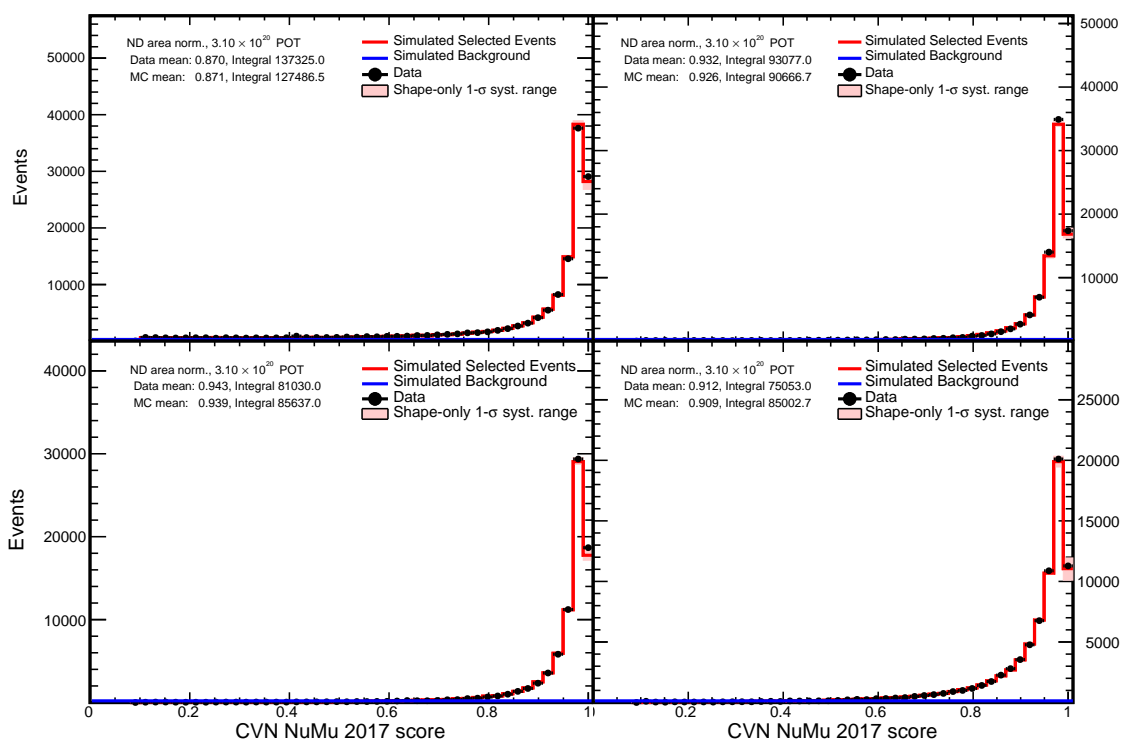

Figure 7.32: Area normalized RHC distributions of the CVN 2017 for each $E_{h a d} / E_{\nu}$ quantile. MC and data events passing selection are shown by the red histogram and black points respectively. The MC systematic uncertainty is shown by the shaded red region. The MC background after selection is shown by the blue histogram. 


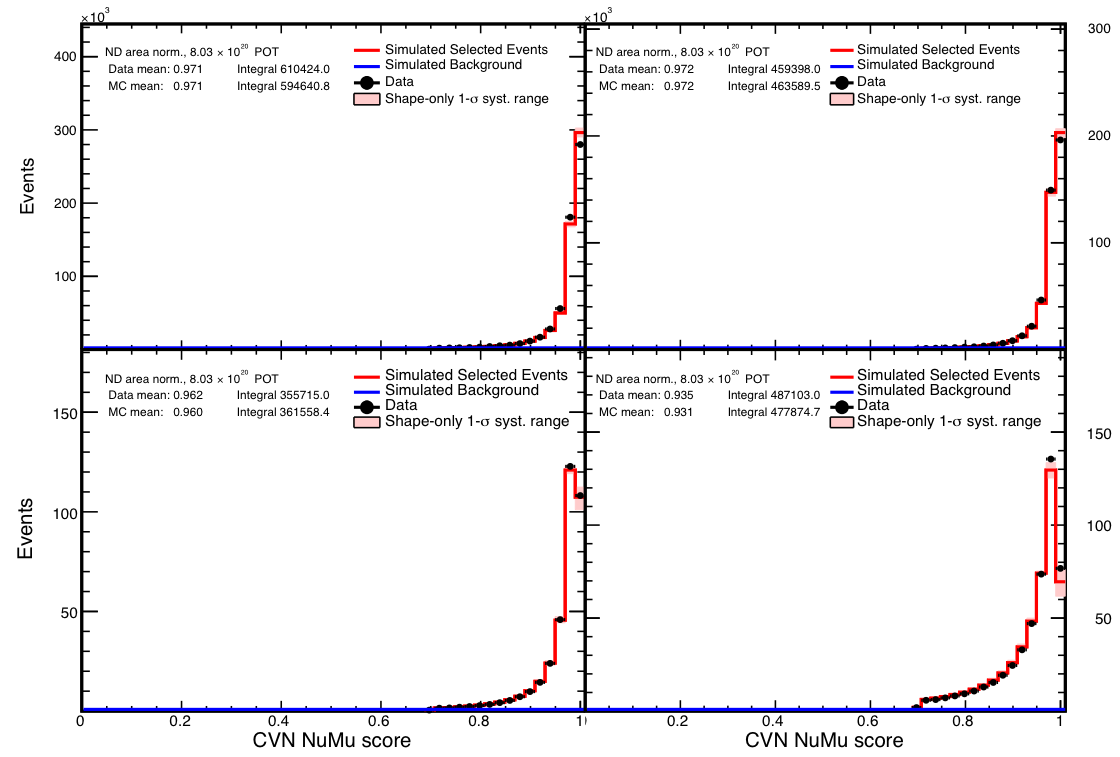

Figure 7.33: Area normalized FHC distributions of the CVN 2018 for each $E_{\text {had }} / E_{\nu}$ quantile. $\mathrm{MC}$ and data events passing selection are shown by the red histogram and black points respectively. The MC systematic uncertainty is shown by the shaded red region. The MC background after selection is shown by the blue histogram.

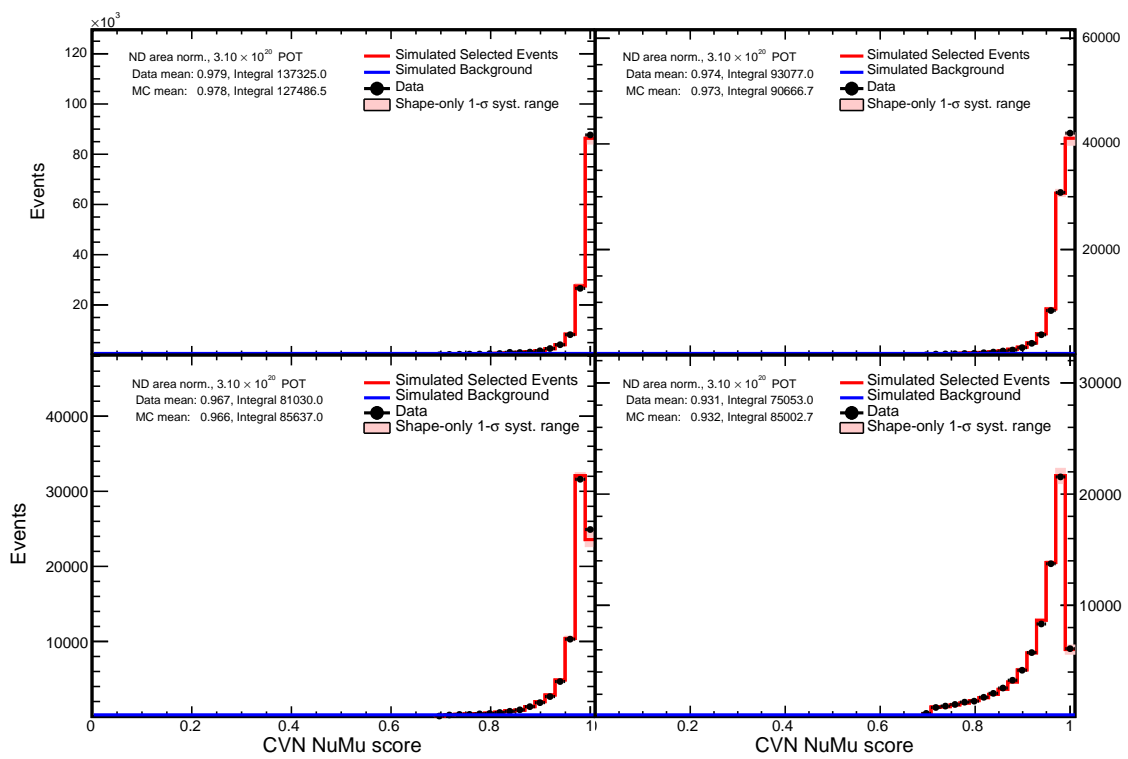

Figure 7.34: Area normalized RHC distributions of the CVN 2018 for each $E_{\text {had }} / E_{\nu}$ quantile. MC and data events passing selection are shown by the red histogram and black points respectively. The MC systematic uncertainty is shown by the shaded red region. The MC background after selection is shown by the blue histogram. 

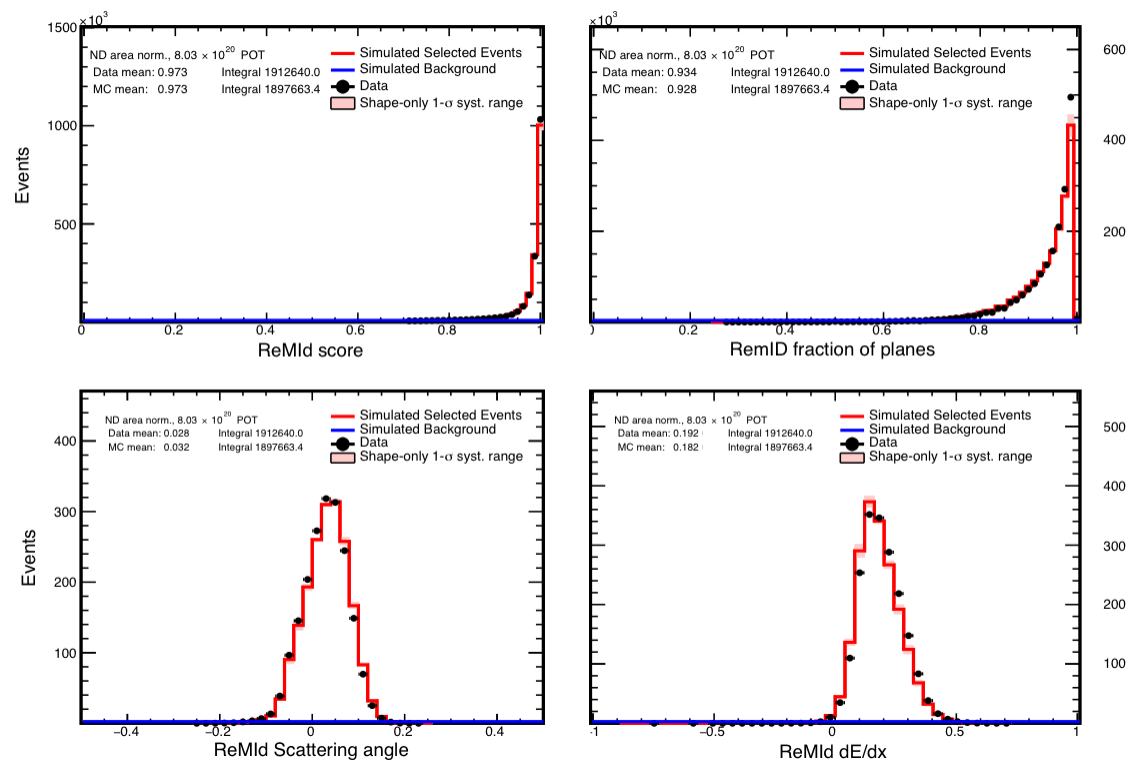

Figure 7.35: Area normalized FHC distributions of ReMId, ReMId fraction likelihood, ReMId scattering angle likelihood \& ReMId $\frac{d E}{d x}$ likelihood for the entire data set. MC and data events passing selection are shown by the red histogram and black points respectively. The systematic uncertainty in the distribution of the simulated events is shown by the shaded red region enclosing the red histogram. The simulated background events passing the selection are shown by the blue histogram.
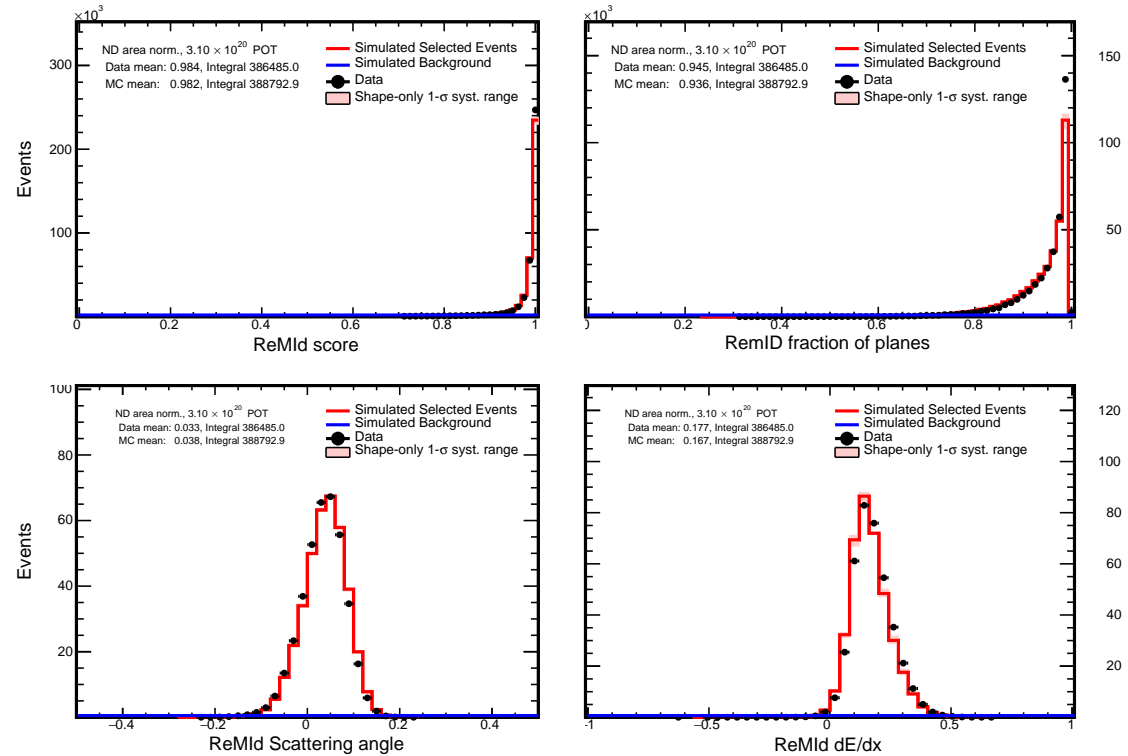

Figure 7.36: Area normalized RHC distributions of ReMId, ReMId fraction likelihood, ReMId scattering angle likelihood \& ReMId $\frac{d E}{d x}$ likelihood for the entire data set. MC and data events passing selection are shown by the red histogram and black points respectively. The systematic uncertainty in the distribution of the simulated events is shown by the shaded red region enclosing the red histogram. The simulated background events passing the selection are shown by the blue histogram. 


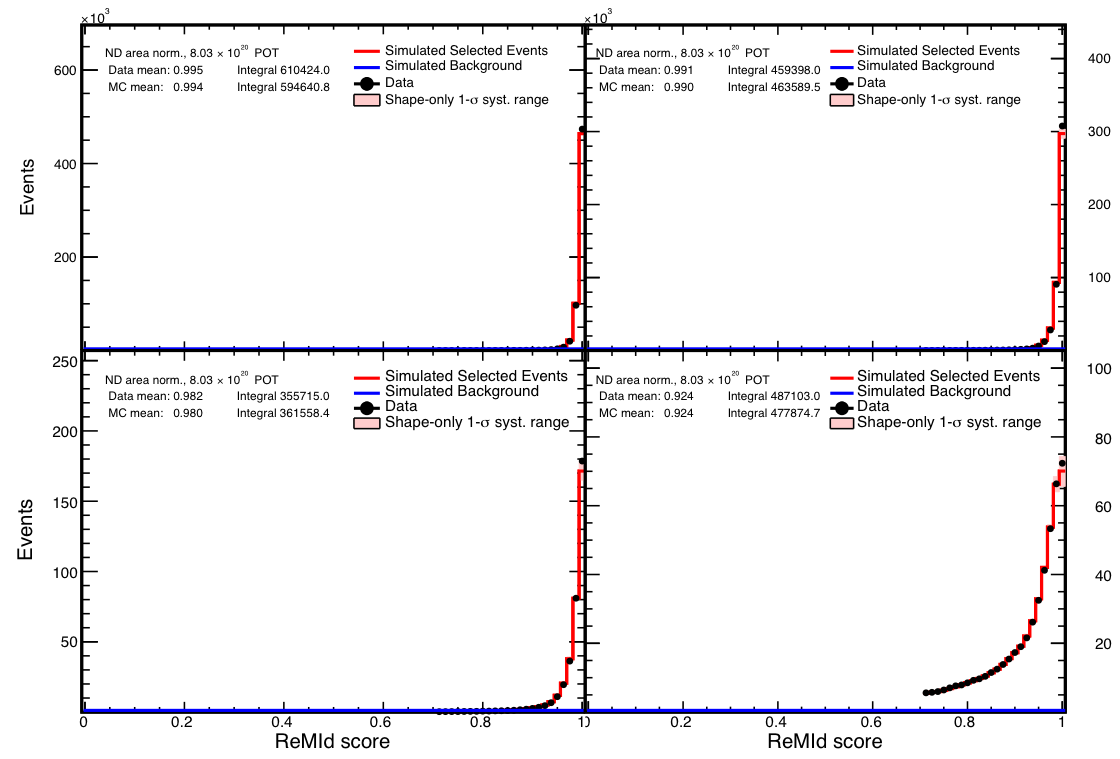

Figure 7.37: Area normalized FHC distributions of the ReMId for each $E_{\text {had }} / E_{\nu}$ quantile. $\mathrm{MC}$ and data events passing selection are shown by the red histogram and black points respectively. The MC systematic uncertainty is shown by the shaded red region. The MC background after selection is shown by the blue histogram.

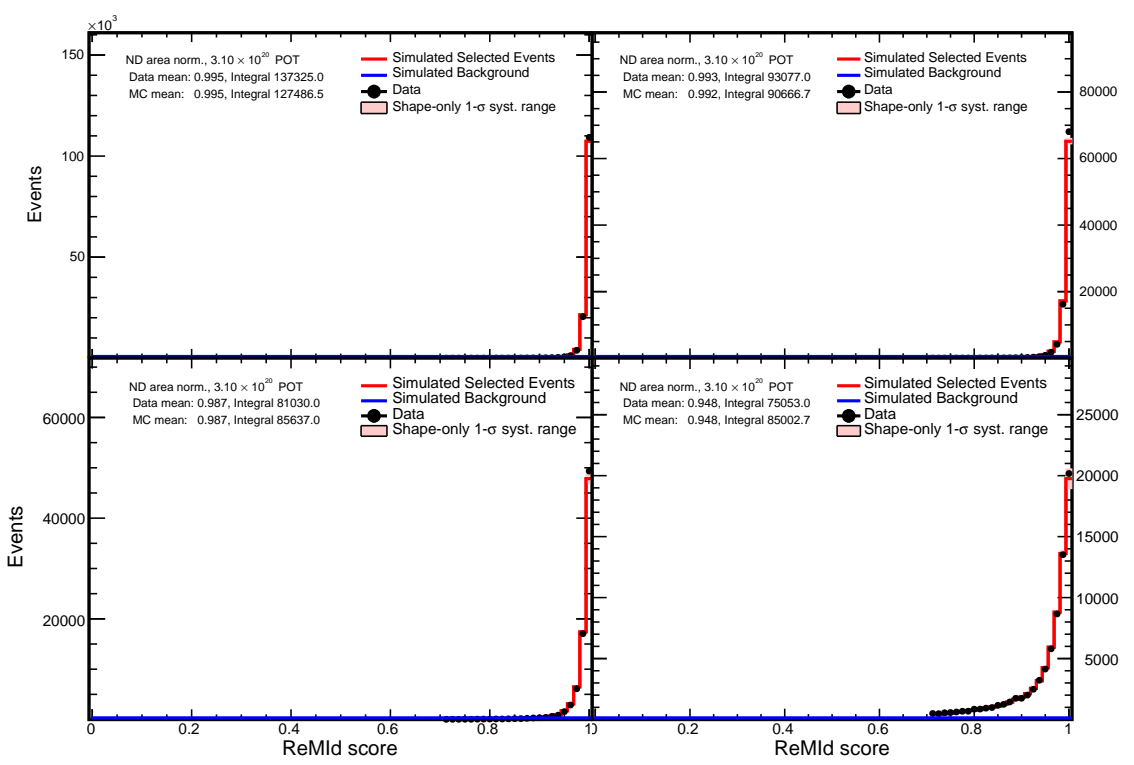

Figure 7.38: Area normalized RHC distributions of the ReMId for each $E_{\text {had }} / E_{\nu}$ quantile. $\mathrm{MC}$ and data events passing selection are shown by the red histogram and black points respectively. The MC systematic uncertainty is shown by the shaded red region. The MC background after selection is shown by the blue histogram. 


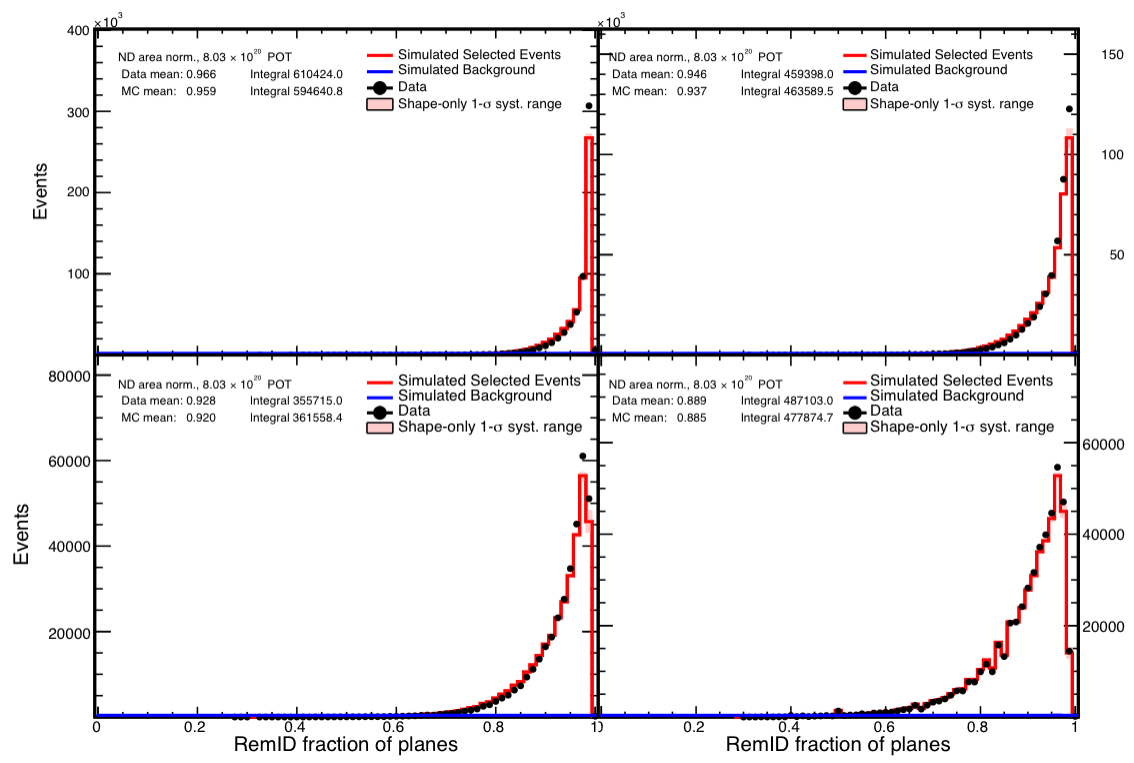

Figure 7.39: Area normalized FHC distributions of the ReMId fraction of planes along the track consistent with having minimum ionizinglike $\frac{d E}{d x}$ for each $E_{h a d} / E_{\nu}$ quantile. MC and data events passing selection are shown by the red histogram and black points respectively. The MC systematic uncertainty is shown by the shaded red region. The MC background after selection is shown by the blue histogram.

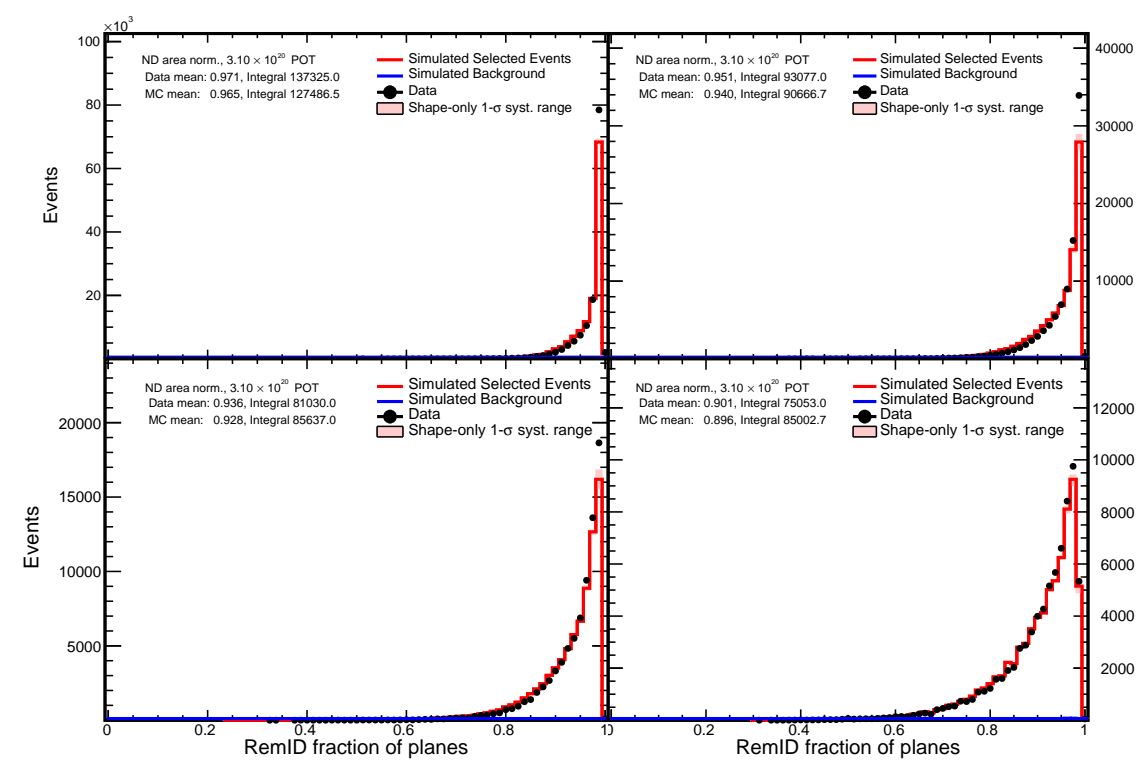

Figure 7.40: Area normalized RHC distributions of the ReMId fraction of planes along the track consistent with having minimum ionizinglike $\frac{d E}{d x}$ for each $E_{h a d} / E_{\nu}$ quantile. MC and data events passing selection are shown by the red histogram and black points respectively. The MC systematic uncertainty is shown by the shaded red region. The MC background after selection is shown by the blue histogram. 


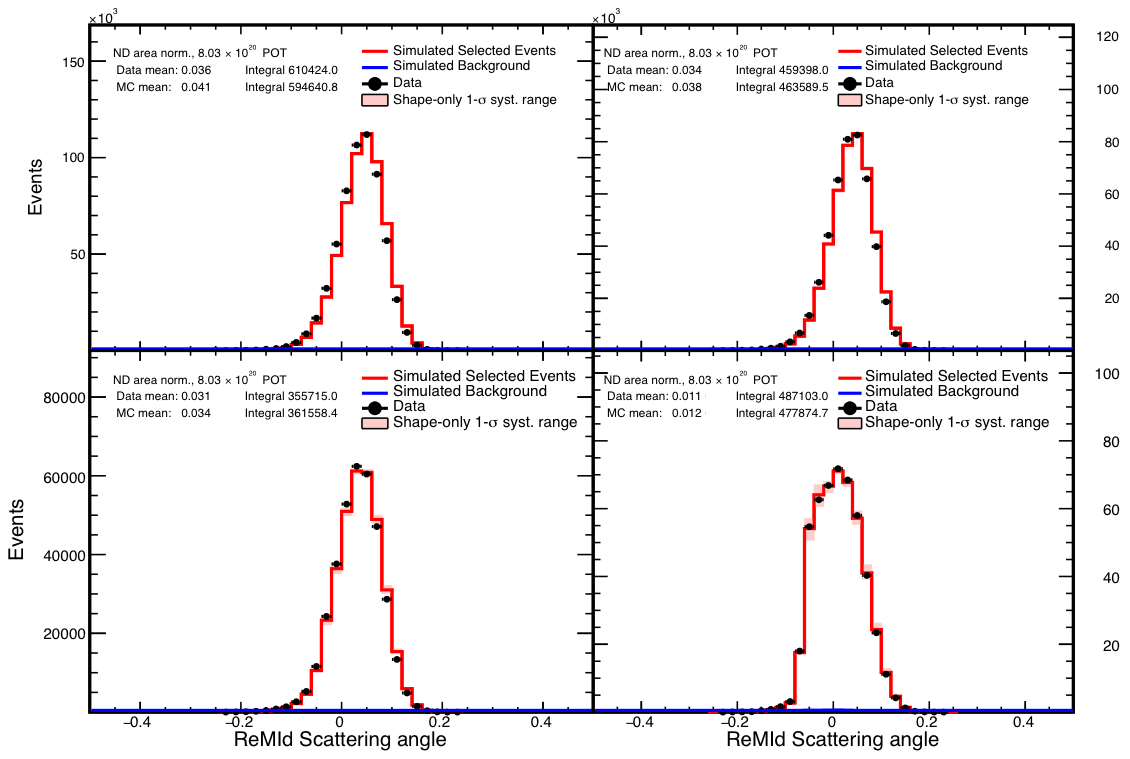

Figure 7.41: Area normalized FHC distributions of the ReMId scattering angle likelihood for each $E_{h a d} / E_{\nu}$ quantile. $\mathrm{MC}$ and data events passing selection are shown by the red histogram and black points respectively. The MC systematic uncertainty is shown by the shaded red region. The MC background after selection is shown by the blue histogram.

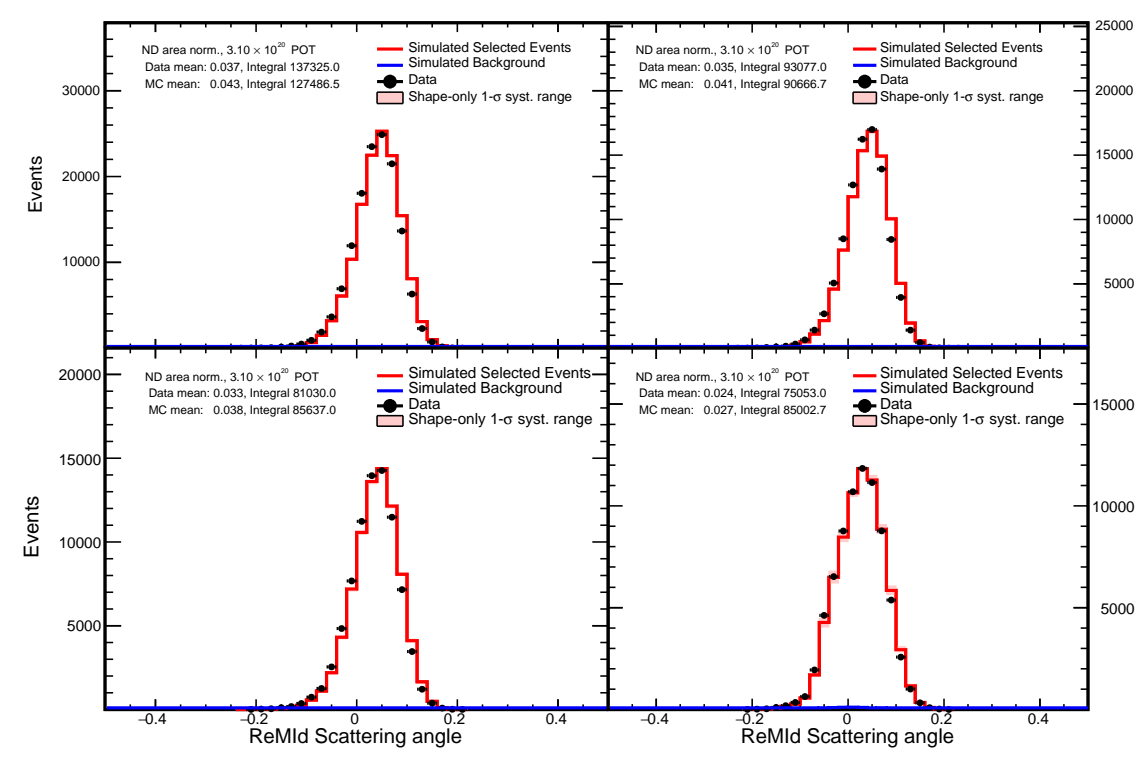

Figure 7.42: Area normalized RHC distributions of the ReMId scattering angle likelihood for each $E_{h a d} / E_{\nu}$ quantile. MC and data events passing selection are shown by the red histogram and black points respectively. The MC systematic uncertainty is shown by the shaded red region. The MC background after selection is shown by the blue histogram. 


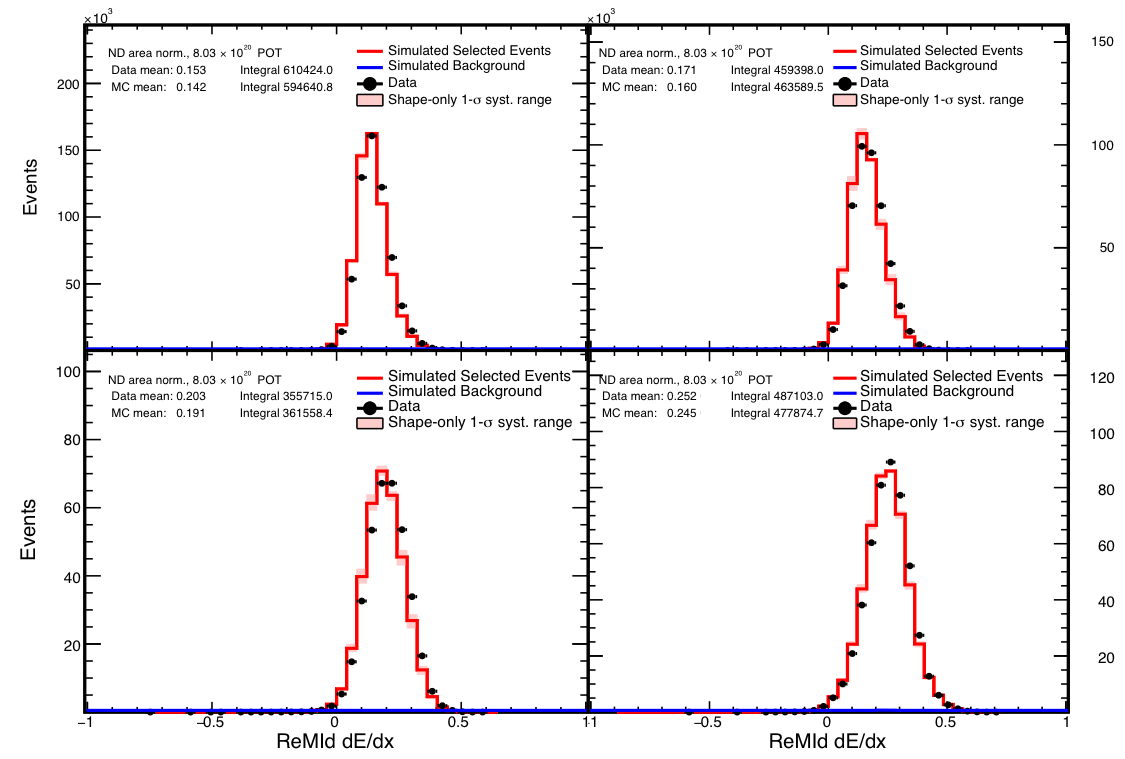

Figure 7.43: Area normalized FHC distributions of the ReMId $\frac{d E}{d x}$ likelihood for each $E_{\text {had }} / E_{\nu}$ quantile. MC and data events passing selection are shown by the red histogram and black points respectively. The MC systematic uncertainty is shown by the shaded red region. The MC background after selection is shown by the blue histogram.

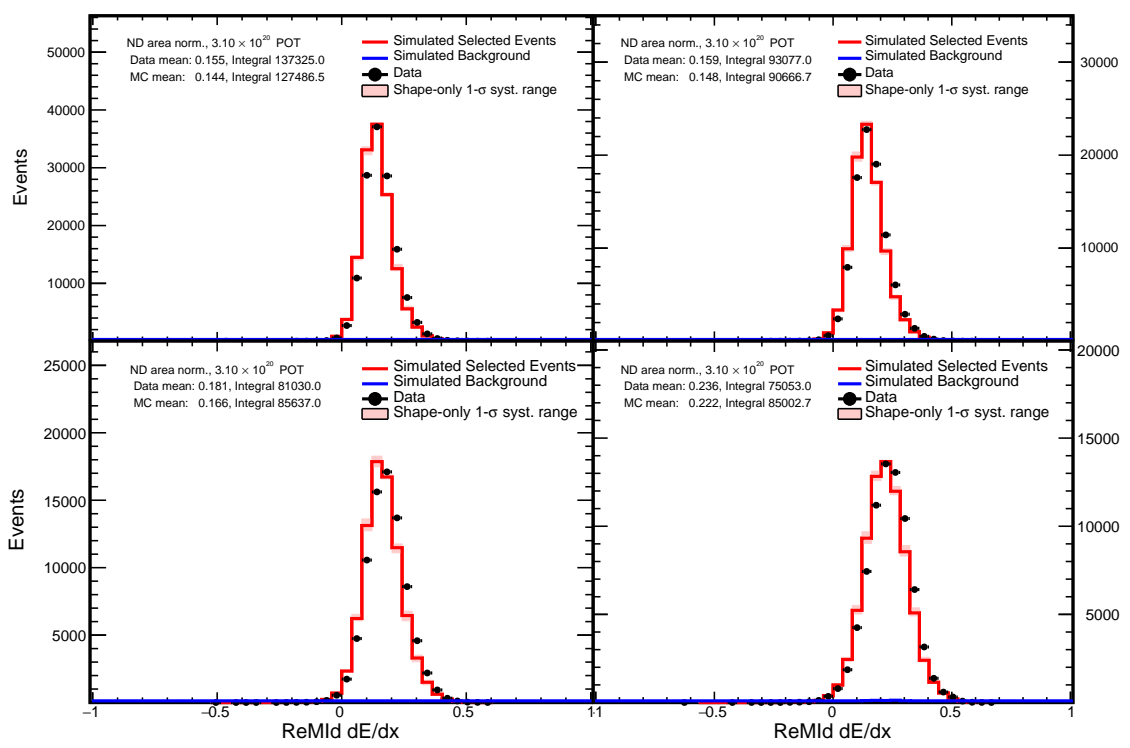

Figure 7.44: Area normalized RHC distributions of the ReMId $\frac{d E}{d x}$ likelihood for each $E_{\text {had }} / E_{\nu}$ quantile. MC and data events passing selection are shown by the red histogram and black points respectively. The MC systematic uncertainty is shown by the shaded red region. The MC background after selection is shown by the blue histogram. 


\subsection{Far Detector Data}

Far detector data distributions and analysis results are presented in this section. Distributions of data and expectation are shown first followed by the contours produced by the $\nu_{\mu}+\bar{\nu}_{\mu}$ disappearance analysis. Contours are shown for both the statistics only and full systematics result as well as for the individual neutrino and antineutrino component contours. The contour produced by the NOvA combined $\nu_{\mu}+\bar{\nu}_{\mu}$ disappearance and $\nu_{e}$ $+\bar{\nu}_{e}$ appearance is also shown. Significance plots of $\Delta \chi^{2}$ vs. $\Delta \mathrm{m}_{32}^{2}$ and $\Delta \chi^{2}$ vs. $\sin ^{2} \theta_{23}$ are also presented, produced by profiling the other oscillation parameter that is not shown.

After $\nu_{\mu}+\bar{\nu}_{\mu}$ CC candidate events have been identified, Data-MC comparisons are used to verify the analysis methodology and check for unexpected issues, analogous to the near detector. The distribution of neutrino energy is then studied and the confidence limit contour in oscillation parameter phase space is produced using the CAFAna fitting framework.

The predicted far detector FHC and RHC oscillation channel breakdown is detailed in Table 7.4. The breakdowns are given for each $E_{\text {had }} / E_{\nu}$ quantile in Figure 7.5 and Figure 7.6 for FHC and RHC respectively. 


\begin{tabular}{|c|c|c|c|c|c|c|c|}
\hline \multicolumn{4}{|c|}{ FHC } & \multicolumn{4}{|c|}{ RHC } \\
\hline Channel & Selected events & Channel & Selected events & Channel & Selected events & Channel & Selected events \\
\hline$\nu_{\mu} \rightarrow \nu_{\mu}$ & 109.696 & $\nu_{e} \rightarrow \nu_{\mu}$ & 0.234 & $\nu_{\mu} \rightarrow \nu_{\mu}$ & 12.577 & $\nu_{e} \rightarrow \nu_{\mu}$ & 0.028 \\
\hline$\nu_{\mu} \rightarrow \nu_{e}$ & 0.082 & $\nu_{e} \rightarrow \nu_{e}$ & 0.022 & $\nu_{\mu} \rightarrow \nu_{e}$ & 0.003 & $\nu_{e} \rightarrow \nu_{e}$ & 0.004 \\
\hline$\nu_{\mu} \rightarrow \nu_{\tau}$ & 0.345 & $\nu_{e} \rightarrow \nu_{\tau}$ & 0.001 & $\nu_{\mu} \rightarrow \nu_{\tau}$ & 0.107 & $\nu_{e} \rightarrow \nu_{\tau}$ & 0.000 \\
\hline $\bar{\nu}_{\mu} \rightarrow \bar{\nu}_{\mu}$ & 7.216 & $\bar{\nu}_{e} \rightarrow \bar{\nu}_{\mu}$ & 0.011 & $\bar{\nu}_{\mu} \rightarrow \bar{\nu}_{\mu}$ & 36.090 & $\bar{\nu}_{e} \rightarrow \bar{\nu}_{\mu}$ & 0.053 \\
\hline $\bar{\nu}_{\mu} \rightarrow \bar{\nu}_{e}$ & 0.001 & $\bar{\nu}_{e} \rightarrow \bar{\nu}_{e}$ & 0.000 & $\bar{\nu}_{\mu} \rightarrow \bar{\nu}_{e}$ & 0.005 & $\bar{\nu}_{e} \rightarrow \bar{\nu}_{e}$ & 0.002 \\
\hline $\bar{\nu}_{\mu} \rightarrow \bar{\nu}_{\tau}$ & 0.345 & $\bar{\nu}_{e} \rightarrow \bar{\nu}_{\tau}$ & 0.001 & $\bar{\nu}_{\mu} \rightarrow \bar{\nu}_{\tau}$ & 0.107 & $\bar{\nu}_{e} \rightarrow \bar{\nu}_{\tau}$ & 0.000 \\
\hline$\nu_{\mu}+\bar{\nu}_{\mu}$ signal & $\mathrm{NC}$ & Other beam bkg & Cosmics & $\nu_{\mu}+\bar{\nu}_{\mu}$ signal & $\mathrm{NC}$ & Other beam bkg & Cosmics \\
\hline 117.158 & 1.188 & 0.497 & 2.074 & 48.748 & 0.386 & 0.224 & 0.459 \\
\hline
\end{tabular}

Table 7.4: MC oscillation channel breakdown for the total FHC dataset and for the total RHC dataset. The $20188.85 \times 10^{20}$ POT FHC-only analysis best fit is used. 


\begin{tabular}{|c|c|c|c|c|c|c|c|}
\hline \multicolumn{4}{|c|}{ Lowest $E_{\text {had }} / E_{\nu}$ quantile (Q1) } & \multicolumn{4}{|c|}{ Second-Lowest $E_{\text {had }} / E_{\nu}$ quantile (Q2) } \\
\hline Channel & Selected events & Channel & Selected events & Channel & Selected events & Channel & Selected events \\
\hline$\nu_{\mu} \rightarrow \nu_{\mu}$ & 27.057 & $\nu_{e} \rightarrow \nu_{\mu}$ & 0.057 & $\nu_{\mu} \rightarrow \nu_{\mu}$ & 25.953 & $\nu_{e} \rightarrow \nu_{\mu}$ & 0.056 \\
\hline$\nu_{\mu} \rightarrow \nu_{e}$ & 0.000 & $\nu_{e} \rightarrow \nu_{e}$ & 0.000 & $\nu_{\mu} \rightarrow \nu_{e}$ & 0.001 & $\nu_{e} \rightarrow \nu_{e}$ & 0.000 \\
\hline$\nu_{\mu} \rightarrow \nu_{\tau}$ & 0.065 & $\nu_{e} \rightarrow \nu_{\tau}$ & 0.000 & $\nu_{\mu} \rightarrow \nu_{\tau}$ & 0.087 & $\nu_{e} \rightarrow \nu_{\tau}$ & 0.000 \\
\hline $\bar{\nu}_{\mu} \rightarrow \bar{\nu}_{\mu}$ & 3.138 & $\bar{\nu}_{e} \rightarrow \bar{\nu}_{\mu}$ & 0.005 & $\bar{\nu}_{\mu} \rightarrow \bar{\nu}_{\mu}$ & 1.754 & $\bar{\nu}_{e} \rightarrow \bar{\nu}_{\mu}$ & 0.003 \\
\hline $\bar{\nu}_{\mu} \rightarrow \bar{\nu}_{e}$ & 0.000 & $\bar{\nu}_{e} \rightarrow \bar{\nu}_{e}$ & 0.000 & $\bar{\nu}_{\mu} \rightarrow \bar{\nu}_{e}$ & 0.000 & $\bar{\nu}_{e} \rightarrow \bar{\nu}_{e}$ & 0.000 \\
\hline $\bar{\nu}_{\mu} \rightarrow \bar{\nu}_{\tau}$ & 0.065 & $\bar{\nu}_{e} \rightarrow \bar{\nu}_{\tau}$ & 0.000 & $\bar{\nu}_{\mu} \rightarrow \bar{\nu}_{\tau}$ & 0.087 & $\bar{\nu}_{e} \rightarrow \bar{\nu}_{\tau}$ & 0.000 \\
\hline$\nu_{\mu}+\bar{\nu}_{\mu}$ signal & $\mathrm{NC}$ & Other beam bkg & Cosmics & $\nu_{\mu}+\bar{\nu}_{\mu}$ signal & $\mathrm{NC}$ & Other beam bkg & Cosmics \\
\hline 30.257 & 0.052 & 0.080 & 0.612 & 27.767 & 0.097 & 0.102 & 0.204 \\
\hline \multicolumn{4}{|c|}{ Total: 31.001} & \multicolumn{4}{|c|}{ Total: 28.170} \\
\hline \multicolumn{4}{|c|}{ Second-Highest $E_{\text {had }} / E_{\nu}$ quantile (Q3) } & \multicolumn{4}{|c|}{ Highest $E_{\text {had }} / E_{\nu}$ quantile (Q4) } \\
\hline Channel & Selected events & Channel & Selected events & Channel & Selected events & Channel & Selected events \\
\hline$\nu_{\mu} \rightarrow \nu_{\mu}$ & 26.599 & $\nu_{e} \rightarrow \nu_{\mu}$ & 0.059 & $\nu_{\mu} \rightarrow \nu_{\mu}$ & 30.087 & $\nu_{e} \rightarrow \nu_{\mu}$ & 0.063 \\
\hline$\nu_{\mu} \rightarrow \nu_{e}$ & 0.008 & $\nu_{e} \rightarrow \nu_{e}$ & 0.002 & $\nu_{\mu} \rightarrow \nu_{e}$ & 0.072 & $\nu_{e} \rightarrow \nu_{e}$ & 0.020 \\
\hline$\nu_{\mu} \rightarrow \nu_{\tau}$ & 0.102 & $\nu_{e} \rightarrow \nu_{\tau}$ & 0.000 & $\nu_{\mu} \rightarrow \nu_{\tau}$ & 0.090 & $\nu_{e} \rightarrow \nu_{\tau}$ & 0.000 \\
\hline $\bar{\nu}_{\mu} \rightarrow \bar{\nu}_{\mu}$ & 1.498 & $\bar{\nu}_{e} \rightarrow \bar{\nu}_{\mu}$ & 0.002 & $\bar{\nu}_{\mu} \rightarrow \bar{\nu}_{\mu}$ & 0.825 & $\bar{\nu}_{e} \rightarrow \bar{\nu}_{\mu}$ & 0.002 \\
\hline $\bar{\nu}_{\mu} \rightarrow \bar{\nu}_{e}$ & 0.000 & $\bar{\nu}_{e} \rightarrow \bar{\nu}_{e}$ & 0.000 & $\bar{\nu}_{\mu} \rightarrow \bar{\nu}_{e}$ & 0.000 & $\bar{\nu}_{e} \rightarrow \bar{\nu}_{e}$ & 0.000 \\
\hline $\bar{\nu}_{\mu} \rightarrow \bar{\nu}_{\tau}$ & 0.102 & $\bar{\nu}_{e} \rightarrow \bar{\nu}_{\tau}$ & 0.000 & $\bar{\nu}_{\mu} \rightarrow \bar{\nu}_{\tau}$ & 0.090 & $\bar{\nu}_{e} \rightarrow \bar{\nu}_{\tau}$ & 0.000 \\
\hline$\nu_{\mu}+\bar{\nu}_{\mu}$ signal & $\mathrm{NC}$ & Other beam bkg & Cosmics & $\nu_{\mu}+\bar{\nu}_{\mu}$ signal & $\mathrm{NC}$ & Other beam bkg & Cosmics \\
\hline 28.158 & 0.224 & 0.123 & 0.170 & 30.976 & 0.815 & 0.192 & 1.088 \\
\hline \multicolumn{4}{|c|}{ Total: 28.675} & \multicolumn{4}{|c|}{ Total: 33.071} \\
\hline
\end{tabular}

Table 7.5: FHC MC oscillation channel breakdown for each $E_{\text {had }} / E_{\nu}$ quantile under the assumption the $20188.85 \times 10^{20}$ POT FHC-only analysis best fit. 


\begin{tabular}{|c|c|c|c|c|c|c|c|}
\hline \multicolumn{4}{|c|}{ Lowest $E_{\text {had }} / E_{\nu}$ quantile (Q1) } & \multicolumn{4}{|c|}{ Second-Lowest $E_{\text {had }} / E_{\nu}$ quantile (Q2) } \\
\hline Channel & Selected events & Channel & Selected events & Channel & Selected events & Channel & Selected events \\
\hline$\nu_{\mu} \rightarrow \nu_{\mu}$ & 2.065 & $\nu_{e} \rightarrow \nu_{\mu}$ & 0.004 & $\nu_{\mu} \rightarrow \nu_{\mu}$ & 2.676 & $\nu_{e} \rightarrow \nu_{\mu}$ & 0.006 \\
\hline$\nu_{\mu} \rightarrow \nu_{e}$ & 0.000 & $\nu_{e} \rightarrow \nu_{e}$ & 0.000 & $\nu_{\mu} \rightarrow \nu_{e}$ & 0.000 & $\nu_{e} \rightarrow \nu_{e}$ & 0.000 \\
\hline$\nu_{\mu} \rightarrow \nu_{\tau}$ & 0.011 & $\nu_{e} \rightarrow \nu_{\tau}$ & 0.000 & $\nu_{\mu} \rightarrow \nu_{\tau}$ & 0.023 & $\nu_{e} \rightarrow \nu_{\tau}$ & 0.000 \\
\hline $\bar{\nu}_{\mu} \rightarrow \bar{\nu}_{\mu}$ & 11.170 & $\bar{\nu}_{e} \rightarrow \bar{\nu}_{\mu}$ & 0.016 & $\bar{\nu}_{\mu} \rightarrow \bar{\nu}_{\mu}$ & 9.527 & $\bar{\nu}_{e} \rightarrow \bar{\nu}_{\mu}$ & 0.014 \\
\hline $\bar{\nu}_{\mu} \rightarrow \bar{\nu}_{e}$ & 0.000 & $\bar{\nu}_{e} \rightarrow \bar{\nu}_{e}$ & 0.000 & $\bar{\nu}_{\mu} \rightarrow \bar{\nu}_{e}$ & 0.000 & $\bar{\nu}_{e} \rightarrow \bar{\nu}_{e}$ & 0.000 \\
\hline $\bar{\nu}_{\mu} \rightarrow \bar{\nu}_{\tau}$ & 0.011 & $\bar{\nu}_{e} \rightarrow \bar{\nu}_{\tau}$ & 0.000 & $\bar{\nu}_{\mu} \rightarrow \bar{\nu}_{\tau}$ & 0.023 & $\bar{\nu}_{e} \rightarrow \bar{\nu}_{\tau}$ & 0.000 \\
\hline$\nu_{\mu}+\bar{\nu}_{\mu}$ signal & $\mathrm{NC}$ & Other beam bkg & Cosmics & $\nu_{\mu}+\bar{\nu}_{\mu}$ signal & $\mathrm{NC}$ & Other beam bkg & Cosmics \\
\hline 13.255 & 0.009 & 0.031 & 0.042 & 12.222 & 0.021 & 0.047 & 0.083 \\
\hline \multicolumn{4}{|c|}{ Total: 13.336} & \multicolumn{4}{|c|}{ Total: 12.372} \\
\hline \multicolumn{4}{|c|}{ Second-Highest $E_{\text {had }} / E_{\nu}$ quantile (Q3) } & \multicolumn{4}{|c|}{ Highest $E_{\text {had }} / E_{\nu}$ quantile (Q4) } \\
\hline Channel & Selected events & Channel & Selected events & Channel & Selected events & Channel & Selected events \\
\hline$\nu_{\mu} \rightarrow \nu_{\mu}$ & 3.111 & $\nu_{e} \rightarrow \nu_{\mu}$ & 0.007 & $\nu_{\mu} \rightarrow \nu_{\mu}$ & 4.726 & $\nu_{e} \rightarrow \nu_{\mu}$ & 0.011 \\
\hline$\nu_{\mu} \rightarrow \nu_{e}$ & 0.000 & $\nu_{e} \rightarrow \nu_{e}$ & 0.000 & $\nu_{\mu} \rightarrow \nu_{e}$ & 0.003 & $\nu_{e} \rightarrow \nu_{e}$ & 0.004 \\
\hline$\nu_{\mu} \rightarrow \nu_{\tau}$ & 0.033 & $\nu_{e} \rightarrow \nu_{\tau}$ & 0.000 & $\nu_{\mu} \rightarrow \nu_{\tau}$ & 0.040 & $\nu_{e} \rightarrow \nu_{\tau}$ & 0.000 \\
\hline $\bar{\nu}_{\mu} \rightarrow \bar{\nu}_{\mu}$ & 8.241 & $\bar{\nu}_{e} \rightarrow \bar{\nu}_{\mu}$ & 0.013 & $\bar{\nu}_{\mu} \rightarrow \bar{\nu}_{\mu}$ & 7.152 & $\bar{\nu}_{e} \rightarrow \bar{\nu}_{\mu}$ & 0.011 \\
\hline $\bar{\nu}_{\mu} \rightarrow \bar{\nu}_{e}$ & 0.000 & $\bar{\nu}_{e} \rightarrow \bar{\nu}_{e}$ & 0.000 & $\bar{\nu}_{\mu} \rightarrow \bar{\nu}_{e}$ & 0.005 & $\bar{\nu}_{e} \rightarrow \bar{\nu}_{e}$ & 0.002 \\
\hline $\bar{\nu}_{\mu} \rightarrow \bar{\nu}_{\tau}$ & 0.033 & $\bar{\nu}_{e} \rightarrow \bar{\nu}_{\tau}$ & 0.000 & $\bar{\nu}_{\mu} \rightarrow \bar{\nu}_{\tau}$ & 0.040 & $\bar{\nu}_{e} \rightarrow \bar{\nu}_{\tau}$ & 0.000 \\
\hline$\nu_{\mu}+\bar{\nu}_{\mu}$ signal & $\mathrm{NC}$ & Other beam bkg & Cosmics & $\nu_{\mu}+\bar{\nu}_{\mu}$ signal & $\mathrm{NC}$ & Other beam bkg & Cosmics \\
\hline 11.372 & 0.058 & 0.062 & 0.083 & 11.899 & 0.298 & 0.084 & 0.250 \\
\hline \multicolumn{4}{|c|}{ Total: 11.576} & \multicolumn{4}{|c|}{ Total: 12.531} \\
\hline
\end{tabular}

Table 7.6: RHC MC oscillation channel breakdown for each $E_{\text {had }} / E_{\nu}$ quantile under the assumption the $20188.85 \times 10^{20}$ POT FHC-only analysis best fit. 
NOvA's $20188.85 \times 10^{20}$ POT FHC-only [3] best fit predicts 121 candidate events, 730 in the absence of oscillations. 113 candidate events were observed. The cosmic and beam event backgrounds are 2.1 and 1.2 events respectively. The RHC best fit predicts 50 candidate events, 266 in the absence of oscillations. 65 candidate events were observed. The cosmic and beam event backgrounds are 0.5 and 0.6 events respectively.

Table 7.7 shows a quantitative breakdown of the data and oscillated prediction far detector event counts for each $E_{\text {had }} / E_{\nu}$ quantile. FHC is shown at the top, and RHC is shown at the bottom. Oscillation predictions are produced using NOvA's $20188.85 \times$ $10^{20}$ POT FHC-only [3] best fit point.

For FHC data the lowest and highest $E_{h a d} / E_{\nu}$ quantiles contain the most events at 32 and 30 respectively. The second lowest and second highest $E_{\text {had }} / E_{\nu}$ quantiles contain 25 and 26 events respectively. The expectation in each quantile, assuming oscillations at NOvA's $20188.85 \times 10^{20}$ POT FHC-only [3] best fit point, is greater than the actual number of events. This is most apparent in the 3 highest $E_{\text {had }} / E_{\nu}$ quantiles which each have 4 events fewer in data than in the prediction. The event counts are consistent with expectation within the $1 \sigma$ Poisson error.

For RHC data the lowest and second highest $E_{h a d} / E_{\nu}$ quantiles contain the most events at 17 and 19 respectively. The second lowest and highest $E_{\text {had }} / E_{\nu}$ quantiles contain 14 and 15 events respectively. The expectation in each quantile, assuming oscillations at NOvA's $20188.85 \times 10^{20}$ POT FHC-only [3] best fit point, is lower than the actual number of events. This is true in all $4 E_{h a d} / E_{\nu}$ quantiles. Each quantile holds 1-7 events more in data than predicted. The event counts are consistent with expectation within the $1 \sigma$ Poisson error.

The $\nu_{\mu}+\bar{\nu}_{\mu}$-CC energy distributions are shown for the MC prediction assuming no oscillation, for the MC prediction assuming the $20188.85 \times 10^{20}$ POT FHC-only [3] best fit point and for the FD data in Figure 7.45 for FHC and in Figure 7.46 for RHC. The unoscillated prediction, oscillated prediction and data distributions are shown for each $E_{\text {had }} / E_{\nu}$ quantile in Figure 7.47 for FHC and in Figure 7.48 for RHC. The $\frac{\text { oscillated }_{\text {Data/MC }}}{\text { unoscillated }}$ ratio plots are shown in Figure 7.49 for FHC and Figure 7.50 for RHC. Strong data-MC agreement is shown in all distributions. The statistical uncertainty in the $\frac{\text { oscillated }_{\text {Data }}}{\text { unoscillated }}$ ratio plot entirely covers the predicted $\frac{\text { oscillated }_{M C}}{\text { unoscillated }}$ ratio. 


\begin{tabular}{|c|cc|cc|}
\hline FHC & \multicolumn{2}{|c|}{ Events per quantile } & \multicolumn{2}{c|}{$\frac{2}{\text { Tonts per quantile }}$} \\
\hline$E_{\text {had }} / E_{\nu}$ quantile & Data & MC & Data & MC \\
\hline Lowest & 32 & 31.00 & 0.28 & 0.26 \\
Second Lowest Q2 & 25 & 28.17 & 0.22 & 0.23 \\
Second Highest Q3 & 26 & 28.68 & 0.23 & 0.24 \\
Highest Q4 & 30 & 33.07 & 0.27 & 0.27 \\
\hline Total & 113 & 120.92 & 1 & 1 \\
\hline
\end{tabular}

\begin{tabular}{|c|cc|cc|}
\hline RHC & \multicolumn{2}{|c|}{ Events per quantile } & \multicolumn{2}{c|}{$\frac{2}{\text { Total per quantile }}$} \\
\hline$E_{\text {had }} / E_{\nu}$ quantile & Data & $\mathrm{MC}$ & Data & $\mathrm{MC}$ \\
\hline Lowest & 17 & 13.34 & 0.26 & 0.27 \\
Second Lowest Q2 & 14 & 12.37 & 0.22 & 0.25 \\
Second Highest Q3 & 19 & 11.58 & 0.29 & 0.23 \\
Highest Q4 & 15 & 12.53 & 0.23 & 0.25 \\
\hline Total & 65 & 49.82 & 1 & 1 \\
\hline
\end{tabular}

Table 7.7: $\nu_{\mu}+\bar{\nu}_{\mu}$-CC candidate events in each $E_{h a d} / E_{\nu}$ quantile in data and MC. FHC is shown in the top table, RHC in the bottom. The predicted events are calculated assuming neutrino oscillations at NOvA's $20188.85 \times 10^{20}$ POT FHC-only [3] best fit point. The predicted events include both the beam and the cosmic ray background. 
Neutrino beam

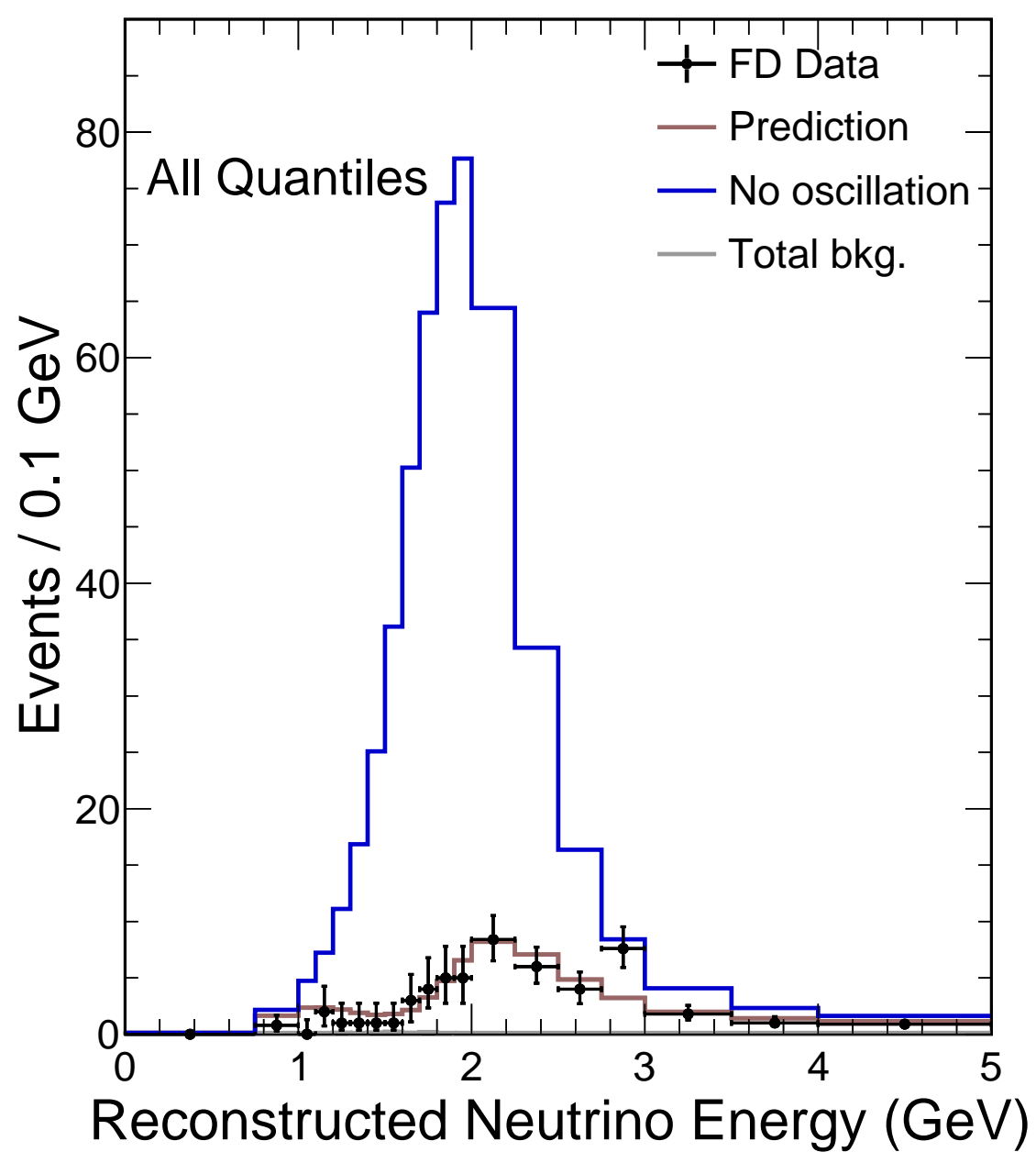

Figure 7.45: The FHC $\nu_{\mu}+\nu_{\mu}$-CC energy distributions shown for MC prediction assuming no oscillation, for MC prediction assuming the $20188.85 \times 10^{20}$ POT FHC-only [3] best fit point and for FD data. 
Antineutrino beam

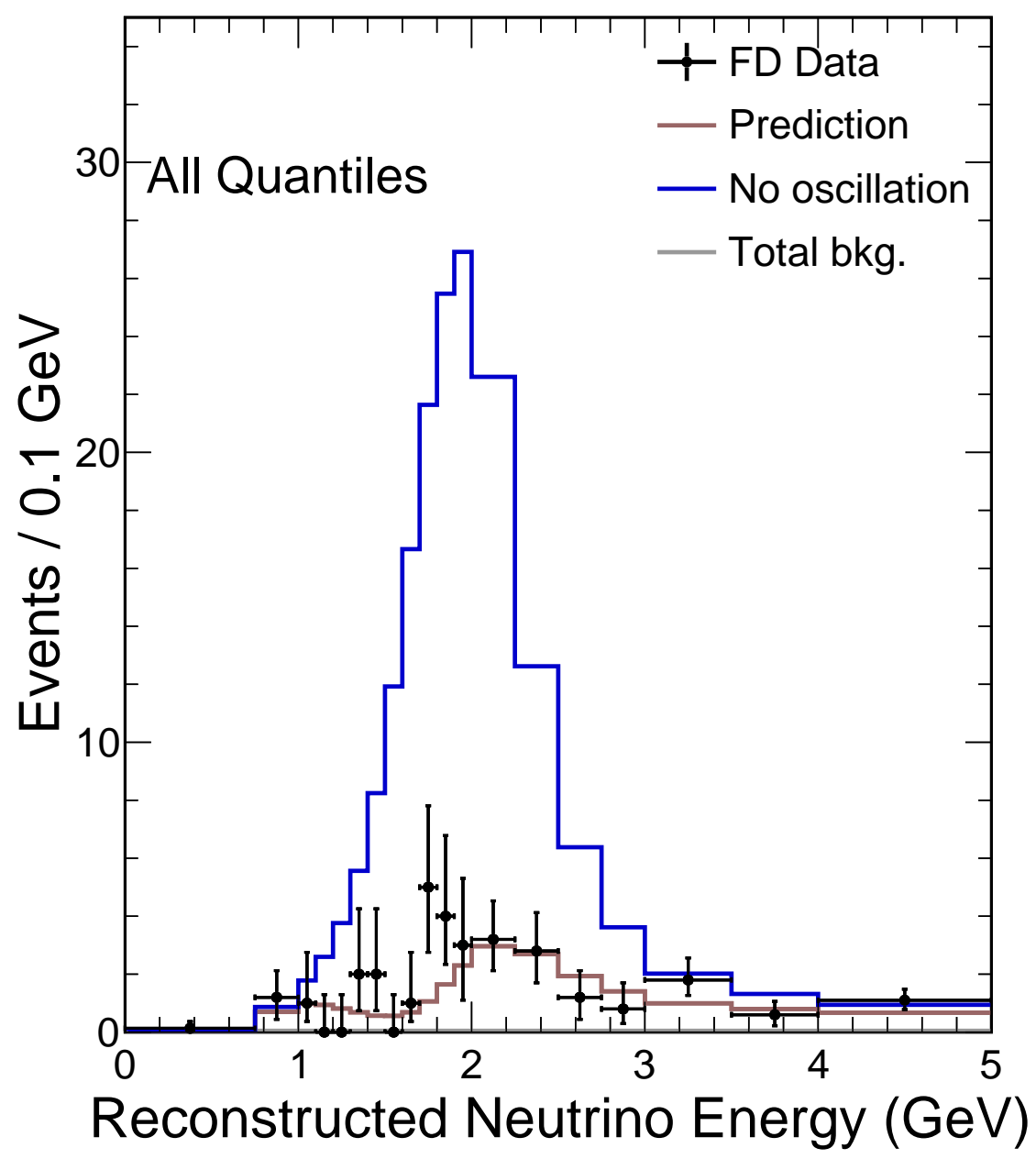

Figure 7.46: The RHC $\nu_{\mu}+\nu_{\mu}$-CC energy distributions shown for MC prediction assuming no oscillation, for MC prediction assuming the $20188.85 \times 10^{20}$ POT FHC-only [3] best fit point and for FD data. 


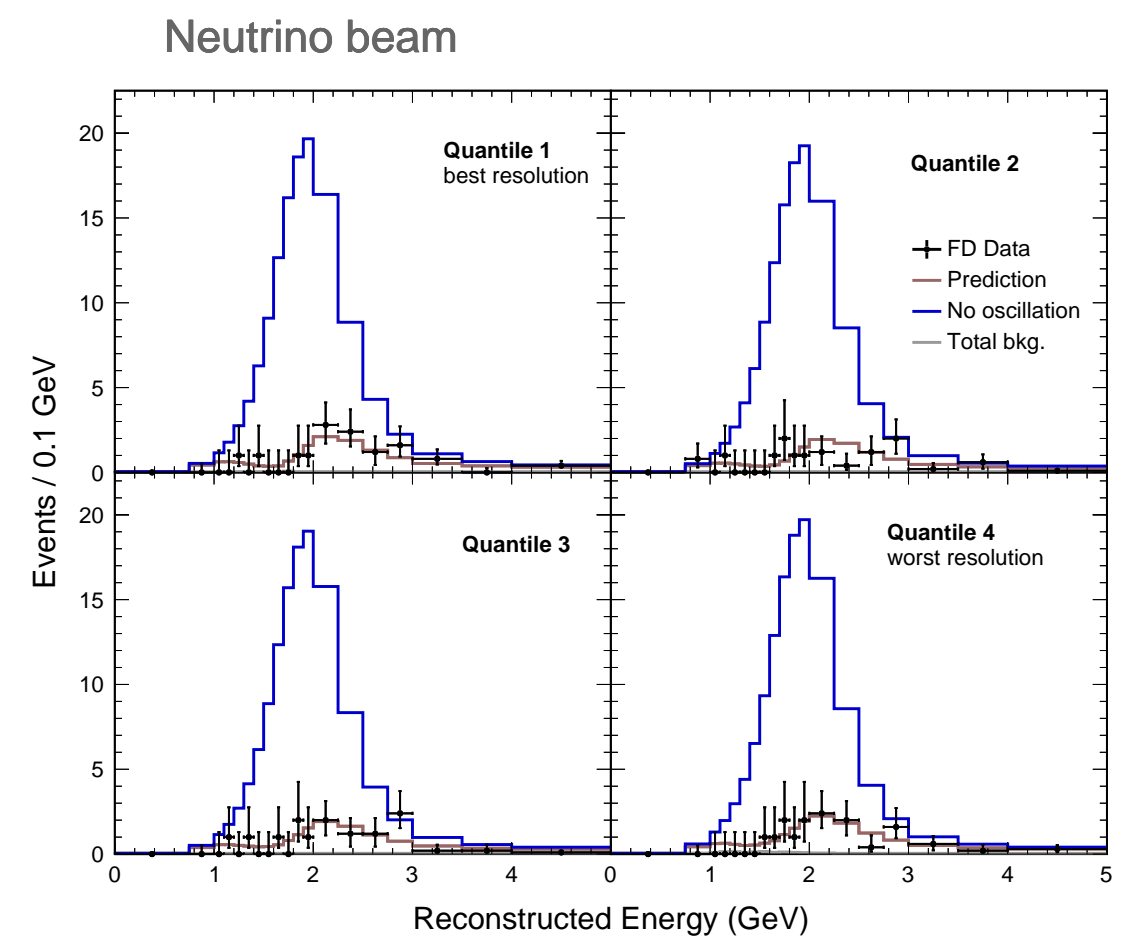

Figure 7.47: The RHC $\nu_{\mu}+\nu_{\mu}$-CC energy distributions shown for MC prediction assuming no oscillation, for MC prediction assuming the $20188.85 \times 10^{20}$ POT FHC-only [3] best fit point and for FD data.

\section{Antineutrino beam}

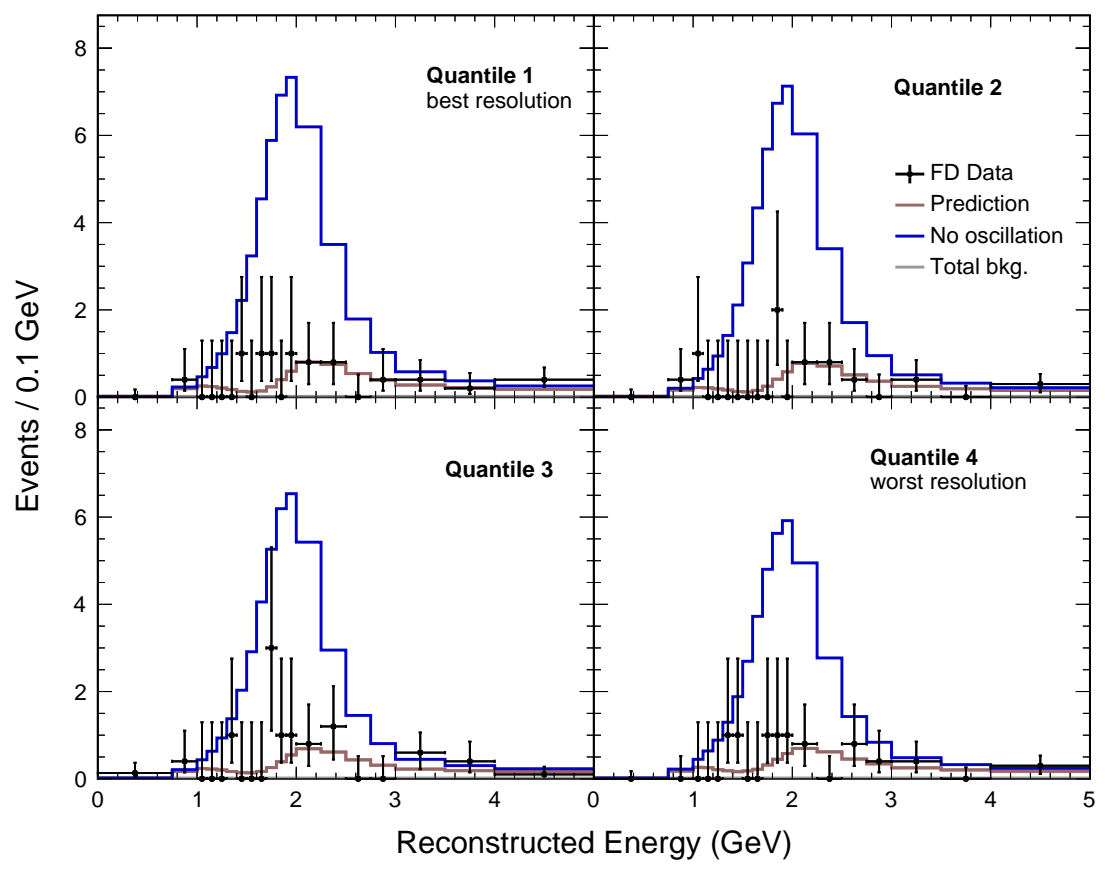

Figure 7.48: The RHC $\nu_{\mu}+\nu_{\mu}$-CC energy distributions shown for MC prediction assuming no oscillation, for MC prediction assuming the $20188.85 \times 10^{20}$ POT FHC-only [3] best fit point and for FD data. 


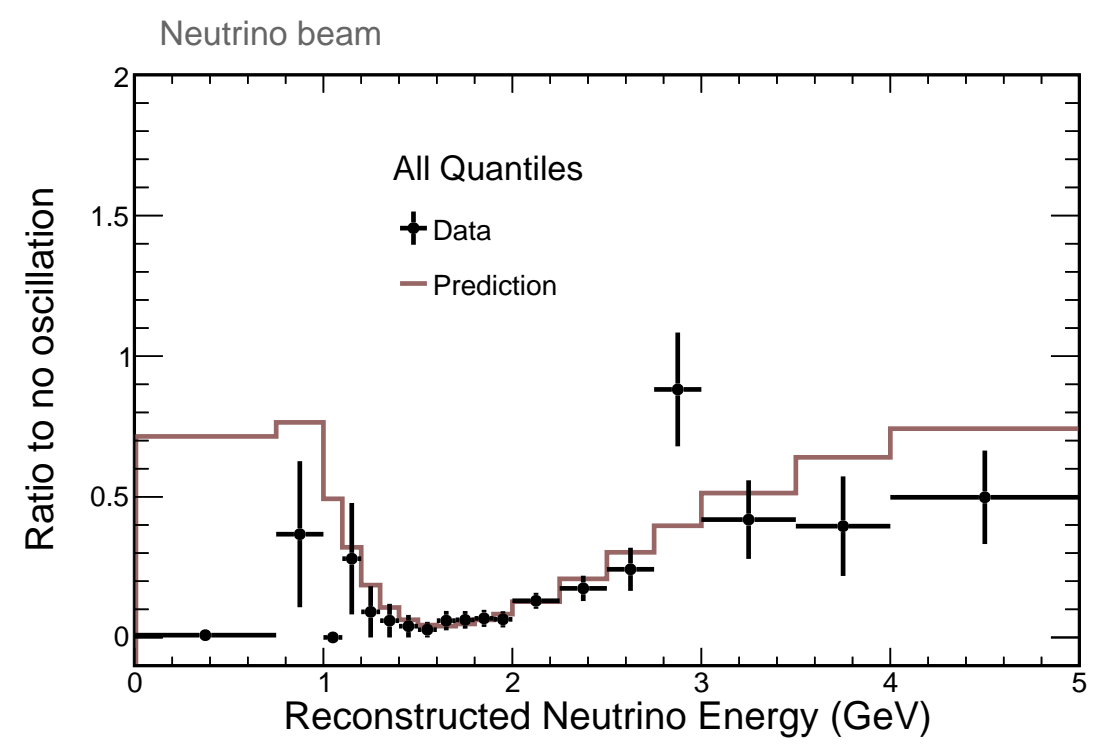

Figure 7.49: FHC FD ratios of the $\frac{\text { oscillated }_{M C}}{\text { unoscillated }} \nu_{\mu}+\nu_{\mu}$-CC distributions and of the $\frac{\text { oscillated }_{\text {Data }}}{\text { unoscillated }} \nu_{\mu}+\nu_{\mu}$-CC distributions.

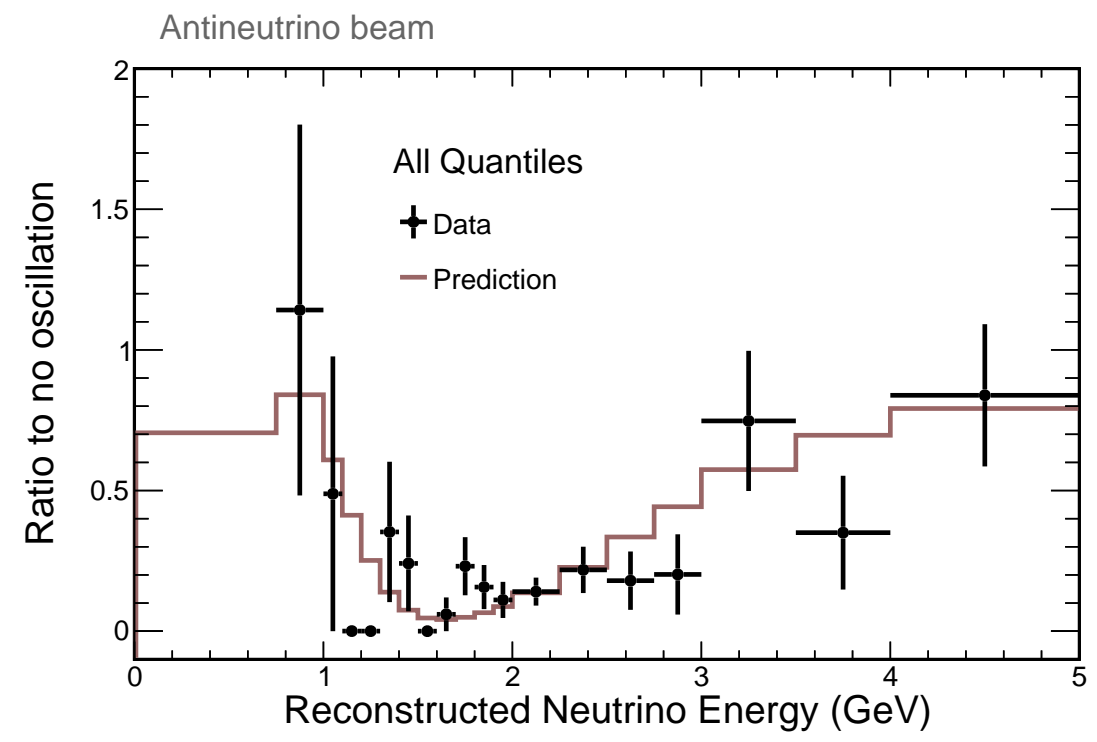

Figure 7.50: RHC FD ratios of the $\frac{\text { oscillated }_{M C}}{\text { unoscillated }_{\mu}} \nu_{\mu}+\nu_{\mu}$-CC distributions and of the $\frac{\text { oscillated }_{\text {Data }}}{\text { unoscillated }} \nu_{\mu}+\nu_{\mu}$-CC distributions. 


\subsubsection{Far Detector Distributions}

An oscillated MC prediction is used for comparison with the far detector data. The MC prediction is produced by means of near detector data-MC extrapolation. The simulated oscillations uses the best fit from NOvA's $20188.85 \times 10^{20}$ POT FHC-only [3] analysis.

Figures 7.51 through Figure 7.104 show a series of distributions corresponding to measurements of far detector data and MC. The most relevant analysis variables are assessed and distributions corresponding to the FHC and RHC data are shown separately. The overall distribution is given first, followed by the $E_{\text {had }} / E_{\nu}$ breakdown for both FHC and RHC. Each plot shares a common legend - far detector data is shown by the black points, simulation is shown by the red histogram, wrong-sign beam component is shown in blue, total background in gray and cosmic background in green. The systematic error band is not shown in the FD plots. This is due to the untenable computational load required to generate this error for the the large number of plots shown, for each quantile and for each horn current. Additionally, in the FD the statistical error in data far exceeds the prediction uncertainty due to the low statistics nature.

The presented plots are grouped into energy, track and particle identification distributions, which is the respective order in which they are presented. The energy plots concern the reconstructed neutrino, muonic and hadronic energies of candidate events. Also included are plots of the muonic energy per hit, the hadronic energy per hit and the hadronic fraction of total energy. The track plots concern the number of Kalman tracks identified and the angle of the primary Kalman track with respect to the beam. The particle identification plots concern the CVN and ReMId scoring variables. The 2017 and current CVN distributions are shown as both scores are selected on. The ReMId score and the likelihood variables that inform it are the last plots shown.

Energy Variables Figures 7.51 and 7.52 show the reconstructed neutrino energy distributions for the total FHC and RHC datasets respectively. The $E_{\text {had }} / E_{\nu}$ quantile distributions are shown in Figures 7.53 and 7.54 for FHC and RHC respectively. Both RHC and FHC exhibit good MC-data agreement, with almost all data errors encompassing the simulated prediction. The individual $E_{\text {had }} / E_{\nu}$ quantiles also exhibit good agreement for both RHC and FHC. For FHC the disagreement in the second-lowest $E_{h a d} / E_{\nu}$ quantile is worse than in the other quantiles with data consistently short of prediction in the $2-3 \mathrm{GeV}$ region.

Figures 7.55 and 7.56 show the reconstructed muon energy distributions for the total 
FHC and RHC datasets respectively. Figures 7.57 and 7.58 show the muon energy distributions for the individual $E_{\text {had }} / E_{\nu}$ quantiles for FHC and RHC respectively. Both RHC and FHC exhibit good MC-data agreement, with almost all statistical errors in data overlapping with the simulated prediction. For the individual $E_{h a d} / E_{\nu}$ quantiles agreement is also good for both RHC and FHC.

Figures 7.59 and 7.60 show the muon energy per hit distributions for the total FHC and RHC datasets respectively. The individual $E_{\text {had }} / E_{\nu}$ quantile breakdowns are shown in Figures 7.61 and 7.62 for FHC and RHC respectively. FHC exhibits good MC-data agreement, with almost all data errors encompassing the simulated prediction whereas RHC shows weaker agreement, with the peak of the data distribution slightly below that of prediction. The disparity observed in the ND muon energy per hit distribution cannot be resolved in the FD with the limited statistics available. For the individual $E_{h a d} / E_{\nu}$ quantiles agreement is good for both RHC and FHC. However, the two highest RHC quantiles show disagreement wherein the data is significantly above the prediction left of the peak.

Figures 7.63 and 7.64 show the hadronic energy distributions for the total FHC and RHC datasets respectively. Figure 7.65 and Figure 7.66 show the hadronic energy distributions for the individual $E_{\text {had }} / E_{\nu}$ quantiles for FHC and RHC respectively. For both the RHC and FHC total distributions the MC-data agreement is good. The individual $E_{\text {had }} / E_{\nu}$ quantiles also exhibit good agreement for both horn currents.

Figures 7.67 and 7.68 show the hadronic energy per hit distributions for the total FHC and RHC datasets respectively. Figures 7.69 and 7.70 show the FHC and RHC hadronic energy per hit distributions for the individual $E_{\text {had }} / E_{\nu}$ quantiles. For both RHC and FHC the total distribution agreement between MC and data is good. As is the case for the muonic component, the disparity observed in the ND hadronic energy per hit distribution cannot be resolved in the FD with the limited statistics available. For the individual $E_{\text {had }} / E_{\nu}$ quantiles agreement is also consistent with the statistical uncertainty.

Figures 7.71 and 7.72 show the $E_{h a d} / E_{\nu}$ distributions for the total FHC and RHC datasets respectively. Figures 7.73 and 7.74 show the $E_{h a d} / E_{\nu}$ distributions for the individual $E_{\text {had }} / E_{\nu}$ quantiles for FHC and RHC respectively. RHC and FHC both exhibit good agreement in the overall distributions. The individual $E_{h a d} / E_{\nu}$ quantiles also exhibit good agreement with no discernible drop in agreeement in any given quantile.

Track Variables Two track variables are shown - the number of tracks in the slice and the angle of the primary Kalman track with respect to the beam. 
The number of tracks in the slice is shown in Figure 7.75 and 7.76 for FHC and RHC respectively. The per $E_{h a d} / E_{\nu}$ quantile breakdowns are given in Figure 7.77 and 7.78 for FHC and RHC respectively

The distributions of the angle of the primary Kalman track with respect to the beam is shown in Figure 7.79 and 7.80 for FHC and RHC respectively. The per $E_{h a d} / E_{\nu}$ quantile breakdowns are given in Figure 7.81 and 7.82 for FHC and RHC respectively

For both horn currents the two track variables show decent data-MC agreement across all quantiles with few instances of the statistical error not encompassing the prediction.

PID Variables Two particle identification scores, CVN and ReMId, are assessed. Both the 2017 and 2018 CVN variables are shown. The ReMId score is shown as well as three of the variables that are used as inputs to it - the $\frac{d E}{d x}$ likelihood, the scattering angle likelihood and the fraction of planes along the track consistent with having minimum ionizinglike $\frac{d E}{d x}$.

The 2018 CVN score is shown for FHC and RHC in Figure 7.83 and Figure 7.84 respectively. The per quantile breakdowns follow for FHC and RHC in Figure 7.85 and Figure 7.86 respectively. There is universal mild to good agreement between data and simulation in all quantiles for both horn currents with few statistical errors not encompassing the prediction.

The 2017 CVN score is shown for FHC and RHC in Figure 7.87 and Figure 7.88 respectively. The per quantile breakdowns follow for FHC and RHC in Figure 7.89 and Figure 7.90 respectively. There is universal good agreement between data and simulation in all quantiles for both horn currents. As with the 2018 CVN score few statistical errors do not encompass the prediction.

The ReMId score distributions are shown for FHC and RHC in Figure 7.91 and Figure 7.92 respectively. The $E_{h a d} / E_{\nu}$ quantile plots follow in Figure 7.93 and Figure 7.94 for FHC and RHC. Agreement between data and simulation is good across the board with few instances of the prediction not being covered by the statistical error.

The three variables used as inputs to the ReMId score - the scattering likelihod, the $\frac{d E}{d x}$ likelihood, and the fraction of planes along the track consistent with having minimum ionizinglike $\frac{d E}{d x}$ are shown for FHC(RHC) in Figure 7.95(Figure 7.96), Figure 7.99(Figure 7.100) and Figure 7.103(Figure 7.104) respectively.

The scattering likelihood, $\frac{d E}{d x}$ likelihood, and fraction of planes along the track consistent with having minimum ionizinglike $\frac{d E}{d x}$ distributions are shown for each $E_{\text {had }} / E_{\nu}$ quantile in Figure 7.97(Figure 7.98), Figure 7.101(Figure 7.102) and Figure 7.105(Fig- 
ure 7.106) for $\mathrm{FHC}(\mathrm{RHC})$ respectively.

The ReMId training variables generally exhibit mild to good data-MC agreement but there are notable disparities. The FHC and RHC total distributions and per quantile scattering likelihood plots all exhibit mild to good data-MC agreement but the agreement is slightly weaker for RHC. In the overall plots the prediction mildy overestimates scattering angle likelihood for RHC. Agreement in the less statistically limited FHC data is tighter. The overall and per quantile $\frac{d E}{d x}$ likelihood plots all exhibit a good data-MC agreement in FHC and mild to weak agreement in RHC. The total and per quantile fraction of planes along the track consistent with having minimum ionizinglike $\frac{d E}{d x}$ plots all exhibit a good data-MC agreement in FHC and in RHC.

Summary Generally all FD distributions show good data-MC agreement. The reconstructed neutrino, muon and hadronic energy variables, which are chiefly important to the analysis, exhibit agreement across both horn currents and all quantiles. Disparities in the ReMId training variables are not manifest in the agreement of the ReMId score itself, which is good for both horn currents.

In general, the RHC distributions show more tension between data and MC. However no disagreements are significant enough for concern and the RHC exposure is less than half of the equivalent FHC exposure. Any disagreement is likely to lessen as the experiment collects more data and upgrades to the beam and to the analysis are performed. The most important variables to accurately reconstruct in a LBL neutrino experiment are the neutrino energy, hadronic energy and muonic energy. Within the statistical limit of the data there is currently good agreement in all of the reconstructed energy variables. 


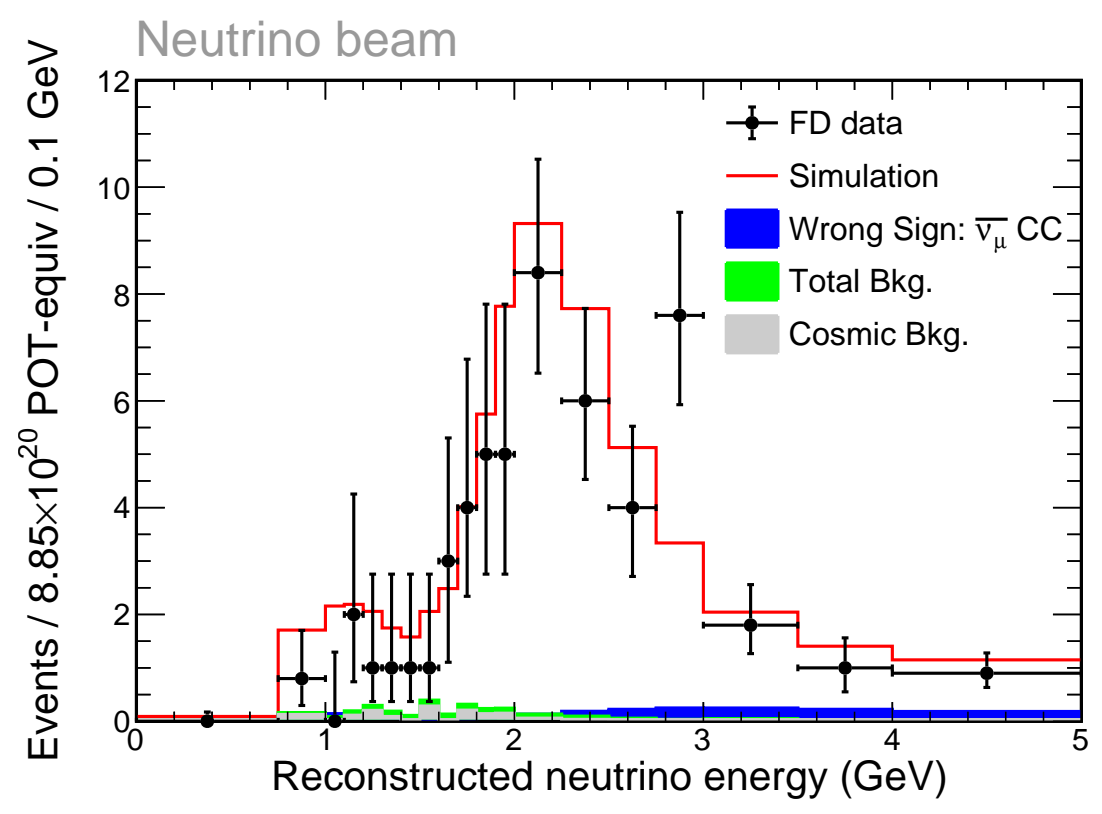

Figure 7.51: FHC FD reconstructed $\nu_{\mu}$-energy distributions for the total dataset. Data is shown in black and simulation in red. Also shown is the wrong-sign $\bar{\nu}_{\mu}$ component in blue, the cosmic background in grey and the total background in green.

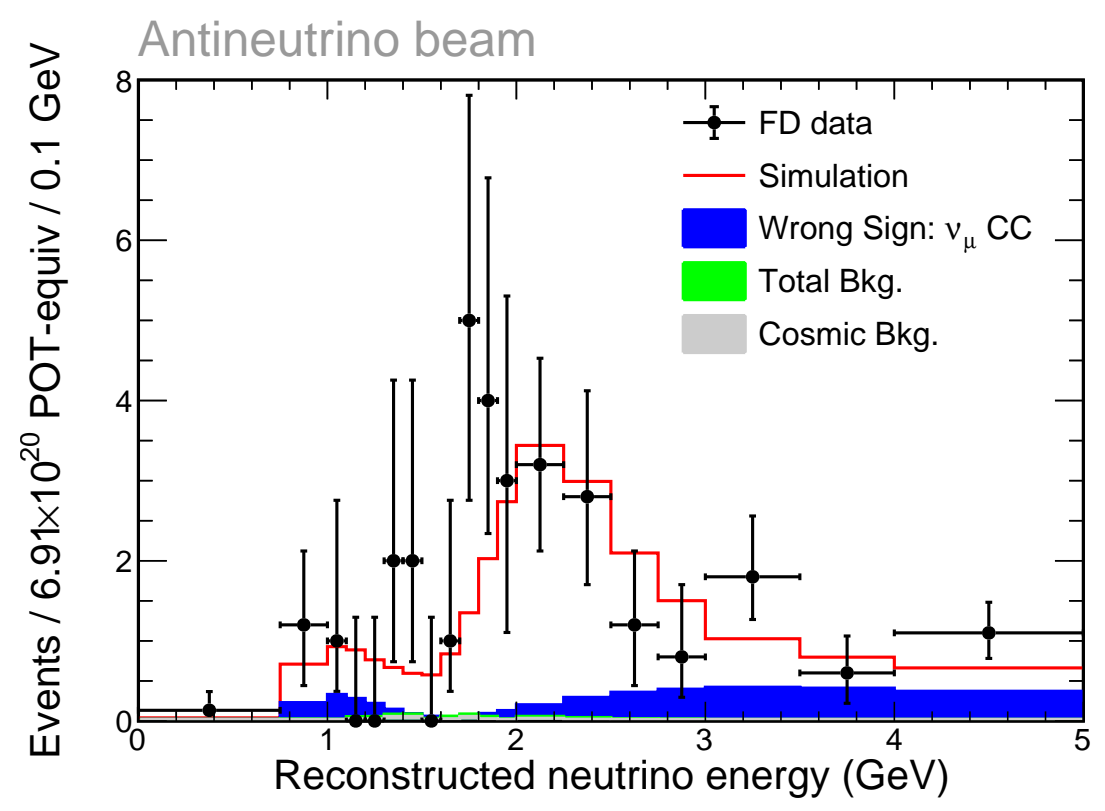

Figure 7.52: RHC FD reconstructed $\bar{\nu}_{\mu}$-energy distributions for the total dataset. Data is shown in black and simulation in red. Also shown is the wrong-sign $\nu_{\mu}$ component in blue, the cosmic background in grey and the total background in green. 


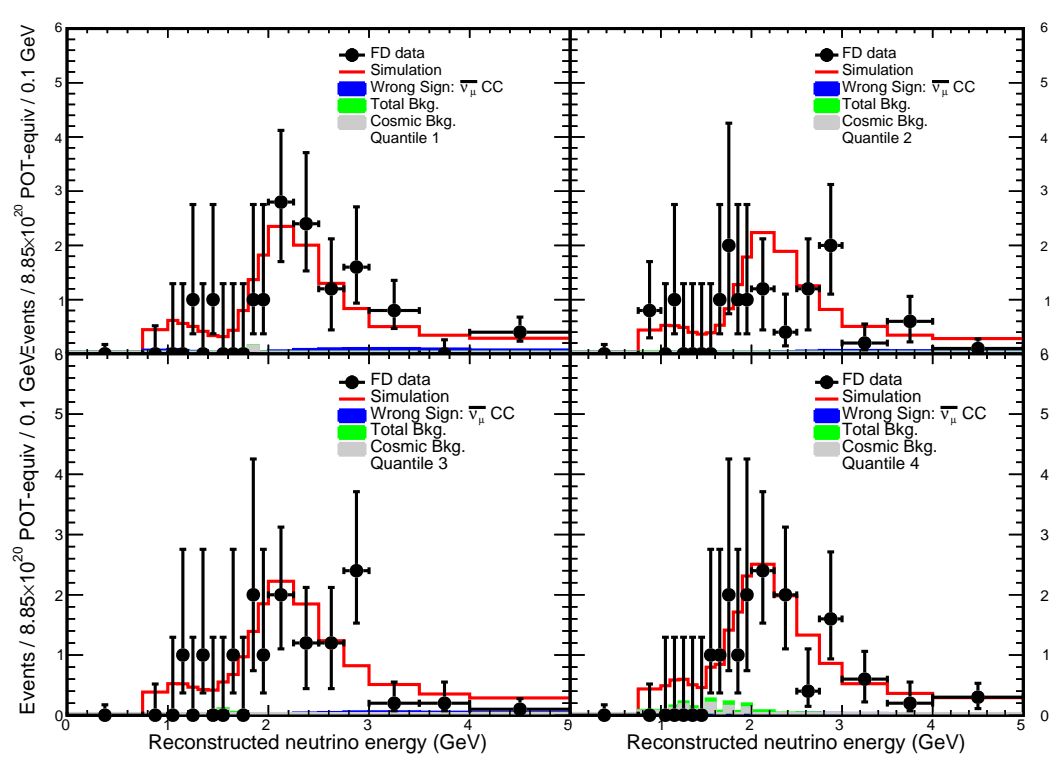

Figure 7.53: FHC FD reconstructed $\nu_{\mu}$-energy distributions for each $E_{h a d} / E_{\nu}$ quantile. Data is shown in black and simulation in red. Also shown is the wrong-sign $\bar{\nu}_{\mu}$ component in blue, the cosmic background in grey and the total background in green.

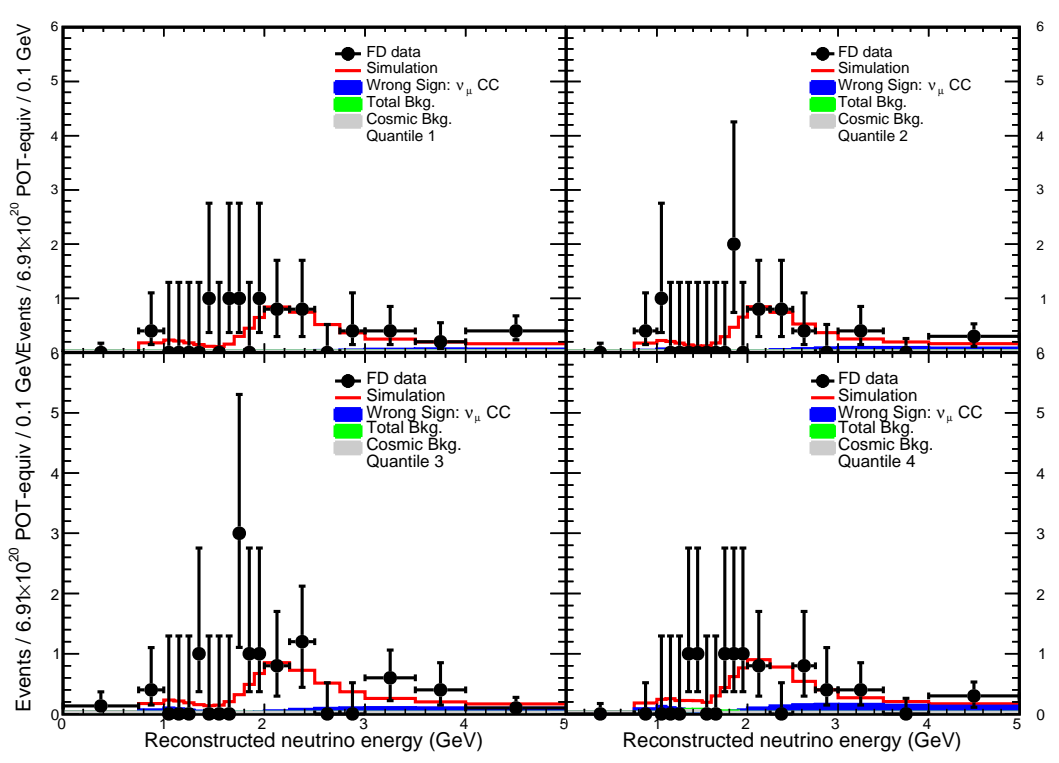

Figure 7.54: RHC FD reconstructed $\bar{\nu}_{\mu}$-energy distributions for each $E_{h a d} / E_{\nu}$ quantile. Data is shown in black and simulation in red. Also shown is the wrong-sign $\nu_{\mu}$ component in blue, the cosmic background in grey and the total background in green. 


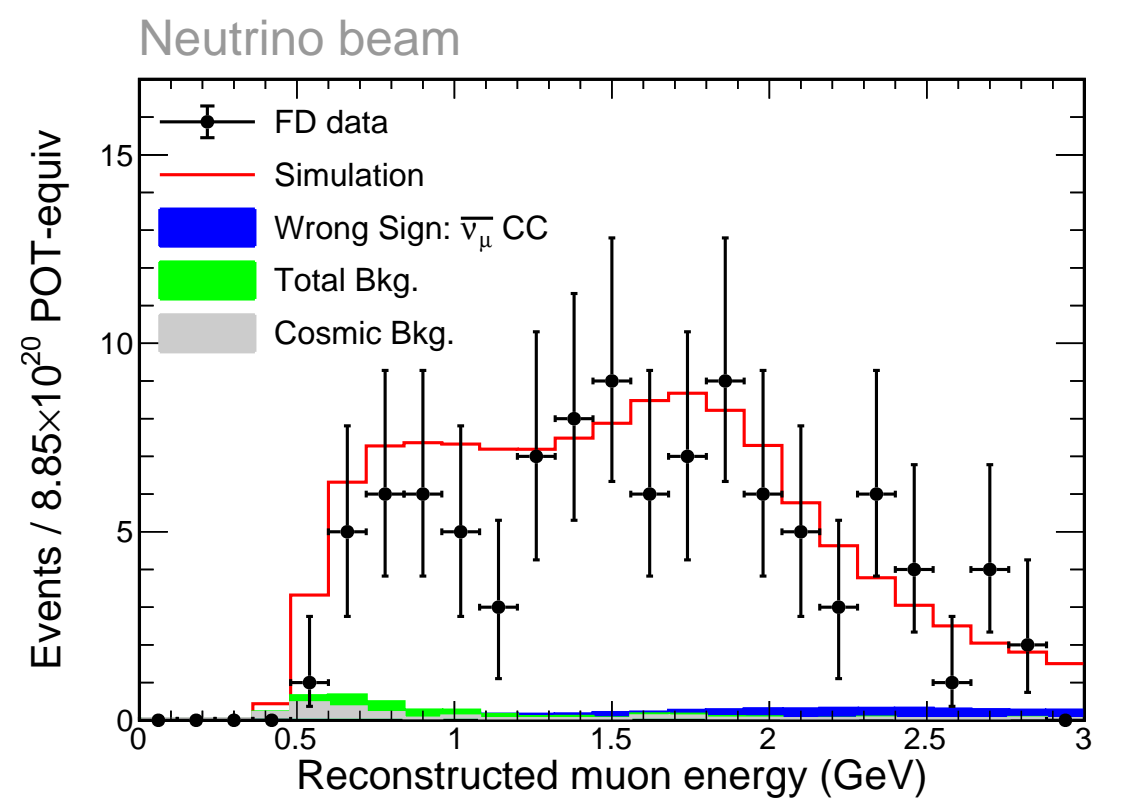

Figure 7.55: FHC FD reconstructed $\mu^{-}$-energy distributions for the total dataset. Data is shown in black and simulation in red. Also shown is the wrong-sign $\bar{\nu}_{\mu}$ component in blue, the cosmic background in grey and the total background in green.

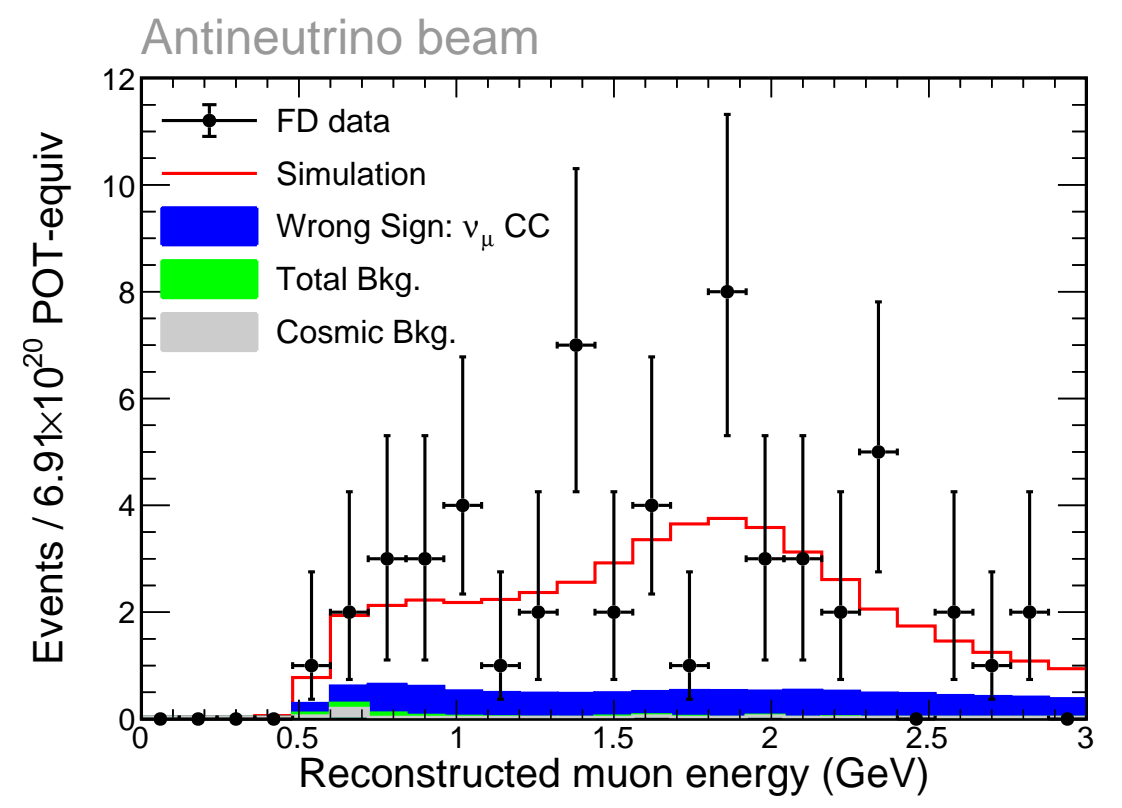

Figure 7.56: RHC FD reconstructed $\mu^{+}$-energy distributions for the total dataset. Data is shown in black and simulation in red. Also shown is the wrong-sign $\nu_{\mu}$ component in blue, the cosmic background in grey and the total background in green. 


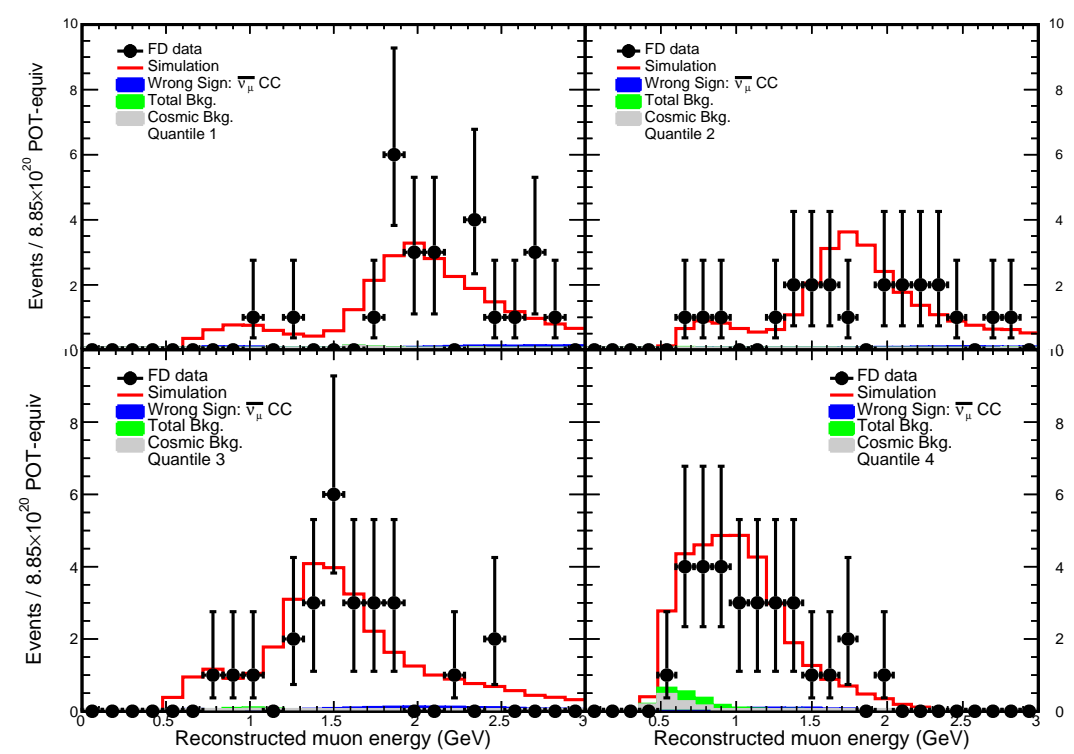

Figure 7.57: FHC FD reconstructed $\mu^{-}$-energy distributions for each $E_{h a d} / E_{\nu}$ quantile. Data is shown in black and simulation in red. Also shown is the wrong-sign $\bar{\nu}_{\mu}$ component in blue, the cosmic background in grey and the total background in green.

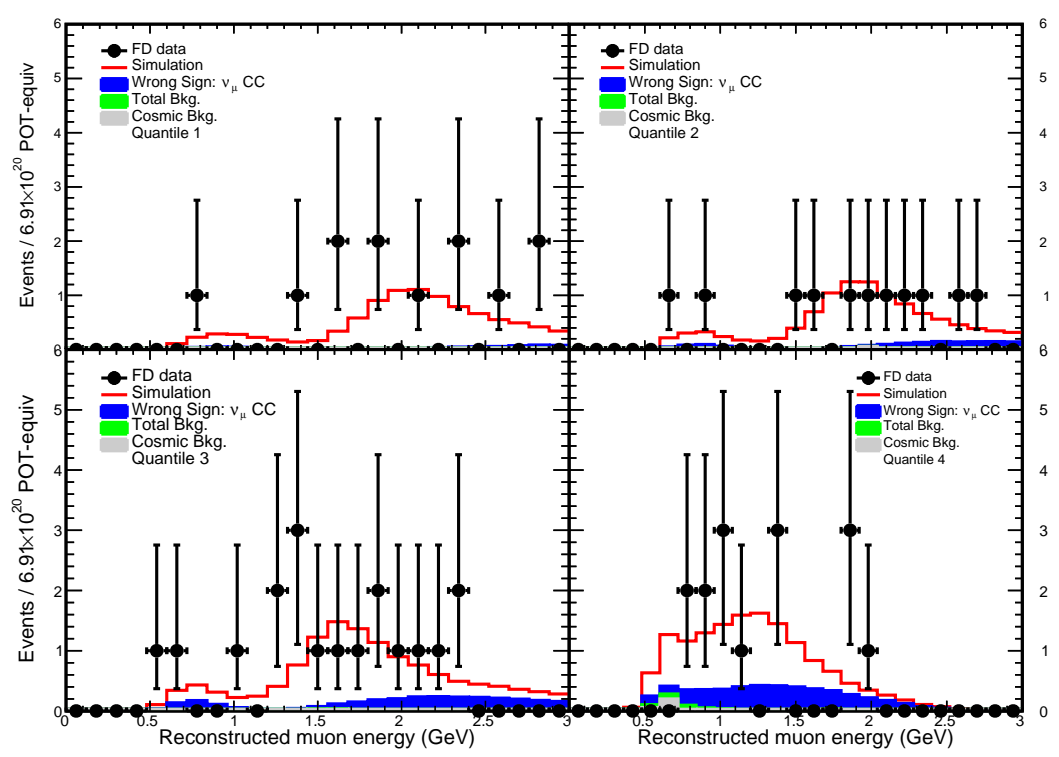

Figure 7.58: RHC FD reconstructed $\mu^{+}$-energy distributions for each $E_{\text {had }} / E_{\nu}$ quantile. Data is shown in black and simulation in red. Also shown is the wrong-sign $\nu_{\mu}$ component in blue, the cosmic background in grey and the total background in green. 


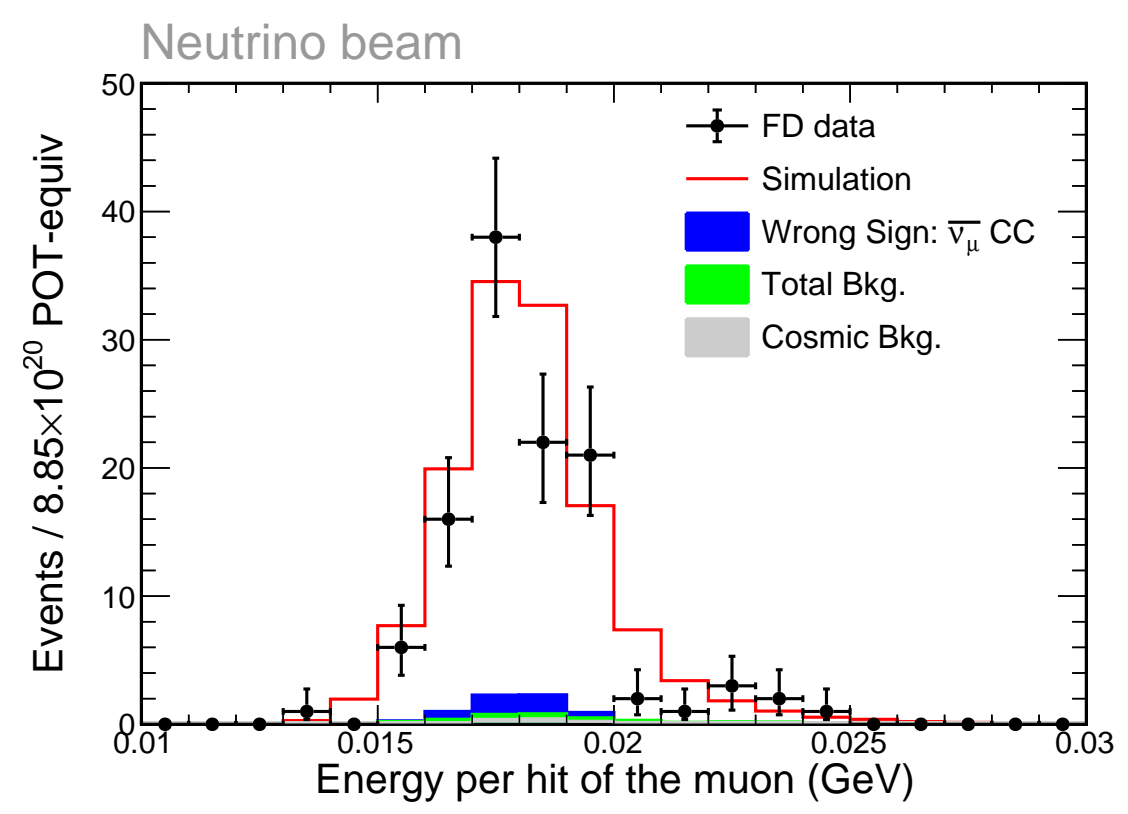

Figure 7.59: FHC FD reconstructed $\mu^{-}$-energy per hit distributions for the total dataset. Data is shown in black and simulation in red. Also shown is the wrong-sign $\bar{\nu}_{\mu}$ component in blue, the cosmic background in grey and the total background in green.

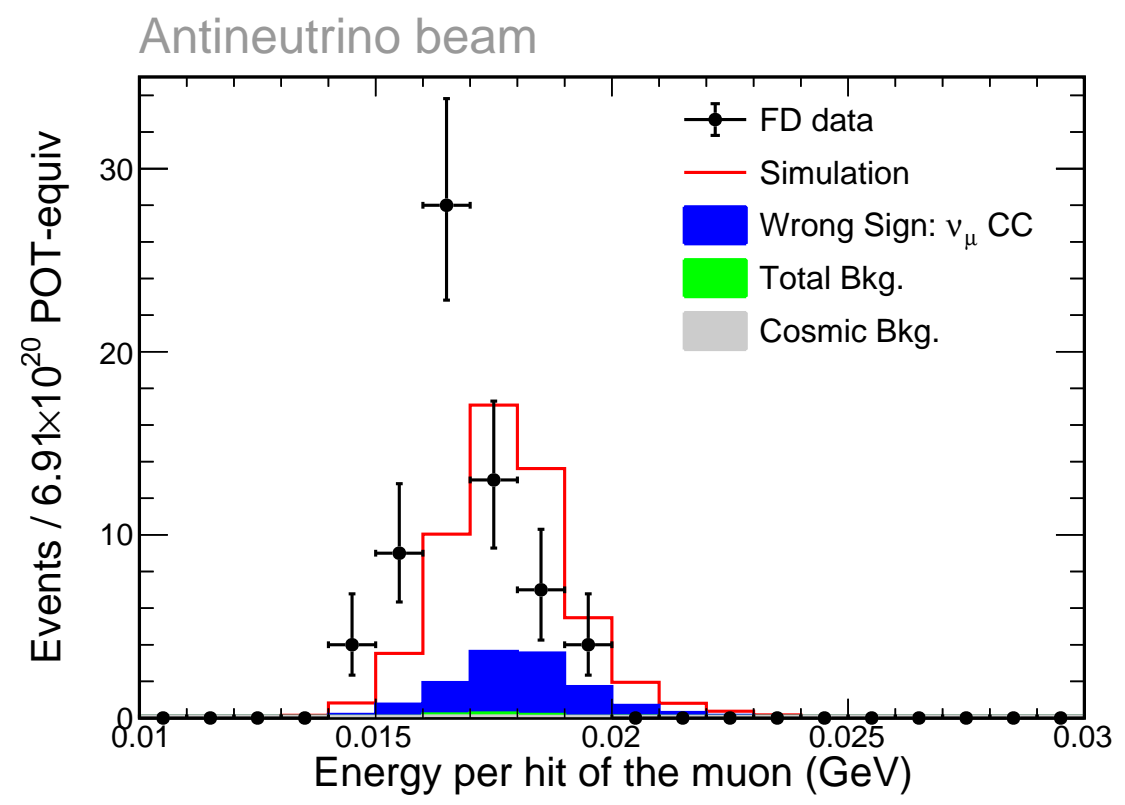

Figure 7.60: RHC FD reconstructed $\mu^{+}$-energy per hit distributions Data is shown in black and simulation in red. Also shown is the wrong-sign $\nu_{\mu}$ component in blue, the cosmic background in grey and the total background in green. 


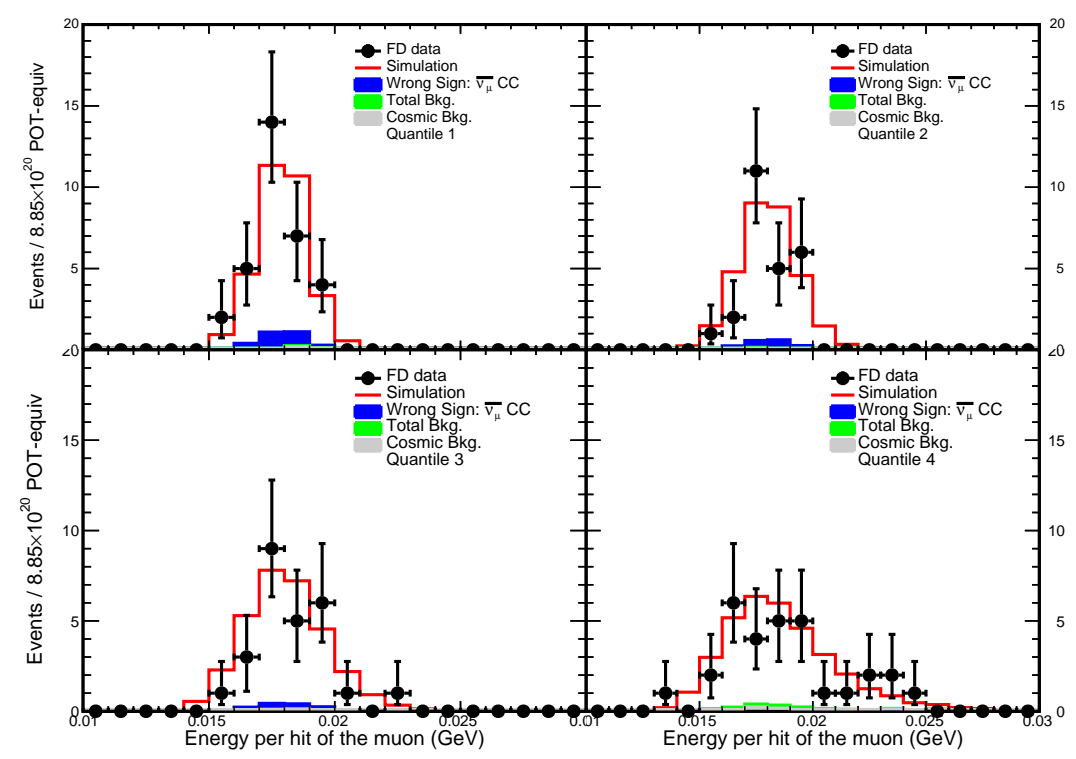

Figure 7.61: FHC FD reconstructed $\mu^{-}$-energy per hit distributions for each $E_{h a d} / E_{\nu}$ quantile. Data is shown in black and simulation in red. Also shown is the wrong-sign $\bar{\nu}_{\mu}$ component in blue, the cosmic background in grey and the total background in green.

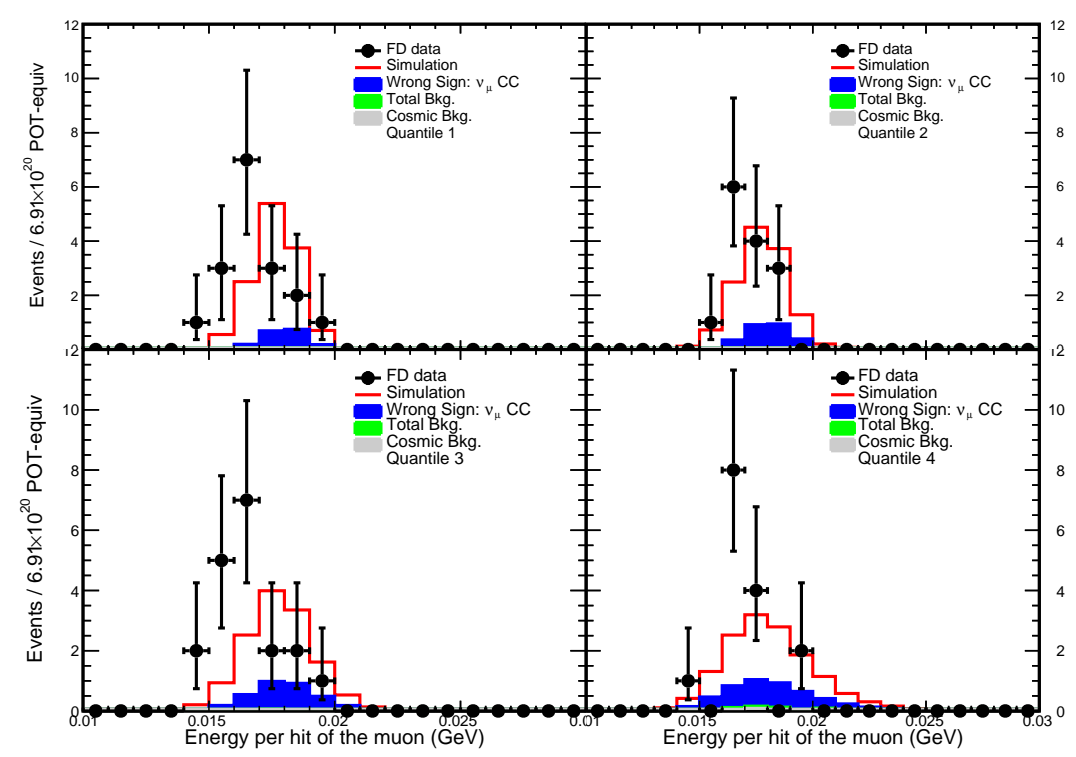

Figure 7.62: RHC FD reconstructed $\mu^{+}$-energy per hit distributions for each $E_{h a d} / E_{\nu}$ quantile. Data is shown in black and simulation in red. Also shown is the wrong-sign $\nu_{\mu}$ component in blue, the cosmic background in grey and the total background in green. 


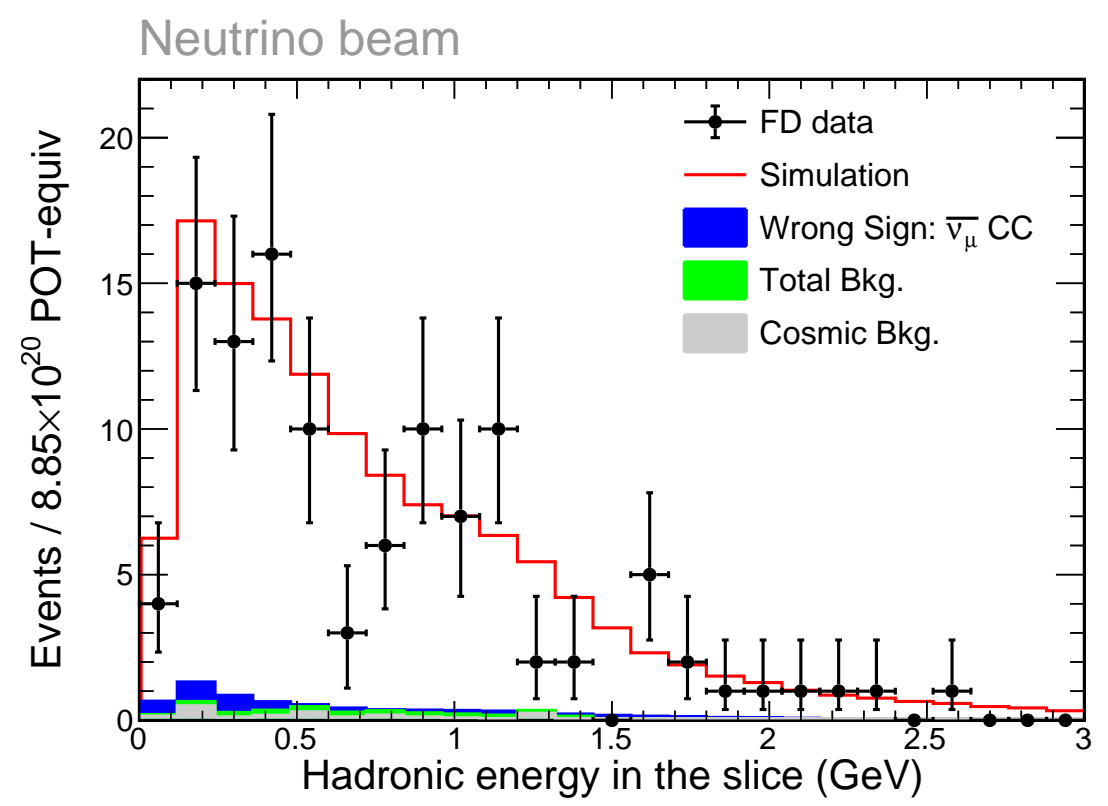

Figure 7.63: FHC FD reconstructed hadronic-energy distributions for the total dataset. Data is shown in black and simulation in red. Also shown is the wrong-sign $\bar{\nu}_{\mu}$ component in blue, the cosmic background in grey and the total background in green.

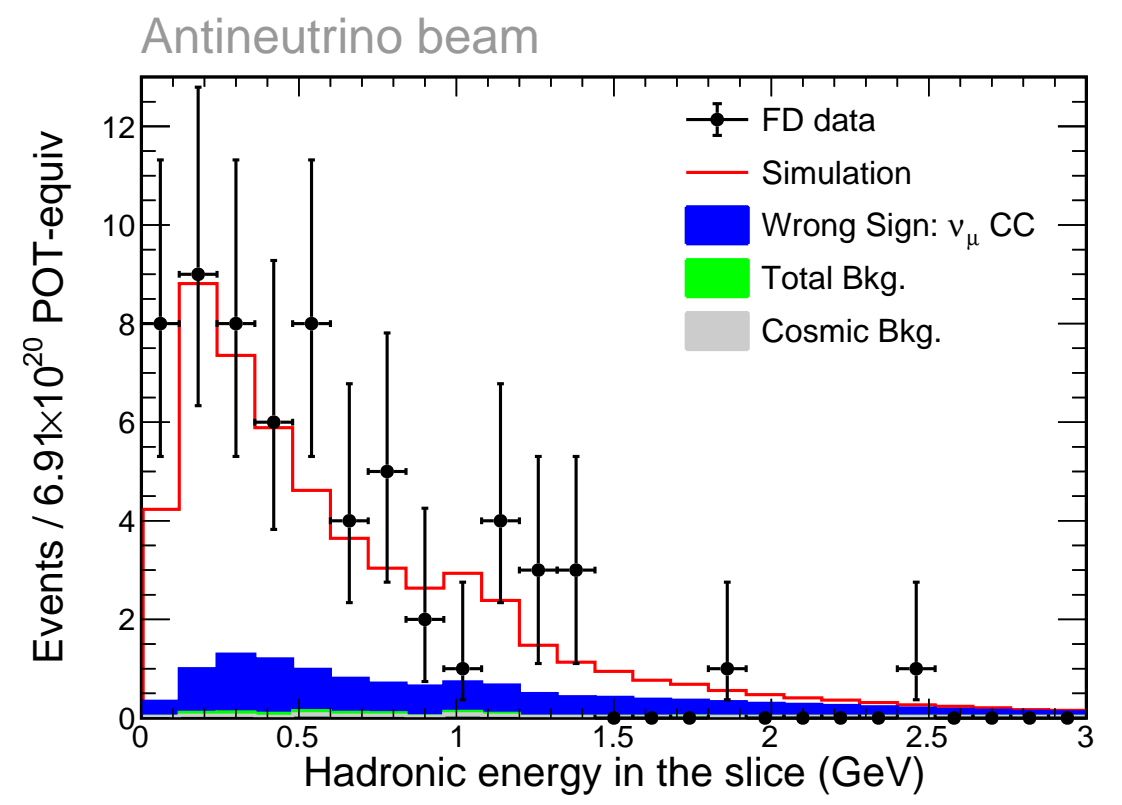

Figure 7.64: RHC FD reconstructed hadronic-energy distributions for the total dataset. Data is shown in black and simulation in red. Also shown is the wrong-sign $\nu_{\mu}$ component in blue, the cosmic background in grey and the total background in green. 


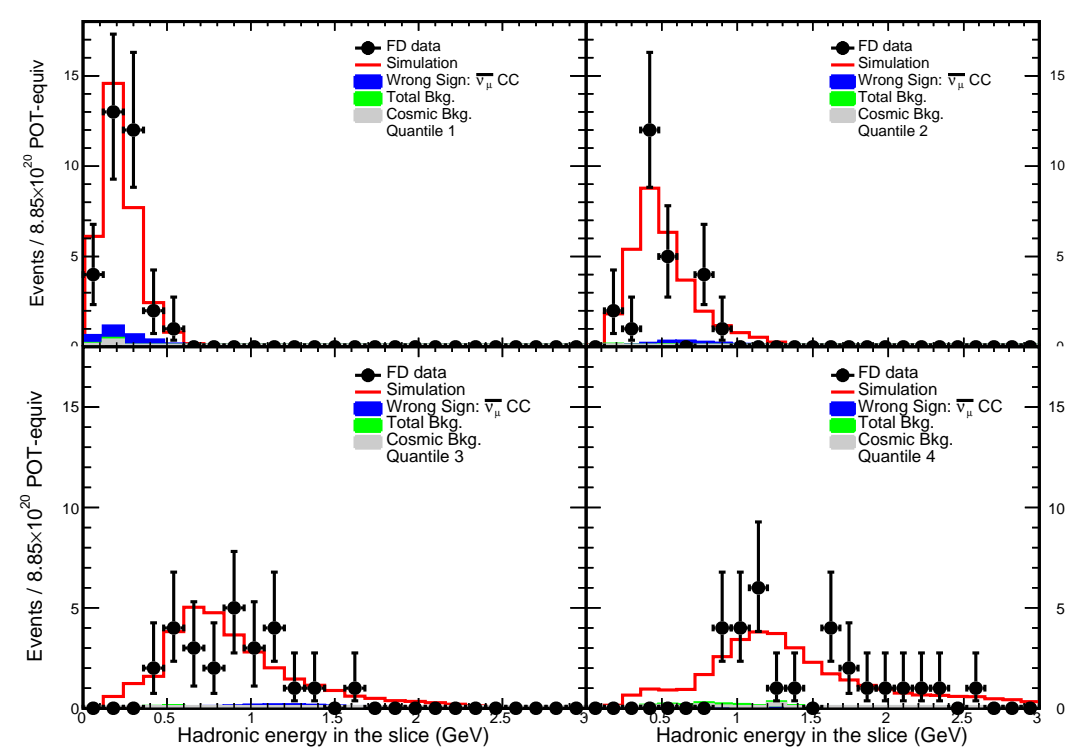

Figure 7.65: FHC FD reconstructed hadronic-energy distributions for each $E_{h a d} / E_{\nu}$ quantile. Data is shown in black and simulation in red. Also shown is the wrong-sign $\bar{\nu}_{\mu}$ component in blue, the cosmic background in grey and the total background in green.

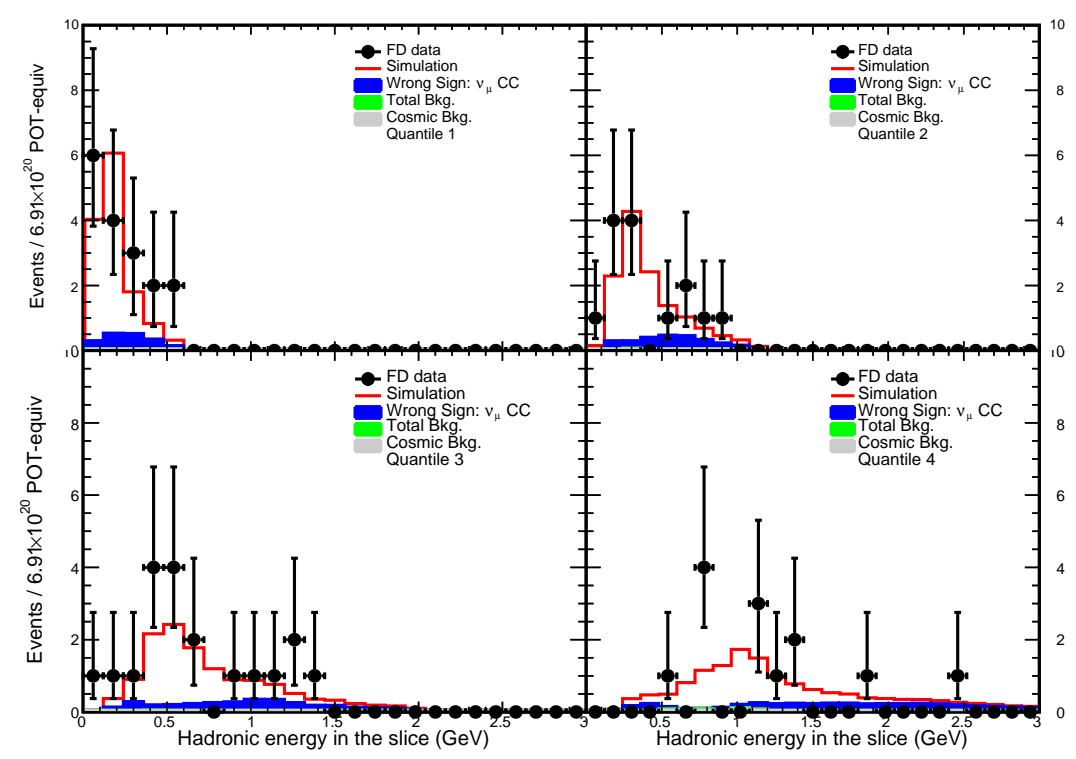

Figure 7.66: RHC FD reconstructed hadronic-energy distributions for each $E_{h a d} / E_{\nu}$ quantile. Data is shown in black and simulation in red. Also shown is the wrong-sign $\nu_{\mu}$ component in blue, the cosmic background in grey and the total background in green. 


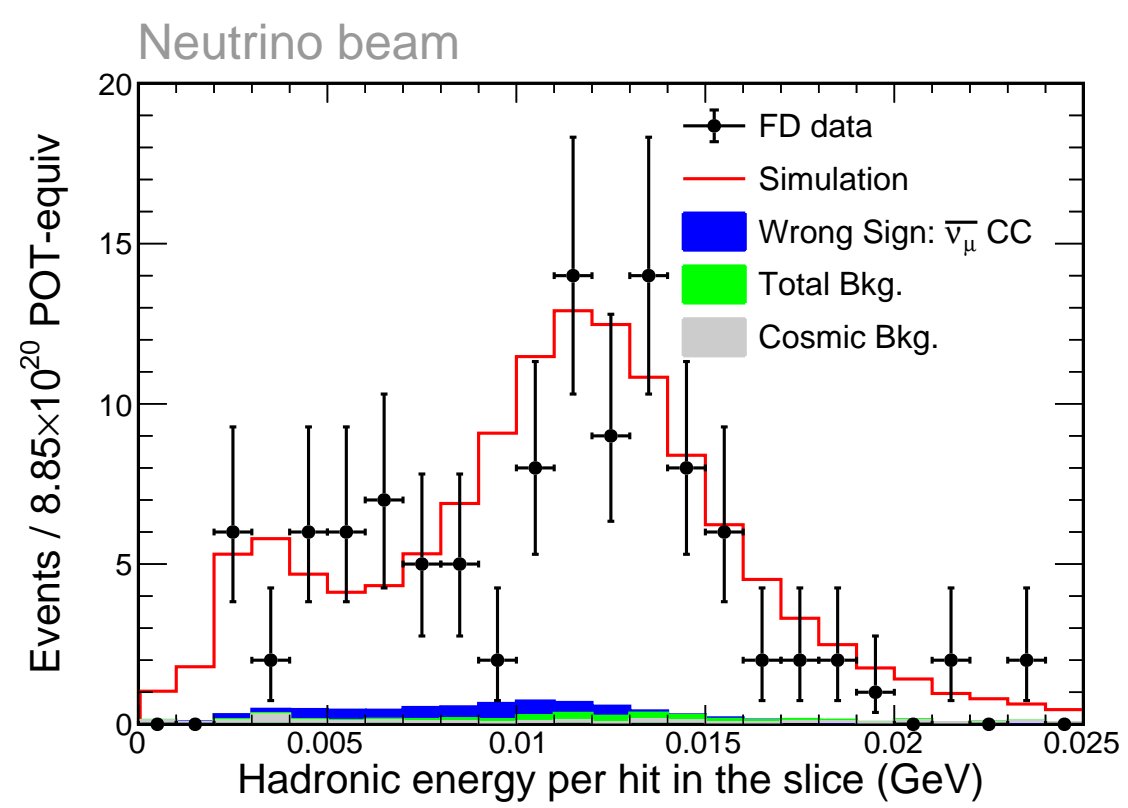

Figure 7.67: FHC FD reconstructed hadronic-energy per hit distributions for the total dataset. Data is shown in black and simulation in red. Also shown is the wrong-sign $\bar{\nu}_{\mu}$ component in blue, the cosmic background in grey and the total background in green.

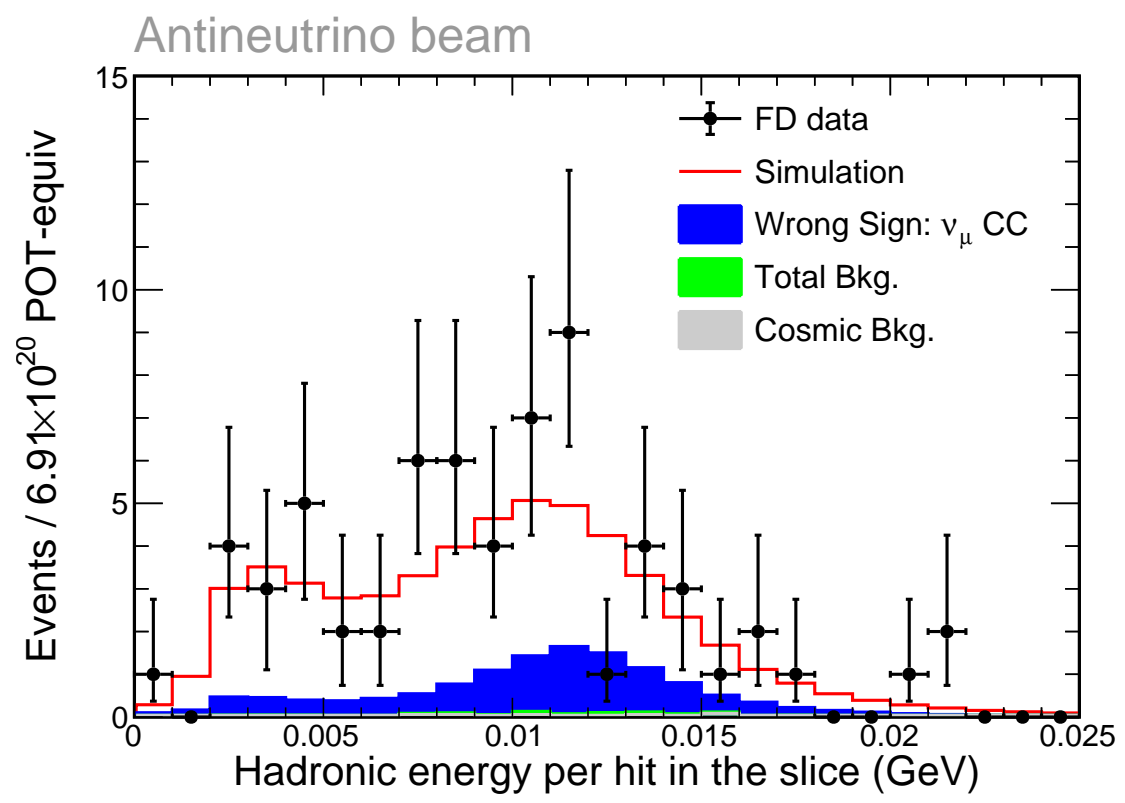

Figure 7.68: RHC FD reconstructed hadronic-energy per hit distributions for the total dataset. Data is shown in black and simulation in red. Also shown is the wrong-sign $\nu_{\mu}$ component in blue, the cosmic background in grey and the total background in green. 


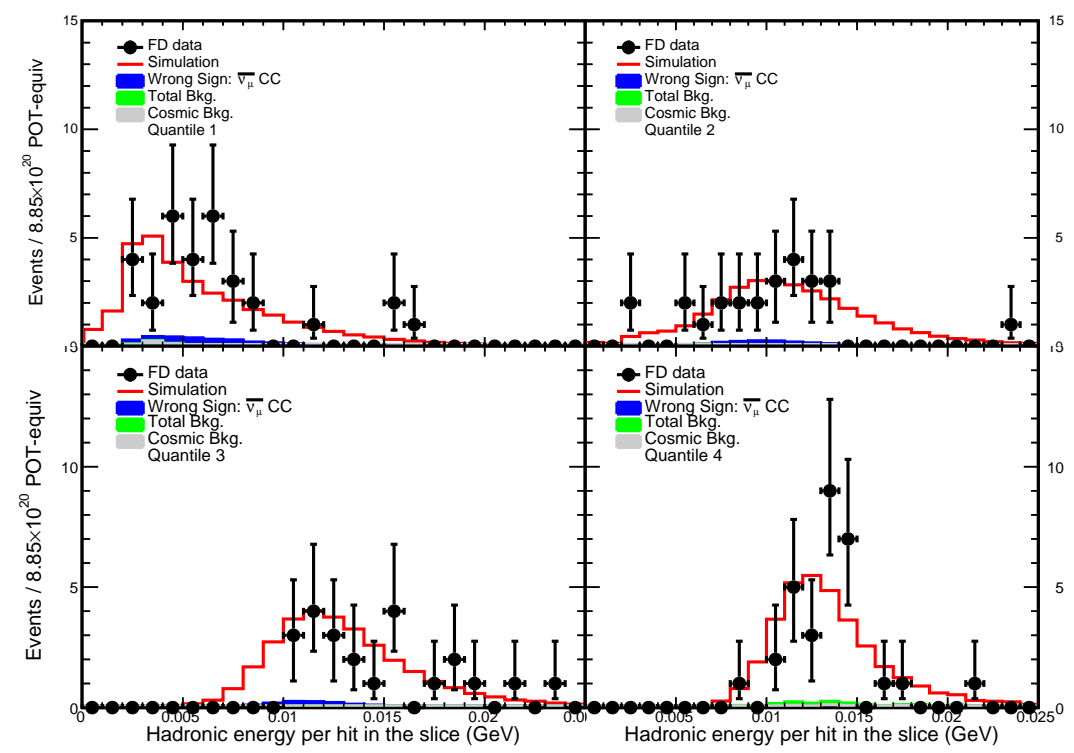

Figure 7.69: FHC FD reconstructed hadronic-energy per hit distributions for each $E_{h a d} / E_{\nu}$ quantile. Data is shown in black and simulation in red. Also shown is the wrong-sign $\bar{\nu}_{\mu}$ component in blue, the cosmic background in grey and the total background in green.

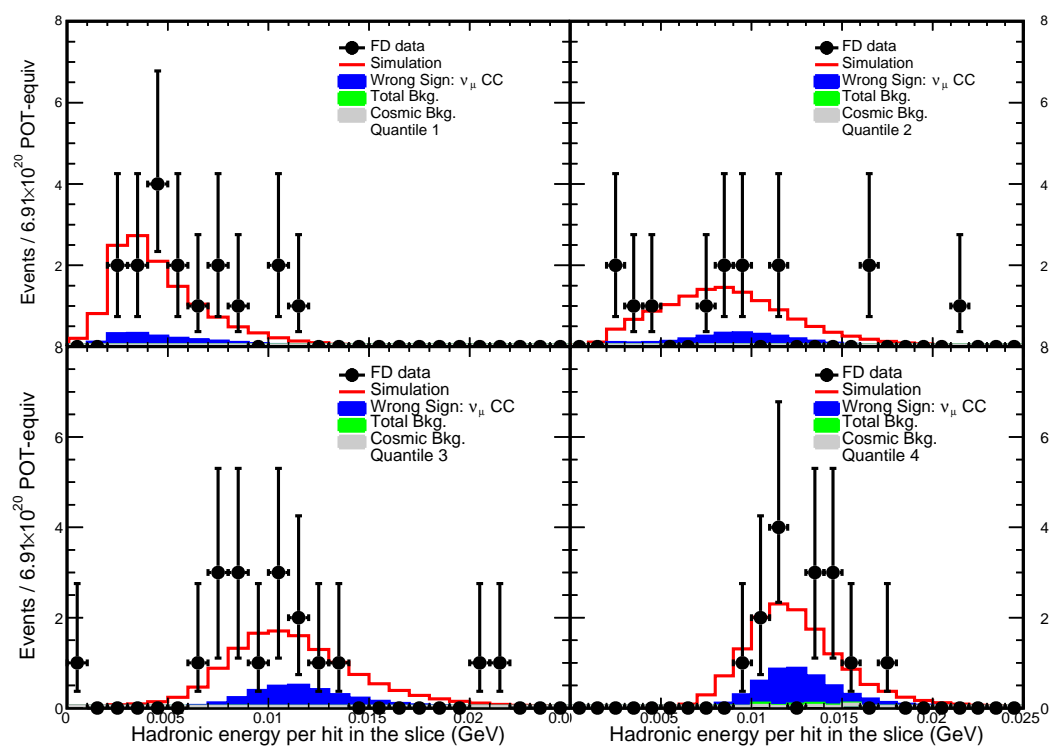

Figure 7.70: RHC FD reconstructed hadronic-energy per hit distributions for each $E_{\text {had }} / E_{\nu}$ quantile. Data is shown in black and simulation in red. Also shown is the wrongsign $\nu_{\mu}$ component in blue, the cosmic background in grey and the total background in green. 


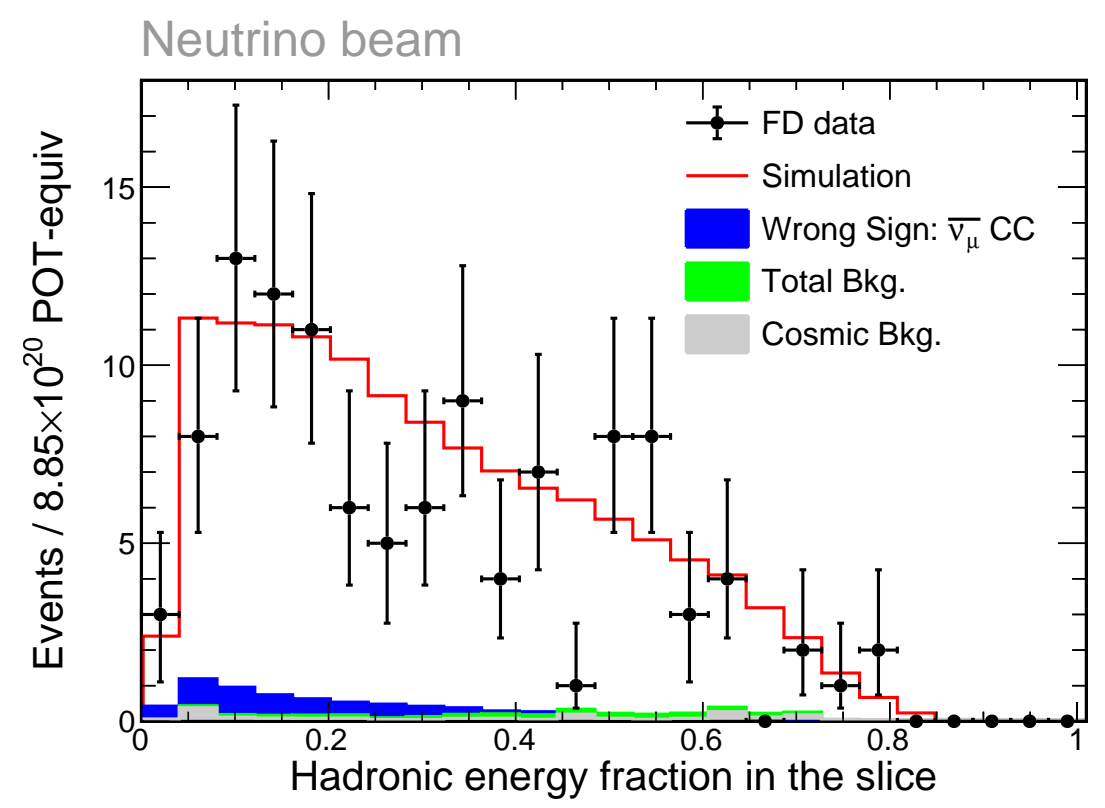

Figure 7.71: FHC FD hadronic fraction of total energy distributions for the total dataset. Data is shown in black and simulation in red. Also shown is the wrong-sign $\bar{\nu}_{\mu}$ component in blue, the cosmic background in grey and the total background in green.

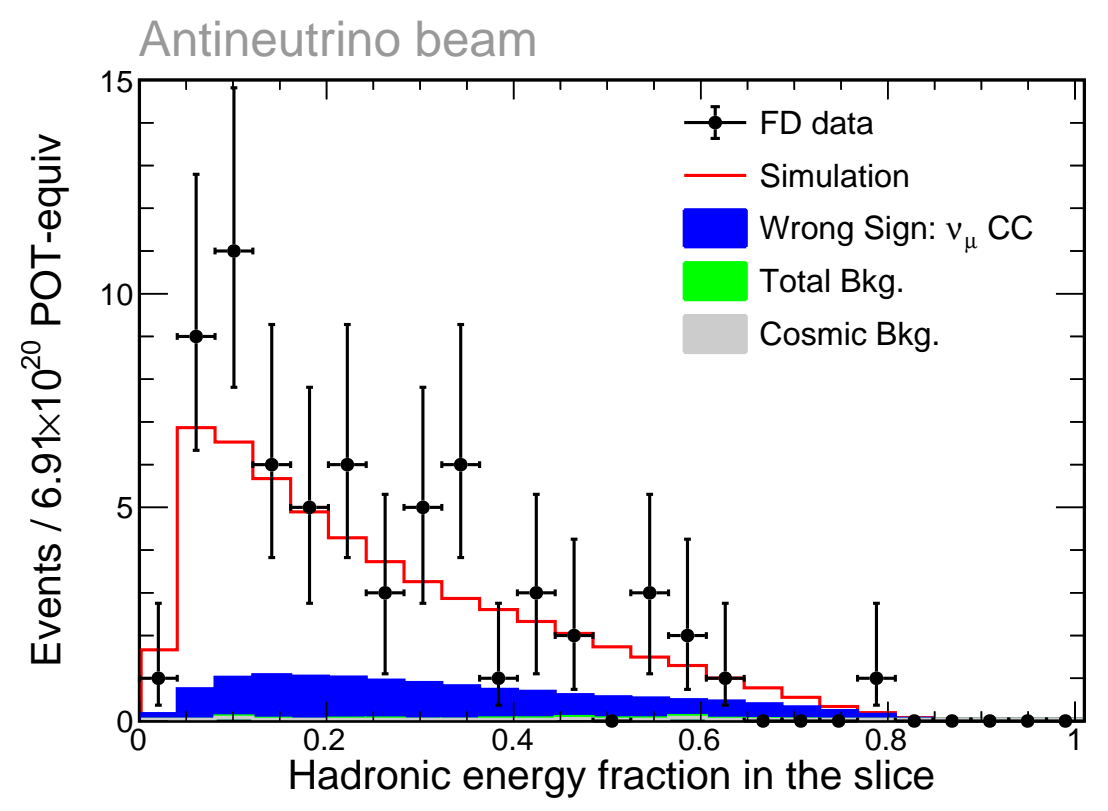

Figure 7.72: RHC FD hadronic fraction of total energy distributions for the total dataset. Data is shown in black and simulation in red. Also shown is the wrong-sign $\nu_{\mu}$ component in blue, the cosmic background in grey and the total background in green. 


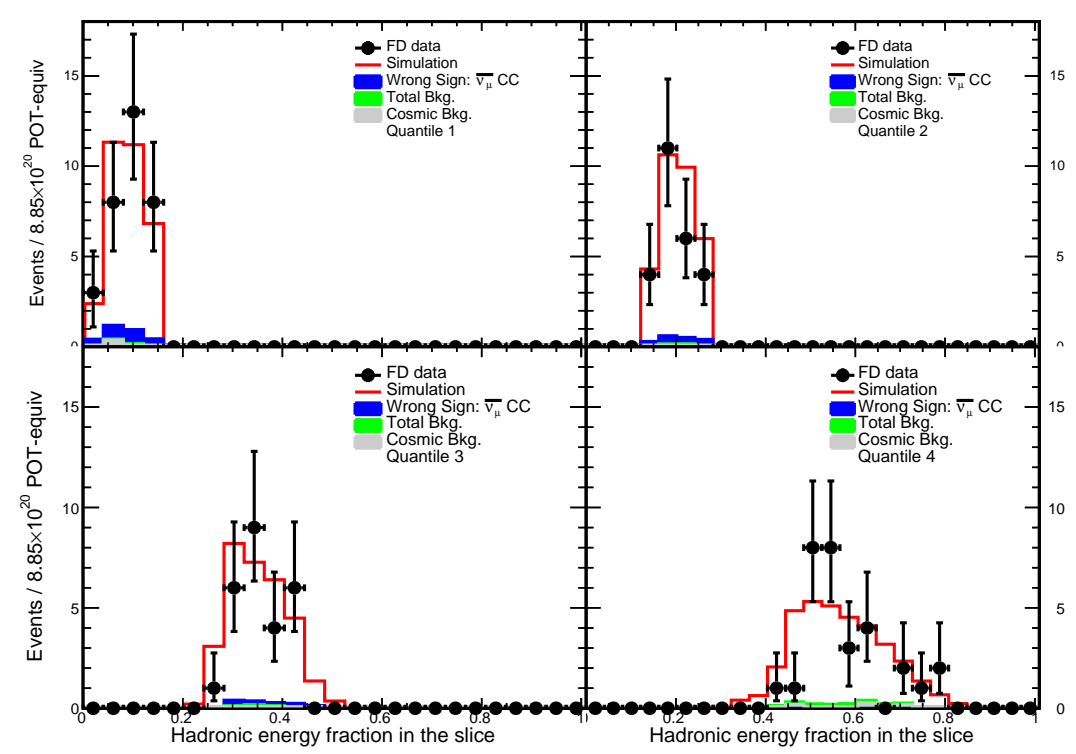

Figure 7.73: FHC FD hadronic fraction of total energy distributions for each $E_{h a d} / E_{\nu}$ quantile. Data is shown in black and simulation in red. Also shown is the wrong-sign $\bar{\nu}_{\mu}$ component in blue, the cosmic background in grey and the total background in green.

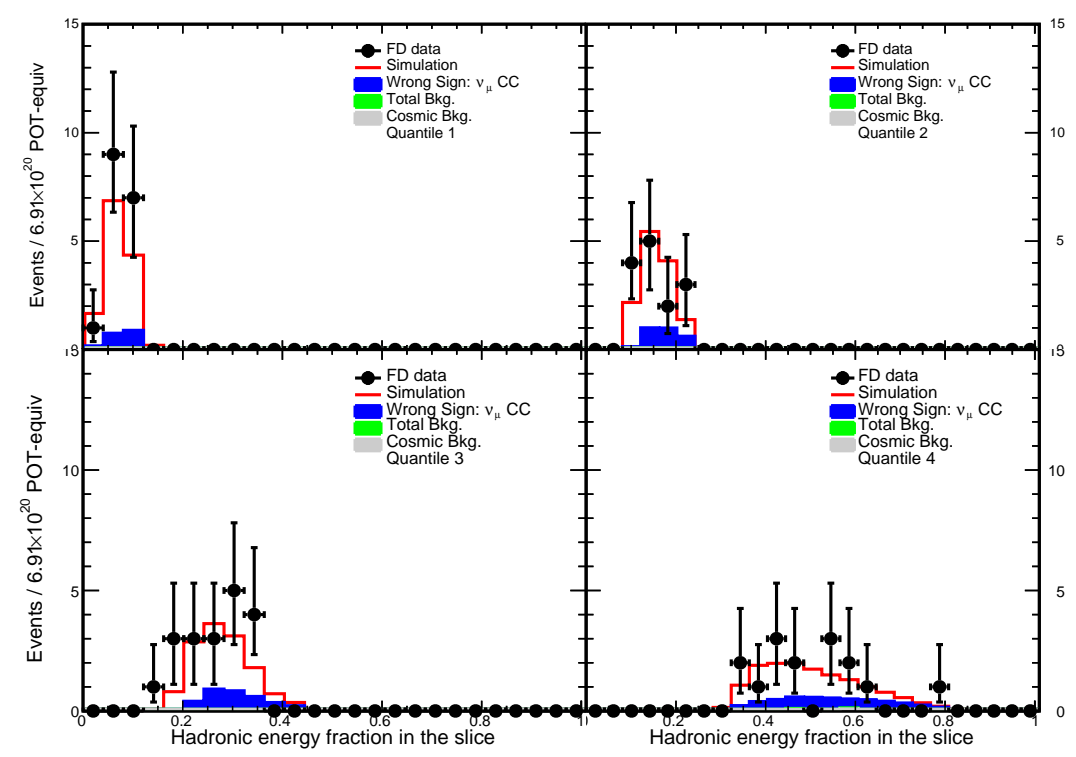

Figure 7.74: RHC FD hadronic fraction of total energy distributions for each $E_{h a d} / E_{\nu}$ quantile. Data is shown in black and simulation in red. Also shown is the wrong-sign $\nu_{\mu}$ component in blue, the cosmic background in grey and the total background in green. 


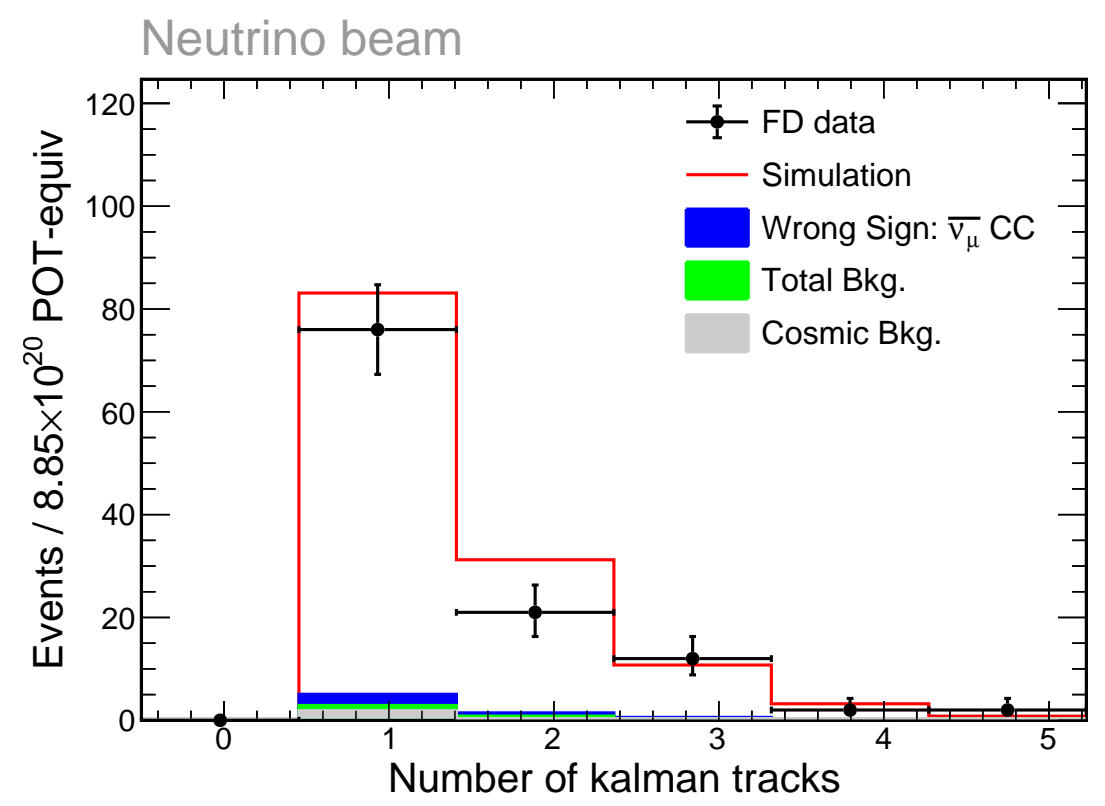

Figure 7.75: FHC FD distributions of the number of Kalman tracks for the total dataset. Data is shown in black and simulation in red. Also shown is the wrong-sign $\bar{\nu}_{\mu}$ component in blue, the cosmic background in grey and the total background in green.

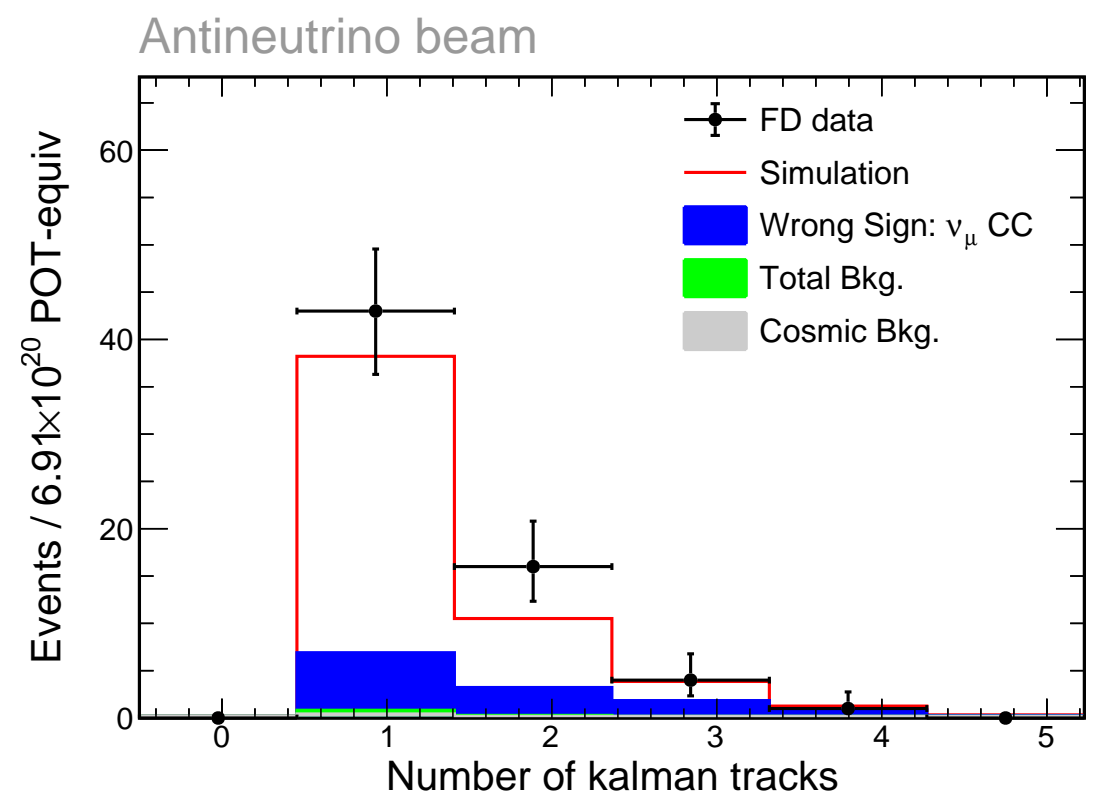

Figure 7.76: RHC FD distributions of the number of Kalman tracks for the total dataset. Data is shown in black and simulation in red. Also shown is the wrong-sign $\nu_{\mu}$ component in blue, the cosmic background in grey and the total background in green. 


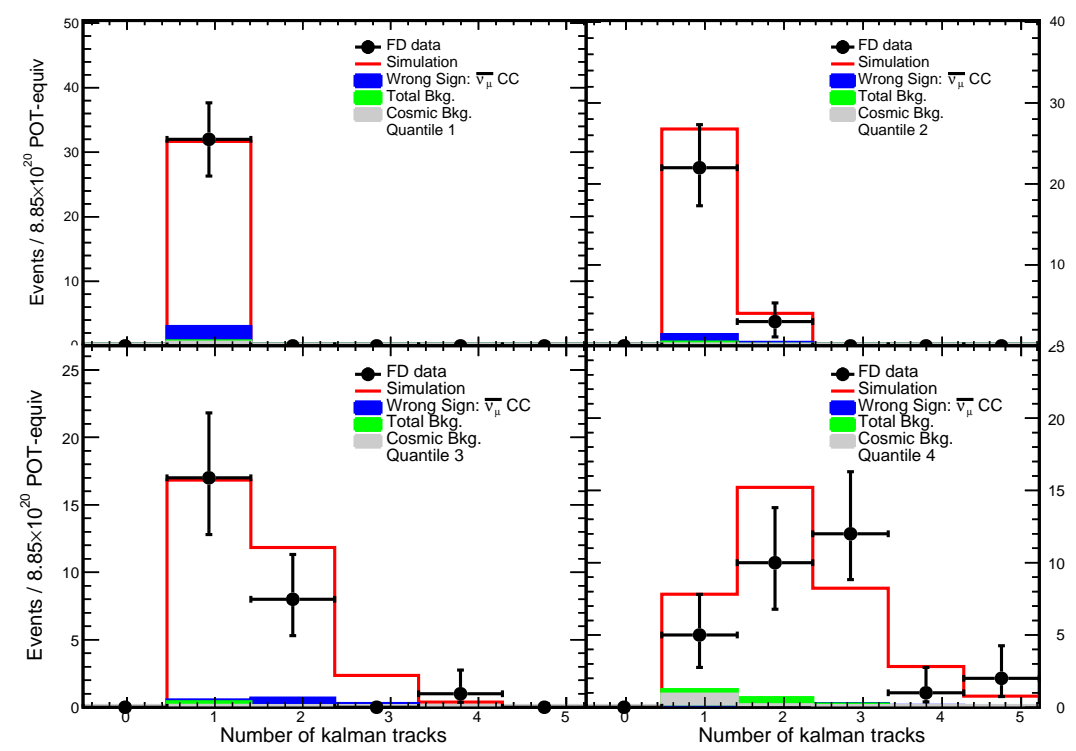

Figure 7.77: FHC FD distributions of the number of Kalman tracks for each $E_{h a d} / E_{\nu}$ quantile. Data is shown in black and simulation in red. Also shown is the wrong-sign $\bar{\nu}_{\mu}$ component in blue, the cosmic background in grey and the total background in green.

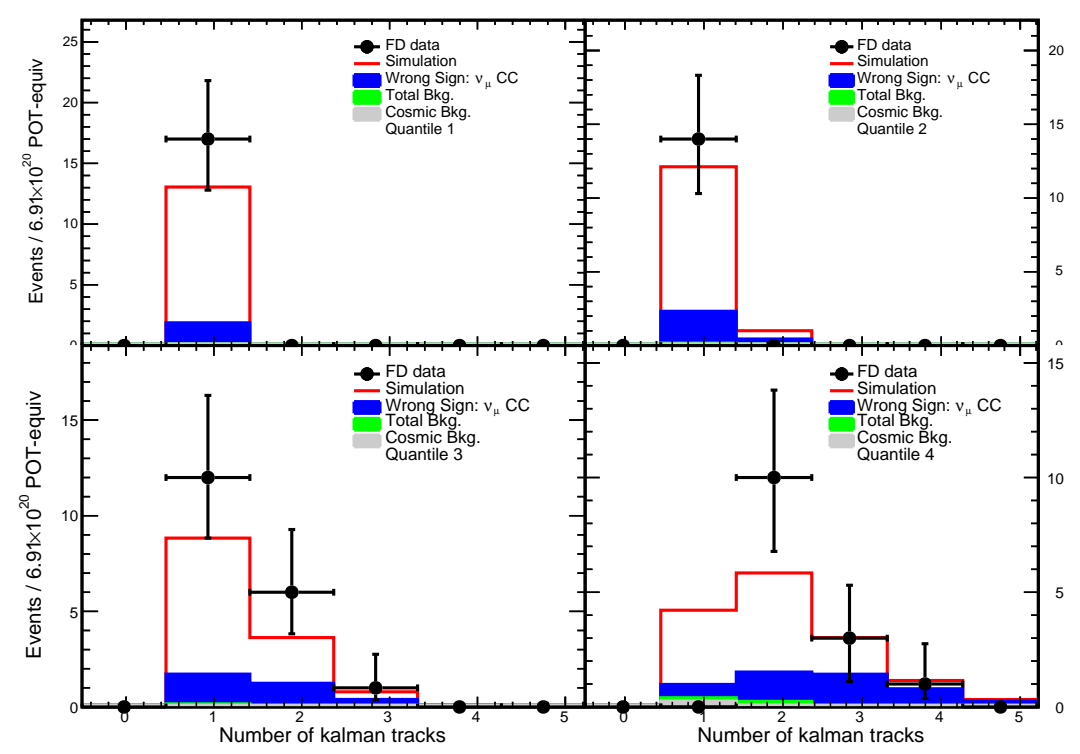

Figure 7.78: RHC FD distributions of the number of Kalman tracks for each $E_{h a d} / E_{\nu}$ quantile. Data is shown in black and simulation in red. Also shown is the wrong-sign $\nu_{\mu}$ component in blue, the cosmic background in grey and the total background in green. 


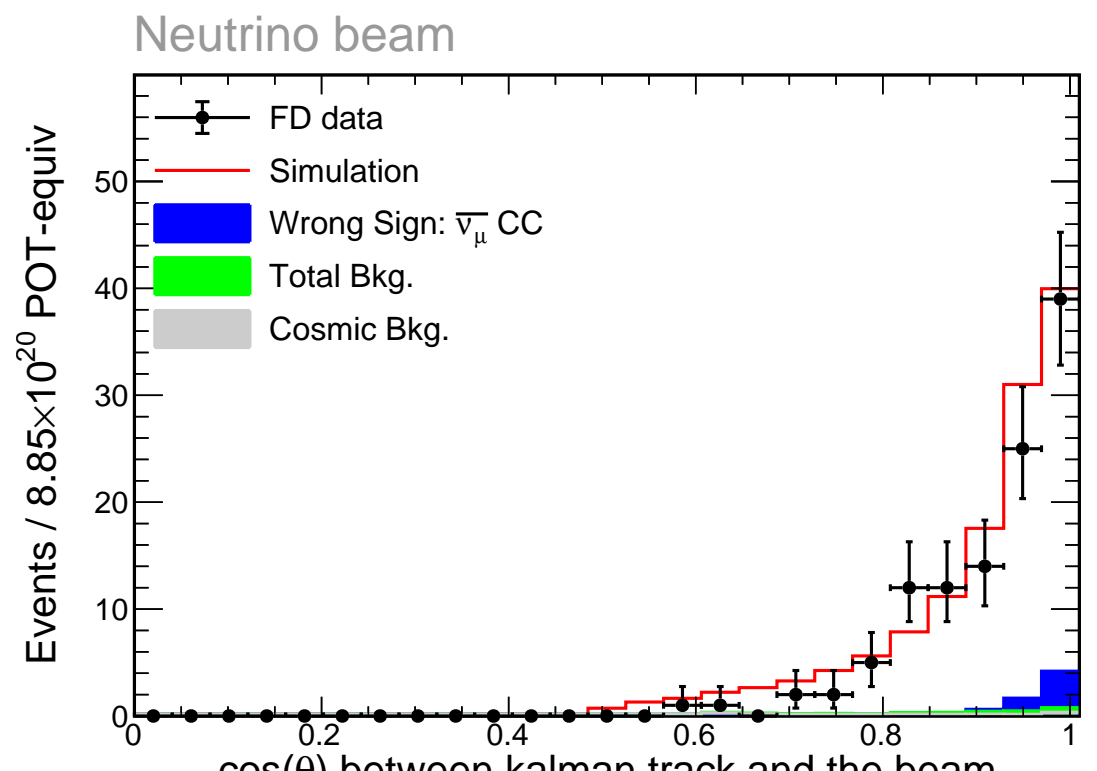

Figure 7.79: FHC FD distributions of the Kalman track angle with respect to the beam for the entire data set. Data is shown in black and simulation in red. Also shown is the wrong-sign $\bar{\nu}_{\mu}$ component in blue, the cosmic background in grey and the total background in green.

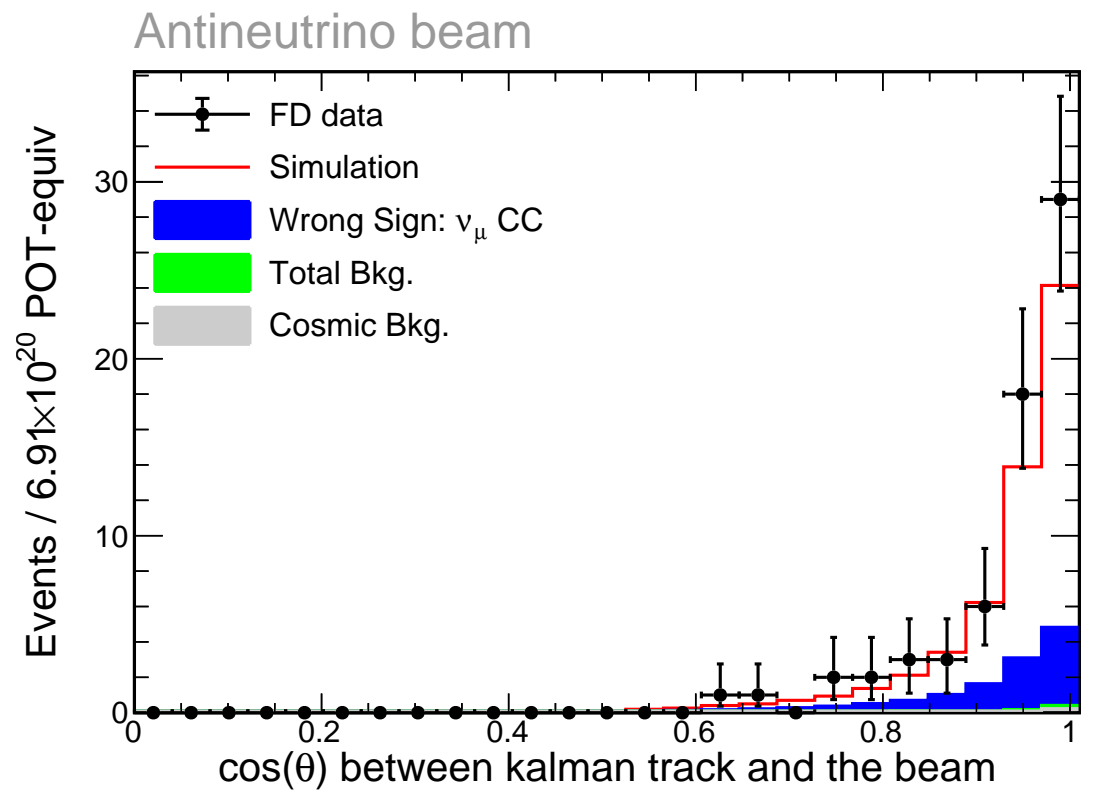

Figure 7.80: RHC FD distributions of the Kalman track angle with respect to the beam for the entire data set. Data is shown in black and simulation in red. Also shown is the wrong-sign $\nu_{\mu}$ component in blue, the cosmic background in grey and the total background in green. 


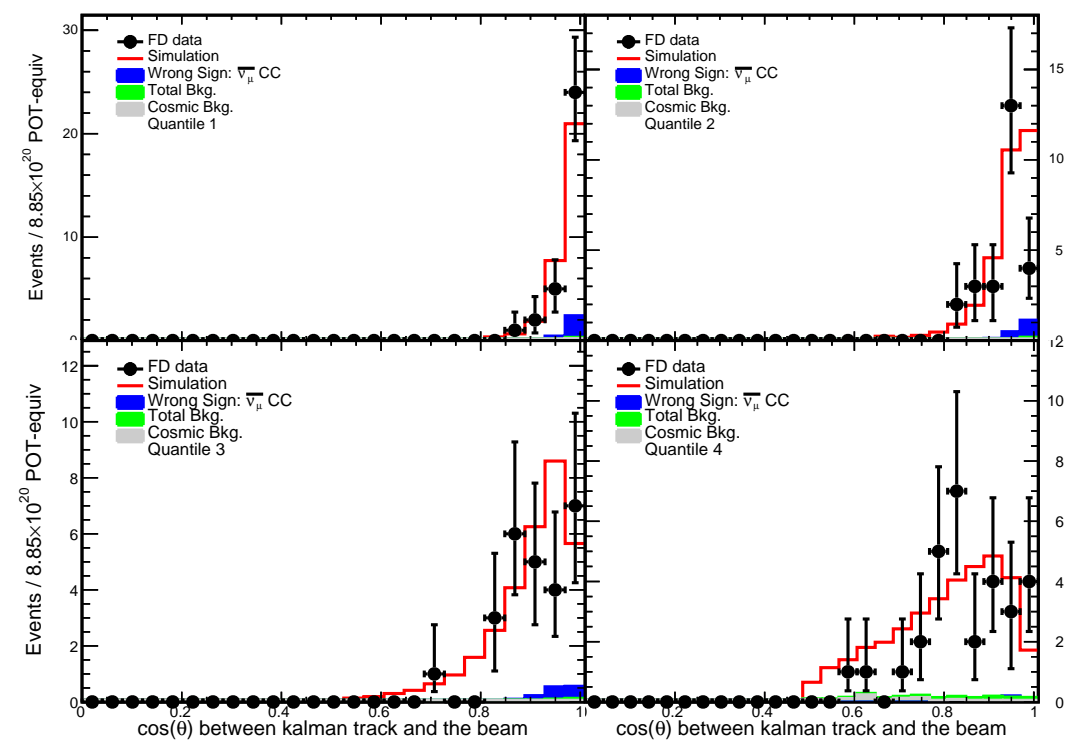

Figure 7.81: FHC FD distributions of the Kalman track angle with respect to the beam for each $E_{h a d} / E_{\nu}$ quantile. Data is shown in black and simulation in red. Also shown is the wrong-sign $\bar{\nu}_{\mu}$ component in blue, the cosmic background in grey and the total background in green.

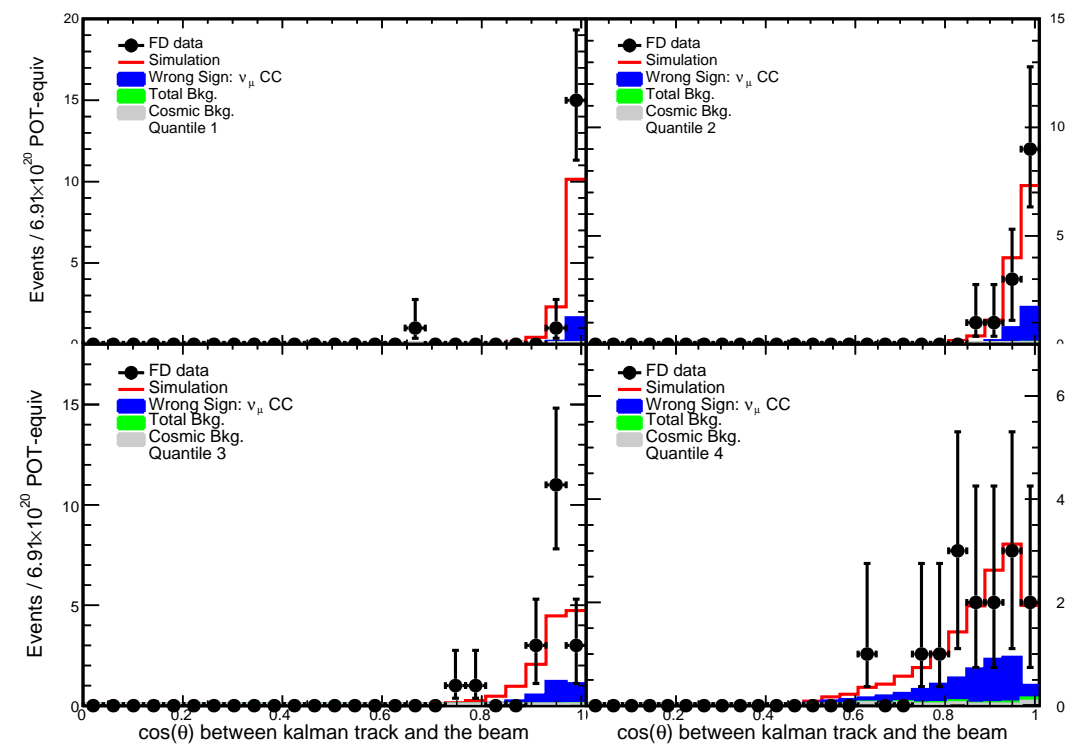

Figure 7.82: RHC FD distributions of the Kalman track angle with respect to the beam for each $E_{h a d} / E_{\nu}$ quantile. Data is shown in black and simulation in red. Also shown is the wrong-sign $\nu_{\mu}$ component in blue, the cosmic background in grey and the total background in green. 


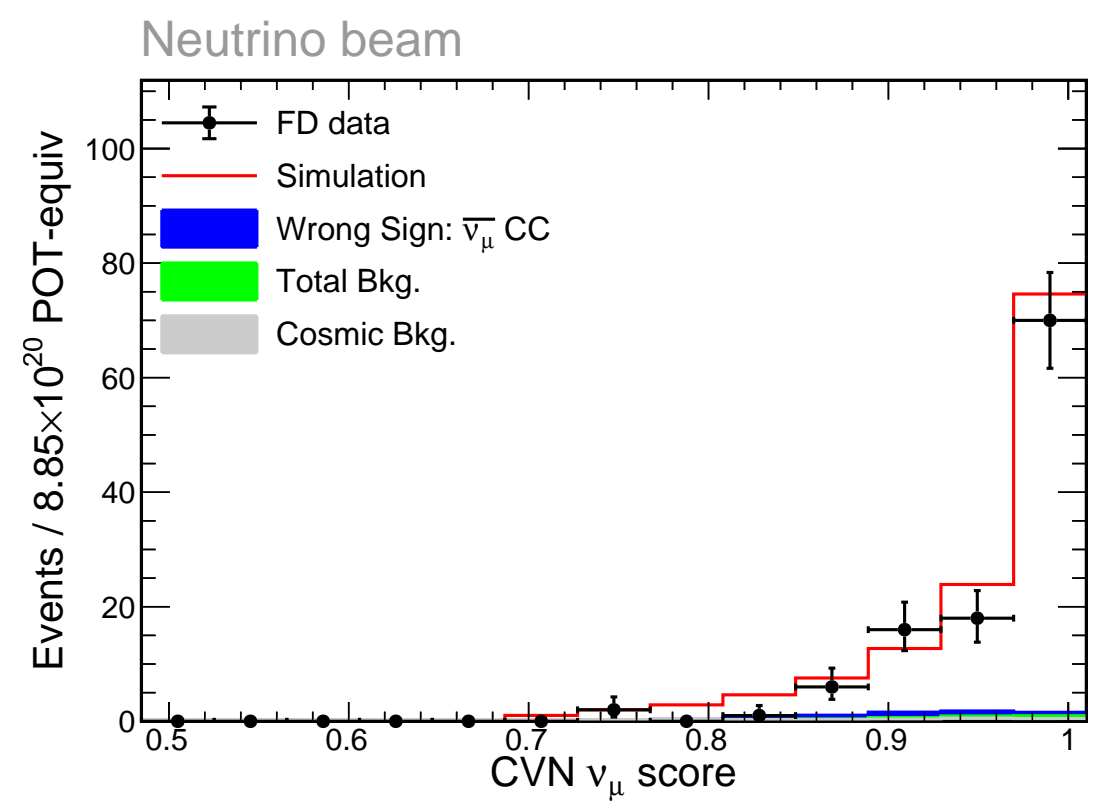

Figure 7.83: FHC FD distributions of the CVN PID score for the total dataset. $E_{h a d} / E_{\nu}$ quantiles. Data is shown in black and simulation in red. Also shown is the wrong-sign $\bar{\nu}_{\mu}$ component in blue, the cosmic background in grey and the total background in green.

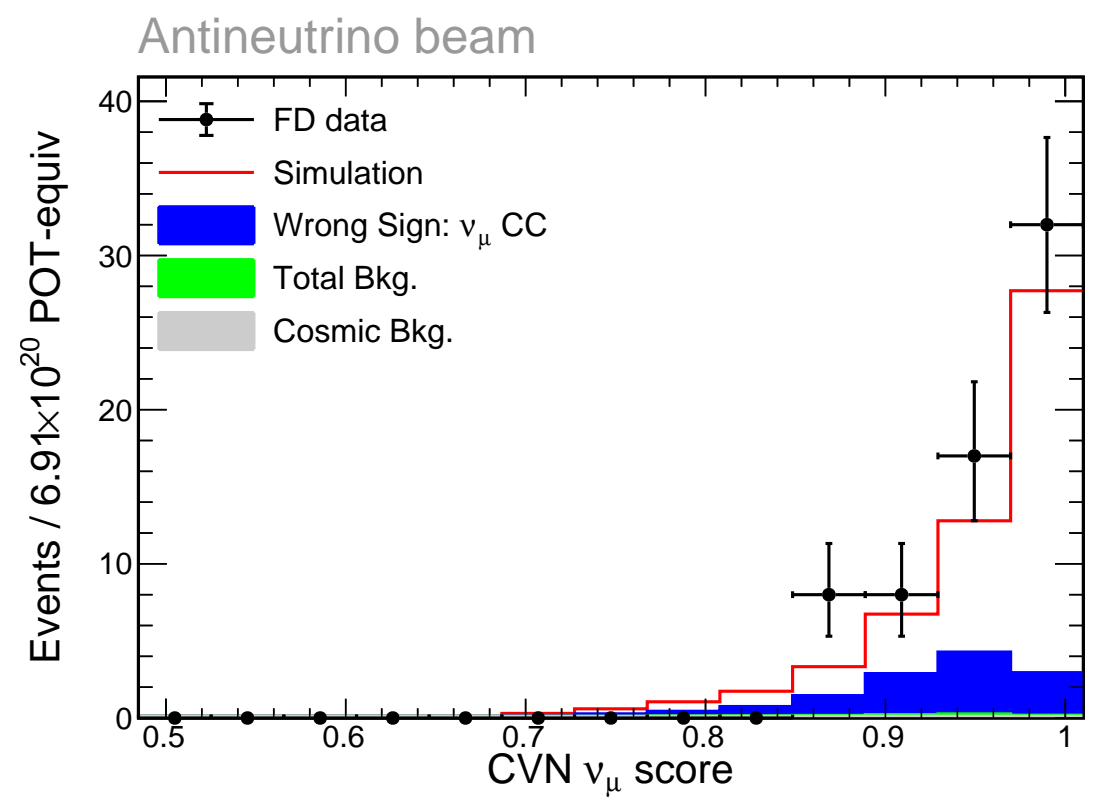

Figure 7.84: RHC FD distributions of the CVN PID score for the total dataset. $E_{\text {had }} / E_{\nu}$ quantiles. Data is shown in black and simulation in red. Also shown is the wrong-sign $\nu_{\mu}$ component in blue, the cosmic background in grey and the total background in green. 


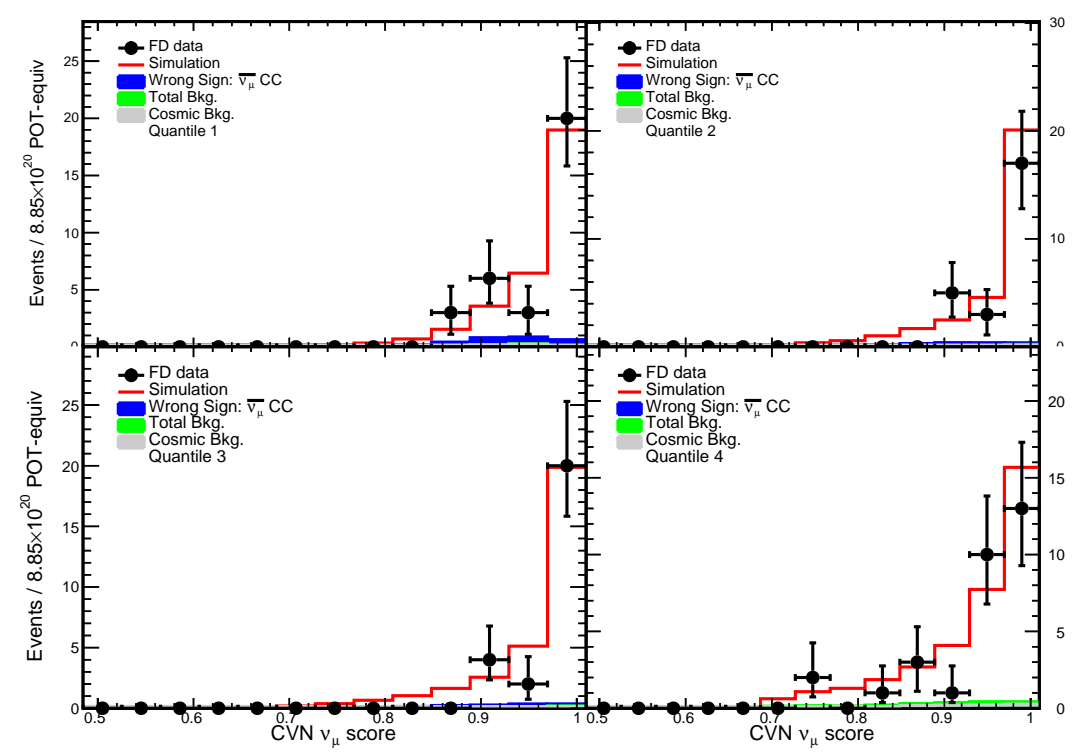

Figure 7.85: FHC FD distributions of the CVN PID score for each $E_{h a d} / E_{\nu}$ quantile. Data is shown in black and simulation in red. Also shown is the wrong-sign $\bar{\nu}_{\mu}$ component in blue, the cosmic background in grey and the total background in green.

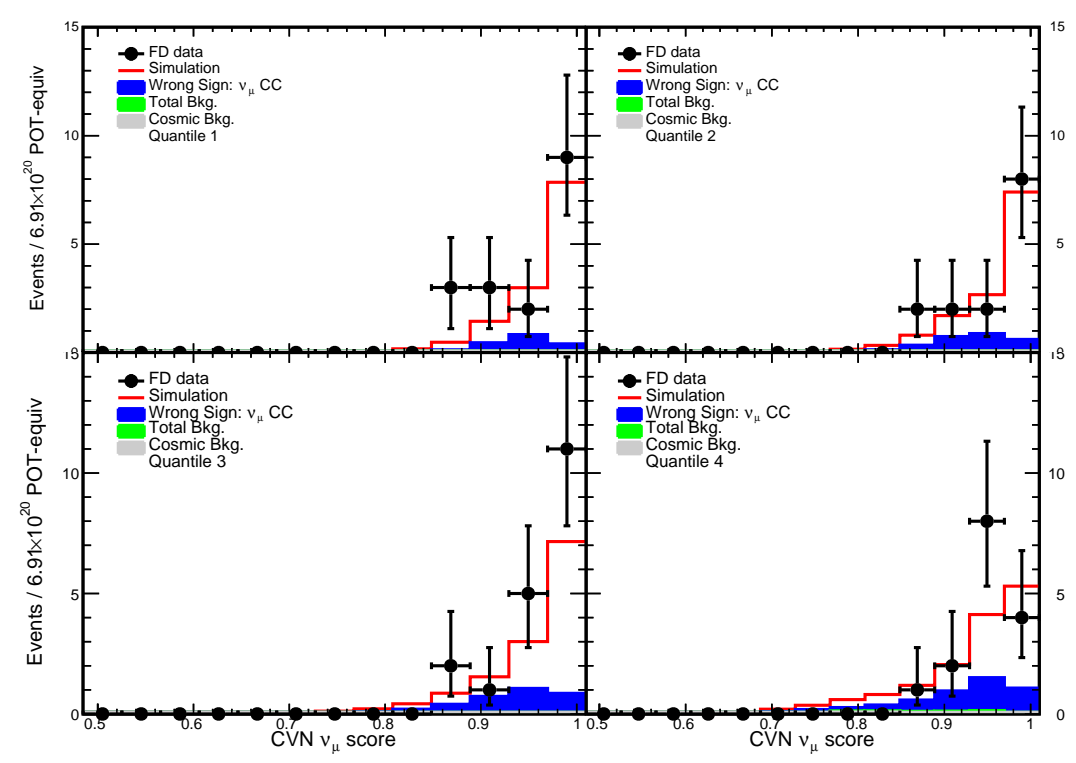

Figure 7.86: RHC FD distributions of the CVN PID score for each $E_{h a d} / E_{\nu}$ quantile. Data is shown in black and simulation in red. Also shown is the wrong-sign $\nu_{\mu}$ component in blue, the cosmic background in grey and the total background in green. 


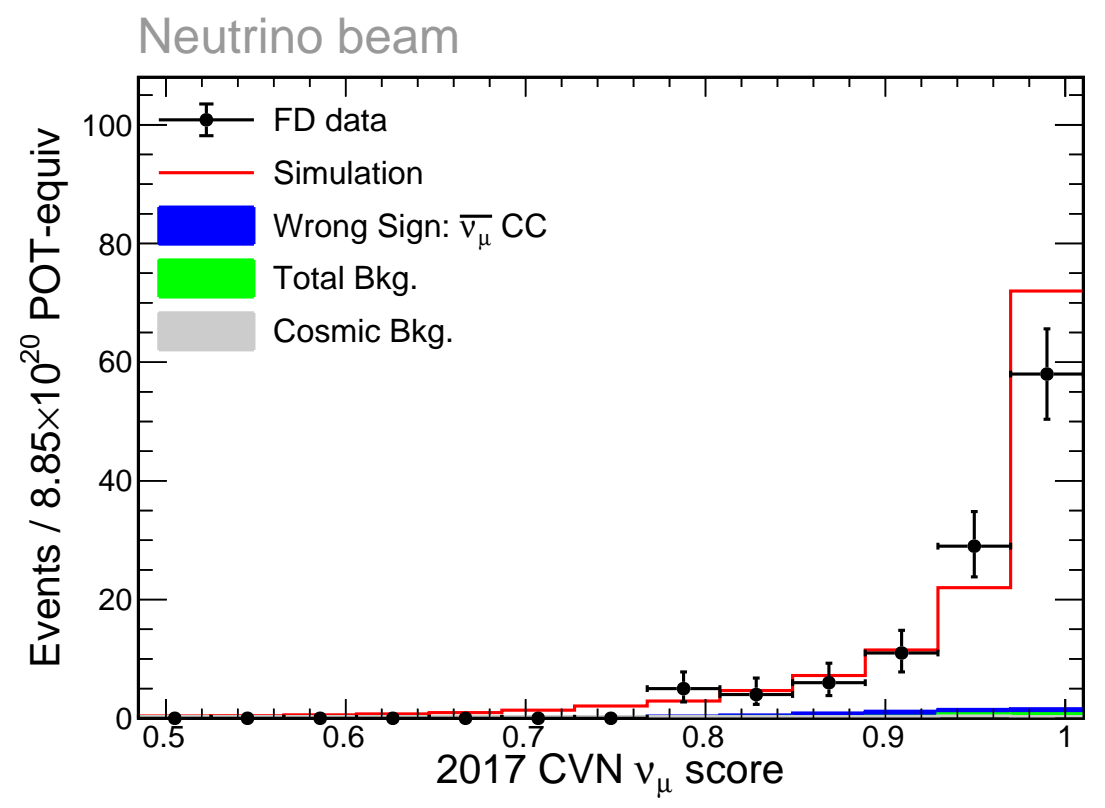

Figure 7.87: FHC FD distributions of the 2017 (older) CVN PID score for the total dataset. Data is shown in black and simulation in red. Also shown is the wrong-sign $\bar{\nu}_{\mu}$ component in blue, the cosmic background in grey and the total background in green.

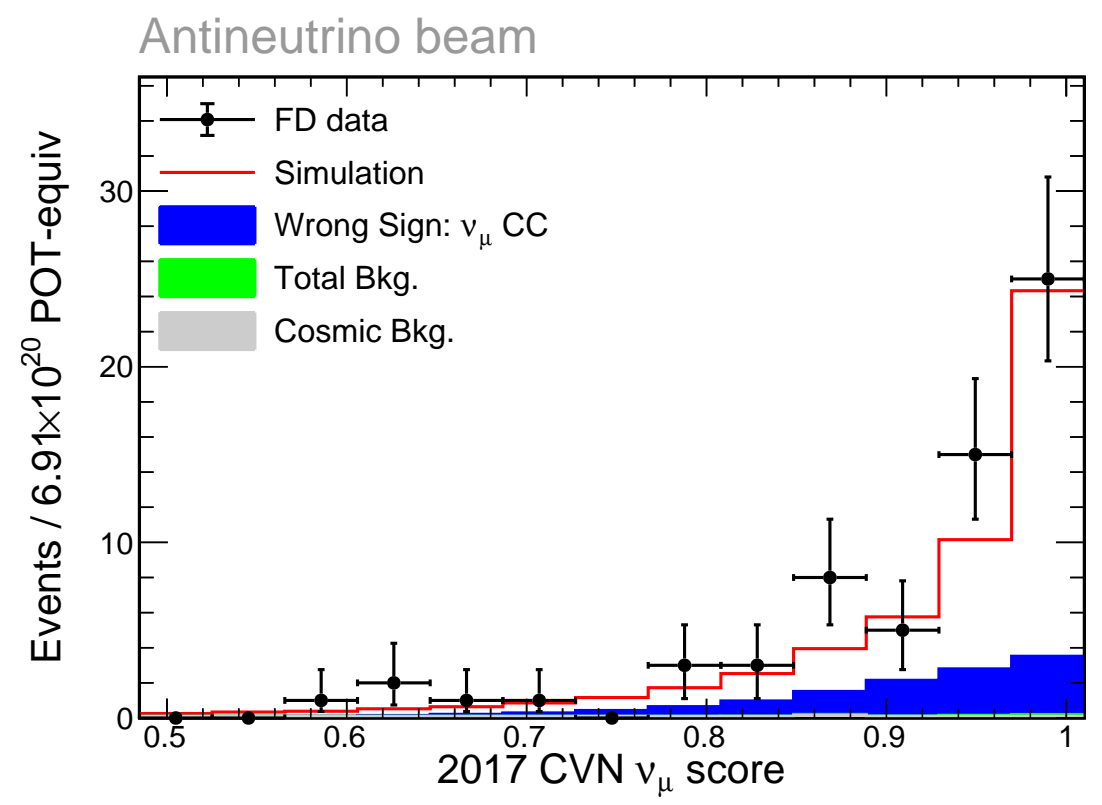

Figure 7.88: RHC FD distributions of the 2017 (older) CVN PID score for the total dataset. Data is shown in black and simulation in red. Also shown is the wrong-sign $\nu_{\mu}$ component in blue, the cosmic background in grey and the total background in green. 


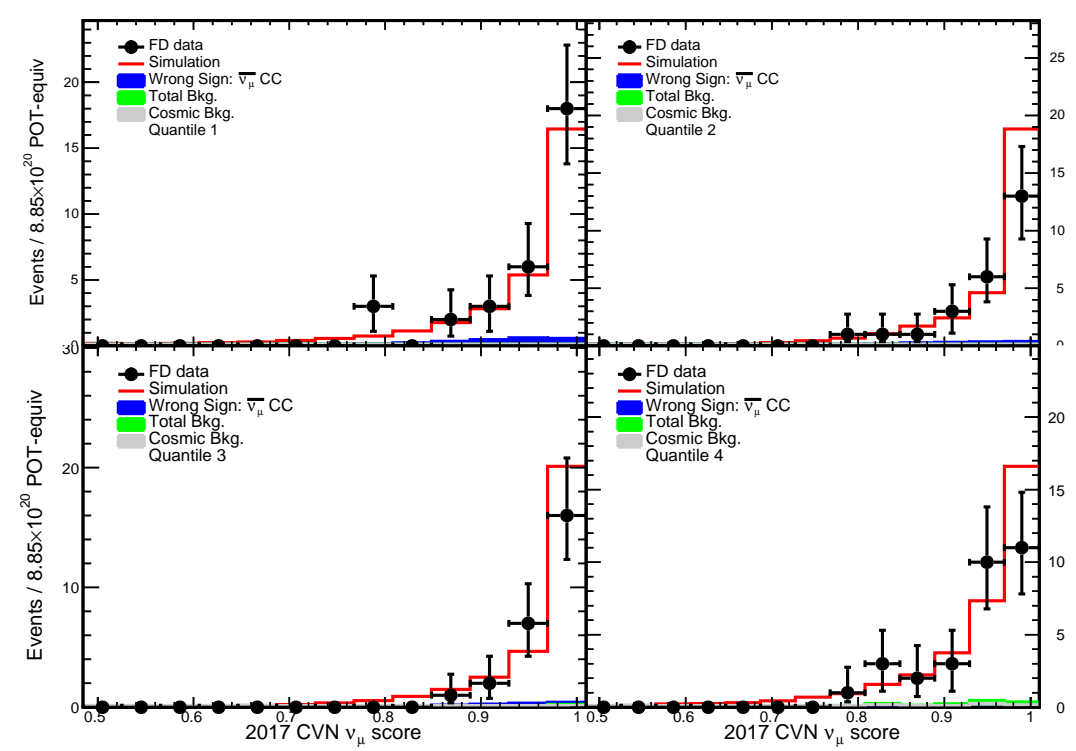

Figure 7.89: FHC FD distributions of the 2017 (older) CVN PID score for each $E_{h a d} / E_{\nu}$ quantile. Data is shown in black and simulation in red. Also shown is the wrong-sign $\bar{\nu}_{\mu}$ component in blue, the cosmic background in grey and the total background in green.

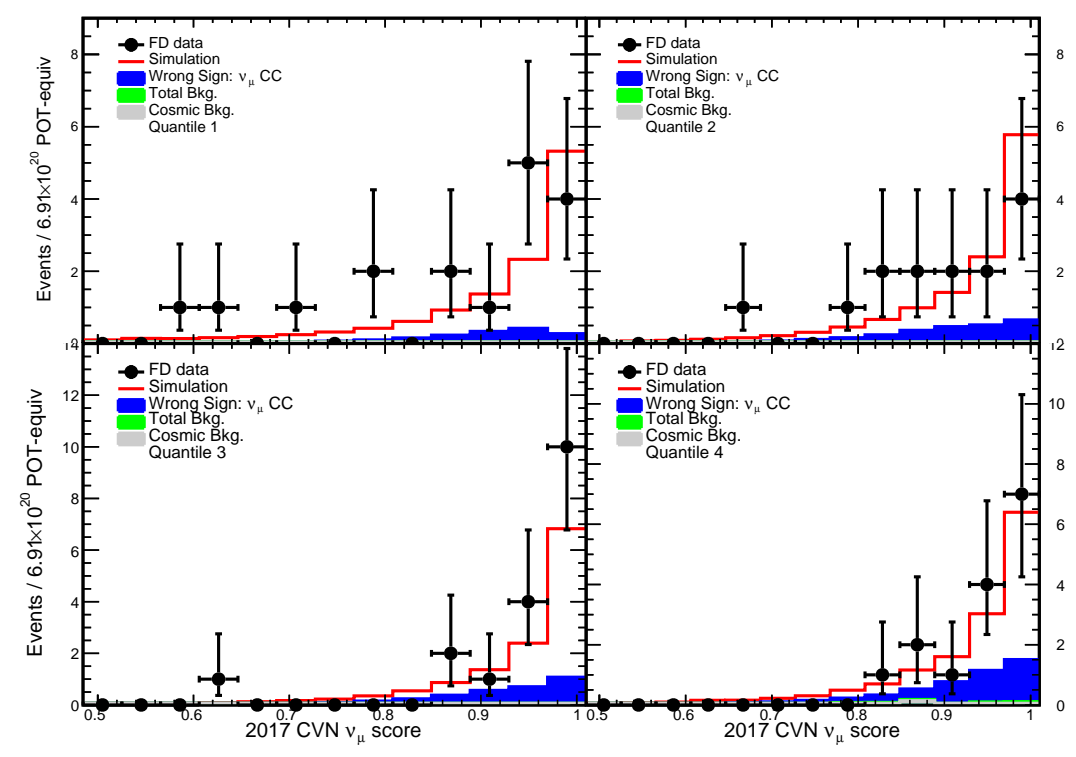

Figure 7.90: RHC FD distributions of the 2017 (older) CVN PID score for each $E_{h a d} / E_{\nu}$ quantile. Data is shown in black and simulation in red. Also shown is the wrong-sign $\nu_{\mu}$ component in blue, the cosmic background in grey and the total background in green. 


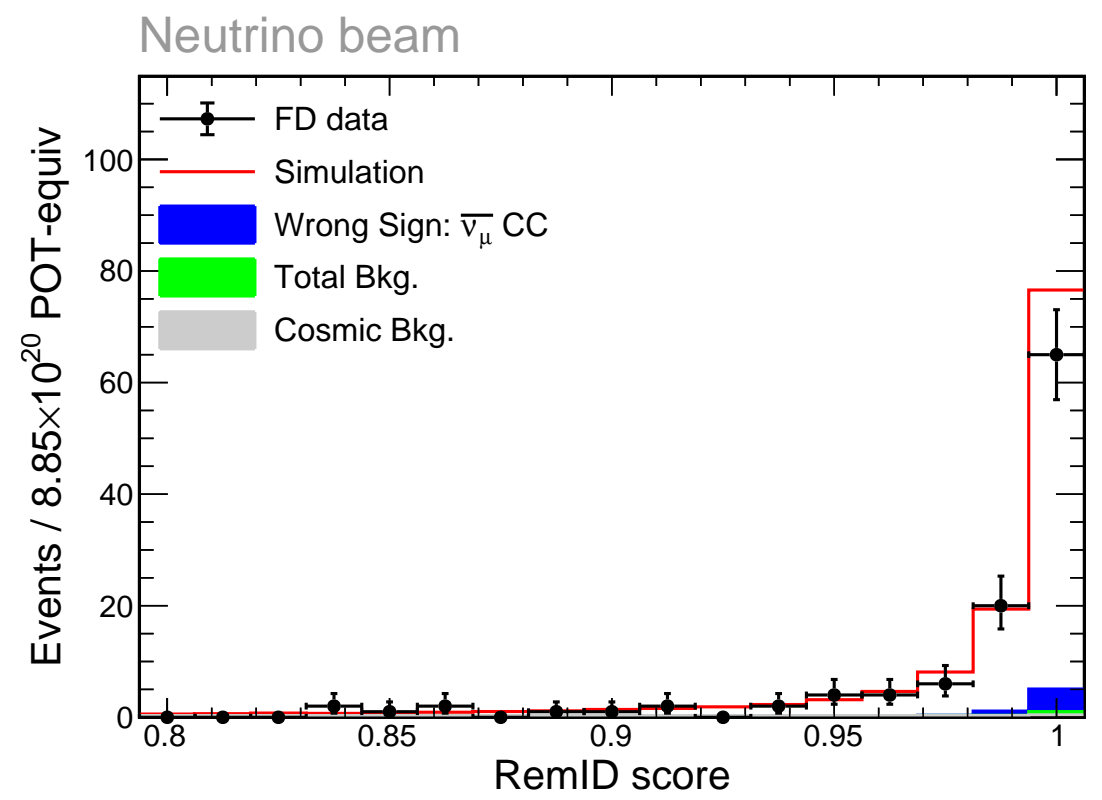

Figure 7.91: FHC FD distributions of the ReMId PID score for the total dataset. $E_{\text {had }} / E_{\nu}$ quantiles. Data is shown in black and simulation in red. Also shown is the wrong-sign $\bar{\nu}_{\mu}$ component in blue, the cosmic background in grey and the total background in green.

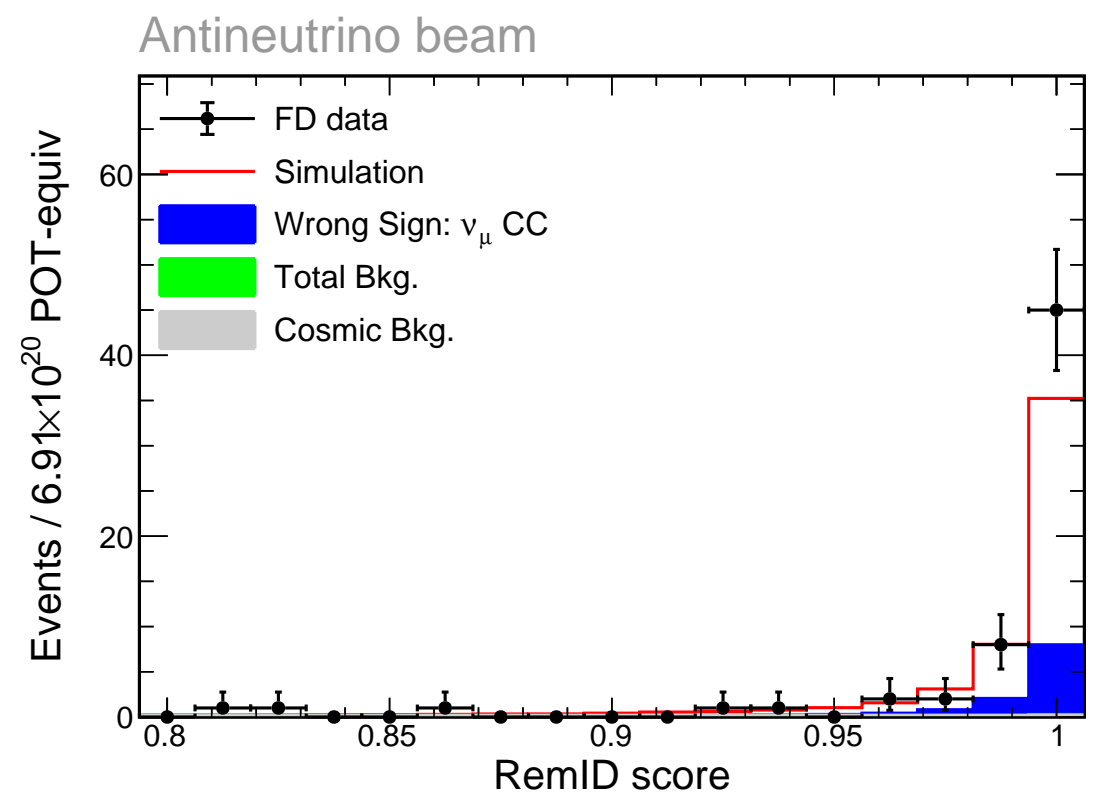

Figure 7.92: RHC FD distributions of the ReMId PID score for the total dataset. $E_{\text {had }} / E_{\nu}$ quantiles. Data is shown in black and simulation in red. Also shown is the wrong-sign $\nu_{\mu}$ component in blue, the cosmic background in grey and the total background in green. 


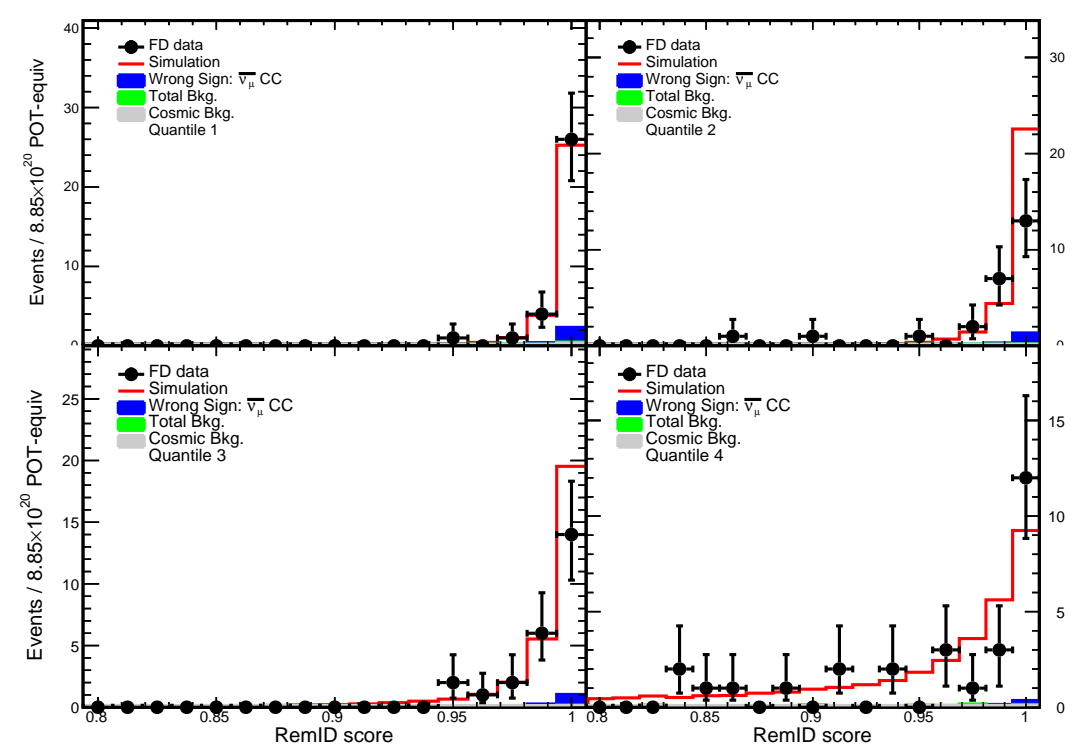

Figure 7.93: FHC FD distributions of the ReMId PID score for each $E_{h a d} / E_{\nu}$ quantile. Data is shown in black and simulation in red. Also shown is the wrong-sign $\bar{\nu}_{\mu}$ component in blue, the cosmic background in grey and the total background in green.

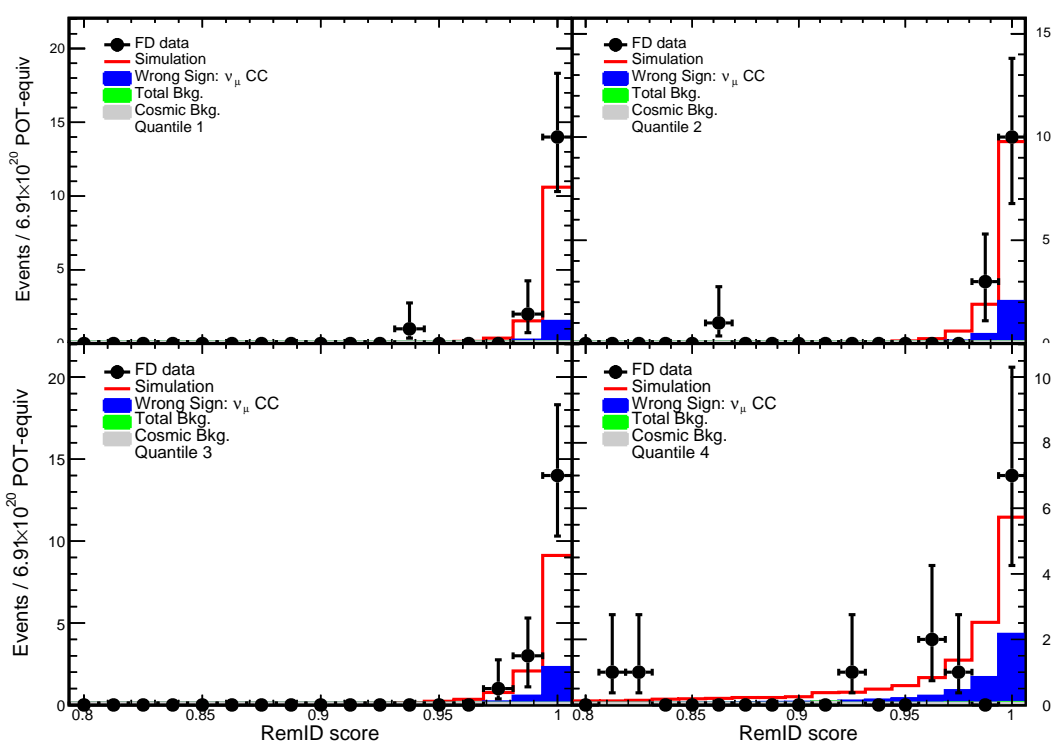

Figure 7.94: RHC FD distributions of the ReMId PID score for each $E_{h a d} / E_{\nu}$ quantile. Data is shown in black and simulation in red. Also shown is the wrong-sign $\nu_{\mu}$ component in blue, the cosmic background in grey and the total background in green. 


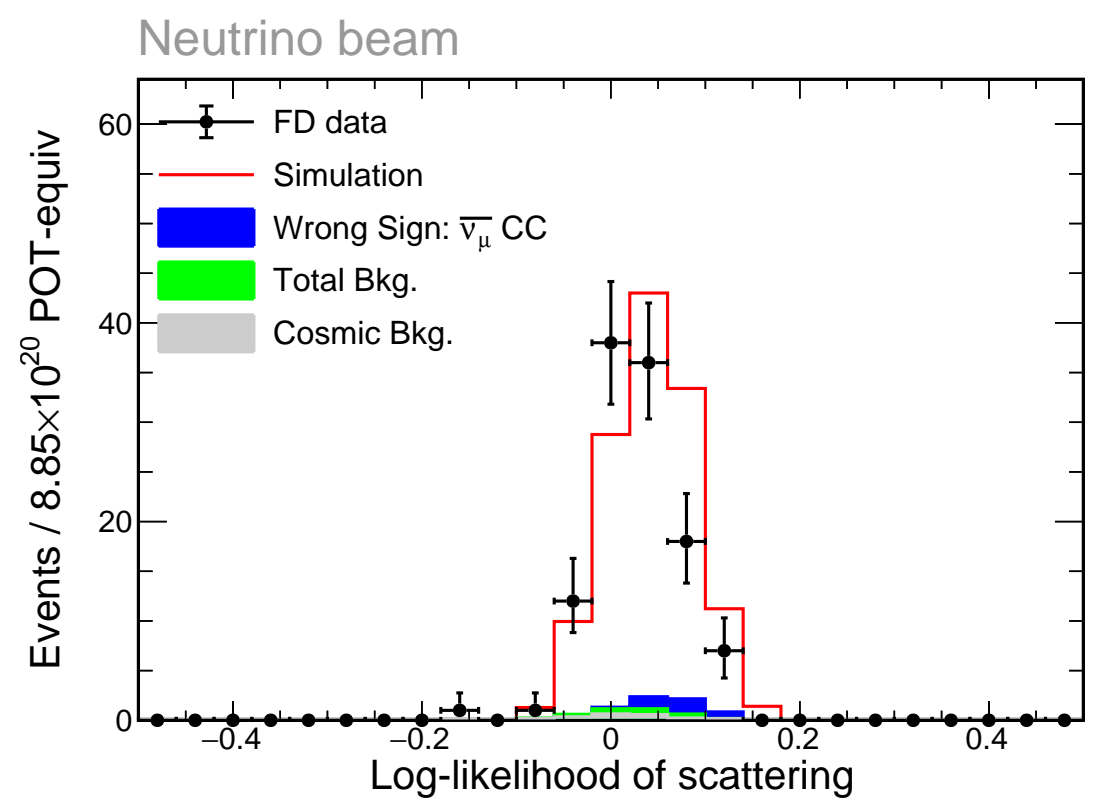

Figure 7.95: FHC FD distributions of the ReMId scattering angle likelihood for the entire data set. Data is shown in black and simulation in red. Also shown is the wrong-sign $\bar{\nu}_{\mu}$ component in blue, the cosmic background in grey and the total background in green.

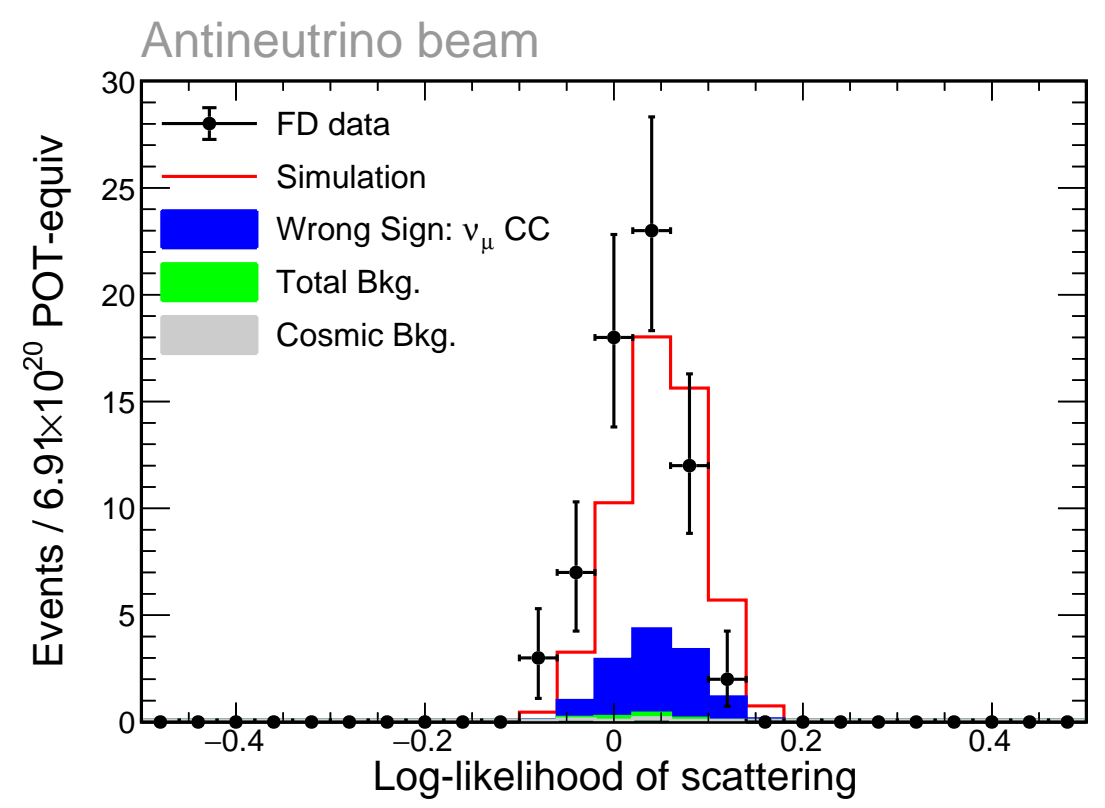

Figure 7.96: RHC FD distributions of the ReMId scattering angle likelihood for the entire data set. Data is shown in black and simulation in red. Also shown is the wrong-sign $\nu_{\mu}$ component in blue, the cosmic background in grey and the total background in green. 


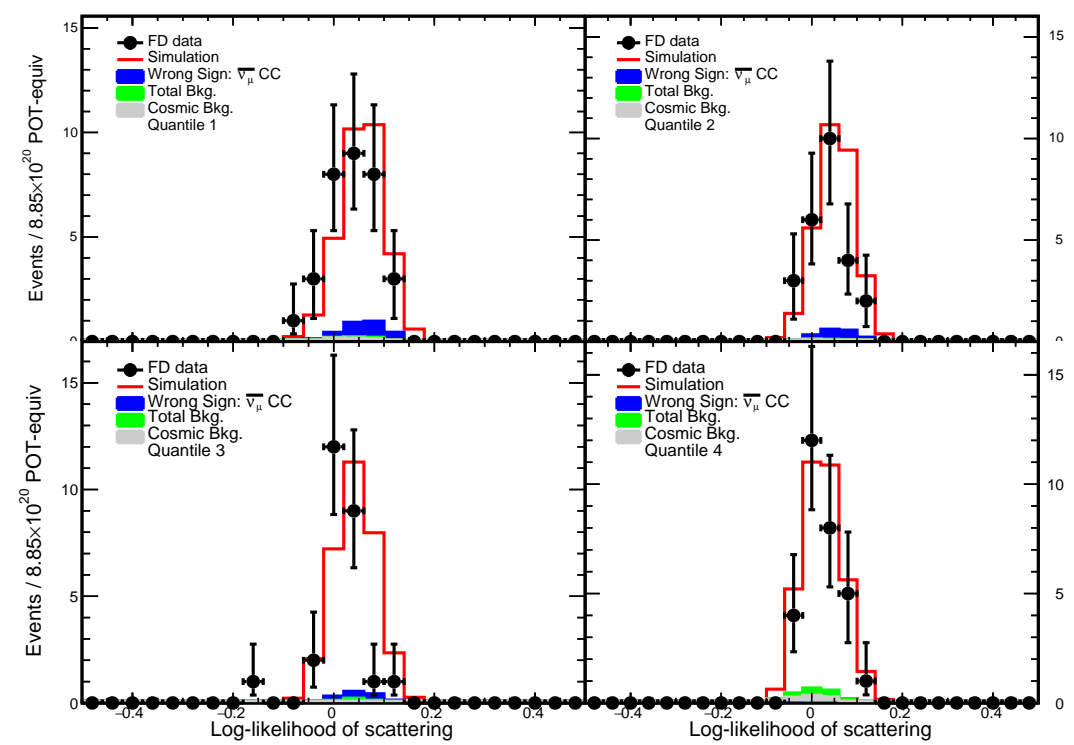

Figure 7.97: FHC FD distributions of the ReMId scattering angle likelihood score for each $E_{\text {had }} / E_{\nu}$ quantile. Data is shown in black and simulation in red. Also shown is the wrongsign $\bar{\nu}_{\mu}$ component in blue, the cosmic background in grey and the total background in green.

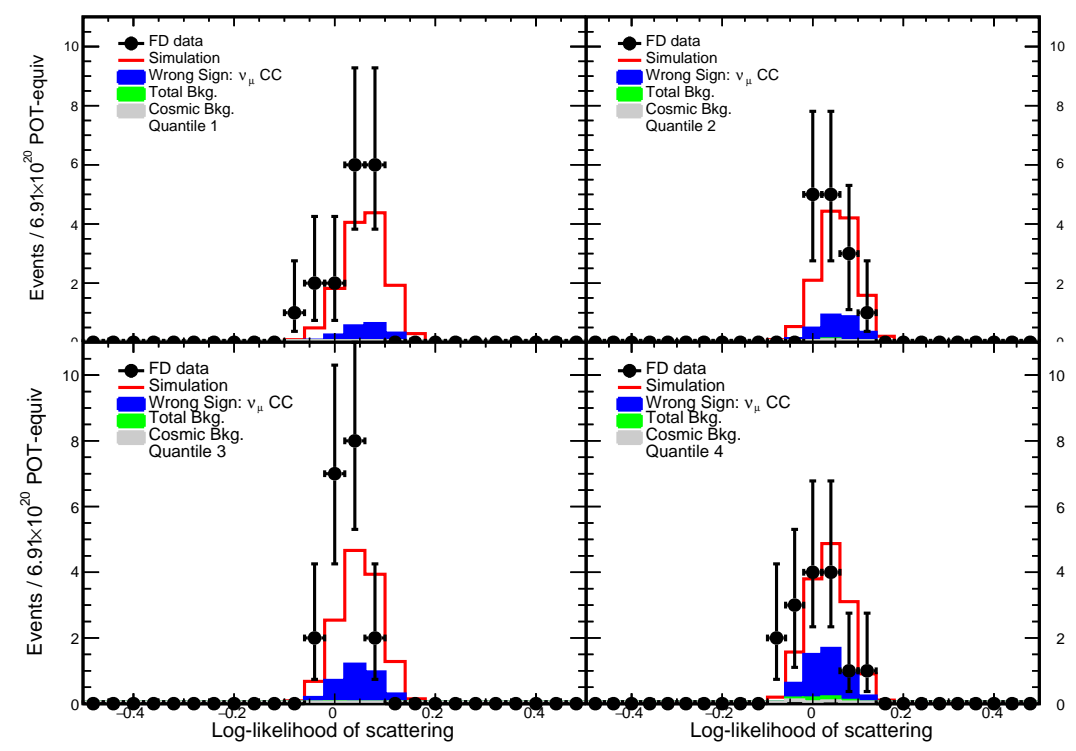

Figure 7.98: RHC FD distributions of the ReMId scattering angle likelihood score for each $E_{\text {had }} / E_{\nu}$ quantile. Data is shown in black and simulation in red. Also shown is the wrong-sign $\nu_{\mu}$ component in blue, the cosmic background in grey and the total background in green. 


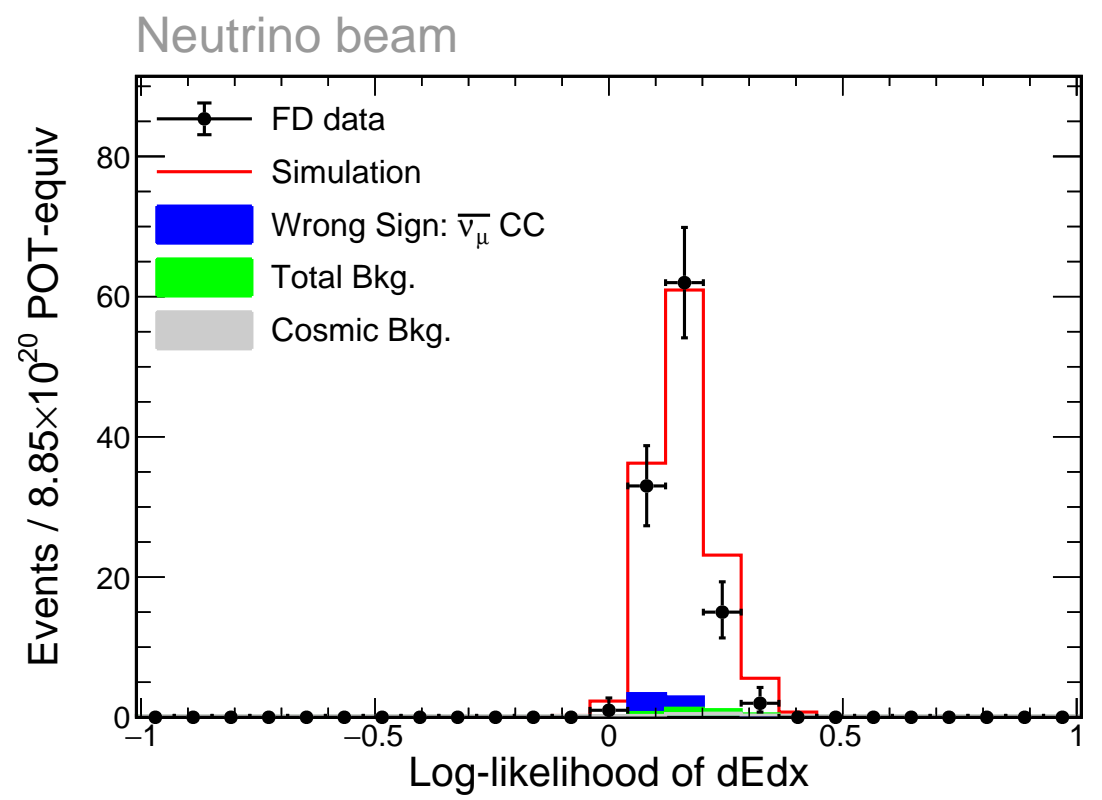

Figure 7.99: FHC FD distributions of the ReMId $\frac{d E}{d x}$ likelihood for the total dataset. Data is shown in black and simulation in red. Also shown is the wrong-sign $\bar{\nu}_{\mu}$ component in blue, the cosmic background in grey and the total background in green.

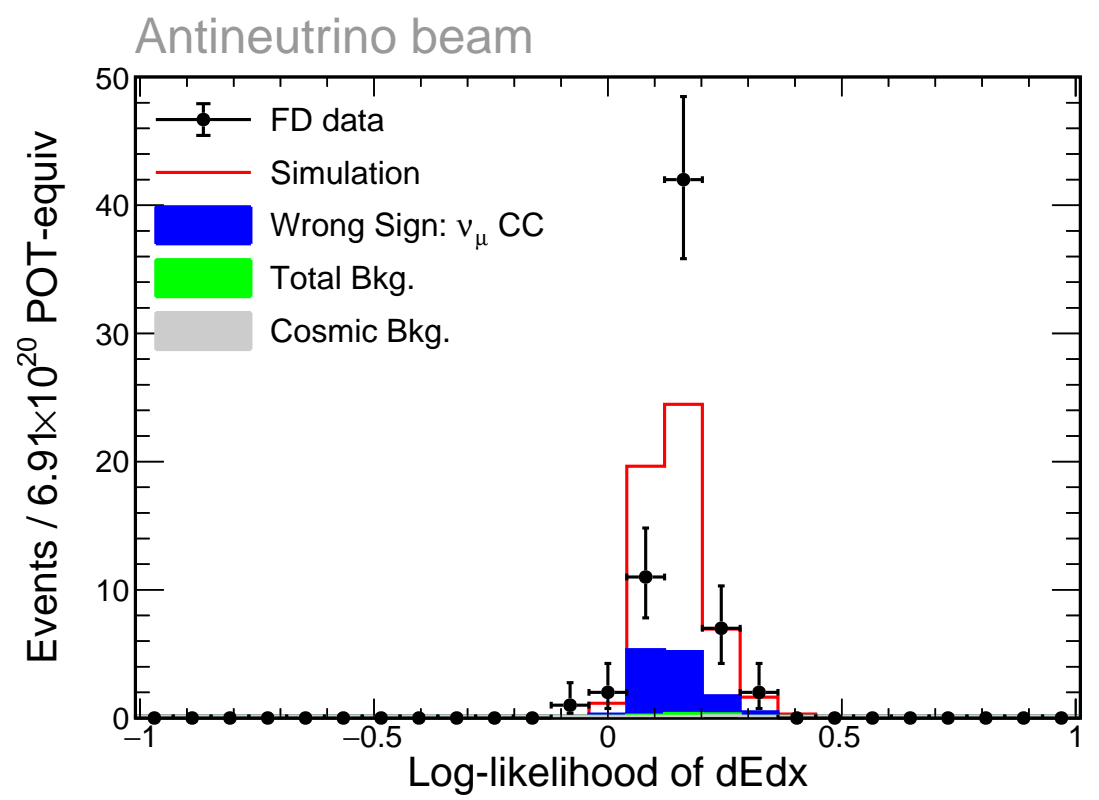

Figure 7.100: RHC FD distributions of the ReMId $\frac{d E}{d x}$ likelihood for the total dataset. Data is shown in black and simulation in red. Also shown is the wrong-sign $\nu_{\mu}$ component in blue, the cosmic background in grey and the total background in green. 


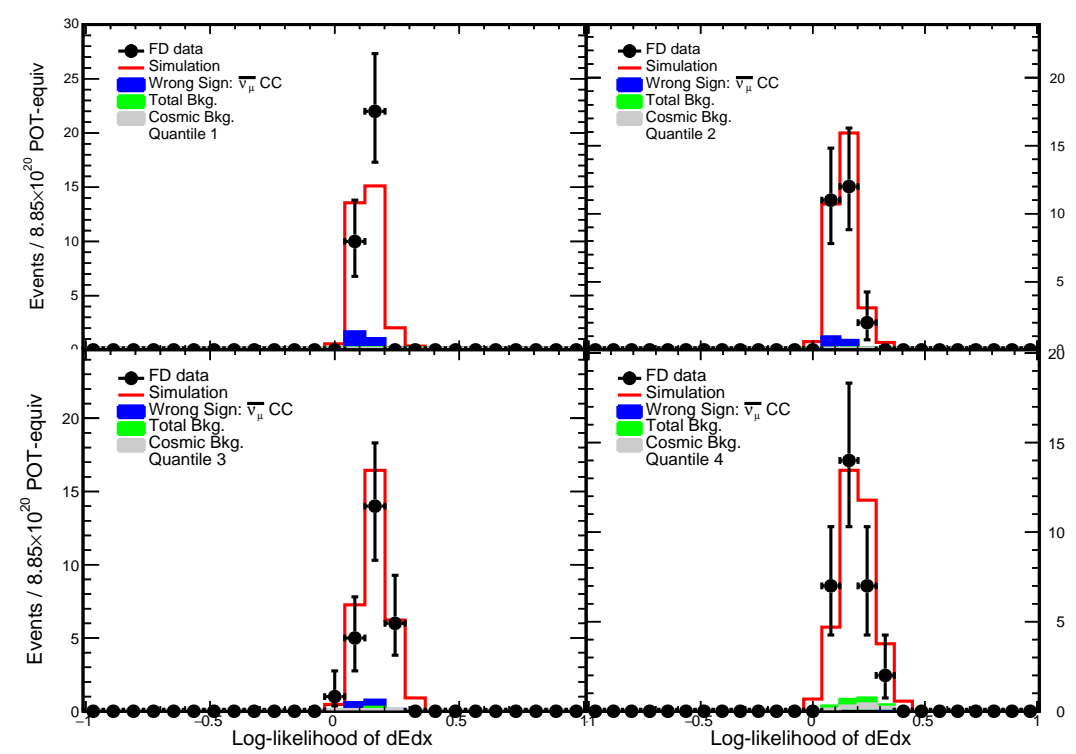

Figure 7.101: FHC FD distributions of the ReMId $\frac{d E}{d x}$ likelihood for each $E_{h a d} / E_{\nu}$ quantile. Data is shown in black and simulation in red. Also shown is the wrong-sign $\bar{\nu}_{\mu}$ component in blue, the cosmic background in grey and the total background in green.

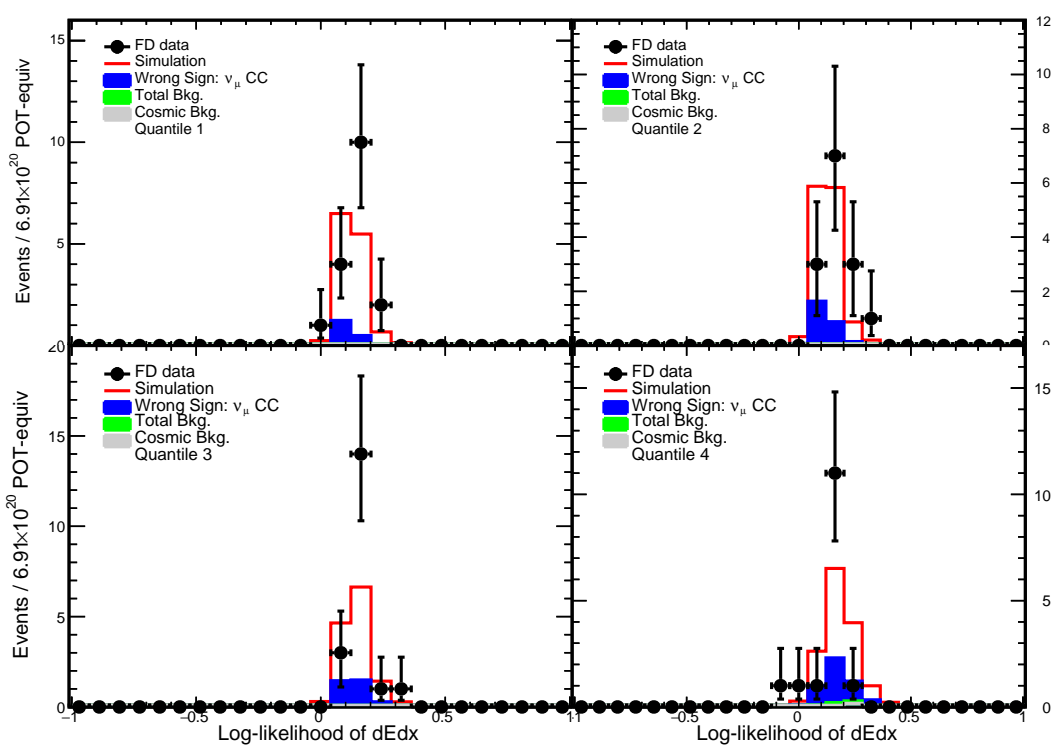

Figure 7.102: RHC FD distributions of the ReMId $\frac{d E}{d x}$ likelihood for each $E_{h a d} / E_{\nu}$ quantile. Data is shown in black and simulation in red. Also shown is the wrong-sign $\nu_{\mu}$ component in blue, the cosmic background in grey and the total background in green. 


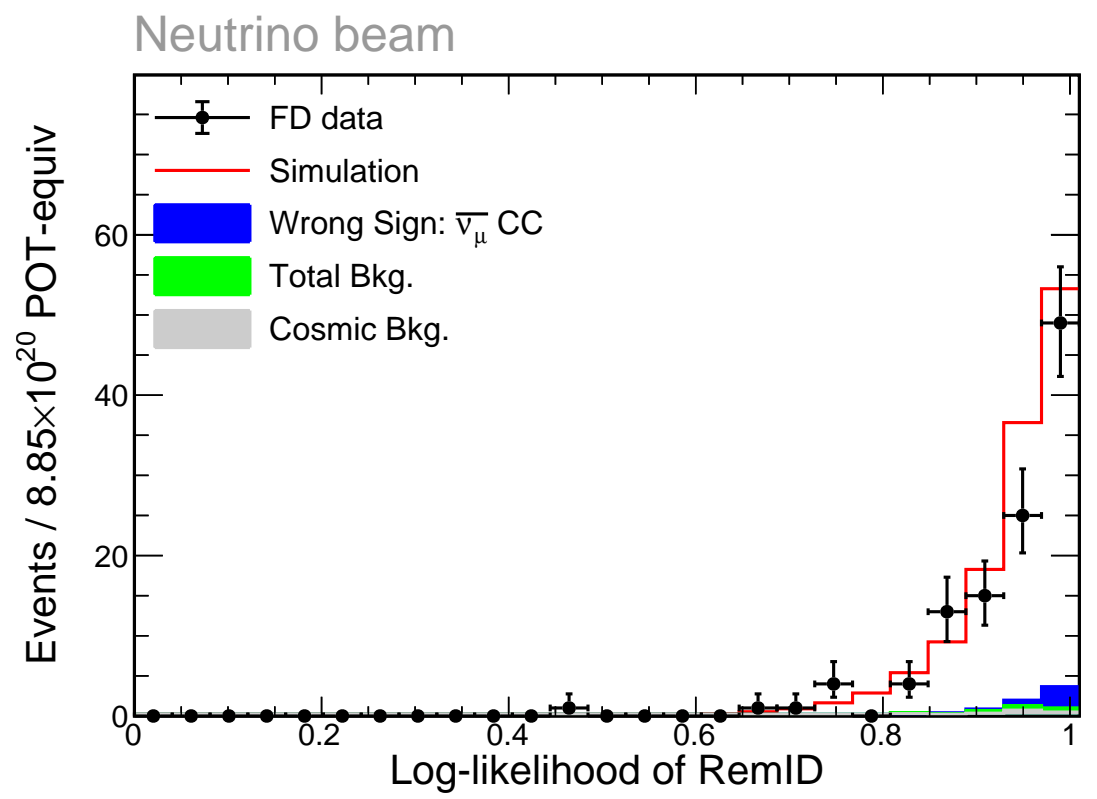

Figure 7.103: FHC FD distributions of the ReMID fraction of planes along the track consistent with having minimum ionizinglike $\frac{d E}{d x}$ likelihood for the total dataset. $E_{\text {had }} / E_{\nu}$ quantiles. Data is shown in black and simulation in red. Also shown is the wrong-sign $\bar{\nu}_{\mu}$ component in blue, the cosmic background in grey and the total background in green.

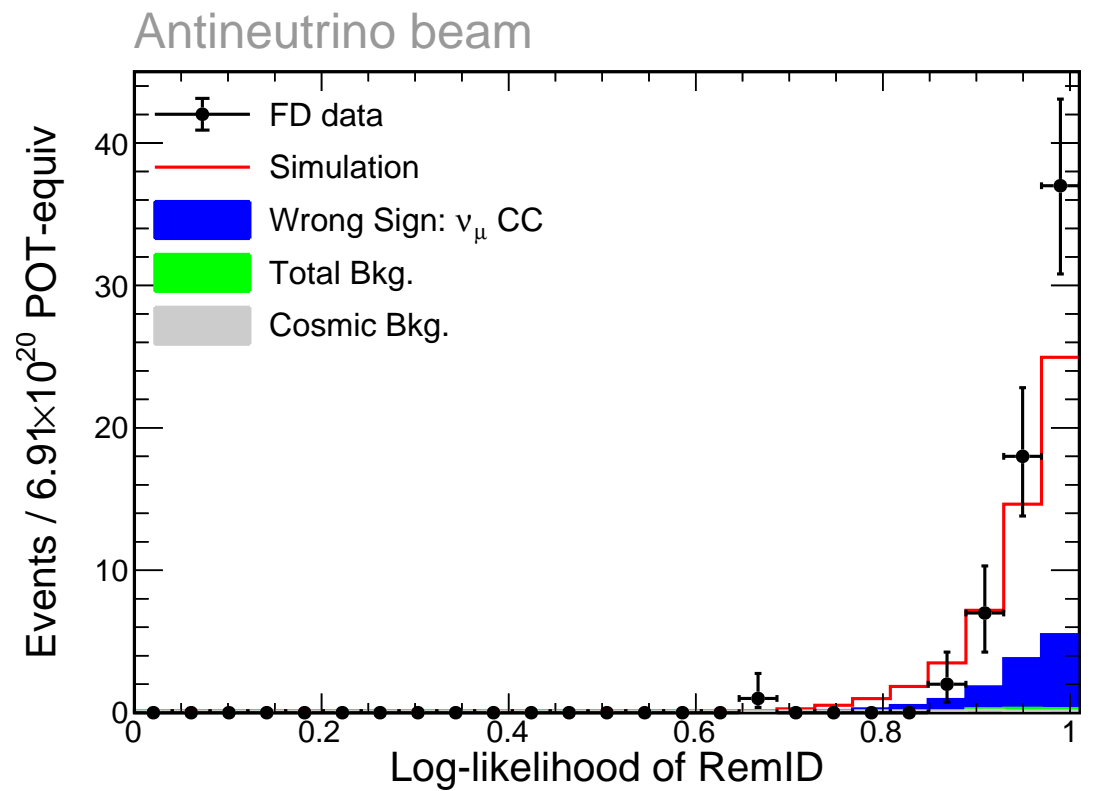

Figure 7.104: RHC FD distributions of the ReMID fraction of planes along the track consistent with having minimum ionizinglike $\frac{d E}{d x}$ likelihood for the total dataset. $E_{h a d} / E_{\nu}$ quantiles. Data is shown in black and simulation in red. Also shown is the wrong-sign $\nu_{\mu}$ component in blue, the cosmic background in grey and the total background in green. 


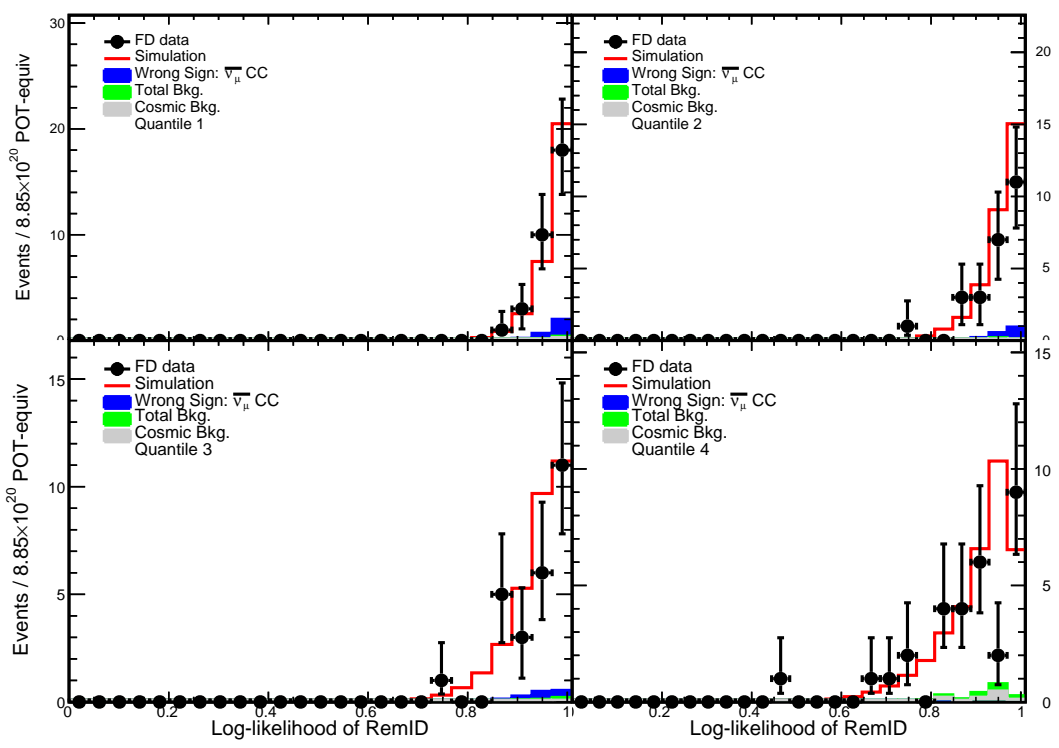

Figure 7.105: FHC FD distributions of the ReMId fraction of planes along the track consistent with having minimum ionizinglike $\frac{d E}{d x}$ likelihood for each $E_{h a d} / E_{\nu}$ quantile. Data is shown in black and simulation in red. Also shown is the wrong-sign $\bar{\nu}_{\mu}$ component in blue, the cosmic background in grey and the total background in green.

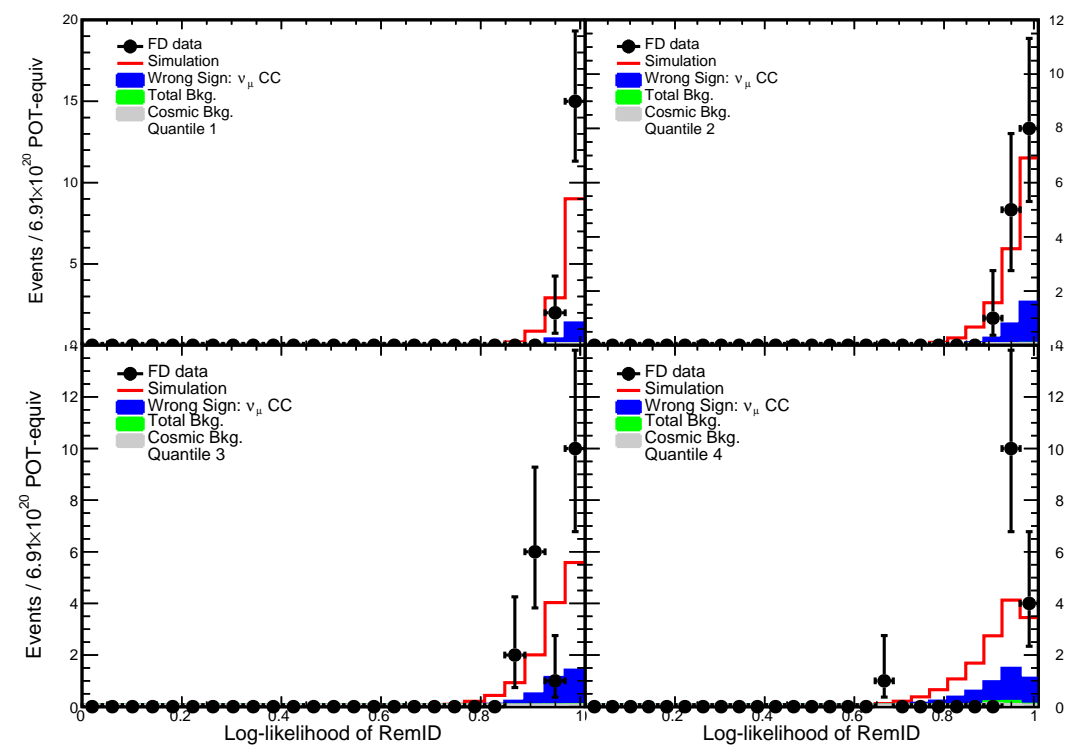

Figure 7.106: RHC FD distributions of the ReMId fraction of planes along the track consistent with having minimum ionizinglike $\frac{d E}{d x}$ likelihood for each $E_{h a d} / E_{\nu}$ quantile. Data is shown in black and simulation in red. Also shown is the wrong-sign $\nu_{\mu}$ component in blue, the cosmic background in grey and the total background in green. 


\subsubsection{Fitting the Far Detector Neutrino Energy Spectrum}

Constraints on oscillation parameters, confidence limit contours and significance plots are presented in this section. The $\nu_{\mu}+\bar{\nu}_{\mu}$ disappearance analysis $\Delta m_{32}^{2}$ vs. $\sin ^{2} \theta_{23}$ contour produced from the combined $\mathrm{FHC}+\mathrm{RHC}$ fit is shown first. Both the statistical uncertainty only and systematics inclusive contour are shown. Contours are then given individually for FHC data, for RHC data and for the combined FHC-RHC fit. All contours are drawn at the $90 \%$ confidence level.

The measurements and $1 \sigma$ bounds of the mixing angle and the mass splitting under the assumption of a normal (inverted) neutrino mass hierarchy are $\Delta m_{32}^{2}=+2.49_{-0.07}^{+0.09} \times$ $10^{-3} \mathrm{eV}^{2}\left(\Delta m_{32}^{2}=-2.54 \pm 0.08 \times 10^{-3} \mathrm{eV}^{2}\right)$ and $\sin ^{2} \theta_{23}=0.59 \pm 0.03\left(\sin ^{2} \theta_{23}=\right.$ $0.44 \pm 0.03)$. The $1 \sigma$ bounds quoted are the combined systematic and statistical uncertainty bounds. The best fit is found with the parameter $\delta_{C P}$ set to $3 \pi / 2$. Maximal mixing $\left(\sin ^{2} \theta_{23}=0.5\right)$ is disfavoured at the $1.7 \sigma$ level.

The $\chi^{2}$ significance is calculated from the $-2 \ln \mathrm{L}$ where $\mathrm{L}$ is the likelihood function calculated using Poisson statistics with Gaussian penalty terms for the systematic uncertainties. The $\Delta \chi^{2}$ defines the difference between the $\chi^{2}$ at a given point in $\Delta m_{32}^{2}$ vs. $\sin ^{2} \theta_{23}$ and the best fit point. The $\chi^{2}$ for the overall best fit is 84.6 for 150 degrees of freedom.

Official NOvA results use the Feldman-Cousins (FC) correction to determine confidence limit boundaries by inspecting the range of likelihood ratios found in pseudoexperiments [90]. Application of the FC correction is very computationally intensive and so confidence limit contours presented in this chapter do not have it applied. As in previous analyses, the FC correction is expected to slightly improve analysis sensitivity when compared to results shown in this thesis.

Figure 7.107 shows the normal hierarchy $90 \%$ confidence limit contours in $\Delta m_{32}^{2}$ vs. $\sin ^{2} \theta_{23}$ produced by the statistics only and systematics inclusive $\nu_{\mu}+\bar{\nu}_{\mu}$ disappearance analysis. The $8.85 \times 10^{20}$ POT FHC-only contour is also shown for comparison.

The normal hierarchy $90 \%$ confidence limit contours for neutrino beam data, antineutrino beam data and for the combined neutrino+antineutrino data are shown in Figure 7.108. The corresponding significance plots in $\sqrt{\Delta \chi^{2}}$ vs. $\Delta m_{32}^{2}$ and $\sqrt{\Delta \chi^{2}}$ vs. $\sin ^{2} \theta_{23}$ are shown in Figure 7.109 and Figure 7.110 respectively. As for the contour, the FHC fit, RHC fit and combined FHC+RHC fit are shown individually on the significance plots. Figure 7.107 through Figure 7.110 all use modified fitting scripts originally presented in [91]. 


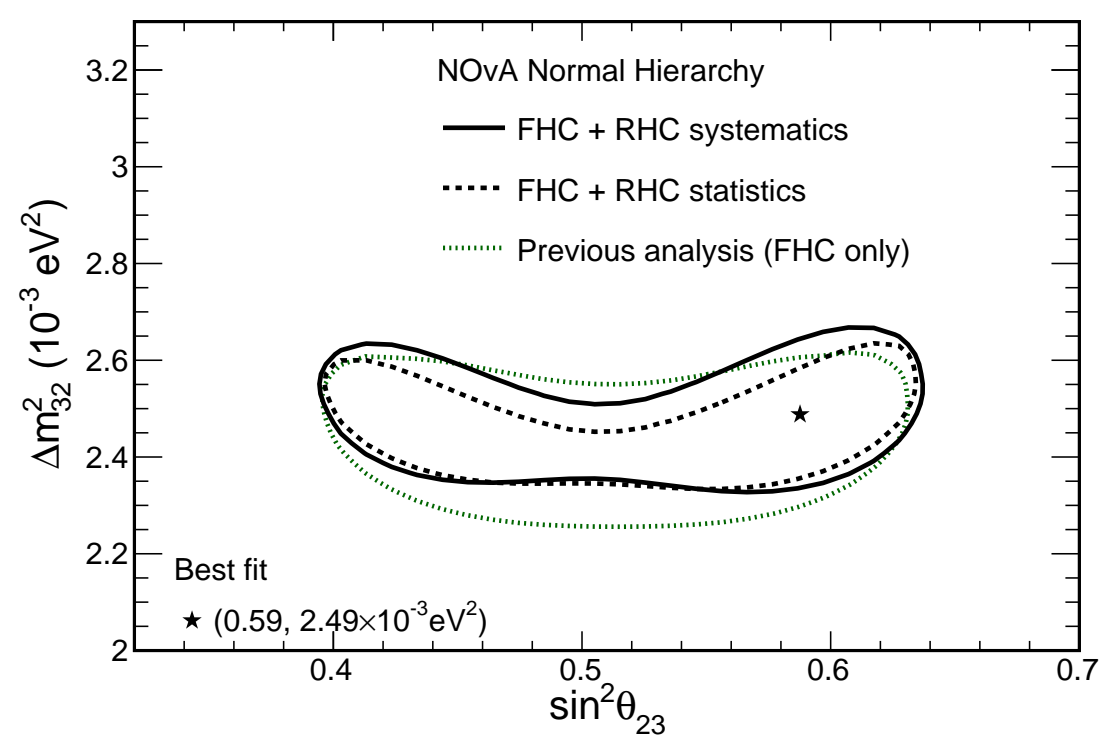

Figure 7.107: The statistics only and full systematics $90 \%$ confidence limit contours in $\Delta m_{32}^{2}$ vs. $\sin ^{2} \theta_{23}$ for the $\nu_{\mu}+\bar{\nu}_{\mu}$ disappearance analysis. The full systematics contour is shown by the solid black line and the statistics only contour is shown by the black dashed line. The $8.85 \times 10^{20}$ POT FHC-only previous analysis result is shown by the green dashed line for comparison.

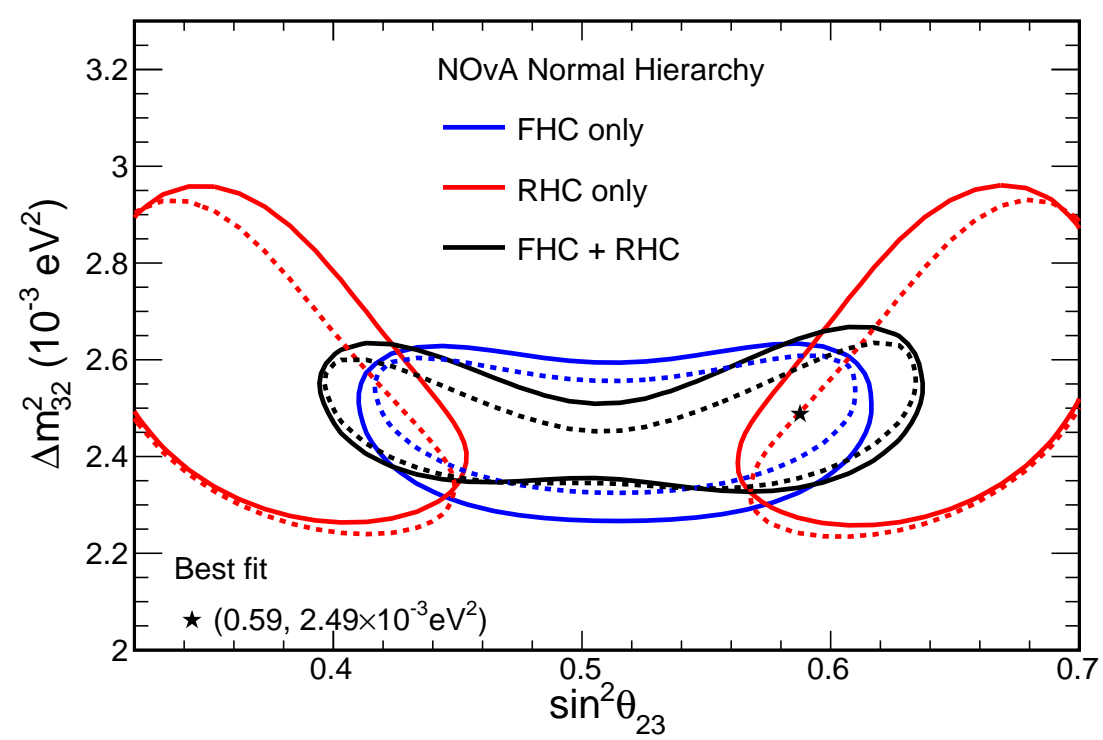

Figure 7.108: The $90 \%$ full systematic contour in $\Delta m_{32}^{2}$ vs. $\sin ^{2} \theta_{23}$ produced from the FHC fit (blue), RHC fit (red) and combined FHC+RHC fit (black). Solid lines show systematics-inclusive contours and dashed lines represent statistics-only contours. The best fit point is shown in the bottom left. 


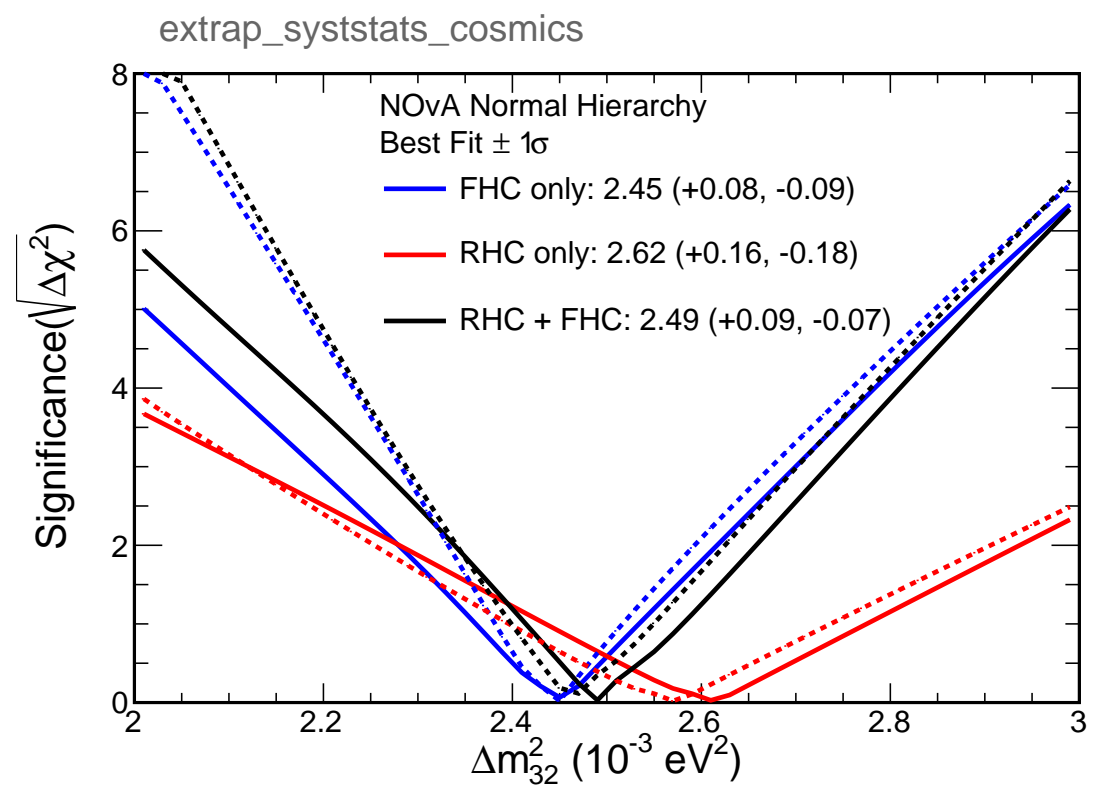

Figure 7.109: The significance vs. $\Delta m_{32}^{2}$ for the FHC fit (blue), for the RHC fit (red) and for the combined FHC+RHC fit (black), assuming normal hierarchy. Solid lines show the systematics-inclusive result and dashed lines represent the statistics-only equivalent. The numbers in the legend correspond to the best fit values and associated $1 \sigma$ bounds for each dataset.

The results presented in this chapter are from the $\nu_{\mu}+\bar{\nu}_{\mu}$ disappearance analysis, part of the official NOvA results that comprise $\nu_{\mu}+\bar{\nu}_{\mu}$ disappearance and $\nu_{e}+\bar{\nu}_{e}$ appearance. The joint $\Delta m_{32}^{2}$ vs. $\sin ^{2} \theta_{23}$ contour produced from analysis of NOvA disappearance and appearance data is shown in Figure 7.111 alongside the contours produced from other leading experiments - T2K, SuperK, MINOS and IceCube.

\subsubsection{The Matter Effect \& Combined Octant-Hierarchy Sensitivity}

Previous analyses have given measurements for each of the two possible octants that $\sin ^{2} \theta_{23}$ may occupy. The combined $\nu_{\mu}+\bar{\nu}_{\mu}$ disappearance results exclude the lower octant and upper-octant to a greater than $1 \sigma$ significance in the normal and inverted hierarchy respectively. Constraints on the octant-hierarchy combination can be extracted from analysis of the measured value of $\sin ^{2} \theta_{23}$ when using each horn current dataset. This is possible because matter effects introduce false CPT-violation meaning $\bar{\nu}_{\mu}$ and $\nu_{\mu}$ disappear differently ${ }^{3}$ - in vacuum CPT symmetry leads to identical $\nu_{\mu}$ and $\bar{\nu}_{\mu}$ disappearance.

A discussion of the theory of neutrino oscillation is provided in Section 2.2.3. It can

\footnotetext{
${ }^{3}$ The matter effect is described in Section 2.4.1.
} 


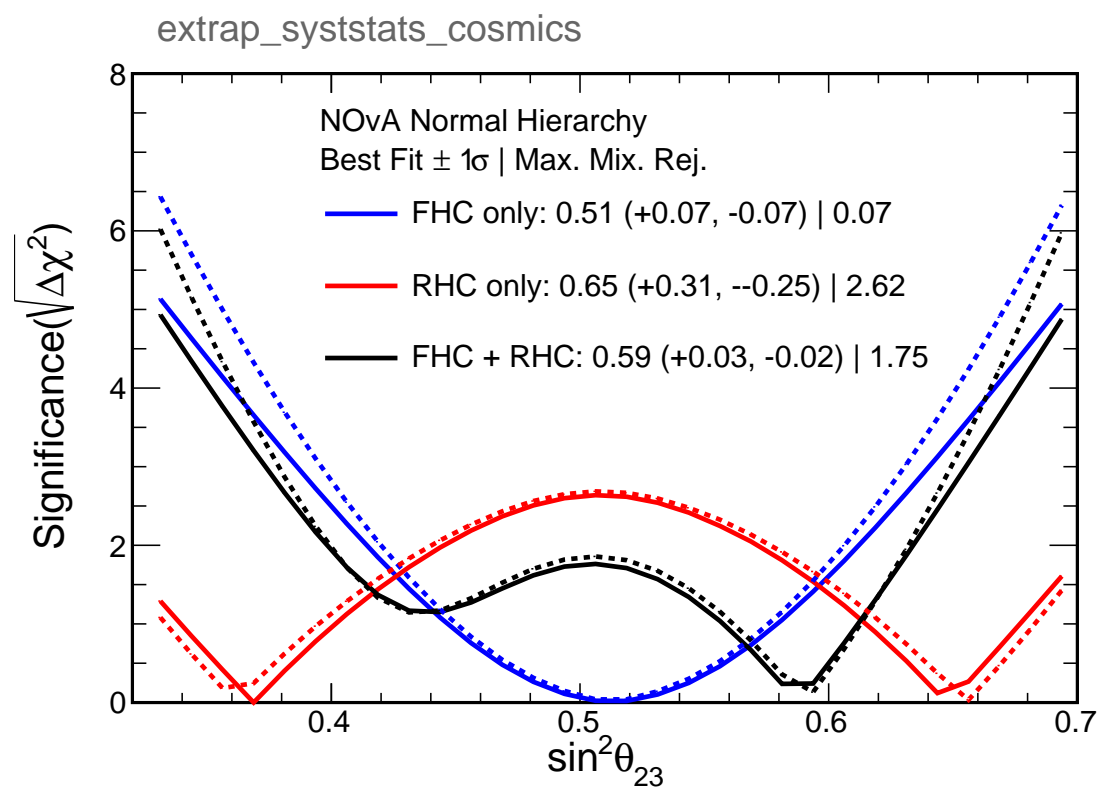

Figure 7.110: The significance vs. $\sin ^{2} \theta_{23}$ for the FHC fit (blue), for the RHC fit (red) and for the combined FHC+RHC fit (black), assuming normal hierarchy. Solid lines show the systematics-inclusive result and dashed lines represent the statistics-only equivalent. The numbers in the legend correspond to the best fit values and associated $1 \sigma$ bounds for each dataset. The second number, right of the 'I' symbol, is the $\sigma$ level to which maximal-mixing $\left(\sin ^{2} \theta_{23}=0.5\right)$ is rejected by the labelled dataset.

be shown that maximal disappearance in a vacuum occurs at $\left.\sin ^{2} \theta_{23} \simeq \frac{1}{\left(2-2 \sin ^{2} \theta_{13}\right.}\right)$ and that the probability of disappearance is symmetric in $\sin ^{2} \theta_{23}$ about this point. From this, it can be seen that maximal disappearance occurs at $\sin ^{2} \theta_{23}=0.5$ when $\sin ^{2} \theta_{13}=0$ a possibility no longer allowed by recent measurements of a non-zero $\sin ^{2} \theta_{13}$. Maximal disappearance occurs at a different point to maximal 23-sector mixing but the exact value is subject to matter effects. This opens a door for the $\nu_{\mu}+\bar{\nu}_{\mu}$ disappearance analysis to have sensitivity to the octant-hierarchy combination.

Matter effects boost (suppress) $\nu_{e}$ appearance for normal (inverted) hierarchy and vice versa for $\bar{\nu}_{e}$. This has a direct effect on NOvA constraints as the number of selected candidate events is directly affected by this suppression or boosting of $\nu_{e}$ appearance. The additional normal hierarchy $\nu_{e}$ appearance increases $\nu_{\mu}$ disappearance and/or decreases $\nu_{\tau}$ appearance. $\mathrm{CP}$-violation can occur because the e/ $\tau$ appearance split may be different for neutrinos and antineutrinos. At maximal $\nu_{\mu}$ disappearance, additional $\nu_{e}$ must be contributed to solely from less $\nu_{\tau}$ appearance.

Assuming the normal hierarchy scenario, maximal $\nu_{\mu}$ disappearance occurs at higher value of $\sin ^{2} \theta_{23}(0.514)$ than the no-matter-effect value (0.511). Maximal $\bar{\nu}_{\mu}$ disappearance 


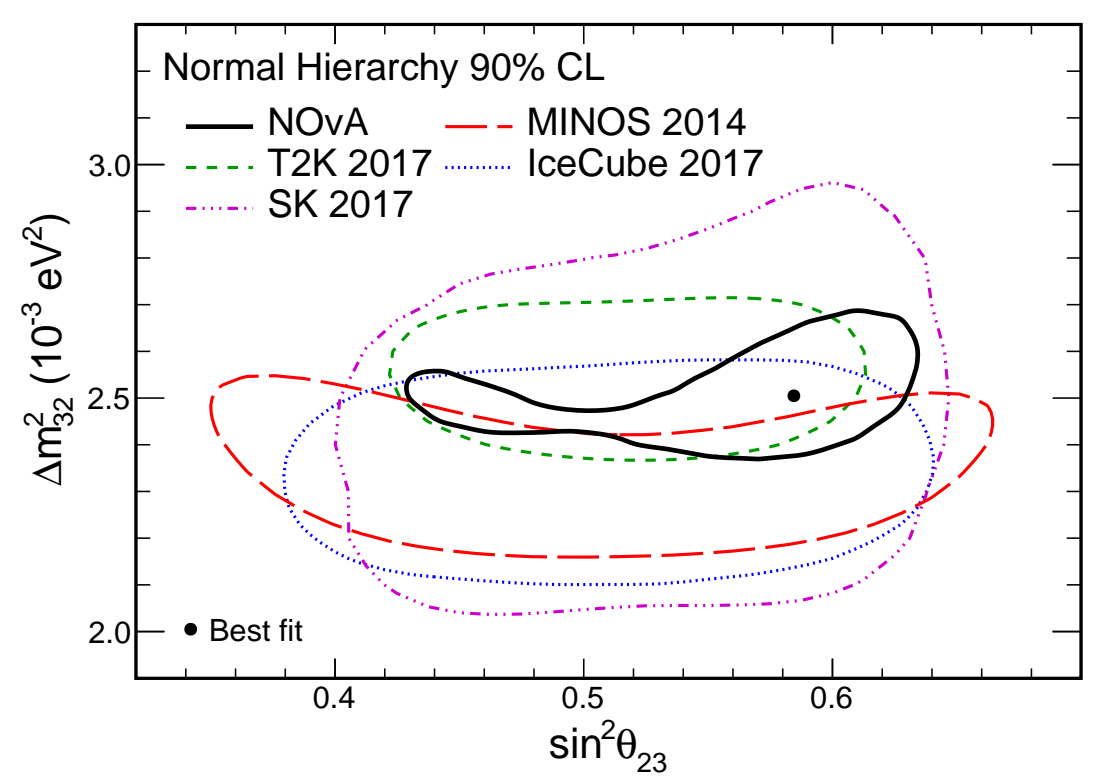

Figure 7.111: The $90 \%$ confidence limit contour in $\Delta m_{32}^{2}$ vs. $\sin ^{2} \theta_{23}$ produced by various experiments. The NOvA joint $\left(\nu_{\mu}+\bar{\nu}_{\mu}\right)$ disappearance and $\left(\nu_{e}+\bar{\nu}_{e}\right)$ appearance contour is shown in black, the T2K contour in green [92], the SuperK contour in purple [93], the MINOS contour in red [39] and the Icecube contour in blue [94]. The NOvA best fit point is also shown as the black dot. This plot was first shown at NEUTRINO2018 by Diana Mendez [95].

occurs at a lower value of $\sin ^{2} \theta_{23}(0.508)$. The reverse is true for the inverse hierarchy with the maximal disappearance point decreasing for $\nu_{\mu}$ and increasing for $\bar{\nu}_{\mu}$. In the current results $\bar{\nu}_{\mu}$ disappearance data, for both the inverted and normal hierarchy scenario, maps to two almost identical points in $\sin ^{2} \theta_{23}$ in the lower octant $(\approx 0.36)$ and to two different points in the upper octant $(\approx(0.65$ and 0.66$))$. The $\nu_{\mu}$ disappearance data maps to values of $\sin ^{2} \theta_{23}$ close to maximal disappearance in both hierarchy scenarios at approximately $\sin ^{2} \theta_{23}=0.51$ with $\sin ^{2} \theta_{23} I H<\sin ^{2} \theta_{23} H$. The convolution of the inverted and normal hierarchy fits that produce each of the $\sin ^{2} \theta_{23}$ values in a single octant gives an exclusion significance at that value of $\sin ^{2} \theta_{23}$. Disappearance is symmetric about the maximal disappearance point and, because of the hierarchy-dependent measurement of $\sin ^{2} \theta_{23}$, an octant-hierarchy sensitivity can be extracted by analysis of the individual FHC and RHC measurements of $\sin ^{2} \theta_{23}$ and the respective agreement with each hierarchies' maximal disappearance offset. 


\begin{tabular}{|ccc|}
\hline Source of uncertainty & $\begin{array}{c}\text { Uncertainty in } \\
\sin ^{2} \theta_{23}\left(\times 10^{-3}\right)\end{array}$ & $\begin{array}{c}\text { Uncertainty in } \\
\Delta m_{32}^{2}\left(10^{-6} \mathrm{eV}^{2}\right)\end{array}$ \\
\hline Absolute hadronic energy scale & \pm 7.4 & $+21 /-26$ \\
Absolute muon energy scale & \pm 1.4 & $+9.1 /-10$ \\
Relative hadronic energy scale & \pm 3.6 & $+11 /-13$ \\
Relative muon energy scale & \pm 1.2 & $+2.6 /-1.8$ \\
Cross sections and final-state interaction & \pm 3 & $+13 /-15$ \\
Detector response & \pm 1.4 & $+3.1 /-3$ \\
Neutrino flux & \pm 0.52 & $+1.6 /-1.6$ \\
Neutron uncertainty & \pm 4.3 & $+6.3 /-11$ \\
Normalisation & \pm 1.3 & $+1.9 /-2.7$ \\
$\Delta_{C P}(0-2 \pi)$ & \pm 0.14 & $+12 /-12$ \\
\hline Statistical Total & \pm 28 & $+62 /-59$ \\
Systematic Total & \pm 9.6 & $+26 /-33$ \\
\hline
\end{tabular}

Table 7.8: Breakdown of systematic and statistical uncertainty contributions on the values of $\sin ^{2} \theta_{23}$ and $\Delta m_{32}^{2}$ as measured by the $\nu_{\mu}+\bar{\nu}_{\mu}$ disappearrance analysis.

\subsection{Systematic Uncertainty Analysis}

The NOvA analysis addresses a number of systematic uncertainties. Table 7.8 shows the the positive and negative uncertainty associated with each systematic. Values are given in units of the relevant oscillation parameter. The absolute hadronic energy scale, relative hadronic energy scale, neutron uncertainty and cross section uncertainty contribute most significantly to the overall systematic uncertainty in the analysis. Figure 7.112 and Figure 7.113 graphically show the calculated magnitudes of each systematic uncertainty on the measurements of $\Delta m_{32}^{2}$ and $\sin ^{2} \theta_{23}$ respectively. 


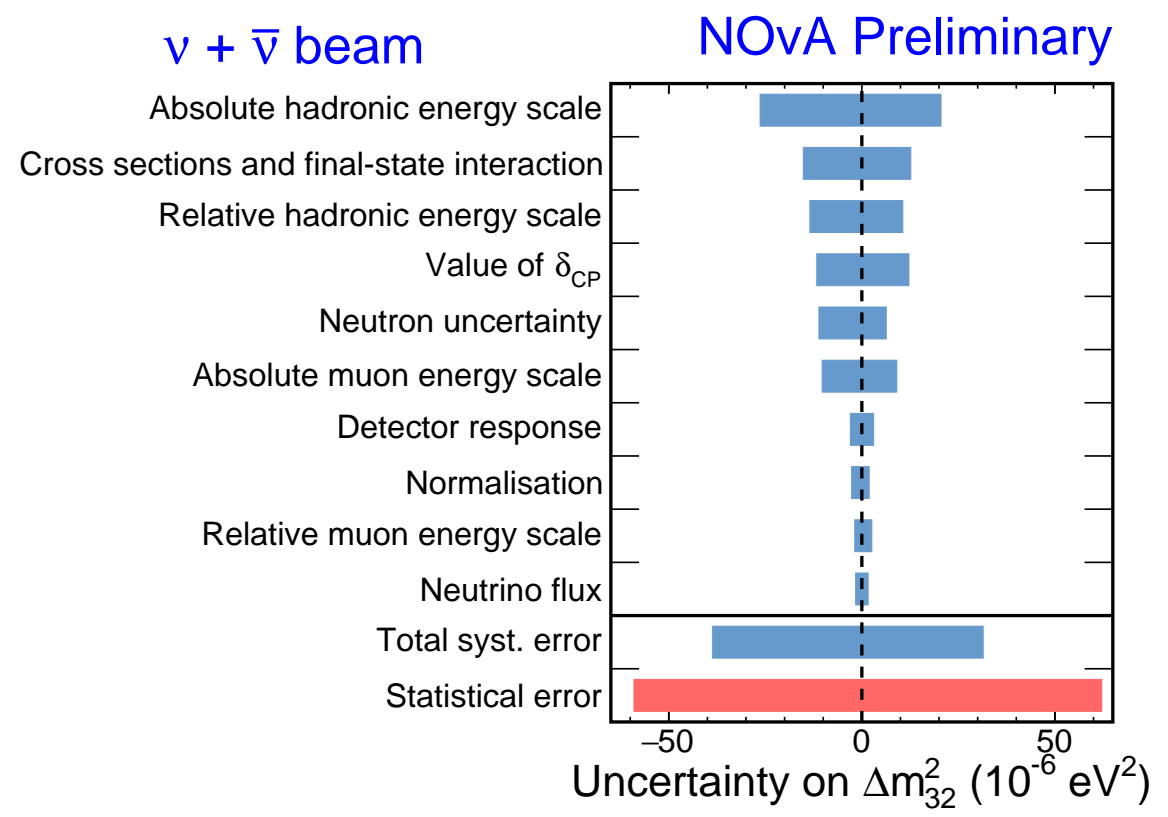

Figure 7.112: Uncertainty bar chart for $\Delta m_{32}^{2}$ showing each systematics positive and negative uncertainty on the measurement. Fake data was used and oscillated assuming the $8.85 \times 10^{20}$ POT FHC-only best fit [3] and normal hierarchy.

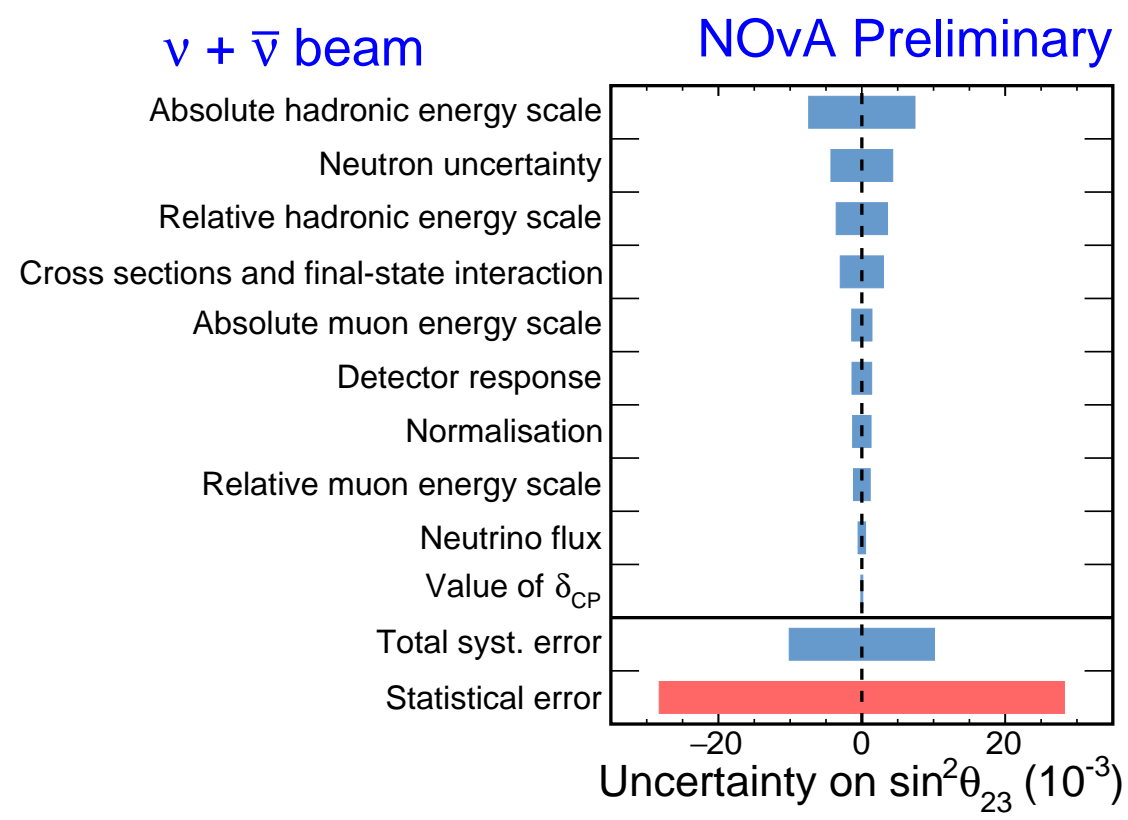

Figure 7.113: Uncertainty bar chart for $\sin ^{2} \theta_{23}$ showing each systematics positive and negative uncertainty on the measurement. Fake data was used and oscillated assuming the $8.85 \times 10^{20}$ POT FHC-only best fit [3] and normal hierarchy. 
Figure 7.114 shows the total best fit shift caused by each systematic for the individual fits produced from FHC data, RHC data and the combined FHC+RHC result. The Cherenkov uncertainty arises from the effects of Cherenkov radiation on the light yield. The relative calibration uncertainty arises from imperfections in modelling cell variance and the absolute calibration uncertainty arises from the application of the overall scale factor. The neutron uncertainty arises due to the potential presence of neutral particles in the hadronic system that leave no energy signature in the detector. The MEC uncertainty is due to imperfect modelling of meson exchange current processes. The calibration shape uncertainty is due to the difference in true and reconstructed cell response to light in the fibre. The absolute calibration and Cherenkov radiation systematics have the largest pulls at the best-fit. The most significant systematic pulls at the best-fits produced by the individual FHC (neutrino) and RHC (antineutrino) data are given in Table 7.9. The most significant systematic pulls at the best-fit point for the joint $\mathrm{FHC}+\mathrm{RHC}$ analysis are given in Table 7.10. The calibration and neutron systematics contribute significantly to the uncertainty, and are pulled by $-0.672 \sigma$ and $-0.446 \sigma$ respectively.

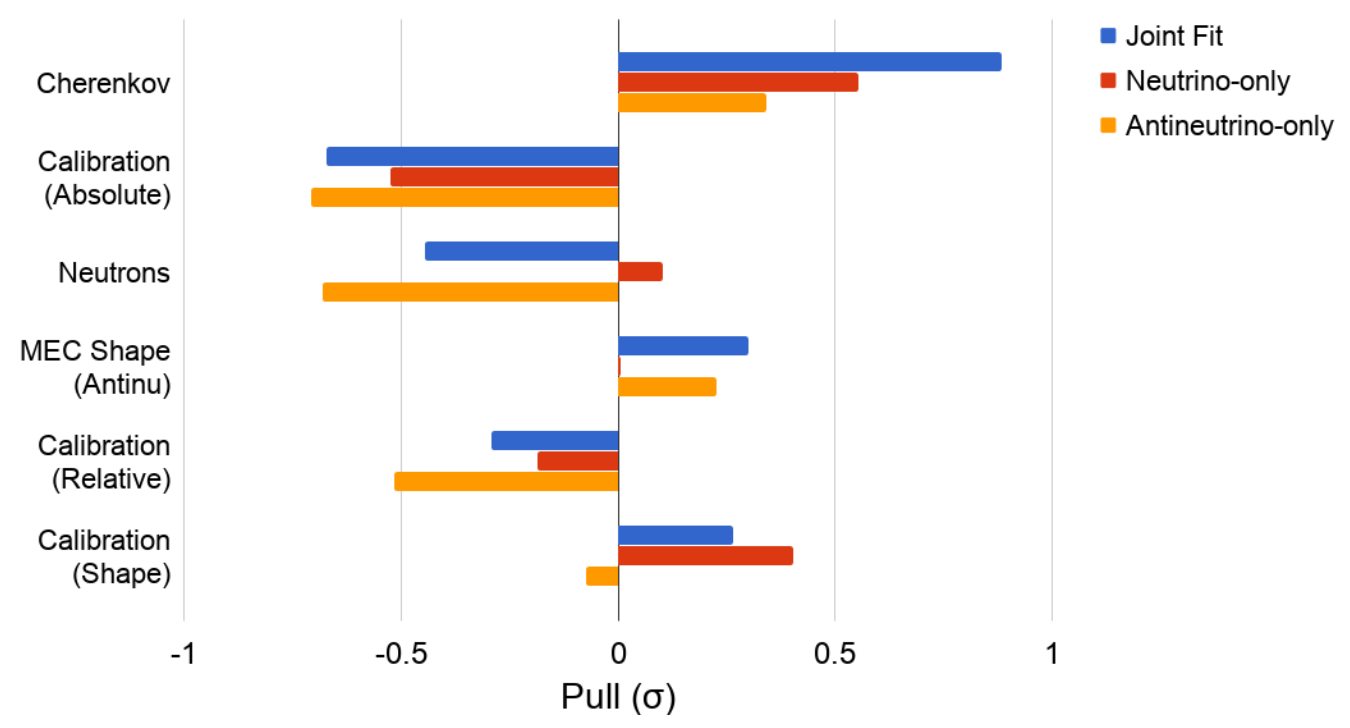

Figure 7.114: Bar chart comparing the pulls of key systematics on the measured best fit from the $\nu_{\mu}+\bar{\nu}_{\mu}$ disappearance analysis for neutrino data only (red), antineutrino data only (yellow) and both neutrino and antineutrino data (blue). 


\begin{tabular}{|cc|cc|}
\hline FHC & & RHC & \\
\hline Systematic & Pull & Systematic & Pull \\
\hline Cherenkov effects & 0.553 & Calibration & -0.708 \\
Calibration & -0.523 & Neutron uncertainty & -0.680 \\
Resonance RPA & 0.460 & Absolute $E_{\mu^{ \pm}}$scale & 0.573 \\
Calibration shape & 0.404 & Relative calibration & -0.515 \\
Scintillator brightness & -0.249 & Cherenkov effects & 0.341 \\
Absolute $E_{\mu^{ \pm}}$scale & -0.230 & Scintillator brightness & 0.266 \\
Relative calibration & -0.186 & Resonance CC $M_{A}$ & 0.227 \\
MEC & -0.150 & MEC & 0.227 \\
Relative $E_{\mu^{ \pm}}$scale & 0.144 & ND to FD kinematics extrapolation & -0.115 \\
Hadronic flux prediction & -0.144 & Resonance CC $M_{V}$ & 0.108 \\
\hline
\end{tabular}

Table 7.9: Ten most significant systematic pulls on the FHC fit (left), and RHC fit (right), ordered by absolute size of the pull. 


\begin{tabular}{|c|c|c|c|}
\hline \multicolumn{4}{|c|}{ Systematic Pulls } \\
\hline Systematic & Pull (FHC+RHC) & Pull (FHC only) & Pull (RHC only) \\
\hline Cherenkov effects & 0.883 & 0.553 & 0.341 \\
\hline Calibration & -0.672 & -0.523 & -0.708 \\
\hline Neutron uncertainty & -0.446 & 0.103 & -0.680 \\
\hline MEC & 0.299 & 0.007 & 0.227 \\
\hline Relative calibration & -0.292 & -0.186 & -0.515 \\
\hline Calibration shape & 0.265 & 0.404 & -0.071 \\
\hline Hadronic flux prediction & -0.227 & -0.144 & -0.057 \\
\hline FHC pileup and noise normalization & -0.207 & -0.132 & 0.000 \\
\hline Resonance RPA & 0.183 & 0.460 & -0.098 \\
\hline Absolute $E_{\mu_{-}^{+}}$scale & 0.162 & -0.230 & 0.573 \\
\hline
\end{tabular}

Table 7.10: Top ten systematic pulls in the combined FHC+RHC fit ordered by absolute size of the pull. The individual RHC and FHC pulls are restated for comparison. 
The shift in the RHC+FHC statistics-only best fit caused by each systematic is shown in Figure 7.115 through Figure 7.121. The $\nu_{\mu}+\bar{\nu}_{\mu}$ disappearance analysis statistics-only $90 \%$ confidence limit contour and best fit point are shown by the black line and star respectively. The best fit produced once all systematics are applied is shown by the red star.

Figure 7.115 shows the best fit shift caused by each of the calibration systematics. The absolute (pink), relative (green) and shape (orange) calibration systematics have the most significant effect. This is true in both $\sin ^{2} \theta_{23}$ and $\Delta m_{32}^{2}$. This is expected as the calibration systematic uncertainties dwarf the light level uncertainties and allow for the largest space in which the best fit can shift.

Figure 7.116 shows the best fit shift caused by each of the flux systematics in $\Delta m_{32}^{2}$ vs. $\sin ^{2} \theta_{23}$. The shifts are very small for all flux systematics with the third (orange) and fifth (teal) primary component causing the largest effect. The NuMI flux is well characterized [42]. It follows that the possible space for a shift on the best fit from the flux uncertainties is very small.

Figure 7.117 shows the best fit shift caused by each of the GENIE principal component analysis systematics in $\Delta m_{32}^{2}$ vs. $\sin ^{2} \theta_{23}$. The fourth (blue) primary component has the greatest effect but, as with the flux systematics, the GENIE shifts to the best fit are very small.

Figure 7.118 shows the best fit shift caused by each of the GENIE reweight systematics in $\Delta m_{32}^{2}$ vs. $\sin ^{2} \theta_{23}$. The systematics from the charged-current resonance axial mass $\left(M_{A}\right)$ and vector mass $\left(M_{V}\right)$ have the biggest effect on the best fit point, shown in pink and orange respectively. The associated uncertainties are significant and it follows that the corresponding shifts on the best fit are large.

Figure 7.119 shows the best fit shift caused by each of the MEC systematics in $\Delta m_{32}^{2}$ vs. $\sin ^{2} \theta_{23}$. The best fit is most affected by the 2018 neutrino flux shaping systematic shown in orange. The initial state neutron fraction systematic has almost no effect on the best fit.

Figure 7.120 shows the best fit shift caused by each of the normalization and energy scale systematics in $\Delta m_{32}^{2}$ vs. $\sin ^{2} \theta_{23}$. The neutron visible energy uncertainty is the biggest contributor to overall shift. This is expected as the amount of energy carried by neutrons, invisible to the unmagnetized detectors, has a large associated uncertainty.

Figure 7.121 shows the best fit shift caused by each of the cross-section systematics. The resonant Random Phase Approximation (RPA) is the biggest contributor to overall 
uncertainty. Resonance events arise when a neutrino excites a struck nucleon - the excited nucleon then decays into a ground state nucleon and single pion. The RPA is an effective theory constructed to address excitation of interactions nucleons in a many body system. The RPA has only been formally calculated for QE events and a coarse approximation based on the QE RPA is used for the resonance RPA. This means that the error associated with it is significant and leads to the large effective space for the best fit to shift. 

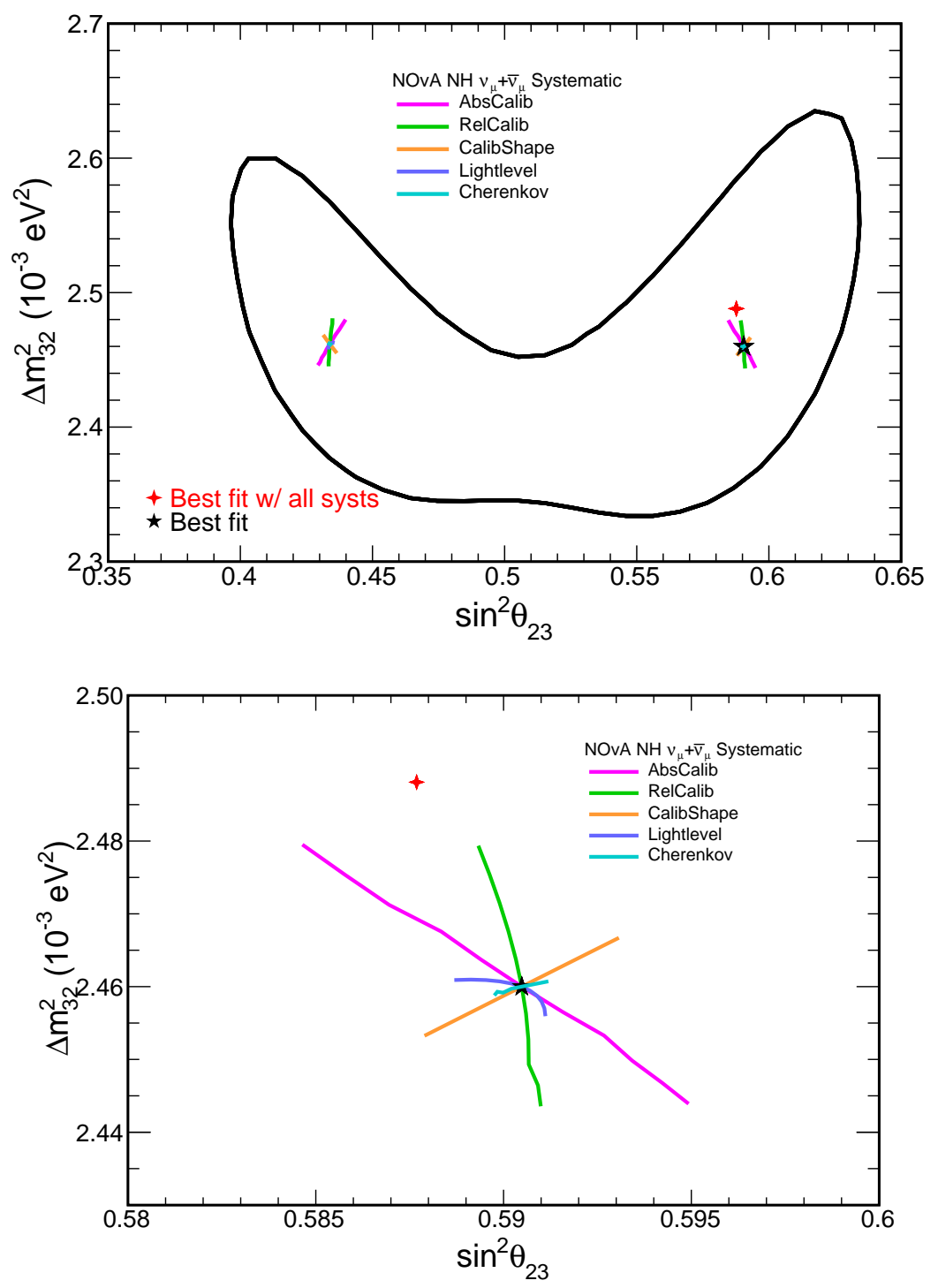

Figure 7.115: Plot of $\Delta m_{32}^{2}$ vs. $\sin ^{2} \theta_{23}$ with the best fit shift caused by each calibration systematic shown by the coloured lines. The effect of the absolute systematic is shown in pink, the relative calibration is shown in green, the calibration shaping systematic is shown in orange, the light level systematic in dark blue and the Cherenkov systematic in teal. The $\nu_{\mu}+\bar{\nu}_{\mu}$ disappearance statistics-only $90 \%$ confidence limit contour and corresponding best fit are shown by the black line and star respectively. The systematicsinclusive $\nu_{\mu}+\bar{\nu}_{\mu}$ disappearance best fit is shown by the red cross. The top figure shows an expanded axis scale and the bottom figure shows a zoomed scale. 

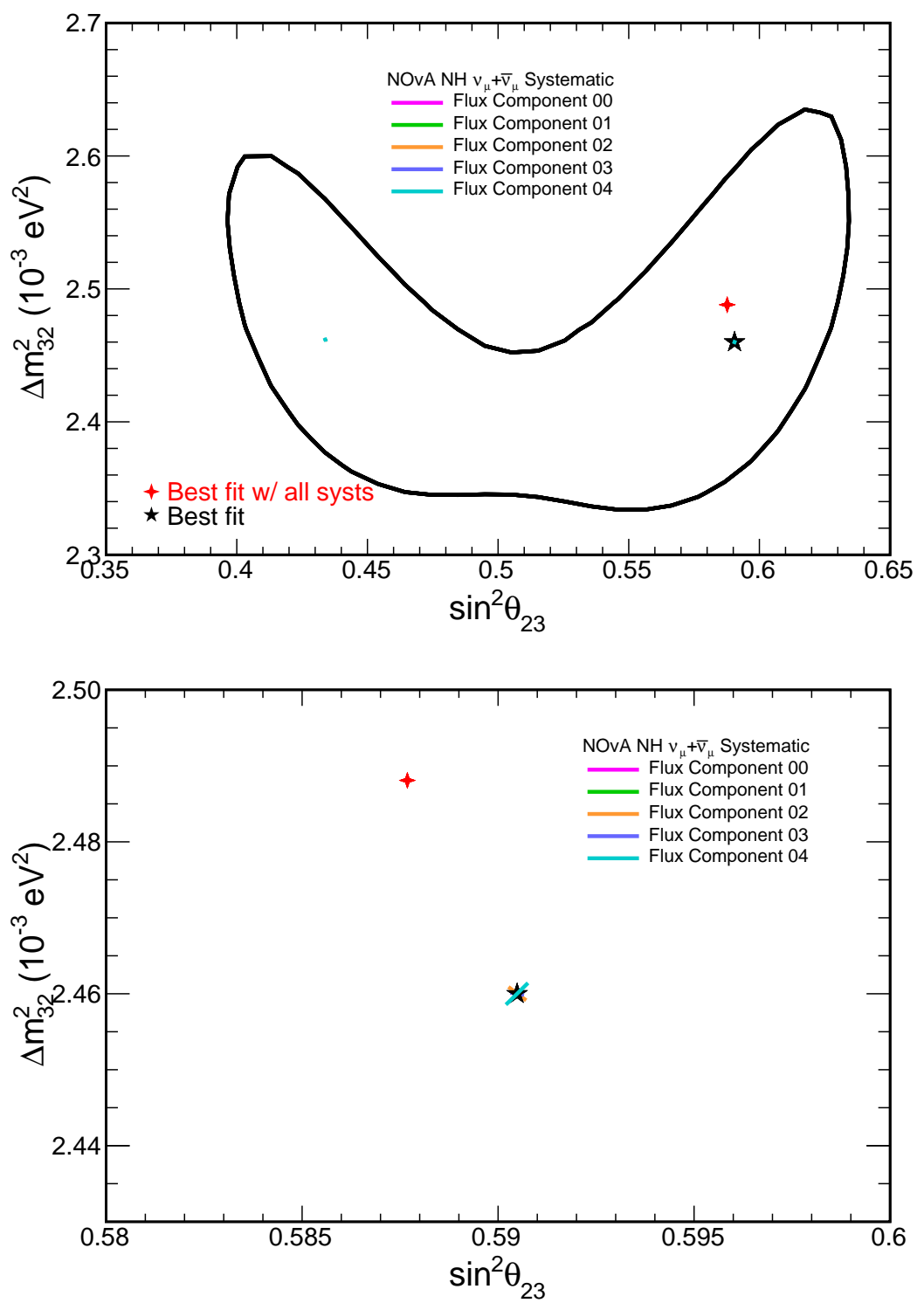

Figure 7.116: Plot of $\Delta m_{32}^{2}$ vs. $\sin ^{2} \theta_{23}$ and the best fit shift caused by each PPFX flux systematic is shown by the coloured lines. The $\nu_{\mu}+\bar{\nu}_{\mu}$ disappearance statistics-only $90 \%$ confidence limit contour and corresponding best fit are shown by the black line and star respectively. The systematics-inclusive $\nu_{\mu}+\bar{\nu}_{\mu}$ disappearance best fit is shown by the red cross. The top figure shows an expanded axis scale and the bottom figure shows a zoomed scale. 

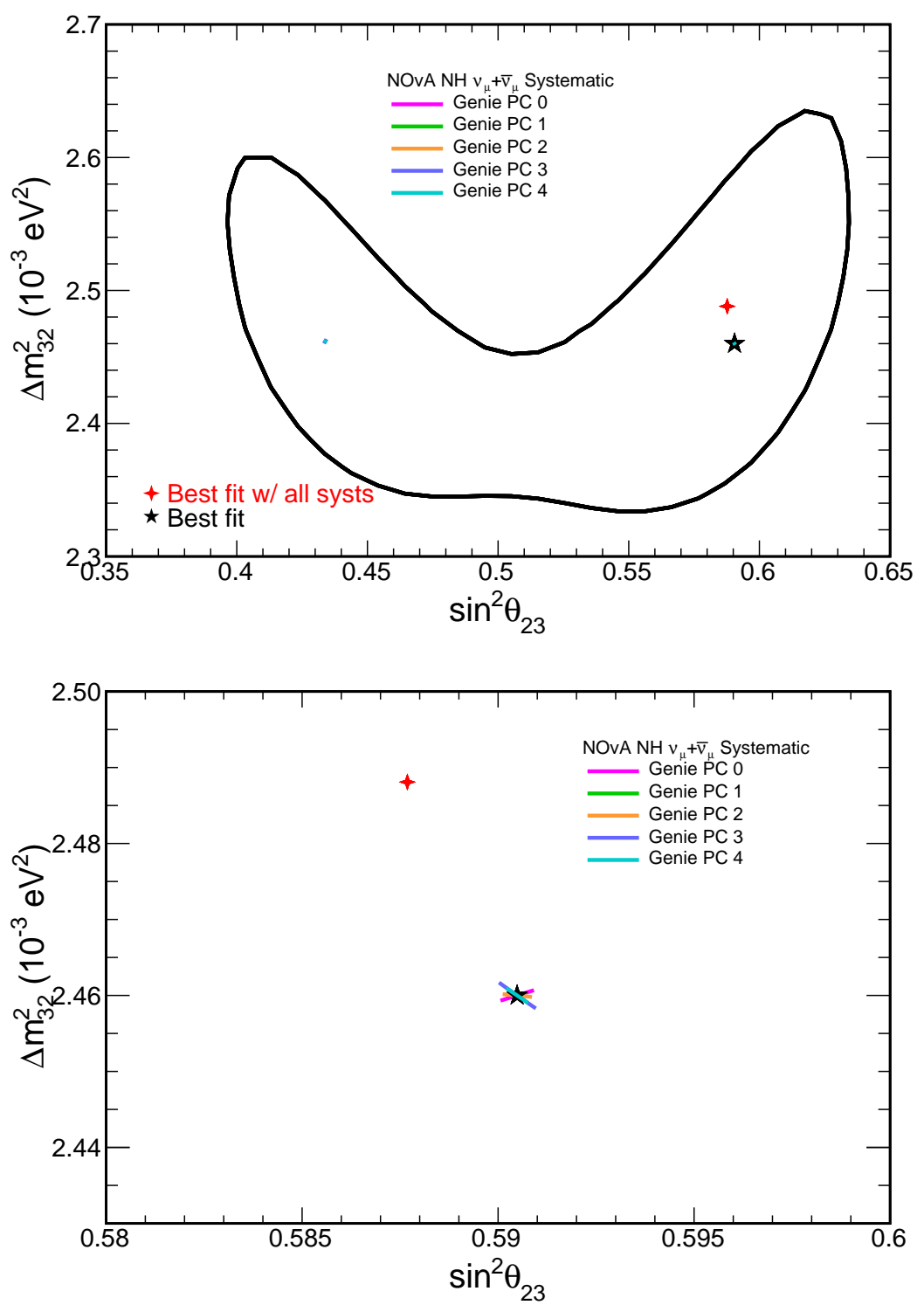

Figure 7.117: Plot of $\Delta m_{32}^{2}$ vs. $\sin ^{2} \theta_{23}$ with the best fit shift caused by each GENIE principal component analysis (PCA) systematic shown by the coloured lines. These systematics address inefficiencies in selection criteria at each detector. The $\nu_{\mu}+\bar{\nu}_{\mu}$ disappearance statistics-only $90 \%$ confidence limit contour and corresponding best fit are shown by the black line and star respectively. The systematics-inclusive $\nu_{\mu}+\bar{\nu}_{\mu}$ disappearance best fit is shown by the red cross. The top figure shows an expanded axis scale and the bottom figure shows a zoomed scale. 

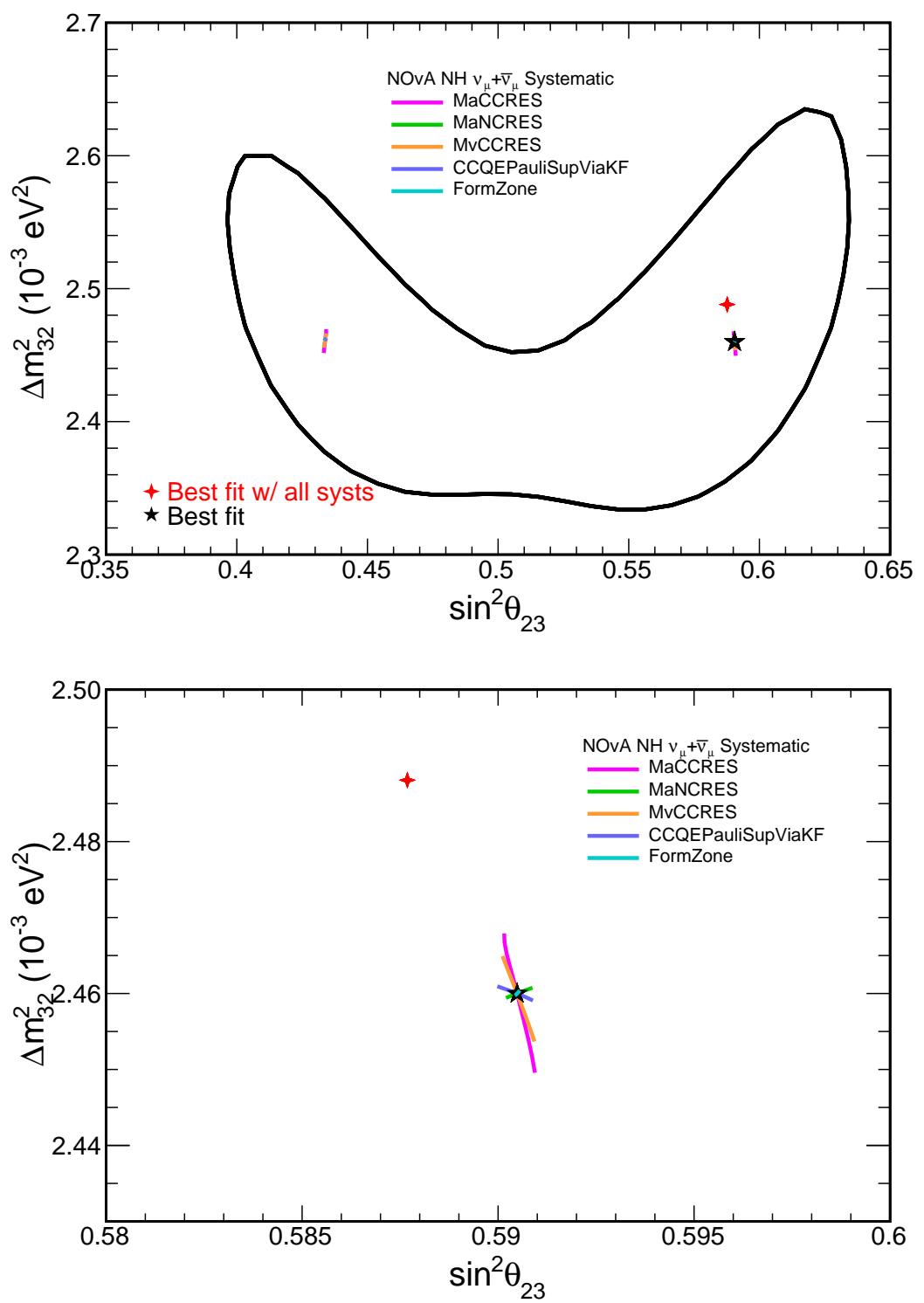

Figure 7.118: Plot of $\Delta m_{32}^{2}$ vs. $\sin ^{2} \theta_{23}$ with the best fit shift caused by each GENIE reweighting systematic shown by the coloured lines. These systematics address the energy resolutions of different interaction types. The $\nu_{\mu}+\bar{\nu}_{\mu}$ disappearance statistics-only $90 \%$ confidence limit contour and corresponding best fit are shown by the black line and star respectively. The systematics-inclusive $\nu_{\mu}+\bar{\nu}_{\mu}$ disappearance best fit is shown by the red cross. The top figure shows an expanded axis scale and the bottom figure shows a zoomed scale. 

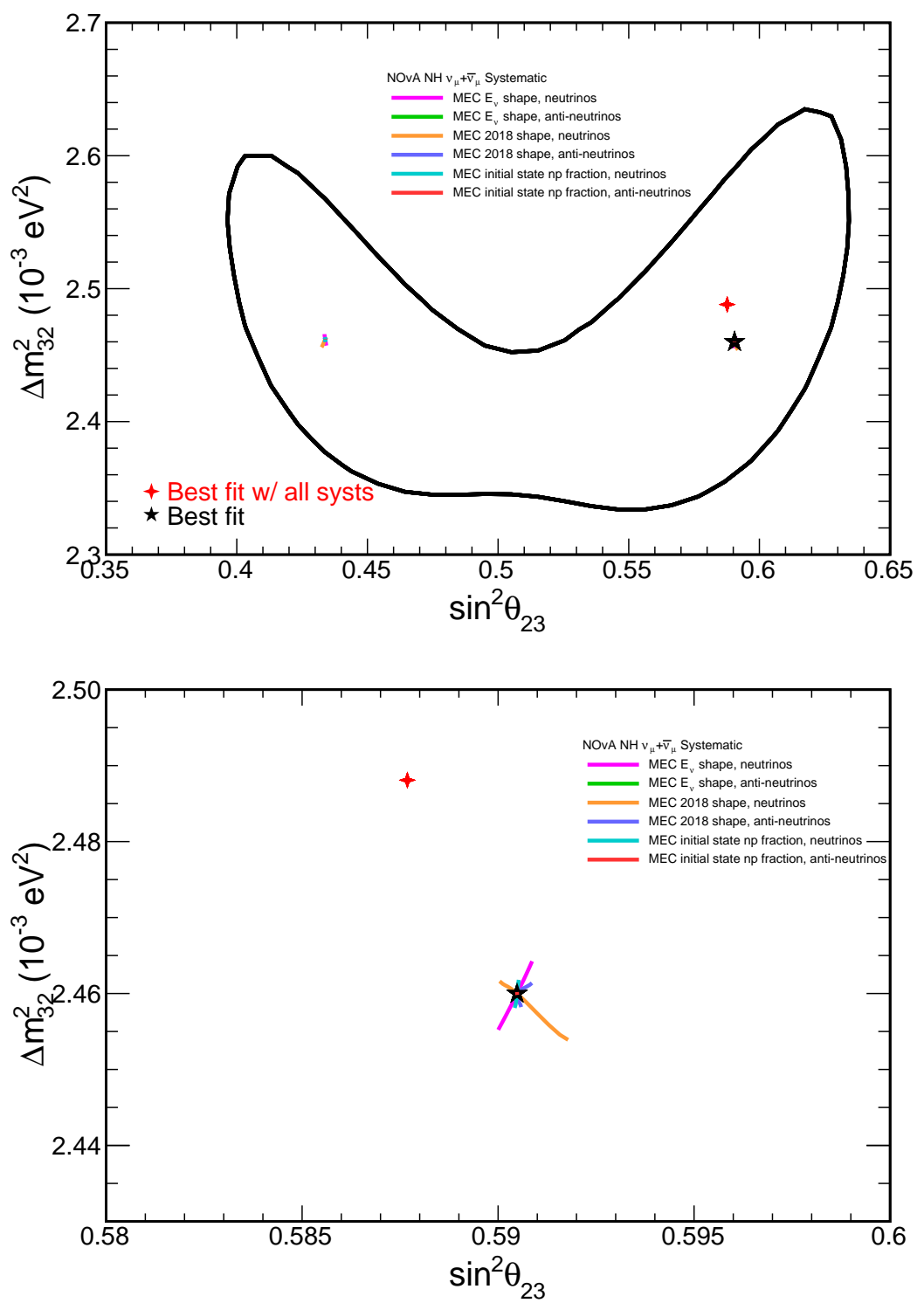

Figure 7.119: Plot of $\Delta m_{32}^{2}$ vs. $\sin ^{2} \theta_{23}$ with the best fit shift caused by each MEC systematic is shown by the coloured lines. These systematics address inaccuracies in modelling of meson exchange current interactions. The $\nu_{\mu}+\bar{\nu}_{\mu}$ disappearance statistics-only $90 \%$ confidence limit contour and corresponding best fit are shown by the black line and star respectively. The systematics-inclusive $\nu_{\mu}+\bar{\nu}_{\mu}$ disappearance best fit is shown by the red cross. The top figure shows an expanded axis scale and the bottom figure shows a zoomed scale. 

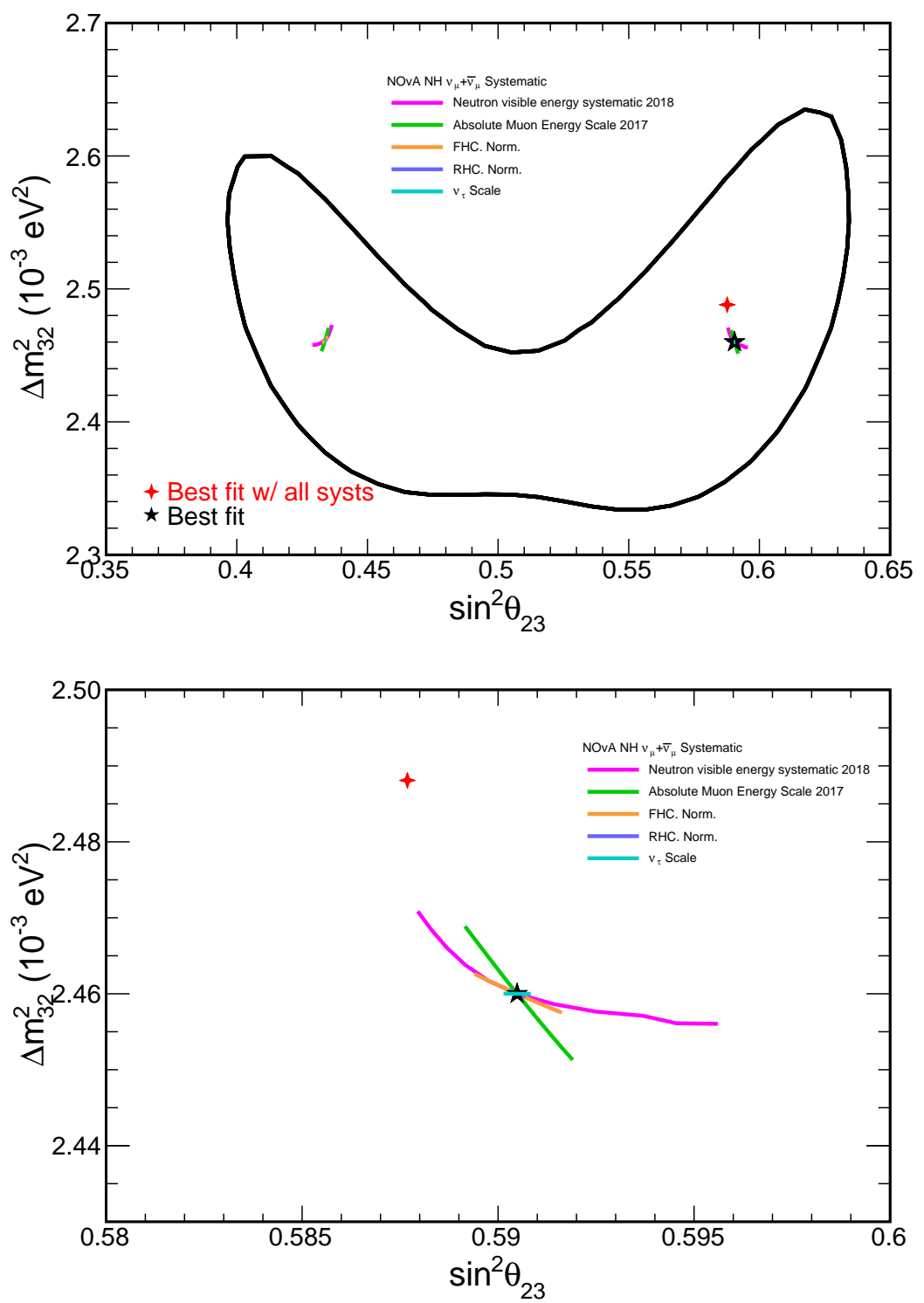

Figure 7.120: Plot of $\Delta m_{32}^{2}$ vs. $\sin ^{2} \theta_{23}$ with the best fit shift caused by each normalization systematic is shown by the coloured lines. The $\nu_{\mu}+\bar{\nu}_{\mu}$ disappearance statistics-only $90 \%$ confidence limit contour and corresponding best fit are shown by the black line and star respectively. The systematics-inclusive $\nu_{\mu}+\bar{\nu}_{\mu}$ disappearance best fit is shown by the red cross. The top figure shows an expanded axis scale and the bottom figure shows a zoomed scale. 

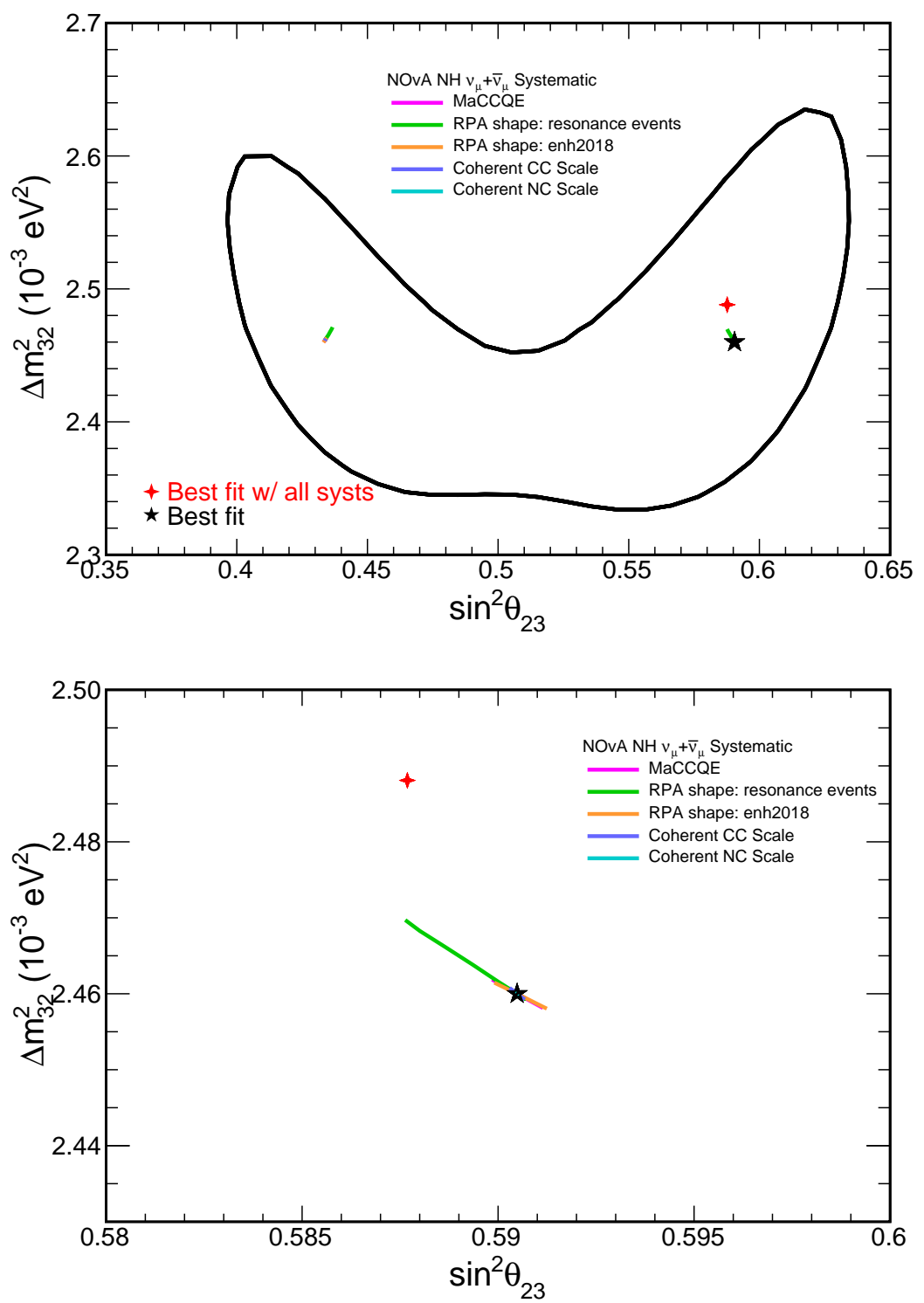

Figure 7.121: Plot of $\Delta m_{32}^{2}$ vs. $\sin ^{2} \theta_{23}$. The best fit shift caused by each cross section systematic is shown by the coloured lines. The $\nu_{\mu}+\bar{\nu}_{\mu}$ disappearance statistics-only $90 \%$ confidence limit contour and corresponding best fit are shown by the black line and star respectively. The systematics-inclusive $\nu_{\mu}+\bar{\nu}_{\mu}$ disappearance best fit is shown by the red cross. The top figure shows an expanded axis scale and the bottom figure shows a zoomed scale. 


\subsection{Future Prospects}

NOvA is continuously looking to reduce systematic uncertainties, increase selection efficiency and further reduce the background. The most significant systematic error in the current analysis comes from the $5 \%$ calibration uncertainty. Calibration uses muons to pin the energy scale in data/MC, a process that can be cross-checked using the forthcoming NOvA test beam program. The test beam, under development at the Fermilab Test Beam Facility, will direct tagged 0.2-2.0 GeV/c electrons, muons, pions, kaons and protons onto a small tracking calorimeter that is functionally identical to the NOvA detector. In doing so, the test beam will be able to characterize detector response to electromagnetic and hadronic showers from well prescribed initial states and thus reduce several analysis uncertainties. In addition to systematic reduction, the test beam can aid the development of analysis techniques. The test beam will likely produce high verbosity data, providing information on a wide range of particles at known energies and angles of incidence. This data can be used to inform machine learning algorithms and cross-check physics assumptions and models. Test beam construction and commissioning is underway with data recording scheduled for the first half of 2019 [96].

NOvA has currently taken about half of the data envisioned in the technical design report [5]. Recently, an extension to the NOvA running period has been proposed for which NOvA has taken $\frac{1}{4}$ of the total data planned by 2024 . The resulting projected exposure of NOvA is $36 \times 10^{20}$ POT for each horn current $-72 \times 10^{20}$ POT total. The experiment is currently taking antineutrino data and from 2019 onwards will collect antineutrino and neutrino data equally. The Proton Improvement Plan - I+ (PIP-I+) is a large scale infrastructure and hardware upgrade to the Fermilab accelerator complex. This upgrade is part of a larger project, called 'PIP-II', which is being principally carried out for the upcoming DUNE experiment. The timeline for the project was accelerated to allow the NOvA experiment to benefit. PIP-I+ is targeting an overall beam power upgrade to $1000 \mathrm{~kW}$ as opposed to the current $700 \mathrm{~kW}$ [97]. A higher power beam increases the exposure per unit time and thus increases the statistical power of all beam measurements. Statistical uncertainties on $\Delta m_{32}^{2}$ and $\sin ^{2} \theta_{23}$ are expected to, approximately, fall with $\sqrt{\text { exposure. }}$

Figure 7.122 shows the projected significance of maximal mixing rejection vs. time for both the normal hierarchy, inverted hierarchy and for the two possible $\sin ^{2} \theta_{23}$ octant possibilities. Close to $5 \sigma$ sensitivity to maximal-mixing rejection is predicted by 2024 under the assumption of $\sin ^{2} \theta_{23}=0.4(0.6)$ for both the normal and inverted hierarchy 
scenarios. The upper octant scenario requires a greater running time to reject as $\sin ^{2} \theta_{23}$ $=0.6$ is closer to maximal-disappearance in matter, which occurs around $\sin ^{2} \theta_{23}=0.511$.

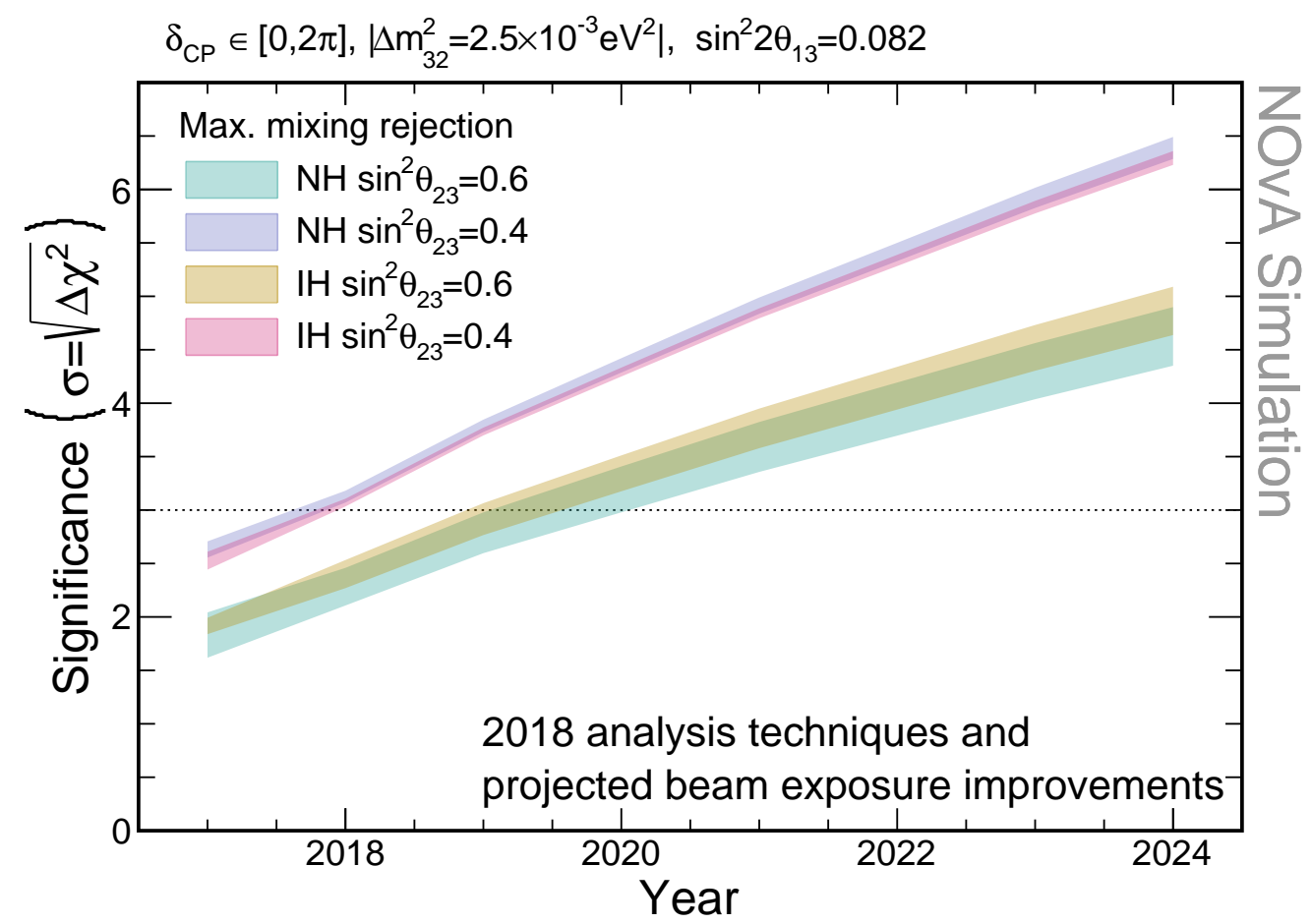

Figure 7.122: Significance vs. time for the rejection of maximal mixing under the assumption of $\sin ^{2} \theta_{23}=0.6$ and $\sin ^{2} \theta_{23}=0.4$ for both hierarchy possibilities. The normal hierarchy upper and lower octant projections are shown in green and blue respectively. The inverted hierarchy upper and lower octant projections are shown in yellow and red respectively. Figure taken from [98]. 


\section{Chapter 8}

\section{Conclusion}

This thesis details improvements to the muon neutrino and antineutrino disappearance analysis that have been performed by the author. To this end, two significant contributions have been described - the re-designing of the containment criteria and the optimization of the particle-identification event selection.

The containment criteria were optimized to reduce the analysis susceptibility to noise, capture more muon (anti)neutrino signal events and reduce the selected background. The 'cells from edge' cut was removed in favour of a series of cuts that discriminate events based on the distance between track-like objects and the detector edges. Further to this, the previous containment criteria were re-optimized. This work yielded a $0.1 \%$ increase in the number of selected signal events, a $55 \%$ reduction in the cosmic background and a reduction in the percentage of events lost due to pile-up with cosmic rays $(3.1 \% \rightarrow 1.4 \%)$.

The machine learning algorithms that NOvA uses for particle identification have been introduced and updated at various points during the last five years. The optimization of the selectors for the latest analysis was performed by the author using a large scale 3D integral technique that maximized the figure of merit for analysis sensitivity when looking at the combined effect of the three cuts. The result of this work was a total background reduction of approximately $65 \%$ in $\mathrm{FHC}$ and $75 \%$ in RHC. In addition to this, RHC data saw a $2 \%$ increase in selected neutrino signal and the FHC data saw a $5 \%$ decrease.

The full-detector equivalent neutrino and antineutrino beam exposures used for this thesis are $8.85 \times 10^{20}$ and $6.91 \times 10^{20}$ protons on target respectively. Under the assumption of a normal (inverted) neutrino mass hierarchy, analysis of the data gives $\Delta m_{32}^{2}=+2.49_{-0.07}^{+0.09} \times 10^{-3} \mathrm{eV}^{2}\left(\Delta m_{32}^{2}=-2.54 \pm 0.08 \times 10^{-3} \mathrm{eV}^{2}\right)$ and $\sin ^{2} \theta_{23}=0.59 \pm 0.03$ $\left(\sin ^{2} \theta_{23}=0.44 \pm 0.03\right)$. Maximal mixing $\left(\sin ^{2} \theta_{23}=0.5\right)$ is disfavoured at the $1.7 \sigma$ level. The measurements of $\Delta m_{32}^{2}$ and $\sin ^{2} \theta_{23}$ are competitive with the latest T2K and Daya 
Bay $\left(\Delta m_{32}^{2}\right.$ only) results.

The systematic and statistical uncertainty on the measurement in $\sin ^{2} \theta_{23}$ is \pm 0.010 and \pm 0.028 respectively. The systematic and statistical uncertainty on the measurement in $\Delta m_{32}^{2}$ is ${ }_{-0.033}^{+0.026} \times 10^{-3}$ and ${ }_{-0.059}^{+0.062} \times 10^{-3}$ respectively. As in previous analyses, the uncertainty is dominated by the statistical component. The most significant systematic uncertainty on the measurements of both of $\Delta m_{32}^{2}$ and $\sin ^{2} \theta_{23}$ comes from the absolute and relative hadronic energy scale uncertainties. The cross-section systematics also have a significant impact on both measurements, as does the neutron uncertainty. The impact of the neutron systematic on the $\sin ^{2} \theta_{23}$ measurement is relatively large due to the uncertainty it introduces to the RHC analysis. The absolute muon energy scale systematic has approximately the same magnitude impact on the $\Delta m_{32}^{2}$ measurement as the neutron uncertainty does but a smaller effect on the measurement on $\sin ^{2} \theta_{23}$.

NOvA has taken about $\frac{1}{4}$ of the total data it plans to. The experiment is scheduled to record total beam exposures of $36 \times 10^{20}$ POT for FHC and RHC, producing a total beam exposure of $72 \times 10^{20}$ POT by 2024. Systematic uncertainty in $\sin ^{2} \theta_{23}$ and in $\Delta m_{32}^{2}$ is projected to remain below the statistical error at NOvA's best fit point. Current projections indicate close to $5 \sigma$ sensitivity to maximal-mixing rejection by 2024 under the assumption of $\sin ^{2} \theta_{23}=0.4(0.6)$ for both the normal and inverted hierarchy scenarios. The upper octant scenario requires a greater running time to reject as $\sin ^{2} \theta_{23}=0.6$ is closer to maximal-disappearance in matter, which occurs around $\sin ^{2} \theta_{23}=0.511$.

The collaboration is presently doing much work to reduce systematic uncertainties, increase selection efficiency and further reduce the background. Currently, both the absolute and relative calibration uncertainties are $5 \%$. The test beam data will characterize the detector response to electromagnetic and hadronic showers from well prescribed initial states, helping reduce several analysis uncertainties. The current calibration process uses muons to pin the energy scale in data and MC and so can be cross-checked using the test beam data. The neutron uncertainty is being targeted in a number of ways, for example through a detailed investigation of the models and external data used in GEANT4 and studies of neutron rich event samples collected in the ND. As well as NOvA's own future constraints on neutrino interaction cross-sections, measurements from other experiments will also aid in driving down the associated systematic uncertainty.

The future of NOvA is promising and the author is personally grateful for being able to contribute towards the experiment and for working alongside the people who make up the NOvA collaboration. 
Appendices 


\section{Appendix A}

\section{Containment and Pile-Up From Cosmics}

A discrepancy was observed between the number of $\nu_{\mu}$ events captured by our reconstruction chain when the analysis was run over the standard monte-carlo data, as opposed to the cosmic overlay monte-carlo data. This appendix contains a series of plots which follow on from the containment work outlined in Chapter 5 to show the effect of the updated containment criteria on this discrepancy.

\section{A.0.1 2017 Analysis Datasets}

Figure A.1 through Figure A.10 show the 2017 (second) analysis energy distributions for events passing selection criteria. The plots run through each data taking epoch, chronologically labelled. The selection criteria are given explicitly in each plot caption. Each data period comprises 3 plots. The first plot shown uses the 2017 analysis selection, varying the containment criteria only. The second plot shown uses the second analysis hybrid selection, varying containment criteria only. The third plot shown uses the updated analysis hybrid selection, varying containment criteria only. The final three plots show the summed total for all data taking periods. In each period, and for the overall distribution, the discrepancy between cosmic overlay and standard MC is reduced by migration from previous containment criteria to the criteria outlined Chapter 5. This is indicative of a more robust analysis, as the introduction of cosmic events impacts the number of signal events selected less. 


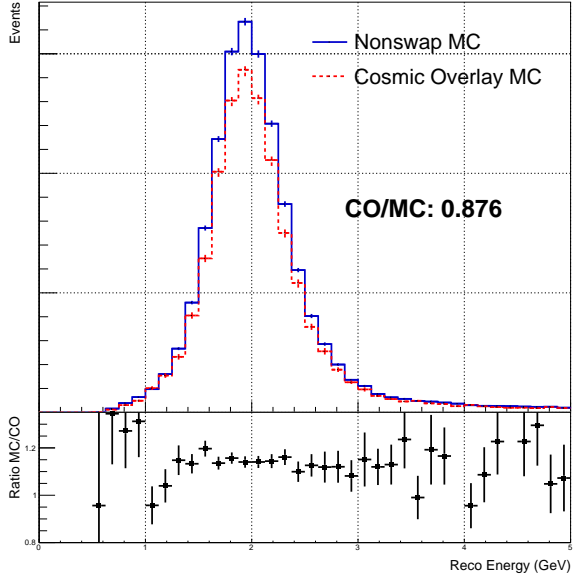

(a) $\nu_{\mu}$ energy distributions using 2017 analysis containment

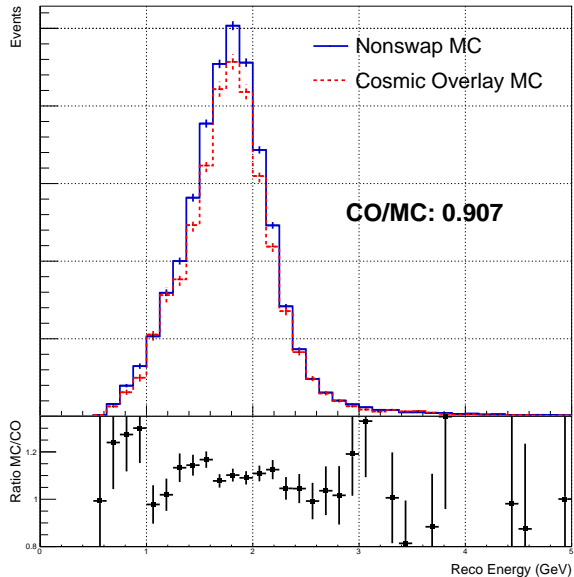

(b) $\nu_{\mu}$ energy distributions using 2018 analysis containment

Figure A.1: $\nu_{\mu}$ energy distributions in Period 1. The blue line shows the distribution when run over the nonswap standard MC dataset. The red dashed line shows the distribution when run over the cosmic overlay dataset. The discrepancy in each case is given below the legend as the integral ratio of the cosmic overlay distribution with respect to the standard MC distribution. Common cuts employed in these plots are kNumuQuality, kNumuNCRej and kNumuCosmicRej.

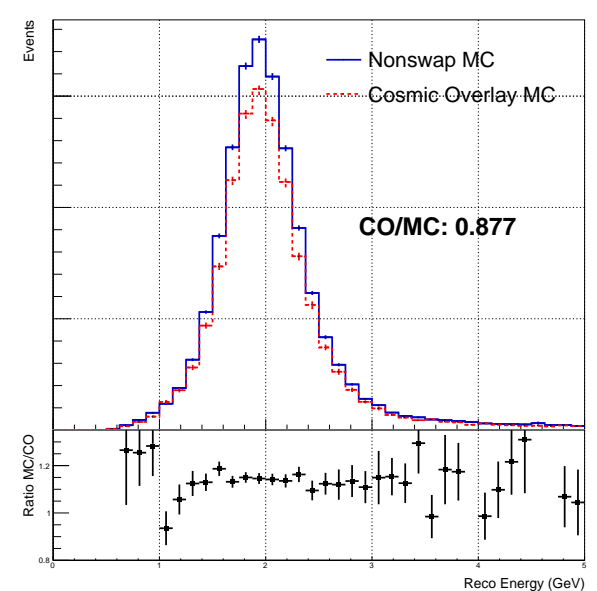

(a) $\nu_{\mu}$ energy distributions using 2017 analysis containment

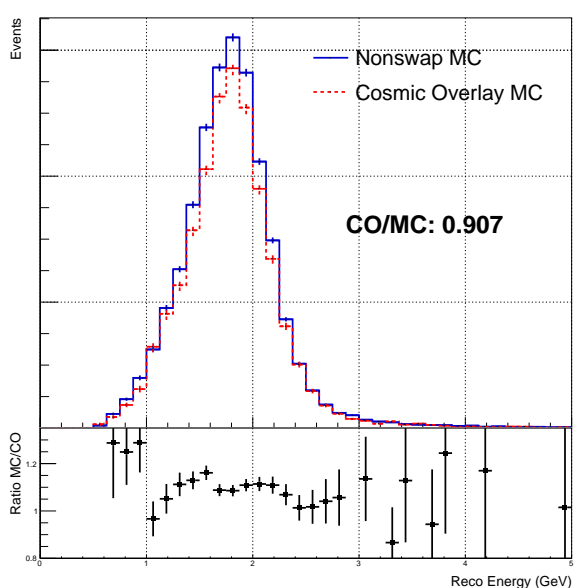

(b) $\nu_{\mu}$ energy distributions using 2018 analysis containment

Figure A.2: $\nu_{\mu}$ energy distributions in Period 1. The blue line shows the distribution when run over the nonswap standard MC dataset. The red dashed line shows the distribution when run over the cosmic overlay dataset. The discrepancy in each case is given below the legend as the integral ratio of the cosmic overlay distribution with respect to the standard MC distribution. Common cuts employed in these plots are kNumuQuality and kKirkSATuneFD (using 2016 BDT). 


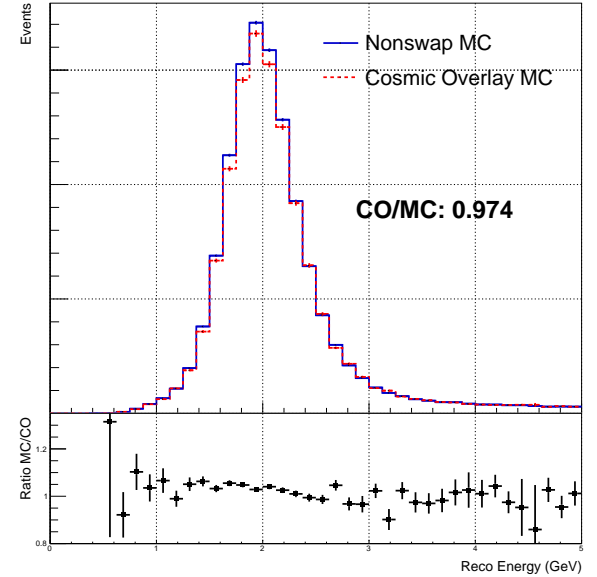

(a) $\nu_{\mu}$ energy distributions using 2017 analysis containment

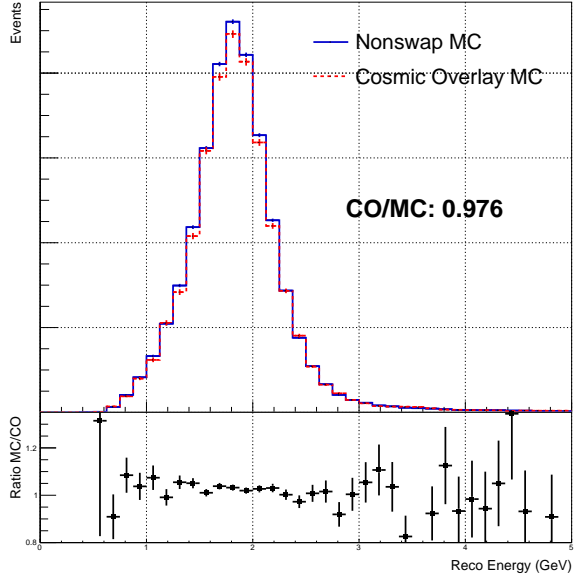

(b) $\nu_{\mu}$ energy distributions using 2018 analysis containment

Figure A.3: $\nu_{\mu}$ energy distributions in Period 2. The blue line shows the distribution when run over the nonswap standard MC dataset. The red dashed line shows the distribution when run over the cosmic overlay dataset. The discrepancy in each case is given below the legend as the integral ratio of the cosmic overlay distribution with respect to the standard MC distribution. Common cuts employed in these plots are kNumuQuality, kNumuNCRej and kNumuCosmicRej.

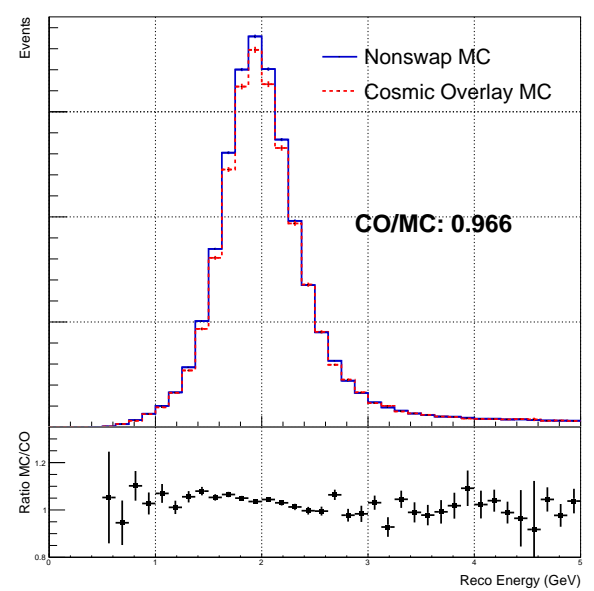

(a) $\nu_{\mu}$ energy distributions using 2017 analysis containment

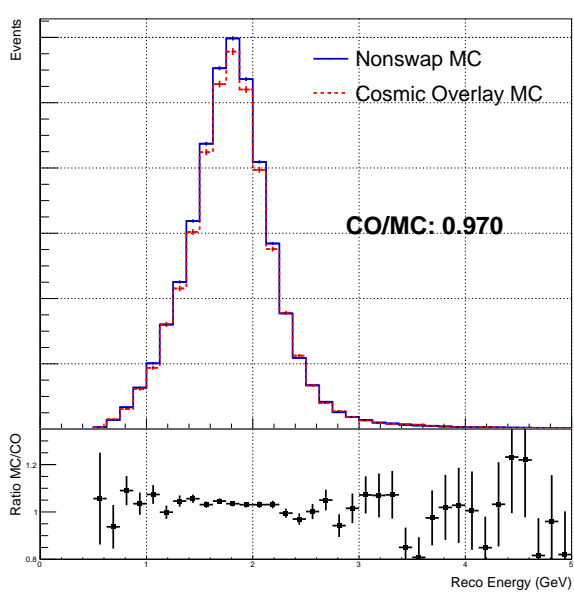

(b) $\nu_{\mu}$ energy distributions using 2018 analysis containment

Figure A.4: $\nu_{\mu}$ energy distributions in Period 2. The blue line shows the distribution when run over the nonswap standard MC dataset. The red dashed line shows the distribution when run over the cosmic overlay dataset. The discrepancy in each case is given below the legend as the integral ratio of the cosmic overlay distribution with respect to the standard MC distribution. Common cuts employed in these plots are kNumuQuality and kKirkSATuneFD (using 2016 BDT). 


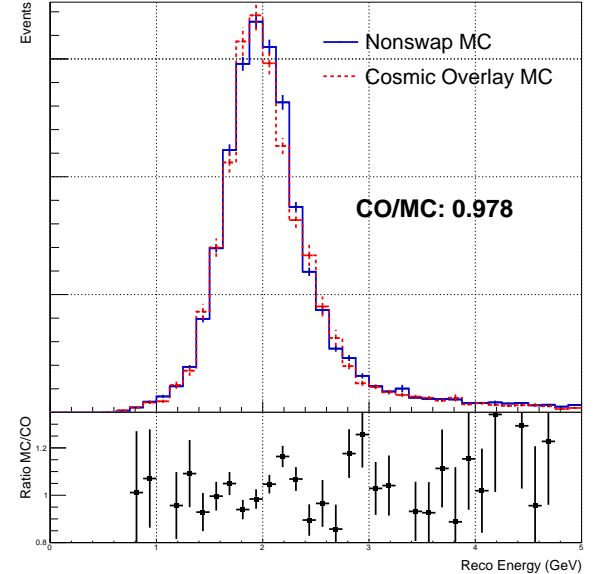

(a) $\nu_{\mu}$ energy distributions using 2017 analysis containment

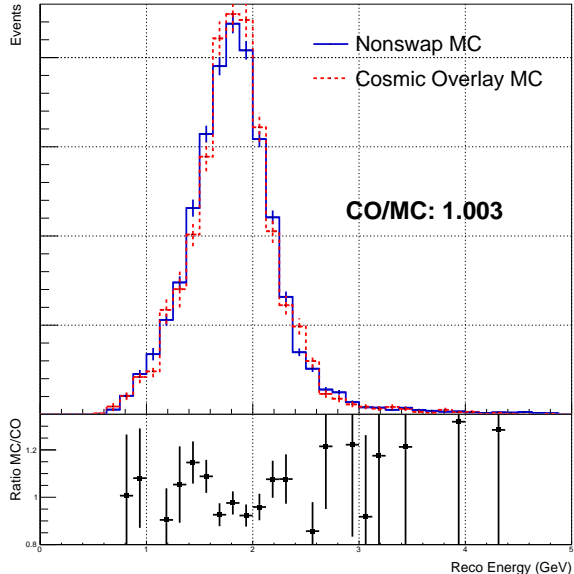

(b) $\nu_{\mu}$ energy distributions using 2018 analysis containment

Figure A.5: $\nu_{\mu}$ energy distributions in Epoch 3b. The blue line shows the distribution when run over the nonswap standard MC dataset. The red dashed line shows the distribution when run over the cosmic overlay dataset. The discrepancy in each case is given below the legend as the integral ratio of the cosmic overlay distribution with respect to the standard MC distribution. Common cuts employed in these plots are kNumuQuality, kNumuNCRej and kNumuCosmicRej.

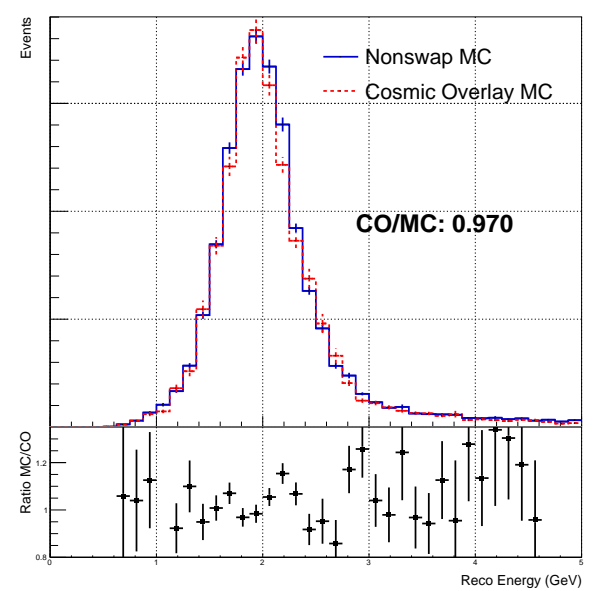

(a) $\nu_{\mu}$ energy distributions using 2017 analysis containment

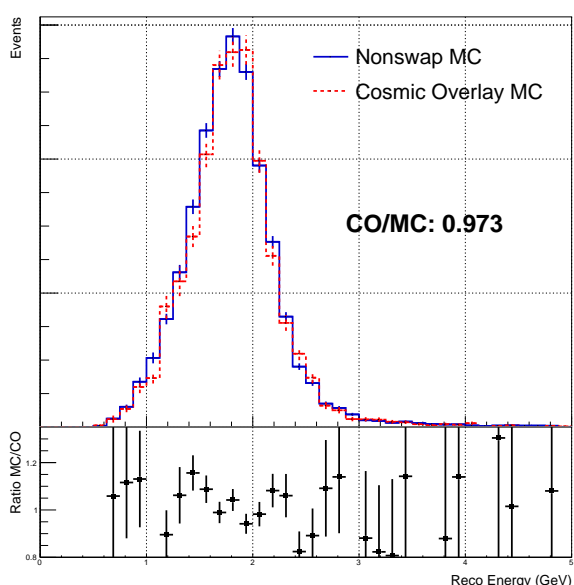

(b) $\nu_{\mu}$ energy distributions using 2018 analysis containment

Figure A.6: $\nu_{\mu}$ energy distributions in Epoch 3b. The blue line shows the distribution when run over the nonswap standard MC dataset. The red dashed line shows the distribution when run over the cosmic overlay dataset. The discrepancy in each case is given below the legend as the integral ratio of the cosmic overlay distribution with respect to the standard MC distribution. Common cuts employed in these plots are kNumuQuality and kKirkSATuneFD (using 2016 BDT). 


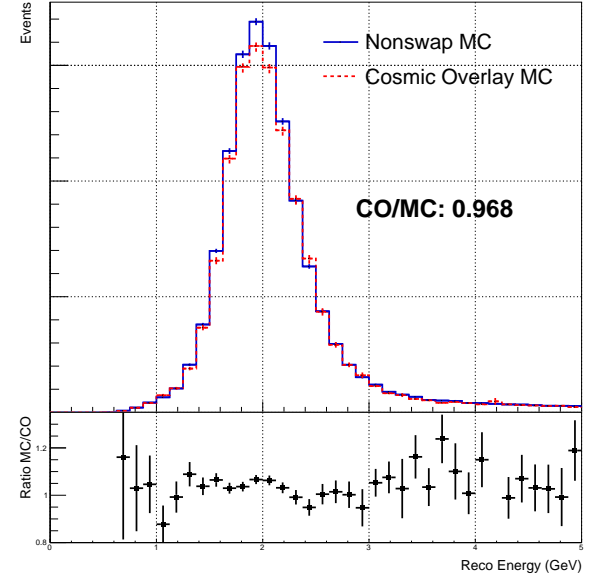

(a) $\nu_{\mu}$ energy distributions using 2017 analysis containment

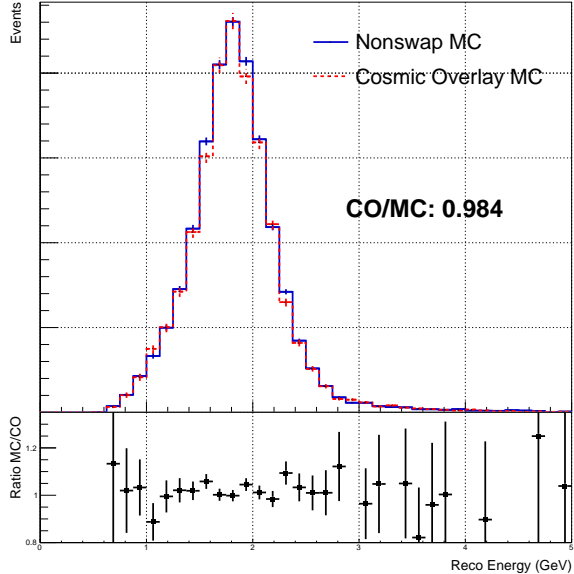

(b) $\nu_{\mu}$ energy distributions using 2018 analysis containment

Figure A.7: $\nu_{\mu}$ energy distributions in Epoch 3c. The blue line shows the distribution when run over the nonswap standard MC dataset. The red dashed line shows the distribution when run over the cosmic overlay dataset. The discrepancy in each case is given below the legend as the integral ratio of the cosmic overlay distribution with respect to the standard MC distribution. Common cuts employed in these plots are kNumuQuality, kNumuNCRej and kNumuCosmicRej.

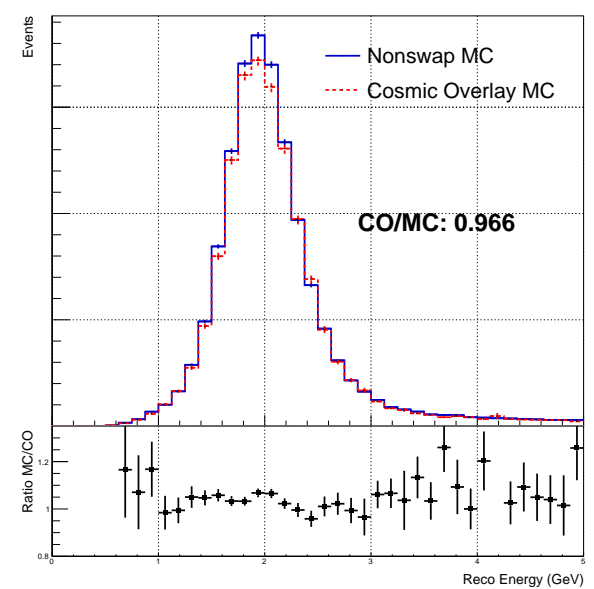

(a) $\nu_{\mu}$ energy distributions using 2017 analysis containment

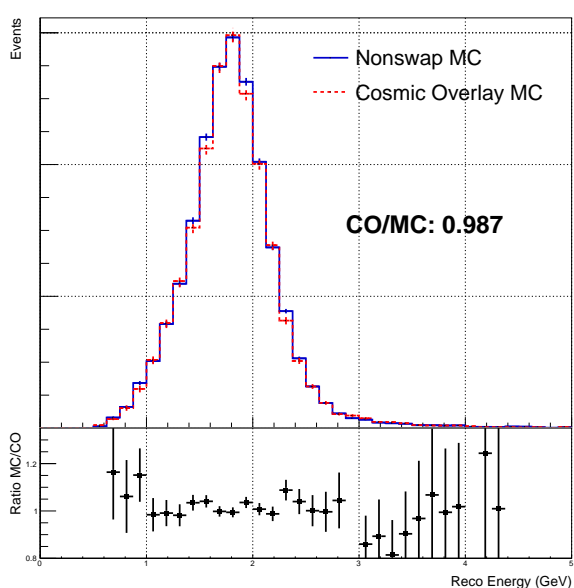

(b) $\nu_{\mu}$ energy distributions using 2018 analysis containment

Figure A.8: $\nu_{\mu}$ energy distributions in Epoch 3c. The blue line shows the distribution when run over the nonswap standard MC dataset. The red dashed line shows the distribution when run over the cosmic overlay dataset. The discrepancy in each case is given below the legend as the integral ratio of the cosmic overlay distribution with respect to the standard MC distribution. Common cuts employed in these plots are kNumuQuality and kKirkSATuneFD (using 2016 BDT). 


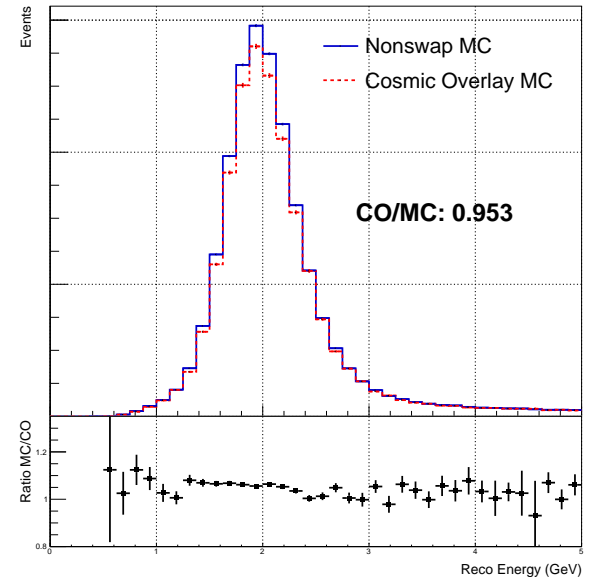

(a) $\nu_{\mu}$ energy distributions using 2017 analysis containment

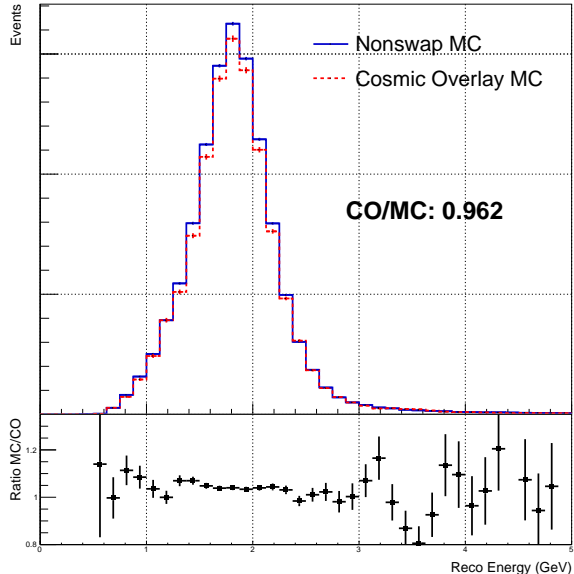

(b) $\nu_{\mu}$ energy distributions using 2018 analysis containment

Figure A.9: $\nu_{\mu}$ energy distributions across all periods. The blue line shows the distribution when run over the nonswap standard MC dataset. The red dashed line shows the distribution when run over the cosmic overlay dataset. The discrepancy in each case is given below the legend as the integral ratio of the cosmic overlay distribution with respect to the standard MC distribution. Common cuts employed in these plots are kNumuQuality, kNumuNCRej and kNumuCosmicRej.

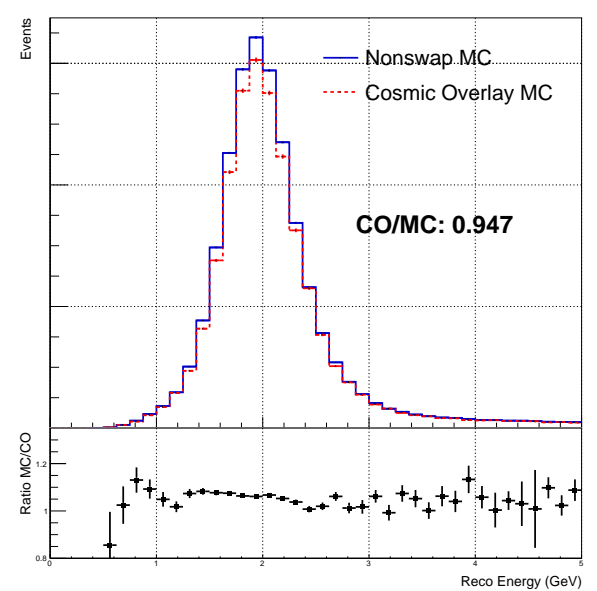

(a) $\nu_{\mu}$ energy distributions using 2017 analysis containment

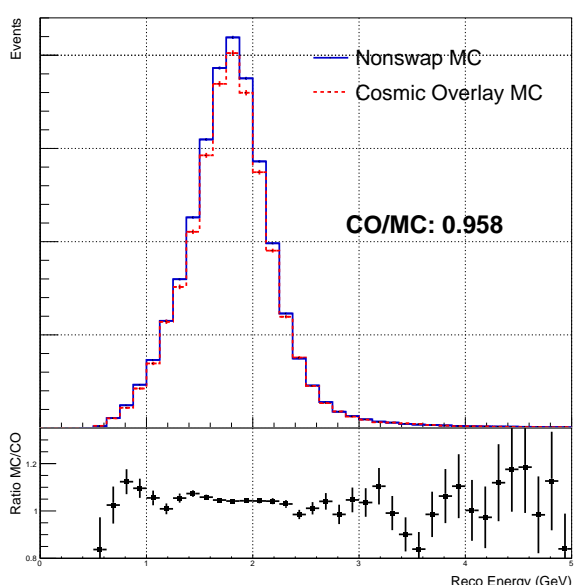

(b) $\nu_{\mu}$ energy distributions using 2018 analysis containment

Figure A.10: $\nu_{\mu}$ energy distributions across all periods. The blue line shows the distribution when run over the nonswap standard MC dataset. The red dashed line shows the distribution when run over the cosmic overlay dataset. The discrepancy in each case is given below the legend as the integral ratio of the cosmic overlay distribution with respect to the standard MC distribution. Common cuts employed in these plots are kNumuQuality and kKirkSATuneFD (using 2016 BDT). 


\section{A.0.2 2018 Analysis Datasets}

Figure A.11 through Figure 5.21 show the 2018 analysis energy distributions for events passing selection criteria. The plots run through each data taking epoch, chronologically labelled. The selection criteria are given explicitly in each plot caption. Each data period comprises 3 plots. The first plot shown is employs the 2017 analysis selection, varying the containment criteria only. The second plot shown uses the second analysis hybrid selection, varying containment criteria only. The third plot shown uses the updated analysis hybrid selection, varying containment criteria only. The final three plots show the summed total for all data taking periods. In each period, and for the overall distribution, the discrepancy between cosmic overlay and standard MC is reduced by migration from previous containment criteria to those outlined in this chapter. This is indicative of a more robust analysis, as the introduction of cosmic events impacts the number of signal events selected less. 


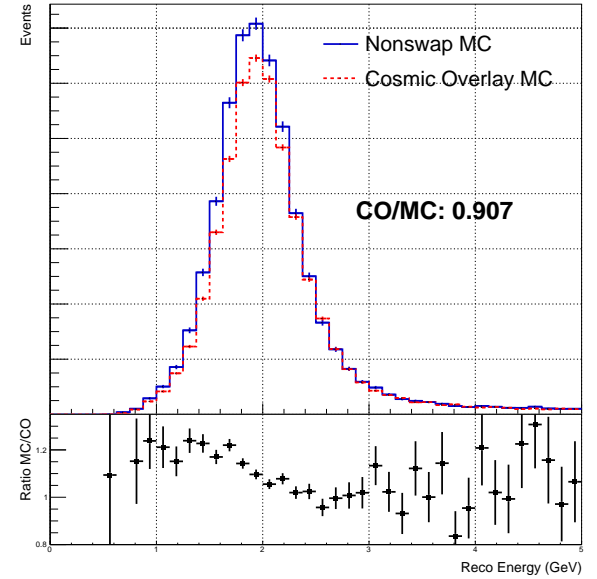

(a) $\nu_{\mu}$ energy distributions using 2017 analysis containment

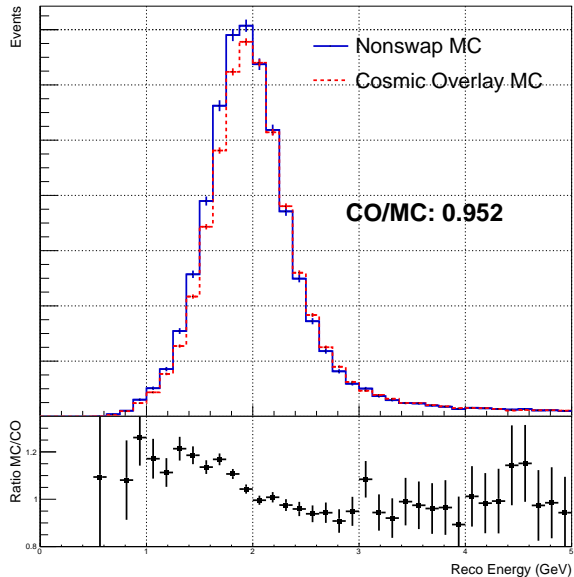

(b) $\nu_{\mu}$ energy distributions using 2018 analysis containment

Figure A.11: $\nu_{\mu}$ energy distributions in Period 1. The blue line shows the distribution when run over the nonswap standard MC dataset. The red dashed line shows the distribution when run over the cosmic overlay dataset. The discrepancy in each case is given below the legend as the integral ratio of the cosmic overlay distribution with respect to the standard MC distribution. Common cuts employed in these plots are kNumuQuality, kNumuNCRej and kNumuCosmicRej.

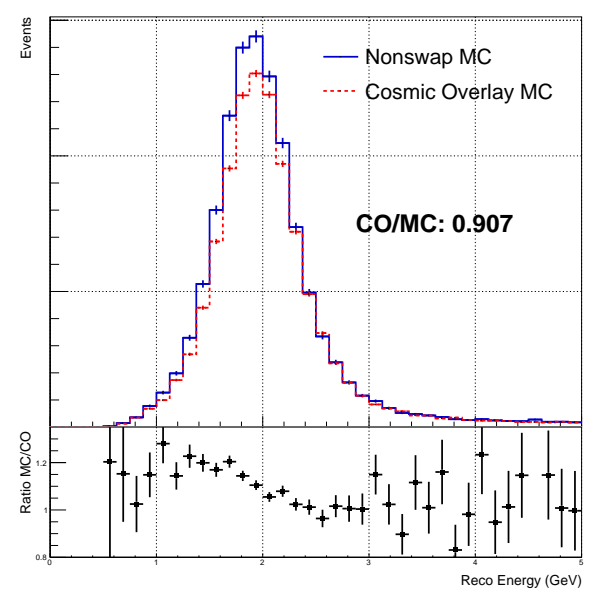

(a) $\nu_{\mu}$ energy distributions using 2017 analysis containment

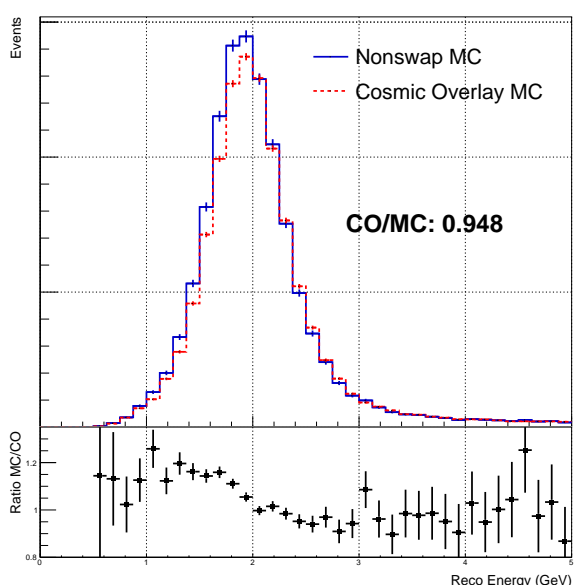

(b) $\nu_{\mu}$ energy distributions using 2018 analysis containment

Figure A.12: $\nu_{\mu}$ energy distributions in Period 1. The blue line shows the distribution when run over the nonswap standard MC dataset. The red dashed line shows the distribution when run over the cosmic overlay dataset. The discrepancy in each case is given below the legend as the integral ratio of the cosmic overlay distribution with respect to the standard MC distribution. Common cuts employed in these plots are kNumuQuality and kKirkTATuneFD (using 2016 BDT). 


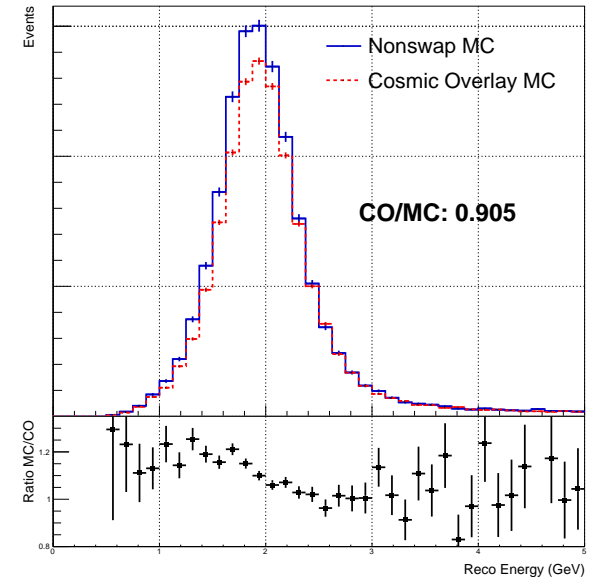

(a) $\nu_{\mu}$ energy distributions using 2017 analysis containment

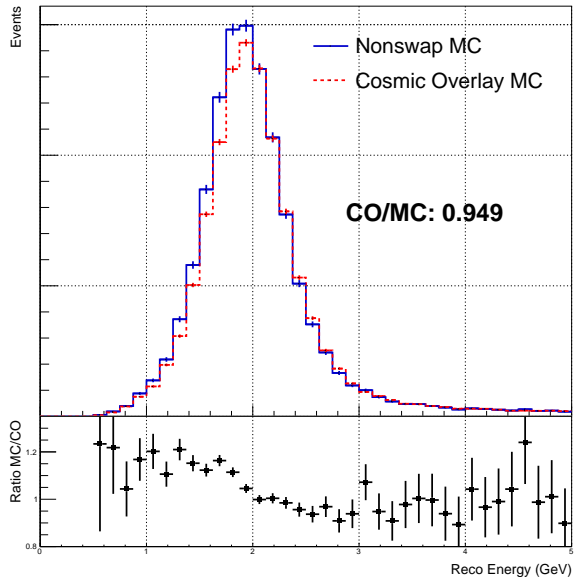

(b) $\nu_{\mu}$ energy distributions using 2018 analysis containment

Figure A.13: $\nu_{\mu}$ energy distributions in Period 1. The blue line shows the distribution when run over the nonswap standard $\mathrm{MC}$ dataset. The red dashed line shows the distribution when run over the cosmic overlay dataset. The discrepancy in each case is given below the legend as the integral ratio of the cosmic overlay distribution with respect to the standard MC distribution. Common cuts employed in these plots are kNumuQuality and kKirkTATuneFD2017 (using 2017 BDT).

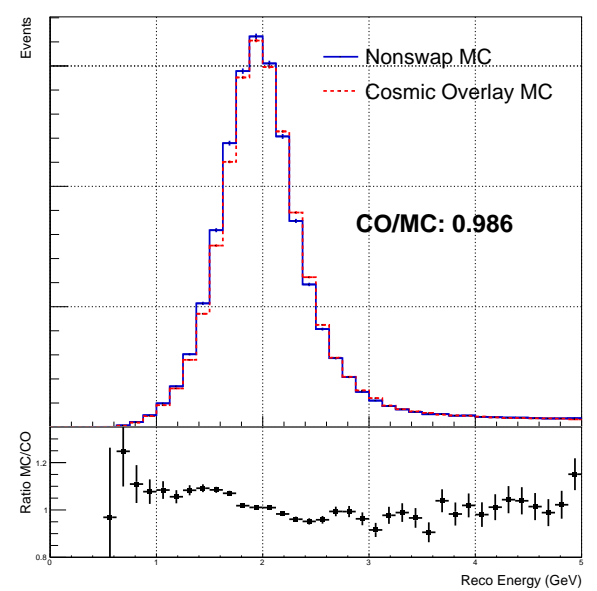

(a) $\nu_{\mu}$ energy distributions using 2017 analysis containment

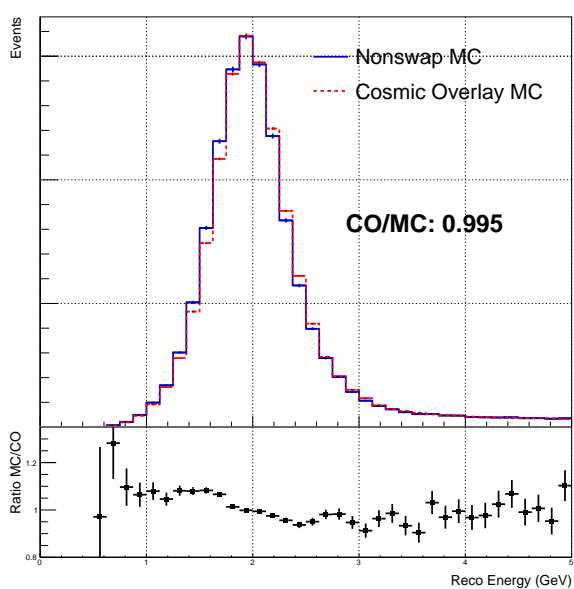

(b) $\nu_{\mu}$ energy distributions using 2018 analysis containment

Figure A.14: $\nu_{\mu}$ energy distributions in Period 2. The blue line shows the distribution when run over the nonswap standard MC dataset. The red dashed line shows the distribution when run over the cosmic overlay dataset. The discrepancy in each case is given below the legend as the integral ratio of the cosmic overlay distribution with respect to the standard MC distribution. Common cuts employed in these plots are kNumuQuality, kNumuNCRej and kNumuCosmicRej. 


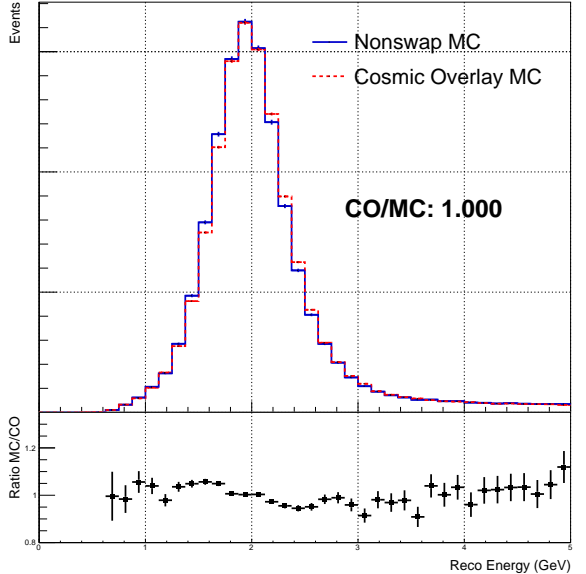

(a) $\nu_{\mu}$ energy distributions using 2017 analysis containment

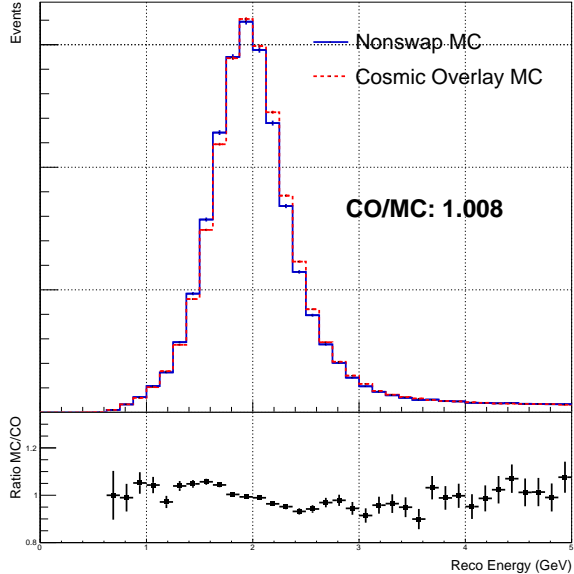

(b) $\nu_{\mu}$ energy distributions using 2018 analysis containment

Figure A.15: $\nu_{\mu}$ energy distributions in Period 2. The blue line shows the distribution when run over the nonswap standard MC dataset. The red dashed line shows the distribution when run over the cosmic overlay dataset. The discrepancy in each case is given below the legend as the integral ratio of the cosmic overlay distribution with respect to the standard MC distribution. Common cuts employed in these plots are kNumuQuality and kKirkTATuneFD (using 2016 BDT).

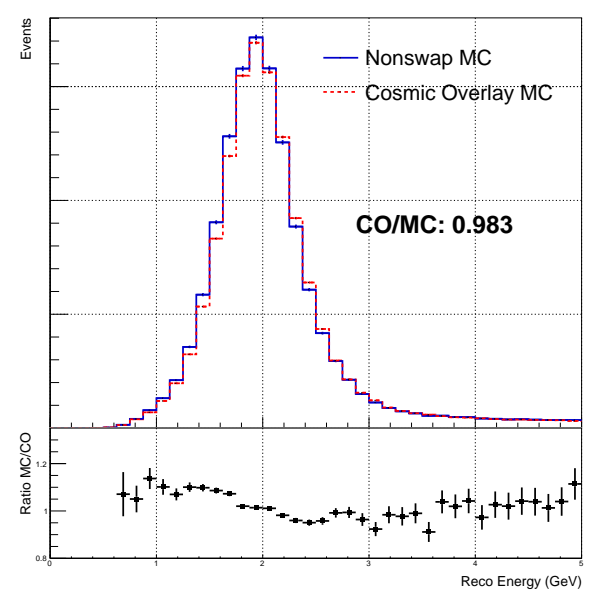

(a) $\nu_{\mu}$ energy distributions using 2017 analysis containment

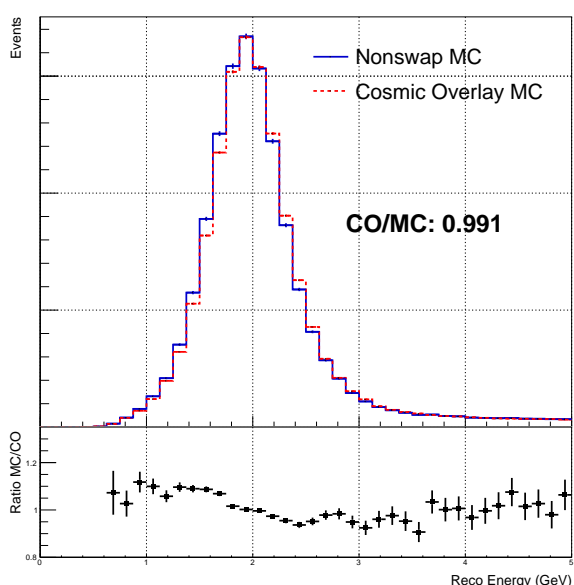

(b) $\nu_{\mu}$ energy distributions using 2018 analysis containment

Figure A.16: $\nu_{\mu}$ energy distributions in Period 2. The blue line shows the distribution when run over the nonswap standard MC dataset. The red dashed line shows the distribution when run over the cosmic overlay dataset. The discrepancy in each case is given below the legend as the integral ratio of the cosmic overlay distribution with respect to the standard MC distribution. Common cuts employed in these plots are kNumuQuality and kKirkTATuneFD2017 (using 2017 BDT). 


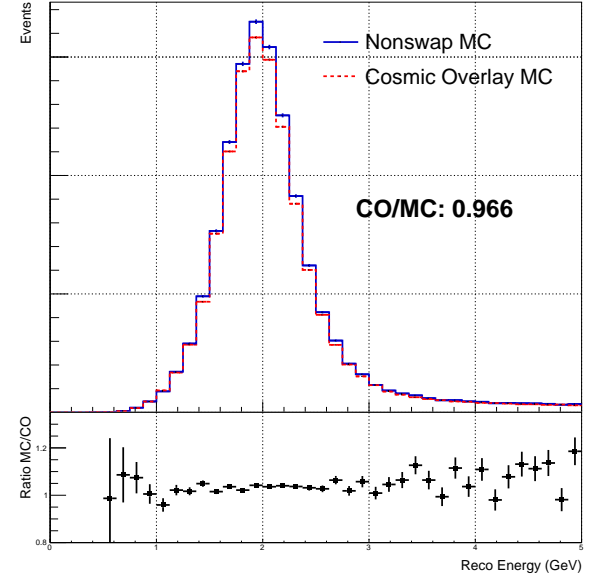

(a) $\nu_{\mu}$ energy distributions using 2017 analysis containment

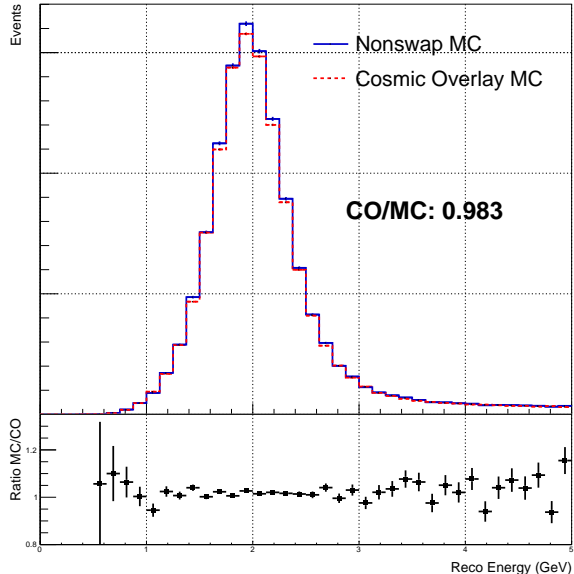

(b) $\nu_{\mu}$ energy distributions using 2018 analysis containment

Figure A.17: $\nu_{\mu}$ energy distributions in Period 3. The blue line shows the distribution when run over the nonswap standard MC dataset. The red dashed line shows the distribution when run over the cosmic overlay dataset. The discrepancy in each case is given below the legend as the integral ratio of the cosmic overlay distribution with respect to the standard MC distribution. Common cuts employed in these plots are kNumuQuality, kNumuNCRej and kNumuCosmicRej.

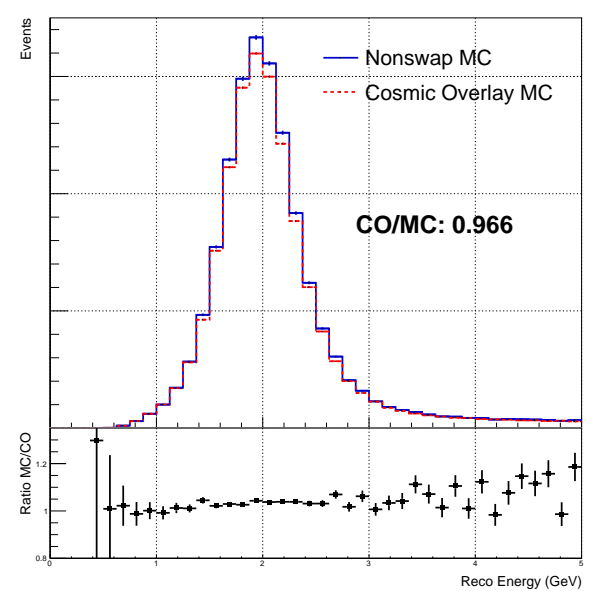

(a) $\nu_{\mu}$ energy distributions using 2017 analysis containment

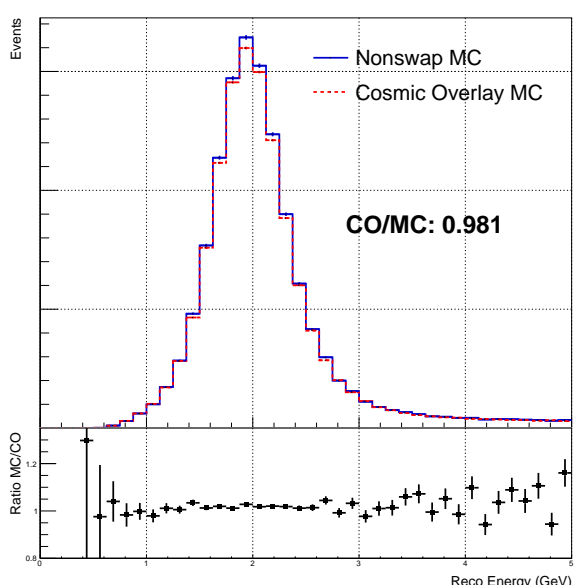

(b) $\nu_{\mu}$ energy distributions using 2018 analysis containment

Figure A.18: $\nu_{\mu}$ energy distributions in Period 3. The blue line shows the distribution when run over the nonswap standard MC dataset. The red dashed line shows the distribution when run over the cosmic overlay dataset. The discrepancy in each case is given below the legend as the integral ratio of the cosmic overlay distribution with respect to the standard MC distribution. Common cuts employed in these plots are kNumuQuality and kKirkTATuneFD (using 2016 BDT). 


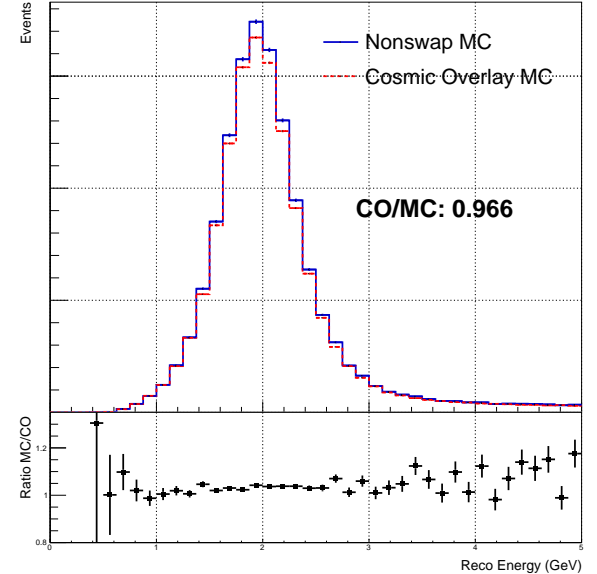

(a) $\nu_{\mu}$ energy distributions using 2017 analysis containment

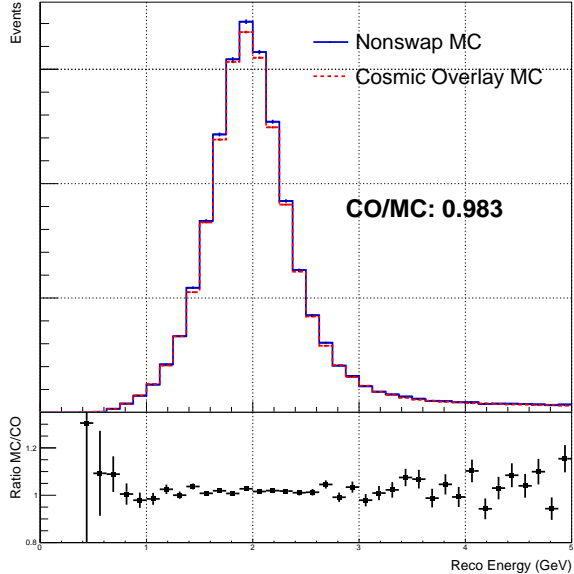

(b) $\nu_{\mu}$ energy distributions using 2018 analysis containment

Figure A.19: $\nu_{\mu}$ energy distributions in Period 3. The blue line shows the distribution when run over the nonswap standard MC dataset. The red dashed line shows the distribution when run over the cosmic overlay dataset. The discrepancy in each case is given below the legend as the integral ratio of the cosmic overlay distribution with respect to the standard MC distribution. Common cuts employed in these plots are kNumuQuality and kKirkTATuneFD2017 (using 2017 BDT).

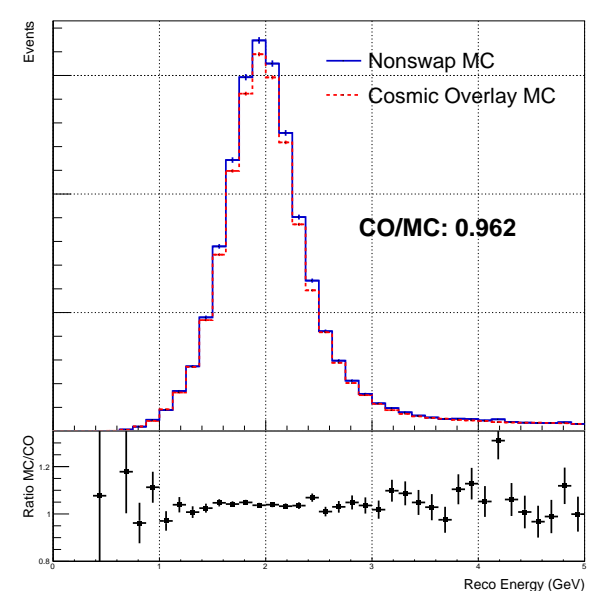

(a) $\nu_{\mu}$ energy distributions using 2017 analysis containment

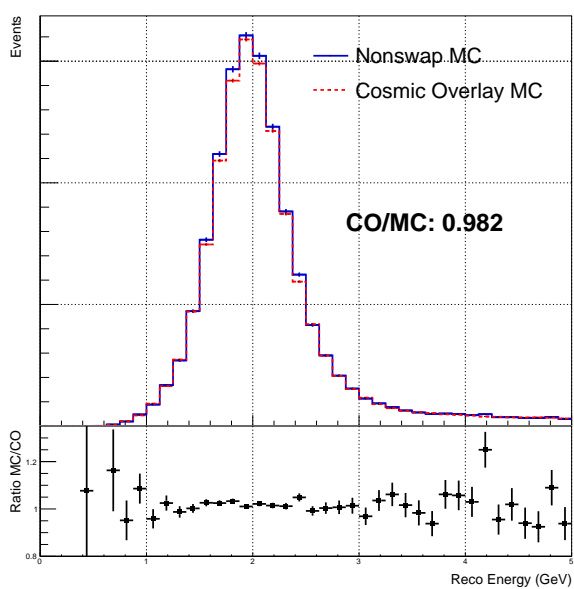

(b) $\nu_{\mu}$ energy distributions using 2018 analysis containment

Figure A.20: $\nu_{\mu}$ energy distributions in Period5. The blue line shows the distribution when run over the nonswap standard MC dataset. The red dashed line shows the distribution when run over the cosmic overlay dataset. The discrepancy in each case is given below the legend as the integral ratio of the cosmic overlay distribution with respect to the standard MC distribution. Common cuts employed in these plots are kNumuQuality, kNumuNCRej and kNumuCosmicRej. 


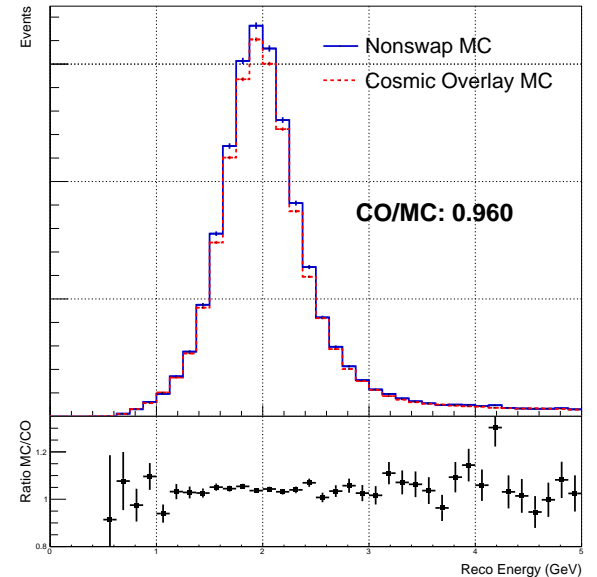

(a) $\nu_{\mu}$ energy distributions using 2017 analysis containment

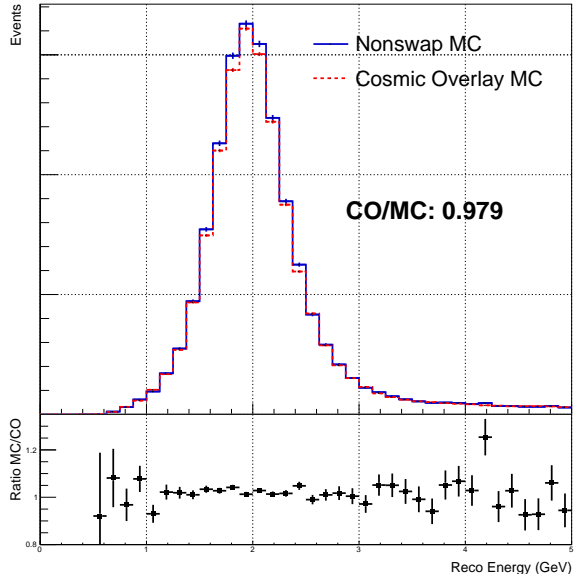

(b) $\nu_{\mu}$ energy distributions using 2018 analysis containment

Figure A.21: $\nu_{\mu}$ energy distributions in Period5. The blue line shows the distribution when run over the nonswap standard MC dataset. The red dashed line shows the distribution when run over the cosmic overlay dataset. The discrepancy in each case is given below the legend as the integral ratio of the cosmic overlay distribution with respect to the standard MC distribution. Common cuts employed in these plots are kNumuQuality and kKirkTATuneFD (using 2016 BDT).

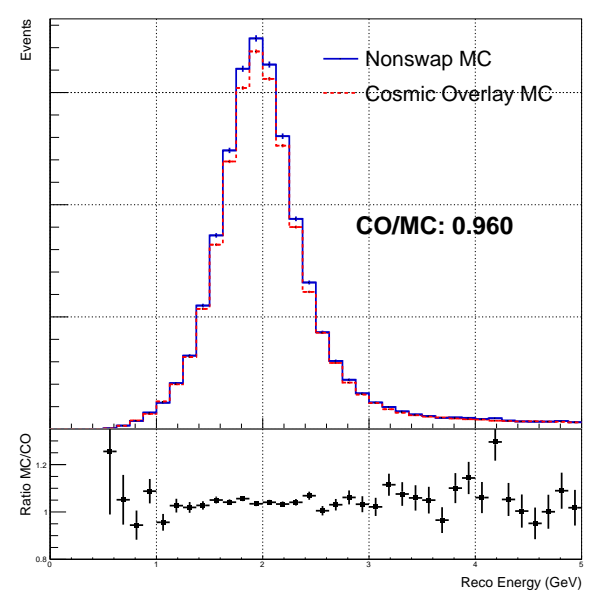

(a) $\nu_{\mu}$ energy distributions using 2017 analysis containment

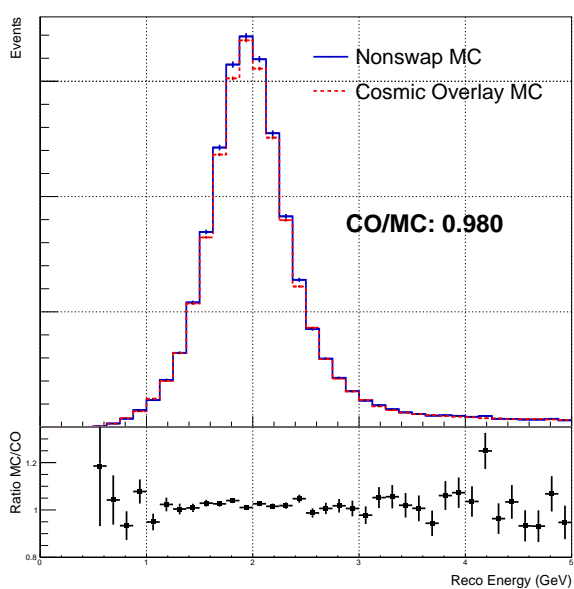

(b) $\nu_{\mu}$ energy distributions using 2018 analysis containment

Figure A.22: $\nu_{\mu}$ energy distributions in Period5. The blue line shows the distribution when run over the nonswap standard MC dataset. The red dashed line shows the distribution when run over the cosmic overlay dataset. The discrepancy in each case is given below the legend as the integral ratio of the cosmic overlay distribution with respect to the standard MC distribution. Common cuts employed in these plots are kNumuQuality and kKirkTATuneFD2017 (using 2017 BDT). 


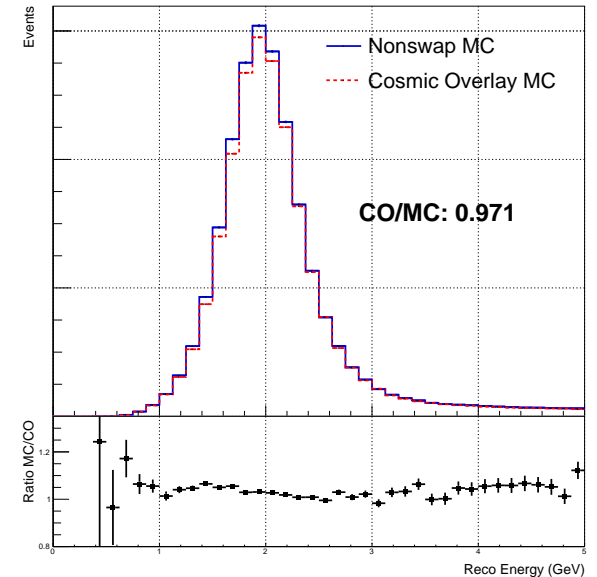

(a) $\nu_{\mu}$ energy distributions using 2017 analysis containment

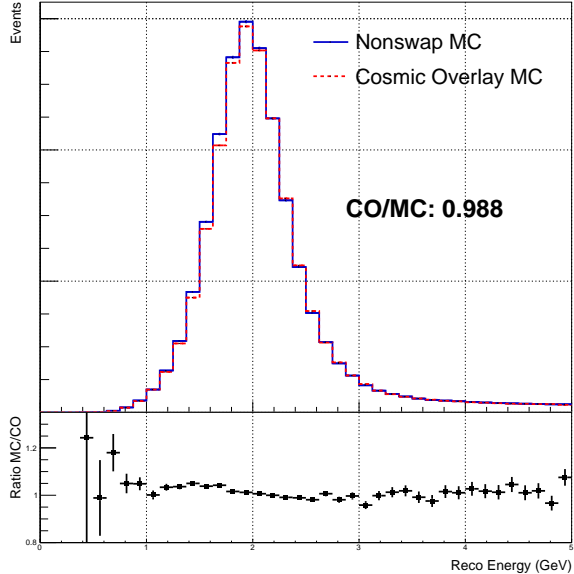

(b) $\nu_{\mu}$ energy distributions using 2018 analysis containment

Figure A.23: $\nu_{\mu}$ energy distributions across all periods. The blue line shows the distribution when run over the nonswap standard MC dataset. The red dashed line shows the distribution when run over the cosmic overlay dataset. The discrepancy in each case is given below the legend as the integral ratio of the cosmic overlay distribution with respect to the standard MC distribution. Common cuts employed in these plots are kNumuQuality, kNumuNCRej and kNumuCosmicRej.

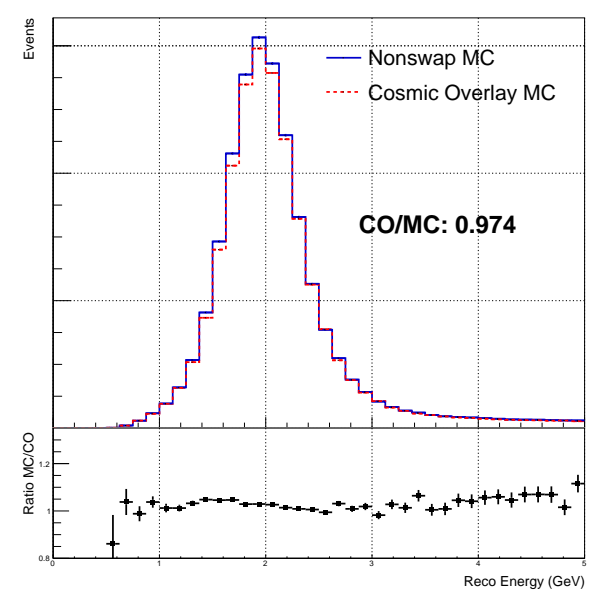

(a) $\nu_{\mu}$ energy distributions using 2017 analysis containment

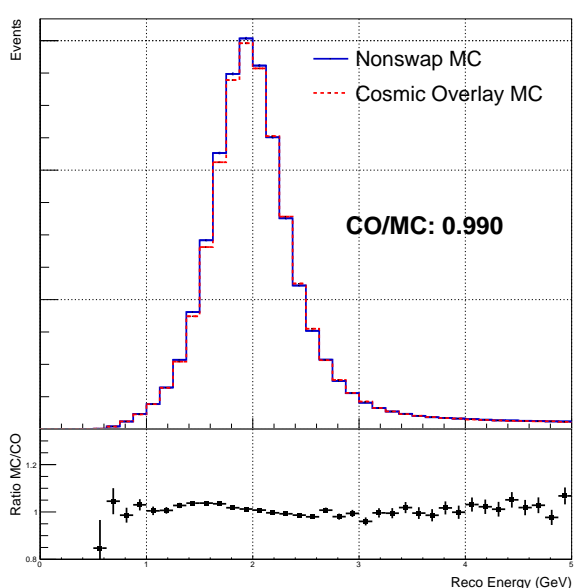

(b) $\nu_{\mu}$ energy distributions using 2018 analysis containment

Figure A.24: $\nu_{\mu}$ energy distributions across all periods. The blue line shows the distribution when run over the nonswap standard MC dataset. The red dashed line shows the distribution when run over the cosmic overlay dataset. The discrepancy in each case is given below the legend as the integral ratio of the cosmic overlay distribution with respect to the standard MC distribution. Common cuts employed in these plots are kNumuQuality and kKirkTATuneFD (using 2016 BDT). 


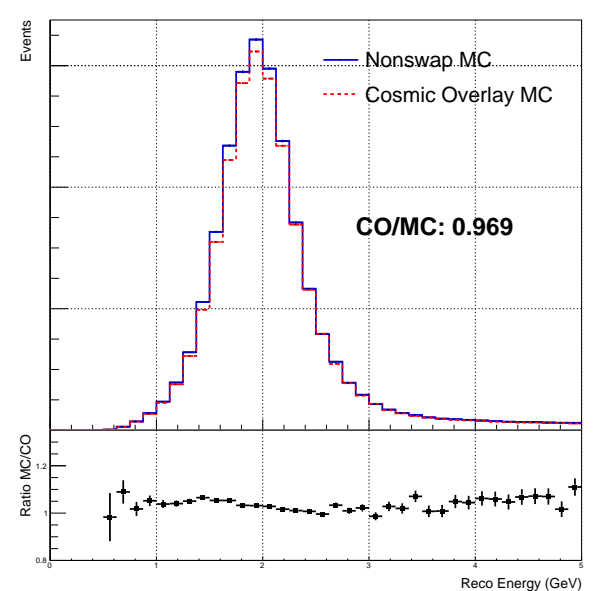

(a) $\nu_{\mu}$ energy distributions using 2017 analysis containment

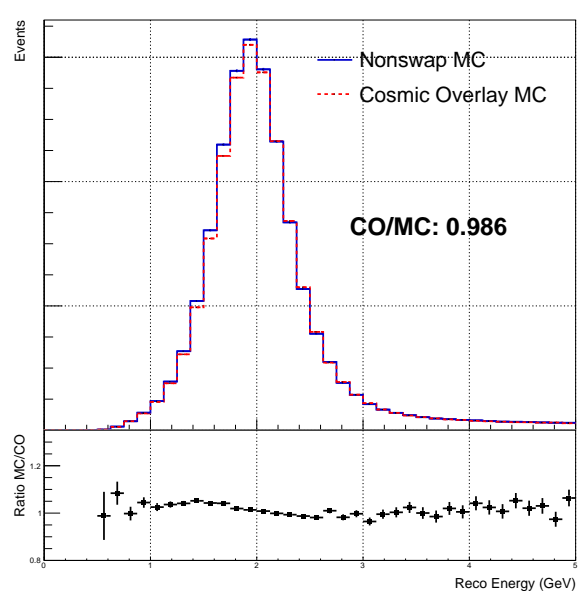

(b) $\nu_{\mu}$ energy distributions using 2018 analysis containment

Figure A.25: $\nu_{\mu}$ energy distributions across all periods. The blue line shows the distribution when run over the nonswap standard MC dataset. The red dashed line shows the distribution when run over the cosmic overlay dataset. The discrepancy in each case is given below the legend as the integral ratio of the cosmic overlay distribution with respect to the standard MC distribution. Common cuts employed in these plots are kNumuQuality and kKirkTATuneFD2017 (using 2017 BDT). 


\section{Appendix B}

\section{List of Software Used for the PID Cut Optimization}

This appendix describes the full software environment used for the PID analysis described in Chapter 6.

NOvASoft version details:

- $\mathrm{S} 18-02-09$

MC datasets(Where 'Tag' refers to the RHC (e) or FHC (d) definition label tag and 'Horn' is 'rhc' or 'fhc'):

- 'prod_caf_R17-11-14-prod4reco.'+Tag+'_fd_genie_nonswap_'+Horn+'_nova_v08_full_v1'

- 'prod_caf_R17-11-14-prod4reco.'+Tag+'_fd_genie_fluxswap_'+Horn+'_nova_v08_full_v1'

Cosmic Datasets:

- RHC - 'prod_caf_R17-11-14-prod4reco.a_fd_cosmic_rhcTune_HighGain_v1_goodruns_snapshot_170116'

- FHC - 'prod_caf_R17-11-14-prod4reco.b_fd_cosmic_fhcTune_full_v1_goodruns'

Full set of cuts employed:

- slc.nhit $>20$

- trk.ncosmic $>0$

- sel.remid.pid >0

- slc.ncontplanes $>4$

- sel.contain.kalfwdcell $>6$

- sel.contain.kalbakcell $>6$ 
- sel.contain.cosfwdcell $>0$

- sel.contain.cosbakcell $>7$

- planestofront $>1$

- planestoback $>1$

- sel.nuecosrej.distallpngTop $>60(\mathrm{~cm})$

- sel.nuecosrej.distallpngBottom > 12(cm)

- sel.nuecosrej.distallpngEast $>16$

- sel.nuecosrej.distallpngWest $>12(\mathrm{~cm})$

- sel.nuecosrej.distallpngFront $>18(\mathrm{~cm})$

- sel.nuecosrej.distallpngBack $>18(\mathrm{~cm})$

- sel.cosrej.anglekal > 0.5

- slc.nhit $<400$

- sel.nuecosrej.pngptp $<0.9$

- sel.cosrej.numucontpid $>0.10$

- sel.remid.pid $>0.10$

- sel.cvnProd3Train.numuid $>0.10$

Additional PID cuts optimized over:

- ReMId - 0.10 to 0.90 in 0.05 steps (17 possible values)

- CVN - 0.10 to 0.90 in 0.05 steps (17 possible values)

- BDT - 0.40 to 0.60 in 0.01 steps (21 possible values)

Hadronic Energy Bin Quantiles and Weights:

Quantile bins were generated using fixed acceptances of ReMId, BDT and CVN scores at $>0.5$. For validation, hadronic energy quantile cuts were generated for each set of PID cuts employed. ' $k X S e c C V W g t 2018 * k P P F X F l u x C V W g t^{\prime}$ weights are used throughout. 


\section{Bibliography}

[1] M.C. Gonzalez-Garcia, Michele Maltoni, and Thomas Schwetz. Global analyses of neutrino oscillation experiments. Nuclear Physics B, 908:199 - 217, 2016. Neutrino Oscillations: Celebrating the Nobel Prize in Physics 2015. x, 31

[2] Stuart L. Mufson. Scintillator update. Internal NOvA document, DocDB-8541, 2013. $\mathrm{x}, 41$

[3] M. A. Acero et al. New constraints on oscillation parameters from $\nu_{e}$ appearance and $\nu_{\mu}$ disappearance in the NOvA experiment. Phys. Rev., D98:032012, 2018. xi, xii, xxiv, 25, 26, 93, 107, 124, 132, 133, 134, 136, 137, 163, 164, 165, 166, 167, 169, 207

[4] P. Adamson et al. The NuMI Neutrino Beam. Nucl. Instrum. Meth., A806:279-306, 2016. xiv, $5,12,33,34,45$

[5] D. S. Ayres et al. The NOvA Technical Design Report. Fermilab Publication, 2007. xiv, $32,35,37,39,40,220$

[6] J Chadwick. Distribution in intensity in the magnetic spectrum of the $\beta$-rays of radium. Ver. Dtsch. Physik. Ges, 16:383-391, 1914. 3

[7] Enrico Fermi. Tentativo di una teoria dei raggi $\beta$. Il Nuovo Cimento (1924-1942), 11(1):1-19, 1934. 3

[8] Hans Bethe and Rudolph Peierls. Theneutrino. Nature, 133(3362):532, 1934. 3

[9] Frederick Reines and Clyde L. Cowan. The neutrino. Nature, 178:446-449, 1956. 3

[10] G. Danby et al. Observation of high-energy neutrino reactions and the existence of two kinds of neutrinos. Phys. Rev. Lett., 9:36-44, Jul 1962. 4

[11] K. Kodama et al. Observation of tau neutrino interactions. Phys. Lett., B504:218-224, 2001. 4 
[12] D. Decamp et al. Determination of the Number of Light Neutrino Species. Phys. Lett., B231:519-529, 1989. 4

[13] S. Schael et al. Precision electroweak measurements on the $Z$ resonance. Phys. Rept., 427:257-454, 2006. 4

[14] M. Goldhaber, L. Grodzins, and A. W. Sunyar. Helicity of neutrinos. Phys. Rev., 109:1015-1017, Feb 1958. 5

[15] P. Anselmann et al. Solar neutrinos observed by GALLEX at Gran Sasso. Phys. Lett., B285:376-389, 1992. 6, 7

[16] Raymond Davis, Don S. Harmer, and Kenneth C. Hoffman. Search for neutrinos from the sun. Phys. Rev. Lett., 20:1205-1209, May 1968. 6

[17] J.N. Bahcall, N.A. Bahcall, W.A. Fowler, and G. Shaviv. Solar neutrinos and lowenergy nuclear cross sections. Physics Letters B, 26(6):359-361, 1968. 6

[18] A. I. Abazov et al. Search for neutrinos from the sun using the reaction ${ }^{71} \mathrm{Ga}\left(\nu_{e}, e^{-}\right)^{71}$ ge. Phys. Rev. Lett., 67:3332-3335, Dec 1991. 7

[19] B. Pontecorvo. Neutrino Experiments and the Problem of Conservation of Leptonic Charge. Sov. Phys. JETP, 26:984-988, 1968. [Zh. Eksp. Teor. Fiz.53,1717(1967)]. 7

[20] V. N. Gribov and B. Pontecorvo. Neutrino astronomy and lepton charge. Phys. Lett., B28:493, 1969. 7

[21] Ziro Maki, Masami Nakagawa, and Shoichi Sakata. Remarks on the unified model of elementary particles. Prog. Theor. Phys., 28:870-880, 1962. 7

[22] Y. Fukuda et al. Evidence for oscillation of atmospheric neutrinos. Phys. Rev. Lett., 81:1562-1567, Aug 1998. 8

[23] QR Ahmad, RC Allen, TC Andersen, JD Anglin, JC Barton, EW Beier, M Bercovitch, J Bigu, SD Biller, RA Black, et al. Direct evidence for neutrino flavor transformation from neutral-current interactions in the sudbury neutrino observatory. Phys. Rev. Lett., 89(1):011301, 2002. 8

[24] C. Patrignani et al. Chin. phys. c, 40, 100001. Particle Data Group, 2016. 8, 10, 11, 38

[25] R. N. Mohapatra et al. Theory of neutrinos: A White paper. Rept. Prog. Phys., 70:1757-1867, 2007. 8 
[26] Mark Thomson. Modern particle physics. Cambridge University Press, 2013. 9

[27] Hiroshi Nunokawa, Stephen Parke, and Jose W. F. Valle. Cp violation and neutrino oscillations. Prog. Part. Nucl. Phys, 2007. 10

[28] Boris Kayser. On the quantum mechanics of neutrino oscillation. Physical Review D, 24(1):110, 1981. 11

[29] A. D. Sakharov. Violation of cp in variance, c asymmetry, and baryon asymmetry of the universe. Phys. Usp., 34(5):392-393, 1991. 12

[30] S. P. Mikheyev and A. Yu. Smirnov. Resonant amplification of $\nu$ oscillations in matter and solar-neutrino spectroscopy. Il Nuovo Cimento C, 9(1):17-26, 1986. 14

[31] L. Wolfenstein. Neutrino oscillations in matter. Phys. Rev. D, 17:2369-2374, May 1978. 14

[32] M. V. Diwan, V. Galymov, X. Qian, and A. Rubbia. Long-Baseline Neutrino Experiments. Ann. Rev. Nucl. Part. Sci., 66:47-71, 2016. 15

[33] T. Araki et al. Measurement of neutrino oscillation with kamland: Evidence of spectral distortion. Phys. Rev. Lett., 94:081801, Mar 2005. 15

[34] A. Gando et al. Reactor On-Off Antineutrino Measurement with KamLAND. Phys. Rev., D88(3):033001, 2013. 16, 17

[35] F. P. An et al. Observation of electron-antineutrino disappearance at Daya Bay. Phys. Rev. Lett., 108:171803, 2012. 16

[36] F. P. An et al. New measurement of antineutrino oscillation with the full detector configuration at daya bay. Phys. Rev. Lett., 115:111802, Sep 2015. 18, 19

[37] P Adamson, I Anghel, A Aurisano, G Barr, M Bishai, A Blake, GJ Bock, D Bogert, SV Cao, CM Castromonte, et al. Combined analysis of $\nu_{\mu}$ disappearance and $\nu_{\mu} \rightarrow \nu_{e}$ appearance in minos using accelerator and atmospheric neutrinos. Phys. Rev. Lett., 112(19):191801, 2014. 20, 21

[38] Kou Abe, J Adam, H Aihara, T Akiri, C Andreopoulos, S Aoki, A Ariga, S Assylbekov, D Autiero, M Barbi, et al. Measurements of neutrino oscillation in appearance and disappearance channels by the t2k experiment with $6.6 \times 1020$ protons on target. Physical Review D, 91(7):072010, 2015. 21, 22 
[39] P. Adamson et al. Combined analysis of $\nu_{\mu}$ disappearance and $\nu_{\mu} \rightarrow \nu_{e}$ appearance in minos using accelerator and atmospheric neutrinos. Phys. Rev. Lett., 112:191801, May 2014. 43, 205

[40] Andrew Norman. Nova timing verification records. NOvA Internal Document, DocDB-15216, 2014. 43

[41] Evan Niner. Timing calibration technical note. NOvA Internal Document, DocDB12570, 2015. 43

[42] L. Aliaga et al. Neutrino Flux Predictions for the NuMI Beam. Phys. Rev., D94(9):092005, 2016. [Addendum: Phys. Rev.D95,no.3,039903(2017)]. 48, 50, 52, 211

[43] Costas Andreopoulos, A Bell, D Bhattacharya, F Cavanna, J Dobson, S Dytman, H Gallagher, P Guzowski, R Hatcher, P Kehayias, et al. The genie neutrino monte carlo generator. Nuclear Instruments and Methods in Physics Research Section A: Accelerators, Spectrometers, Detectors and Associated Equipment, 614(1):87-104, 2010. 48,51

[44] Adam Aurisano. The NOvA Detector Simulation. NOvA Internal Document, DocDB13577, June 2015. 48

[45] A Aurisano et al. CHEP2015 Proceeding: The NOvA Simulation Chain. NOvA internal document, DocDB 13370, May 2015. 48

[46] Chris Hagmann, David Lange, and Douglas Wright. Cosmic-ray shower generator (cry) for monte carlo transport codes. In 2007 IEEE Nuclear Science Symposium Conference Record, volume 2, pages 1143-1146. IEEE, 2007. 49

[47] PA Rodrigues, J Demgen, E Miltenberger, L Aliaga, O Altinok, L Bellantoni, A Bercellie, M Betancourt, A Bodek, A Bravar, et al. Identification of nuclear effects in neutrino-carbon interactions at low three-momentum transfer. Phys. Rev. Lett., 116(7):071802, 2016. 52

[48] Jeremy Wolcott. Genie tune and uncertainty band for second analysis. NOvA Internal Document, DocDB-15214, 2016. 52

[49] P. Adamson et al. Measurement of the neutrino mixing angle $\theta_{23}$ in nova. Phys. Rev. Lett., 118:151802, Apr 2017. 52, 64, 73, 74, 94, 132, 137 
[50] Omar Benhar, Donal Day, and Ingo Sick. Inclusive quasielastic electron-nucleus scattering. Reviews of Modern Physics, 80(1):189, 2008. 52

[51] Jackie Schwehr, Dan Cherdack, and Rik Gran. GENIE implementation of IFIC Valencia model for QE-like 2p2h neutrino-nucleus cross section. 2016. 52, 70

[52] Kirk Bays. 2018 Cross-Section Tuning Tech Note. NOvA Internal Document, DocDB27755, 2018. 53, 54

[53] Michael David Baird. An Analysis of Muon Neutrino Disappearance from the NuMI Beam Using an Optimal Track Fitter. PhD thesis, Indiana U., 2015. 53, 54

[54] Martin Ester, Hans-Peter Kriegel, Jörg Sander, Xiaowei Xu, et al. A density-based algorithm for discovering clusters in large spatial databases with noise. In $K d d$, volume 96, pages 226-231, 1996. 53

[55] Rudolph Emil Kalman et al. A new approach to linear filtering and prediction problems. Journal of basic Engineering, 82(1):35-45, 1960. 55

[56] Nicholas Raddatz. Kalmantrack technical note. NOvA Internal Document, DocDB13545. 55

[57] Louise Suter. Second analysis data quality summary tech note. NOvA Internal Document, DocDB-15307, 2016. 56, 95

[58] Jeff Hartnell and Greg Pawloski. Summary of the 2016 (second analysis) $\nu_{\mu}$ disappearance analysis. NOvA Internal Document, DocDB-15232, May 2016. 58

[59] Jeff Hartnell and Alexander Radovic. Numu analysis package summary for boxopening review october 2017. NOvA Internal Document, DocDB-22562, 2017. 58, 94

[60] Nicholas Raddatz. Remid technical note. NOvA Internal Document, DocDB-11206. $58,59,60$

[61] Adam Aurisano. Deep learning techniques. NOvA Internal Document, DocDB-16303, 2016. 58

[62] Naomi S Altman. An introduction to kernel and nearest-neighbor nonparametric regression. The American Statistician, 46(3):175-185, 1992. 58 
[63] Nick Raddatz. Measurement of Muon Neutrino Disappearance with Non-Fiducial Interactions in the NOvA Experiment. PhD thesis, University of Minnesota, 2016. 58,59

[64] A Aurisano, A Radovic, D Rocco, A Himmel, MD Messier, E Niner, G Pawloski, F Psihas, A Sousa, and P Vahle. A convolutional neural network neutrino event classifier. Journal of Instrumentation, 11(09):P09001, 2016. 59, 60, 61

[65] Kirk Bays. Nova cosmic rejection package and algorithms technical note. NOvA Internal Document, DocDB-11205. 60

[66] Louise Suter and James Musser. SA Data Quality updates. NOvA Internal Document, DocDB-15307, April 2016. 62

[67] Sarah R Phan-Budd and Lisa Goodenough. Technical note on the nova beam monitoring for 2015 summer analysis. NOvA Internal Document, DocDB-13572, July 2015. 62

[68] Kanika Sachdev and Bu Xuebing. Spill level data quality. NOvA Internal Document, DocDB-12437, July 2015. 62

[69] Susan Lein. DCM Edge Metric. NOvA Internal Document, DocDB-13527, June 2015. 62

[70] NOvA authors. Nova numu blessed plots. http://nusoft.fnal.gov/nova/ blessedplots/Numu_SA.html, February 2017. 63

[71] Christopher Backhouse. The CAFAna Framework - Technote. NOvA Internal Document, DocDB-9222, 2014. 63

[72] Fred James and MINUIT Roos. Minuit-a system for function minimization and analysis of the parameter errors and correlations. Computer Physics Communications, 10(6):343-367, 1975. 64

[73] Glen Cowan, Kyle Cranmer, Eilam Gross, and Ofer Vitells. Asymptotic formulae for likelihood-based tests of new physics. Eur. Phys. J., C71:1554, 2011. [Erratum: Eur. Phys. J.C73,2501(2013)]. 65

[74] Luke Vinton. Sensitivity studies. hybrid selection and number of neutrino energy bins. NOvA Internal Document, DocDB-16292, 2016. 67 
[75] Strait, Matthew. Muon Energy Scale Systematic. NOvA Internal Document, DocDB20816, Jan 2019. 69, 133

[76] Saqib Shadman Bashar. Available Hadronic Energy Estimator for AntiNumu-CH reactions in NOvA. NOvA Internal Document, DocDB-34309, Nov 2018. 70

[77] Susan Lein. Summary of de/dx plots for muons/protons. NOvA Internal Document, DocDB-15028, 2016. 70

[78] Vladimir Bychkov. Technote for genie systematics. NOvA Internal Document, DocDB-15234, 2016. 70

[79] Richard Gran. Model Uncertainties for Valencia RPA Effect for MINERvA. 2017. 70

[80] R. Gran, J. Nieves, F. Sanchez, and M. J. Vicente Vacas. Neutrino-nucleus quasielastic and 2p2h interactions up to $10 \mathrm{GeV}$. Phys. Rev., D88(11):113007, 2013. 70

[81] Backhouse, C and Radovic, A and Singh, P and Campbell, M. TA Attenuation and Threshold. NOvA Internal Document, DocDB-13579, Sep 2017. 71

[82] Kirk Bays. Cosmic background numu 2018. NOvA Internal Document, DocDB-27878, 2018. 75,106

[83] Jeff Hartnell and Alexander Radovic. Numu analysis package summary for boxopening review spring 2018. NOvA Internal Document, DocDB-26702, 2018. 94, 106

[84] Kirk Bays. Executive summary for the first numu cc analysis. NOvA Internal Document, DocDB-13641, Jul 2015. 94

[85] Jeff Hartnell and Alexander Radovic. Numu analysis package summary for boxopening review may 2016. NOvA Internal Document, DocDB-15232, 2016. 94

[86] Mayly Sanchez. NOvA Results and Prospects. Jun 2018. Talk at XXVIII International Conference on Neutrino Physics and Astrophysics, 4-9 June 2018, Heidelberg, Germany, DOI: 10.5281/zenodo.1286758, URL: https://doi.org/10.5281/zenodo.1286758. 124

[87] Luke Vinton. Measurement of Muon Neutrino Disappearance with the NOvA Experiment. PhD thesis, Sussex U., 2018. 135, 136

[88] P. Adamson et al. First measurement of muon-neutrino disappearance in nova. Phys. Rev. D, 93:051104, Mar 2016. 137 
[89] P. Adamson et al. First measurement of electron neutrino appearance in nova. Phys. Rev. Lett., 116:151806, Apr 2016. 137

[90] Gary J Feldman and Robert D Cousins. Unified approach to the classical statistical analysis of small signals. Physical Review D, 57(7):3873, 1998. 201

[91] Hartnell, Jeff and Mendez, Diana and Radovic, Alexander. NuMu Results and Plans 1st plenary May 2018. NOvA Internal Document, DocDB-29135, Jun 2018. 201

[92] K. Abe et al. Measurement of neutrino and antineutrino oscillations by the T2K experiment including a new additional sample of $\nu_{e}$ interactions at the far detector. Phys. Rev., D96(9):092006, 2017. [Erratum: Phys. Rev.D98,no.1,019902(2018)]. 205

[93] K. Abe et al. Atmospheric neutrino oscillation analysis with external constraints in Super-Kamiokande I-IV. Phys. Rev., D97(7):072001, 2018. 205

[94] M. G. Aartsen et al. Measurement of Atmospheric Neutrino Oscillations at $656 \mathrm{GeV}$ with IceCube DeepCore. Phys. Rev. Lett., 120(7):071801, 2018. 205

[95] Mendez, Diana Patricia. First $\nu_{\mu}+\bar{\nu}_{\mu}$ Disappearance Results from the NOvA experiment, Jun 2018. Poster at XXVIII International Conference on Neutrino Physics and Astrophysics, 4-9 June 2018, Heidelberg, Germany, DOI: 10.5281/zenodo.1300655, URL: https://doi.org/10.5281/zenodo.1300655. 205

[96] Karol Lang. The NOvA Test Beam Program, Jun 2018. DOI: 10.5281/zenodo.1300576, URL: https://doi.org/10.5281/zenodo.1300576. 220

[97] Vladimir Shiltsev. Fermilab Proton Accelerator Complex Status and Improvement Plans. Mod. Phys. Lett., A32(16):1730012, 2017. 220

[98] Erika Catano-Mur. Projected significances "reach" - joint nue + numu - NEUTRINO 2018. NOvA internal document, DocDB-30027, Jun 2018. 221 